
THE ASCENT OF PREHISTORIC MAN

MAN'S CULTURAL STAGES AND PHYSICAL DEVELOPMENT TO THE END OF THE OLD STONE AGE

[DIAGONAL LINES INDICATE RACIAL PERTODS,
DOT SHOWS CONUECTURED DATE OF EXTINCTION]

ICE AGES

${ }_{0.600}^{\text {POST-CLACIAL }}$

ATS GLACIAL

ose

3ra INTERCLACIAL

PILTDOWNMAN

$\log _{0}$

3 GLACIAL

200.000

2NA INTERGLACIAL

His:200

\section{and INTERGLicial}

203000

and CLACIAL
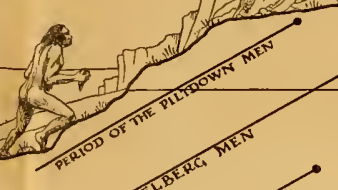

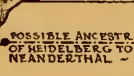
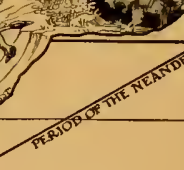

as

(a)

PALEO

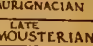

ARLY

HEULEAN

$$
\frac{5230}{200}
$$

PRE-PALFOLITHIC

EOLITHIC ?
PLEISTOCENE $f$

PLIOCENE ᄀ 





\section{THE BRAIN FROM APE TO MAN}

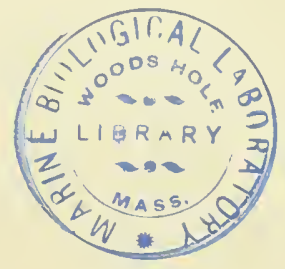





\section{THE BRAIN}

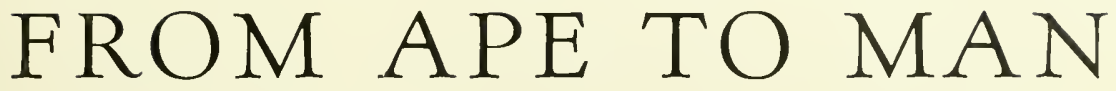

A CONTRIBUTION TO THE STUDY OF THE EVOLUTION AND DEVELOPMENT OF THE HUMAN BRAIN

BY FREDERICK TILNEY, PH.D.,M.D.

Professor of Neurology. Columbia University

With Chapters on the Reconstruction of the Gray

Matter in the Primate Brain Stem by

HENRY ALSOP RILEY, A.M.,M.D.

Associate Professor of Neurology, Columbia University

FOREWORD BY

HENRY FAIRFIELD OSBORN, SC.D.,LL.D.

Research Professor of Zoology, Columbia Unversity

557 ILlUSTRATIONS, MANY IN COLOR

VOLUME ONE

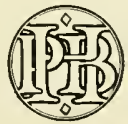

PAUL B $\cdot$ HOEBER $\cdot$ INC

NEW YORK, MCMXXVIII 
COPYRIGHT 1928 BY PAUL B. HOEBER, INC. ALL RIGHTS RESERVED - PUBLISHED MAY I928 PRINTED IN THE UNITED STATES OF AMERICA 
IN APPRECIATIVE ADMIRATION THIS BOOK

IS DEDICATED TO

\section{PROFESSOR HENRY FAIRFIELD OSBORN}

\section{UNDER WHOSE INSPIRED LEADERSHIP THE}

DIM PATHWAYS OF EVOLUTION AND

THE OBSCURE APPROACHES TO

THE FUTURE HAVE BECOME

MORE ACCESSIBLE TO

THOSE SEEKING

THE TRUTH

*ำ

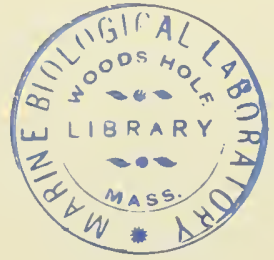





\section{PREFACE}

7 HE study of the brain in all of its evolutionary aspects is a task requiring the labors of many investigators. Such studies are urgently

I needed because the questions surrounding this subject are assuming increasing prominence in modern thought. No single work may even aspire to a satisfactory completeness in dealing with the entire problem; therefore only certain aspects of it are approached in these volumes.

Since brain power admittedly is the secret of human success, the brain itself must be the pioneer hewing the path of man's progress; hence the genesis of this dominant organ is a vital issue which calls for most intensive research. The answers to the questions "whence came the human brain, and how" have become indispensable to modern understanding. Their value lies in the light thrown by them upon the course of man's long ascent and in their searching penetration through the dense veil which hides his future.

Upon directing our attention toward this future, there are many reasons for asking whither we are trencling as a race. Does the path lead forward through repetitious ancient cycles of disaster, toward wars and revolutions, toward civic frustrations amid the preparations for future war in times of peace, toward further stupidities in the management of social organizations, toward grosser misconceptions of man and humanity, toward greater depths of superstitious delusion and human unworthiness with their full and final penalty of ultimate extinction? Catastrophe such as this has not infrequently overtaken many races of men long since extinct. It is possible, however, that the supreme organ which created what there is of human succeding has still further benefits to confer.

And yet no satisfactory appraisal of these possibilitics could be attempted without a thorough study of the processes by which the brain came into existence. To this study must be added some comprehension of the vii 
manner in which this master organ has progressively adapted itself for the effective distribution of its predominant powers.

These volumes dealing with the evolution of the brain of primates represent approximately fifteen years of preparation, collection and study. They cmbody the neurological evidence based upon structural and behavioral studies of Lemur, Tarsius, Marmoset, Howling Monkey, Baboon, Macacus, Gibbon, Orang, Chimpanzee, Gorilla and Man. In this investigation, it has been my good fortune to have access to the unusually complete collection of vertebrate brains gathered under the direction of Prof. George S. Huntington in Columbia University. Still more fortunate was my privilege of studying for many years under his personal supervision. Indeed, most of this work is the direct result of his inspiration, instruction and guidance. Each page bears some affectionate momory of his influence, and the entire effort could have no finer reward than to be, if only in some small way, a pupil's tribute to his great master.

To Prof. Henry Fairfield Osborn I am also decply indebted for his gracious counsel and his generosity in putting at my disposal many of the valuable collections of the American Museum of Natural History. The New York Zoological Garden and the New Iork Aquarium have both been constant and liberal contributors of valuable material. Many fine brain specimens, particularly of the great anthropoid apes, were presented to me by the late Mr. Carl Akeley after his last African expedition. One of the most valuable specimens in the collection is the brain of the young gorilla, John Daniel I, an animal which lived for a relatively long time in captivity and offered an exceptional opportunity for the study of its behavioral reactions under the influences of domestic life.

It gives me especial pleasure to acknowledge my gratitude to President Nicholas Murray Butler and to Dean William Darrach for their interest in this research and particularly for having made possible the photographic reproductions. 
The purpose of this work is to assemble and discuss the evidence of evolution contained in the brains of primates.

The present volumes are the first part of a cerebral survey subdivided as follows:

1. The Brain from Ape to Man.

II. Brain Evohution from Mammals to Man.

III. The Brain from Fish to Man.

The treatise setting forth this evidence of cerebral evolution is unavoidably lengthy. It necessarily contains a large amount of technical detail. The full presentation of the facts, however, has been deemed essential in order to substantiate the arguments and conclusions drawn from them. On the other hand, the convenience of those who would not be burdened by the tedium of detailed recitals has not been overlooked.

The text has been arranged in five parts. The first part recounts the observations made upon the Lower Primates; the second, upon the Intermediate Primates; the third, upon the three Great Anthropoids; the fourth, upon Man; and the fifth summarizes alt of the evidence adduced in the preceding parts.

At the end of the first, second and third parts are sectional summaries including interpretations and discussions of the facts as they bear upon the correlative evolution of structures in the brain stem and the behavioral development of the animals described.

In the fifth part a summary in conclusion discusses the evidence afforded by the entire group of primates. It gives the author's views concerning the significance of the evolutionary process which, it is believed, reveals itself clearly in this order of mammals.

In dealing with the technical problems involved in a discussion of the brain, it is recognized that many features might well have been treated more extensively. This recognition applies notably to the cerebral hemi- 
spheres, especially the cerebral cortex. It is doubtless unfortunate that such a condition exists, hut an adequate treatise on the neopallium alone would necessitate the addition of at least several volumes to the present work. The chicf emphasis in this description and analysis has been laid upon the brain stem, an element in the cerebrum which has hitherto been too little dwelt upon. In the brain stem are included the medulla oblongata, the cerebellum, the pons Varolii, the midbrain and the interbrain. Even of these parts the more intimate details concerning the structure and evolution of the cerebellum and of the interbrain have not been discussed in extenso. These regions of the primate brain are at present being studied by my colleague, Prof. Henry Alsop Riley, and will shortly appear as separate publications.

In submitting the brain stem to study, several methods of analysis have been employed. Not only have the gross appearances in this part of the neuraxis been analyzed, but careful microscopic studies of serial sections of the brain have been made at the following critical levels:

Level of the pyramidal decussation

Level of the caudal extremity of the dorsal sensory nuclei

Level of the caudal extremity of the inferior olivary body

Level through the middle of the inferior olivary body

Level of the vestibular nuclei

Level of the cerebellar nuclei

Level of the inferior portion of the pons Varolii and the emergence of the sixth cranial nerve

Level through the middle of the pons Varolii

Level of the emergence of the fourth cranial nerve (nervus trochlearis)

Level of the inferior colliculus

Level of the superior colliculus

Level of the optic chiasm

Level of the anterior commissure. 
In addition to these microscopic studies, reconstructions according to the Bourne method have been employed. Such reconstructions, made at a magnification of 10 or 12 diameters, reveal the intimate characters and dimensional relations of the gray matter. By no other means is it possible to acquire so comprehensive a conception of the complex nature of the internal structure in the brain stem. Dr. Riley has devoted himself to this work for a number of years and as a result has produced a series of most illuminating reconstruction models which present this aspect of the subject so as to disclose beyond all question the major nuclear movements in the evolutionary process of the primate brain. I am indebted to Dr. Riley not merely for the production of this collection of unsurpassed models, but equally for the careful descriptions which he has given of them in the cleven chapters dealing with this phase of the subject.

For the purpose of further checking the proportional relations of the various structures in the brain stem, several methods of mensuration have been employed. On the basis of these measurements, two series of coeflicients were established: first, planimetric coefficients in which the transverse proportions of a given structure were estimated in relation to the entire cross section by means of a planimeter. This method produced a series of figures which afford a basis of metric comparison of homologous structures. For the painstaking work in these protracted planimetric calculations I am indebted to Mrs. Seymour Basch, whose repeated measurements and remeasurements of each structure required the most exacting application and produced results which are probably as close an approximation to actual facts as may be obtained. Second, estimates for Iongitudinal coefficients were made with the purpose of determining the relative length of each structure to the entire length of the brain stem.

While it is probably true that no method of mensuration may express adequately the relative proportions of the several structures considered 
xii

in the brain stem, it seems fair to presume that by means of these two sets of calculations a fair degree of accuracy has been attained.

Even though every method which reasonably could be applied to the material has been used in controlling these observations, the fact still remains that this study lacks detailed investigation of the cellular elements of the various muclear components. The addition of this detail must be left for later consideration, as the inclusion of such studies would of necessity too greatly expand the size of the present work.

In dealing with the brain stem the treatment has not been that of an atlas, but rather that of a critical review with especial emphasis upon the structures having the greatest evolutional significance. In the selection of such structures two groups were recognized:

First, those more plastic elements of recent acquisition which have been especially susceptible to adaptive influences.

Second, the more inflexible, archaic components of the brain stem, which because of their great antiquity have acquired fundamental stability and respond but little to the influences of adaptation. Chicf attention is directed to the first group from which a surprisingly rich harvest of evidence has been gathered.

The addition of the chapters dealing with the cultural phases of human development and the brain of prehistoric man was made upon the suggestion and with the assistance of Professor Osborn.

Prof. William K. Gregory of Columbia University has given me most generous assistance and I am especially grateful to him for his careful reading and criticism of the concluding chapters.

I also wish to express my appreciation to Miss Christine D. Matthews of the American Museum of Natural History for her revisions in the tabulation showing the fossil remains of prehistoric man.

Throughout the entire preparation of the manuscript I have had the 
able and sympathetic assistance of Mrs. Alice G. Margulies, whose efforts in assembling the bibliography have provided valuable addenda for those desiring further references to the literature.

To make due acknowledgment for all of the assistance I have received would necessitate setting forth a long list of my friends and associates. Among these, however, I cannot fail to mention Mr. Frank N. Doubleday, whose invaluable advice has led to many important revisions in and additions to the text.

It also gives me great pleasure to mention the indispensable services of Miss Regina Unger in preparing the serial sections of the primate brains upon which this study is based. Miss Unger has for a number of years been engaged in the technical production of brain series, particularly Pal-Ileigert preparations, which constitute one of the most highly valued accessions of the Neurological Department in Columbia University. Without these specimens, neither the microscopic nor reconstruction studies would have been possible.

In the preparation of the illustrations and text for publication, Miss Florence Fuller of the publishers' staff has given most enthusiastic and helpful attention.

And, finally, to my publisher, Mr. Paul B. Hoeber, I wish to acknowledge my deep indebtedness for his generosity and for the encouragement and unfailing inspiration which he has given me at all times.

New YORK, N. Y.

F. T.

March, I 928. 



\section{FOREWORD}

7 HE discoveries in astronomy during the last decade have not only widened the boundaries of our own universe but have revealed the

L cxistence of universes far beyond our own. These discoveries prove that in our own and the outer universes cxactly the same physical and chemical principles prevail, namely, gravitation, heat, light, the genesis of new compounds, development from nebulous and active phases into dark and inert phases of death.

Marvelous as are these recent discoveries in astronomy, they are becoming comprchensible because of the uniformity of the laws and principles revealed to man through centuries of research. In brief, physics, astronomy and chemistry are alike coming within the field of exact science capable of measurement, calculation, prediction and prophecy.

What a contrast is presented in the biological sciences, ancient and modern! With a wide circle of astronomic friends and with the most intense admiration for the achievements of astronomy and pure mathematics, I yet believe that their problems are not nearly so difficult or so baffling as our problem. In anatomy, in physiology, in pathology, in heredity we have not yet reached even the threshold of exactitude. I ith increasing energy, refinement and ingenuity, we know all the organs revealed in comparative and human anatomy, in both their grosser and their finer structure. We know also the history of the rise of many of these organs in the course of past time and what their functions and relations are, but there is always the Great Beyond of the unknown, and perhaps unknowable, which is summed up in the word life.

Of all incomprehensible things in the universe Man stands in the front rank, and of all incomprehensible things in Man the supreme difficulty centers in human intelligence, human memory, human aspirations, human powers of 
discovery, researeh, and conquest of obstacles. The approach to this unknown field of future human advanee - the seat of the human mind and the constitution of the human mind - is along the great paths of human and comparative anatomy and of human and comparative psychology.

This volume contains the basis of what to our knowledge is the first profound study of the genesis of the intimate or internal strueture of the human brain in comparison with the brains of animals more or less nearly related to man. It is a summary of Frederiek Tilney's lifework along largely new and original paths, pursued with the most unremitting intelligence and energy and yielding a result of exceptional breadth, precision and exactitude which affords a new and strong ground on which neurologists, psychologists, pathologists, and students of animal and human behavior may advance further into the unknown.

May we not add a further word of welcome to this splendid monograph at a time when we are mourning the loss of George Sumner Huntington, who laid the foundations of eomparative anatomy for the School of Anatomy of Columbia University and who unfortunately passed away without witnessing the consummation of the rescarch and endeavors of one of his most distinguished students, namely, the linking of Man in all his parts and functions with the long lines of his aneestry. 


\title{
CONTENTS
}

\author{
VOLUME I
}

Preface.

Page

vii

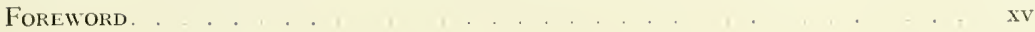

List of IllLUStrations. . . . . . . . . . . . . . . . . . . . . . . . . . . . . . Xix

Introduction. The Primates: Lemurs, Monkeys, Apes and Man, Their Place in

Nature . . . . . . . . . . . . . . . . I

Part I. The Lower Primates

INTRODUCTION . . . . . . . . . . . . . . . . . . . . . 2I

Chapter

I. Lemur Mongoz, Its Brain and Behavior. . . . . . . . . . 23

II. Reconstruction of the Gray Matter in the Brain Stem of Lemur Mongoz . $\quad 73$

III. Tarsius Spectrum, Its Brain and Behavior. . . . . . . . . . 85

IV. Reconstruction of the Gray Matter in the Brain Stem of Tarsius Speetrum 135

V. Callithrix Jacchus, the Marmoset, Its Brain and Behavior. . . . . . . 153

VI. Reconstruction of the Gray Matter in the Brain Stem of Callithrix Jacchus I83

VII. Mycetes Seniculus, Its Brain and Behavior . . . . . . . . . I9I

VIII. Reconstruction of the Gray Matter in the Brain Stem of Mycetes Seniculus 233

IX. Comparative Summary of Structures Having Erolutional Significance in the

Brain Stems of the Lower Primates. . . . . . . . . . . . 243

\section{Part II. The Internediate Prinates}

INTRODUCTION . . . . . . . . . . . . . . . . . . . . . . . . 287

Chapter
X. Papio Cynocephalus, the Common Dog-Headed Baboon, Its Brain and Behavior . . . . . . . . . . . . . . . . . . 289

XI. Reconstruction of the Gray Matter in the Brain Stem of Papio Cynocephalus 335

XII. Pithecus Rhesus, Macacus Rhesus, Its Brain and Behavior . . . . . . 349

XIII. Reconstruction of the Gray Matter in the Brain Stem of Pithecus Rhesus 39I

XIV. Hylobates Hoolock, the Gibbon, Its Brain and Behavior . . . . . . . 405

XV. Reconstruction of the Gray Matter in the Brain Stem of Hylobates Hoolock +47

XVI. Comparative Summary of Structures Having Erolutional Significance in the Brain Stems of the Intermediate Primates. . . . . . . 457

$$
\text { xvii }
$$




\section{VOLUME II \\ Part III. The Higher Anthropolds}

INTRODUCTION

PAGE

$4^{--}$

Chapter

XVII. Simia Satyrus, the Orang-Outang, Its Brain and Behavior

XVII. Reconstruction of the Gray Matter in the Brain Stem of Simia Satyrus.

49

533

XIX. Troglodytes Niger, the Chimpanzee, Its Brain and Behavior.

$5+5$

XX. Reconstruction of the Gray Matter in the Brain Stem of Troglodytes Niger

XXI. Troglodytes Gorilla, Its Brain and Behavior.

609

623

XXIl. Reconstruction of the Gray Matter in the Brain Stem of Troglodytes Gorill

$68 ;$

XXII. Comparative Summary of Structures Having Evolutional Significance in the Brain Stems of the Higher Anthropoids

699

$$
\text { PART IV. MAN }
$$

INTRODLCTION

Chapter

XXIV. From Primitive to Modern Man.

XXV. The Brain of Modern Man.

XXVI. Reconstruction of the Gray Matter in the Iluman Brain Stem

XIVII. The Brain of Prehistoric Man

XXVIII. Man-Past, Present and Future

925

Part V. Evolutional Modifications of the Primate Cerebrla Culinating iN THE HUMAN BraiN

INTRODECTION

Chapter

XXIX. The Significance of the Structural Homogeneity and Specific Modifications in the Primate Brain. Their Relations to the Progressive Adaption of Behavior . . . . . . . . . . . . . . . . . 941

XXX. The Internal Structure of the Brain Stem of the Primates. Its Evolutional Modification in Relation to the Development of Behavior. Essential Similarities in Internal Elements.

References for Further Reading.

INDEX TO VOLUMES I AND II . 


\section{LIST OF ILLUSTRATIONS}

Figuke

1. Brains of lower vertebrates compared with the human brain.

2. Brains of mammals compared with the human brain .

3. Brains of apes compared with the human brain .

4. Comparison of sheletal structures, from fish to man

5. Lemur group in Madagascan habitat.

PAGE

8,9

$6,-7$ Two views of Lemur mongoz .

25

27

30,31

8-11. Hand and foot of Lemur mongoz.

12-15. Hand and foot of Lemur potto

32,33

16. Dorsal surface of brain, Lemur mongoz

1-. Base of brain, Lemur mongoz

18. Left lateral surface of brain, Lemur mongoz .

19. Right lateral surface of brain, Lemur mongoz.

20. Ventral surface of brain stem, Lemur mongoz

21. Dorsal surface of brain stem, Lemur mongoz.

22. Lemur mongoz. Level of the piramidal decussation.

23. Lemur mongoz. Level of caudal extremity of inferior olive

24, 25. Lemur mongoz. Level through middle of inferior olive.

26. Lemur mongoz. Level of the restibular complex.

27. Lemur mongoz. Level of the cerebellar nuclei.

28. Lemur mongoz. Level of emergent fibers of sisth nerve .

29. Lemur mongoz. Level of caudal extremity of pons Varolii

30. Lemur mongoz. Level of the middle of the pons Varolii .

31. Lemur mongoz. Level of emergence of trochlear nerve.

32. Lemur mongoz. Level of the inferior colliculus

33. Lemur mongoz. Level of the superior colliculus.

34. Lemur mongoz. Level of the optic chiasm .

35. Lemur mongoz. Level of the anterior commissure.

36. Ventral surface of gray matter of brain stem, Lemur mongoz (color)

37. Dorsal surface of gray matter of brain stem, Lemur mongoz (color).

38. Lateral surface of gray matter of brain stem, Lemur mongoz (color).

39,40 . Two views of Tarsius spectrum.

$41-44$. Hand and foot of Tarsius spectrum

45. Dorsal surface of brain of Tarsius spectrum

46. Base of brain, Tarsius spectrum .

$4^{-}$. Right lateral surface of brain, Tarsius spectrum

48. Ventral surface of brain stem of Tarsius spectrum.

49. Dorsal surface of brain stem of Tarsius spectrum.

jo. Tarsius spectrum. Level of the first cervical segment

vix

\section{4}

35

36

$3-$

38

39

$+2$

46

48,49

30

52

55

37

60

$6 I$

62

66

(o)

$-0$

$-5$

$-7$

$-9$

86

88,89

95

96

98

100

I01

102 
51. Tarsius spectrum. Level of the pyramidal decussation.

52. Tarsius spectrum. Level of the dorsal sensory nuclei.

53. Tarsius spectrum. Level of caudal tip of inferior olive.

108

54. Tarsius spectrum. Level through middle of inferior olive.

55. Tarsius spectrum. Level of the restibular complex

56. Tarsius spectrum. Level of caudal extremity of trapezoid body

5-. Tarsius spectrum. Level of the cerebellar nuclei.

58. Tarsius spectrum. Level of the trochlear emergence.

50. Tarsius spectrum. Level of the inferior colliculus

6o. Tarsius spectrum. Level of the superior colliculus.

61. Tarsius spectrum. Level of the optic chiasm .

62. Tarsius spectrum. Level of the anterior commissure.

63. Ventral surface of the gray matter of the brain stem, tarsius spectrum (color)

64. Dorsal surface of the gray matter of the brain stem, tarsius spectrum (color).

65, 66. Lateral surface of the gray matter of the brain stem, tarsius spectrum (color) I4I, I 43

$6^{-}$, 68. Callithrix jacchus (marmoset).

$60-2$. Hand and foot of marmoset.

73. Dorsal surface of brain, Callithrix jacchus (marmoset).

74. Base of brain, Callithrix jacchus (marmoset).

7. Left lateral surface of brain, Callithrix jacchus (marmoset).

-6. Right lateral surface of brain, Callithrix jacchus (marmoset).

-7. Ventral surface of brain stem, Callithrix jacchus (marmoset)

-8. Dorsal surface of brain stem, Callithrix jacchus (marmoset)

70. Marmoset. Level of the prramidal decussation .

8o. Marmoset. Level of the dorsal sensory nuclei.

8ı. Narmoset. Level of the tip of the inferior olive.

83. Marmoset. Level of the vestibular nuclei and tuberculum acusticum. . . . . I

$8_{4}$. Narmoset. Level of the cerebellar nuclei.

$17 \mathrm{I}$

85. Marmoset. Level at the middle of the pons Varolii

86. Narmoset. Level of the inferior colliculus

87. Marmoset. Level of the superior cerebellar peduncular decussation

88. Narmoset. Level of the superior colliculus.

89. Narmoset. Level of the optic chiasm .

9o. Narmoset. Level of the anterior commissure.

I 8 I

91. Ventral surface of the gray matter of the brain stem, Callithrix jacchus (color). 
I09, Iro. Distal extremities of prehensile tails, Mycetes seniculus and Ateles ater. . . 203

II I. Dorsal surface of brain, Mycetes seniculus .

I 3. Left lateral surface of brain, Mycetes seniculus

115. Ventral surface of brain stem, Mycetes seniculus

1 6 . Dorsal surface of brain stem, Mycetes seniculus.

I 7 . Mrcetes seniculus. Level of the pyramidal decussation

I 8 . Mycetes seniculus. Level of the caudal extremity of the inferior olive.

119. Mycetes seniculus. Level through the middle of the inferior olive.

I2 1 . Mycetes seniculus. Level of the cercbellar nuclei.

122. Mycetes seniculus. Level of the vestibular nuclei.

123. Mycetes seniculus. Level of emergence of sixth nerve.

222

124. Mycetes seniculus. Level through middle of pons Varolii

224

125. Mycetes seniculus. Level of the inferior colliculus

226

126. Mycetes seniculus. Level of the superior colliculus

228

127. Mycetes seniculus. Level of the optic chiasm .

229

128. Mycetes seniculus. Level of the anterior commissure

230

129. Ventral surface of the gray matter of the brain stem, Mycetes seniculus (color)

235

130. Dorsal surface of the gray matter of the brain stem, Mycetes seniculus (color).

237

131. Lateral surface of the gray matter of the brain stem, Mycetes seniculus (color)

239

132. Habitat group. Baboon at the water hole.

290

133. Papio cynocephalus (baboon) .

$29 \mathrm{I}$

$134^{-137}$. Hand and foot of Papio cynocephalus

I38. Dorsal surface of brain, Papio cynocephalus.

139. Base of brain, Papio cynocephalus. . . . .

$29^{-}$

I 40. Right lateral surface of brain, Papio cynocephalus.

I41. Left lateral surface of brain, Papio cynocephalus.

142. Ventral surface of brain stem, Papio cynocephalus.

I 43. Dorsal surface of brain stem, Papio cynocephalus

I 44. Baboon. Level of the pyramidal decussation.

I 45. Baboon. Level of caudal extremity of nucleus of Burdach.

146. Baboon. Level of the caudal extremity of the inferior olivary nucleus

$I^{-}$. Baboon. Level through the middle of the inferior olive

14 . Baboon. Level of the vestibular nuclei

I 49. Baboon. Level of the cerchellar nuclei

I jo. Baboon. Level of emergent fibers of sixth nerve and caudal fibers of pons.

I 5 r. Baboon. Level at middle of pons, showing entering trigeminal fibers .

I52. Baboon. Level of inferior colliculus showing emergence of fourth nerve . 
Figure Page

I 54 . Baboon. Level of the superior colliculus. . . . . . . . . . $33^{\circ}$

I 55. Baboon. Level of the optic ehiasm. . . . . . . . . . . 332

I 56 . Baboon. Level of the anterior commissure. . . 333

15-. Dorsal surface of the gray matter of the brain stem, Papio cynocephalus (color). 33-

1;8. Lateral surface of the gray matter of the brain stem, Papio cynocephalus (color). 330

150. Ventral surface of the gray matter of the brain stem, Papio cynocephalus (color). $34^{1}$

I60. Macacus rhesus. Full-grown monkey and young. . . . . . . . . . . . . . 35 I

I6 $\mathrm{I}-164$. Hand and foot of Macacus rhesus. . . . . . . . . . . . . . . . . 352, 353

165. Dorsal surface of brain of Macacus rhesus . . . . . . . . . . . . . . . . . 358

I66. Base of brain of Macacus rhesus. . . . . . . . . . . . . . . . . . . 350

167. Left lateral surface of brain, Macacus rhesus . . . . . . . . . . . . . . . 360

I68. Right lateral surface of brain, Macacus thesus. . . . . . . . . . . . 361

160. Ventral surface of brain stem, Macacus rhesus . . . . . . . . . . . . . . . 362

i-o. Dorsal surface of brain stem, Macacus rhesus . . . . . . . . . . . . 363

I $\rightarrow \mathbf{1}$. Macacus. Level of the pyramidal decussation . . . . . . . . . . . . . . 366

172. Macacus. Level of caudal limit of dorsal sensory nuelei. . . . . . . . . . . 36-

$I^{-3}$. Macacus. Level of caudal limit of inferior olivary nucleus. . . . . . . . . 36

i 4. Macacus. Ievel through middle of inferior olivary nucleus . . . . . . . . . . 3 3

$\mathbf{I}^{-5}$. Macacus. Level of the vestibular nuclei. . . . . . . . . . . . . . . . . . . $3^{-3}$

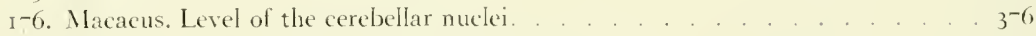

I $^{-\cdots}$. Nacacus. Level of the emergence of the sixth nerve . . . . . . . . . . . . . $3^{-8}$

1-8. Macacus. Level through the middle of the pons Varolii. . . . . . . . . . 38 i

1-9. Macacus. Level of the emergence of the trochlear nerve. . . . . . . . . . 382

180. Macacus. Level of the inferior colliculus . . . . . . . . . . . . . . . 38+

181. Macacus. Level of the superior colliculus . . . . . . . . . . . . . . . . . 386

I82. Macacus. Level of the optic chiasm . . . . . . . . . . . . . . . . . . 38 $8^{-}$

I83. Nacacus. Level of the anterior commissure . . . . . . . . . . . . . . . . 388

184. Ventral surface of gray matter of brain stem, Pithecus rhesus (color). . . . 393

IS5. Dorsal surface of gray matter of brain stem, Pithecus rhesus (color). . . . 395

186. Lateral surface of gray matter of brain stem, Pithecus rhesus (color). . . . . 39-

I8-, 188. Hylobates syndactylus (gibbon). . . . . . . . . . . . . . . . $406,40^{-}$

189-192. Hand and foot of Hylobates syndactylus . . . . . . . . . 408, to9

193. Dorsal surlace of brain, llylobates hoolock . . . . . . . . . . . . . . + 44

194. Base of brain, 1lylobates hoolock. . . . . . . . . . . . . . . . . 4 t

195. Left lateral surface of brain, Hylobates hoolock . . . . . . . . . + + . . . . . . . .

Ig6. Right lateral surface of brain, Hylobates hoolock . . . . . . . . . . . . . $t^{\mathrm{I}}$

r97. Ventral surface of brain stem, Hylobates hoolock . . . . . . . . . . . . . 418

I98. Dorsal surface of brain stem, Hylobates hoolock. . . . . . . . . . . . . + + +19

199. Gibbon. Level of the pyramidal decussation. . . . . . . . . . . . . . . . 422

200. Gibbon. Level of the caudal extremity of the inferior olive . . . . . . . . . 425

20I, 202. Gibbon. Level through the middle of the inlerior olive. . . . . . $\quad+428,429$

203. Gibbon. Level of the vestibular nuclei . . . . . . . . . . . . . . . . . 43 I

204. Gibbon. Level of the cerebellar nuelei . . . . . . . . . . . . . . . . . + + . . . . . . . . 
205. Gibbon. Level of the emergence of the sixth cranial nerve. . . . . . . . . . 436

206. Gibbon. Level through the middle of the pons. . . . . . . . . . . . . . . . 43-

207. Gibbon. Level of the emergence of the trochlear nerve . . . . . . . . . . 438

208. Gibbon. Level of the inferior colliculus . . . . . . . . . . . . . . . 4to

209, 210 . Gibbon. Level of the superior colliculus . . . . . . . . . . . . . . $4+2,+43$

21 . Gibbon. Level of the optic chiasm . . . . . . . . . . . . . . . . . $4+4$

2г2. Gibbon. Level of the anterior commissure. . . . . . . . . . . . . . . . . 4t5

213. Ventral surface of gray matter of brain stem, Hylobates hoolock (color). . . . 4 49

214. Dorsal surface of gray matter of brain stem, Hylobates hoolock (color). . . . 451

215. Lateral surface of gray matter of brain stem, Hylobates hoolock (color) . . . + +53

2 I6. Habitat group, orang-outang, Sadong River, Borneo. . . . . . . . . . . . . 48 I

2 $\mathrm{I}^{\text {. }}$. Orang-outang, erect posture, showing disproportion of arms and legs. . . . . . 482

2 I8. Orang-outang, showing anthropomorphous tendencies in head and face. . . . +83

219-222. Hand and foot of the orang-outang . . . . . . . . . . . . . 484,48 ;

$223 \mathrm{~A}$ and B. Dorsal surface of brain, orang-outang. . . . . . . . . . . . . . 488, 489

$224 \mathrm{~A}$ and B. Base of brain, orang-outang. . . . . . . . . . . . . . . . 490, 491

$225 \mathrm{~A}$ and в. Left lateral surface of brain, orang-outang . . . . . . . . . . . . 494, 495

226.4 and B. Right lateral surface of brain, orang-outang . . . . . . . . . . . 496, $49^{-}$

227. Ventral surface of brain stem of orang-outang. . . . . . . . . . . . . . . . 500

228. Dorsal surface of brain stem, orang-outang . . . . . . . . . . . . . 50 I

220. Orang-outang. Level of the pyramidal decussation. . . . . . . . . . . . . 502

230. Orang-outang. Level of the caudal extremity of the dorsal sensory nuclei . . . 505

231. Orang-outang. Level of the caudal extremity of the inferior olive. . . . . . . . jo8

232. Orang-outang. Level through middle of inferior olive. . . . . . . . . . . . 3i I

233. Orang-outang. Level of the vestibular nuclei. . . . . . . . . . . . . . . 3it

234. Orang-outang. Level of the cerebellar nuclei. . . . . . . . . . . . 316

23;. Orang-outang. Level near caudal limits of pons, emergent sixth nerve fibers. . $\quad 318$

236. Orang-outang. Level of the middle of the pons Varolii . . . . . . . . . . . 521

$23^{-}$. Orang-outang. Level of emergence of trochlear nerve. . . . . . . . . . . . . . $\$ 22$

238. Orang-outang. Level of the inferior colliculus . . . . . . . . . . . . . . . . 524

239. Orang-outang. Level of the superior colliculus. . . . . . . . . . . . . . . . 326

2 to. Orang-outang. Level of the optic chiasm . . . . . . . . . . . . . . . 330

241. Ventral surface of gray matter of brain stem, Simia satyrus (color) . . . . . 535

242. Dorsal surface of gray matter of brain stem, Simia satyrus (color) . . . . . . . 53-

243. Lateral surface of gray matter of brain stem, Simia satyrus (color). . . . . . . 539

2+t. Habitat group, chimpanzee. . . . . . . . . . . . . . . . . . . . . . $54^{-}$

245,246 . Chimpanzee Susie. . . . . . . . . . . . . . . . . . . . 548,549

$24^{-2}-250$. I land and foot of chimpanzee. . . . . . . . . . . . . . 552,553

$251 \mathrm{~A}$ and в. Dorsal surface of brain, chimpanzee . . . . . . . . . . . 560, 561

$252 \mathrm{~A}$ and B. Base of brain, chimpanzee . . . . . . . . . . . . . . 562,563

253.4 and B. Left lateral surface of brain, chimpanzee . . . . . . . . . . . ;68, 569

2j+. Ventral surface of the brain stem, chimpanzee. . . . . . . . . . . . . . . . . . . . .

255. Dorsal surface of brain stem, chimpanzee. . . . . . . . . . . . . . . 53 
274. Lateral surface of gray matter of brain stem, Troglodytes niger (color). 
Figure

313. Dorsal surface of gray matter of brain stem, Troglodytes gorilla (color)

319. Prof. Osborn's estimates of man's antiquity, industries, arts and races

320. Restoration of Pithecanthropus compared with human and anthropoid skulls.

321. Restorations of Heidelberg and Piltdown man by Professor McGregor.

322. The Ncanderthal race

323. The Cro-magnon race

741

324. Stone implements representing the several stages of Paleolithic culture

$7+2$

25. "Eoliths" from Piltdown, Sussex

326. Contrasts between implements of the Paleolithic and Neolithic Ages

$-48$

32-. Evolution of lance point through several stages of the Old Stone Age.

332. African pygmy group from the Bclgian Congo. 
358, 359. Lateral surface of gray matter of brain stem, Homo sapiens (color) .

360. Dorsal surface of gray matter of brain stem, Homo sapiens (color)

$8+3$

36r. Ventral surface of gray matter of brain stem, Homo sapiens (color)

$8+5$

362-36,5. Four vicws of an endocranial cast of a modern human skull.

863

$366-3^{-1}$. Six views of the endocranial cast of Pithecanthropus erectus (Javan Apeman)

$3^{-2} 3^{-4}$. Comparison of endocranial casts of gorilla, Pithecanthropus and Homo sapiens

37. Functional localization of the brain outlined upon the left hemisphere of the endocranial cast of Pithecanthropus erectus

3-6-380. Five views of the endocranial cast of Eoanthropus dawsoni (Dawn man of Piltdown)

381. Functional localization of the brain outlined upon the left hemisphere of the endocranial cast of Eoanthropus. (Piltdown.)

$382-38-$. Six views of the endocranial cast of Homo neanderthalensis.

388. Neanderthal tint workers

389-303. Five views of the endocranial cast of the La Quina skull

394 390 . Six views of the endocranial cast of the Gibraltar skull.

40o. Functional localization of the brain outlined upon the left hemisphere of Homo neanderthatensis (La Chapelle aux Saints)

for to6. Six views of the endocranial cast of Homo rhodesiensis (Rhodesian man)

to-. Functional localization outlined on left hemisphere of flomo rhodesiensis.

$408+13$. Six views of the endocranial cast of Homo sapiens of the Predmost race

4. F. Functional localization of the brain outlined upon the left hemisphere of the Predmost endocranial cast

4 5. Cro-magnon artists making fresco in cave of Font-de-Gaume

$+1^{-422}$. Comparison of the endocranial casts of Pithecanthropus, Piltdown, Rhodesian, Neanderthal and Predmost with modern flomo sapiens . . . . . . 934

$+23+26$. Size and configuration of the dorsal and lateral surfaces of the human brain compared with those of Lemur mongoz.

$42^{-}+3^{\circ}$. Size and configuration of the dorsal and lateral surfaces of the human brain compared with those of Callithrix jacchus, the marmoset.

431 434. Size and configuration of the dorsal and lateral surfaces of human brain com- 949
pared with those of Mycetes seniculus.

431 434. Size and configuration of the dorsal and lateral surfaces of human brain com-
pared with those of Mycetes seniculus.

$435-438$. Size and configuration of the dorsal and lateral surfaces of the human brain compared with that of Papio crnocephalus, the baboon.

430 442. Size and configuration of the dorsal and lateral surfaces of the human brain compared with those of Pithecus rhesus, the macacus.

$443-446$. Size and configuration of the dorsal and lateral surfaces of the human brain compared with those of hylobates hoolock, the gibbon

$447^{-450}$. Size and configuration of the dorsal and lateral surfaces of the human brain compared with those of Simia satyrus, the orang-outang and configuration of the dorsal and lateral surfaces of the human brain

$45 \mathrm{I}-454$. Size and configuration of the clorsal and lateral surfaces of the human brain compared with those of Troglodytes niger, the chimpanzee

$+55-458$. Size and configuration of the dorsal and lateral surfaces of the human brain

compared with those of Gorilla gorilla
450. Principal primate horizons, showing evolutional expansion of neopallium (color)

459. Principal primate horizons, showing crolutional expansion of neopallium (color) 989 
Figure

$4^{60-} 4^{-0}$. Cross section at level of pyramidal decussation in the comparative prinate series. . . . . . . . . . . . . . og6, $99^{-}$

$47 \mathrm{I}-48 \mathrm{~s}$. Cross section at the level of the caudal extremity of inferior olive in the comparative primate series.

482-492. Cross section at the level of the middle of the inferior olive in the com-

IOOO, $100 \mathrm{I}$

\section{parative primate series.}

493. Graphs constructed on the basis of the planimetric indices. 1004,1005

494-504. Cross section at the level of the vestibular nuclei in the comparative primate series

1012,1013

505-515. Cross section at the level of the emergence of the trochlear nerve in the comparative primate series. . . . . . . . . . . . . . IoI6, I0I7

516. Graph based on planimetric indices of the pontile nuclei. . . . . . 1018

517-527. Cross section at the level of the inferior colliculus in the comparative primate scries. . . . . . . . . . . . . . . . 1022,1023

528-538. Cross section at the level of the superior colliculus in the comparative primate series . . . . . . . . . . . . . . . . . 1026,1027

539. Graph based on planimetric indices of cerebral peduncle. . . . . . . . . 1028 

INTRODUCTION

THE PRIMATES: LEMURS, MONKEYS, APES AND MAN THEIR PLACE IN NATURE 



\section{THE BRAIN FROM APE TO MAN}

\section{INTRODUCTION}

Relative Importance of the Brain as Erolutional Eridence. Its Significance Compared with Other Parts of the Body.

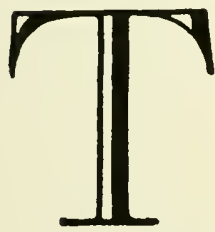

HE brain is conceded to be the master organ of the body, the regulator of life, the source of human progress. In this capacity how has it contributed to the evolution of mankind? Has it, as some authorities assume, passed through successive evolutional stages to reach its highest development?

Both of these questions imply the acceptance of the Evolutionary Theory. If this hypothesis be correct, the human brain should prove an important witness in its favor. It may be expected to retain conclusive evidence of the evolutional process in at least three particulars:

I. It should manifest signs of its primitive origin from the lowest vertebrates (Fig. I).

2. It should bear identifying marks of intimate association with animals of its own class, the mammals (Fig. 2).

3. It should also have many specific details in common with members of the Primate Order to which man belongs, together with lemurs, tarsiers, monkeys and apes (Fig. 3).

A comparison of the human brain with that of its primate coordinates may thus become of utmost importance by disclosing the exact structural basis of such evolution as has occurred in man from his early prehuman beginnings. 


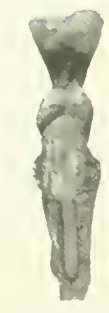

DOG FISH
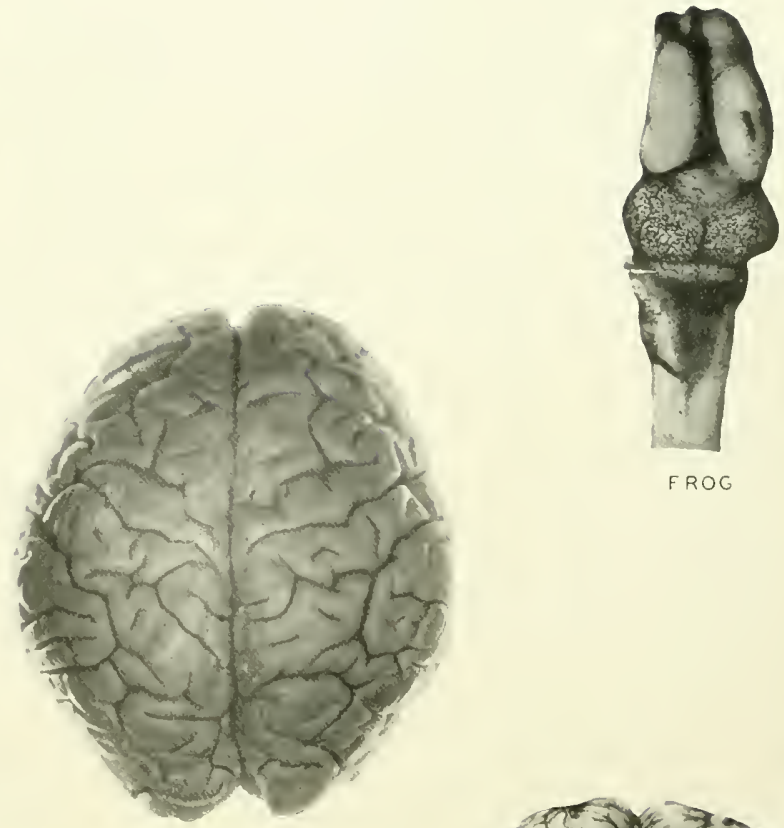

$$
\text { FROG }
$$

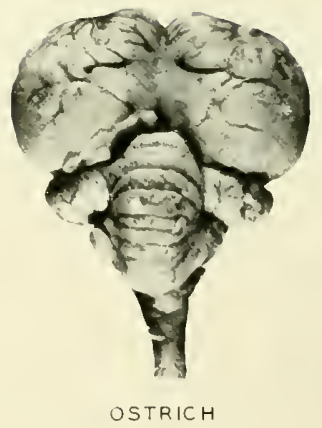

FIG. I. BRAINS OF LOWER VERTEBRATES COMPARED WITH THE HUMAN BRAIN. 


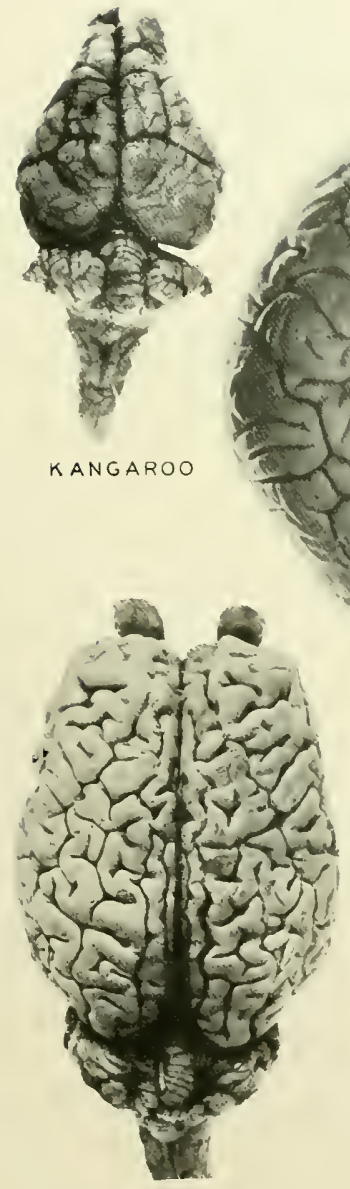

HORSE

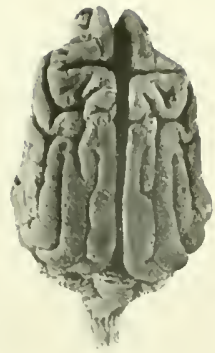

$D O G$

HUMAN
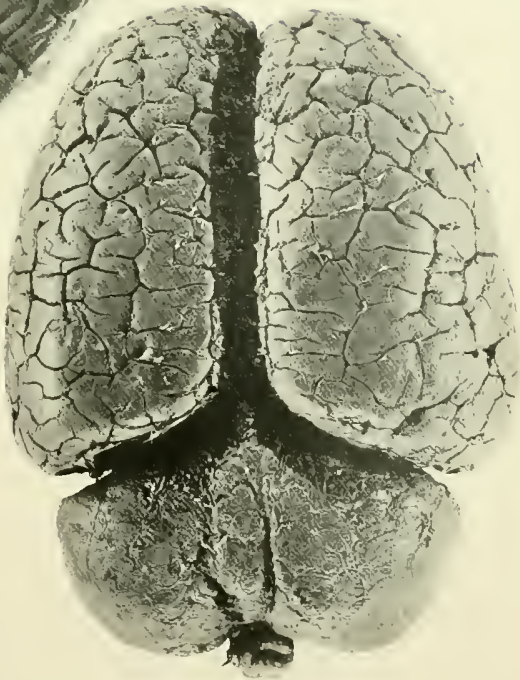

FLEPHANT

FIG. 2. BRAINS OF MAMMALS COMPARED WITH THE HLMAN BRAII. 

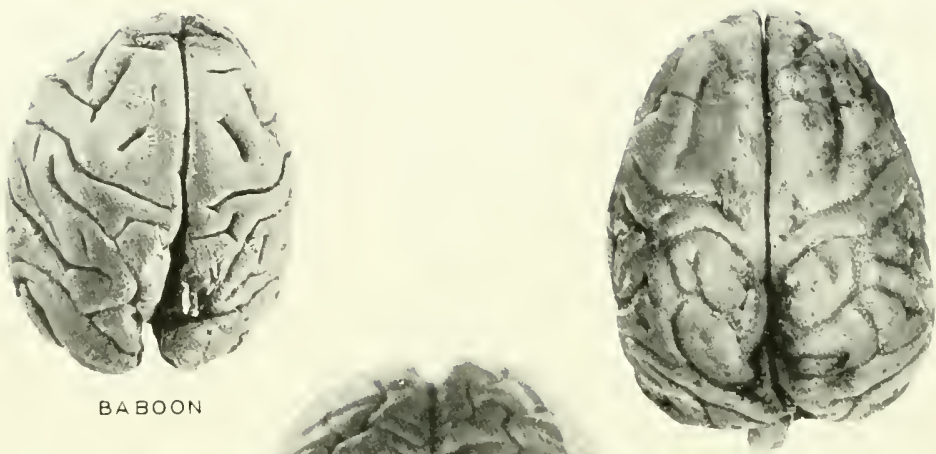

GIBBON

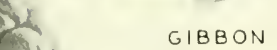

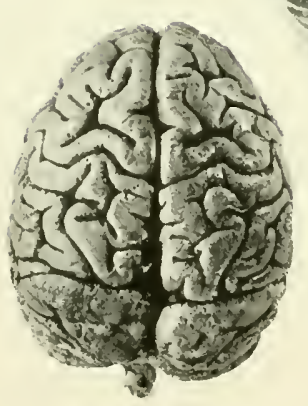

CHIMPANZEE

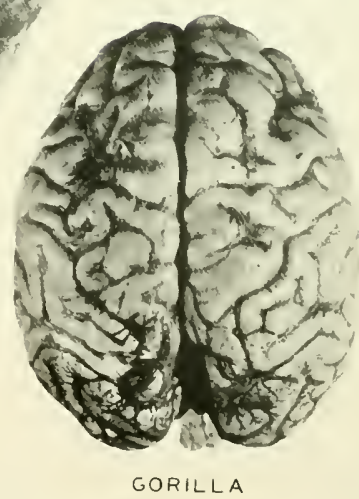

FIG. 3. BRAINS OF APES COMPARED WITH THE HUMAN BRAIN. 


\section{INTRODUCTION}

\section{Other Body Tisse which Reveal Evolutionary Kinsuip THE BLOOD}

The actual existence of evolutionary kinship has already been revealed by many other organs and tissues of the body. The blood, for example, has been most outspoken in this respect. Human blood serum injected into the rabbit produces what is known as anti-buman serum. This latter serum affords an exceedingly delicate test for human blood, either fresh or in the form of old and dried clots. If the blood tested be that of a domestic animal, such as the horse, sheep, pig or fowl, no reaction results from this serum. Anti-horse serum, anti-sheep serum, anti-pig serum and anti-fowl serum may be prepared in a similar manner. These are sensitive agents for detecting the presence of horse, sheep, pig or fowl blood. An anti-serum may also be made for all animals and tests thus provided to detect the blood of similar species.

Investigations have shown that these blood tests may be employed to determine the degree of relationship existing between different kinds of animals. A prompt and often strong reaction is obtained only from the blood of the same species, while the blood of closely allied species, such as that of the horse and donkey, gives a weaker, slower precipitation.

These precipitin tests support the supposition that there is some distant relation between the old-world monkeys, apes and man. They also make clear a less intimate relationship between the new-world monkeys and the human stock. The lowest animals classified among the primates, the lemurs, give no sign of blood relationship with man, although there is some indication of such a kinship with the lower new-world monkeys. Tarsius, however, is an exception to this rule. It bears a closer blood relation to man, simia and gibbon than it does to macacus, the new-world monkeys or the cat.

Hemal tests demonstrate a relationship among all carnivora closer than exists between carnivores and ungulates, while cetacean forms (whales and 

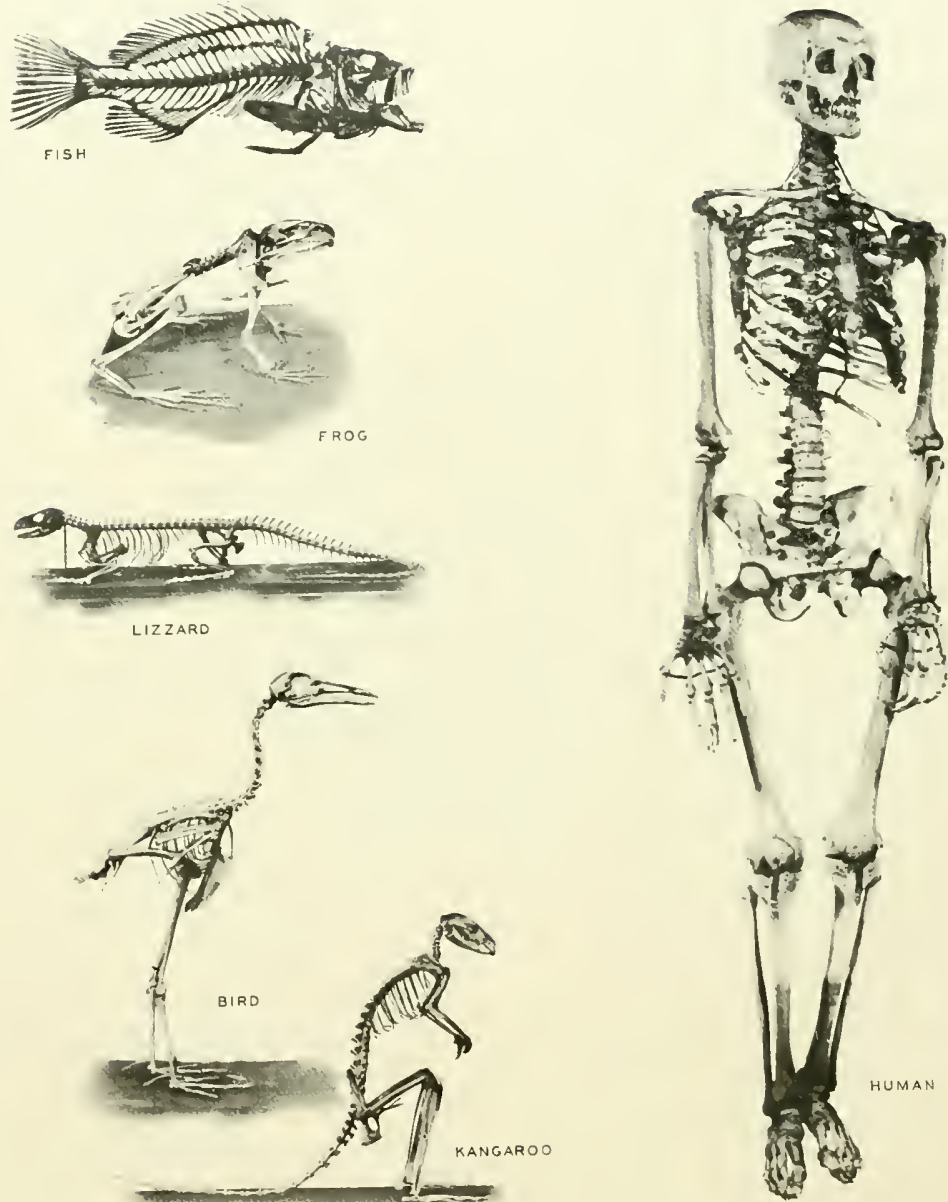

FIG. 4. COMPARISON OF VERTEBRATES SHOWING THE ESSENTIAL 

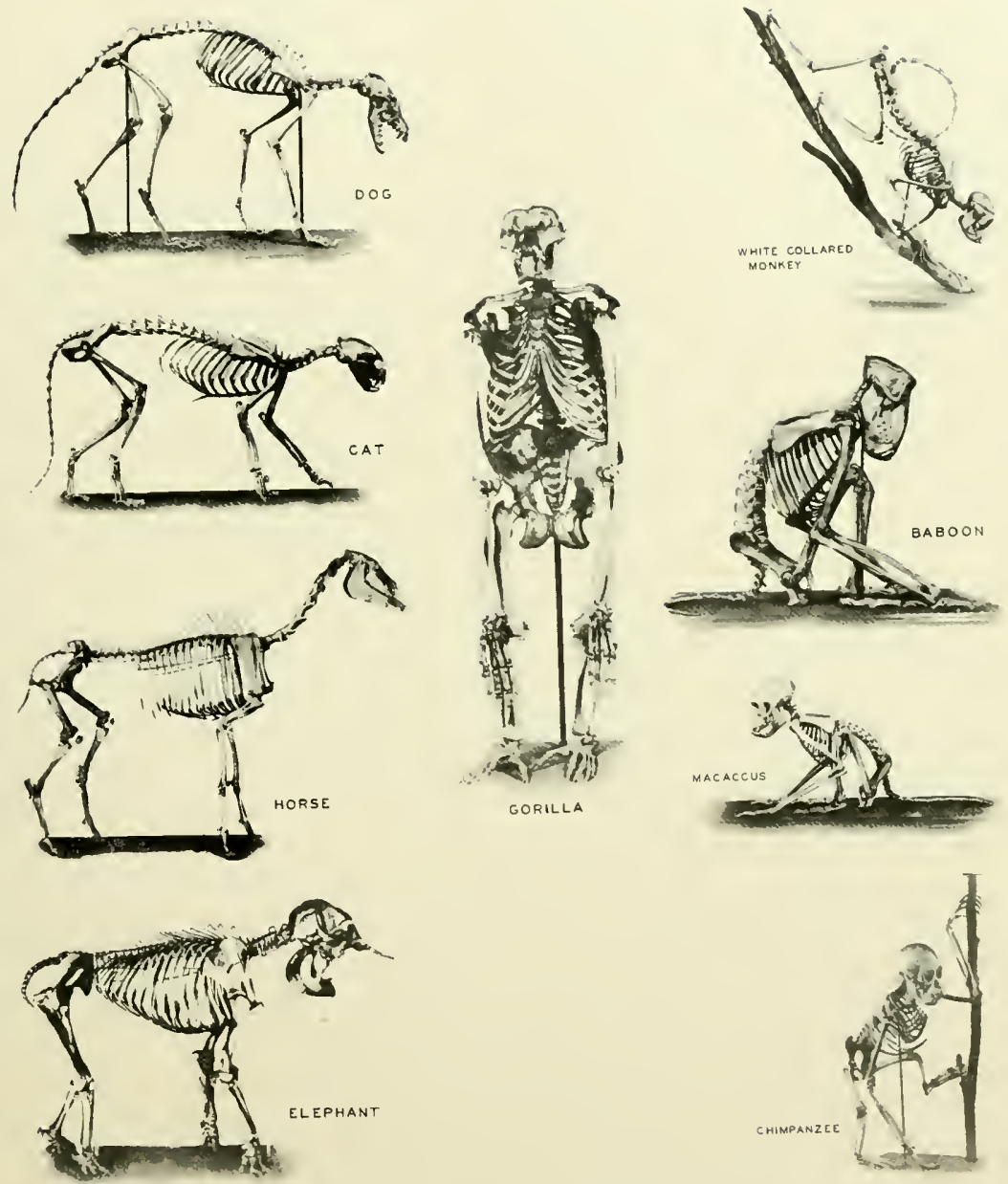

SIMILARITIES IN THE SKELETAL STRUCTLRES, FROM FISH TO MAN. 
porpoises) are more closely allied to cud-chewing animals (ruminants) and pigs.

Blood reactions indicate that a strong interrelationship characterizes all reptilian forms, although there is some serial grading in these affinities as among turtles, crocodiles, lizards and snakes respectively.

Avian blood tests show a striking similarity in the hemal constituency of all birds. This fact stands out in contrast to the mammalian blood which has a specific variability in the intensity of its reactions dependent upon the order and family of the animals tested.

In general, the precipitin blood tests are among the most convineing proofs put forward in support of the evolutionary hypothesis.

\section{TIIE BONY SYSTEM}

The bony system of the body (Fig. 4 ) is also an illuminating recorder of evolutional kinship. The skeleton of the fore- and hindlimbs sheds much light on the modifications connected with the locomotor apparatus. In accordance with the limb specialization as fins, paddles, wings, hoofs, paws, claws, hands or feet, the general propinguities in kinship may to a certain extent be estimated. The size and shape of the skull are equally significant in determining an exact classification of species.

\section{THE MUSCULAR SYSTEM}

Another significant index of a generalized ground-plan in vertebrate organization is the muscular system. This system, while it demonstrates the great stability underlying the motor apparatus, discloses a flexibility capable of providing for the special needs of aquatic, amphibian, terrestrial and acrial life. It thus reveals propinquities in kinship amongst animals which have adjusted their locomotor apparatus to similar or closely allied conditions of environment. 


\section{THE IIEART AND LUNGS}

The heart and the lungs afford important evidence of vertebrate relationship and evolution. The increase in specialization of the respiratory apparatus, from the gill stages of the fish to the conditions of the mammalian pumonary organs, is a most reliable means of distinction between classes and orders.

\section{Enibriogenesis as Evidence of Evolutionary Kinship}

And finally, embryogenesis, coming as a culmination of reproductive activity, discloses the fact that all vertebrates-fishes, amphibians, reptiles, birds and mammals however different their form, habitat, mode of life and behavior - are cast in a structural mold of development laid down in accord with a common plan.

Each department of structural organization in the body contributes adequate testimony of a common ancestry. But no one of them portrays more than a single phase of vertebrate adaptation. Thus the blood represents the metabolic and biochemical adjustment of the animal. The osseous system and the muscles are indicative only of motor and locomotor capabilities. The teeth reveal the feeding habits and part of the protective mechanism. The genito-urinary system bears witness to the variety of exeretory differentiation and the type of reproductive specialization. Embryogenesis summarizes the process of structural unfolding and thus discloses the general morphological plan of organization.

\section{The Brain as the Most Comprehensive Record of an Evolutional Process}

The reasons why the brain contains the most comprehensive record of the evolutional process are readily perceived. As an organ its influences pervade and dominate all other systems of the body. It is the great trans- 
former of energy which integrates all parts into a harmoniously acting machine. It interprets all impressions from without. It projects all impulses from within. In its dual capacity of servant and master the brain has been peculiarly susceptible to the influence of that combination of factors deseribed by Professor Osborn in his tetraplastic theory of evolution. The cerebrum has felt more, perhaps, than any other organ, the effects of action, reaction and interaction. It has responded more extensively to its inorganic and vital environment because it comprises the most highly differentiated tissue of the organism. It has been equally sensitive to the influence of heredity-chromatin and life (ecological) environment.

\section{The Comparison of the Human Brain with the Brains of Other Prinates}

A structural comparison of the human brain with the brains of other members of the primate order illustrates in detail the progressive modifications in this evolutionary process. In this comparison the brain of man for many obvious reasons is placed at the head of this order. It has been compared with the brains of Troglodytes gorilla (gorilla), Troglodytes niger (chimpanzee), Simia satyrus (orang-outang), Hylobates hoolock (gibbon), Papio cynocephahus (baboon), Macacus rhesus (common old-world monkey), Mycetes seniculus (South American howling monkey), Callathrix jacchus (marmoset), Tarsius spectrum (tarsier) and Lemur mongoz (lemur). These species comprise representatives of the highest and lowest primates.

The lemur represents the transition from some mammalian form which was predominantly arboreal. The weight, size and celerity of this animal adapt it especially to a lofty arboreal habitat.

In most of the new-world monkeys the development of a prehensile tail greatly extends the range of motor activity. Due to increasing size and weight of body, the larger apes have shown a progressive tendency to desert their 
more inacessible arboreal retreats and approach nearer to the ground. The transition from semi-arboreal habitat to completely terrestrial life has culminated in the development of the erect posture and plantigrade locomotion with tree climbing but an incident in bipedal adjustment.

\section{CERTAIN FUNDAMENTAL ADJUSTMENTS OF BEHAVIOR}

In making the comparison of these primate brains, the chief object has been to note the structural changes whereby the evolutional process has been advanced. Certain fundamental components of behavior have been simultaneously studied in relation to these structural modifications. These components include the following:

The development of the prehensile tail, its recession and final disappearance

The progressive adaptation of the hand for manual performances other than those concerned in locomotion

The adjustment of simultancous movements in the eyes, head and hands necessary to the exccution of skilled acts

The gradual assumption of the erect posture

The increase of volitional control of the arms and the legs

The amplification of postural regulation in the body and extremities

The development and perfection of binocular vision

The progressive readjustments of visual, auditory and equilibratory reactions in passing from arboreal to ground-living habits

The modification of the automatic associated movements induced by such adjustments.

In comparing these species one with another, it is possible to observe the reflection of certain behavioral adjustments as they are mirrored by the organic constituents of the brain. In some cases, the range of variation in these behavioral reactions is so wide as to create most outspoken differences. 
Again, in closely allied species the contrast is not pronounced or convineing; but by comparing members in the primate group standing phyletically far apart from each other, the essential differences between them gather the full foree of their evolutional signifieance. They leave no room to doubt that a progressive functional development has gone hand in hand with impressive evolutional modifications in the structure of the brain.

\section{Bonds of Kinship among the Primates}

No attempt has been made to arrange the species in exact serial order. Such classification would be open to numerous objections. A brief panoramic view of the primates may, however, diselose the broad bonds of kinship which link them together in the foremost place of the vertebrate phylum. One feature in this panorama is especially noteworthy. There has been a strong tendency among the vertebrates to take refuge in the trees, either to escape predacious contemporaries or otherwise to foster the opportunity to live. Its obvious purpose is to increase the measure of safety by extending the radius of retreat and making ascent from the ground a real biological advantage.

\section{MODIFICATIONS INDUCED BY TREE-DWELLING}

With the advent of the primates, this arboreal tendency was diverted into still another channel. Here it took the turn of affording an almost complete permanency of abode rather than a refuge in time of emergeney. Such a change to more or less permanent tree-dwelling could but induce farreaching modifications. Not only did this life create a new type of habitat; it at once enforced a new mode of transit over the leafy highways of the tree tops. In order to obtain proper adjustment for such transportation, both hand and foot aequired the qualities of prehensile organs. Locomotion of this kind eventuated in the development of quadrumanal characters, with the foot 
departing from its more ancient patterns of hoof, paw or claw and assuming the nearly complete grasping powers of the hand. The tail also, in certain instances, participated in this specialization for arborcal Iocomotion.

These physical modifications actuated by tree life were quite as emphatically augmented by psychic influences arising from the new habitat. The background of the lives of almost all of the primates has played a dominant rôle in molding their behavior. The primeval forests and jungles, in their perpetual semi-darkness, created a domain whose subtle powers made themselves felt in all primate reactions. It might be that the forest stood on the edge of a wide plain with a clear opening from which to look across toward the distant hills. The psychological effect of these factors, it must be clear, determined certain decisive attitudes on the part of the animals toward their environment. For example, there could not fail to be an alluring temptation in the green plain and its cxpansive freedom. To venture into this open space, however, the early primates required mechanisms which they did not as yet possess. Certain dangers lurked in and over the plain. Preclatory ereatures of every hind were there - reptile, mammal and bird-lying in wait for just such an excursion. For the time, at least, the semi-clarkmess was safer with its limited view and real security in the upper highways out of reach of these preying enemies.

\section{THE LEMILS AND TARSIERS, SHOWING THE EARLIEST}

\section{MODIFICATIONS OF PRIMATE STRLCTURE}

With the lemurs, perhaps, there came the first modifications of structure leading to the more conspicuous groups of the primate kind. These animals, with their slender, furry bodies, their fox-like heads, their widely separated eyes and bushy tails, showed in both hands and feet the beginning differentiation of fingers and toes. This adaptation marks the transition from some lower form of mammal to the primates. It was in an epochal modification 
such as this that the primate order had its origin. Then began a tenancy of the trees which profoundly influenced these animals as they passed through their many and varied adaptations. Tarsius, in many respects even more than the lemurs, illustrates the effects of these new influences.

\section{THE CEBIDAE FORESHADOWERS OF A NEW RACE}

It is diffieult to discern the exact point at which the Hapalidae, most familiarly represented by the diminutive marmosets, took departure from the early beginnings of this primate line. Their appearance on the scene was probably a retrogressive step in the development of the new-world monkeys. Their small size, their lack of power both in body and brain, would scarcely permit of a dominating adjustment to their environment. In many respects their organization was inferior to that of the lemurs. This is particularly true in their manual differentiation, since the development of their fore- and hindlimbs tencled much more toward claws than hands. Nevertheless, for all their insignificance, there was that about them which suggested the coming of a new race. The configuration of the head, the expression of the face, the relation of the eyes, the shape of the nose, the position of the mouth, all were prophetic of the more definitely ape-like tribes which were to follow. The arrival of the new-world monkeys at length introduced all of the major simian characters. Throughout the large family of the Cebidae, quadrumanal development is well established. Most of the members of this group have also acquired prehensile tails.

\section{INFLUENCES ACTIVATING MODIFICATION WHICH PRODLCED OLD-WORLD}

$$
\text { CONGENERS OF PRIMITIVE SIMIANS }
$$

What influences activated the modification which produced the oldworld congeners of these South American simians is not clearly understood. It may have been a progressive tendency to increase in weight, added to the 
waning importance of the tail. In any event, the old-world monkeys do not develop prehensile functions in their tails, and many of them, like the baboons, have ventured out of the trees to live more upon the ground. Nost of the latter show a distinct dog-like development in mode of life, in size and in form of body. In the gibbons for the first time is established the ability to walk upright, but with these animals forest life is still predominant in its influence. By means of their long arms they are capable of swinging from branch to branch with a marvelous agility. The absence of the tail in them, and the partial assumption of the erect posture, are most significant as forerumners of developments in the larger anthropoids. In these latter a great increase of weight enforced the habit of living nearer to the ground.

The first of these large anthropoids, the orang-outang, still shows its ancestral adherence to the forest, although it is able at times to stand and run. The chimpanzee, while it has great cleverness in climbing, seeks the ground oftener and has learned to walk upon all fours, using the knuckles of the hand as a support in this act. Finally, the gorilla, often attaining the great weight of nearly four hundred pounds in adult life, has been compelled by this reason to seek the support of the earth and only at times makes use of the heavier portions of the trees for refuge or retreat. Still, so indomitable is the arboreal tendency, eren in these great animals, that they have made but imperfect terrestrial adaptation and also at the same time they are only partially suited to tree life. Were it not for their prodigious strength, their limited arboreal activities would be even further curtailed.

All of the man-like apes are capable of the upright posture, of standing and walking on the hind legs but in an awkward, inefficient manner. This is due to the fact that the foot in all three of these animals maintains predominant characters of the hand and affords at best an imperfect basis for bipedal locomotion. Yet, notwithstanding such limitations as long, dragging arms and inadequate feet, the great anthropoids are able to venture beyond the limit 
of their forest home. They are partially equipped to cope with some of the dangers lurking without. Their forclimbs are in part freed from the responsibilities of Jocomotion and so made available for acts of self defense and even, to a considerable degree, for exploration.

Actual progress in the direction of human specialization had its beginnings in this recession of superarboreal tendencies and the establishment of a terrestrio-arboreal mode of life. Such a gradual recession may be seen in this panorama of the primates. It is apparent in the first indecisive yet promising steps that Ied the anthropoid out of his ancient forest dominion toward the inviting plain. And finally, with the complete recession of arboreal life, there began that long journey destined to lead over every sea and into every land, until no region of the earth remained for further conquest; until the complete acquisition of the up ight posture and the full development of the hand had more and more bent the forees of nature to the designs of the races of man.

\section{Brain Development Paralleling the Progressive}

\section{Development of Behayior}

Step by step the brain has kept pace with these progressive alterations. Its newer, recently acquired portions reveal a much more conspicuous response to this progress than its less plastic, more archaie elements. But old parts and new alike, according to their varying degrees of susceptibility, bear the certain imprint of adaptive change. Among the primates the biological formula determined by quadrumanal specialization and arboreal habitat has worked itself out through graded stages from lemur to man. The evolutional process of this gradual transition is disclosed by structural modifications of the nervous system. Beginning with the Lower Primates, such as the tarsiers, the advance may be traced through many intermediate phases of cerebral development to its ultimate goal in the human brain. 


\section{PART I}

THE LOWER PRIMATES 



\section{INTRODUCTION TO PART I}

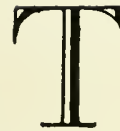

HOSE mammalian forms occupying the upper extremity of the vertebrate phylum have, since the time of Linnaeus, been known as Primates, or chief of mammals. It was Linnacus who, recognizing the anthropomorphous characteristies of this large group, created the term to designate their order. He further subdivided them into Humans, Simians and Prosimians.

\section{The Slborders in the Order of Printates}

At present the order of primates is arranged in three suborders: ( $\mathrm{I}$ ) Lemuroidea, (2) Tarsioidea and (3) Anthropoidea. The first of these includes all of the prosimian forms of the lemur kind, white the third comprises all families of actual simians and man. According to this arrangement the tarsiers occupy an intermediate position between the other two suborders.

The following are the accepted families of these three suborders:

Suborder Lemuroiclea:

Daubentonidae

Nycticebidae

Suborder Tarsioidea :

Tarsiidae
Suborder Anthropoidea:

Hapalidae

Cebidae

Lasiopygidae

Hylobatidae

Simiidae

Hominidae

In this discussion a slight rearrangement has been made to serve the purposes of presentation and at the same time to observe the dictates imposed by certain morphological similarities in the brain. Accordingly, the primates are here considered in four distinct divisions: First, the lower primates which comprise the lemurs, tarsiers and alf of the new-world monkeys (including

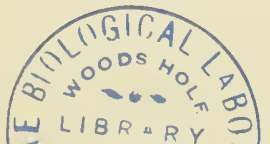


marmosets and the Cebidae). The second group, or intermediale primates, includes all of the old-world Catarrhine monkeys with the exception of the three great anthropoid apes. The third group, called the bigher antbropoids, includes the orang, the chimpanzee and the gorilla; while the sole occupant of the fourth group is Homo sapiens, although reference is made to certain features in the development of the several races of prehistoric man. 


\section{Chapter I}

\section{LENIUR MONGOZ, ITS BRAIN AND BEHAVIOR}

Its Position among the Primates; Measurements and Brain Indices; Surface Appearance of the Brain; Internal Structure of the Brain Stem in Cross Section

7 HE lemurs, so called because of their nocturnal and ghostly habits, represent the lowest level of primate organization. In all, there are I some fifty species of these animals referable to seventeen genera. Thirty-six of these species are indigenous to Madagascar and its small adjacent islands. The remaining species have their habitat in Ethiopian and oriental regions. It is a fact of much interest that the rest of the world is quite devoid of these animals, although fossil remains indicate that the lemurs were much more widespread throughout the globe in some earlier geological period.

\section{External Appearance of the Lemur (Figs, ; 6 and 7)}

The external appearance of the lemurs justifies the establishment of a suborder to contain these animals. They are readily distinguished from the apes and monkeys constituting the higher suborder of primates. One important distinguishing feature is the head, which in Iemurs is more like that of the fox and is drawn out into a long muzzle. The face lacks the humanoid expression characteristic even of the lower apes. The tail, which may be long, is not prehensile; check pouches do not develop, nor do integumental callosities occur, although all these characters are frequently met with in the apes.

The lemurs have certain striking resemblances to other primates, especially in the opposable fingers and toes, flattened digits and pectoral mammae. In all lemurs the second toe is provided with a sharp nail entirely 
unlike the other fingers and toes; while in tarsius the third toe also is furnished with a similar nail (Figs. 9 and 11 ).

Certain important divergences exist between the suborders of primates, which involve the digits of the feet and hands. In lemurs the thumb and great toe are always well developed, while the second or third digit constantly manifests some abnormality; as, for example, a remarkable elongation of the third digit in Chiromys, or the complete absence of the index in Potto (Figs. I 2 and 13). In the Anthropoidea, on the other hand, it is the hallux and the pollex which are subject to most marked variation.

In size the lemur is about equal to the domestic eat. The fur is thick and of ten woolly in texture. The eyes are large and prominent, the ears long, with tufts of hair on their upper portions. The arms are not quite as long as the legs. The tail, usually about half as long as the body, is sometimes inclined to be bushy. Fleshy pads appear on the palms of the hands and feet as well as upon the palmar surface of the fingers, thus permitting the animal to grasp the branches of trees with great tenacity.

\section{The Lemur's Habits in the Wild State}

Little is known of the lemur's habits in the wild state. It is not strictly nocturnal, for some species are seen in search of food during the clay as well as at night. They go in troupes often consisting of many individuals. They are very noisy and live in the forests. One species alone frequents rocky places destitute of trees; namely, Lemur catta. The animals live upon fruits of various kinds, insects, birds' eggs and birds themselves when they can catch them. During the heat of the day they sleep with the head placed beneath the arm and the tail curled about the neck. When walking they go upon the hands and the feet both on the ground and in the trees, the tail being carried above the body in the manner of a balancing or steering organ. Their four limbs are primarily used in locomotion and only to a limited 


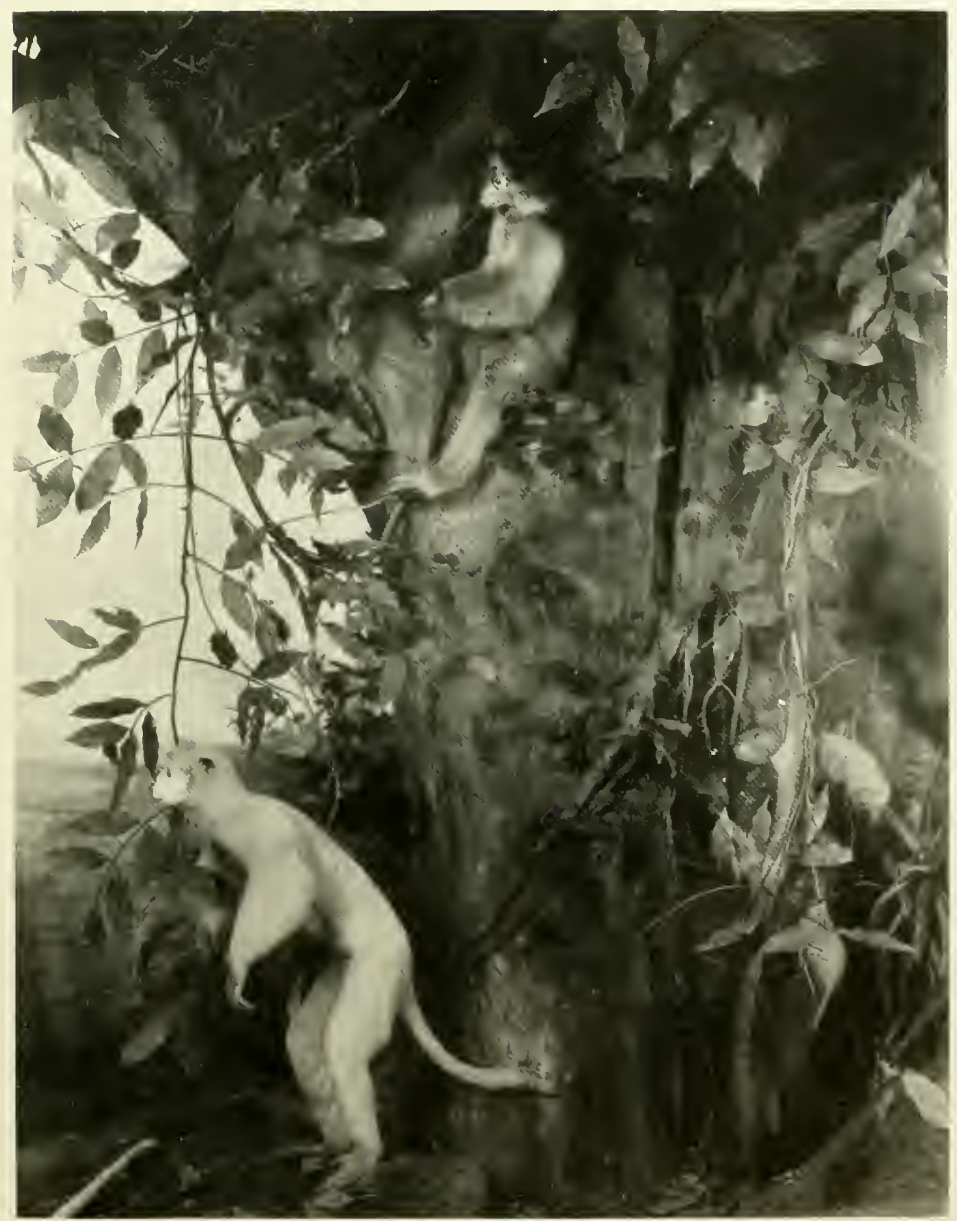

Courtest, American Museum of Natural History

FIG. j. LEMUR GROUP IN MADAGASCAN HABITAT.

$[2 ;]$ 
degree serve the purposes of other volitional aets. The simultaneous movements in their eyes, head and hands necessary to the execution of skilled acts show a much more limited range of adaptation than in some of the higher apes. The tendeney to assume the ereet position or to acquire a sitting posture, even in some partial degree, is not pronouneed. Their development of binocular vision is much less adraneed than in many of the Anthropoids.

\section{Activities of Lemur Mongoz}

The first specimen of the Primates considered in this series is one of the subfamily Lemurinae, Lemur mongoz.

This animal is distributed along the northwest coast of Madagasear, from Baly to Marinda, and is also found in the islands of Anjouan, Comorro, Mohilla and Nossi-Bé. It inhabits the forests and goes in large trompes, keeping to the uppermost branches of the highest trees. Its agility in leaping from tree to tree is most remarkable and so rapid that it ean with difficulty be followed by the eye. Hunters say that it is easier to kill a bird on the wing than a lemur when leaping. If pursued, it has a habit of dropping suddenly from the topmost branches into the bushes, giving the hunter the impression that the animal has been killed. This impression, however, is soon dissipated upon seeing the lemur in another tree at a considerable distance from the spot where it fell.

In the wild state the animals subsist largely upon bananas, but they are also fond of the brains of birds which they are most skillful in capturing. After fracturing the bird's skull with the teeth, as they might a nutshell, they suck the brain out of the brain-case. The lemur, however, does not eat the rest of the bird.

From this description of its activities, the great agility of the animal is evident, as is also the faet that it lives in an environment requiring the utmost 

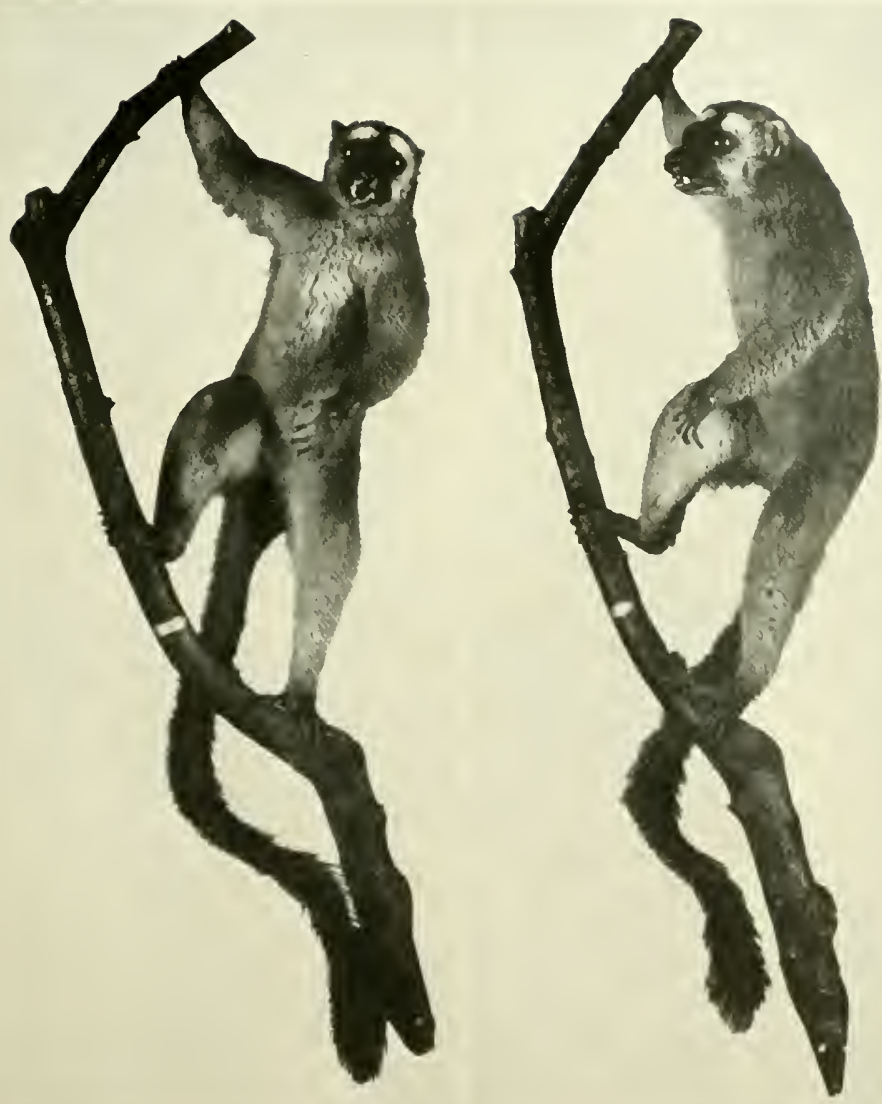

Courtesy, American Museum of Natural History FIGS. 6 AND - TWO VIEWS OF LEMLR MONGOZ. 
nicety in balancing and the greatest precision in all movements.

$$
\text { Measurements and Indices of Lemlr Mongoz }
$$

The measurements of Lemur mongoz are:

Total length.................. 8-6 to $906 \mathrm{~mm}$.

Skull in its occipito-nasal diameter....... 76 to $82 \mathrm{~mm}$.

Skull in its bitemporal diameter........ 27 to $31 \mathrm{~mm}$.

Width of the brain case........... $3 ; \mathrm{mm}$.

The dimensions of the brain, including the cerebellum and brain stem, are $4 ; \mathrm{mm}$. Iongitudinally and $32.5 \mathrm{~mm}$. transversely.

Total weight of the brain............ 18 gms.

Total water displacement............ I7 c.c.

IVeight of the forebrain............ I $4.5 \mathrm{gms}$.

(including endbrain and interbrain)

Weight of the midbrain. ........... I $\mathrm{gm}$.

Weight of the hindbrain ........... 2.5 gms.

Upon the basis of these figures the following encephalic indices have been computed for the several divisions of the brain:

Forebrain index................ 8 I per cent

Midbrain index. ................ ; per cent

Hindbrain index................. $1+$ per cent

A forebrain index of $8 \mathrm{r}$ per cent definitely aligns this anmal with those forms in which the inception of manual development is already under way. The highest forebrain index of what may be called the submanual group, such as the dog, cat and horse, is 80 per cent. The Iemur marks the transition line between the manual and submanual groups. 
Surface Appearance of the Brain in Lenur Mongoz

THE FISSURAL PATTERN

Since it is the purpose of this comparative review to deal with the structural evidence of an adaptive unfolding in the brain, it will be impossible to present or discuss the morphological details with that completeness which might be expected in an atlas on the subject. Especial emphasis is laid upon structural features deemed to possess the greatest evolutional significance. On the other hand, the effort has been made to present the necessary topographical correlations appearing in the several different levels of the brain.

Thus, certain prominent features on the external surface of the brain appear to have such decisive evolutional significance as to require extended consideration. Other features are, so to speak, structurally incidental and are mentioned largely for purposes of identification and topography.

The type of the cerebral hemisphere of Lemur mongoz (Figs. 16 and 17) is gyrencephalic. It presents a relatively simple fissural pattern. There is a general teridency for the major fissure lines to arrange themselves about the Sylvian sulcus, as is the rule in many of the gyrencephalic mammals of the lower orders. On the other hand, the circumsylvian disposition of the sulci in lemur shows a distinct tendency to depart from that concentric arcuate arrangement so characteristic, for example, of the carnivores. The angulation of the fissure of Sylvius in lemur with the base line of the brain is between $55^{\circ}$ and $60^{\circ}$. The defmite circumferential arrangement of the other fissures which surround the fissure of Sylvius disappears to the extent that there may be found only a remnant of the postsylvian sulcus in the form of the sulcus parallelus or superior temporal fissure. The sulcus lateralis also has lost much of its circumsylvian disposition and now exists as the main portion of the interparietal fissure. A well-defined sulcus in the area of transition between the frontal and orbital surfaces constitutes the fronto-orbital sulcus 
and indicates in a general way the position of the inferior frontal sulcus of the higher primates. A faint indentation on the boundary between the parietal and the frontal lobes marks the position of the sulcus centralis, but no
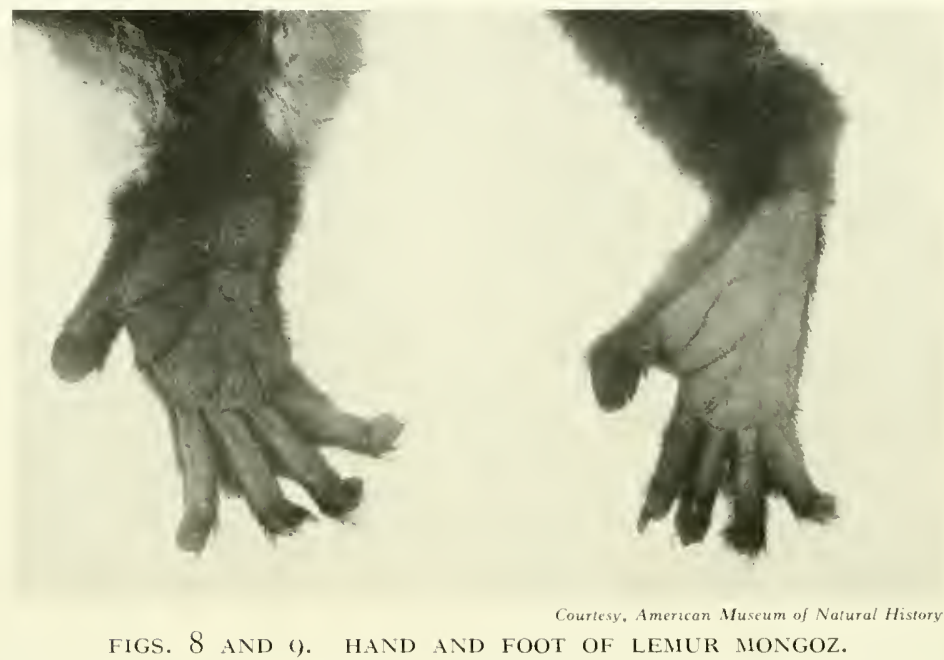

FIGS. 8 AND (). HAND AND FOOT OF LEMUR MONGOZ.

LEFT. Palmar surface of hand showing digitation, palmar creases, phalangeal pads and opposable thumb. Right. Plantar surface of foot showing plantar creases, digitation, long toes, plantar pads, distal phalanges and opposable great toe.

fissural marking corresponding to the suleus simiarum or any oceipital marking on the lateral aspect of the brain is apparent. In fact, such expansion as has occurred in the differentiation of the oceipital lobe confmes itself almost exclusively to the mesial surface of the hemisphere. This primitive development of the occipital lobe leaves a large portion of the cerebellum still uncovered by the overhanging eerebral hemisphere. It thus determines a condition intermediate between the cerebral development in all the monkeys and apes (in which the cerebellum is completely overhung by the cerebral hemisphere) and in lower mammals such as the carnivores (in which the cere- 
bellum is not so covered). The hemisphere of lemur is in many respects intermediate and transitional between the lower mammals and the higher primates (Fig. I 8).

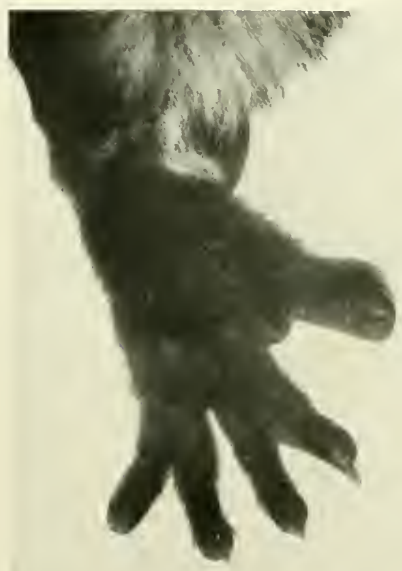

FIGS. IO AND II.

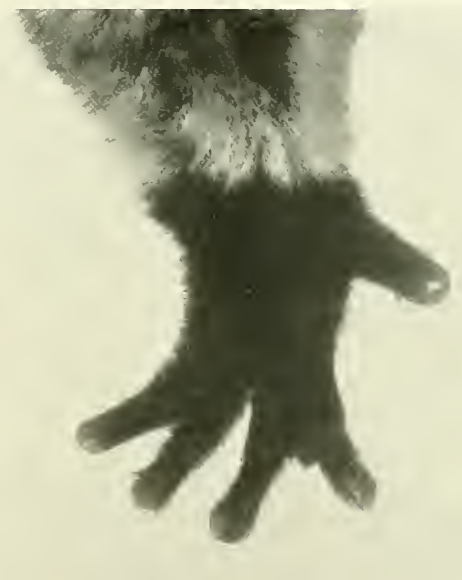

Courtesy, American Museum of Natural History

LEFT. Dorsum of hand showing discrete digitation with tendency to syndactyle.

Right. Dorsum of foot showing toe-nails, digitation with marked syndactyle, and opposable great toe.

CONIOLUTIONS, LOBES AND OTHER SURFACE CHARACTERISTICS

In the convolutions of the lemur brain only the first tendeney may be seen to develop that gyral arrangemnet which is characteristic of the apes. The lobation of the hemispheres is correspondingly limited, the boundary between the frontal and parietal lobes existing as a faint indentation indicating the inception of the sulcus centralis. The division between the parictal and temporal lobes is more clearly defined in the boundary line established by the Sylvian fissure.

The temporal lobe has shown but slight advance over what is characteristic of the carnivores, with the possible exception of the disappearance of 
the rhinal fissure on the lateral aspect, and a slight further protrusion downward of the tip of the temporal Iobe. No actual distinction of an occipital lobe can be made upon the lateral surface although the markings on the mesial
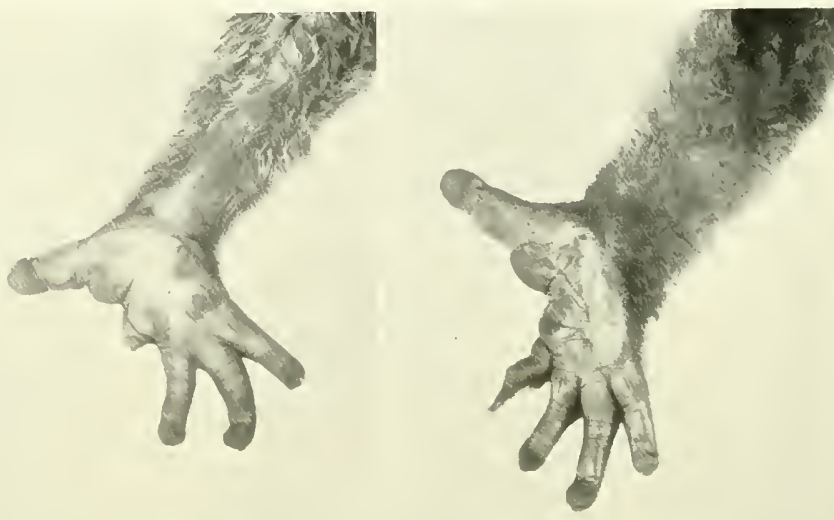

Courtesy, American Museum of Natural Hestory

FIGS. I 2 AND I 3. HAND AND FOOT OF LEMUR POTTO.

LEFT. Palnar surface of hand showing rudimentary development of index finger, palmar creases and opposable thumb.

Righr. Plantar surface of foot showing rudimentary second toe and opposable hallux.

surface show that this specialization of the neopallium has already begun to make definite expansions toward the oceipital pole of the hemisphere.

On the orbital surface of the brain the two orbital concavities are fairly well marked. This surface has a general obliquity outward, which increases the prominence of this concavity and permits the orbital plane to pass over into the frontal surface without sharp angulation in the region of transition. The interorbital keels are well marked. The olfactory bulb is large and detachable for a certain distance, although the olfactory tract is short and not detachable. The latter shows its greatest development in its lateral roots 
extending backward into the Sylvian fossa and exposing at trigonum olfactorium larger than in the higher primates. In the oecipital region there is a deep occipital concavity, particularly about the midline, where the superior
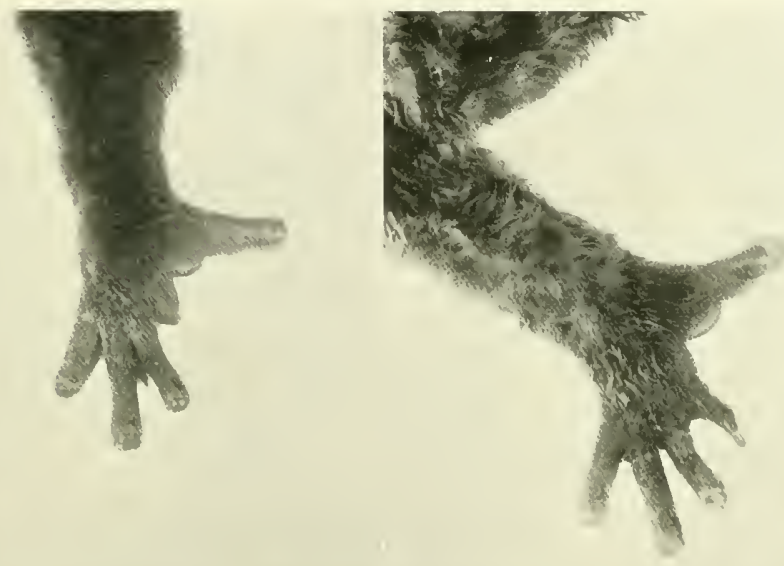

Courtesy, Americun Museum of Nolural History

FIGS, 14 AND Ij. HAND AND FOOT OF LEMUR POTTO.

LEFT. Dorsum of hand showing finger-nails and moderate syndactyle.

Right. Dorsum of foot. Syndactyle not so conspicuous as in Lemur mongoz.

vermis of the ecrebellum is lodged in what may be called the postsplenial fossa. The cerebellum in its tentorial surface presents a prominent superior vermis which appears as a ridge-pole in this sharply gabled surface. Its lateral extension is short because of the limited cerebellar hemisphere. The occipital surface of the cercbellum shows the vermal portion as the most pronounced structure in this area, with two rather insignificant lateral extensions forming the hemispheres (Fig. I 8).

The cerebellum of the lemur shows none of the tendency to form a vallecula into which the vermis gradually sinks on account of the increasing 
prominence of the lateral cerebellar lobes. In other words, the vermis cerebelli is equally as conspicuous as the lateral lobes.

In the tentorial surface, the folial sulci extend without interruption from
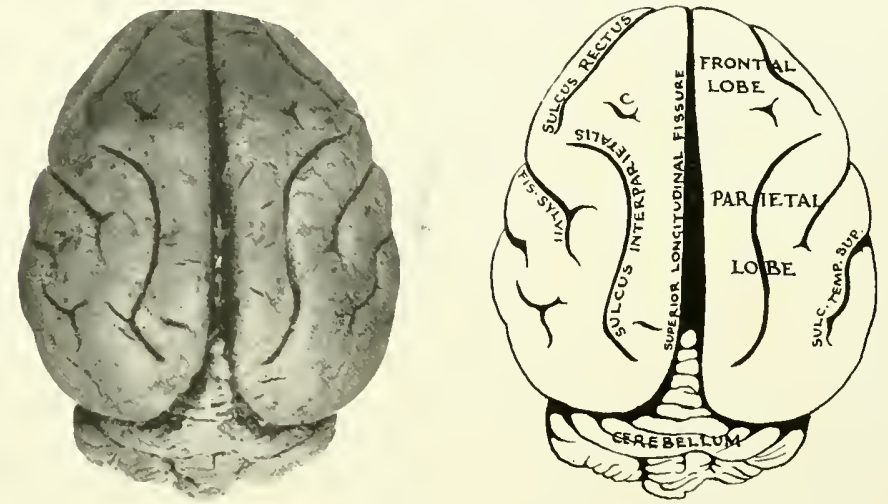

FIG. 16. DORSAL SURFACE OF BRAIN, LEMLR MONGOZ.

[Actual length, $+3 \mathrm{~mm}$.]

Key to Diagran. C., Sulcus Centralis; ris. silvi, Fissure of SyIvius; sulc. Te MP. Sup., Sulcus

Temporalis Superior.

the vermis to the lateral lobes. On the occipital surface, however, two lateral paramedian sulci interrupt the course of the interfolial grooves, as they pass from the vermis to the lateral lobes.

\section{THE BRAIN STEM}

The markings on the several surfaces of the brain stem are not so decisive as in many other species of primates (Fig. 20).

The Oblongata. The oblongata upon its ventral surface presents a ventromedian sulcus and two ventrolateral sulci. In the more cephalic portion of the ventral surface the ventromedian sulcus is flanked on either side by the pyramids which, in turn, are separated from the inferior olivary eminences by the ventrolateral sulci. The pyramids become progressively less well marked as they approach their caudal extremity, at which point 
there are faint indications of the pyramidal decussation. Somecrossing pyramidal strands remain close to the surface and these partially interrupt the ventromedian sulcus at this level.
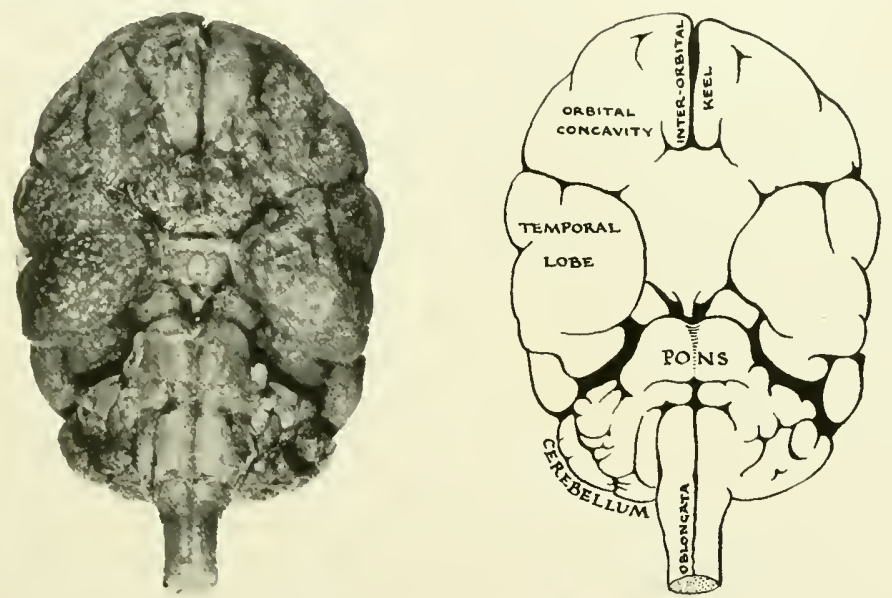

FIG. IT. BASE OF BRAIN, LEMUR MONGOZ.

(II hite dots are due to salt precipitation in fixative.)

The rather feeble relief both of the pyramids and the inferior olives is taken to indicate a comparatively low organization in spheres of action which these two structures represent. In the case of the relatively insignificant pyramids the cortico-spinal connection between the motor cortex and spinal cord must be regarded as correspondingly small and volitional control of motion but poorly developed; likewise, in the case of the inferior olive, the lack of surface prominence suggests a relatively low degree of organization in the reflex control of simultaneous movements in eyes, head and hands, and in the facilitation of the coordination of all skilled learned performances.

The cephalic extremity of the oblongata on its ventral surface comes to an abrupt termination in the slight elevation produced by the pons Varolii, 
the line of demarcation between the pons and the oblongata giving rise to the bulbopontile sulcus.

At the point where the ventromedian suleus meets the bulbopontile
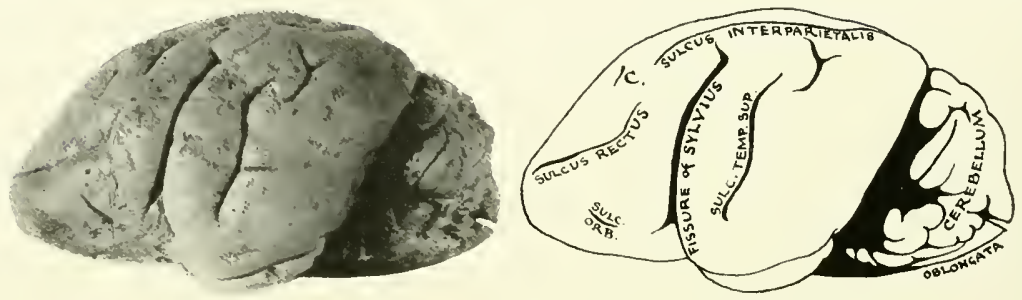

FIG. 18. LEFT LATERAL SURFACE OF BRAIN, LEMLR MONGOZ. [Actual Length, $45 \mathrm{~mm}$.]

hey to Dragran. C., Sulcus Centralis; sulc. orb., Sulcus Orbitalis; stlc. tevip. sup., Sulcus Temporalis Superior.

sulcus, there is a small blind pocket underlying the pons, the foramen cecum posticum. In the sulcus between the inferior olive and the pyramid the twelfth nerve emerges from the oblongata (Fig. 20).

The Pons Varolı. Continuing further cephalad, the brain stem is characterized by the presence of a relatively narrow and flat transverse band, the pons Varolii, lying in front of which is the optico-peduncular space. This space is bounded cephalically by the optic chiasm and optic tracts, and caudally by the convergent fibers of the cerebral peduncles. It contains the tuber cinereum, the region of attachment of the infundibular stalk, the postinfundibular eminence and the mammillary bodies.

A fairly well-pronounced median groove in the pons marks the position of the basilar artery.

In general, the degree of prominence attained by the pons Varolii has been regarded as indicative of neopallial development. The pons provides ultimate connection between the cerebral cortex and cerebellum, and is 
necessary to the coordinative regulation of skilled movements. The small size of the pons in lemur thus suggests a poor capacity on the part of the animal to acquire or execute even a comparatively simple series of skilled acts.
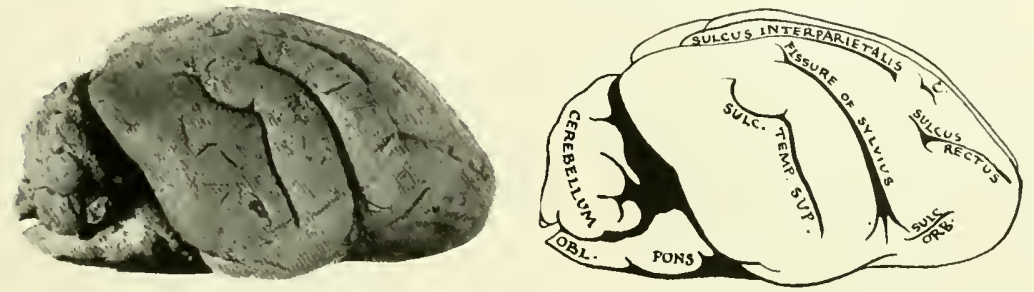

FIG. IO. RIGHT LATERAL SURFACE OF BRAIN, LEMUR MONGOZ.

[Actual Length, $4 ; \mathrm{mm}$ ].

Key to Diagram. C, Sulcus Centralis; obl., Oblongata; sulc, orb., Sulcus Orbitalis; sulc, temp. Sup., Sulcus Temporalis Superior.

The Dorsal Surface of the Brain Stem. The dorsal surface of the brain stem in its axial portion is concealed by the overlapping cerebellum which presents a vermis with two small but well-marked lateral lobes. These lobes are clearly separated from the vermis by two distinct lateral paramedian sulci.

The small cerebellum of lemur emphasizes its limited range of skilled performances, especially as regards skilled acts of the upper extremities in purposes other than those essential to locomotion (Fig. I6).

When the cerebellum is removed by section through its peduncles, the dorsal aspect of the brain stem in its axial portion is revealed. The oblongata presents its typical ventricular and infraventricular portions. In the infraventricular area the dorsomedian seam indicates the line of fusion between the two alar plates of the bulb. The eminences of the clava and cuneus are not prominent. In the ventricular portion of the oblongata the gradual divarication of the alar plates exposing the floor of the fourth ventricle follows the same general outline as in other mammals. The inferior triangle of the 
fourth ventricle is bounded laterally by a fairly prominent clava but a much less conspicuous cuneus. Both of these structures are most prominent in their caudal portions. At their cephalic extremities they become less conspic-
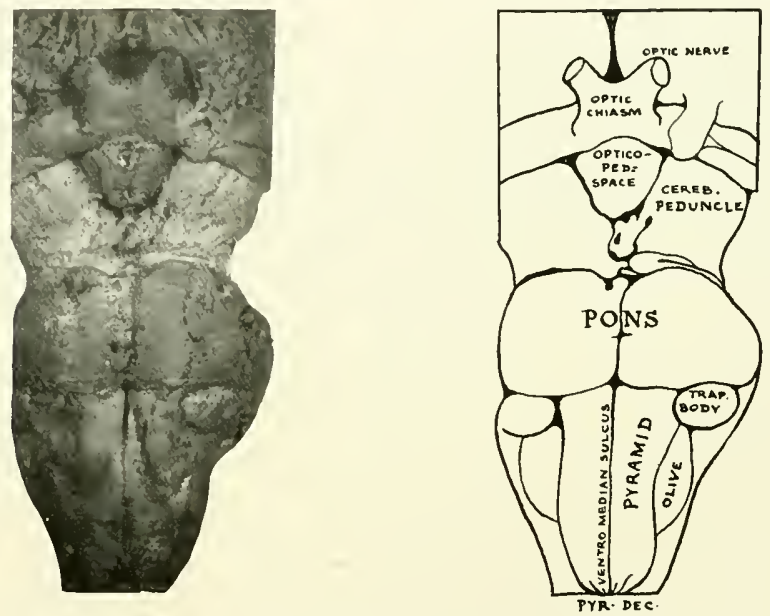

FIG. 20. VENTRAL SLRFACE OF BRAIN STEM, LEMUR MONGOZ. [Actual Length, $3 ; \mathrm{mm}$.]

hey to Diagrais. cereb. Peduncle, Cerebral Peduncle; optico.-Ped.-SPace, Opticopeduncular Space; PYr. DEC, Pyramidal Decussation; trap. Body, Trapezoid Body.

uous as elevations. The decrease in surface relief is more marked in the cuneus than in the clava (Fig. 21 ).

The relatively slight development of the cuneus may be interpreted as due to a sensory representation of the forelimb which, as yet, has gained no marked prominence. The cuneus itself consists of nerve fibers and cells related to the transmission of sensory impulses arising in the upper trunk and forelimb. The types of sensory impulses passing over this conduction path pertain to the discriminative quality of sensibility, i.e., tactile, muscle, 
joint and posture discrimination. Narked prominence of the cuncus is significant particularly of highly developed discriminative sensibility in the hand. Conversely, a lack of such prominence indicates an upper extremity
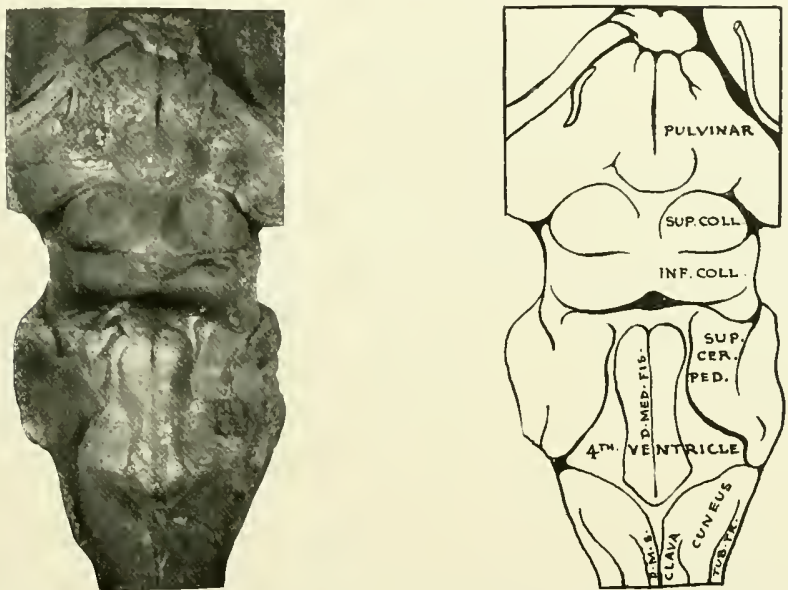

FIG. 21. DORSAL SURFACE OF BRAIN STEM, LEMUR MONGOZ.

[Actual Length, $35 \mathrm{~mm}$.]

Key to Diagray. D. Med. FIS., Dorsomedian Fissure; D.M.S., Dorsomedian Sulcus; inf. Coll., Inferior Colliculus; Sup. CER. PED., Superior Cerebellar Peduncle; SeP. COll., Superior Colliculus; tuB. Tr., Tuberculum Trigemini.

and hand still mainly employed in Iocomotion. Of the two dorsal columns in lemur, the clava and cuneus, the former seems to be structurally emphasized because it represents the path of sensory conduction not only from the leg but also from the important steering and balancing organ, the tail. The cuneus in lemur is indicative of a forelimb as yet wholly inadequate for advanced manual adaptation.

A fairly well-defined dorsolateral sulcus extends upward upon either side of the dorsomedian seam, becoming most prominent in the region between 
the cuneus and the clava. Other markings on the dorsal surface, as well as upon the lateral surface of the oblongata, are faint and may only with difficulty be identified.

The Hindbrain. The axial portion of the metencephalon presents upon its dorsal surface the cephalic continuation of the fourth ventricle, bounded upon either side by the middle and superior cerebellar peduncles. The markings on the floor of the fourth rentricle are not easy to discern. The median sulcus is prominent, running from the region just below the obex cephalad to the beginning of the iter. Well-defined striae acusticae cross the floor of the ventricle at the level of the lateral recesses. Their direction from their point of entry toward the midline is almost at right angles to the median suleus in the floor of the ventricle. Some fibers, however, have an obliquity caudad from their point of entrance toward the midline. In the inferior triangle of the fourth ventricle a shallow depression upon either side of the median sulcus indicates the position of the nucleus hypoglossus, lateral to which is a faintly marked fovea vagi. It is impossible to discover any distinctly demarcated region corresponding to the area postrema or area plumiformis.

The cephalic triangle of the fourth ventricle is devoid of distinct markings, although immediately above the striae acusticae may be seen a slight elevation, the eminentia abducentis. There is no evidence of a locus coerulcus.

The Midbrax. The dorsal aspect of the midbrain presents the characteristic quadrigeminal plate with the median sulcus intersected at right angles by the intercollicular sulcus. At the cephalic extremity of the median sulcus there is a triangular expansion, the fovea pincalis, which lodges the epiphysis cerebri.

The superior colliculi are somewhat larger than the inferior colliculi, but both collicular elevations are well-defined and prominent tectal features. 
Near the cephalic extremity of the midbrain on the lateral aspect of the diencephalon are the mesial and lateral geniculate bodies.

The surface prominences on the ventral aspect of the midbrain, due to the presence of the two cerebral peduncles, present a relatively low relief. This fact is dependent upon the development in this animal of a small pyramidal system. It also indicates that the fibers coming from the cerebral cortex to reach the cerebellum, in the interest of maintaining the proper coordination of the animal's limited skilled movements, are not numerous.

The markings upon the lateral aspect of the midbrain, with the exception of the mesial geniculate body, are not pronounced. In fact, the entire relief of the structures appearing upon the mesencephalic surfaces, with the exception of those features already mentioned, conveys the impression that this is a particularly generalized portion of the neuraxis devoid of many of the striking features characteristic of the higher forms.

Internal Structure of the Brain Stem in Lemur Mongoz

Some features which have a certain degree of prominence in surface outline assume more significant proportions when seen in cross sections of the brain stem. The sections of the axis in the Icmur about to be discussed provide a survey of the internal structure at all of its critical levels. These descriptions begin with the sections at the lower end of the stem and proceed to higher levels of the more expanded portions of the brain.

\section{LEVEL OF THE PYRAMIDAL DECUSSATION (FIG. 22)}

At the level of the pyramidal decussation the outstanding feature of the oblongata is the crossing of the pyramidal fibers (Py $\mathrm{x}$ ) with the consequent separation of the ventral gray column $(V e n)$ from the central gray matter ( Cen) by the prramidal fasciculi ( $\mathrm{Py}_{\mathrm{y}}$ ). 
The pyramidal bundles forming this decussation are relatively narrow, in this respect bearing out the impression gained from the surface relief of the pyramid which indicates a limited capacity for volitional control over

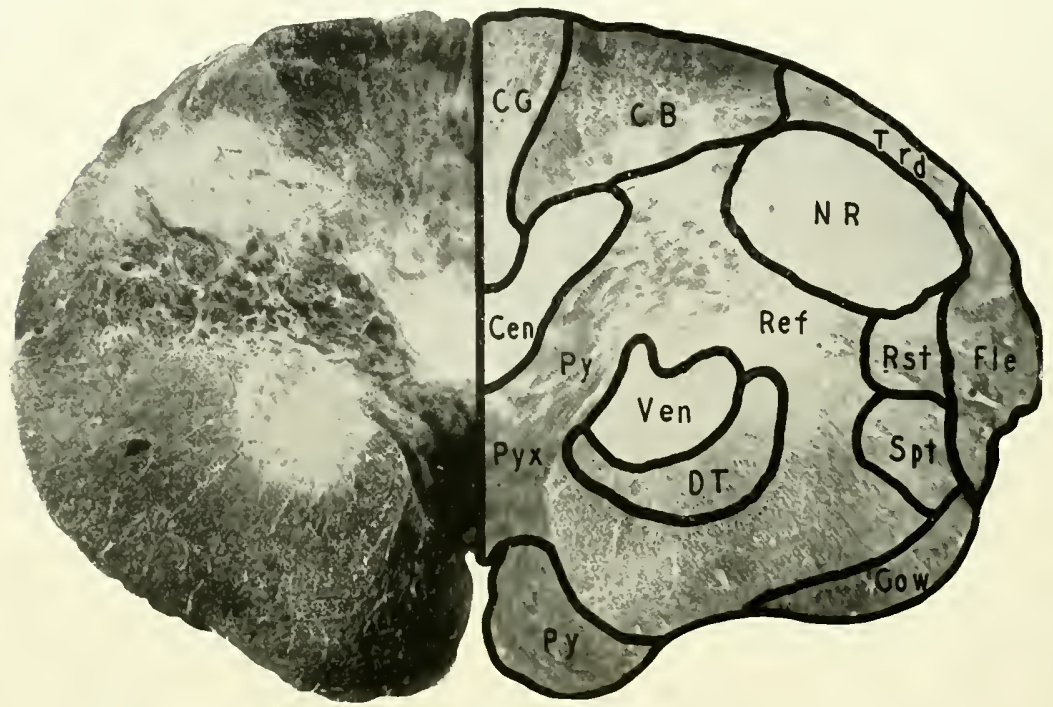

FIG. 22. LEMUR MONGOZ. LEVEL OF THE PYRAMIDAL DECUSSATION.

CB, Column of Burdach; CEN, Central Gray Matter; CG, Column of Goll; DT, Deiterso-spinal Tract; FLE, Dorsal Spinocerebellar Tract; cow, Ventral Spinocerebellar Tract; NR, Nucleus of Rolando; Py, PyramidaI Tract, Pyramidal Fibers; Pyx, Pyrumidal Decussation; REF, Reticular Formation; Rst, Rubrospinal Tract; SPT, Spinothalamic Tract; TRD, Descending Trigeminal Tract; vex, Ventral Gray Matter. [Accession No. 147. ${ }^{*}$ Section 57. Actual Size, $9 \times 5 \mathrm{~mm}$. In ill actual measurements fractions of the millimeter have been disregarded.]

the animal's voluntary muscles. As a corollary it may be inferred that the small pyramidal system bespeaks a small ability on the part of the animal to acquire Icarned reactions. From observation of lemurs in captivity, it is apparent that they learn little more, either by imitation or repetition, than in the free state.

* The numbers mentioned at the end of each caption in this and the following cross sections refer to the Study. Collection of the Columbia University Neurological Department. 
Another feature is the appearance of the caudal extremity of the nucleus of Goll together with an extensive bundle of fibers which surrounds it and forms the column of Goll (CG). This broad field of myelinized axons makes a long ascent from the spinal cord, arising in those levels which represent the dermatomes and proprioceptors of the tail and lower extremity. In Iemur, the nucleus of Goll and the surrounding fasciculus have their special significance in the fact that the adjacent bundle of fibers in the column of Burdach is relatively more extensive. From this it may be inferred that the parts of the body represented by the colımn of Burdach possess more functional prominence than the parts represented by the more mesial collection of fibers in Goll's column. This idea gains support from the fact that the column of Burdach represents the upper extremity. In lemur the manual differentiation of the forelimb is still far from complete. Although all of the principal elements entering into the formation of the hand are present, the upper extremity is in a distinctly low manual stage. The disparity in size of these two columns speaks in favor of a less highly developed functional adaptation in the use of the hindlimb and the tail than in the forelimb and hand.

Another feature in the dorsal region of the section is the marked expansion of the substantia gelatinosa trigemini (nucleus of Rolando) (NR) which serves as a relay station for all fibers bearing impulses from the trigeminal areas of the face and head. This innervation includes regions anterior to the interparietal line drawn across the vertex of the skull from one external auditory meatus to the other. In an animal which has not yet developed a hand to serve in capturing prey for food, the head, and particularly the mouth, affords an important organ of offense and defense. Such an organ must be provided with an adequate sensory apparatus to guide it in these essential efforts of life. Its sensory rôle in directing the course of the animal as it makes its way through the leafy parts of the trees or through 
the underbrush supplements the sense of sight and thus facilitates passage amid obstacles in the environment. For this reason the head and face are amply provided with a sensory equipment.

The entire dorsal field of the section is fundamentally sensory in its significance. Thus a line drawn transversely through the central canal bounds a territory which represents the animal's capacity in discriminative sensibility for the whole budy. This territory may be spoken of as the dorsal field of sensory discrimination. It is the area of the central nervous system which aflords the best index of an animal's capacity in discriminative sensibility. Beginning at the more lateral portion of this sensory freld are the descending tract of the lifth nerve ( $\mathrm{Trd}$ ) and the substantia gelatinosa (NR). These structures represent the head and face. The sensory representation of the neck and arm lies contiguous to the substantia gelatinosa (NR), while occupying a mesial position, adjacent to the dorsomesial septum, is the area concerned in sensory transmission from the tail and the leg (CG). Analysis of this sensory field in lemur indicates that innervation is more generously provided for the leg, tail, face and head than for the forelimb with its slightly differentiated hand. The type of sensibility which this area serves is preeminently discriminative. It is particularly involved in finer adjustments of body posture and contact relations with the outside world. Hence it not only plays an important part in affording discrimination in the analysis of elements encountered in the environment, but must also be regarded as the sensory substratum upon which the complexity of the skilled reactions depends.

Other structures in this level need mention for topographical identification. Among these are the ventral gray column ( Ven) which, because of the decussating bundles of the pyramid ( $P_{y x}$ ), has been detached from the central gray matter $(\mathrm{Cen})$, the latter now lying in contact with the modian line and surrounding the small central canal. Dorsolateral 
to the ventral gray column are the scattered bundles of the pyramidal tract $\left(P_{y}\right)$ as they begin to assemble in the position occupied by them in their descent through the oblongata and in to the spinal cord. On the periphery of the section is a narrow band of medullary substance, the circumferential zone, which contains the two large ascending spinocerebellar tracts (Gow, Fle). Mesial to this zone and bordering upon the gray matter of the ventral gray column is the intermediate zone which in its ventral portion contains the two descending Deiterso-spinal tracts (DT) and, in its lateral portion, the spinothalamic and rubrospinal tracts ( $\left.S_{p} t, R_{s}\right)$.

LEVEL OF THE CALDAL EXTREMITY OF THE INFERIOR OLIVE (FIG. 23)

At the level of the caudal extremity of the inferior olive the general appearance of the section has changed considerably. The chief alteration is due to the presence in the ventral field of a new collection of gray matter. This is the inferior olivary nucleus (IO) which lies immediately dorsolateral to the fiber bundles constituting the pyramid $\left(\mathrm{P}_{\mathrm{y}}\right)$. Dorsal to the olive is a smaller mass, the dorsal accessory olive. Situated in front of the central gray matter $(\mathrm{Cen})$ are fibers (Fai) arising in the dorsal nuclear masses, which pass inward and forward to the midline where they undergo decussation. At this point they turn upward and constitute the mesial fillet. These decussating axons are internal arcuate fibers. Most of them take origin in the nucleus of Goll (NG) and contribute to the formation of the lowest portion of the mesial fillet which here represents the lower extremity and tail. The pyramid $\left(P_{y}\right)$ appears as a compact bundle occupying the most ventromesial portion of the section. Its transverse and anteroposterior diameters give it the proportion of about I to I to the entire section. This ratio conveys some impression as to the relative importance of the pyramidal system in the 
regulation of volitional movements. The dorsal columns of Goll and Burdach ( CG, CB) are considerably increased in extent due to the presence in them of nuclear masses constituting the nucleus of Goll (NG) and the

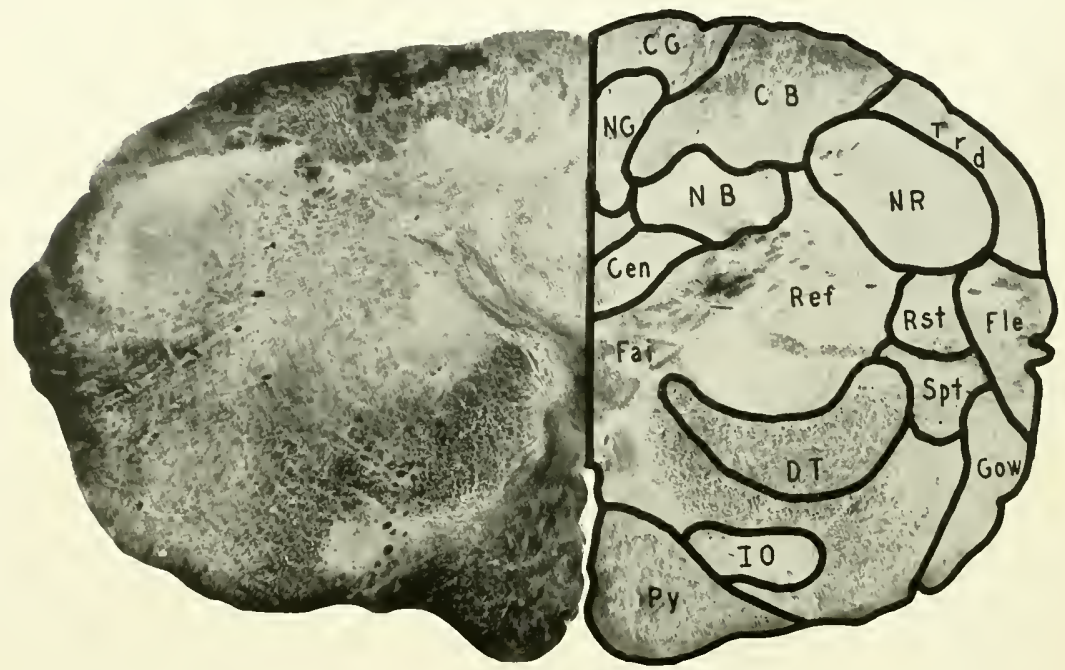

FIG. 23. LEMLR MONGOZ. LEVEL OF CALDAL EXTREMITY OF INFERIOR OLIVE.

$\mathrm{CB}$, Column of Burdach; CEN, Central Gray Matter; CG, Column of Goll; DT, Deiterso-spinal Tract; FAI, Internal Arcuate Fibers; FLe, Dorsal Spinocerebellar Tract; cow. Ventral Spinocercbellar Tract; Io, Inferior Olive; NB, Nucleus of Burdach; NG, Nucleus of Goll; NR, Nucleus of Rolando; PY, Pyramidal Tract and Fibers; REF, Reticular Formation; RST, Rubrospinal Tract; SPT, Spinothalamic Tract; TRD, Descending Trigeminal Tract. [Accession No. 147. Section 75. Actual Size, ro $\times 6 \mathrm{~mm}$.]

nucleus of Burdach (NB). It is evident that the nucleus of Goll is smaller than that of Burdach. This appearance bears out the point already made as to the sensory predominance of the more lateral of the two bundles in the dorsal field. The fibers representing the hand and arm are more numerous and constitute a more conspicuous bundle than those representing the leg. In a similar manner, the substantia gelatinosa of Rolando (NR), as well 
as the descending tract of the fifth nerve ( $\mathrm{Trd}$ ), stand out as prominent elements and add to the preeminent sensory character of the clorsal field.

Dorsal to the pyramidal tract and ventral to the fillet is an important bundle of fibers, namely the posterior longitudinal fasciculus, which is concerned with the automatic rellexes for the regulation of certain primitive movements. Lateral to the fasciculus longitudinalis posterior is a considerable bundle representing the Deiterso-spinal tracts (DT), whose function is the conduction of those impulses necessary to the adjustments of equilibrium.

\section{LEVEL THROLGH THE WIDDLE OF THE INFERIOR OLIVI}

$$
\text { (FIGS. } 24 \text { AND } 2 j \text { ) }
$$

At the level through the middle of the inferior olive, the outstanding feature is the appearance of an irregular mass of gray matter lying in the ventral field near the pyramid. This is the inferior olivary nucleus (IO). Its significance has already been touched upon in relation to the automatic regulation of simultancous movements of the head, eyes and hand and to the facilitation of the coordination of all skilled learned performances. This structure has a direct connection, through the central tegmental tract, with the oculomotor nuclei and, through the olivary fasciculus of Helweg, with the cervical levels of the spinal cord. By means of many arcuate fibers it is also connected with the cerebellum. While there is no difficulty in identifying this structure in lemur, it is smaller and much more indefinite in outline than in most of the other species considered. The lemur thus appears to be provided with but a small amount of automatic regulation of simultaneous movements of hands, head and eyes. Mesial to the olive and adjacent to the midline of the section is the mesial fillet ( $\mathrm{If}$ ). This structure represents the continuation of the discriminative sensory pathway, being made up largely of fibers for sensation in the extremities. The substantia gelatinosa (NR) and the accompanying descending trigeminal tract 
( Trd) are still prominent, while the dorsal columns of Goll and Burdach again give evidence that the more mesial of these fasciculi, representing the leg and tail, is larger than the lateral one representing the upper extremity.

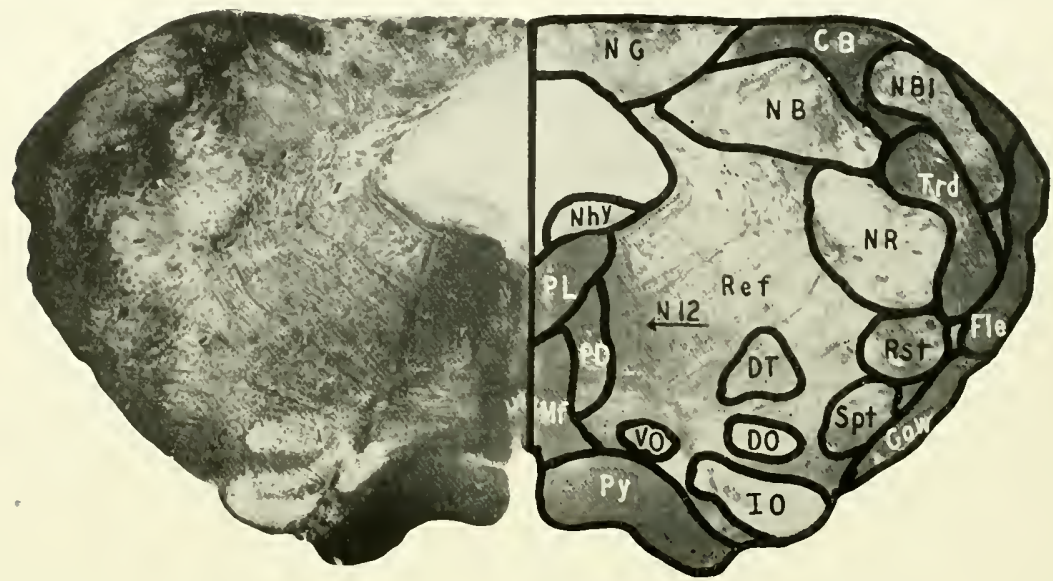

FIG. 24. LEMUR MONGOZ. LEVEL THROUGH MIDDLE OF INFERIOR OLIVE.

CB, Column of Burdach; Do, Dorsal Accessory Olive; DT, Deiterso-spinal Tract; FLE, DorsaI Spinocerebellar Tract; Gow, Ventral Spinocerebellar Tract; to, Inferior Olive; MF, Mesial Fillet; NB, Nucleus of Burdach; vis, Nucleus of Blumenau; NG, Nucleus of Goll; NHy, Hypoglossal Nucleus; NR, Nucleus of Rolando; Nı2, Hypoglossal Nerve; PD, Predorsal Bundle; PL, Posterior Longitudinal Fasciculus; Py, Pyramid; REF, Reticular Formation; RST, Rubrospinal Tract; sPT, Spinothalamic Tract; TRD, Descending Trigeminal Tract; vo, Ventromesial Accessory Olive. [Accession No. 147. Section 102. Actual Size, I I X $5 \mathrm{~mm}$.]

LEIEL OF THE VESTIBULAR NUCLEI (FIG. 26)

At the level of the vestibular muelei some noteworthy changes have occurred. They result primarily from the introduction of two additional sensory systems. One of these additional elements in the sphere of sensation, namely, the tuberculum acusticum $(T u b)$ is related to the sense of hearing; the other, connected with the function of balancing, is the vestibular 
area. The tuberculum acusticum receives the fibers which arise in the cochlear portion of the internal ear. In lemur it is particularly large, thus indicating a highly developed auditory sense.

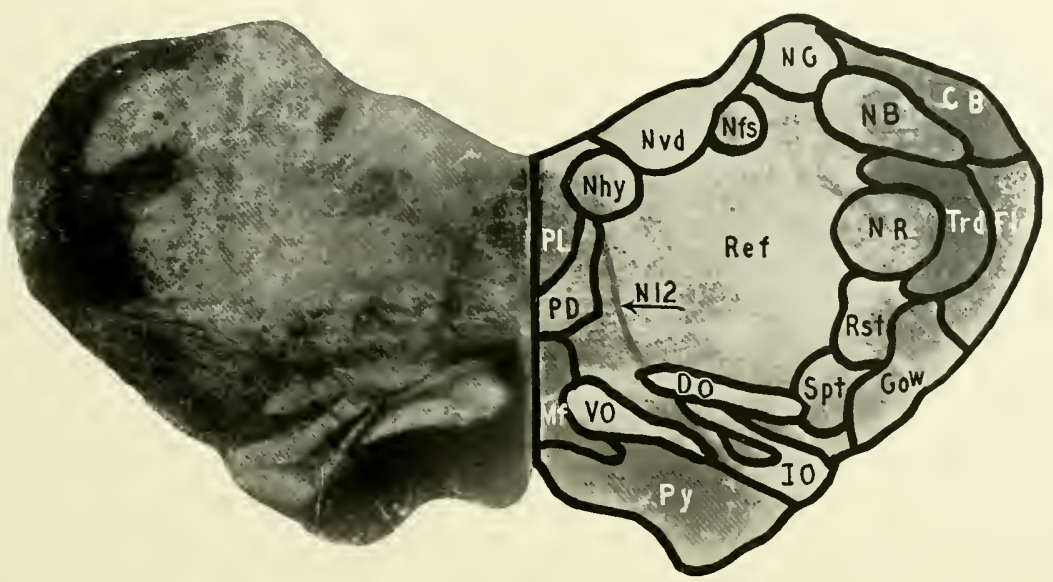

FIG. 2j. LEMUR MONGOZ. LEVEL THROLGH MIDDLE OF INFERIOR OLIVE.

$\mathrm{CB}$, Column of Burdach; Do, Dorsal Accessory Olive; FLE, Dorsal Spinocerebellar Tract; Gow; Ventral Spinocerebellar Tract; 10, Inferior Olive; $\mathrm{MF}$, Mesial Fillet; NB, Nucleus of Burdach; . NFs, Nucleus Fasciculus Solitarius; NG, Nucleus of Goll; NHr, Hypoglossal Nucleus; NR, Nucleus of Rolando; NvD, Dorsal V'agal Nucleus; NI 2 Hypoglossal Nerve; PD, Predorsal Bundle, PL, Posterior Longitudinal Fasciculus; Py, Pyramid; REF, Reticular Formation; RST, Rubrospinal Tract; SPT, Spinothalamic Tract; TRD, Descending Trigeminal Tract; vo, Ventromesial Accessory Olive. [Accession No. 147. Section 117. Actual Size it $X+\mathrm{mm}$.]

In the region formerly occupied by the tracts and the nuclei for the conduction of impulses coming from the skin, the joints, the muscles and the bones of the extremities, there now appear two groups of cells and fibers for the reception of impulses arising in the proprioceptive organs of the restibule; i.e., the semicireular canals, utricle and saceule. These highly specialized parts of the internal ear play a fundamental rôle in equilibrium. Near the midline, in the dorsal freld, immediately beneath the floor of the fourth 
ventricle, is the triangular nucleus of Schwalhe (NSc), and lateral to this a large-celled nucleus known as Deiters' nucleus (ND). Together they constitute the restibular area. Dispersed among the cells of Deiters'nucleus

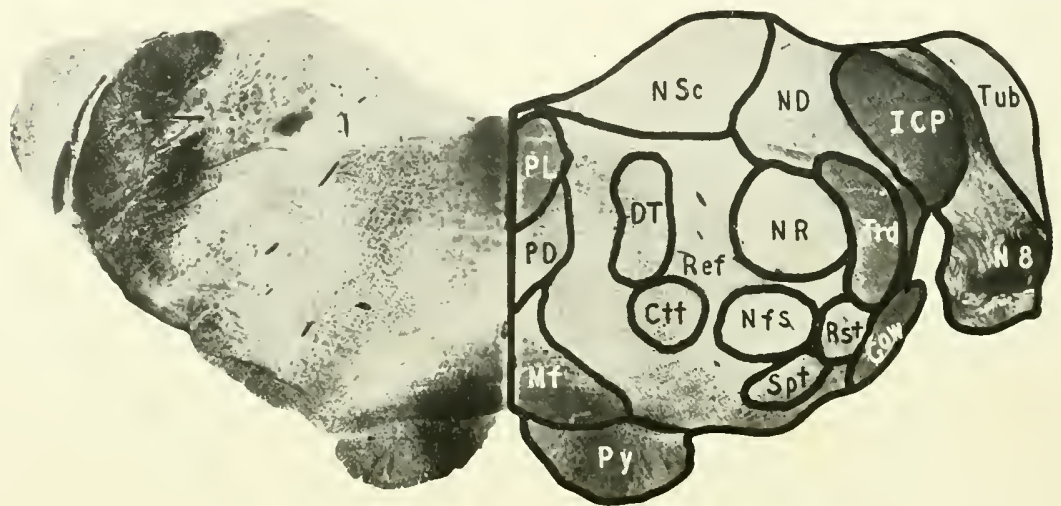

FIG. 26. LEMUR MONGOZ. LEVEL OF THE VESTIBULAR COMPLEX.

CтT, Central Tegmental Tract; DT, Deiterso-spinal Tract; Gow, Ventral Spinocerebellar Tract; 1CP, lnferior Cerebellar Peduncle; mF, Mesial Fillet; ND, Nucleus of Deiters; nfs, Facial Nucleus; nr, Nucleus of Rolando; isc, Nucleus of Schwalbe (Triangularis); . 8, Auditory Nerve; pD, Predorsal Bundle; PI., Posterior Longitudinal Fasciculus; PY, Pyramid; REF, Reticular Formation; RST, Rubrospinal Tract; SPT, Spinothalamic Tract; TRD, Descending Trigeminal Tract; TUB, Tubereulum Acusticum. [Accession No. 147. Section 138. Actual Size, $1+x+\mathrm{mm}$.]

are many scattered bundles of nerve fibers representing essential connections of this nucleus. The relative dimensions of the two vestibular nuclei to the rest of the cross section arc of especial internst. They provide a basis for estimating the degree of balancing function possessed by the lemur, whose life is spent primarily in the loftiest parts of trees and whose locomotion is adapted to the constantly varying movements of the tree tops. The animal is able to execute difficult locomotor feats with great precision and finds as much security in its balancing under these most difficult circumstances as the quadruped does upon terra firma. It is quite in keeping, therefore, with the inherent behavioral requirements, to find the central representation of 
this important proprioceptive system so highly developed in these tree-living animals.

Situated between the tuberculum acusticum ( Tub) and Deiters' nucleus is the restiform body. (ICP) which represents an aggregation of ascending fibers from the spinal cord and oblongata on their way to the cerebellum. These ascending cerebellar fibers come from several different sources and serve the purpose of conveying to the cerebellum afferent impulses necessary, as it were, to orient the cerebellum in its activity of transmitting to the muscles those impulses essential to coordination. An exact adjustment of action exists between the various groups of muscles in the body in order to maintain such coordination. Since the cerebellum is specialized to regulate the factors cntering into this function, it naturally follows that the organ must at all times be in possession of information concerning the varying tensional status of all the muscles of the body. Nere this not the case it would be impossible for the cerebellum to transmit to the muscles the tensional control essential to the execution of all muscular acts. Thus the significance of the afferent cerebellar connections as indicated by the restiform body may be clearly discerned. The degree of development of this structure discloses the extent to which the animal is dependent upon its coordinating mechanism.

\section{LEVEL OF THE CEREBELLAR NUCLEI (FIG. 27)}

At the level of the cerebellar nuclei certain structures which furnish a criterion concerning the functional capacity of the cerebellum make their appearance for the first time. These are the cerebellar nuclei. Only a portion of the cerebellum is shown in this cross section. Structurally, the organ consists of a central division, the vermis ( Ver), and two lateral lobes or hemispheres. The beginning of the lateral lobe is indicated in the cross section. Immediately mesial to the mass of medullary substance 
of the lateral lobe is a large, irregular and rather diffuse collection of eclls, the nucleus dentatus $(\mathrm{Ndt})$. From this nucleus arise the fibers which make their way out of the cerebellum, providing the efferent pathway for

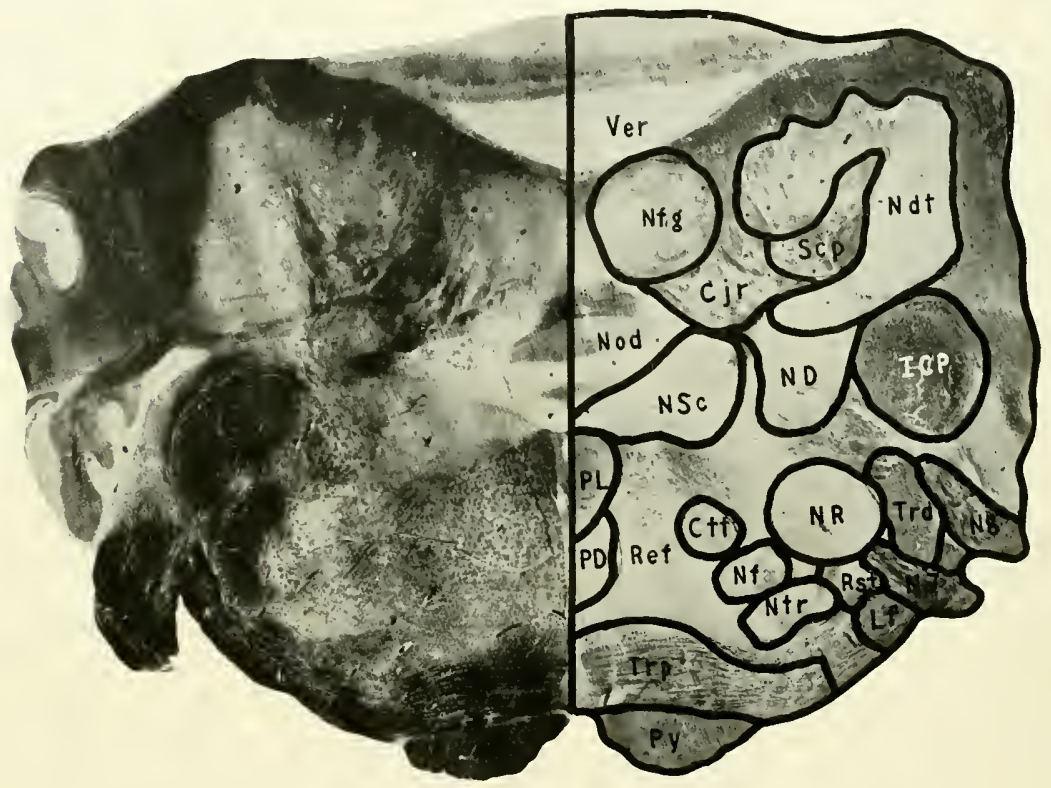

FIG. 2-. LEMUR MONGOZ. LEVEL OF THE CEREBELLAR NLCLEI.

UR, Juxtarestiform Body; CTT, Central Tegmental Tract; ICP, Inferior Cerebellar Peduncle; LF, Lateral Fillet; ND, Nucleus of Deiters; NDr, Dentate Nucleus; NF, Facial Nucleus; NFG, Nucleus Fastigii; NoD, Vermis Cerebelli; NR, Nucleus of Rolando; NsC, Nucleus of Schwalbe (Triangularis); NTR, Trapezoid Nucleus; N7. Facial Nerve; N8, Auditory Nerve; PD, Predorsal Bundle; PL, Posterior Longitudinal Fasciculus; PY, Pyramid; REF, Reticular Formation; RST, Rubrospinal Tract; SCP, Superior Cerebellar Peduncle; TRD. Descending Trigeminal Tract; Trp, Trapezoid Body; VER, Vermis. [Accession No. 147. Section 163. Actual Size, $15 \times 10 \mathrm{~mm}$.]

cerebellar impulses which regulate the coordinative control of the muscles. The relative size of the dentate nucleus is consequently of much significance, as it indicates to what degree the cerebellum contributes to the function of 
coordination. The nucleus may be, as in this instance, an irregular, dilluse mass of gray matter, or it may present itself as a complexly convoluted structure whose convolutions serve to increase its functional capacity. An animal having a small dentate nucleus should be capable of a relatively limited range of highly coordinated acts. It is of interest to note that in lemur this nucleus is neither well defmed nor extensive in size, from which it may be inferred that the degree of coordinative control dependent upon the cerebellum is relatively less in this animal than in some of the higher primates.

Mesial to the dentate nucleus is another aggregation of nerve cells. This is the nucleus fastigii $(\mathrm{Nfg})$. By means of fibers constituting the juxtarestiform body $(\mathrm{Cjr})$, it is connected with those nuclei in the floor of the fourth ventricle which receive impulses from the semicircular canals, utricle and saccule. The nucleus fastigii is, therefore, a structure intimately concerned with the function of balancing and may be regarded as one of the higher elaborating stations for equilibratory control. Its comparatively Jarge size in lemur has a significance similar to that of the large nuclei of Schwalbe and Deiters, unquestionably indicating the great need on the part of the animal for a highly organized balancing control.

It is presumed that functionally the vermis ( $V$ er ) is concerned with the maintenance of coordinative control of the axial and paraxial musculature of the body. This is a portion of the cerebellum which manifests the greatest phyletic constancy, whereas the Jateral lobes, because they are functionally related to the muscles of the extremities, vary according as the upper and lower extremities are capable of more or less complex performances. Another feature at this level is the trapezoid body $(\operatorname{Tr} p)$, and adjacent to it the trapezoid nucleus ( $\mathrm{Ntr}$ ). The trapezoid body is situated immediately dorsal to the py ramid $\left(\mathrm{P}_{\mathrm{y}}\right)$ and consists of a complex system of decussating fibers. These crossing fibers represent the decussation in the 
secondary auditory pathway. They provide the means for the conveyance of impressions received by the cochlea of the internal ear to the higher auditory centers. Immediately lateral to the trapezoid body is a dense bundle of fibers, the lateral fillet (Lf), constituting the secondary pathway for auditory impulses. The nucleus of the seventh nerve which supplies motor innervation to the facial muscles ( $\mathrm{Nf}$ ) and a spray of libers, the first part of the seventh nerve, which extends dorsomesially toward the floor of the ventricle, are also features of this level.

LEVEL OF THE EMERGENT FIBERS OF THE SINTH NERIE (FIG. 28)

At the level of the emergent fibers of the sixth nerve, some minor changes have occurred which are noteworthy as preparing the way for the marked transition observed in the next succeeding sections. One significant feature is the appearance of these emergent fibers of the abducens nerve, the sixth nerve of the cranial series, which arises in a nucleus situated near the midline beneath the floor of the fourth ventricle. These emergent fibers proceed forward and outward, penctrating the corpus trapezoideum in their course and funally emerging at the junction of the ventrolateral and bulbopontile sulci. The abducens nucleus supplies the external rectus muscle of the cyeball and has special signifreance in that it sets the pace for all movements of lateral gaze involving both eyeballs. The appearance of the superior cercbellar peduncle, taking origin in the dentate nucleus, adds another feature to this level. This bundle represents the diserete aggregation of nerve fibers by means of which impulses arising in the cerebellum leave this organ on their way to the several levels of the neuraxis. They have their ultimate distribution to the muscles in the interest of proper coordination. The superior cerebellar peduncle is the one great pathway out of the cerebellum; hence its relative size furnishes a valuable index of cercbellar function. Another peduncular structure connected with 
the cerebellum also appears at this level, the middle cerebellar peduncle. The significance of this peduncle becomes more evident at the higher levels of the stem. Functionally it is the final connecting link between the cerebral

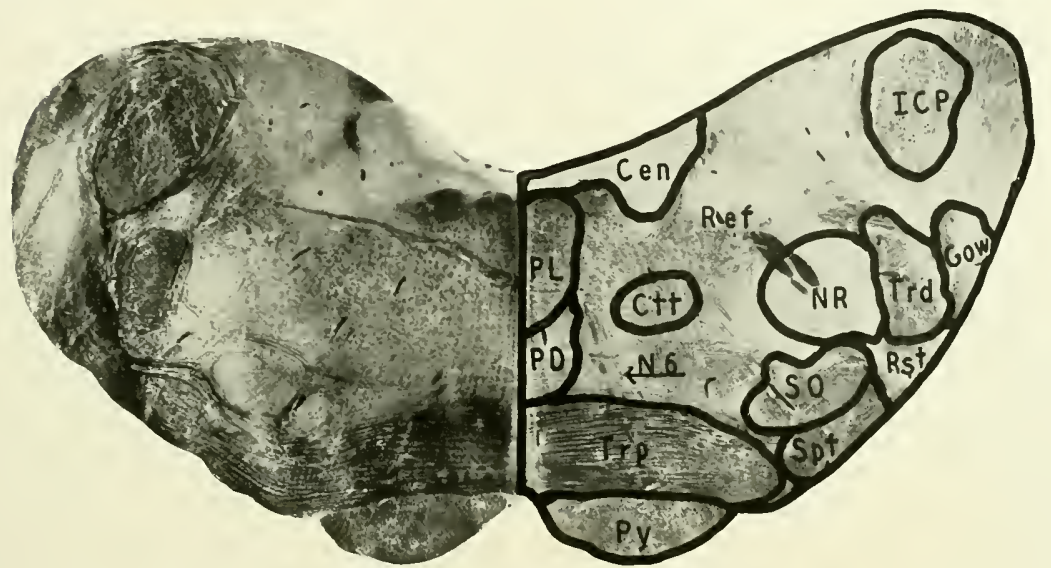

FIG. 28. LEMUR MONGOZ. LEVEL OF EMERGENT FIBERS OF SINTH NERVE.

CEN, Central Gray Matter; ctr, Central Tegmental Tract; Gow, Ventral Spinocerebellar Tract; ICP, Inferior Cerebellar Peduncle; NR, Nucleus of Rolando; N6, Abducens Nerve; PD, Predorsal Bundle; PL, Posterior Longitudinal Fasciculus; Pr. Pyramid; ReF, Reticular Formation; RST, Rubrospinal Tract; so, Superior Olive; SPT, Spinothalamic Tract; TRD, Descending Trigeminal Tract; TRP, Trapezoid Body. [Accession No. 14-. Section 173. Actual Size, $1 ; \times ; \mathrm{mm}$.]

hemispheres and the lateral lobes of the cerebellum. The inferior eerebellar peduncle has begun to spread out its fasciculi like a fan as it enters the medullary substance of the cerebellum immediately lateral to the dentate nucleus. Much significance attaches to the physiological importance of these three cerebellar pechuncles. Collectively they constitute a reliable index for estimating the clegree of coordination possessed by the animal, and thus reveal the extent to which the more complex muscular performances have been developed. The size of the inferior cerebellar peduncle provides a 
scale for measuring the relative amount of afferent inflow to the cerebellum. The superior cerebellar peduncle gives a similar opportunity regarding the relative outflow from the cerebellum, while the middle cerebellar peduncle furnishes the means of estimating the degree of communicational capacity between the cerebral cortex (where all courses of sustained volitional action take origin) and the cerebellum which coordinates the movements of such action.

LEYEL OF THE CAUDAL EXTREMity OF THE PONS VAROLII (Fig. 29)

At the level of the caudal extremity of the pons Varolii, several marked changes have occurred. Most conspicuous among these is the appearance of a fairly wide band of transverse frbers extending across the ventral surface of the brain stem and constituting the stratum superficiale of the pons Varolii. This layer has now become the most ventral element in the neuraxis. It lies in front of the pyramidal fibers ( Py) which, in consequence, have lost their surface position and are no longer seen in relief along the ventral aspect of the stem. The appearance of the pyramidal tract itself is also altered in such a manner that it no longer maintains its compact solidarity. It now appears as a collection of seattered bundles. This dissemination of the pyramidal fasciculi is due to a number of transverse pontile fibers which weave themselves among the descending pyramidal bundles and thus constitute a complex layer of the pons Varolii known as the stratum complexum. The appearance in this stratum of numerous nerve cells still further complicates the structure. These cellular elements form an irregular mass of gray matter of considerable size scattered amidst the pyramidal and transverse pontile fibers. They constitute the pontile nuclei $(\mathrm{PN})$. From the degree of development, both in the pontile nuclei and the transverse pontile fibers, inference may be drawn regarding the range of skilled volitional movements with which the animal is endowed. The anatomi- 
cal and physiological reasons for this inference are not far to seek. Such fibers as constitute the transverse fasciculi of the pons have their origin in the cerebral cortex of the frontal, parietal, occipital and temporal lobes. Descend-

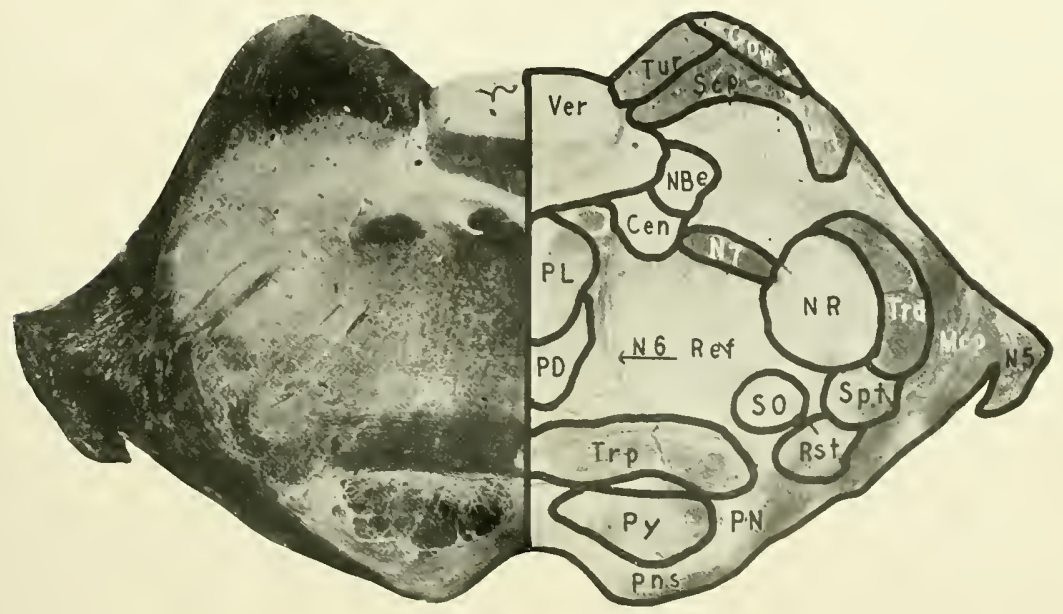

FIG. 29. LEMLR MONGOZ. LEVEL OF CAUDAL EXTREMITY OF PONS VAROLII.

Cen, Central Gray Matter; Gow, Ventral Spinocerebellar Tract; MCP, Middle Cerebellar Peduncle; NBE, Nucleus of Bcchterew; NR, Nucleus of Rolando; N5, Trigeminal Nerve; N6, Abducens Nerve; N7, Facial Nerve; PD, Predorsal Bundle; PL, Posterior Longitudinal Fasciculus; PN, Pontile Nuclei; PNS, Pons; Pr, Pyramid; ReF, Rețicular Formation; RST, Rubrospinal Tract; sCP, Superior Cerebellar Peduncle; so, Superior Olive; SPT, Spinothalamic Tract; TRD, Descending Trigeminal Tract; TRP, Trapezoid Body; TUR, Tractus Uncinatus of Russel (Hook Bundle); VER, Vermis. [Accession No, 147. Section 188. Actual Size, [5 $57 \mathrm{~mm}$.

ing from these origins they make their entrance into the cerebral peduncle and ultimately reach the pons. At this point they alter their direction from the vertical to the horizontal plane, thereafter pursuing a transverse course. Many of these fibers end in the pontile nuclei of the same side for a relay here. The relaying axons then undergo decussation across the midline and eventually enter the middle cerebellar peduncle ( $\mathrm{lcp}$ ). Some pallio- 
pontile fibers, however, cross the midline and receive their relay in the contralateral pontile nuclei whose axons in turn pass into the middle cerebellar peduncle. This peduncle extends into the cercbellum and its fibers finally ramify among the various lobulcs constituting the lateral cerebellar lobes. By means of the pallio-pontile fibers the major functional divisions of the cerebral hemispheres establish chirect communication with the lateral lobes of the cerebellum. Experimental and clinico-pathological observations warrant the opinion that these connections are essential to proper coordination in the more complex motor performances of the animal. The pathway between the cerebral cortex and the cerebellum, in a functional sense, parallels the conduction tract which conveys impulses necessary to the voluntary control of action. The latter pathway provides for the transmission of nervous energy involved in the purpose and pattern of the act to be performed. This includes the incentive, initiation, design, direction and ultimate inhibition of the act. The pallio-pontile connections provide for concurrent impulses which regulate the coordinative and postural attributes necessary to the execution of such voluntary performances.

The slight degree of development in the transverse fibers of the pons, as well as in the pontile nuclei and the middle cerebellar peduncle in lemur, points conclusively to a motor organization capable of but a limited range and variety of the more highly synthetized voluntary performances. This fact is corroborated by the rather feeble development of the pyramidal system. In this sense the pons Varolii and its several constituents may be accepted as an index of the extent to which the cerebral cortex has developed. They provide a structural basis for estimating the range of adaptation and degree of volitional adjustment of which the animal is capable.

The position of the cerebellum is similar to that of the lower Ievels, and the cross section shows espccially well the medullan vestibule consisting of all the frbers assembled from the middle and inferior cerebellar pedun- 
cles, which near its center contains the much reduced cephalic extremity of the nucleus dentatus. At a considerable distance ventrally from the dentate nucleus is the superior cerebellar peduncle ( $S c p$ ). At the lateral extremity of the section and penetrating the superficial pontile fibers, the fifth cranial nerve $\left(N_{j}\right)$ makes its way into the brain stem. These fibers constitute the motor and sensory roots of the trigeminal nerve, the motor root of which may be traced to its nucleus of origin close in the angle of the fourth ventricle, the nucleus masticatorius of the fifth nerve supplying the muscles of mastication.

\section{LEVEL AT THE MIDDLE OF THE PONS VAROLII (FIG. 30)}

At the level at the middle of the pons Varolii this structure attains its full dimensions and displays its three major layers, the stratum superficiale, the stratum profundum and the intermediate stratum complexum. Scattered among the transverse fibers of the stratum complexum are the much separated bundles of the pyramidal system (Py) and also the accumulated mass of gray matter constituting the pontile nuclei (PN). The fibers emerging from the pontile nuclei become collected both in the superficial and deep layers of the pons and pass laterally to enter the bundle constituting the middle cerebellar peduncle ( $\mathrm{Icp}$ ). As already indicated, the pontile nuclei, together with the fibers entering into and forming the middle cerebellar peduncle, may be accepted as evidence concerning the degree of cooperative innervation existing between the portions of the brain having to do with skilled movements and the coordination of such movements. The relatively small size of these structures in lemur points to a correspondingly limited range of skilled performances.

That portion of the axial stem, which comprises the several layers of the pons and contains the pontile nuclei together with the scattered fibers of the pyramidal fasciculi, constitutes what is known as the basis, whose 
dorsal boundary is created by a narrow band of transversely disposed fasciculi forming the mesial fillet (Mf). This line of boundary indicates the division between the basis pontis situated ventrally and the tegmentum

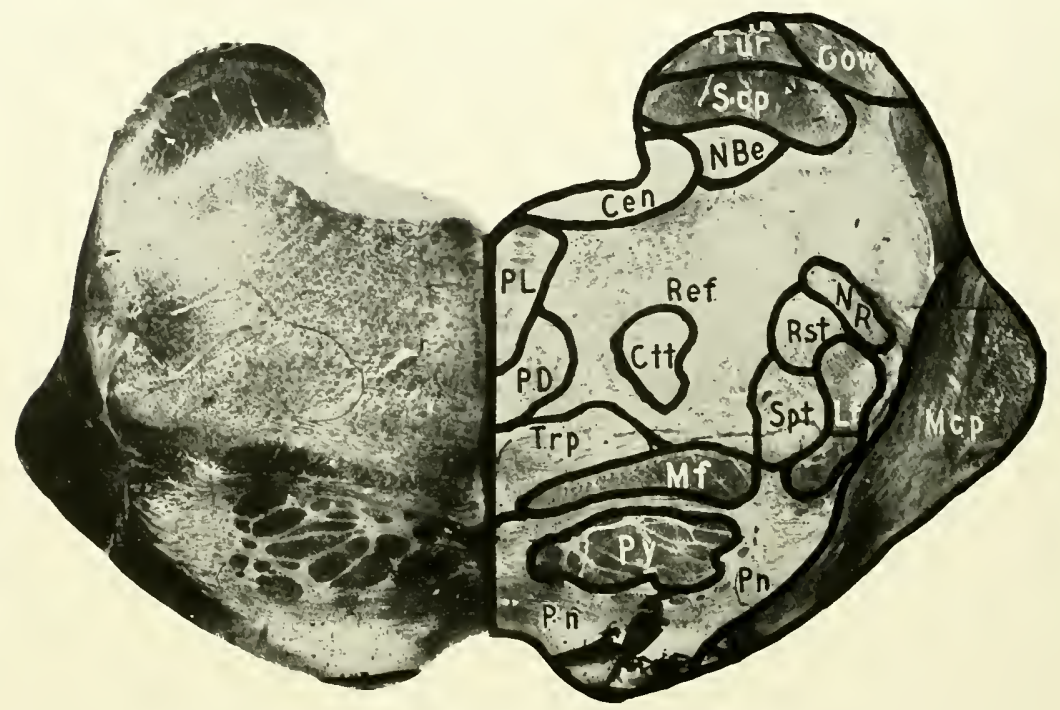

FIG. 3O. LEMLR MONGOZ. LEVEL OF THE MIDDLE OF THE PONS VAROLII. Cen, Central Gray Matter; ctr, Central Tegmental Tract; Gow, Ventral Spinocerebellar Tract; LF, Lateral Fillet; MF, Mesial Fillet; MCP, Middle Cerebellar Pcduncle; NBE, Nuclers of Von Bechterew; NR, Nucleus of Rolando; PD, Predorsal Bundle; PL, Postcrior Longitudinal Fasciculus; PN, Pontile Nuclci; PY, Pyramid; REF, Reticular Formation; RST, Rubrospinal Tract; SCP, Superior Cerebellar Peduncle; SPT, Spinothalamic Tract; TRP, Trapezoid Body; Tur, Tractus Uncinatus of Russel (Hook Bundle). (Accession No. 1.7. Section 208. Actual Size, $14 \times 5 \mathrm{~mm}$.]

pontis, situated dorsally. In the dorsolateral aspect of the section are three conspicuous tracts, the ventral spinocerebellar tract (Gow) making its way toward the vermis, ventral to which is the tractus uncinatus of Russel ( Tur), while immediately in front of this is the superior cerebellar peduncle $(S c p)$. In the angle between the superior cerebellar peduncle 
and the central gray matter (Cen), immediately beneath the floor of the fourth rentricle, is a small collection of medium-sized nerve cells constituting the nucleus of ron Becbterew (NBe) which is one of the group of

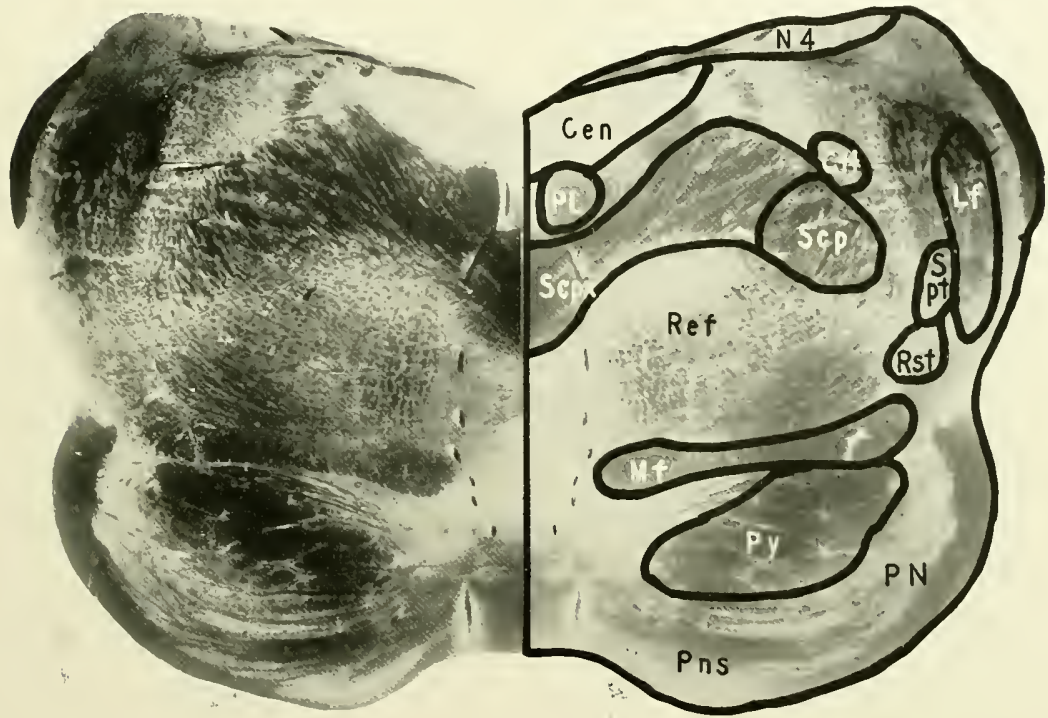

FIG. 31. LEMUR MONGOZ. LEVEL OF EMERGENCE OF TROCHLEAR NERTE.

CEN, Central Gray Matter; ctт, Central Tegmental Tract; LF, Lateral Fillet; MF, Mesial Fillet; N4, Trochlear Nerve; PL, Posterior Longitudinal Fasciculus; PN, Pontile Nuclei; PNS, Pons; PY, Pyramid; REF, Reticular Formation; RST, Rubrospinal Tract; SCP, Superior Cerebcllar Peduncle; scPX, Crossing of the Superior Cerebellar Peduncle; Spt, Spinocerebellar Tract. [Accession No. 147. Section 245. Actual Size, i $\times 6$ mm.]

vestibular nuclei. Ventral to this nucleus are the ascending root fibers of the trigeminal nerve known as the tractus mesencepbalici trigemini. A large collection of small nerve cells lies at the border of the tegmentum opposite the middle cerebellar peduncle. This is the dorsal nucleus of the lateral fillet. 
LEIEL OF THE INFERIOR COLLICULUS (FIG. 32)

At the level of the inferior colliculus, the appearance of certain striking features alters the external configuration of the stem. Among these

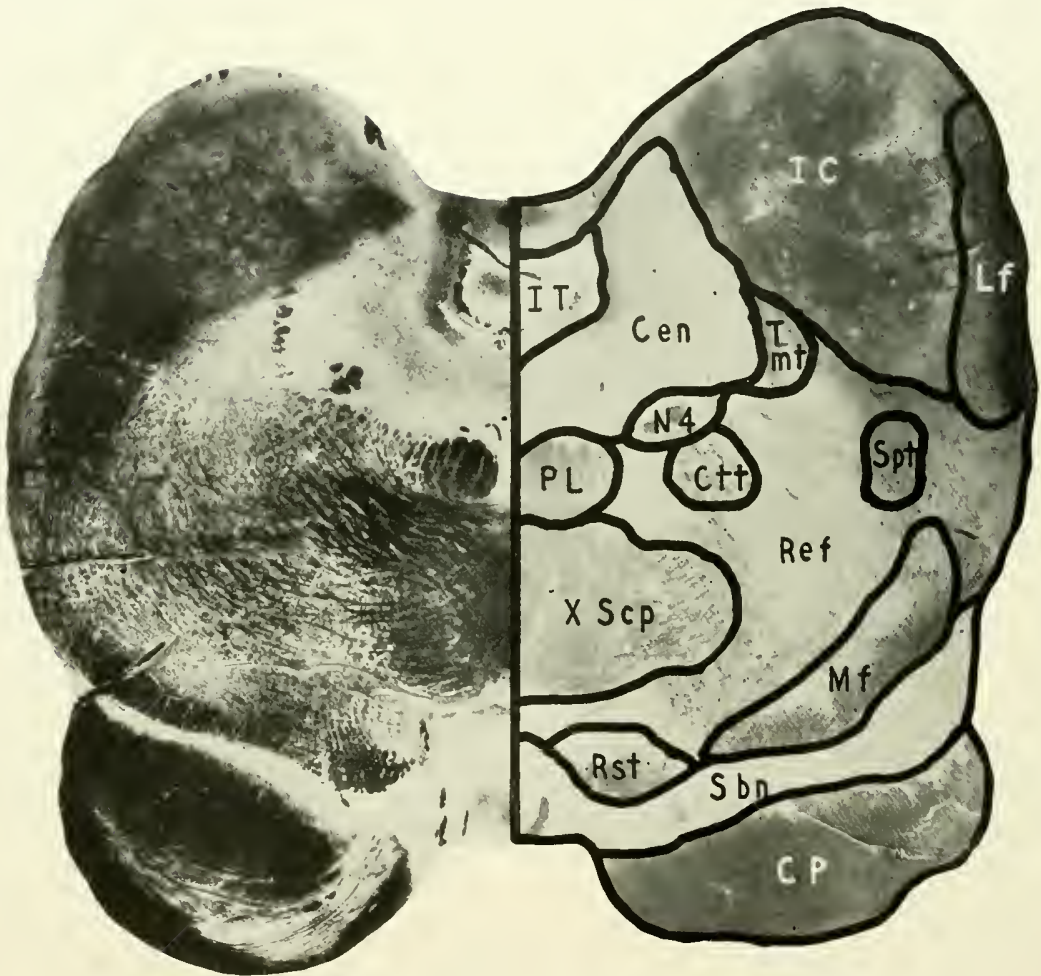

FIG. 32. LEIIUR MONGOZ. LEVEL OF THE INFERIOR COLLICULUS.

CEN, Central Gray Matter; CTT, Central Tegmental Tract; $C P$, Cerebral Peduncle; IC, Inferior Colliculus; IT, Aqueduct of Sylvius; LF, Lateral Fillet; MF, Mesial Fillet; N4. Trochlear Nerve; PL, Posterior Longit udinal Fasciculus; REF, Reticular Formation; RST, Rubrospinal Tract; sB., Substantia Nigra; SPT, Spinothalamic Tract; тмт, Tractus Mesencephalici Trigemini; xscP, Crossing of Superior Cerebellar Peduncle. [Accession No. 147. Section 255. Actual Size, in $\times>\mathrm{mm}$.] 
features may be noted the narrowing of the ventricular space to form the beginning of the Sylvian aqueduct and the appearance of two large nuclear masses situated in the dorsolateral portion of the field. These structures constitute the inferior collieuli (IC), distinguishing characteristics of the quadrigeminal plate in the midbrain. Ventrally the transverse continuity of the pons Varolii is interrupted by the disappearance of the decussating pontile fibers, causing a sulcus to appear on the ventral aspect of the sten which marks the beginning of the cerebral peduncles (CP) and interpeduncular space. The inferior colliculi are significant because they represent primordial receiving stations for the sense of hearing. In many of the lower forms of vertebrates they are the chief centers for auditory sense. In most of the higher forms, especially in mammals, they have delegated much of their original dominance in auditory function to cortical areas of the cerebral hemispheres. Their conspicuous size in lemur indicates that they have here retained much of their primordial significance. They appear to provide a correlating center for auditory impulses essential in determining rapid automatic motor responses incited by auditory stimuli. While these lower centers of hearing have lost much of their autonomy during the process of telencephalization (that is, the progressive advancement of higher synthetic control to the endbrain), they have not entirely surrendered their auditory functions. Through the inferior colliculi the animal is able to respond to auditory stimuli by adequate motor reactions without submitting these stimuli of hearing to the supervision of the higher and more deliberative centers of the cerebral cortex. The fundamental need in the life of these animals for an apparatus which reacts immcdiately to threatening sounds by automatic movements of escape or attitudes of defense may readily be understood. At the same time, this very tendency for auditory stimulation to be shortcircuited in the interest of producing immediate and definitely crystallized automatic reactions cannot fail to create a certain degrce of limitation in the 
adaptability of motor responses exeited by auditory stimuli. It does, as it were, deprive the reaction of a deliberative quality, a period of lateney and reflection in which the acts in response to sound may be made more complete, more precise and more effectively adjusted to the several alternatives of action developing in a given situation.

Lateral to the inferior collieulus is the brachium conjunctivum posticum which constitutes a connecting link in the auditory pathway to the mesial geniculate body. Ventral to the colliculus is the lateral fillet (Lf), making its entrance into the primordial receiving station for the sense of hearing. A large mass of transversely disposed fibers sweeping toward the midline comprises the two major divisions of the superior cerebellar peduncle ( $\mathrm{XScp}$ ) now about to undergo decussation preparatory to entering the red nucleus.

The pyramidal tracts, together with the descending fibers which constitute the pallio-pontile tracts, are situated along the ventral aspeet of the axis in a discretely collected bundle of fibers (CP). A few of the more cephalie transverse fibers of the pons, together with some of the pontile nuclei, are shown ventral to the pyramidal fibers and the fibers of the pallio-pontile system. Dorsal to the pyramidal fibers and stretching transversely across the section from the midline to the periphery is a mass of gray matter containing cells of several sizes and constituting the caudal extremity of an important nucleus known as the substantia nigra (Sbri). It is presumed that this nuelear mass is essential to the regulation of automatic associated movements. Since it assumes such striking proportions in lemur, the inference seems warranted that this type of motor reaction is especially characteristic of these animals. The central gray matter (Cen) has become greatly enlarged and surrounds the caudal extremity of the aqueduct of Sylvius, the roofplate of which is formed by the superior medullary velum which supports the cephalic extremity of the vermis 
cerebelli. At the lateral extremity of the central gray matter are several scattered bundles of nerve fibers, the mesencepbalic root of the trigeminal nerve $(\mathrm{T} \mathrm{m} \mathrm{t})$, while along the ventral border of the central gray matter are two or three small bundles of fibers, the descending fasciculi of the trochlear nerve $\left(\mathrm{N}_{4}\right)$.

\section{LEVEL OF THE SUPERIOR COLLICULUS (FIG. 33)}

At the level of the superior colliculus, prominent tectal structures of the mesencephalon make their appearance. They are the much reduced remnants of the optic lobes which form the most conspicuous elements in the midbrain of the lower classes of vertebrates. Here they retain a certain degree of stratification reminiscent of the optic lobes. Three distinct layers or strata of alternating cell and fiber distribution may be discerned microscopically in the area indicated as stratum griseum superficiale.

Still other layers may be differentiated in this tectal region of the midbrain. The optic lobes have been progressively superseded in the importance of their visual function as the cortex of the cerebral hemispheres expanded and became more intimately concerned in those higher associational syntheses necessary for a fuller sensory adjustment to the external world. This supersedence on the part of the cerebral cortex involves not only the sense of vision and the sense of hearing, but quite as much the general body sense in all those various modalities which are essential to the production of highly organized volitional movements. That all of these specialized forms of sensation, including those which put the animal in touch with elements in its environment more or less remote from its own body, as well as those requiring actual contact with the body surfaces, have in their more primitive states been represented by parts of the brain less highly organized than the cerebral cortex, there can be no doubt. By a slow and gradual process, proceeding step by step from species to species, and only incompletely 
consummating its progress as it sought new sensory fields for further expansion, the functions related to sensibility have attained their fullest representation in the pallium of the cerebral hemispheres. This stepping-up process

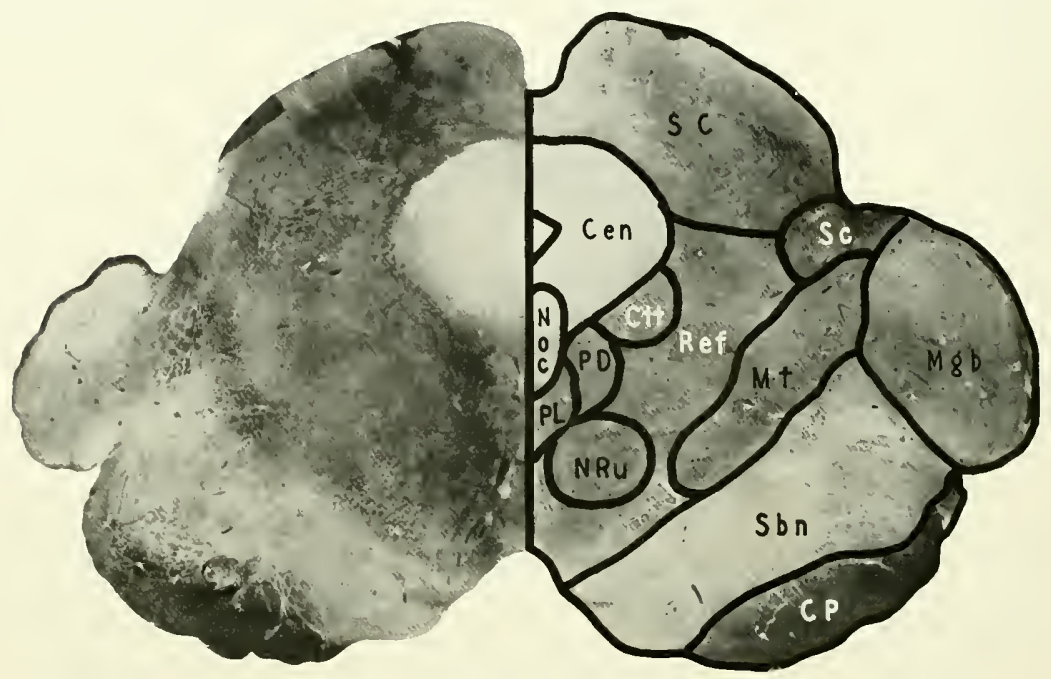

FIG. 33. LEIIUR MONGOZ. LEIEL OF THE SUPERIOR COLLICULUS.

CEN, Central Gray Natter; CP, Cerebril Peduncle; CTt, Central Tegmental Tract; MGB, Mesial Geniculate Body; Mf, Mesial Fillet; Noc, Nucleus Oculomotorius; NRU, Nucleus Ruber; PD, Predorsal Bundle; PL, Posterior Longitudinal Fasciculus; Ref, Reticular Formation; sBn, Substantia Nigra; sc, Superior Colliculus. [Accession No. 147. Section 300. Actual Size, $19 \times 9 \mathrm{~mm}$.]

from a lower, less highly organized region to a higher and more expansible territory in the end-brain, is spoken of as telencepbalization. Yet in no case has this transference upward been made at one such stride that the entire allegiance of a certain form of sensibility is advanced from its more lowly sphere of structural organization to its new domain in a higher region. The gradual projection from lower to higher centers is clearly demonstrated in the phyletic series of the vertebrates. Nowhere is there a more marked instance 
of this partial and graded telencephalization than in the case of the optic lobes which ultimately give over their dominance in visual function to the occipital area of the cerebral hemispheres. Even within the limited range of the primate order there is still evidence of this slow transference from a highly organized visual region in the midbrain to the more highly developed visual centers of the occipital lobe. The stratification already noted in the superior collicular region which retains, even in this high form of mammal, at least ten of the original fourteen cortical layers of the optic lobe, indicates that telencephalization has by no means attained its full expression in lemur. It must be inferred, therefore, that some at least of the primordial visual function is vested in these still highly differentiated tectal structures of the midbrain. This supposition is further borne out by the large number of optic fibers which end in the superior colliculi. Some of these fibers are shown immediately lateral to the stratum griseum superficiale.

Another feature at this level of the brain stem is the oculomotor nucleus (Noc). This nucleus gives rise to the third cranial nerve whose fibers supply all but two of the muscles which move the eyeball within the orbit. It also innervates the intrinsic muscles of the eye, the constrictor iridis and the muscle of the ciliary body which regulates the convexity of the lens. In addition, the oculomotor nucleus sends fibers to the levator palpebrac muscle which produces elevation of the upper eyelicl. This portion of the midbrain therefore is preeminently related to the visual sense. Not only is this true as to the structures concerned with the actual receipt of visual impressions, but quite as much in the transmission of motor impulses requisite to the muscular adjustments of the eyes in risual fixtaion and in visual pursuit of objects. The fact that this oculomotor nucleus (Noc) shows a striking simplicity in its development indicates a relatively low degree of oculomotor organization. It implies that the lemur's vision is as yet only partially binocular; that in the main the animal is still using its eyes 
as far-distance receptors. The adjustment of ocular movement is as yet so incomplete that the animal has not learned the many advantages arising from a more exact stereoscopic vision for objects near at hand. Ocular convergence brings the visual axes into such a position as to make possible the more discriminating scrutiny of objects near the animal. It secures the more exact effects of perspective and proportion characteristic of complete binocular vision. The relatively small size of the oculomotor nucleus in lemur indicates an imperfectly developed binocular vision, while the lack of internuclear commissures implies that the muscles of the two eyes have not as yet acquired the intimate intermuscular cooperation characteristic of animals which have developed a high degree of binocular fusion and stereoscopic vision.

Another feature is the appearance of the red nucleus ( $N R u$ ), a collection of nerve cells in which at least two great systems of fibers receive relay. One of these systems is especially concerned in the efferent conduction of impulses arising in the cerebellum and destined for distribution in the lower levels of the axis. The nucleus ruber $(\mathrm{NRu})$ in lemur is a relatively small structure. Its size is in proportion to the correspondingly small number of fibers forming the superior cerebellar peduncle. This small red nucleus signifies a limited functional capacity for the distribution of impulses essential to the coordinative control, more particularly the coordinative control of complex motor reactions in the upper and lower extremities.

The two cerebral peduncies (CP) extend along the ventral surface and are becoming more divergent as each approaches its corresponding cerebral hemisphere. At the basis of the peduncle are the collected fibers of the pyramidal system and also of the pallio-pontile system. Immediately dorsal to these bundles of fibers is an extensive mass of gray matter, the substantia nigra $(\mathrm{Sbn})$, the significance of which has already been discussed in connection with the control of certain automatic associated move- 
ments of a primordial character largely vested in the midbrain. Immediately dorsal to the substantia nigra is the mesial fillet (Mf), while occupying positions in front of the oculomotor nuclei are two decussations. One of

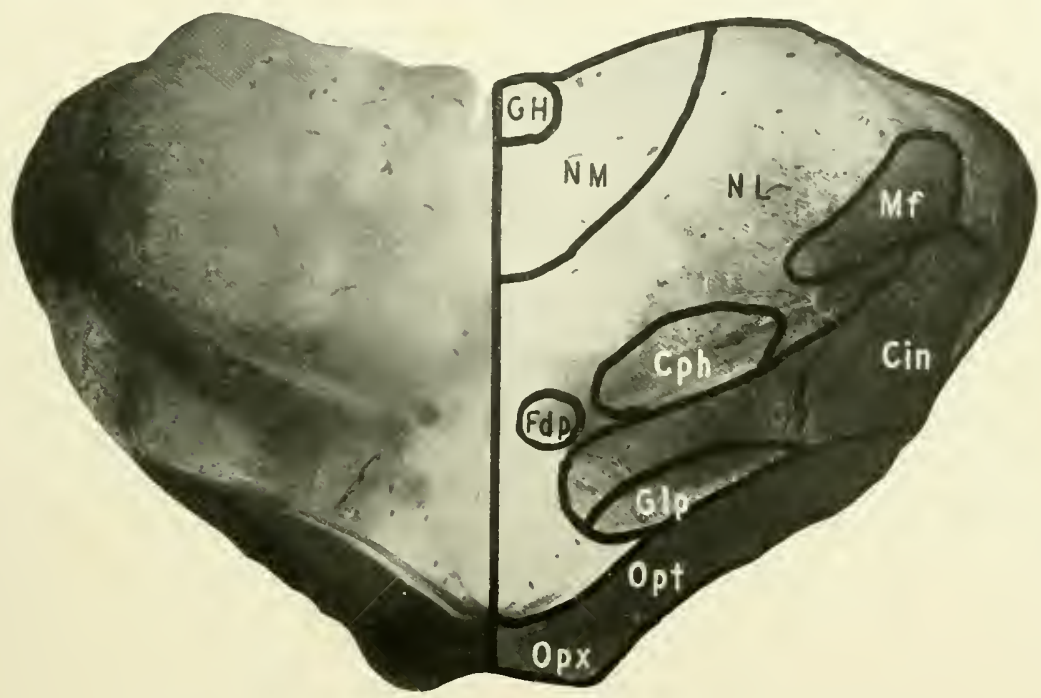

FIG. 34. LEMUR MONGOZ. LEVEL OF THE OPTIC CHIASM.

CIN, Internal Capsule; CPH, Corpus Hypothalamicum; FDP, Descending Pillar of Fornix; Gæ, Ganglion Habenulae; GLP, Globus Pallidus; MF, Mesial Fillet; NL, Nucleus Lateralis Thalami; Nu, Nucleus Medialis Thalami; OPT, Optic tract; OPX, Optic Chiasm. [Accession No. 1 47 . Section 363. Actual Size, $25 \times 15 \mathrm{~mm}$.]

these is the dorsal decussation of Meynert through which the emergent fibers of the third nerve make their way toward the surface. Ventral to the dorsal decussation of Meynert is the smaller decussation of Forel. These decussations represent respectively the crossing of the fibers arising in the reticular formation of the midbrain to enter into the predorsal bundle (PD) and the decussating descending fibers arising in the red nucleus to form the rubrospinal tract. 
LEIEL OF THE OPTIC CHIASM (FIG. 34)

At the level of the optic chiasm the decussation in the optic pathway is the identifying feature $(\mathrm{Opx})$. Another salient element is the optic

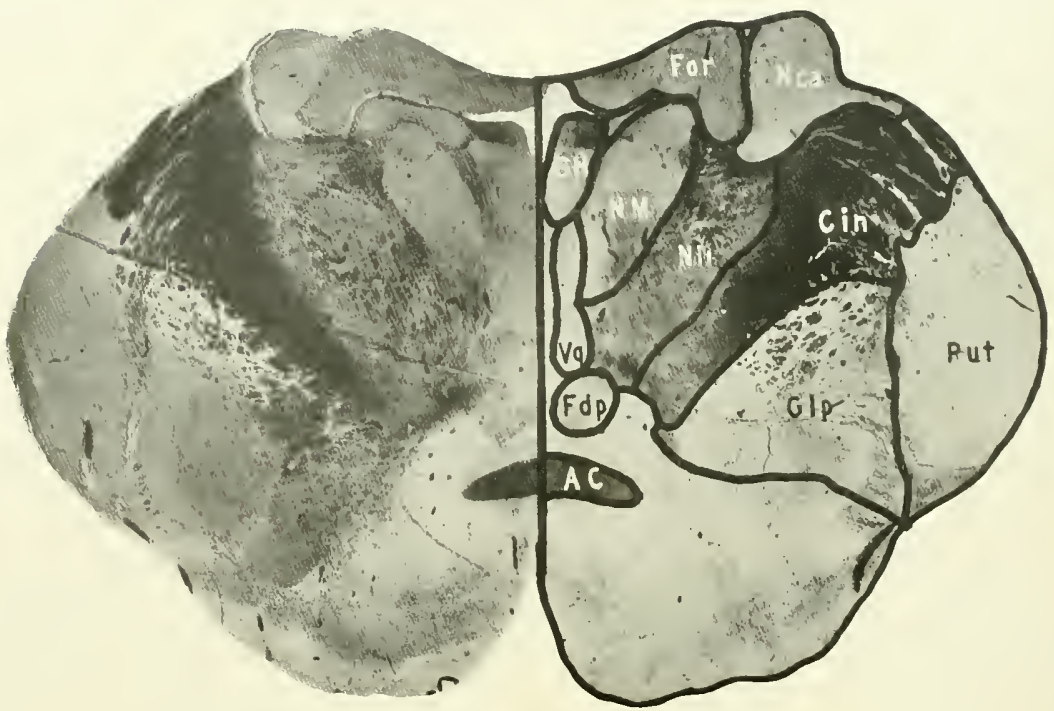

FIG. 35. LEIIUR MONGOZ. LEVEL OF THE ANTERIOR COMMISSSUR.

AC, Anterior Comnissure; civ, Internal Capsule; fDP, Descending Piflar of Fornix; For, Fornix; GH, Ganglion llabenulae; GLP, Globus Pallidus; NM, Nuclcus Medialis Thalami; NL, Nucleus Lateralis Internus Thalami; NCA, Caudate Nucleus; Pur, Putamen; vo, Fasciculus of Vicq d'Azyr. [Accession No. 147. Section 417. Actual Size, $20 \times 10 \mathrm{~mm}$.

thalamus which represents the last great Iclay station in the pathway of all types of sensibility with the exception of olfactory sense. It completes the conduction pathway of body sensibility. Dorsal to the optic chiasm are crossing fibers which constitute the supra-optic commissure of Meynert. Immediately dorsal to this is a large mass of gray matter containing cells for the most part of the motor type and forming the globus 
pallidus $(\mathrm{Glp})$. This is one of the most important portions of the endbrain. Being especially concerned with the regulation of automatic associated movements, it constitutes an outstanding division of the basal telencephalic ganglia. Resting upon and dorsal to the globus pallidus is a fairly large mass of myelinated nerve fibers representing axons which arise in the motor cortex and descend as the pyramidal system. Associated with these are the many fibers which form the main part of the pallio-pontile system. The mass of fibers situated between the thalamus and the globus palliclus constitutes a portion of the internal capsule ( $\mathrm{C}$ in). In contact with the dorsal aspect of the internal capsule is a mass of gray matter surrounded by a considerable capsule of myelinized fibers. This is the corpus hypothalamicum $\left(\mathrm{C}_{\mathrm{ph}}\right.$ ) which contains the hypothatamic nucleus of Luys and the adjacent medullary substance comprising the two fields of Forel known as $\mathrm{H}_{\mathrm{I}}$ and H2. Mesial to the corpus hypothalamicum is the fasciculus of Vicq d'Azyr and ventral to this a bundle of small, weakly staining fibers, the descending pillar of the fornix ( $\mathrm{Fdp}$ ).

LEVEL OF THE ANTERIOR COMMISSURE (FIG. 35)

At the level of the anterior commissure (AC) the section illustrates the cephatic limit of the brain stem, the last remaining structure of which is the anterior portion of the optic thalamus. Other structures of topographical interest at this level are indicated in the caption. 

Chapter II

\section{RECONSTRUCTION OF THE GRAY MATTER IN THE BRAIN STEM OF LEMUR MONGOZ}

7 PE impression conveyed by a survey of cross sections of the brain stem does not give any such realistic idea of the nuclear masses as that obtained from a study of reconstructions by the Bourne method. Such reconstructions of the gray matter are here employed to make more comprehensible the proportions of those nuclear structures which bear the most significant evidence of evolutional unfolding in the brain stem. It is to be noted, however, that these reconstructions do not disclose the dimensions or relations of the nuclear masses which occupy positions within the reticular formation. It is equally noteworthy that these deeper lying cellular collections constitute the more archaically fixed and least variable elements in the composition of the axial gray matter, while the superficial nuclear aggregations represent the recently acquired, more plastic structures of the stem. The tri-dimensional demonstration of the gray matter afforded by reconstructions facilitates the visualization of these elements and establishes for each structure a discrete morphological entity of its own.

In the following descriptions, only the structures seeming to have salient evolutional significance have been selected for discussion. Any complete anatomical analysis of the constituents of the brain stem belongs more properly to the ream of an anatomical atlas. The structures to be considered here include: the dorsal sensory nuclei, the inferior olivary nucleus, the reticular formation, the pontile nuclei, the vestibular nuclei, the cochlear nucleus, the inferior and superior colliculi, the substantia nigra and the red nucleus.

\section{The Dorsal Sensori Nuclei}

In lemur the sensory nucleus of Goll begins as a stender extension from the central gray column close to the midline and separated from 
its fellow of the opposite side by the dorsal septum. The base remains narrow while the distal portion presents a tendency to swing and spread laterally.

At a slightly higher level the first indication of the sensory nucleus of Burdach appears as a flat, bilateral thickening in the dorsolateral portion of the eentral gray column. The two nuclei then extend dorsally with the same lateral swing which is characteristic of the dorsal gray masses and of the substantia gelatinosa Rolandi preparatory to the opening of the fourth ventricle. As these nuclei of Goll and Burdach increase in size, they develop at their dorsal extremities overhanging masses of nuclear material, almost arboreal in form, but limited to the lateral aspects of the main trunks of the nucleus. The more lateral of these nuclear appendages forms the external nucleus of Burdach. Its leaf-like appearance is produced by the breaking-up of the nuclear material by the bundles of fibers of the column of Burdach which are here seeking their cells of relay. The substantia gelatinosa of Rolando which forms the cap surmounting the dorsal horns passes insensibly into the substantia gelatinosa trigemini with a marked lateral inclination in accordance with the common lateral swing of these nuclear columns, until it almost reaches the lateral meridian. As this swing takes place, the substantia gelatinosa Rolandi, slender in the spinal cord, rapidly increases in size to become the substantia gelatinosa trigemini of the oblongata. These three dorsal columns of gray matter expand and diverge in almost parallel parabolic rows. The substantia gelatinosa trigemini approaches the lateral surface of the cord and, losing its intimate contact with the reticular formation, extends upward in a position so constant that it may be used as an orienting structure in the study of the stem. The nuclear gray column reaches upward to the midpontine level where dorsomesial to it there appears the motor nucleus of the trigeminus nerve. At the midolivary level the substantia gelatinosa trigemini presents a defmite constriction which has been 
termed the "waist of the trigeminal nucleus." At its cephalic extremity the nucleus increases in all its diameters as it prepares to meet the entering mass of fibers constituting the dorsal root of the Gasserian gan-
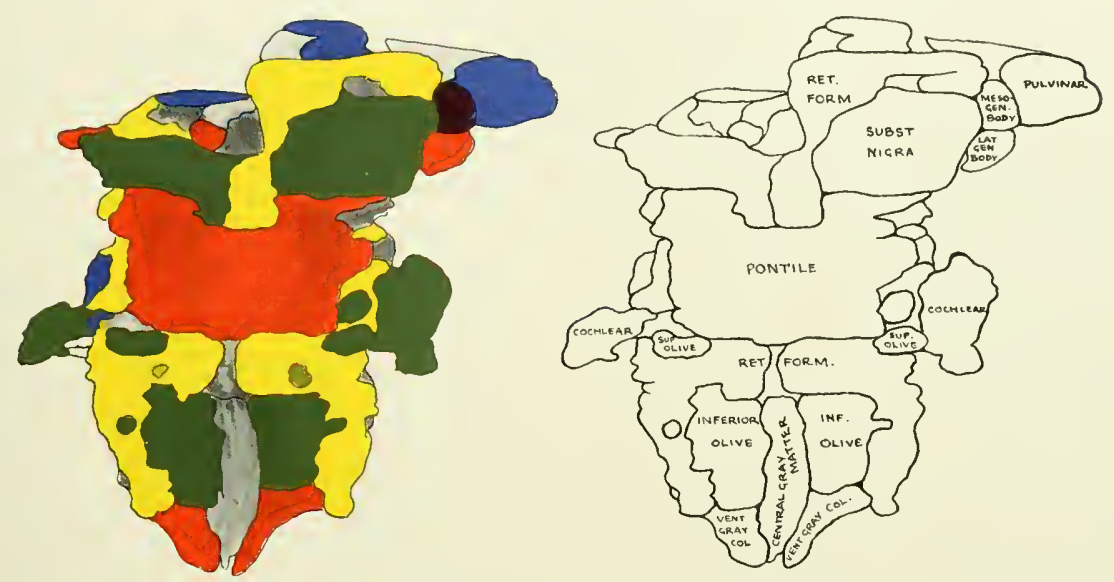

FIG. 36. VENTRAL SURFACE OF GRAY MATTER OF BRAIN STEM, LEMLR MONGOZ.

Kex to Diagran. cochlear, Cochlear Nucleus; inf, ol.1ve, Inferior Olive; lat. gen. body, Lateral Geniculate Body; meso-gen. Bont; Mesial Geniculate Body; pontıle, Pontile Nuclei; Ret. ForM., Reticular Formation; subst. Nigra, Substantia Nigra; sup. Olive, Superior Olive; VENT. Gray col., Ventral Gray Column.

glion. The nucleus extends somewhat above the entering fibers in order to afford relay cells for the short ascending arms of the incoming axons.

\section{The Inferior Olivary Body}

The reconstruction of the inferior olivary body in lemur consists in large part of the dorsal and ventral accessory olivary nuclei. The main mass of the inferior olive seems to be a Iater addition, developing in a position between the two accessory nuclei as a new structure presenting in the higher forms a saccular fundus and two branches. 
The ventral accessory (paleo-olive) nucleus appears somewhat below the mid-decussational level of the oblongata as an oval mass directed mesially and slightly dorsally. It presents no secondary plications and after appearing as a small oval collection of nerve cells it extends transversely to form a flattened band of nuclear material. Mesially it fuses with the extremity of the dorsal accessory nucleus. The dorsal accessory nucleus appears as a round nuclear accumulation imbedded in this ventral surface of the reticular formation. It spreads rapidly into a thin lamina which fuses with the mesial extremity of the ventral accessory olive. The chief olivary nucleus appears as a loop between the lateral extremities of the accessory olivary nuclei with which it fuses. There are no reduplications in this loop.

\section{The Reticular Formation}

In the reconstruction, the reticular formation presents an extensive mass of nuclear material traversed by great numbers of scattered nerve fibers. It is roughly quadrilateral in shape and forms the main portion of the tegmentum of the oblongata, pons and midbrain. In it develop the various nuclei of termination and origin of the medullary and pontile cranial nerves. Upon its surface it presents the indentations made by the various ascending and descending tracts of the brain stem; while at various levels it is pierced by the wide swinging bundles of decussating fibers constituting the mesial and lateral fillets and the inferior cerebellar peduncles. It forms a matrix a long the mesial septum for the posterior longitudinal fasciculus, the predorsal bundle and other fongitudinal fasciculi.

The reticular formation, in affording passage to the various white fiber tracts, seems to serve as a semi-fluid medium which surrounds these tracts on all sides, supporting them in a soft, gelatinous matrix. It begins at about the level of origin of the decussation of the pyramidal tracts and, extending ventrally, dorsally and laterally, receives the cephalic termination of the 
ventral gray columm. It affords attachment to the nuclei of Goll and Burdach dorsally and to the substantia gelatinosa trigemini laterally, which, however, becomes separated from the reticular formation at a higher level by the
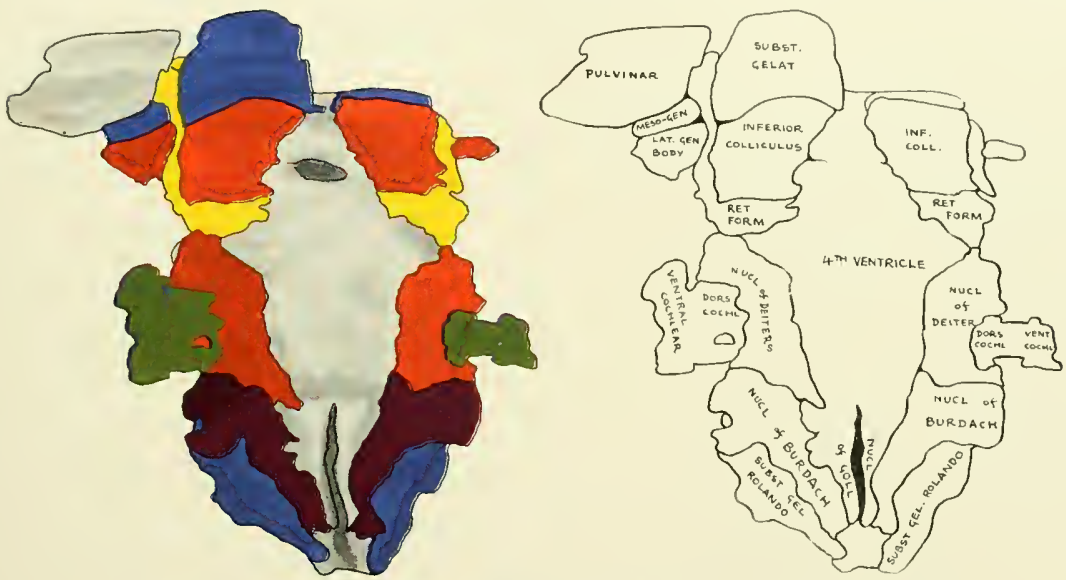

FIG. 37. DORSAL SURFACE OF GRAY MATTER OF BRAIN STEM, LEMUR MONGOZ.

hey to Diagran. dors. Cochl., Dorsal Cochlear Nucleus; inf. Coll., Inferior Colliculus; LAt. Gen, Body, Lateral Geniculate Body; meso-GeN., Mesial Geniculate Body; NcCl. OF Burdach, Nucleus of Burdach; NUCL. OF DEITER, Nucleus of Deiters; NUCL. OF GOLL, Nucleus of Goll; RET. FORM., Reticular Formation; subst. Gelat., Substantia Gelatinosa; subst. Gel. Rolando, Substantia Gelatinosa of Rolando; vent. cochı., Ventral Cochlear Nucleus.

entering vestibular and cochlear nerves and the lateral frllet.

Arising from the dorsal and ventral cochlear nuclei of both the homolateral and contralateral nuclear complexes, the fibers of the trapezoid body develop as a boundary between the reticular formation and the deep layer of the pontile nuclear masses. This body lies in a recess in the ventral surface of the reticular formation. Passing laterally from the superior olive, the trapezoid body forms the lateral fillet which then skirts the edge of the reticular formation, piereing it in some areas as its fibers pass upward to end 
in the inferior colliculus. From the inferior colliculus arises the inferior brachium which occupies a groove on the lateral surface of the mesencephatic reticular formation until it reaches and ends in the mesial geniculate body. The reticular formation is most extensive at the level of the inferior olivary nuclei which develop in its ventral portion. It forms the main mass of the gray matter of the brain stem.

Between the levels of the vestibular complex and the inferior colliculus, the reticular formation sends out a Iong, slender process on the lateral surface of the pons and midbrain to form an enveloping Iayer over the superior cerebellar peduncle.

The reticular formation contains the rentral portions of the twelfth, sixth, fourth and third nerve nuclei, while the dorsal portions of these muclei project into the central gray matter. In the upper medullary and Iower pontile regions the reticular formation presents the specialized condensations forming the vestibular nuclei.

In the mesencephalon the reticular formation approaches but does not come into actual fusion with the substantia nigra. Laterally it forms a support for the mesial geniculate body, while dorsolaterally it becomes continuous with the inferior and superior colliculi. At its cephalic extremity it is continuous with the zona incerta of the diencephalon and fuses with the thalamic gray matter.

\section{The Pontile Nuclei}

Reconstruction of this mass of gray matter provides one of the most striking differential features in the brain stem of primates. This nuclear aggregation, forming with the pallio-spinal and pallio-pontile tracts the basilar portion of the metencephalon, presents a relatively simple arrangement. The pontile nuclei begin rather suddenly at the trapezoid level and show but little tendency to expansion caudally into the ob ongata. The 
nuclear mass is divisible into a superficial, ventral layer and a deep, dorsal layer, both of which are confluent laterally and mesially in an area surrounding the descending pallio-pontile and pallio-spinal tracts. The pyramidal
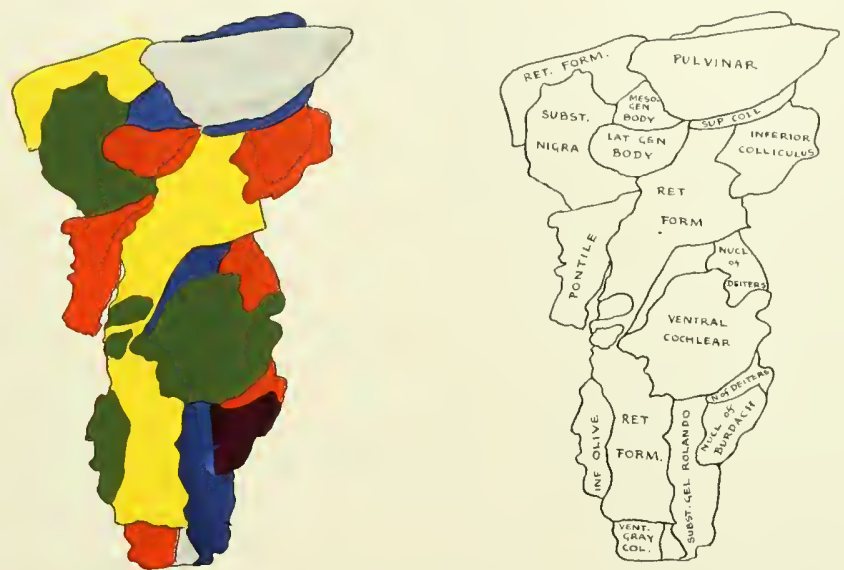

FIG. 38. LATERAL SURFACE OF GRAY MATTER OF BRAIN STEM, LEMUR MONGOZ.

Key to Diagram. INF. Olne, Inferior Olive; LAT. Gen. Body, Lateral Geniculate Body; Meso-gen. Body, Mesial Geniculate Body; NUCl. OF BURDach, Nucleus of Burdach; NUCl. OF DEITERs and N. OF deiters, Nucleus of Deiters; ret. Form., Reticular Formation; SUbst. gel. rolando, Substantia Gelatinosa of Rolando; suest. Nigra, Substantia Nigra; sup. Coll., Superior Colliculus; Vent. gray col., Ventral Gray Column.

tract is separated into fasciculi by the transverse pontile fibers only to a limited extent and in the main appears as a single heavy mass of fibers. The pontile nuclear masses are large and their continuity is not materially interrupted by the decussating ponto-cerebellar fibers. The deep layer of the nuclear mass is continuous at its cephalic extrenity with the substantia nigra. Marked condensations of nuclear material appear laterally and mesially. They connect the superficial and deep layers of the pontile nucleus and form lateral and mesial buttresses for the substantia nigra. 


\section{The Vestibular Nuclei}

In reconstruction this mass of gray matter first makes its appearance as the large nucleus of Deiters at a point a little above the mid-decussational pyramidal level. It appears as a small, wedge-shaped mass of gray matter located between the upper extremity of the nucleus of Burdach, the nucleus of Goll and the central gray matter. It also participates in the general lateral swing occasioned by the opening of the fourth ventricle and rapidly increases in size. It occupies a position beneath the central gray matter and in relation with the cephalic extremity of the nucleus of Burdach. In the mid-ventricular region the triangular nucleus of Schwalbe makes its appearance mesial to the nucleus of Deiters and in relation with the central gray matter. The nucleus of Deiters gradually dwindles as it extends cephalad, while the triangular nucleus is continued upward as a large, irregular mass in the lateral reticular formation dorsal to the substantia gelatinosa trigemini. Dorsally and cephalically the nucleus of von Bechterew appears as a small, indefnite mass of gray matter in the lateral wall of the fourth ventricle close to the thin subependymal layer of central gray matter.

\section{The Cochlear Núclei}

The reconstruction of this nuclear mass shows a large ventral cochlear nucleus which extends for a considerable distance into the lower pontile regions of the brain stem. It is separated from the ventrolateral angle of the tegmentum of the stem by the collected mass of the middle and inferior cerebellar peduncles and the descending trigeminal tract. It is connected with the stem only by the fibers of the cochlear nerve.

The large dorsal cochlear nucleus is situated at the extreme dorsolateral angle of the tegmentum in the extremity of the lateral angle of the fourth ventricle. It begins below the recess and extends about an equal distance above the recess. It is limited in extent by the pontile peduncular fibers but 
extends for some distance mesially under the subependymal layer of the central gray matter of the fourth ventricle. It lies immeciately dorsal to the vestibular nuclei, separating this mass from contact with the gray matter of the floor of the fourth ventricle. Between the ventral and dorsal cochlear nuclei are connecting strands of nuclear material as well as the root fibers of the cochlear nerve and scondary fibers of the cochlear tract.

\section{Tile Colliculi}

In reconstruction, these masses of gray matter are about equal in size and extent. They appear as rounded elevations on the tectum of the midbrain, occupying the entire width of the dorsal aspect of the axis. They are continuous laterally with the dorsal extension of the reticular formation of the mesencephakn. Mesially and dorsally they are continuous across the midline with each other by means of the dorsal gray matter which serves as a matrix for the superior and inferior collicular commissures. The colliculi are separated from the central gray matter by these collicular commissures and by the peripheral fiber condensation which is clisposed as a fringe along the line of contact between the central gray matter and the reticular formation. The inferior colliculus is sharply separated from the large superior colliculus by a narrow, funger-like process sent out by the mesencephalic reticular formation, while the superior colliculus merges insensibly into the subpineal region of the cpithalamus. The surface of the mesencephalic reticular formation is traversed in this region by the passage of the two brachia connecting the colliculi with the genic ulate bodies.

\section{The Substantia Nigra}

As reconstructed, this mass of gray matter appears as a gradual transformation in the deep layer of the pontile nuclear mass, assuming its characteristic coloration and form at the junction of the isthmus and midbrain. It 
is supported not only by the deep pontile layer, but also rests upon the two buttresses which form laterally and mesially in the pontile gray matter. It is dense aud heavy in lemur and is connected mesially to its fellow of the opposite side by means of the interpeduncular gray matter which appears to be quite undifferentiated. This interpeduncular gray matter is continuous with the structures forming the hypeneephalon. In the lateral portion of the substantia nigra there develops a discrete nucleus containing many tangled nerve fibers which pass dorsally into the tegmentum. The substantia nigra gradually attenuates and disappears. It is not continuous with any definite gray mass in the thalamic region of the brain.

\section{The Nucleus Ruber}

The reconstruction of this nuelear mass, so predominant in sections of the mesencephalon in man and the higher primates, is relatively insignificant in size. The nucleus is located in the most ecphalic portion of the mesenecphalon extending into the reticular formation of the diencephalon. It is relatively indistinct and poorly demarcated from the surrounding tissue by the eneapsulating fibers of the superior cerebellar peduncle.

\section{The Central Grai Matter}

In the upper regions of the spinal eord this nuclear mass is roughly quadrilateral, receiving the bases of the two dorsal gray columns and ventrolaterally the two ventral gray columns. Passing upward, the central gray matter receives the bases of the developing nuclei of Goll and Burdach and then gives off dorsally a narrow, tongue-like extension which passes along the dorsal median septum. As the caudal extremity of the fourth ventricle is approached, the entire central gray matter migrates dorsally, earrying the central canal with it, and as the dorsal gray columns begin to diverge, the quadrilateral body of gray matter gradually flattens dorsoventrally and 
the central canal emerges dorsally in to the fourth ventricle, thus disposing the central gray matter as two lateral halves connected across the midline. This lateral displacement continues until at last the gray matter becomes flattened out in an almost transverse plane as the floor of the fourth ventricle. From the edges of the lateral walls of the fourth ventricle the ependymal lining continues across the roof of the fourth ventricle which eaudally is formed by the inferior medullary velum. In this locality the roof is invaginated by the chorioidal plexuses of the fourth ventricle and here the layer of central gray matter, which at best is extremely attenuated on the roof of the fourth ventricle, practically ceases to exist.

In the midpontile portion of the fourth ventricle the cerebellum enters into the roof of the rentricle and cephalad to the cerebellum the superior medullary velum contains the superior cerebellar peduncles. Approaching the upper portion of the metencephalon the lateral walls gradually become convergent until they meet just caudad to the inferior colliculus. At this point the fourth cranial nerves decussate in this reconstituted roof of the ventricular cavity. The lateral walls of the fourth ventricle are formed from below upward by the nuclei of the columns of Goll and Burdach, the muclei of Deiters and Schwabe and the inferior cerebellar peduncle. The lateral walls fail at the lateral recesses but appear again as the middle cerebellar peduncle. Cephalically the superior cerebellar peduncles lie in the rapidly converging lateral walls of the cephalic portion of the fourth ventricle.

The floor of the fourth ventricle is comparatively smooth and presents but little modelling. The underlying nuclear masses and fiber tracts produce scarcely any impression on the floor which is stretched evenly from side to side and from below upward. It is in general diamond-shaped, beginning from the opening of the central canal caudally, spreading to its greatest width at the level of the lateral recesses and then rapidly narrowing as the aqueduct of Sylvius is approached. 
In the oblongata the rounded dorsal surface of the hypoglossal nucleus passes backward and invades to a limited extent the central gray matter. In the metencephalon the dorsal surface of the abducens nerve passes somewhat further dorsally, while in the mesencephalon the trochlear and oculumotor nuclei extend deeply into the central gray matter. The central gray matter in the regions of the mesencephaton forms a complete investment for the central canal and as the dicncephalon is approached, it becomes continuous with the eentral gray matter underlying the ependymal lining of the ventricle of the diencephalon. Dorsally at the junction of the tectal region with the habenular region, the ecntral gray matter is penetrated by the habenular and the posterior commissures. 


\section{Chapter III}

\section{TARSIUS SPECTRUM, ITS BRAIN AND BEHAVIOR}

Its Position among the Primates; Measurements and Brain Indices; Surface Appearance of the Brain; Internal Structure of the Brain Stem

7 ARSIUS occupies a unique position among the primates. It has been singled out by eminent authorities to carry upward the line of human derivation from some Iower mammalian form. In this rôle it deserves closest scrutiny regarding its structure and behavior.

\section{Appearance and Behavior of Tarsius}

In size, the animal is about as large as a small squirrel. It is peculiar in appearance because of its closely set, protruding eyes, its long tufted tail, its protruding ears, and the disc-like pads upon the ends of its fingers and toes.

The tarsiers inhabit some of the Nalay Islands. They are noted for two curious habits: they can rotate their heads until they look directly backwards; and they leap with astonishing speed among the trees from bough to bough in pursuit of insects. Mr. Le Gros Clarh has recently given the most detailed description of the tarsier's behavior in captivity. He discusses the affinities of tarsius to lemurs and the insectivores on the one hand, and the anthropoids on the other. In this cliscussion he recalls the opinion of the Royal Zoological Society of London, previously expressed, to the effect that whatever its definitive allocations, tarsius should be placed in a suborder of primates (Tarsioidea) which is intermediate between I.cmuroidea and Anthropoidea. 
Clark's observation on tarsius covers a period of thrce years in Sarawak. During this time he was never fortunate enough to see one of the animals in the wild state. Such specimens as he observed were captured by the Dyaks

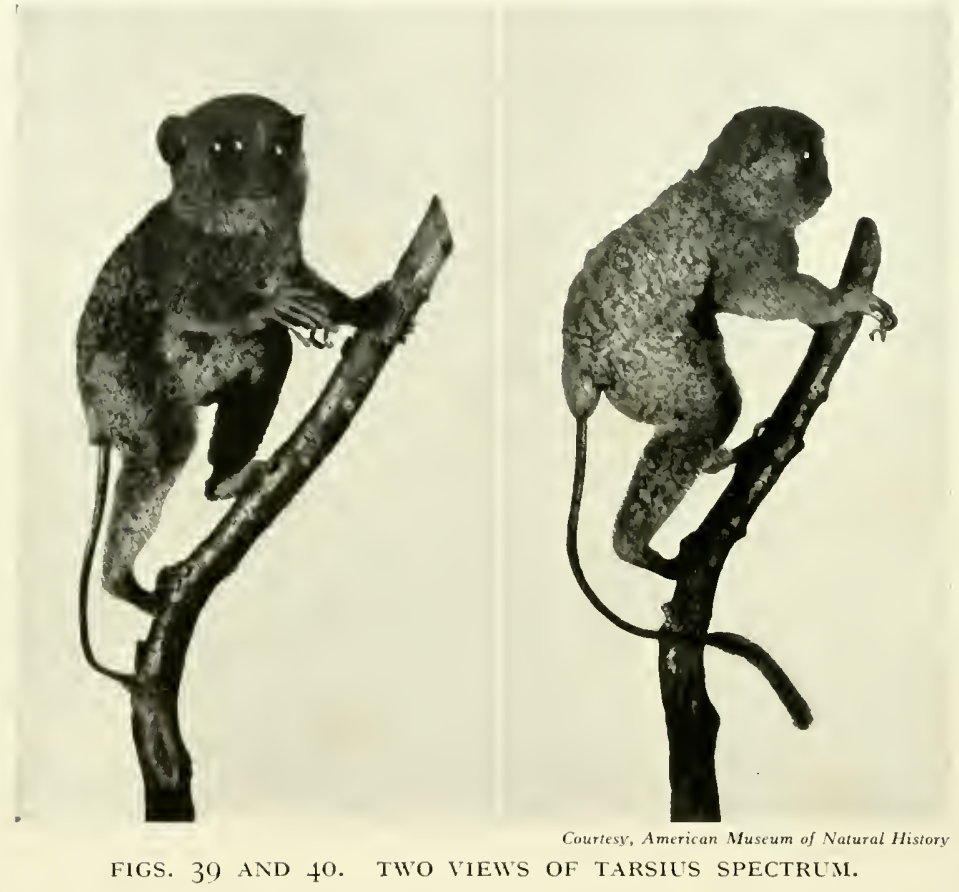

while lelling trees. The animal was easily caught during the daytime. It acted almost stupidly, as though its diurnal vision was imperfect. In the main, it made most ineffectual efforts to escape, at the most pivoting on a branch in such a way as to put the latter between itself and its pursuer.

It lives in the jungle of secondary type, particularly in a low country, and for the most part passes its time clinging in a vertical position to branches 
of trees and underbrush. The manner in which it supports itself in this vertical posture is interesting and peculiar. With the fingers and toes it holds the branch in prehensile grasp, meantime pressing its long slender tail against the branch very much in the manner of a spring. If the tail is pulled away from its support, tarsier tends to sag downward. The tail, however, is in no sense prehensile, nor is it used, with the exception above mentioned, in other functions than as a balancing and steering organ during locomotion.

Tarsius is humanoid in reproducing a single offspring at a time. It is not gregarious, as many of the other primates are, but goes in pairs. After the breeding season the female and her young usually live alone together. There is no evidence that these animals build nests, or even live in the holes of trees. When sleeping, the head sinks downward, much as that of an old man asleep in his chair. Often the young tarsius will perch upon the mother's head while she is thus sleeping, and in this position fall asleep itself. This is an interesting motor combination, plainly showing that the mechanism for maintaining the vertical clinging posture operates perfectly even though the animal slecps.

The general behavior of tarsius seems extremely stereotyped. It is capable of but little acquisition under training, and in captivity is apparently unable to make new adaptations. In spite of its enormous eyes, it has difficulty in securing its food during the daytime, grasping awkwardly at grasshoppers or other insects offered to it. This no doubt is due to the fact that its visual apparatus is specialized for nocturnal hunting, and also because the retina possesses no macula.

Characteristic in the motor activity of the tarsier are its marvelous leaps from branch to branch, which are so swift as almost to defy the human eye. It is said that the animal is able to capture small insects on the wing in its leaps. On the ground it leaps also like a frog, but awkwardly. When it lands it sprawls, and then hops away again. Only occasionally does 
it walk and then its gait is ineffective and ungainly. Insects, especially grasshoppers, are the main staple of its food supply, but it is also partial to milk and drinks water.
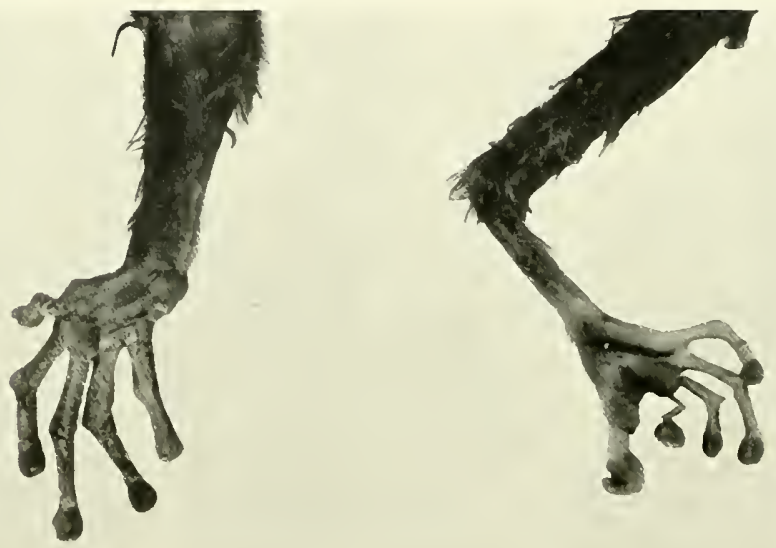

Courtesy, American Museum of Notural History.

FIGS. +I AND +2. HAND AND FOOT OF TARSIUS SPECTRLM.

LEFT. Palmar surface of hand showing rudimentary development of the palm, palmar pads, pronounced digitation, disc-like specialization on the distal phalanx of each finger.

Right. Plantar surface of foot showing rudimentary sole and heel, long hallux partially opposable, and disc-like specialization on the end of each toe.

Tarsius performs its toilet much as a cat does. When it comes to the hind legs it grasps one and then the other with its hands, and thus keeps itself scrupulously clean. It is not known to make vocal sounds indicative of fear or anger, and only on rare occasions has it been heard to squeak, most particularly when young. It is probably not the case that the mother carries her offspring with her teeth in the manner of a cat. This act Clark has never observed. As with many other primates, the infant tarsius grasps and clings 
to the hair on the abdominal wall of the mother. The eyes are open at birth, and many reactions appear at once which are often long delayed in such animals as the rat, cat, $\operatorname{dog}$ and higher primates.
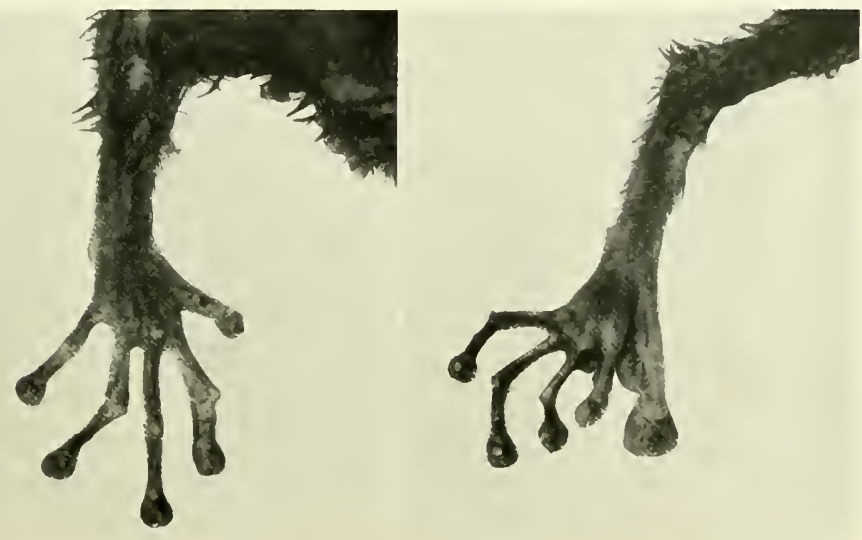

Courtesy, American Museum of Natural History

FIGS. 43 AND 44 . HAND AND FOOT OF TARSIUS SPECTRUM.

Left. Dorsum of hand showing disc-fike specialization on finger tips, with claws instead of nails. Thumb partly opposable.

Rıgнт. Dorsum of fout. Each toe shows the marked disc-like specialization on the distal phalanx; the great toe is short with little or no opposability.

The contention that tarsius may be in the direct line of human ancestry is borne out by its blood tests. Clark has shown that tarsius has a defmite blood relationship with man, chimpanzec and gibbon. On the other hand, the blood reactions are negative with macacus, nycticebus, the cat and the squirrel. The hands are peculiar for their long and slender fingers and short thumbs; the nails resemble claws. The feet are long, the toes slender; the index and middle digits have true claws. The palms of the hands and soles of the feet are provided with arch-like pads. 
In a personal communication, Mr. Harry Raven tells of hunting tarsius both in Dutch Borneo and Celebes. The animals were usually in second growth of the forest, or the scrubby forest along river banks. They are entirely nocturnal and frequently found in clumps of bamboo or in vines that surround the trunks of large forest trees. He hunted at night with a jacklight. The first time he saw tarsius, two or three of them were together and he caught the reflection of the eyes of one, shot and wounded it. When it was picked up it squeaked, a sharp, piereing squeak. The other two answered the call and when the wounded one was shaken it would repeat the squeaking until the others came up close. The animals are extremely active, probably the quickest jumpers of all mammals. When they are grasping a small branch, they can twist their heads in the direction they are going to jump, so quickly that it is almost impossible to see it-more quickly than the eye can follow. It is as though they were looking in one direction and jumping in another, they turn their heads with such great speed. In captivity they are pugnacious and cannot be tamed, although it is difficult to keep them for long. In habits tarsius is close to the galago of South Africa, which lives in thickets and dense forests; in fact, their habits are nearly identical.

\section{Affinities of Tarsius to Other Prinates}

G. Elliot Smith, who has given much attention to the alfinities of the primates, says: "It is well to recall the fact that the brain of tarsius exhibits decisive evidence of the lemuroid status in the calcarine region, in the Sylvian fissure and in numerous traits which have been enumerated. In the degree of caudal extension of its hemispheres, it is even further removed from the insectivora and more pithecoid than the lemurs. But the evidence of cerebral anatomy lends no more support than I believe the structure of the rest of the body does to the view that the approximation of tarsius to the apes implies its separation from the lemurs. So far as its brain is concerned, Tarsius is a 
lemur of the lemurs, to use an expression of Professor Howe's. It is certainly more nearly related to the apes than most other lemurs. But on the other hand, all the apes and lemurs are linked by a much closer bond of affinity, the one to the other, than are any of them to the other mammals. Tarsius is unquestionably the most primitive living primate."

A. A. W. Hubrecht, on the other hand, maintains that "tarsius is not a lemur at all. It should never have been placed among the lemurs. Its position is somewhere between an unknown type of insectivores and our modern monkeys and man."

Memoirs published within recent years by Forsyth-Major, Earle and Standing have made it perfectly clear that the demonstration of the affinities of tarsius to the apes does not in any way affect the recognition of the fact that it is at least as nearly related to the lemurs. So that Hubrecht's proposal to restrict the term primates to tarsius and the apes lacks any adequate justification.

G. Elliot Smith speaks again even more emphatically concerning the position of tarsius. He has, in fact, come to the conclusion that tarsius is much more primitive and at the same time distinctly more pithecoid than the lemurs. He believes that the primate stem flowed from its source among a group of tarsius-like mammals. The apes and the lemurs were merely divergent branches of this stem and the latter, the lemurs, as a suborder although definitely specialized in structure, remained nearer to the Tarsiidae than to the apes.

The primates, he asserts, consist of three divergent phyla which have all departed in varying degrees and in different ways from their original common ancestor which must have been a creature in many respects like tarsius, but more macrosmatic and possessed of a small and less highly specialized visual cortex.

Woollard, on the basis of an exhaustive anatomical study, con- 
chudes that tarsius is a lemur annectant to the early Eocene primitive placentals. Standing at the base of the primate stem, it reaches forth to the simian forms and is annectant to the Anthropoidea.

In stating his views concening the affnities of tarsius, E. D. Cope elaimed that "the genus Anaptomorpbus is the most simian lemur yet discovered and probably represents the family from which the anthropoid monkeys and men were derived. The animal was nocturnal in its habits and had a number of resemblances to Tarsius which is perhaps its nearest ally among the lemurs."

Mathew and Granger both are in general aceord with this view when they say that "There are several characters in addition to the larger braincase in which the skull of Tarsius is more modernized than that of the Lower Eocene Anaptomorphus, but in some other genera of this group the dentition is much nearer to Tarsius and the skult construction may likewise have been nearer."

In regard to tarsius, Earle likewise believes that this is evidently a type nearly between the lemurs and the apes, but with many essential characters belonging to the former group. Some of its anthropoid characters are naseent, so to speak. They are just developing, and as in the case of the orbit of tarsius, it is not yet fully differentiated into the higher type of true anthropoids. The anthropoids diverged from a lemurine stock probably not earlier than the Upper Eocene. This deduction is supported by the fact that the first lemurs to appear are insectivorous in their affnities.

Sonntag also believes that the Eocene Anaptomorphidac gave rise to tarsius and the monkeys arose from a tarsioid ancestor.

Wood-Jones, however, has assumed what is perhaps the most radical attitude in holding that tarsius "like man shows primitive cranial architecture. His kidney is formed on human lines, his aortic arch is arranged as in man, and in a word he shows with man the basal mammalian simplieity of the primate group. He is a most highly specialized little creature on his own cur- 
ious lines and yet he retains with man a host of those astonishingly primitive features that place this odd couple at the base of the primate stem. He lingers today a specialized primitive primate nearer akin to man than any other animal known to the zoologist. Tarsius dates right back in the form of Anaptomorphus to the base of the Eocene period and at that astonishingly early epoch he had already gained his own peculiar specializations. His companion in primitiveness - homo-has his own specialization."

The paleontological evidence reviewed by Professor Gregory seems to be against Wood-Jones' view that the existing tarsius is the nearest living relative of man. "Tarsius may well parallel the human condition in construction of the placenta and in a few other points noted by Mood-Jones, but its relationships to man are plainly very indireet and must be traced backward along gradually converging lines to the primitive Tarsioid stocks which gave rise at different times and at diflerent places to the higher groups of Tarsius."

"Neither tarsius itself nor its own Eocene relatives mentioned above appear to be directly ancestral either to the platyrrbine or to the catarrbine divisions of the Anthropoidea. Nevertheless, tarsius parallels the higher primates in so many characters of the brain, skull, reproductive organs and other parts that a very remote common ancestry of the three suborders seems highly probable."

\section{Measurements of Tarsius}

The average measurements of tarsius are:

Body length.

$149 \mathrm{~mm}$.

Tail. $208 \mathrm{~mm}$.

Head length. 40 $\mathrm{mm}$.

Head breadth. $32 \mathrm{~mm}$.

Upper extremity I $09 \mathrm{~mm}$.

Lower extremity $177 \mathrm{~mm}$.

Total weight $923 \mathrm{gms}$. 


\section{Surface Appearaice of the Brain in Tarsics}

The cerebral hemisphere of tarsiers is lissencephalic, the fissure of Sylvius being the sole indication of suleal marking. In general contour the brain of tarsius is elongate, its greatest length being $21 \mathrm{~mm}$., its greatest width $20 \mathrm{~mm}$. The length is further augmented by the addition of a pronounced bubus olfactorius which projects forward for a considerable distance beyond the frontal region. In this regard the tarsial brain is as primitive as many of the lower mammals and even approaches reptilian conditions. None of the primates possesses a bulbar development comparable in prominenee to that of tarsius. In fact, it is in exactly this olfactory detail that all of the Anthropoidea manifest that marked involution significant of a progressive microsmatic development.

\section{THE LISSENCEPHALIC CHARACTER OF TIE CEREBRAL CORTEX}

The Sylvian fissure, although clearly distinguishable, has none of those features which characterize it in the apes and man. It unquestionably corresponds to that suprarhinal fold which forms the mammalian pseudo-Sylvian fissure. Lobation of the tarsier's hemisphere is almost negligible. The Sylvian indentation separates a poorly developed frontal lobe from a feebly delineated temporal area. The occipital pole has extended caudad so that the cerebral hemisphere covers a large portion of the tentorial surface of the cerebellum. This is a definitely pithecoid character. On the other hand, that total absence of boundary between the oceipital and parietal as well as between the parietal and frontal areas bespeaks a neopallium of such pronounced generalization that it might well serve for the lowest of mammalian forms. With the exception of the marmoset, no such lissencephalic condition of the cerebral cortex is encountered among the primates. In the Hapalidae, how- 
ever, the lack of fissures in the neopallium may be attributed to a retrograde process in the cortex which, according to one interpretation at least, represents a secondary degenerate stage produced during the evolution of the
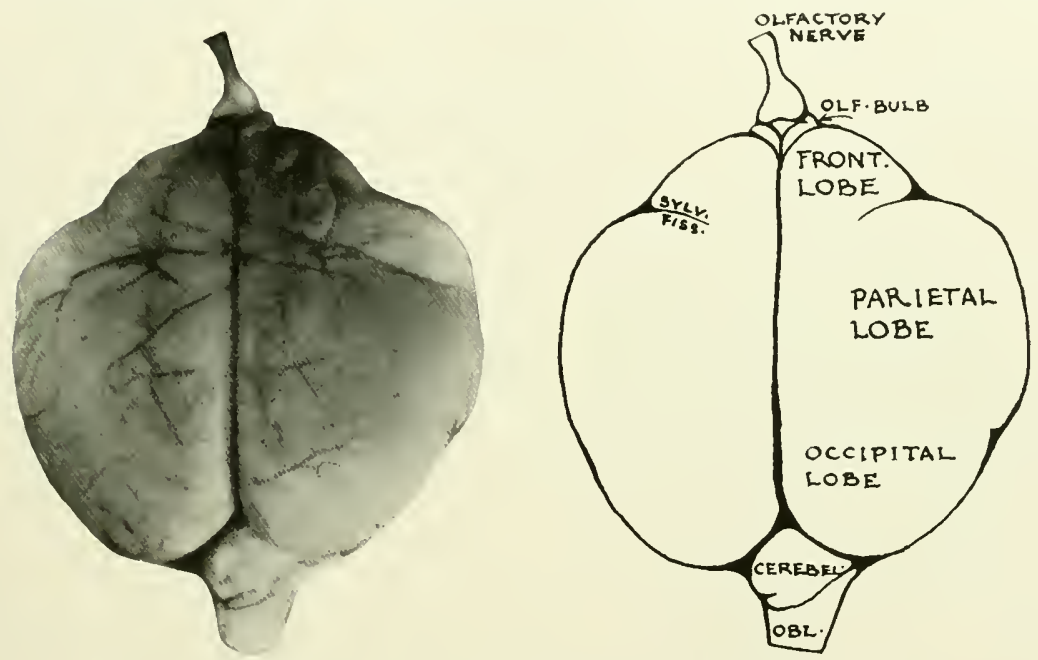

FIG. 45. DORSAL SURFACE OF BRAIN OF TARSIUS SPECTRLM. [Actual Length, $2 \mathrm{I} \mathrm{mm}$.]

Key to Dlagrat. Cerebel., Cerebellum; front. lobe, Frontal Lobe; obl., Oblongata; olf. be lb, Olfactory Bulb; sriv. Fiss., Sylvian Fissure.

Cebidae. The simple condition of the hemispheres of tarsius would seem, on the other hand, to represent the retention of definitely primitive characters in a brain in many other respects affected by marked progressive tendencies.

ORBITAL CONCAVITIES AND OLFACTORY BULB

The basal surface of the hemisphere presents two ill-defined orbital concavities although the orbits in tarsius are extremely large. The lack of prominence of these concavities is due to the fact that the frontal lobe is poorly developed. The olfactory bulb is large, protruding rostrad in front of the 
frontal pole and passing backward into a short stubby olfactory tract which terminates in a prominent tuberculum olfactorium. This protraction of the bulb is not found in lemurs. The tuberculum is embraced by the olfactory
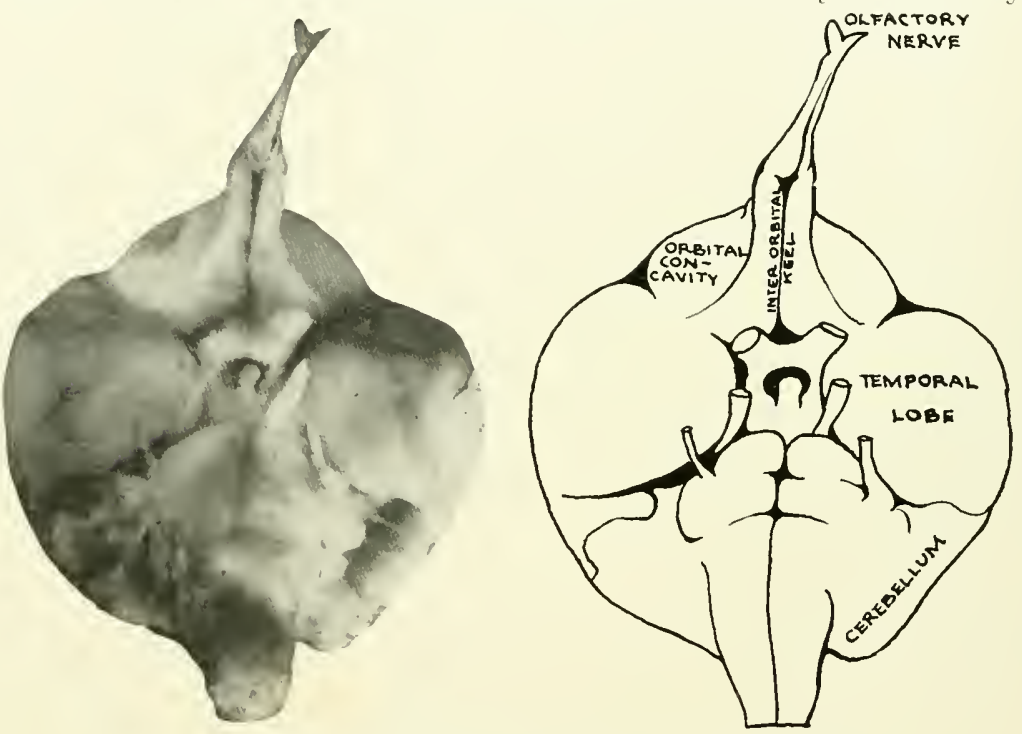

FIG. +6. BASE OF BRAIN, TARSIUS SPECTRUM.

[Actual Length, 2\& $\mathrm{mm}$.]

striae and bounded posteriorly by a well-defined perforated space and diagonal band of Broca. The two olfactory bulbs and tracts together constitute a most pronounced interorbital keel.

The olfactory bulb is further identifed in tarsius by its connection with the olfactory portion of the nasal mucosa through a single olfactory nerve. The fila olfactoria, undoubtedly because of the large size of the orbits, have coaleseed to form a single strand, although near the entrance into the olfactory bulb there is some evidence of the spreading-out characteristic of other primates. 
THE OPTIC CHIASM

The optic chiasm is llat and broad and gives the impression of generally greater thickness than in other forms, due to the fact that almost all of the optic fibers undergo decussation. Very few optic fibers in tarsius are uncrossed. The interpeduncular space is small, bounded by feebly developed cerebral peduncles, while the temporal lobes are broad and flat, each filling a more or less irregular quadrate area in the lateral portion of the middle fossa.

THE VENTRICLES AND THE VISUAL CORTEX

The ventricular system of the hemispheres is particularly significant, inasmuch as the lateral ventricle develops a posterior horn extending into the occipital region, which is not the case in any of the lcmurs. The ventricular extension of the olfactory peduncle characteristic of many mammals is, however, being obliterated. The visual cortex of the cercbral hemisphere shows a marked increase as compared with Iower mammals and even the lemurs. It is easily detected by the naked eye and occupies nearly one-third of the entire neopallium.

\section{THE CEREBELLUM}

The cerebellum is notable as indicating those lateral expansions which in higher primates become the most important part of the organ, namely, the IateraI cerebellar lobes. Tarsius in many respects is an excellent prototype in most simple terms for the subsequent extensive development of the primate cerebellum. Its tentorial surface lies at an angle of about $-0^{\circ}$ with the axis of the stem, thus showing the early tendencies which eventually carry this surface more nearly into the horizontal. The occipital lobe covers most of the tentorial surface which consists largely of the superior vermal 
portion of the cerebellum. A well-defined floceulus and parafloceulus are attached along the region of confluence with the axis.

The occipital surface also occupies a vertical position and as yet has not
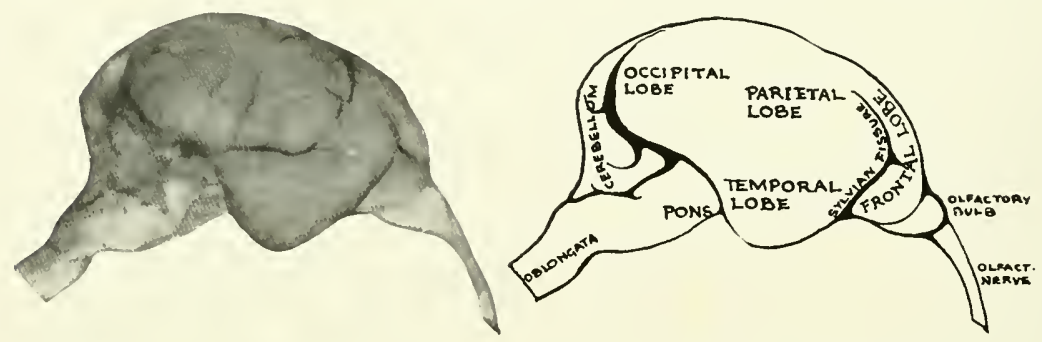

FIG. 47. RIGHT LATERAL SURFACE OF BRAIN, TARSIUS SPECTRUM.

[Actual Length, 2 I mm.]

been tipped backward, as is the case in higher primates. This fact accounts for the exposed position of the urula and nodule which have not yet undergone that introversion which produces their ultimate intraventricular relation characteristic of most of the anthropoids.

The fissures of the cerebellum have an almost diagrammatic clearness in producing the three major subdivisions of the cerebelhum. The fissura prima appears upon the tentorial surface and extends outward from the vermis upon the lateral lobe without sulcal interruption. This is likewise true of several other fissures of this surface. The fissura secunda appears in relation with the occipital surface but, as in other mammals, it does not extend out upon the Iateral expansion of the cerebellum, being interrupted by the presence of a definite paramedian sulcus. All things considered, the cerebellum of tarsius is the most primitive of all primates, and yet it foreshadows in so many notable details the future development of this organ in apes and man that it must be regarded as inherently anthropoid. 


\section{The Brain Sten in Tarsius}

THE VENTRAL SURFACE

The ventral appearance of the brain stem in tarsius is peculiar because of the relatively small size of the pons Varolii, the pyramids, the inferior olivary eminences and the cerebral peduncles. All of these are evidences of a low degree of development in the neokinetic organization of the animal. Its voluntary control of motor activity, its dispensation of coordinative control of the muscles, its measure of simultaneous regulation in the movements of the head, eyes and hands, could not be other than extremely simple and generalized on the basis of these structures. The pyramicis appear as two narrow bands extending caudad from the lower border of the pons toward the upper cervical region of the spinal cord. The pons itself is a narrow, flat band of very little surface prominence. Caudal to it the corpus trapezoideum occupies a transverse position upon the surface of the axis. Tausius is the only primate in which the corpus trapezoideum is entirely exposed in this position. This fact does not imply any greater organization in the auditory apparatus of the animal, but does denote how poorly the pons Varolii has developed.

The olive makes a very small protuberance lateral to the pyramid and is separated from it by a wide area hardly justifying the term preolivary sulcus. The emergent fibers of the hypoglossal nerve, however, make their appearance on the surface in close relation to the olivary eminence. Two small peduncles form the caudal boundary of a limited optico-peduncular space whose cephalic boundary is provided by the massive optic chiasm and tracts.

THE DORSAL SURFACE

The dorsal surface, upon removal of the cerebellum, shows a poorly defined clava, cuneus and tuberculum trigemini. The floor of the fourth 
ventricke has its usual boundaries but shows in a most indefinite way these surface markings which characterize it in Anthropoidea. This dorsal surface of the brain stem, however, assumes great prominenee because of the develop-
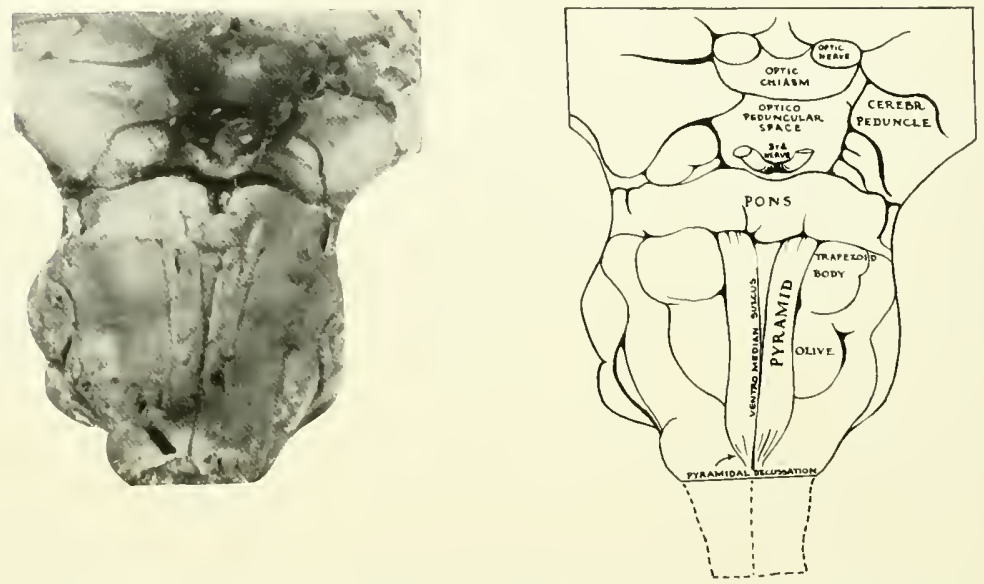

FIG. 48. VENTRAL SURFACE OF BRAIN STEM OF TARSIUS SPECTRUM. [Actual Length, $16 \mathrm{~mm}$.]

Key to Dlagran. Cerebr.-Peduncle, Cerebral Peduncle.

ments in the mesencephalie roofplate. Here the inferior colliculus and mesial genieulate body are larger and better defined than in all other primates. The superior colliculus attains dimensions almost warranting the designation of optic lobe, but in any event much more conspicuous than in lemurs, monkeys, apes or man.

Thus, in the fecble development of eertain features whose progressive expansion characterizes the line of evolutional development in the primates, tarsius appears to be an expression of this process in its simplest terms. It indicates this primitiveness quite as much in the superior and inferior colliculi of the midbrain. In spite of the fact that the animal has made such notable 
gains in the teleneephalization of vision, the primordial receiving centers for this sense still maintain a high degree of prominence in the midbrain. This primitive feature becomes progressively more prominent in descending
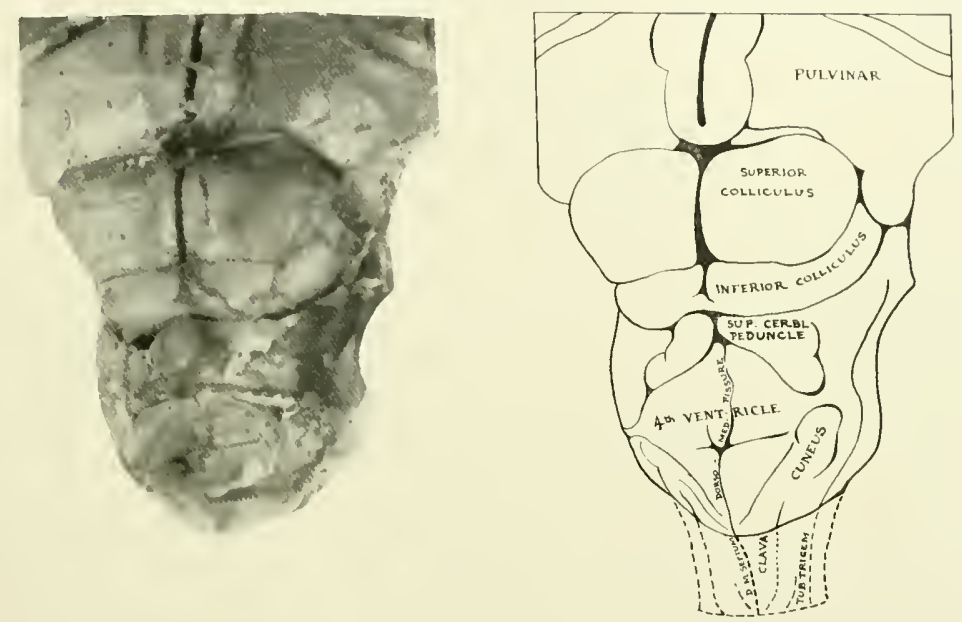

FIG. 49. DORSAL SURFACE OF BRAIN STEM OF TARSIUS SPECTRLII. [Actual Length, $16 \mathrm{~mm}$.]

Key to Diagrair. dorso-Mfed. fissure, Dorsomedian fissure; D. M. Septum, Dorsomedian Septum; sup. Cerebl. Peduncle, Superior Cerebellar Peduncle; tub. Trigem. Tuberculum Trigemini.

the vertebrate scale to its convergence upon the reptilian basis of mammalian differentiation, at which level it reaches its full expansion in the optic lobes.

\section{Internal Structure of the Brain Steil}

The impression obtained from a microscopic review of the brain stem is that of a primitive mammalian organization. The structural details are, so to speak, still in the crude. They have none of that refined definition which distinguishes the higher primates and man. All of the topographical features 
throughout the stem show an indecisive differentiation. This feature is particularly noted in the cranial nerves whose emergent fibers by comparison with other primates seem coarse and heavy. The nuclear territories and tract

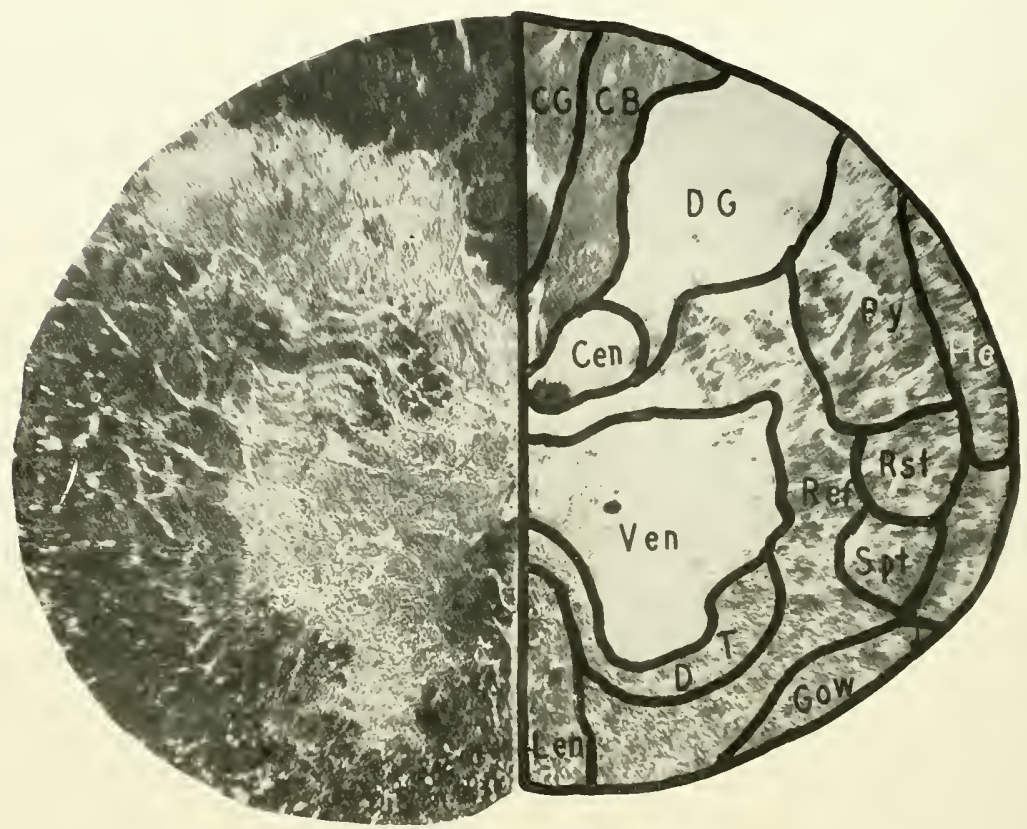

FIG. jO. TARSIUS SPECTRUM. LEVEL OF TIIE FIRST CERVICAL SEGMENT. Cen, Central Gray Matter; CB, Column of Burdach; CG, Column of Goll; DG, Dorsal Gray Column; DT, Deiterso-spinal Tract; FlE, Dorsal Spinoccrebellar Tract; gow, Ventral Spinocerebellar Tract; PY, Crossed and Uncrossed Pyramidal Tract; REF, Reticular Formation; RST, Rubrospinal Tract; SPT, Spinothalamic Tract; ven, Ventral Gray Column. [Accession No. 210 . Section 26. Actual Size, $3 \times 2 \mathrm{~mm}$.]

regions alike are indefinite in their boundaries. Some noteworthy specializations appear in particular mechanisms, such, for example, as the pronounced expansion of the ventral gray column connected with the origin of the spinal 
accessory nerve, and the large dimensions of the vestibular area. These features will be especially dealt with in the interpretation of their physiological significance.

LEVEL OF THE PYRAMIDAL DECUSSATION (FIG, jI)

At this level the characteristic feature is the crossing from one side to the other of pyramidal fibers $(\mathrm{Pyx})$. This is much less regular than in other primates. The crossing strands are somewhat indiscriminate in their disposition. The bundles tend to interlace and have little of that successive crossing first from one side and then from the other, notable in higher primates. There is a suggestion, particularly at the caudal end of the decussation, that some fibers may make their way into the dorsal columns after the manner of certain lower mammals. This observation needs experimental confirmation. PalWeigert preparations scarcely more than suggest this possibility. In all details the pyramidal crossing seems more primitive than in any other primates. If some fibers actually do enter the dorsal columns, the conception of tarsius as a much generalized intermediate form gains further support. The central gray matter ( Cen) is large and rectangular in outline, containing near its center the central canal. Its greatest diameters are in the antero-posterior direction. It is connected with a large dorsal gray column by a cervix of somewhat irregular outline, but separated from the ventral gray column by the crossing pyramidal fibers which assume a juxtagriseal position in dense bundles after they have decussated. The large size of the ventral gray column $(\mathrm{Ven})$ is notable, particularly as there appear to arise in it many fibers which take a course backward and outward, characteristic of the spinal accessory nerve. These fibers constitute a nerve of much larger size and generally more conspicuous than in any of the apes. This fact, in conjunction with the great prominence of the ventral gray column, suggests the probable specialization in connection with one of the peculiar habits of 
tarsius, namely, the turning of its head completely around so that it can look backward as well as to the front. The animal moves its head rather than its eyes in its visual pursuit of objects. This requires a highly specialized cervical

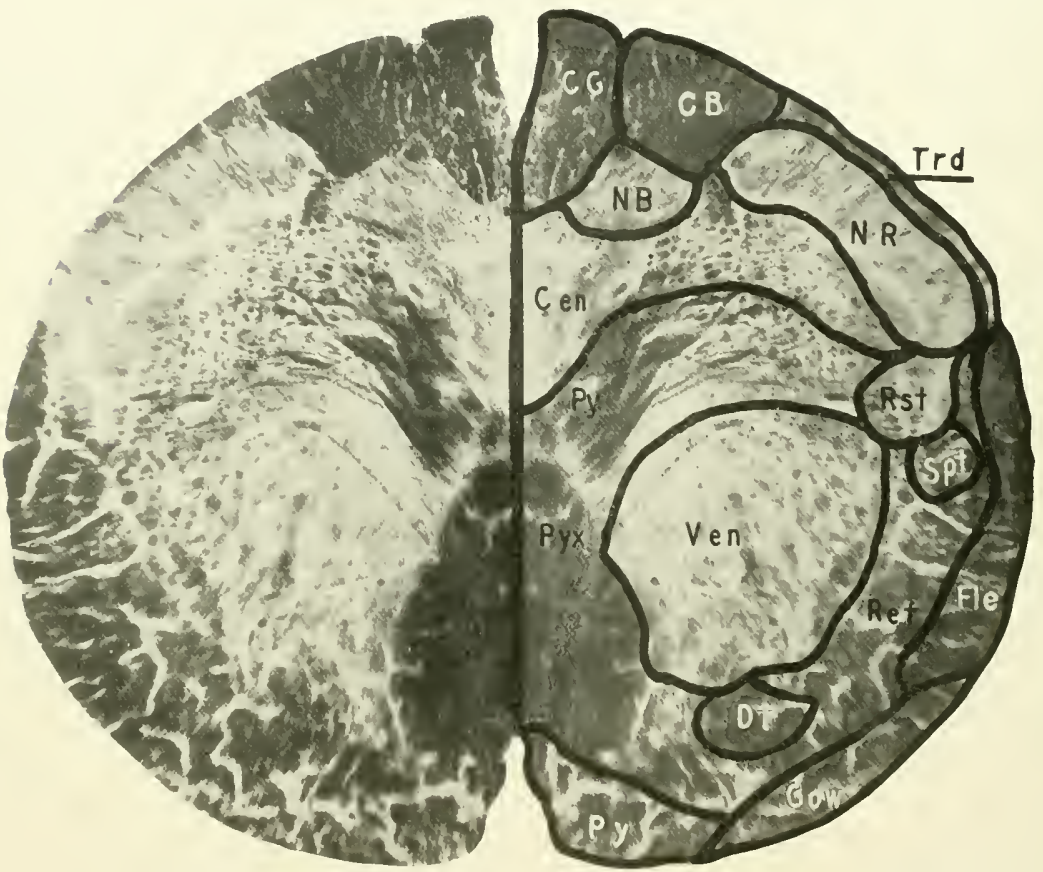

FIG. jI. TARSIUS SPECTRLM. LEIEL OF THE PYRAMIDAL DECLSSATION. CB, Column of Burdach; CEN, Central Gray Matter; CG, Column of Goll; DT, Deiterso-spinal Tract; ILE, Dorsal Spinocercbellar Tract; gow; Ventral Spinocerebellar Tract; NB, Nucleus of Burdach; Nr, Nucleus of Rolando; PY, Pyramid; PYX, Pyramidal Decussation; REF, Reticular Formation; Rst, Rubrospinal Tract; SPT, Spinothalamic Tract; TRD, Descending Trigeninal Tract; VI, Ventral Gray Matter. [Accession No. 210. Section 65. Actual Size $+\times 3 \mathrm{~mm}$.]

musculature, which no doubt determines the prominent spinal accessory feature in this region of the brain stem. 
The dorsal field consists of a small column of Goll (CG), a large column of Burdach (CB) and an extensive substantia gelatinosa (NR). Adjacent to the latter is a well-defined descending trigeminal tract ( $\mathrm{Trd})$. The separation of the dorsal gray column is Icss pronounced than in many of the higher forms. The general proportions of the elements entering in to this field suggest at once at tail and lower extremity which give rise to a sensory influx much less than that from the hand and arm. It is probably the case that in tarsius differentiation of the upper cxtremity is more effective than of the lower extremity. The great speed and accuracy with which the hands are cmployed in the capture of insects, and at the same time for prehensile purposes in alighting after its remarkable leaps, would secm to imply a speciatization in the upper extremity much superior to that in the hind limbs.

The tail has no prehensile characteristics, and while it acts in a supplementary manner for supporting the clinging posture of the animal, its activities indicate no great increment of sensory influx. The size of the nueleus of Rolando is indicative of facial innervation, which is essential to the animal in guicling its locomotion.

The lateral white column eontains in its circumferential zone the spinocerebellar tracts (FIe, Gow) while in the intermediate zone are the rubrospinal (Rst), Deiterso-spinal (DT) and spinothalamic tracts $\left(S_{p t}\right)$. The pyramidal status denotes a behavioral complex consisting of relatively few components and extremely poor in its pattern for skilled acts.

\section{CAUDAL EXTREMITY OF TIE DORSAL SENSORY NUCLEI (FIG. j2)}

Here the chicf feature is the appcarance of the three nuclear structures representing discriminative sensory transmission from the body. The nucleus of Goll (NG) is well defined but shows no evidence of the median unpaired nucleus of Bischoff. This deficiency in tarsius harmonizes with the fact that the animal's tail is not highly specialized, although it acts in spring- 
106

like capacity, aiding the animal to cling in vertical position. The tail has no prehensile qualities but serves in the general capacity of a steering and balancing organ much as in the tailed monkeys of the old world.

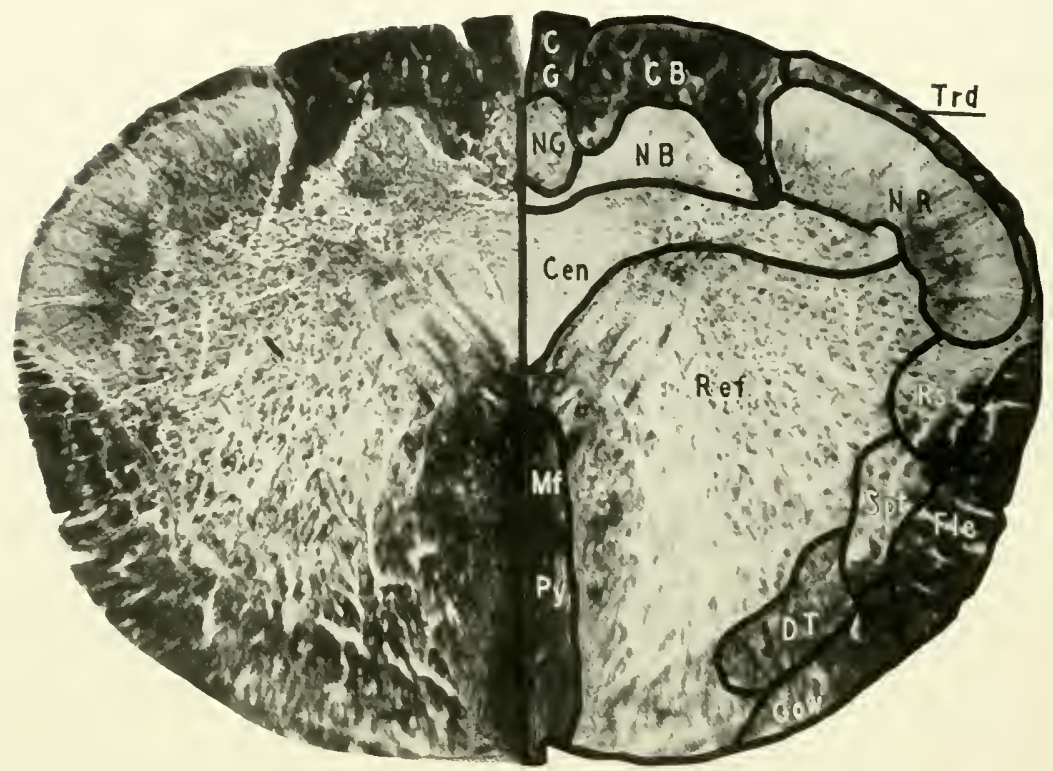

FIG. j2. TARSIUS SPECTRLM. LEVEL OF THE DORSAL SENSORT NUCLEI. CEN, Central Gray Matter; cB, Column of Burdach; CG, Column of Goll; DT, Deiterso-spinal Tract; fLE, Dorsil Spinocerebellar Tract; Gow, Ventral Spinocerebellar Tract; MF, Mesial Fillet; NB, Nucleus of Burdach; NG, Nucleus of Gol]; NR, Nucleus of Rolando; PY, Pyramid; REF, Reticular Formation; RST, Rubrospinal Tract; spt, Spinothalamic Tract; TrD, Descending Trigeminal Tract. [Accession No. 210. Section 72. Actual Size, $6 \times+\mathrm{mm}$.]

The nucleus of Burdach (NB) is considerably larger than the nucleus of Goll. This fact gains considerable weight when the columns of Burdach and Goll are contrasted. Tarsius seems to possess a mechanism for sensory conduction more extensive in the representation of its hands and arms 
than of its feet, legs and tail. In contrast with the lemur and marmoset, this relation approaches nearer to the more highly developed anthropoids. The explanation, however, depends probably not so much upon the high speciatization in the upper extremities, as upon the low specialization in the lower extremities. Tarsius uses the hind legs much as does a frog. It leaps from branch to branch and hops upon the surface of the ground. The specialization of the legs is thus not particularly advanced for purposes of locomotion. It is inferior to that in the forelimbs for such acts as the animal clevelops in connection with procuring food.

The nucleus of Rolando (NR) is particularly prominent as a sensory chement. This is also true of the descending trigeminal tract ( $\operatorname{Trd}$ ), from which it is obvious that the sensory innervation of the head and face plays a conspicuous part in directing the animal's locomotion. The head and face are especially provided with vibrissac about the chin, mouth, and beneath the nose. Supraorbital vibrissae also exist. The central gray matter (Cen) occupies a position in the center of the section but has shown considerable migration dorsad, thus manifesting the general tendency to assume its characteristic position in the floor of the fourth ventricle. The ventral gray column has cntirely disappeared, and its position is occupied by a continuous irregular mass representing the formatio reticularis grisea (Ref). Contiguous with the latter on its lateral aspect is the intermediate medullary substance which contains the rubrospinal (Rst), spinothalamic ( $\mathrm{Spt}$ ) and Deiterso-spinal (DT) tracts.

On the periphery is the circumferential zone which contains the ventral (Gow) and dorsal (Fle) spinocerebellar tracts. Immediately adjacent to the ventromedial sulcus is a dense mass of decussating axons composed of internal arcuate fibers arising in the nucleus of Goll ( $\mathrm{NG}$ ). These decussating fibers form the lower portion of the mesial fillet ( $\mathrm{MT}$ ). 
LEVEL OF THE CALDAL EXTREMITY OF THE INFERIOR OLIVE (FIG. 53 )

At this level the lower tip of the inferior olive makes its appearance ( $\mathrm{IO}$ ). An important fact concerning this structure in tarsius is its indecisive

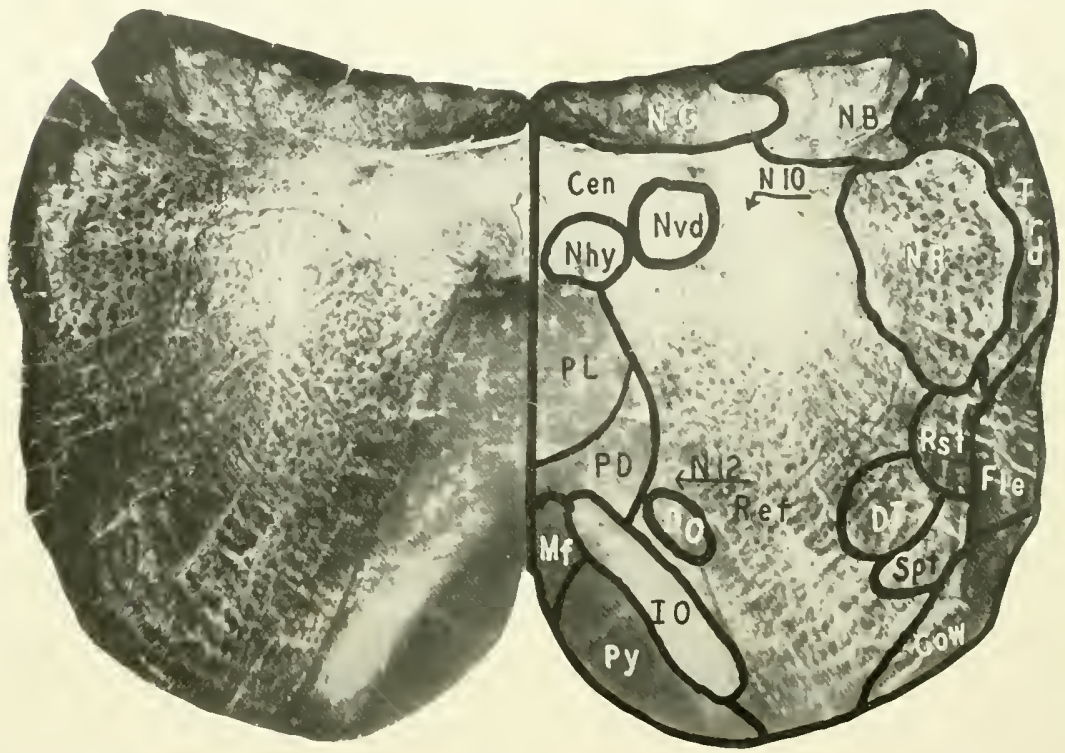

FIC. 53. TARSIUS SPECTREM. LEVEL OF CALDAL TIP OF INFERIOR OLIVE.

CEN, Central Gray Matter; FLE, Dorsal Spinoccrebcllar Tract; Do, Dorsal Accessory Olive; DT, Deitersospinal Tract; Gow, Ventral Spinocercbellar Tract; Io, Inferior Olive; MF, Mesial Fillet; vB, Nucleus of Burdach; NG, Nucleus of Gull; NHy, Hypoglossal Nucleus; NR, Nucleus of Rolando; Nvd, Dorsal Vagal Nucleus; Nio, Vagus Nerve; n 2, l Iypoglossal Nerve; PD, Predorsal Fasciculus; PL, Posterior Longitudinal Fasciculus; Py, Pyramid; ReF, Retieular Formation; RST, Rubrospinal Tract; SPT, Spinothalamic Tract; TRD, Descending Trigeninal Tract. [Accession No. 210. Section 95. Actual Size $6 \times 3 \mathrm{~mm}$.]

outline. Its diminutive size as compared with that of other primates is also notable. Nesial to the olive is the pyramid ( Py) which likewise is small. Dorsomesial to the pyramid is the mesial fillet (Mf), still receiv- 
ing some contributions from the internal arcuate fibers arising in the nucleus of Burdach (N B). By contrast, the extensive size of the posterior longitudinal fasciculus (PL) and of the predorsal bundle (PD) denotes the importance of these colliculo-spinal and other midbrain connections.

The central gray matter ( Cen) is oval in outline and still more dorsal in position. It contains in its center a much enlarged central canal, while in its ventromesial area is the nucleus hypoglossus ( $\mathrm{Nhy}$ ). This nucleus has none of the discrete distinction in its boundaries notable in the higher primates. The emergent fibers passing from it appear in larger and coarser bundles than is the case in any other species examined.

In a position dorsal to the central gray matter are the chief nuclei of the dorsal sensory field. Their relation discloses the preponderance of the macleus of Burdach (NB) as compared with the nucleus of Goll (NG). The large size of the nucleus of Rolando (NR) and of the descending trigeminal tract ( $\mathrm{Trd}$ ) calls attention to the important rôle of the head and face as a sensory director of the animal's locomotion.

The outline of the dorsal aspect of the section shows an increasing concavity due to the presence of the uvula cerebelli in this position. The fibers of the spinal accessory nerve pass ventrolaterally through the nucleus of Rolando (NR) toward their point of emergence. The reticular formation (Ref) occupies the greatest portion of the section at this level. In its lateral periphery is the nucleus ambiguus from which the emergent fibers of the vagus nerve pass backward and inward toward the central gray matter in the first part of their intramedullary course. The reticular formation is further specialized to form the nucleus funiculus lateralis and is penetrated by many long internal arcuate fibers. It is noteworthy that these latter elements have none of the conspicuous appearance characteristic of higher primates. They, like the mesial fillet and the pyramid of 
tarsius, indicate a less highly organized condition in discriminative sensibility and voluntary motor control.

It seems obvious from the known behavior of the animal that its range of skilled movements is comparatively limited. From these structures in the brain stem it is permissible to conclude that tarsiers in general acquire but little increase in their motor attaimments under the influence of training. This level might well pass for the corresponding region in the cat or rabbit; in fact, its components have a close resemblance to marsupial organization. The medullary substance in the intermediate zone contains the rubrospinal and spinothalamic tracts (Rst, Spt), while on the circumference are the two ascending cercbellar fascicles (FIe, Gow).

A small collection of gray matter dorsal to the main body of the olive is the dorsal accessory olivary nucleus (DO).

\section{LEVEL THROUGH THE MIDDLE OF THE INFERIOR OLIVARY NUCLEUS (FIG. $j 4$ )}

At this level the inferior olive is conspicuous because of its structural inferiority as compared with other species. This nucleus in tarsius consists of an aggregation of gray matter, so poorly differentiated from the formatio reticularis that it might appear as an intrinsic part of this formation. The portion of the nucleus which may be discerned appears to correspond to the mesial accessory olive in other forms. A very small mass of cells lateral to this structure represents the inferior olive itself. Thus the most conspicuous portion of the nucleus seems to represent what is usually described as the paleo-olive. Compared with either the cat or the rabbit, the olive in tarsius is distinctly more primitive, and this certainly is the case in comparison with all other primates. Judged alone by the criteria of this structure, it would seem that the tarsier occupies a low position in the organization of its central nervous system; one which might, however, serve most advantageously as a fairly unbiased foundation for extensive further modifications. In the inter- 
pretation of olivary function, tarsius is an important witness concerning the activity of this nucleus. If, as is assumed to be the fact, the olivary nucleus is essential to simultancous coordination of the hands, eyes and head in complex

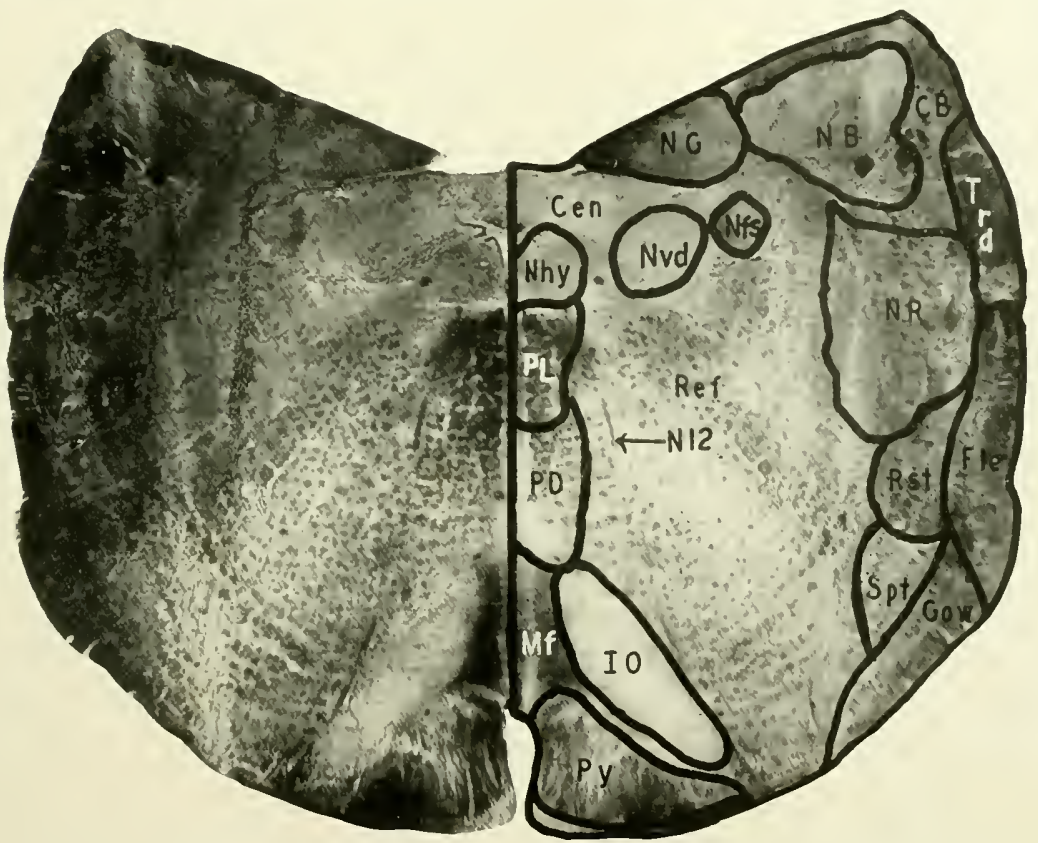

FIG. j4. TARSIUS SPECTRUII. LEVEL THROUGH IIIDDLE OF INFERIOR OLIVE. CB, Column of Burdach; CEN, Central Gray Matter; Fle, Dorsal Spinocerebellar Tract; Gow, Ventral Spinocerebellar Tract; IO, Inferior Olive; MF, Mesial Fillct; NB. Nucleus of Burdach; NFs, Nucleus Fasciculus Solitarius; NG, Nucleus of Goll; vir;, Hypoglossal Nucleus; NR, Nucleus of Rolando; Nvd, Dorsal Vagal Nuclcus; Ni 2, Hypoglossal Nerve; PD, Predorsal Bundle; PL, Posterior Longitudinal Fasciculus; Py, Pyramid; REF, Reticular Formation; RST, Rubrospinal Tract; SPT, Spinothalamic Tract; TRD, Descending Trigeminal Tract. [Accession No. 210. Section [00. Actual Size, $7 \times+\mathrm{mm}$.]

skilled acts and helps to facilitate the coordination of all skilled learned performances, its inferior development in the tarsier implies a low development of such activity. 
It has already been noted that the animal is able to turn its head completely around so that it may in effect look both forward and backward. Such rotation is due to the fact that the animal moves its eyes relatively little in the visual pursuit of objects. If it wishes to cover its visual field it does so in great measure by movements of the head. This predominance of cephalogyric action over ocular morement may account for the extensive derelopment of the ventral gray column in those cervical regions of the spinal cord in which the spinal accessory nerve takes origin. Thus may be explained the preeminence of the neck muscles in acts of visual pursuit, as well as the inconsiderable development of the inferior olive. From the behavior of tarsius it is clear that the animal does not cmploy the muscles of the eyes, hands and head in many acts requiring intimate cooperation. It is essentially a nocturnal hunter, and this fact in itself limits its manual activities to a somewhat restricted range of performance. The remarkable dexterity of the tarsier in capturing insects on the wing, as it is said to do, probably represents the acme of its skilled achievement. This no doubt is a highly specialized act, but it is no more complex than that manifested by many birds which capture their prey while on the wing. Such development of skill would not necessarily make great demand upon the olivary mechanism. Two other facts support the view that tarsiers do not require particular exactitude in the simultaneous control of the head, eye and hand muscles, i.c., the absence of retinal macula and the more or less complete decussation of fibers in the optic chiasm. Both of these important conditions imply the absence of stereoscopic vision, as they also indicate the lack of those visual specializations essential to the development of highly shilled acts. The indispensable relation of simultancous coordination of head, eye and hand in such acts as handwriting is obvious. In acts of this kind the eyes follow the hand, and the head cooperates with the eyes in this exact visual guidance. Tarsius is capable of no reactions comparable in any sense to such a highly developed skilled performance. It manifests no 
motor pattern which necessitates simultaneous association of the ocular, cervical and brachial musculature. It is not surprising in this light that the inferior olive is poorly developed in tarsius and in marked contrast to the proportions and configuration of higher primates.

The other features at this level serve to bear out the observations already made concerning the motor and sensory organization, as these functions are illustrated by the small size of the pyramid and mesial fillet.

The great importance of the animal's automatic associated movements is indicated by the size of its midbrain bundles, the posterior longitudinal and predorsal fasciculi (PL, PD). The central gray matter has assumed its position in the floor of the fourth ventricle, and above the central canal a massive obex marks the point of ventricular transition. The nucleus of the hypoglossal nerve has all the indecisiveness in boundary characteristic of lower levels. Its emergent fibers are coarse and heavy. Lateral to this nucleus is the dorsal vagal nucleus $(\mathrm{Nvd})$. In the dorsolateral angle of the central gray matter is the nucleus of the fasciculus solitarius ( $\mathrm{N} f \mathrm{~s}$ ).

The nuclei of Goll and Burdach (NG, NB) are both prominent and partially invested by fibers forming their corresponding columns. The nucleus of Burdach is remarkable in tarsius for the very high development of the ancillary nucleus of Blumenau. The nucleus of Rolando (NR) and the descending trigeminal tract ( Trd) are further removed from the periphery due to the moving of the dorsal spinocerebellar tract into a more dorsal position. The reticular formation still occupies the largest portion of the section and is characterized by its diffuse arrangement (Ref). In its ventrolateral portion may be discerned the nucleus funiculus lateralis.

\section{LEVEL OF THE VESTIBULAR NUCLEI (FIG. $j j$ )}

At the level of the vestibular nuclei the dorsal sensory freld has changed in character, as is the rule in all primates. It still represents proprioceptors, 
but of a highly specialized order. This field, formerly occupied by the nuclei of Goll and Burdach, has given place to the nucleus of Deiters (ND) and the nucleus of Schwabe (NSc), both of which have acquired remark-

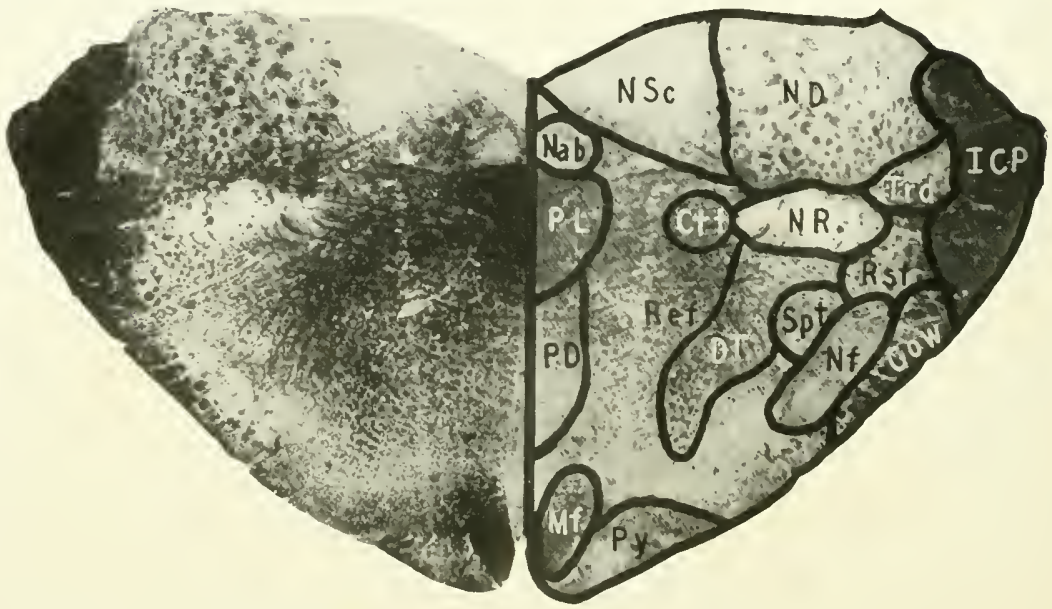

FIG. 5j. TARSIUS SPECTRUM. LEVEL OF THE IESTIBULAR COMPLEX.

Cтт, Central Tegmental Tract; Dr, Deiterso-spinal Tract; cow, Ventral Spinocerebellar Tract; IcP, Inferior Cercbellar Peduncle; MF, Mesial Fillet; NAB, Nucleus Abducentis; ND, Deiters' Nucleus; NF, Facial Nucleus; NR, Nucleus of Rolando; Nsc, Nucleus of Schwalbe; PD, Predorsal Bundle; $P_{L}$, Posterior Longitudinal Fasciculus: PY, Pyramid; ReF, Reticular Formation; RST, Rubrospinal Tract; SPT, Spinothalamic Tract; TRD, Descending Trigeminal Tract. [Accession No. 310. Section 1 fo. Actual Size $7 \times 3 \mathrm{~mm}$.]

able dimensions as compared with other primates. The Deitersal area in tarsius is larger than in the cat, rabbit, kangaroo, horse, dog, or any of the primates. The triangular nucleus of Schwalbe, while large, is perhaps not so striking in comparison with other forms.

To find such extensive development in the balancing mechanism of tarsius implies that the animal requires a most highly efficient apparatus for equilibrium. The behavior of tarsius bears out this supposition. There is nothing perhaps in its postural requirements while at rest which may sug- 
gest extensive equilibratory needs; but the Iong, swift leaps which it makes, not unlike volplaning, would almost certainly require a capable mechanism for maintaining balance. Compared with other primates, tarsius may be considered quite alone in the specialization of this peculiar flight-like type of locomotion. In the case of lemur and marmoset, the leaping propensities have a different character, appearing to be internediate steps in a continuous process of passing from one point of support to another. In this act, all four extremities and the tail participate. The gibbon is another example of flight-like passage through the trees, but in the case of this latter primate, the arms are the principal means of locomotion in the long swings from branch to branch. Tarsius, on the other hand, executes its leaps more in the nature of long dives or upward hops in which it glides through the air for considerable distances in order to reach its next objective. If it is true that in these dives it captures insects on the wing, then the delicate balancing needed for its locomotion is all the more apparent.

The great prominence of the vestibular area must depend upon some such specialization as this in tarsius, although it is to be regretted that no authentic statements are forthcoming concerning the actual equilibratory derelopment of this animal. It is remarkable and noteworthy, however, that tarsius leads all the primates, and for that matter most of the mammals, in the ligh specialization of its vestibular nucki.

At this level the fourth ventricle is widely open and the lateral recesses communicate with it over its dorsolateral angle. The urula fills the entire ventricular cavity. On the lateral margin of Deiters' nucleus (ND) are the collected bundles of the inferior cerebellar peduncle (ICP), while ventral to the nucleus is the substantia gelatinosa of Rolando (NR). Along the outer border of the latter structure lies the descending trigeminal tract $(\operatorname{Trd})$. The rubrospinal ( R s t) and spinothalamic tracts $(S p t)$ occupy a position upon the outer edge of the formatio reticularis ( $\mathrm{Ref}$ ). The posterior 
longitudinal fasciculus (PL) has been augmented by the addition of fibers from Deiters' nucleus, whose course may be traced inward and backward from this nucleus. Some of the caudal fibers of the vestibular division

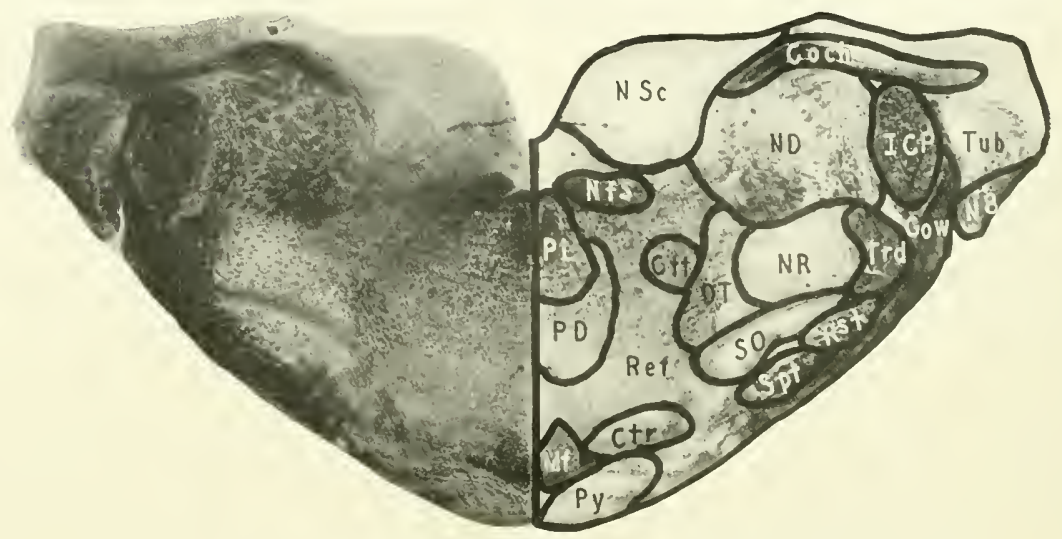

FIG. ;6. TARSIUS SPECTRUM. LEVEL OF CALDAL EXTREMITI OF TRAPEZOID BODY.

coch, Cochlear Fibers; стк, Trapezoid Body; стт, Central Tegmental Tract; DT, Deitersu-spinal Tract; Gow, Ventral Spinocerebellar Tract; ICP, Inferior Cerebellar Peduncle; MF, Mesial Fillet; ND, Deitersal Area; N8, Auditory Nerve; Nfs, Genu of Facial Nerve; NR, Nucleus of Rolando; Nsc, Nucleus of Schwalbe; PD, Prcdorsal Bundle; PL, Posterior Longitudinal Fasciculus; PY, Pyramid; REF, Reticular Formation; RST, Rubrospinal Tract; so, Superior Olive; SPT, Spinothalamic Tract; TRD, Descending Trigeminal Tract; TUB, Tuberculum Acusticum. [Accession No. 210. Section 153. Actual Size $-\times 3 \mathrm{~mm}$.]

of the eighth nerve pass through the descending trigeminal tract and the substantia gelatinosa to enter Deiters' nucleus. A large nuclear structure on the periphery of the reticular formation gives rise to the first portion of the facial nerve. This is the nucleus facialis ( $\mathrm{Nf}$ ). The fibers arising in it pass backward and inward toward the floor of the fourth ventricte in the form of a heavy spray. This mucleus in tarsius is nearer the periphery of the section than in any of the other primates and the fibers constituting the furst portion of the facial nerve are coarser than observed in other species. 
Both of these conditions are in closer accord with the subprimate mammals than with the Anthropoidea.

Mesial to the facial nucleus is a disseminated collection of axons reaching backward toward Deiters' nucleus. These form the beginning of the Deiterso-spinal tracts (DT). Immediately dorsal to the posterior longitudinal fasciculus is the caudal extremity of the nucleus abducentis $(\mathrm{Nab})$. The predorsal bundle (PD) is large and indicates to what degree the animal depends on its automatic movements. This observation becomes more significant in vicw of the small size of the pyramid which calls attention to the relatively meager control of voluntary movement possessed by tarsius. The mesial fillet (Mf) occupying a position dorsal to the pyramid, denotes a low degree of discriminative sensibility in the animal.

All of these facts collectively signify an animal of a simple motor organization and extremely limited in such behavior as is conditioned by learning and imitation.

\section{LEIEL OF THE CEREBELLAR NUCLEI (FIG. 57)}

Characteristic features at this level are the appearance of the heavy bundles constituting the intramedullary portions of the sixth and seventh cranial nerves ( $N 6, N_{7}$ ). The fibers of the vestibular division of the eighth nerve make their way to Deiters' nucleus between the restiform body (ICP) and the descending trigeminal tract ( $\operatorname{Trd}$ ). Some fibers of the acoustic division (N8) of the eighth nerve appear in relation to the tubercuIum acusticum ( $T u b)$. The fourth ventricle is still further reduced in size. Its roof is formed by the median portion of the cerebellum in which is situated the roof nucleus ( $\mathrm{Nfg}$ ). Heavy bundles of fibers entering the juxtarestiform body pass backward and inward from Deiters' nucleus to the nucleus of the roof. Lateral to these fibers in the median vestibule of the cerebellum is a collection of gray matter forming the nucleus dentatus and 
the nucleus emboliform is ( NDt). It is impossible to discern distinctive boundaries between these two nuclear masses. Even by means of reconstruction these nuclei do not disclose any distinct individualization. It is hence deemed

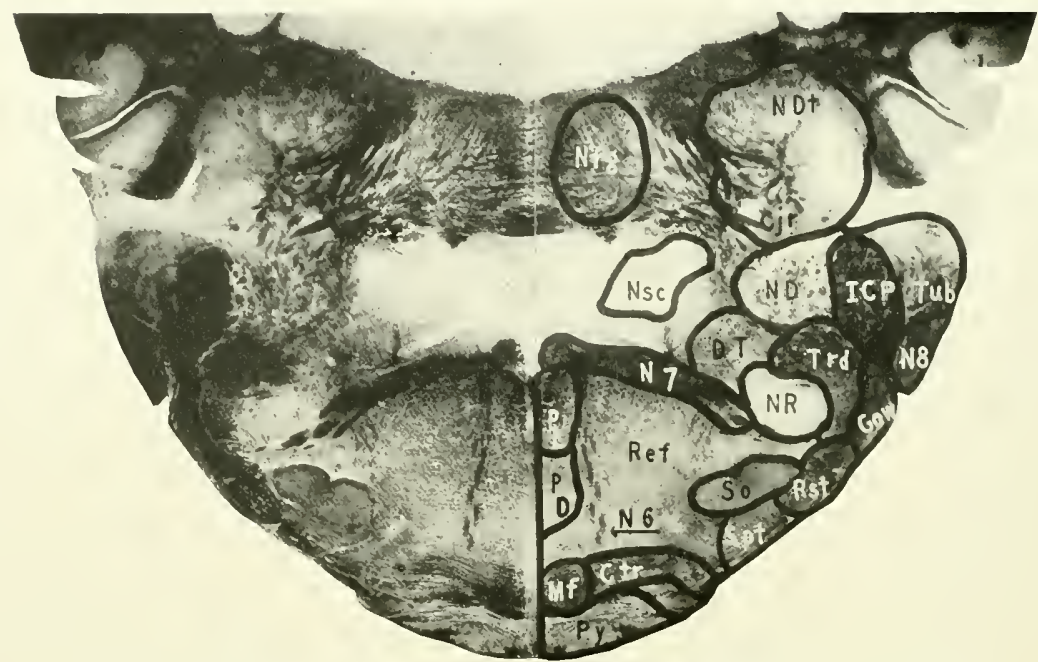

FIG. 5- TARSIUS SPECTRUM. LEVEL OF THE CEREBELLAR NLCLEI.

CJR, Justarestiform Body; CrR, Trapezoid Body; DT, Deitersil Tract; Gow, Ventral Spinocercbellar Tract; IC1, Inferior Cerebellar Peduncle; MF, Mesial Fillet; nd, Deitcrsal Area; NDt, Cerebellar Nuclei, Lateral Group; Nfg, Cerebellar Nuclei, Mesial Group; Nr, Nucleus of Rolando; Nsc, Nucleus of Schwalbe; N6, Abducens Nerve; N7, Facial Nerve; N8, Eighth Nerve; PL, Posterior Longitudinal Fasciculus; PD, Predorsal Bundle; PY, Pyramid; REF. Reticular Formation; Rst, Rubrospinal Tract; so, Superior Olive; Spt, Spinothalamic Tract; TRD, Descending Trigeminal Tract; TUB, Tubcrculum Acusticum. [Accession No. 210. Section 163. Actuil Size $8 \times ; \mathrm{mm}$.]

advisable to designate this collection as the dentate mass. The outstanding feature regarding it is its lack of defunition and the entire absence of any tendency toward convolution which characterizes this nucleus in the higher primates. In tarsius the dentate nucleus is small and, as might be inferred from it, the lateral lobes of the cerebellum are poorly developed. This condition indicates an animal provided with a low degree of coordinative control, 
a fact which permits of the conclusion that the extremities, and particularly the upper extremities, are capable of the simplest motor patterns only This index of motor organization is an added argument supporting the view that tarsius is but meagerly endowed in behavioral reactions.

Adjacent to the ventromedian sulcus of the fourth ventricle, and beneath the ventricular floor, is the dense circular bundle representing the second portion of the seventh nerve $\left(\mathrm{N}_{7}\right)$. Lateral to this facial bundle are the heavy fibers of the abducens nerve (N6), which make their way directly forward toward the trapezoid body (Ctr). They arise in the abducens nucleus which is situated ventral to the triangular nucleus. It is of interest in this connection to note the diffuse character of the triangular nucleus. Its boundaries have none of the definition characteristic of the higher species. Many fibers traverse it, giving the impression of a nuclear territory which has not yet thoroughly established its own autonomy.

The origin of the Deiterso-spinal fibers (DT) is elearly seen. The restiform body (ICP), the substantia gelatinosa of Rolando (NR) and the descending trigeminal tract ( $\mathrm{Trd}$ ) all occupy their usual positions. The trapezoid body $\left(\mathrm{Ctr}\right.$ ), with its large superior olive ( $\mathrm{So}_{\mathrm{o}}$ ) and its extensive decussation, indicates the degree of development in auditory conduction. The reticular formation( $\mathrm{Ref}$ ) is extensive and contains no discretely differentiated territories.

Adjacent to the raphe, the posterior longitudinal and predorsal bundles (PL, PD) appear in the unusually large dimensions characteristic of tarsius. The mesial fillet ( Mf ) is still partially obscured by the crossing fibers of the trapezoid decussation $(\mathrm{Ctr}$ ), while the relatively small size of the pyramid $(\mathrm{Py})$ is decisively shown in comparison to the rest of the section. It is noteworthy that the entire trapezoid body remains without being submerged by pontile fibers. Tarsius is the only one of the primates in which this is the case. Although the corpus trapezoideum is only partially 
exposed in lemur and marmoset, the pons Varolii is so poorly developed in tarsius as to leave this auditory decussation wholly uncovered. This fact again speaks in favor of the low development attained by tarsius in the organization of its skilled performances.

The summary of features in this region of the axis clearly calls attention to the large size of both the auditory and balancing mechanisms of the animal. It is equally emphatic in denoting the relatively low development of voluntary control possessed by tarsius. It clearly indicates the ample provision made for that more fundamental regulation of motion afforded by the posterior longitudinal fasciculus and predorsal bundle. Attention is also called to the rather coarse appearance of both emergent and intersegmental fibers which are in such contrast to the finer architectonics of the higher species.

\section{LEVEL OF THE EMERGENCE OF THE TROCHLEAR NERVE (FIG. ;8)}

At this level the main features of the posterior isthmus are apparent. The roof plate of the fourth ventricle is here formed by the superior medullary velum upon which rests the lingula of the cercbellum. Passing through the velum are the decussating fibers of the trochlear nerve $\left(\mathrm{N}_{4}\right)$ which emerges from this region of the brain stcm. The fourth ventricle is much reduced in size as it approaches the caudal orifice of the aqueduct. The gray matter in its floor ( $\mathrm{Cen}$ ) shows no specialization, and rentral to it are the dense bundles of the posterior longitudinal fasciculus (P L) and the predorsal bundle (PD). Lateral to the central gray matter ( Cen) are the axons constituting the mesencephalic root of the trigeminal nerve, outside of which are the fasciculus uncinatus of Russel (hook bundle) ( $\mathrm{T}$ ur ) and some fibers of the ventrospinal cerebellar tract (Gow). In the dorsolateral position of the section are the fibers forming the lateral fillet (Lf) now ascending on its way to the inferior" colliculus. The fibers of the superior cerebellar peduncle (Sep) occupy a position mesial to the hook bundle of Russel. Some hori- 
zontal fibers are already in process of crossing toward the midline in a position dorsal to the posterior Iongitudinal fasciculus (PL). This forms part of the dorsal decussation of the superior cerebellar peduncle.

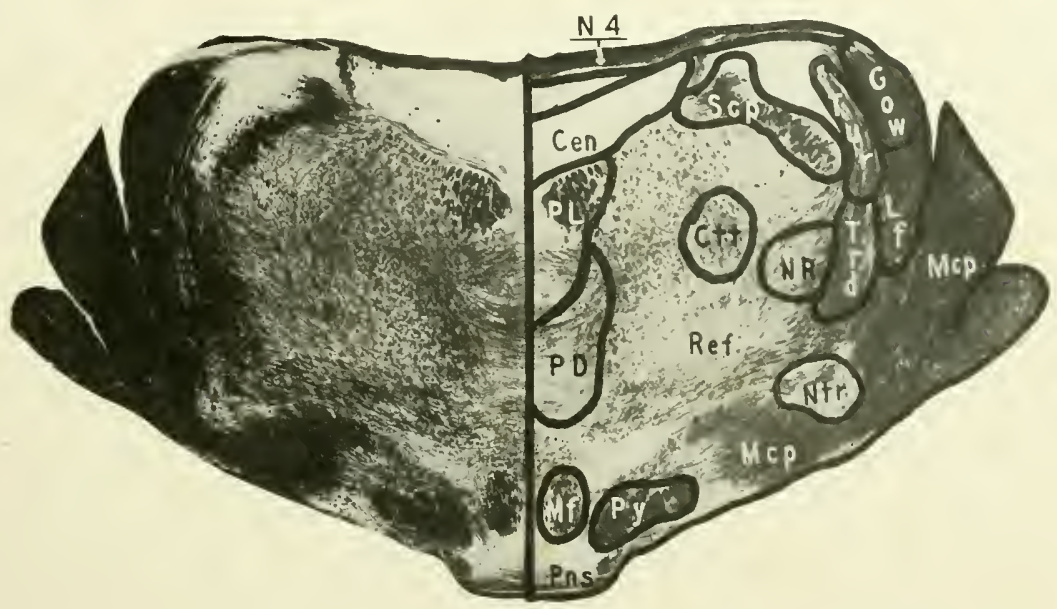

FIG. 58. TARSIUS SPECTRUM. LEVEL OF THE TROCHLEAR EMERGENCE.

CEN, Central Gray Matter; crT, Central Tegmental Tract; gow, Ventral Spinocercbellar Tract; LF, Lateral Fillet; MCP, Middle Cerebellar Peduncle; MF, Mesial Fillet; NR, Nucleus of Rolando; NTR, Trapezoid Nucleus; N4, Trochlear Nerve; PD, Predorsal Bundle; PL, Posterior Longitudinal Fasciculus; PNs, Pons; PY, Pyramid; REF, Reticular Formation; SCP. Superior Cerebellar Peduncle; TRD, Descending Trigeminal Tract; TUR, Tractus Uncinatus of Russel (Hook Bundle). [Accession No. 2 10. Section 195. Actua[ Size $9 \times 5 \mathrm{~mm}$.]

The fibers constituting the ventral peduncular decussation have already moved into a position nearer the midline. This latter disposition in the decussation of the superior cerebellar peduncle is characteristic of all of the primates. The arrangement of the more dorsal fibers in tarsius, however, corresponds more to the conditions in the cat and the rabbit in which the dorsal decussating axons from the superior cerebellar peduncle tend to take up positions dorsal to the bundles of the posterior longitudinal fasciculus. 
In another respect the superior cerebellar peduncles show primitive characteristics in tarsius. This appears in the fact that the crossing of this cerebellar connection begins much lower down than is the case in any of the other primates. As in other instances, the peduncle crosses in two divisions, but the more dorsal division decussates earlier in tarsius than in other forms, leaving a dense ventral bundle in the lateral portion of the reticular formation. These more ventral fibers eventually move inward to complete the superior peduncular decussation. Especial importance attaches to this division of the decussation, as it represents a less highly organized cerebellar comnection than in any of the other primates.

The ventral portion of the section contains a small collection of pontile fibers and nuclei. The number of the crossing fibers in the pons is so limited that it is difficult to distinguish the three typical strata of this portion of the stem. On the other hand, there is a slight separation of the pyramidal fibers by transverse axons passing through them in the pons region. Pyramidal dissemination, however, has none of those characteristic features prominent in all of the other primates. The mesial fillet ( $\mathrm{If}$ ) occupies a position immediately dorsal to the pyramid. In the lateral portion of the section is the upper extremity of the trapezoid nucleus $(\mathrm{Ntr})$. In general appearance this level is notable for the unusually meager representation of the pontile nuclei and the primitive manner in which the supcrior cerebellar peduncle undergoes its decussation.

\section{LEVEL OF THE INFERIOR COLLICULUS (FIG. j9)}

Here, the special features are the marked development of the tectum (IC) and the pons ( $\mathrm{Pns}$ ). This Iatter character of the brain stem in tarsius deserves particular consideration. In several ways it differs from the corresponding structure in all other primates. The lowermost transverse fibers of the pons Varolii do not make their appearance until the level of the 


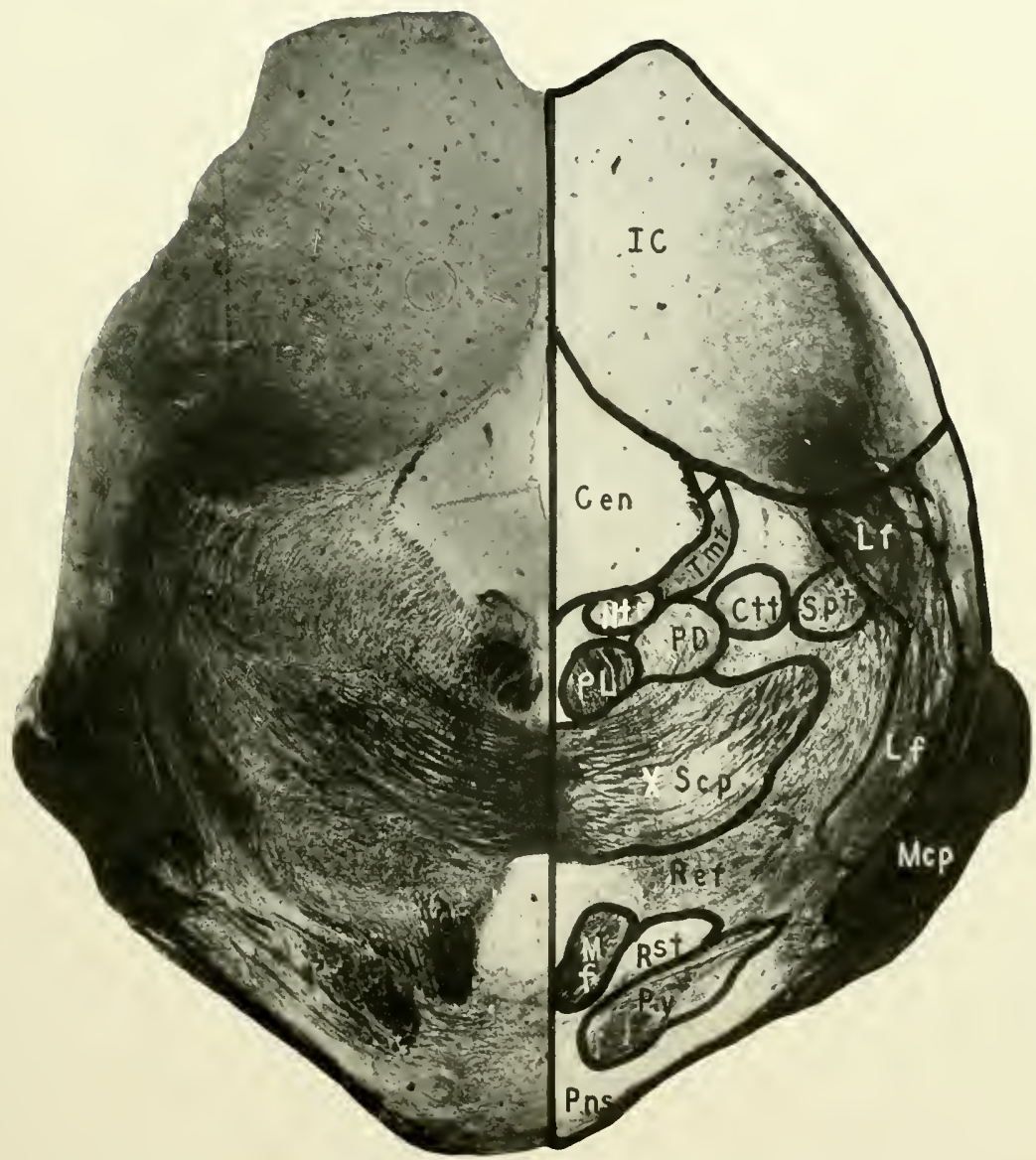

FIG. 59. TARSIUS SPECTRUM. LEVEL OF THE INFERIOR COLLICULUS.

CEN, Central Gray Matter; CtT, Central Tegmental Tract; IC, Inferior Colliculus; LF, Lateral Fillet; Mif, Mesial Fillet; MCP, Middle Cerebellar Peduncle; NTR, Nucleus Trochlearis; PD, Prcdorsal Bundle; PL, Postcrior Longitudinal Fasciculus; pNS, Pons; PY, Pyramid; REF. Reticular Formation; RST, Rubrospinal Tract; SPT, Spinothalamic Tract; TMr, Mesencephalic Root of Fifth Nerve; xscP, Decussation of Superior Cerebellar Peduncle. [Accession No, 210 . Section 205. Actual Size $9 \times 9 \mathrm{~mm}$.] 
trochlear decussation is reached. In other words, the bulbopontile sulcus holds a much more cephalic position than in other species. It is even higher than in the cat and most rodents. This position of the sulcus clearly indicates the inferior development of the pons Varolii. The internal pontile structure, however, does not depart from the type of organization characteristic of it elsewhere. In it may be discerned the three typical strata. A peculiarity in their arrangement is the fact that the stratum superficiale does not, as in other species of primates, appear as the most caudal element in the pons. Fibers from the stratum complexum make their way across the stem in the direction of the middle cerebellar peduncle before there is eviclence of the more superficial transverse axons. This gives rise to an unusual appearance, in that the pyramid maintains its ventral position in the stem athough pontile fibers are already present. It is usually the case that in the caudal portion of the pons, superficial transverse fibers cross in front of the pyramid and thus exclude the latter from a position on the surface. This arrangement of frbers makes the pyramid in tarsius appear much longer than in other species, while the pons is actually much shorter. Nor is the peculiarity of the pontile fibers limited alone to this late appearance of the superficial stratum. Such decussation as the pontile fibers do make, exerts a feeble influence upon the long suprasegmental systems. The pyramid, for example, which in all other primates becomes separated into more or less scattered bundles, shows little of such tendency in tarsius. The manner in which the pyramidal system maintains its integrated constituency and seems in this way to force the pontile fibers to sweep around rather than through it, is another fact emphasizing the low organization of the pons Varolii in tarsius.

The marked tendency of the mesial fillet to stretch out transversely in the pons so as to form the boundary between the tegmentum and the basis, is almost entirely absent in tarsius. The fillet maintains its position relatively near the median line, where it appears as a dense bundle. All of these phenom 
ena, as they affect respectively the appearance of the pyramid and the mesial fillet, denote the slight influence exerted by the pontile fibers upon the course of such suprascgmental systems. Such a pons as that of tarsius could not belong to an animal with more than a meager neokinetic endowment. As a behavioral index it gives a rating lower than the Felidae and about equal to some of the rodents. A capacity so low as this implies a low cerebral organization which might well serve as the stepping stone in the progressive ascent from lower mammalia toward the primates. The surface appearance of the mesencephalic tectum strongly suggests that the inferior colliculus is a functionally important structure. Its general and microscopic characters support this view. It is pronounced in size even when compared with many subprimate forms. Mensuration also indicates that the inferior colliculus plays an important rôle in the sense of hearing. That part of the animal's behavior dictated by auditory stimuli is characterized by prompt reflex responses. Tarsius consumes little time in reflecting upon the nature of sounds which it hears. It employs such stimuli with almost automatic directness to produce such reactions as guarantec its immediate safety or provoke fundamental activities. Furthermore, these auditory stimuli call forth at best only a most limited series of motor reactions. This conception is substantiated by the meager development of auditory areas in the temporal lobe of the brain, as well as by the auditory suprasegmental connections between the midbrain and the endbrain.

Tarsius also retains in the microscopic appearance of its inferior colliculus much of that organization characteristic of lower forms. In the cortex mesencephalica posterior it is possible to identify nine distinct strata. This arrangement harks back to the lower vertebrates, so that the large size of the inferior colliculus, in conjunction with its architectonic specialization signifres a retention of the primordial midbrain control over the function of hearing. 
The central gray matter is quadrilateral in outline surrounded by the collicular eminences and contains a central canal considerably elongated in the dorsoventral direction. It is of interest in this connection to note a number of small diverticula connected with the central canal. Such appearances are common in the adults of many subprimate mammals, but become less conspicuous in the higher members of the primate group. Ontogenetically, these evaginations are boubtless related to those extensions of the Sylvian aqueduct observed in many lower mammals and connected with the development of both the inferior and superior colliculi. Traced further back they are doubtless remnants of the phyletically ancient ventricles of the optic and auditory lobes of the midbrain.

In the ventral aspect of the gray matter is the trochlear nucleus $(\mathrm{Ntr})$, which is notable because of its indefmite boundaries and the profusion of fibers interspersed throughout its entire mass. It is a nuclear collection of unusual size, suggesting that the fourth nerve in tarsius must play an important rôlc. In conjunction with the closely set, protruding eyes, it may be that convergence of the visual axes has assumed great physiological prominence in these animals. Their acquisition of binocular vision has doubtless already set on foot those specializations which culminate in the development of stereoscopic function. On the other hand, the absence of a retinal macula might call the validity of this view in question. However this may be, it is clear that the tarsiers need and employ the superior oblique muscle of the orbit in such a manner as to require a large nucleus for its innervation.

The course of the trochlear nerve is of interest because in its emergence it departs somewhat from the course pursued in other primates. In emerging from its nuclear origin, it passes immediately dorsad following a path restricted to the central gray matter. It has no descending portion characteristic of members of this group. It leaves the dorsal rather than the dorsomesial aspect of its nucleus. Its entire course gives the impression of 
shortening due to compression in the stem, particularly about the region of the posterior isthmus.

Subjacent to the trochlear nucleus are the dense bundle of the posterior longitudinal fasciculus (PL) and the predorsal bundle (PD). On the periphery of the central gray matter is the mesencephalic root of the fifth nerve $(T m t)$. The reticular formation at this level is extensive, but its details are considerably obscured by the decussation of the superior cerebellar peduncle ( $\mathrm{XScp}$ ).

IEVEL OF THE SUPERIOR COLLICULUS (FIG. 6O)

At this Ievel certain features peculiar to Tarsius become apparent. Most conspicuous among these is the unusually large size of the entire tectal region. Yet, in spite of exceptional dimensions, the superior colliculus (SC) presents less stratigraphical specialization than might be expected from its size. The colliculus as a whole appears to be a region extremely rich in cellular elements.

In contrast to the large proportions of the colliculus, the extremely small size of the cerebral peduncle (CP) is another striking feature of the midbrain. Considered together, they impart to this segment of the tarsial stem a characteristic appearance different from all other primates. The small size of the cercbral peduncle is dependent primarily upon the meagre contributions made by the neopallium to the pallio-pontile system. It is also dependent upon the relatively small volume of the pyramidal system.

Thus, the peduncle is indicative of an animal whose behavioral patterns are extremely rudimentary and limited. It is another fact confirming the opinion that tarsius has little capacity in the acquisition of Iearned skilled acts and that its biological formula in adaptive reaction is determined largely by its automatic associative combinations. 
If comparison were made between tarsius and some of the lower mammalian forms-carnivores, rodents and even marsupials - on the basis of the cerebral peduncle, the tarsier would be forced into a position

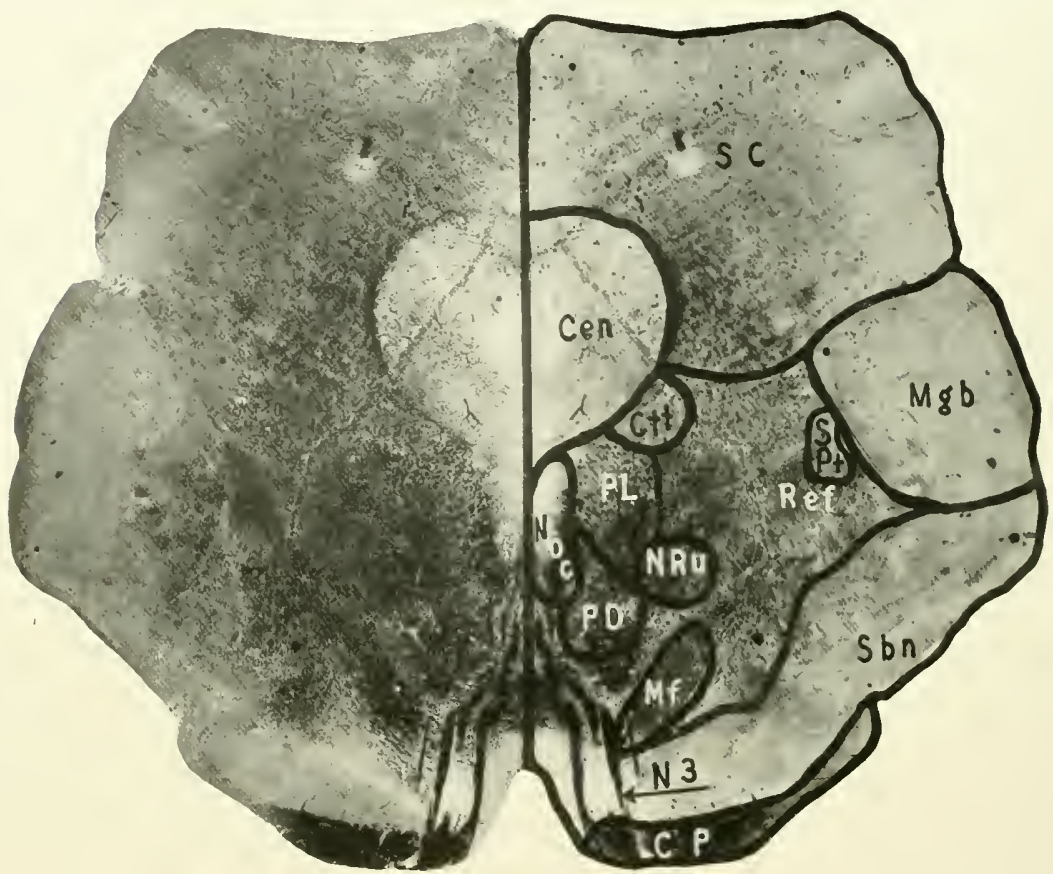

FIG. 60. TARSIUS SPECTRUM, LEVEL OF THE SLPERIOR COLLICULUS. CEN, Central Gray Matter; CTT, Central Tegmental Tract; CP, Cerebral Peduncle; MF, Mesial Fillet; MGB, Mesial Geniculate Body; N3, Oculomotor Nerve; NRU, Nucleus Ruber; Noc, Oculomotor Nucleus; PD, Predorsal Bundle; PL, Posterior Longitudinal Fasciculus; REF, Reticular Formation; sBn, Substantia Nigra; sc, Superior Colliculus; spt, Spinothalamic Tract. [Accession No. 210 . Section 250 . Actual Size $9 \times 7 \mathrm{~mm}$.]

of relative inferiority. It may perhaps, to some extent at least, excel certain of the marsupial species. Its primitiveness is again emphasized by the extreme dimensions of the superior eolliculus. This structure, because of its size, by 
permitting a certain degree of latitude, might be considered an "optic Jobe." The disposition of the central canal in this midbrain region would carry further suggestiveness in this line of interpretation. The large lateral diverticulum extending in the direction of the colliculus is strongly reminiscent of the lateral mesencephalic extension characterizing the brain of many lower vertebrates.

One notable advance in the brain of tarsius toward primate differentiation is the definite progress which it has made in extending the visual cortex. On the other hand, it is improbable that such advance has gone so far as to relieve the superior colliculus of much of its primitive visual function. Such a vicw attributes to the optic portion of the mesencephalic roof a large retention of activities related to vision, a condition which again declares the primitive organization of the brain in this animal.

Accompanying these strikingly primitive characters in the cerebral peduncle and the superior colliculus are other features at this level which bear testimony of similar moment. The substantia nigra ( $\mathrm{Sbn}$ ), for example, is an even more extensive structure than it is in lemurs. It thus stands out in contrast to this structure in all others of the primate order. The reticular formation (Ref) likewise is more conspicuous and much more diffuse. In it may be distinguished a poorly defmed nucleus ruber ( $N R u$ ) of relatively small size. Along the ventrolateral border of the substantia nigra are the two divisions of the mesial fillet (Mf), the ventral portion lying in close proximity to the emergent fibers of the nerve, the dorsal portion bordering the brachium geniculatum and lying ventral to the spinothalamic tract $\left(S_{p t}\right)$. The central gray matter $(C e n)$ is more extensive and also more pyriform than in other primates. It contains in its ventral apex the dorsal and ventral divisions of the oculomotor mucleus (Noc) which is characterized by the fact that but few fibers enter the oculomotor decussation. This latter observation is in harmony with the statement made by the late 
Dr. John Hunter to the effect that the nucleus of Perlia, although present in tarsius, is extremely small. The inference to be drawn from these correlated facts again indicates an animal poorly equipped in the neural mechanisms necessary for binocular stereoscopic vision. Hence in the organization of visual function the tarsier occupies a position befow the lemurs and new-world monkeys.

The importance of that ocular advance which distinguishes the primates from all other mammatian orders cannot be overestimated. It has been a momentous factor in the evolutionary process. What effects must have been produced in consequence of forward-looking eyes- eyes so related to each other that both visual axes could be directed upon an object either nearby or at a distance, eyes which no longer looked more or less independently to one side or to the other-it is difficult to estimate in measurable terms. In the final outcome of constructive development this new ocular relation must have borne in the weightiest manner upon that enormous superstructure of skifled movements which has had its supreme expression in the achievements of man. Whether tarsius represents the carliest hesitating steps in this direction, or whether it has made real progress over some even simpler prototype in visual organization, is a question beside the point. This animal is illustrative of one of those earty steps, if not the earliest, toward that inestimable consummation of visual activity in the acquisition of skilled movements which at length came to be the distinguishing feature in the neokinetic progress of the primates.

Lateral to the nucleus of the third nerve is an extensive fasciculus Iongitudinalis posterior (PL) whose ventral extremity borders upon the large collection of fibers constituting the predorsal bundle (PD). In the concavity of this extensive collection of axons is the central tegmental tract $(\mathrm{Ctt}$ ) now drawn closely in toward its ultimate position of contact with the central gray matter. Bordering this substance in its more dorsal 
region is the ascending trigeminal tract. Arcuate fibers passing inward from the region of this latter tract sweep forward to decussate in the dorsal decussation of Meynert. Decussating fibers occupying a still more ventral

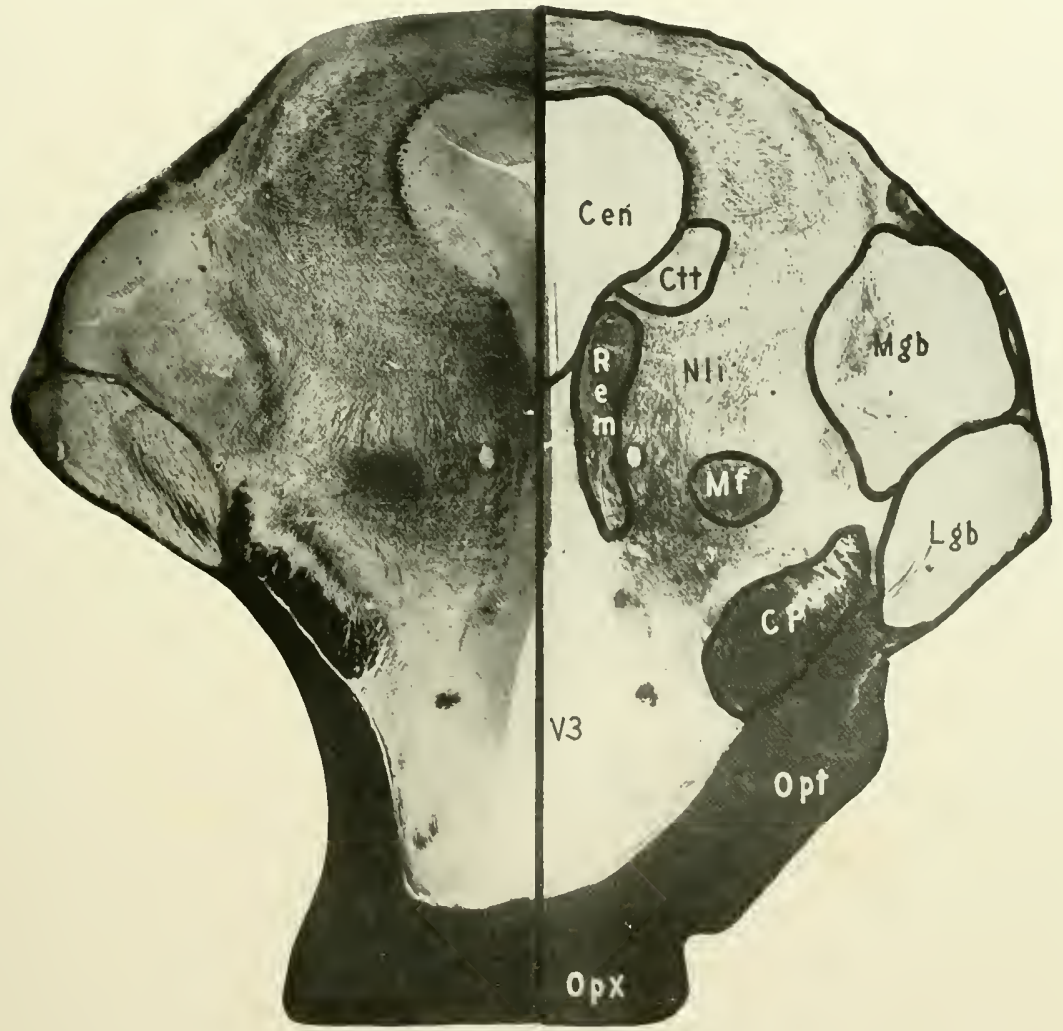

FIG, 6I. TARSIUS SPECTRUM, LEVEL OF THE OPTIC CHIASM.

CEv, Central Gray Natter; CTT, Central Tegmental Tract; CP, Cerebral Peduncle; I.GB, Lateral Geniculate Body; mF, Mesial Fillet; Mgr, Mesial Geniculate Body; NLI, Nucleus Lateralis Internus Thalami; OPT, Optic Tract; opx, Optic Chiasm; REM, Tractus Retroflexus of Meyncrt; v3, Third Ventricle. [Accession No. 210 . Section 261. Actual Size $10 \times 10 \mathrm{~mm}$. 
position constitute the decussation of Forel, many of whose axons appear to take origin in the red nucleus (NRu), cross the midline and enter into that descending bundle which constitutes the rubrospinal tract. On the

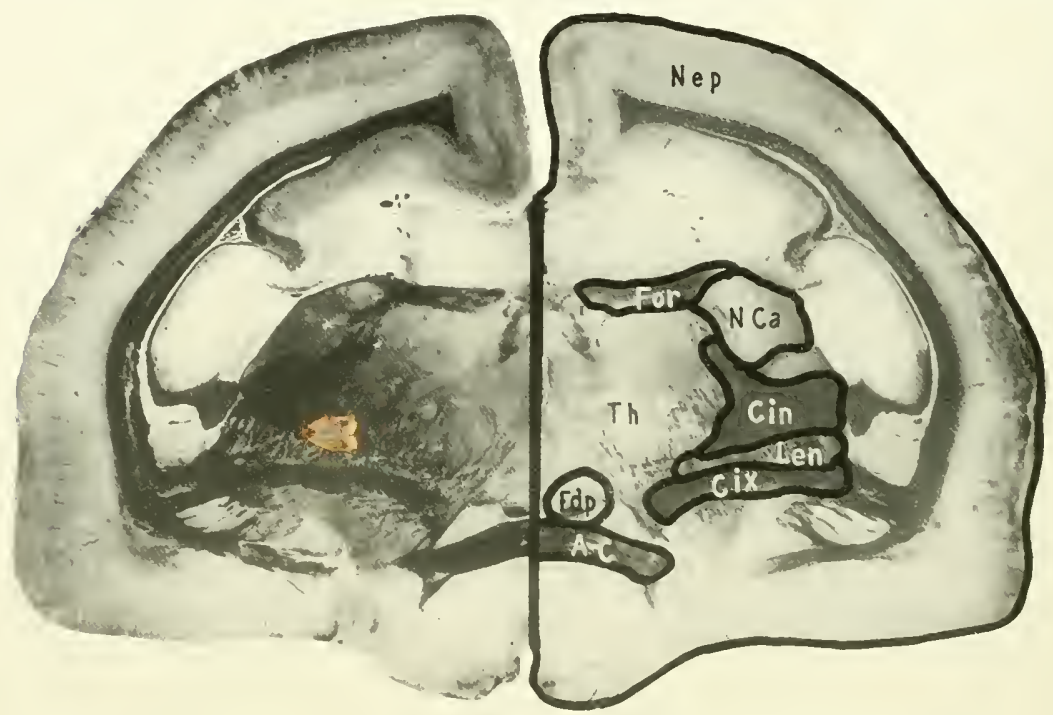

FIG. 62. TARSILS SPECTRUM. LEVEL OF THE ANTERIOR COMMISSLRE. AC, Anterior Commissure; cu, Internal Capsule; cix, External Capsule; FDP, Descending Pillar of Fornix; For, Fornix; lev, Lenticular Nucleus; xca, Nucleus Caudatus; Nep, Neopallium; th, Thalamus. [Accession No. 2 to. Section 305. Actual Size is $\times-\mathrm{mm}$.]

Iateral periphery of the seetion and ventral to the superior colliculus is the mesial geniculate body ( $\mathrm{Igb})$.

LEIEL OF THE OPTIC CHIASII (FIG. 6I)

At the level of the optic chiasm the brain stem is seen approaching its caudal extremity. The third ventricle is here flanked upon either side by the massive thalamic groups of nuclei, and the roof formed by some fibers 
of the posterior commissure. Other elements of interest at this level are indicated by letters in the caption.

LEYEL OF THE ANTERIOR COMMISSURE (FIG, 62)

At the level of the anterior commissure the brain stem has come to its caudal termination. The structures at this level are indicated by letters specified in the caption. 



\section{Chapter IV}

\section{RECONSTRUCTION OF THE GRAY MATTER IN THE BRAIN STEM OF TARSIUS SPECTRUM}

HE reconstruction of the gray matter of tarsius presents a constitu-
tion more elemental in character than that encountered in any other
species of the primate series. In general contour the reconstruction is distinctly pyriform, beginning with the small dimensions at the most cephalic of the cervical levels and expanding rapidly in the medulla, maintaining a uniform width in the pons and midbrain, but presenting at the upper portion of the midbrain near its junction with the diencephalon a marked increase in its lateral development. Viewed from the side the reconstruction has a singularly flat appearance on the dorsal surface which continues upward to the junction of the metencephalon with the mesencephalon. The ventral contour shows a gradual but continued increase in the dorsoventral dimension from the medulla to the isthmus. At the junction of the metencephalon with the mesencephaton, the dorsoventral diameter of the reconstruction approximately doubles itself by the sudden appearance of the colliculi which rise dorsally in a precipitous, almost palisade-like outgrowth from the dorsal surface of the neuraxis. The collieuli almost immediately assume their extreme vertical diameter, producing a right angle transition between the caudal limit of the collicular plate and the plane presented by the floor of the fourth ventricle. This space, empty in the reconstruction, is occupied by the mass of the cerebellum which lies in contact with the collicular plates throughout the major portion of its cephalic surface.

The geniculate bodies make their appearance at a level unusually low in the brain stem, taking on recognizable form on the lateral aspect of the mesencephalon. The mesial geniculate body, developing first at a point 
opposite the middle of the inferior colliculus, continuing upward and increasing gradually in width is joined by the lateral geniculate which rapidly develops into a large mass applied to the lateral surface of the midbrain. The superior colliculus is unusually large and appears as a direct continuation upward of the collicular plate, presenting a flat plateau-like strueture which extends to the upper limit of the reconstruction. The ventral surface presents a marked development of what corresponds to the interpenduncular gray matter, in this case, however, protruding between and ventral to the peduncles. The interpeduncular gray matter continues forward as a protrusion which cxtends ventrally from the surface of the brain stem for a considerable distance. The major portion of the brain stem is formed by the reticular formation which has a particularly undillerentiated appearance and scrves as a support for the more specialized structures of the stem which develop upon its several surfaces.

The presence of a small quantity of reticular formation in the angle between the ventral and dorsal gray horns, indicates that at the lowest levels of the reconstruction the decussation of the pyramidal tract has already begun. The first sections of the reconstruction correspond to the lowermost limit of the medulla oblongat and have an appearance almost typically that of the spinal cord, with the exception of the small mass of the reticular formation already mentioned. The central gray matter is distinctly $\mathrm{V}$-shaped in appearance, presenting definite ventral gray horns and dorsolateral cxtensions with a distinct cap of gray matter, the substantia gelatinosa of Rolando. For a short distance the substantia gelatinosa of Rolando continues upward on both sides of the midline separated by a rather deep groove in which lie the dorsal white columus of the spinal cord and medulla oblongata. The dorsal horns, however, soon show a tendency to deviate ontward, the tips of the horns first turning laterally and then somewhat ventrally, as the clorsal gray horns separate themselves one from the other. The ventral gray column 
presents a typical appearance, is rather irregular in contour and rapidly diminishes in size.

The reticular formation appearing in the angle between the dorsal and
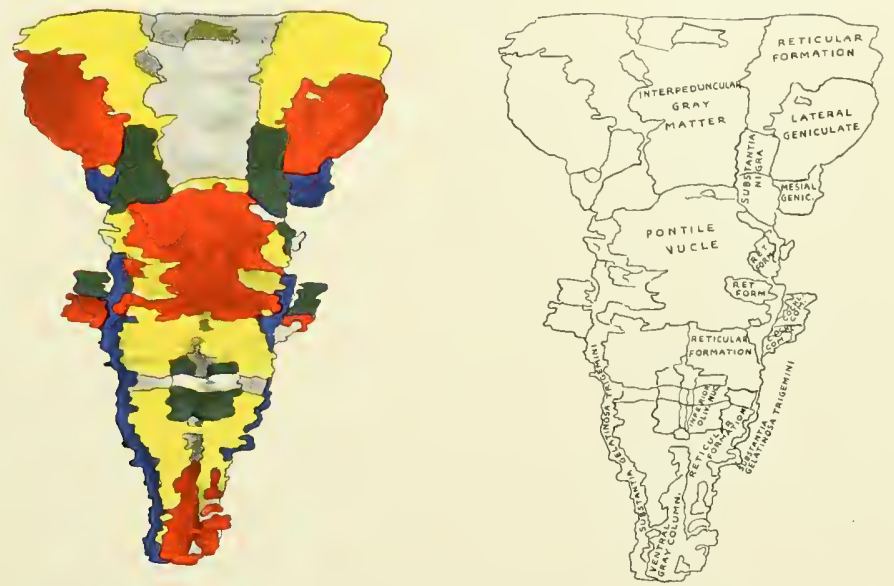

FIG. 63. VENTRAL SURFACE OF THE GRAY MATTER OF THE BRAIN STEM, TARSIUS SPECTRUM.

Key to Diagram. Cochl. Com., Cochlear Complex; inferior oliv. nuc., Inferior Olivary Nuclcus; mesial. GeNic., Mesial Geniculate Body; REt. Form., Reticular Formation.

ventral gray columns gradually increases in size and assumes the position which is being vacated by the ventral gray column. The central gray matter continues upward relatively unchanged until the point at which the nuclei of Rolando begin to diverge one from the other. At this point the ventral gray matter rather rapidly approaches the surface and becomes somewhat flattened out from side to side.

\section{The Dorsal Medullary Nuclei}

The nucleus of Goll is the frrst of the medullary nuclei to appear with, of course, the exception of the nucleus of Rolando. This nucleus appears a short 
distance above the point at which the nuclei of Rolando begin to diverge one from the other. The nucleus appears on either side of the midline as a small protrusion of gray matter from the dorsal surface of the central gray matter. These relatively insignificant dorsal protrusions lie close to the midline and increase very slightly in size as they continue upward. As the opening of the fourth ventricle is approached, these nuclei assume somew hat larger proportions and form a part of the lateral wall of the ventricle, extending laterally but never raising themselves to any extent from the surface. The nuclei of Goll continue upward to at point only slightly above the opening of the fourth ventricle in the lateral ventricular wall, and come to a rather abrupt termination.

The nucleus of Burdach appears at a higher level than the nucleus of Goll, as a llat sessile elevation on the dorsal surface of the central gray matter lateral to the nucleus of Goll. It slowly but gradually increases in size, diverging rather markedly from its fellow of the opposite side as the fourth ventricle opens. It extends upward to about the midventricular level. In the upper portion of its course it arrives at a certain degree of independence from the wall of the ventricle and rises dorsally to a moderate degree in a wellmarked prominence in the dorsolateral angle of the brain stem. It rather suddenly terminates and its place is taken by the nuclei of the vestibular complex.

The substantia gelatinosa Rolandi continues upward as a direct prolongation of the substantia gelatinosa of the spinal cord into the medulla oblongata. These two nuclei show the first indication of the opening of the lourth ventricle by their divergence one from the other, thus allowing the central gray matter to approach the dorsal surface of the oblongata. Continuing to diverge, the nuclei assume considerable lateral proportions, are supported directly on their mesial aspect by the reticular formation, and finally reach the lateral meridian of the brain stem. From this point they 
continue along the lateral surface of the medulla oblongata, present a definite constriction in the lower portion of the metencephalon, the waist of the trigeminal nucleus. They then extend somewhat dorsally and still further
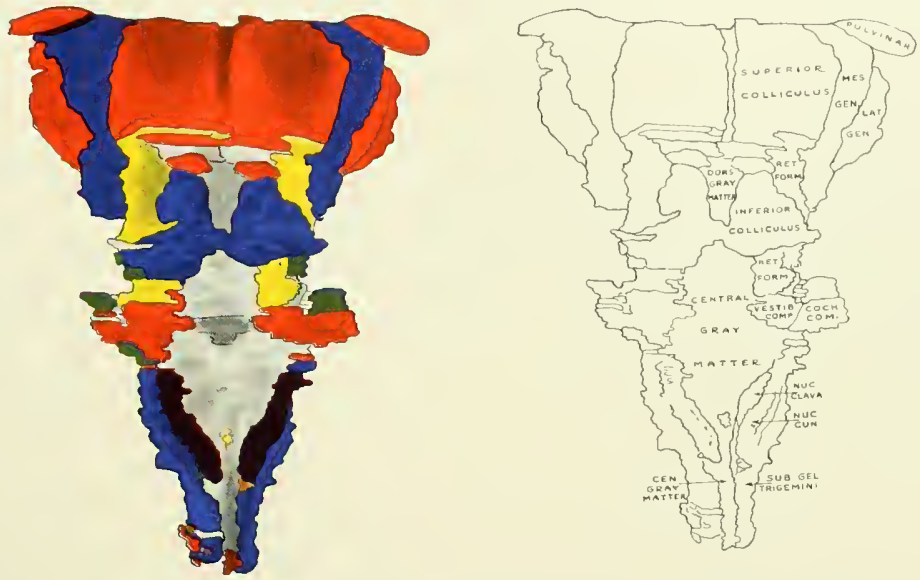

FIG. 64. DORSAL SURFACE OF THE GRAY MATTER OF THEBRAIN STEM, TARSIUS SPECTRUM.

Key to Diagram. cen. gray matter, Central Gray Matter; coch. com., Cochlear Complex; dors. gray matter, Dorsal Gray Matter; Nuc. Clava, Nucleus Clava; Lat. GeN., Lateral Geniculate Body; mes, GeN., Mesial Geniculate Body; Nuc. Cetv., Nucleus Cuneatus; RET. Form., Reticular Formation; sub. GFL. trigemini, Substantia Gelatinosa Trigemini; vestrb. cos., Vestibular Complex.

laterally to come to an end in an expanded upper extremity. In their course upward these nuclei, originally situated in an oblique direction from before, backward and outward, gradually turn upon a vertical axis until their long diameter is directed almost precisely dorsoventrally. They terminate at the mid-metencephatic level above the upper limit of the vestibular complex.

\section{The Inferior Olivary Nuclets}

The inferior olivary nucleus appears at a level corresponding almost exactly with the opening of the fourth ventricle as a narrow, somewhat creseentic lamina of gray matter, situated almost on the ventral surface of the oblongata, and applied to the reticular formation. The two nuclei are 
separated, one from the other, by the pyramidal tracts which lie on the immediate ventral aspect of the brain stem. These nuelei continue upward in this same position, increasing to a moderate extent chiefly by an increment in their most ventral portion which assumes a somewhat club-shaped appearance. There is but little indication of the development of the accessory and main olivary nuclei, the entire nucleus apparently consisting of a single lamina of gray matter. There is not the slightest indication of the formation of a fundus; neither is there any intimation of plication in the arrangement of the lamina. At its upper extremity the inferior olivary nucleus is the most ventral of all of the structures of the brain stem. The nucleus continues upward to a point somewhat below the level of the greatest width of the ventricle. It then rapidly diminishes and comes to an abrupt termination.

\section{The Reticular Formation}

This mass of nuclear material makes its first appearanee in the lowermost level of the reconstruction as a small accumulation of gray matter situated in the angle between the dorsal and ventral gray columns. It continues upward in this location, very gradually increasing in size, and replaces the ventral gray column through the merging of the latter into the undifferentiated reticular formation. From this point upward the reticular formation assumes the position of the major constituent of the brain stem, being relatively massive and supporting the various specializations which appear on its several surfaces. From the point of the disappearance of the ventral gray column, the reticular formation rapidly increases in size both laterally and ventrally being separated in the midline by the longitudinally coursing fiber bundles which form the raphe and by the inferior olivary nucleus which appears as a special condensation in the ventromesial angle of the formation. Above the level of the inferior olivary nucleus, the reticular formation of either side closely approaches the midline, the space occupied by the raphe 
being reduced to a mere slit between the mesial surfaces of the reticular formation extending backward to the central gray matter. The reticular formation on its lateral aspect forms a support for the substantia gelatinosa of
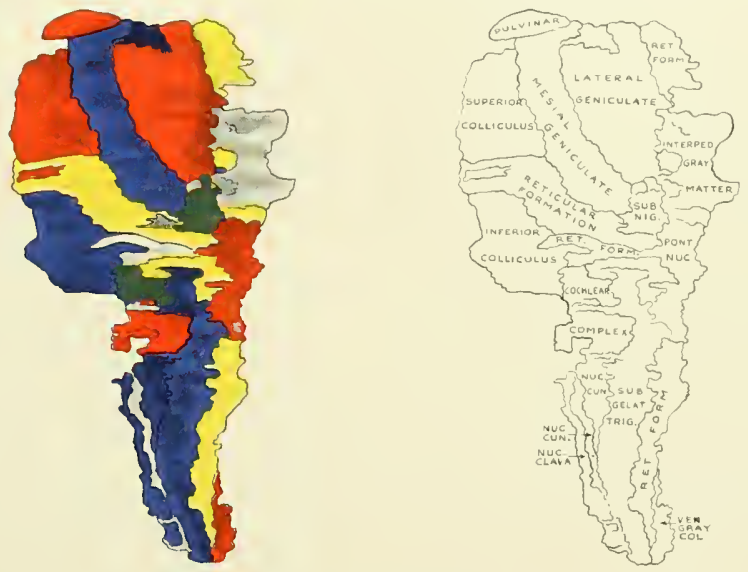

FIG. 6;. LATERAL SURFACE OF THE GRAY MATTER OF THE BRAIN STEM, TARSIUS SPECTRUM.

Key to Diagram. interped. Gray matter, Interpeduncular Gray Matter; Nuc. Clava, Nucleus Clava; Nuc. cun., Nucleus Cuneatus; pont. Nuc., Pontile Nucleus; ret. Form., Reticular Formation; sub, gelat. TRIG., Substantia Gelatinosa Trigemini; sub. Nig., Substantia Nigra; ven. Gray col., V'entral Gray Column.

Rolando which lies in a bed excavated from its lateral surface. Dorsally the reticular formation is in close relationship with the central gray matter forming the floor of the fourth ventricle, the dorsal surface of the reticular formation supporting the central gray matter throughout its entire lateral extent.

The reticular formation in the oblongata presents no definite special nuclear collections on its surface with the exception of the inferior olivary nucleus and the substantia gelatinosa of Rolando. At the junction of the oblongata with the metencephalon, the ventral surface of the reticular formation becomes irregular and recedes from the surface, giving place to the 
nuclear collections which form the very poorly organized system of the pontile nuclei. Its ventral surface, partially covered by the irregular pontile nuclei, shows two grooves, one which proceeds latcrally and dorsally in a somewhat spiral fashion about the lateral surface of the reticular formation toward the inferior colliculus, representing the course of the lateral fillet as this bundle of fibers secks its nucleus of termination in the inferior colliculus. In its more ventral portion, largely covered over and concealed from view by the pontile nuclei, the reticular formation presents another groove which indicates the course of the mesial fillet, which in this form maintains an almost undeviating upward course in the ventromesial angle of the formation throughout the entire length of the brain stem to end in the nuclei of the thalamus. In the upper half of the metencephalon the reticular formation is almost entircly covered by the pontile nuclei ventrally, and in its lateral aspect in this region it presents a particularly irregular outline. As the reticular formation is followed into the mesencephalon, it can be seen to send a prolongation laterally and dorsally which passes backward in a rather massive lamina of gray matter into the space separating the superior and inferior colliculi, extending corsally and mesially in the space separating the two colliculi to join in the midline dorsally. In the mesencephalon the reticular formation is almost entirely obscured from view ventrally by the fusion of the uppermost part of the pontile nuclei with the indifferent gray matter filling the interpeduncular space. Lateral to this there appears the substantia nigra which is separated from the ventral surface of the reticular formation by ascending and descending bundles of fibers. The lateral aspect of the reticular formation in the mesencephalon is entirely obscured by the exceptionally low appearance of the geniculate bodies which develop considerably below the mid-mesencephalic level. In the mesencephaton the only portions of the reticular formation which can be seen are those which form the dorsolateral expansions, extending into the space between the superior and inferior col- 
liculi, and a small ventromesial insinuation which appears between the mesial extremity of the substantia nigra and the interpeduncular gray matter.
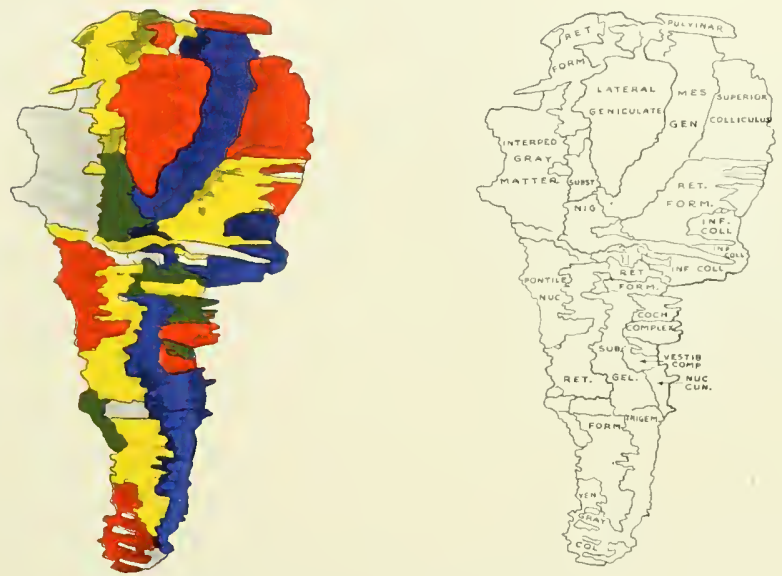

FIG. 66. LATERAL SURFACE OF THE GRAY MATTER OF THE BRAIN STEM, TARSIUS SPECTRUM.

Key to Diagram. coch. complex, Cochlear Conplex; inf. coll., Inferior Colliculus; interped. gray MATIER, Interpenduncular Gray Matter; MES. GEN., Mesial Geniculate Body; NeC, cun., Nucleus Cuneatus; pontile nuc., Pontile Nuclei; ret. form., Reticular Formation; sub. gel. Trigem., Substantia Gelatinosit Trigemini; subst. Nig., Substantia Nigra; ven. gray Col., Ventral Gray Column; restib. Coup., Vestibular Complex.

On the dorsal aspect of the reconstruction, the reticular formation only appears in the region immediately caudal to the isthmus, at which point its dorsolateral angle is seen between the central gray matter mesially, and the substantia gelatinosa of Rolando laterally. In this region, the reticular formation sends dorsally and mesially a thin prolongation which extends around and finally completely surrounds a tunnel which is occupied by the superior cerebellar peduncle. This peduncle in the tarsius is very small, insignificant and occupies a flattened, oval tunnel, direeted upward, inward and forward. The superior cerebellar peduncle continues this course through the reticular formation of the mesencephalon, decussating across the midline to end in the 
special tegmental nuclear condensation of the mescncephalon, the red nucleus. The reticular formation in the mid-mesencephalic region extends around the central gray matter in the form of a thin Iamina which separates the central gray matter from the inferior and superior colliculi, and forms a support for the ventrolateral angle of both colliculi.

Above the level of the substantia nigra the reticular formation again appears between the interpeduncular gray matter and the lateral geniculate as a missive development, extremely irregular in its arrangement and extending upward to become continuous with the nuclei and reticular formation of the diencephalon.

\section{The Pontile Nuclei}

The pontile nuclei appear at about the midventricular level as small, isolated collections of gray matter on the ventral surface of the brain stem. These collections of gray matter develop chielly near the midline, but scattered nuclear masses can be scen extending outward to form small discrete accumulations. Above the midventricular level, the pontile nuclei become somewhat larger and form a definite lamina of gray matter which assumes a fair degree of thickness and a relatively extensive lateral disposition, covering a small segment of the ventral aspect of the gray matter of the brain stem. This mass of pontile nuclei gradually develops out of the isolated groups of gray matter which form the pontile nuclei in the lower portions of the metencephalon. They rapidly extend laterally, continue upward and then rapidly diminish by drawing toward the midline, where they end in contact, if not by fusion with the undifferentiated interpeduncular gray matter. The pontile nuclei, where they assume any proportions at all, appear only as a simple lamina of gray matter forming the ventral contour of the gray matter of the brain stem. They show no tendency whatsoever to establish any degree of complexity and do not interlace with the bundles of the pyramidal tract which lie in a compact group dorsal to the middle of the pontile lamina. 


\section{The Vestibular Núclel}

A relatively short distance above the opening of the fourth ventricle there appears a specialization in the reticular formation which lies between the nucleus of Burdach and the floor of the fourth ventricle. This specialization consists of groups of fibers, rather loosely arranged in a matrix of gray matter, thus presenting the typical appearance of the mucleus of the descending root of the vestibular complex. This mass of gray matter enlarges in size, and very soon assumes the characteristic arrangement of the nucleus of Deiters. As it increases in size, the nucleus of Burdach correspondingly decreases, and as the nucleus of Burdach comes to an end, the nucleus of Deiters assumes its full proportions. In tarsius the nucleus of Deiters is a relatively large collection of gray matter, and occupies the outer third of the dorsal portion of the tegmentum directly ventral to the floor of the fourth ventricle. As the nucleus of Burdach terminates, the nucleus of Deiters comes to the surface and appears on the dorsolateral aspect of the neuraxial tegmentum. The increase in size of the gray matter in this region shows a very definite effect upon the ascending nucleus of Rolando which is displaced to a distinctly ventral position by the increasing mass of the Deitersal complex. The nucleus reaches its maximum proportions at a point somewhat above the mid-ventricular level where, in its mesial portion, there develops a small area somewhat more homogencous in appearance, which is the triangular nucleus of Schwalbe. This nucleus forms part of the lateral wall of the ventricle underlying the ependymal floor and is in contact laterally with the Deitersal complex. At this point there appear the cochlear nuclei in connection with the entrance of the cochlear roots, and the dorsal cochlear nucleus overlies the nucleus of Deiters, hiding it from view. Having assumed its maximum proportions, the nucleus of Deiters rapidly diminishes and disappears, giving place to a rather large collection of reticular substance situated lateral to the central gray matter. As the nucleus of Deiters approaches the 
surface it presents the typical tonguc-shaped prolongation laterally and ventrally in the curve of which is disposed the ascending inferior cerebellar peduncle which at this point passes between the vestibular and the cochlear complex to enter the ecrebellum. No definite evidence of a nucleus of von Bechterew could be obtained in the sections.

\section{The Cochlear Nucle 1}

These nuclei appear at the mid-rentricular level of the brain stem opposite the lateral recess of the fourth ventricle. This nuelear mass in tarsius presents a lateral and a mesial group rather than the generally accepted division of the cochlear mucleus into a ventral and dorsal division. The lateral division of the cochlear nucleus is a well-defoned mass of gray matter which lies lateral to the recess of the fourth ventricle connected by strands of gray matter with the mesial cochlear mucleus. The lateral cochlear nucleus appears to be considerably more extensive than the mesial portion of the nucleus and diflerentiates as a rather definite mass of gray matter almost independent of the gray matter of the stem itself. There could be identifred no definite troughlike form of the nucleus which is the usual arrangement seen in the higher primates. The mesial cochlear nucleus is a rather poorly defined nuclear mass which is situated in the recess of the fourth ventricle and is immediately superimposed upon the Deitersal complex which underlies it. It does not extend upward or downward as far as the lateral division of the nucleus and is a relatively insignificant mass of gray matter.

\section{The Colliculi}

These masses of gray matter, together with the genieulate bodies, form the predominating and outstanding development of the gray matter in the brain stem of tarsius. They are relatively massive in appearance and oceupy the tectal portion of the meseneephalon. The superior collieuli are very much 
larger than the inferior colliculi. The inferior colliculi appear at the dorsolateral angle of the brain stem at first as small rounded collections of gray matter. These expand enormously in sueceeding sections, rapidly assuming their maximum proportions. They are situated lateral to a dorsal prolongation of the central gray matter. They are roughly oval in outline, the axis being directed somewhat from before, backward and inward. Having rapidly assumed their greatest dimensions, the colliculi continue upward for a short distance and then begin to contract. They are in relation laterally with a prolongation of the reticular formation which arises in the clorsolateral angle of the tegmentum, and sweeps around the colliculi sending a prolongation upward to interpose itself between the upper limits of the inferior colliculus and the Iower limits of the superior colliculus. The colliculi are supported ventrally by the reticular formation and mesially by the gray matter of the central formation. As seen in the cross sections the inferior colliculus corresponds fairly accurately with the general conformation of the inferior colliculus in the whole primate series, being represented chielly by a core of white matter, upon which are superimposed successive layers of white and gray matter. The superior colliculus, however, presents a very different. appearance, having a typical cortex with a core of medullary substance. The superior colliculus makes its appearance slightly above the point of termination of the inferior colliculus, as a small, dorsomesially situated mass of gray matter. This rapidly enlarges in size, spreading laterally and presenting a ventral prolongation which continues forward laterally along the outer aspect of the dorsal portion of the brain stem. Central to the superior colliculus, there appears a prolongation of the reticular formation dorsally around the central gray matter which is situated in the center of the reconstruction. As the superior colliculus is followed upward it assumes greater and greater proportions, becoming considerably thicker, and presents a very definite plateau-like appearance on its dorsal aspect, which turns at a sharp angle 
ventrally and Iaterally into the Iateral prolongation which continues forward toward the mesial geniculate body. The superior colliculus continues upward to the point of junction of the mesencephaton with the diencephalon, where it rapidly contracts and comes to a termination.

\section{The Substantia Nigra}

This mass of gray matter appears at about the junction of the metencephalon with the mesencephalon in the ventromesial aspect of the brain stem, as a direct continuation upward of a thin Iamina of reticular formation which is found to underlie the metencephalic pontile nuclei. The substantia nigra is situated obliquely from before, backward and outward, as a rather defmite lamina of gray matter of mediocre dimensions. It continues upward in this position relatively unchanged, being in relationship mesially with the development of the interpechuncular gray matter, and dorsally with the reticular formation of the mesencephalic tegmentum. Latcrally the substantia nigra is in relationship with the origin of the mesial geniculate body and somewhat more cephalically, with the beginning of the lateral geniculate body. In the lateral portion of this lamina of gray matter there develops the lateral nucleus of the substantia nigra which has been found to be present in the brain stems of all of the primates. This forms a rather complicated tangle of nerve fibers arising from a definite nuclear formation, which seems to turn backward into the mesencephalic tegmentum. The substantia nigra continues upward to a point somewhat above the beginning of the superior colliculus, and then comes to an end being replaced by various developments of the reticular formation, which in this region assumes very considerable proportions and extreme complexity.

\section{The Central Gray Matter}

The central gray matter appears in the lowest levels of the model as a more or less $\mathrm{v}$-shaped mass of nuclear material, presenting at its ventrolateral 
angles the ventral gray columns and at its dorsolateral angles the connections of the central gray matter with the substantia gelatinosa of Rolando. Continuing upward, the central gray matter retains very much the same position, the connection between it and the ventral gray colamn being cut across by the decussating fibers of the pyramidal tract. As the dorsal gray columns begin to diverge, the central gray matter migrates dorsally, becoming llattened out, and then gives rise to the dorsal medullary nuclei, while to its ventral aspect is attached the reticular formation. Continuing upward the central gray matter gives rise to the nucleus of the column of Goll, and at a somewhat higher level, to the nucleus of the column of Burdach. As the floor of the fourth ventricle begins to open, the central gray matter comes to the surface and forms the floor of the fourth ventricle which presents a shallow depression, concave from side to side and from above downward. The gray matter of the floor of the fourth ventricle is spread out in a relatively thin sheet, and presents no particular modeling produced by the underlying structures of the floor of the fourth ventricle. It is continued outward into the Iateral recesses and covers the nucleus of Deiters and the triangular nucleus of Schwalbe. It is encroached upon at the angle of the mesial cochlear nucleus which extends considerably toward the midline. Having passed the level of the lateral recess of the fourth ventricle, the gray matter contracts toward the formation of the aqueduct of Sylvius, and the central canal is soon reconstituted by the development of the roof of the fourth ventricle and the lower portion of the aqueduct of Sylvius. It here is in direct relationship with the dorsal prolongation of the reticular formation which surrounds it on either side, and receives the supcrior cerebellar peduncle. Continuing upward, the central gray matter again forms a complete canal which is rather massive and presents walls of considerable thickness. The aqueduct itself is an clongated dorsoventral slit rather than an oval opening, and the central gray matter is continued backward in a long drawn-out projection 
between the mesial aspects of the inferior colliculi. Continuing upward in the mesencephalon, the central gray matter becomes more rounded about the aqueduct of Sylvius, being surrounded by a dorsal prolongation of the reticular formation which separates it from actual contact with the superimposed superior colliculi. In the middle portion of the mesencephalon the central gray matter rapidly begins to extend ventrally forming a long slit-like structure which is surrounded by thick walls of gray matter which then become continuous with the interpeduncular gray matter. Continuing upward, the central gray matter of the mesencephalon becomes continuous with the central gray matter of the diencephalon surrounding the slit-like third ventricle. The interpeduncular gray matter presents a rather massive development immediately above the pontile nuclei. This presents considerable proportions and contains a central ventricle. Continuing upward, with a definite ventral evagination, the interpeduncular gray matter fuses with the ventral prolongation of the central gray matter as the junction of the mesencephalon with the diencephaton is approached. The interpeduncular gray matter presents at a relatively low level the condensations representing the mammillary bodies which appear at a level between the superior and inferior colliculi.

\section{The Geniculate Bodies}

These bodies since they form a definite part of the brain stem of tarsius, are described in connection with the gray matter of the brain stem.

The mesial geniculate body is the first one to appear and the first evidences of its existence are found caudal to the termination of the inferior colliculus. It appears as an oval mass of gray matter, situated lateral to the substantia nigra and directed outward and backward. It increases in size, shifts somewhat laterally and then dorsally and continues up as a rapidly increasing mass of gray matter in contact laterally with the ventral aspect 
of the lateral prolongation of the superior colliculus. It then becomes stretched out laterally in a relatively thin lamina of gray matter which continues upward into the diencephalon.

The lateral geniculate body appears at a slightly higher level than the mesial geniculate body and is situated somewhat ventral to it. It forms the most prominent structure on the ventrolateral aspect of the brain stem. It consists of a relatively massive collection of gray matter, situated lateral and ventral to the mass of the mesial geniculate. It is disposed in an oblique direction from before backward and outward, and presents at its ventral extremity a series of prolongations which receive or give origin to masses of fibers. Continuing upward, the lateral geniculate, rapidly diminishing, ends somewhat caudal to the upper limit of the mesial geniculate which extends further upward. 



\section{Chapter V}

\section{CALLITHRIX JACCHUS, THE MARMOSET, ITS BRAIN AND BEHAVIOR}

Its Position among the Primates; Measurements and Brain Indices; Surface Appearance of the Brain; Internal Structure of the Brain Stem in Cross Section

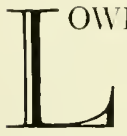

EST among the Anthropoidea are the Hapalidae or mamosets. These animals belong to the group of new-world monkeys. They inhabit South and Central America. Their digits are for the most part clawed, with the exception of the great toe which alone bears a flat nail. The tail is long, bushy and ringed, a condition characteristic of many of the lower groups of mammals but not observed among the higher apes. Unlike most of the South American monkeys, the tail of marmoset is not prehensile. In size they are about as large as a small squirrel and are covered with a thick silky fur. Although naturally timid, they readily become accustomed to those with whom they are familiar in captivity. The female produces two or three young at a birth and in this respect is unlike other Anthropoidea. In facial appearance, in size and shape of head, the Hapalidae convey a much more ape-like impression than do the lemurs. On the other hand, their specialization in limb, particularly with reference to the hand, scems hardly so far advanced as in the Lemuridae. The eyes are much closer together and separated by a flat, narrow nose, suggesting the possibility of some degree, at least, of binocular vision, although it seems probable that even in this respect, the animal's visual function has not attained a high degree of differentiation.

On the whole, the marmosets, because of their very small size, secm but retrograde steps in the differentiation which culminates in the strikingly 
outspoken features of the simian tribes. This relatively large family of newworld primates, however, beeause of its ennvergences and divergenees within this order of mammats, should afford interesting evidenee in the strueture of

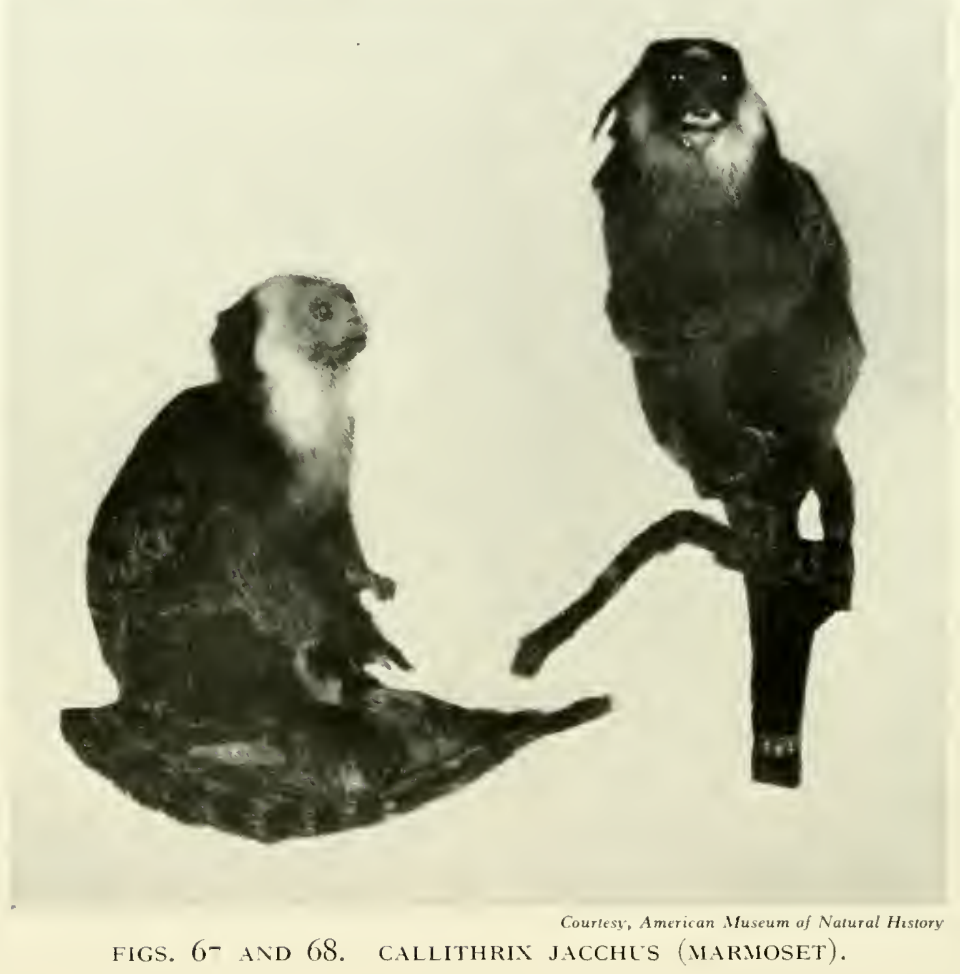

its central nervous system and more particularly in the brain. The species here deseribed is Callithrix jacchus. The greater number of the speeies inchuded in the genus Callithrix are natives of Brazil, one species formerly 
extending its range as far as Bolivia, another being indigenous to Colombia. There are records of Callithrix jacchus having also been found in the island of Marajó, lying between the mouths of the Amazon and Para Rivers.

The marmoset has a black face with white spots. There are cross-bands on the back and tail. The animal lives in the tree tops or smaller underbrush. The claws upon its feet and hands cnable it to climb along the limbs and up the trunks of trees much in the manner of a squirrel. It has a eat-like agility but does not make long and daring leaps like the lemur. It often loses its hold upon the branches and falls from considerable heights to the ground without sustaining apparent injury. For this reason, and perhaps because of its small size, it is not in need of the extreme degree of prehensile power in either fore- or hindlimbs. It may also be because of this fact that its tail has failed to develop prehensile qualities. While in eaptivity it shows little tendency to aequire reactions which it does not already possess in the free state. It does not lend itself to training or the aequisition of tricks, as do many of the other anthropoid forms. The animal lives upon worms, insects and fruits. It is known also to invade the nests of birds and suck the eggs. Only exceptionally, however, does it prey upon bird-life, and in such exceptions it may occasionally be able to overpower one of the smaller birds or unprotected young. It has little of the acquisitive celerity manifested by the lemur.

\section{Measurements and Indices of Callithrix Jacchus}

Total length of the animal............

Length of the tail. . . . . . . . . . . . . $29 ; \mathrm{mm}$.

Length of the foot................ 6i $\mathrm{mm}$.

Diameters of the skull

Occipito-nasal................... $42 \mathrm{~mm}$.

Bitemporal. . . . . . . . . . .

Length of the brain case............. $35 \mathrm{~mm}$. 
Brain, including cerebelfum and brain stem without meninges Longitudinal...................... 3 I $\mathrm{mm}$.

Transverse.................... $23 \mathrm{~mm}$.
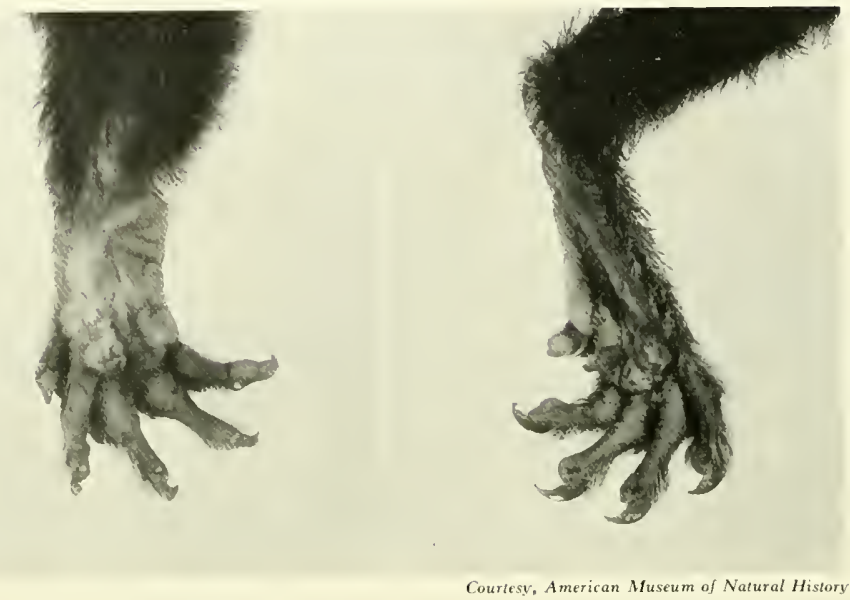

FIGS. 69 AND 70 . HAND AND FOOT OF MARMOSET.

LEFT. Palmar surface of hand showing poorly developed palm, rudimentary thumb, well-defined digitation, with claws instead of finger-nails.

Right. PIantar surface of the foot showing imperfectly developed heel and sole, rudimentary great toe, claws instead of toe-nails.

Total weight of the brain................ $6.2 \mathrm{gms}$.

Total water displacement of the brain........6 c.c.

W'eight of forebrain................ 4.75 gms.

Weight of midbrain................ 0.25 gms.

Weight of hindbrain............... I . 2 gms.

On the basis of these figures the following encephatic indices were computed for the several divisions of the brain: 
Forebrain index

80.5 per cent

Midbrain index

0.5 per cent

Hindbrain index.

I9. o per cent
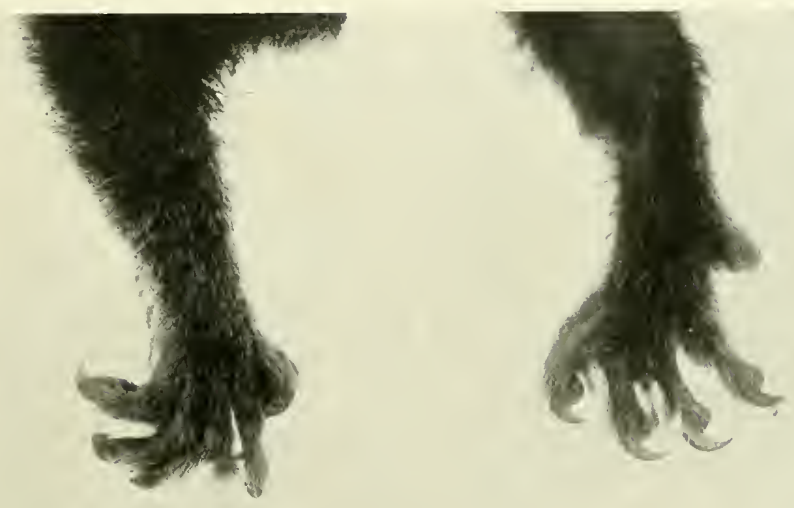

Courtesy, American Museum of Natural History

FIGS. 7 I AND 72. HAND AND FOOT OF MARMOSET.

LEFT. Dorsum of hand showing poorly developed thumb, and claws instead of finger-nails.

Right. Dorsum of foot showing rudimentary toe, claws instead of toe-nails.

These indices align the animal in a definitely submanual group with a forebrain index slightly below that of the lemur and only slightly above the forebrain index characteristic of mammals possessed of paws and claws.

Surface Appearance of the Brain in Callithrix Jacchus

THE FISSURAL PATTERN

The surface appearance of the hemisphere in the marmoset gives the impression at first glance of a lissencephalic brain. Further inspection, however, shows that at least three fissures constitute an indefinite fissural pattern 
in this animal. The Sylvian fissure is a prominent suleus whose angulation with the base line of the brain is slightly less than $0^{\circ}$, showing in this regard a general tendeney to approach the condition of the higher primates. The
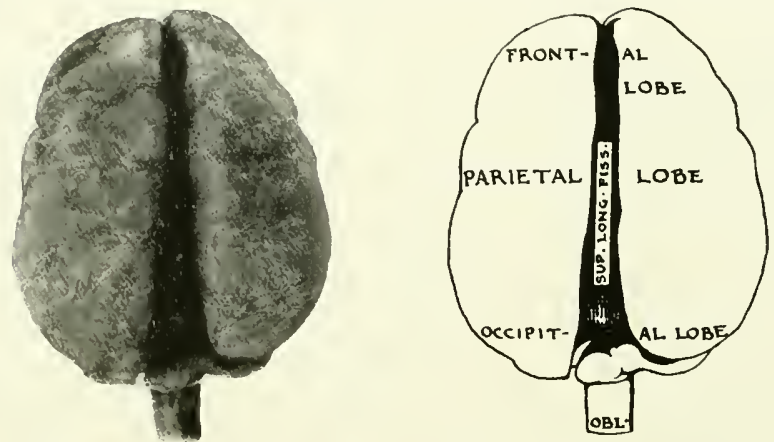

FIG. 73. DORSAL SLRFACE OF BRAIN, CALLITHRIX JACCHLS (MARMOSET). [Actual Length, $29 \mathrm{~mm}$.]

Key to Diagram. obl., Oblongata; Sup. Long. Fiss., Superior Longitudinal Fissure.

Sylvian fissure, however, is relatively short and extends backward and upward for something less than half the distance of the entire lateral surface. Below and behind the Sylvian fissure is a slight indenture indicating the position of the superior temporal fissure, corresponding in its general position to the suleus parallelus in the lemur's brain. A slight indenture above and in front of the Sylvian fissure indieates the position of the sulcus centralis which forms the faint boundary by means of which the limits between the frontal and parietal lobes may be established. There is no evidence of any parietal sulei or of any incisure which may be considered as the homologue of the sulcus simiarum.

\section{LOBATION}

The lobation in the marmoset brain, therefore, is only most rudimentarily outlined. Distinction may be made between the parietal, parieto- 
frontal and temporal regions by the boundary established through the Sylvian fissure. No boundary exists which indicates the actual limits between the parietal and the frontal lobes except the faint indenture already indicated
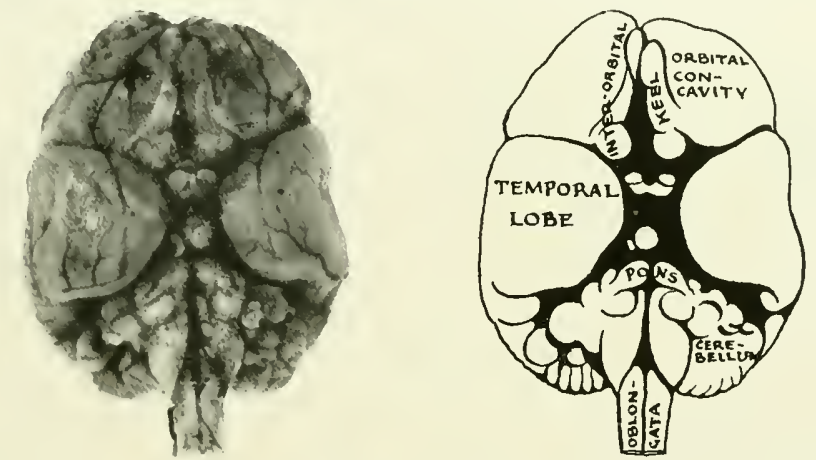

FIG. $\rightarrow$ t. BASE OF BRAIN, CALLITHRIX JACCHUS (MARMOSET).

[Actuit Length, $29 \mathrm{~mm}$.]

as the probable inception of the suleus centralis which, however, is not a constant marking in the marmoset brain. In many species it does not appear at all. As no boundary line exists in the occipital region, it is impossible to describe the limits between the parietal and the occipital lobes. The latter lobe, however, has apparently expanded considerably in its general dimensions because the pole of the brain now completely overhangs the cerebellum and assumes more elosely the general outline of this region in the higher primates. While it is possible to identify the general topography of the frontal, parietal, temporal and occipital lobes in the brain of marmoset, no reliable landmarks may be established as marking the boundaries of these four great hemispheral divisions. The impression conveyed by the survey of the brain in the Hapalidae is that even if this family has defmitely entered the lists of the primate kind, its advance has been a most diffident one. While the hemisphere shows the general outline characteristic of the primate endbrain, it has 
none of the bold markings in the way of fissures and convolutions which ultimately become the identifying (Fig. -5) features of this part of the nervous system in primates. The marmoset brain may be considered as
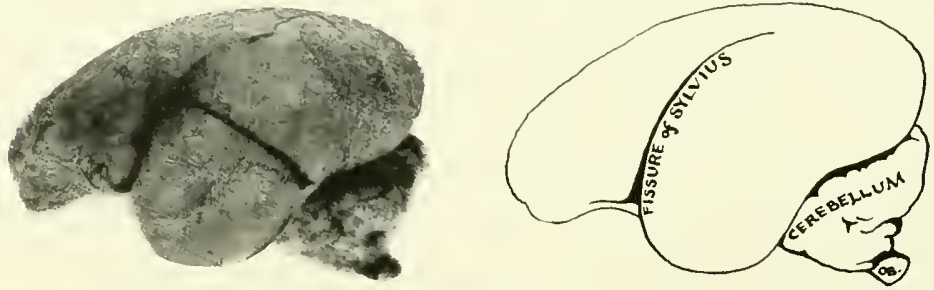

FIG. 73. IEFT LATERAL SURFACE OFBRAIN, CALLITHRIX JACCHUS (MARMOSET). [Actual Length, $31 \mathrm{~mm}$.]

Key to Diagram. ob., Oblongata.

something more than a mere transitional form. It must be taken to represent a transition already achieved from the lower mammals into the primate order but also manifesting certain retrograde changes.

\section{THE ORBITAL SURFACE}

On the orbital surface the brain has all of the typical markings of the lower primates. The two orbital concavities are well defined. The inception of these concavities seen in the lemur's brain is here earried to its ultimate development. The orbital surface now rests upon an expanded orbital plate of the frontal bone. The interorbital keels are also well defmed and more marked than in the lemur brain. The olfactory bulbs, though fairly large, are less pronounced than in the lemur, and the olfactory tract is detachable as far back as the trigonum which is fairly prominent (Fig. 74 ).

THE OCCIPITAL REGION OF THE BASAL SURFACE

In the occipital region of the basal surface a well-marked occipital concavity exists which is most emphasized in and about the midline, particu- 
larly in the region of the postsplenial fossa. This concavity accommodates the upward protrusion of the cerebellum. The cerebellum upon its tentorial surface is almost completely overhung by the occipital pole of the hemisphere.
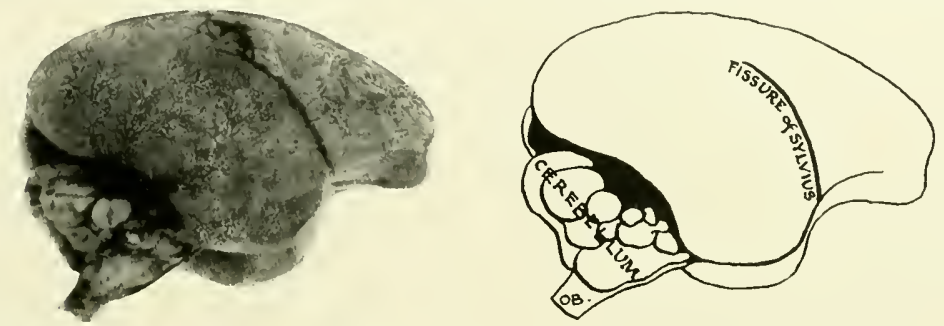

FIG. 6. RIGHT LATERALSLRFACE OFBRAIN, CALLITHRIX JACCHLS (MARMOSET). [Actual Length, $31 \mathrm{~mm}$.]

hey to Diagram. ob., Oblongata.

The superior vermal portion of the cerebellum is more conspicuous than the lateral lobes which show but slight expansion. On the occipital surface the vermis is also the outstanding feature. It represents scarcely less than a third of this surface and stands out conspicuously against the poorly developed lateral expansions of the cerebellum. The two paramedian sulci interrupt the passage of the interfolial fissures from the inferior vermis to the lateral lobe. No such sulcus, however, exists on the tentorial surface. While the cerebral hemispheres of marmoset assign the animal to a definite place among the primates, the simplicity of the brain indicates the relatively low position of the Hapalidae (Fig. -6).

\section{THE SURFACE MARKINGS OF THE BRAIN STEM}

The surface markings of the brain stem are in general much less distinct than in the higher primates. In many respects, they are less impressive than in Lemur mongoz. The oblongata on its ventral surface presents a ventromesial sulcus and two faintly marked ventrolateral sulci. Neither the 
pyramids nor the inferior olivary bodies have any distinct surface relief, nor is it possible to detect any superficial indication of the pyramidal decussation. The relative insignificance of the pyramidal system indicates an
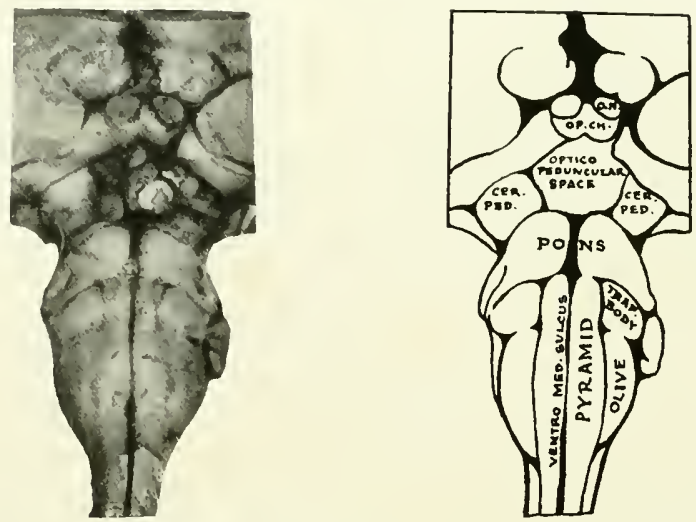

FIG. 7\%, IENTRAL SURFACE OF BRAIN STEM, CALLITHRIX JACCHUS

(MARMOSET).

[Actual Length, $22 \mathrm{~mm}$.]

Key to Diagram. Cek. Ped., Cerebral Peduncle; o.N., Optic Nerve; op. Ch., Optic Chiasm. trap; Bony, Trapezoid Body; veNtro Med, stlcus, Ventromedian Sulcus.

extremely limited range of volitional performances and in this respect places the animal even lower in the scale than the Iemur. The bulbopontile sulcus is difficult to make out except in fresh specimens, and the pons itself is a flat, narrow band conveying the impression that the animal has not developed a pallio-cerebellar connection capable of producing any large degree of coordinative control over skilled movements. On the dorsal aspect of the oblongata there is a slight mesial eminence indicating the presence of the column and nucleus of Goll. No similar lateral prominence adjacent to it gives surface indication of the column of Burdach. This fact seems to indicate that the tail and lower extremity are more prominent functional elements than the forelimb (Fig. -8 ). 


\section{CALLITHRIX JACCHUS, THE MARMIOSET}

THE CEREBELLUN, THE FOURTII VENTRICLE AND THE MIDBRAIN

The cerebellum is small, presenting a large median portion, the vermis, and two limited lateral expansions, the lateral hemispheres. The size of the
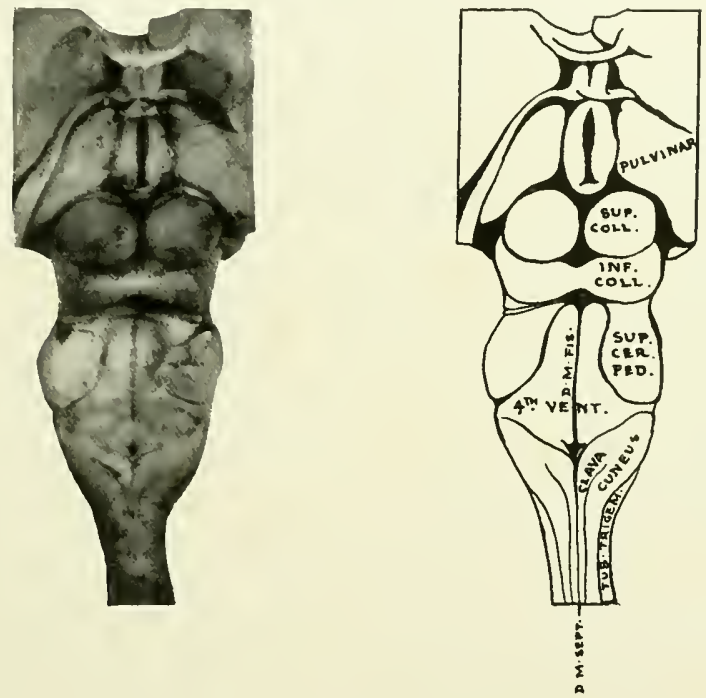

FIG. 78. DORSAL SURFACE OF BRAIN STEM, CALLITHRIX JACCHUS (IARMOSET).

[Actual Length, $22 \mathrm{~mm}$.]

Key to Diagra.i. D. M. Fls., Dorsomedian Fissure; D. M. SePt., Dorsomedian Septum; inf. Coll., Inferior Colliculus; sup. CER. PED., Superior Cerebellar Peduncle; ste. Coll., Superior Colliculus; tub. trigem., Tuberculum Trigemini; 4TH VE.T., 4th Ventricle.

cerebellum, particularly of its lateral lobe, indicates a coordinating organ with a low degree of functional capacity. From this it may be inferred that the animal's attainment in complex voluntary movements is extremely limited. On removal of the cerebellum the fourth ventricle is revealed, bounded upon either side in its inferior triangle by an inconspicuous clava and in its superior triangle by the superior and middle cerebellar peduncles. 
The floor of the ventricke, even with high magnification, shows few of the characteristic markings usually observed in this region of the brain. It is not possible to detect striae acusticae crossing the floor. The lateral recesses are narrow and the configuration of the rhombencephalon is much more compressed in its cephalo-caudal relations than is the case with the fourth ventricle of other primates. In the ventral region of the midbrain the cerebral peduncles may be observed, diverging as they approach the cerebral hemispheres and thus bringing to light the interpeduncular space. The cerebral peduncles, however, make but a faint relief on the ventral mesencephalie surface. On the dorsal aspect of the midbrain the inferior and superior colliculi are well marked and give the impression that the primordial stations for both vision and hearing have a high degree of representation in these primates. The insignificant development of the cerebral peduncles speaks again in favor of a low organization for volitional skilled acts controlled through the agency of the prramidal system.

\section{Internal Structure of the Brain Stem in Callithrix Jacchus}

\section{LEVEL OF THE PYRAMIDAL DECUSSATION (FIG. 70)}

At this level two notable features are present, i.e., the decussation of the pyramidal tracts ( $\mathrm{Py} \mathrm{x}$ ) which has the effect of separating the ventral gray column (Ven) from the central gray matter ( Cen), and the presence of the caudal extremity of the nucleus of Golf (NG). This nucleus is surrounded by a massive tract of fibers, the column of Goll, which ascends from the humbosacral and lower thoracic segments of the cord. From the sensory standpoint, it represents chiefly the tail and lower limb. Immediately adjacent and lateral to the tract of Goll is the tract of Burdach (CB) which, however, presents a size relatively equal in comparison with the more mesial sensory pathway. Lateral to Goll's tract is the large substantia gelatinosa (NR) bordering upon which is the descending tract of the fifth 
nerve ( $\operatorname{Trd})$. This dorsal field of sensory discrimination, embracing the three major sensory territories of the body, the head and face, neck and upper extremity, leg and tail, is almost as conspicuous as in lemur. Its pro-

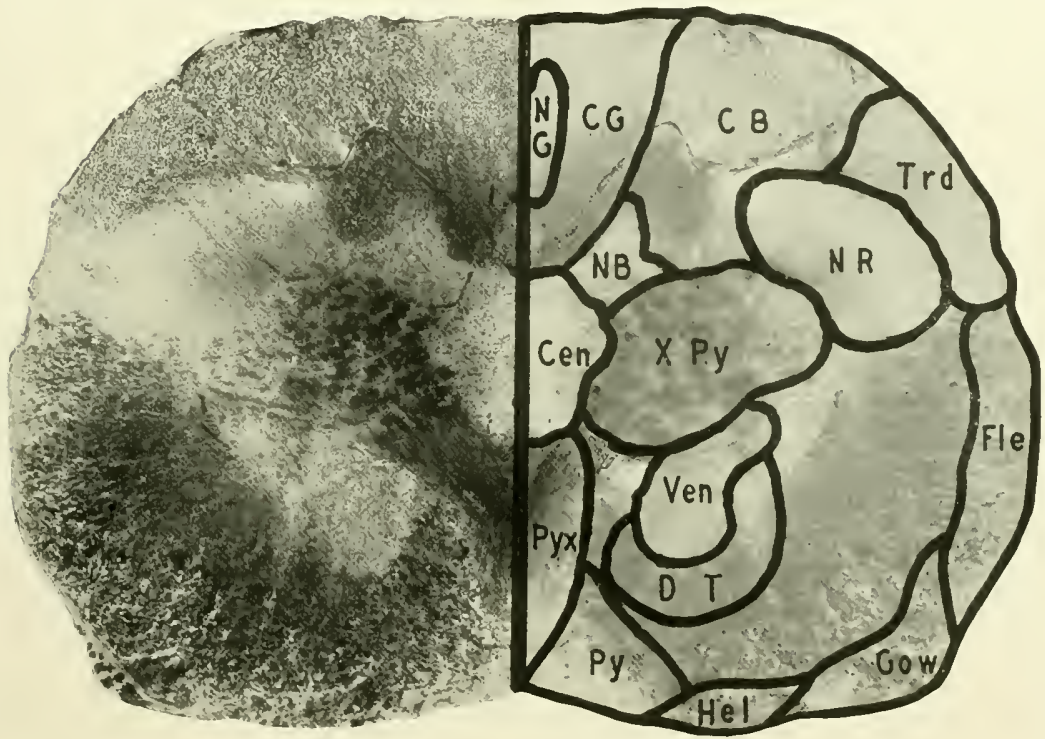

FIG. -9. MARMOSET. LEVEL OF THE PYRAMIDAL DECUSSATION.

CEN, Central Gray Matter; CB, Column of Burdach; CG, Column of Goll; DT, Deiterso-spinal Tract; FLE, Dorsal Spinocerebellar Tract; gow, Ventral Spinocerebellar Tract; HEL, Spino-olivary Tract of Ilelweg; NB, Nucleus of Burdach; NG, Nucleus of Goll, NR, Nucleus of Rolando; Py, Pyramid; Pyx, Pyramidal Decussation; TRD, Descending Trigeminal Tract; VEN, Ventral Gray Matter; xPy, Crossed Pyramidal Tract. [Accession No. 146. Section 4 . Actual Size $5 \times 3 \mathrm{~mm}$.]

portional development of gray matter, as well as the size of the tracts, has practically the same connotation as in the lower form already described. Thus, sensory conduction from the leg and tail, head and face is less amply represented than that from the arm and neck. The significance of this relative disproportion is obvious at a glance. The head, forearm and the hand are 
functionally more prominent than the hind extremity with its poorly differentiated foot. This disproportion is less striking than in lemur, for although marmoset makes but limited use of its hand, its tail and hind leg

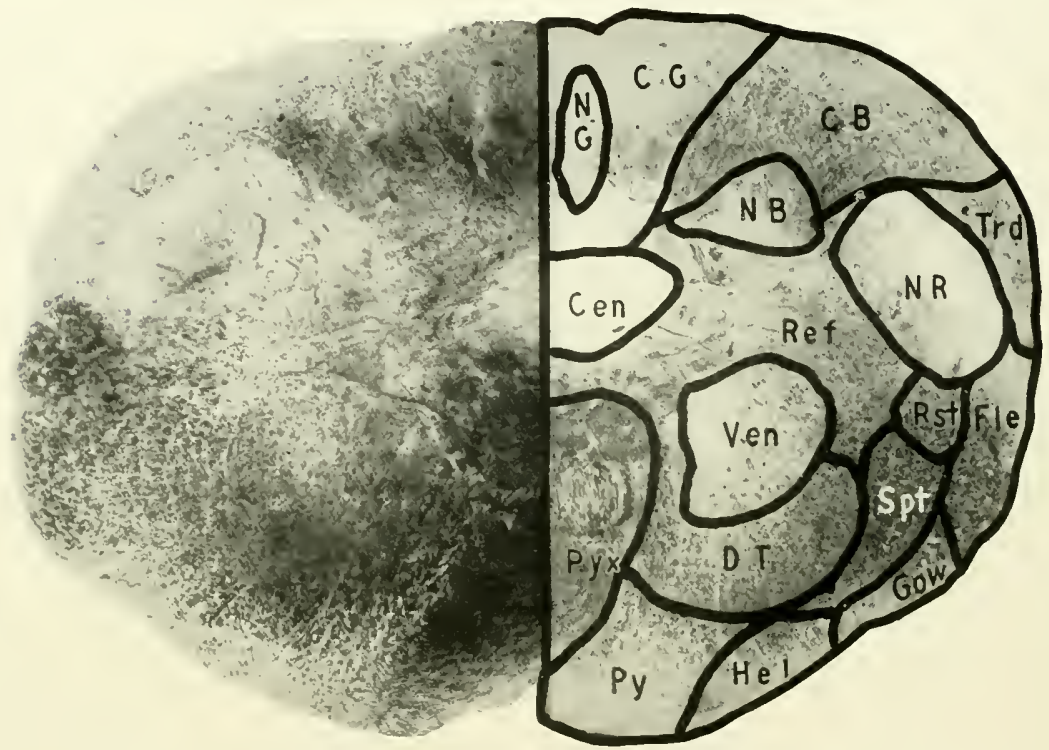

FIG. 8O. MARMOSET. LEVEL OF THE DORSAL SENSORY NUCLEI.

$\mathrm{CB}$, Column of Burdach; CEN, Central Gray Matter; CG, Column of Goll; DT, Deiterso-spinal Tract; FLE, Dorsal Spinocerebellar Tract; Gow, Ventral Spinocerebellar Tract; Hel, Spino-olivary Tract of Helweg; NB, Nucleus of Burdach; NG, Nucleus of Goll; NR, Nucleus of Rolindo; Pr, Pyranid, Pyx, Pyramidal DecusSation; REF, Reticular Formation; RST, Rubrospinal Tract; SPT, Spinothalimic Tract; TRD, Descending Trigeminal Tract; Ven, Ventral Gray Matter. [Accession No. 146. Section 8. Actual Size $6 \times 4 \mathrm{~mm}$.]

manifest as much adaptive significance as in the ease of lemur. That the discriminative type of sensibility has a low representation in marmoset, thus providing a meager substratum for the development of its skilled acts, is further substantiated by the fact that the prramidal tract (Py) is a 


\section{CALLITHRIX JACCHUS, THE MARMOSET}

small bundle of fibers in this animal. This creates the impression that the pyramidal system in its relation to the control of voluntary movement plays but a small part in the animal's somatic behavior.

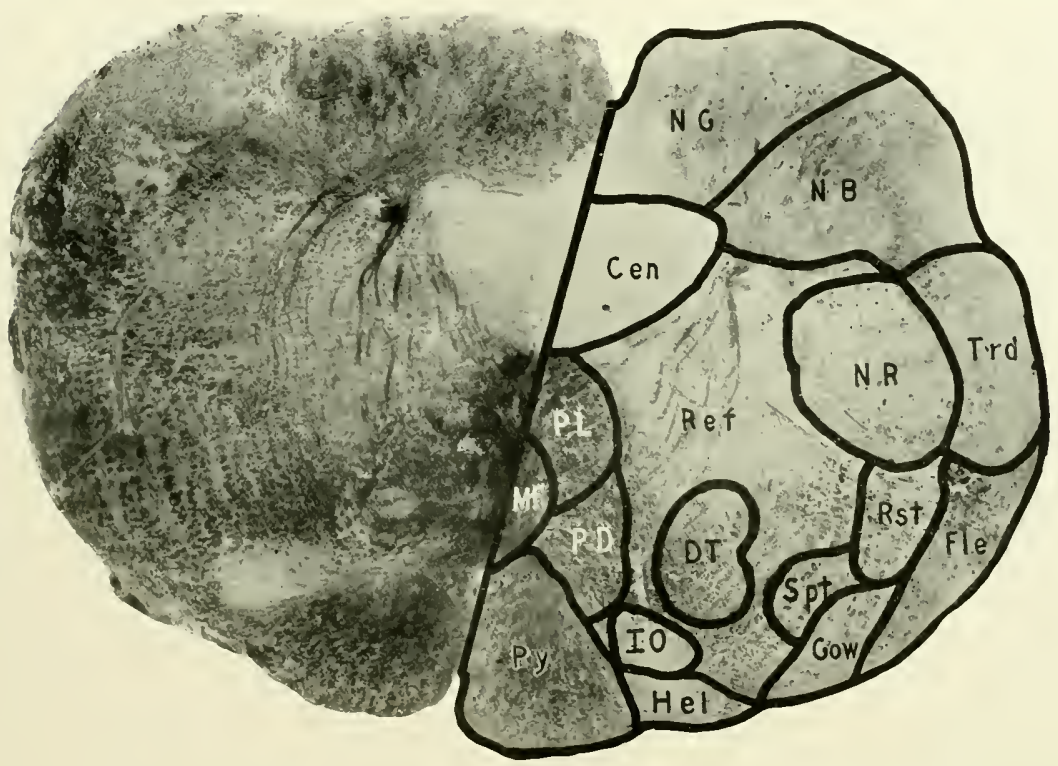

FIG. 8I. MARMOSET. LEIEL OF THE TIP OF THE INFERIOR OLIVE.

CEN, Central Gray Matter; Dt, Deiterso-spinal Tract; fle, Dorsal Spinocerebellar Tract; gow, Ventral Spinocerebellar Tract; Hel, Spino-olivary Tract of Helweg; IO, Inferior Olive; MF, Mesial Fillet; nb, Nucleus of Burdach; ^g, Nucleus of Goll; NR, Nucleus of Rolando; PD, Predorsal Bundle; PL, Posterior LongitudinaI Fasciculus; Pr, Pyramid; REF, Reticular Formation; RST, Rubrospinal Tract; SPT, Spinothalamic Tract; TRD, Descending Trigeminal Tract. [Accession No. ${ }_{4} 46$. Section 22. Actual Size $8 \times 5 \mathrm{~mm}$.]

LEVEL OF THE CAUDAL EXTREMITY OF THE INFERIOR OLIVE (FIG. 8I)

The appearance of the caudal limit of the inferior olivary body (IO) adds another striking feature to the next level. This structure 
occupies its usual position dorsal to the pyramidal bundles. It is of relatively small size and irregular outline. While it has nearly the same prominence as in lemur, in many respects it seems more poorly defined as a distinctive structure in the oblongata. Its edges are not so clearly demarcated. This holds true of the olivary body at all fevels in the marmoset, in which it appears to be a poorly developed constituent indicating a low degree of organization in the olivary sphere of action. Its function, as already described, is related to the automatic control of simultaneous movements of the hand, head and eye in the performance of skilled acts. Certainly there are no acts exccuted by this animal which indicate the necessity of any very close coordinative cooperation between eye, head and hand.

The pyramid ( $\mathrm{Py}_{\mathrm{y}}$ ) here reveals its dimensions in the cross section and again signifies a conduction bundle with rather low capacity for distributing volitional motor impulses. Dorsal to the pyramidal fibers and adjacent to the midline are the first aggregations of fibers which enter into the mesial fillet (M f). This bundle is formed by decussating arcuate fibers arising in the nuclei of Goll and Burdach which sweep forward across the midline where they take a cephalic course toward the higher levels of the brain. It furnishes some idea as to the extensiveness of the secondary sensory pathway.

LEVEL THROUGH THE MIDDLE OF THE INFERIOR OLIVE (FIG. 82)

At the level through the middle of the inferior olivary body, although this body appears in its greatest dimensions, it gives the impression of a poorly developed structure. Its relatively small size supports the supposition that the coordinative control of simultaneous movements of head, eye and hand and the coordination of all skilled learned performances are comparatively slight in this animal. The relative functional significance of the nucleus of Goll as compared with that of Burdach is obvious by the con- 
trast in size of these nuclei as they lie sibe by side in the dorsal portion of the section ( $N G, N B$ ). The mesial fillet ( $\mathrm{If}$ ) has attained more prominence because of the addition to it of a larger number of arcuate fibers.

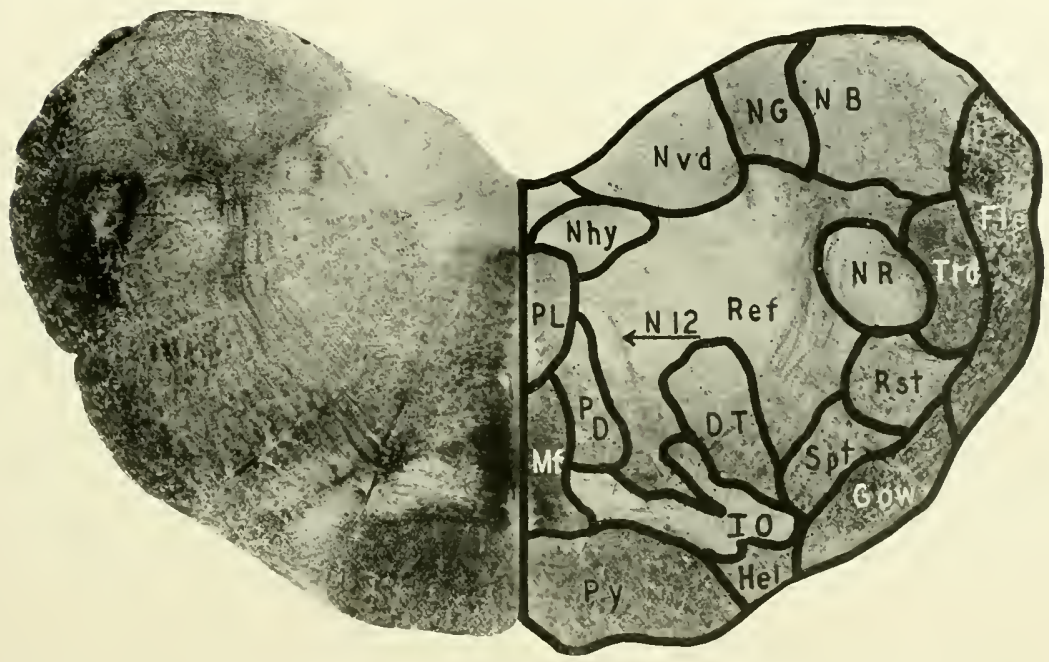

FIG. 82. MARMOSET. LEVEL THROLGH THE MIIDDLE OF THE INFERIOR OLIVE. DT, Deiterso-spinal Tract; fle, Dorsal Spinocerebellar Tract; Gow, Ventral Spinocerebellar Tract; HeL, Spino-olivary Tract of Helweg; 10 , Inferior Olive; MF, Mesial Fillet; NB, Nucleus of Burdach; NG, Nucleus of Goll; NHr, Hypoglossal Nucleus; NR, Nucleus of Rolando; Nvo, Dorsal Vagal Nucleus; Nı2, Hypoglossal Nerve; PD, Predorsal Bundle; PL, Posterior Longitudinal Fasciculus; Pr, Pyramid; Ref, Reticular Formation; Rst, Rubrospinal Tract; SPT, Spinothalamic Tract; TrD, Descending Trigeminal Tract. [Accession No. 146. Section 37. Actual Size $8 \times 4 \mathrm{~mm}$.]

LEVEL OF THE VESTIBLLAR NUCLEI AND TUBERCULUN ACUSTICUM (FIG. 83)

At the level of the restibular nuclei and tuberculum acusticum, the cross sections are characterized by the wide separation of the lateral walls of the fourth ventricle and the appearance of the vermis cerebelli in relation with the ventricle. In the position formerly occupied by the nuclei of Goll and Burdach are two prominent nuclear masses intimately concerned 
with afferent conduction. These nuclei are especially related to a type of conduction which has to do with the maintenance of equilibrium. They receive impulses from the receptive organs of the semicircular canals, utricle and

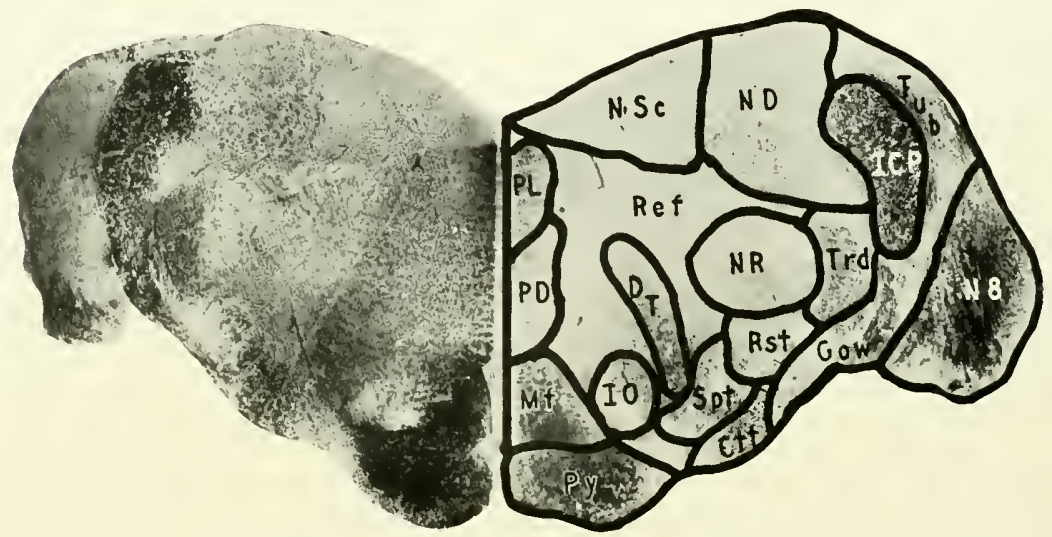

FIG. 83. MARMOSET. LEVEL OF THE VESTIBULAR NUCLEI A.ND TLBERCULUM ACUSTICUM.

CTT, Central Tegmental Tract; DT, Deiterso-spinal Tract; gow, Ventral Spinocerebellar Tract; ICP, Inferior Cerebellar Peduncle; 10 , Inferior Olive; MF, Mesial Fillet; ND, Deiters' Nucleus; NR, Nucleus of Rolando; NSc, Nucleus of Schwalbe: N8, Auditory Nerve; PD, Predorsal Bundle; PL, Posterior Longitudinal Fasciculus; PY, Pyramid; REF, Reticular Formation; Rst, Rubrospinal Tract; SPT, Spinuthalamic Tract; TrD, Descending Trigeminal Tract; тив, Tuberculum Acusticum. [Accession No. 1 46 . Section 67. Actual Sizc $10 \times+\mathrm{mm}$.]

saccule. The two nuclei which have thus replaced the columns of Goll and Burdach are the nucleus triangularis of Schwalbe (NSc) and the nucleus magnocellularis of Deiters (ND). The size of these nuclei and their fiber connections with the vestibular mechanism indicates a high degree of organization in the reflex acts connected with balancing. These restibular nuclei are outstanding features in the section. In their general dimensions they correspond with the similar structures of lemur, a fact which would seem to justify the inference that the marmosets are as well equipped in balancing 
function as the lemurs. The restiform body (ICP), which represents the aggregation of ascending fibers passing to the vermis of the cerebellum, affords a suggestive index of coordinative control. The development of such

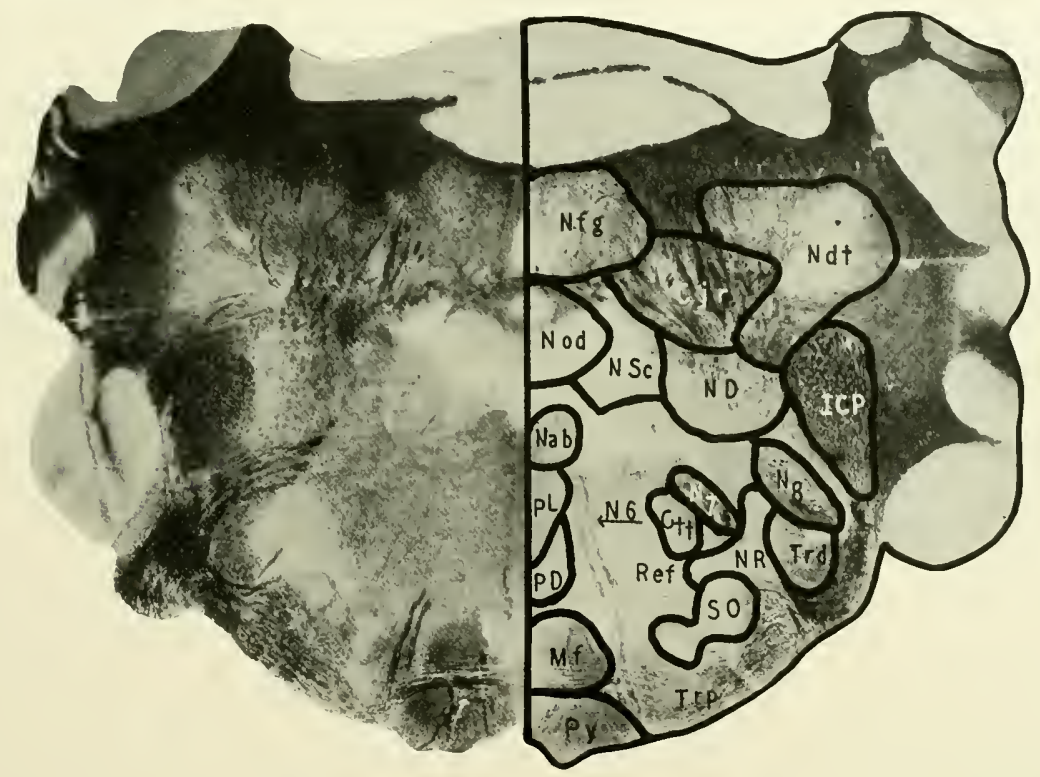

FIG. 84. MARMOSET. LEVEL OF THE CEREBELLAR NUCLEI.

CJR, Juxtarestiform Body; CTT, Central Tegmental Tract; ICP, Inferior Cerebellar Peduncle; MF, Mesial Fillet; NAB, Abducens Nucleus; ND, Deiters' Nueleus; NDT, Dentate Nucleus; NFG, Cerebellar Nuelei, Mlesial Group; von, Vermis Cerebelli; NR, Nucleus of Rolindo; Nsc, Nucleus of Schwalbe; $\wedge 6$, Abducens Nerve; $>7$, Facial Nerve; N8, Auditory Nerve; PD, Predorsal Bundle; PL, Posterior Longitudinal Fasciculus; PY, Pyramid; REF, Reticular Formation; so, Superior Olive; TRD, Deseending Trigeminal Tract; TRP, Trapezoid Body. [Accession No. 146. Section 92. Actual Size $10 \times 7 \mathrm{~mm}$.]

control in marmoset appears to be relatively less than in other primates, a supposition which finds support in the relatively low degree of organization in the cerebellum. 
LEVEL OF THE CEREBELLAR NUCLEI (FIG. 84)

At the level of the cerebellar nuclei the intimate relation between the medulla oblongata and the cercbellum is clear, while an index to the degree of ecrebellar control of motion is furnished in the presence of the main efferent cerebellar nucleus, the nucleus dentatus ( $\mathrm{Ndt}$ ). Occupying the most ventral position in the cross section is the pyramid ( $\mathrm{Py}$ ) immediately dorsal to which is the trapezoid body (Trp) made up of crossing fibers entering the secondary pathway for the conduction of auditory stimuli. The reticular formation ( $R \mathrm{c} f$ ) occupies an extensive area along whose ventral border is the superior olivary body $(\mathrm{SO})$, a way station in the pathway of hearing.

LEVEL AT THE MIDDLE OF THE PONS VAROLII (FIG. 8j)

At the level at the middle of the pons Varolii this structure attains its maximum dimensions and consists of its three typical layers, the stratum superficiale, the stratum complexum and the stratum profundum. The stratum superficiale continues directly into the middle cerebellar peduncle (Mcp). The stratum complexum contains many transverse pontocerebellar fibers together with the scattered fasciculi of the pyramidal system (Py) and the pontile nuclei $\left(\mathrm{Pn}_{\mathrm{n}}\right)$. The general dimensions of all of the structures occupying the basis pontis afford a signiffeant index of the animal's degree of adaptation. The relatively small size of the pyramidal system is clearly demonstrated. The equally small size of the pons, including the pontile contribution of fibers, which goes to make up the middle cerebellar peduncle, is significant of connections which provide but limited means for the animal's organization of a complicated motor adjustment. It may be inferred from this evidence that the marmoset is poorly endowed with motor activities having a high degree of adaptive flexibility and that the entire range of its motor effectiveness is limited to narrowly prescribed motion formulas. The 
animal does not possess the celerity, the dexterity or the muscular power to adapt itself to an extensive natural environment. Neither has it the eapacity to create out of its environmental conditions new combinations which

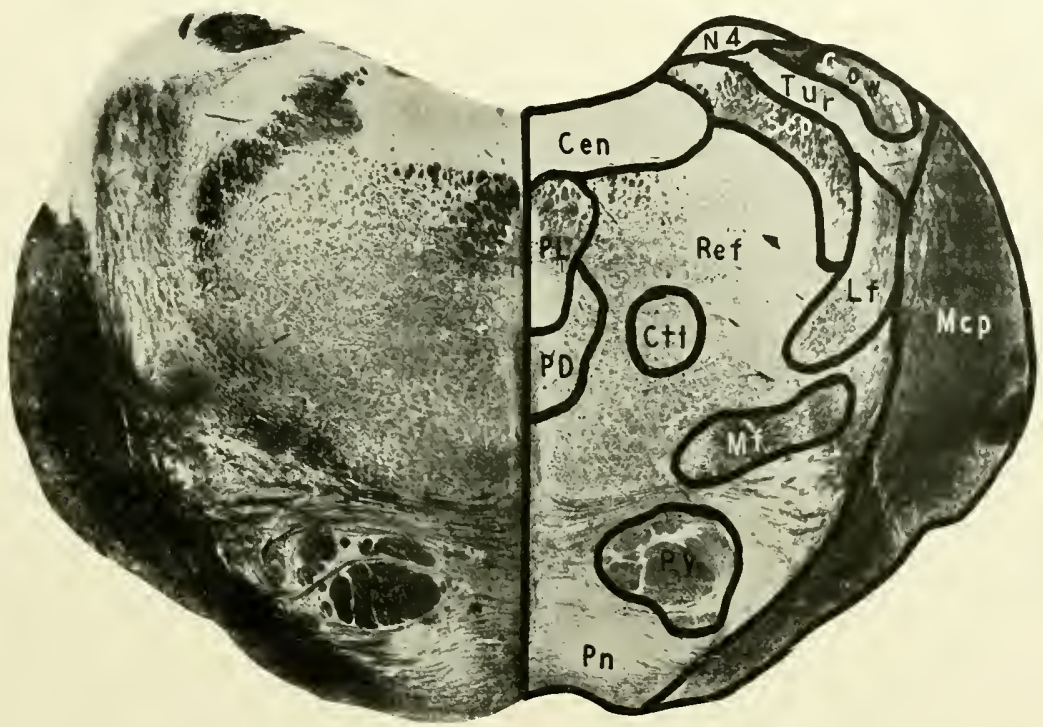

FIG. 8;. MARMOSET, LEVEL AT THE MIDDLE OF THE PONS VAROLII.

CEN, Central Gray Matter; CTT, Central Tegmental Tract; gow, Ventral Spinocerebellar Tract; Lf, Lateral Fillet; mcp, Middle Cerebellar Peduncle; MF, Mesial Fillet; N4, Trochlear Nerve; PD, PredorsaI Bundle; PL, Posterior Longitudinal Fasciculus; PN, Pontile Nuclei; Pr, Pyramid; Ref, Reticular Formation; scP, Superior Cerebellar Pcdunele; rur, Tractus Uncinatus of Russel (Hook Bundle). (Accession No. 146. Section 123. Actual Size $9 \times 5 \mathrm{~mm}$.

would necessitate the development of a more complex motor organization. Hence it lives within a limited sphere of action whose requirements demand a relatively simple motor organization.

LEVEL OF THE INFERIOR COLLICULUS (FIG. 86)

At the level of the inferior colliculus the most significant modification is the appearance of this elevation ( IC), the primordial relay station 
for the sense of hearing. This structure in the marmoset shows a high degree of development which may be taken to indicate an auditory sense still having a large representation at this lower level in the brain. Here, as in

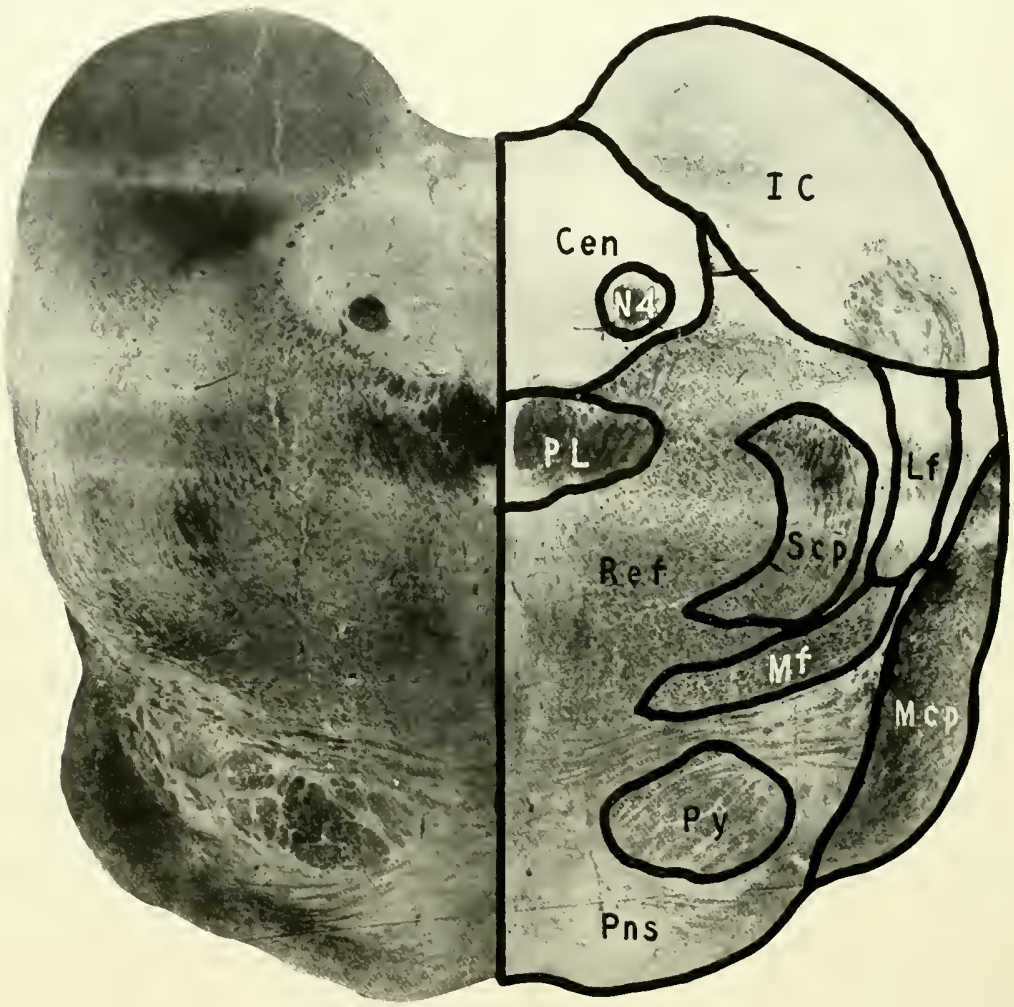

FIG. 86. MARMOSET. LEVEL OF THE INFERIOR COLLICULUS.

CEN, Central Gray Matter; IC, Inferior Coliiculus, IF, Lateral Fillet; MCP, Middle Cerebellar Peduncle; MF, Mesial Fillet; N4, Trochlear Nerve; PL, Posterior Longitudinal Fasciculus; PNS, Pons, P1; Pyramid; REF, Reticular Formation; scr, Superior Cerebellar Peduncle. [Accession No. I 46 . Section i30. Actual Size $9 \times-\mathrm{mm}$.] 
the case of lemur, this primordial auditory organization appears to be in the interest of rapid automatic reactions in response to auditory stimuli. It provides for immediate readjustments of body posture against impending attack or other peril, the approach of which is indicated by sounds. Also, as in the case of Iemur, this provision for immediate reaction in response to sound imposes a definite degree of limitation in the range and scope of motor activity. It deprives the animal of certain deliberative and reflective elements which furnish a period of latency in the selection of alternative courses of action designed to meet emergencies which may develop. The superior cerebellar peduncle $(S c p)$ appears as a crescent extending inward and forward preparatory to its decussation. The pyramid ( $\mathrm{Py}$ ) still presents a disseminated appearance and interspersed among its fasciculi are clusters of the pontile nuclei. Lateral to the mesial fillet (Mf) is a Iong, sweeping mass of fibers on their way to the inferior colliculus, the axons of which constitute the lateral fillet ( $\mathrm{Lf}$ ). Dorsolateral to the mesial fillet is a bundle of fibers constituting the rubrospinal tract and spinothalamic tract. The central gray matter (Cen) has greatly increased in size and now almost surrounds the small cavity constituting the caudal orifice of the Syrian aqueduct whose roof is formed by the superior medullary velum and by the decussating fibers of the trochlear nerve. Between the two inferior colliculi, resting upon the decussation of the trochlear nerve, are the lingular folia of the cerebellum.

LEVEL OF THE SUPERIOR CEREBELLAR PEDUNCULAR DECUSSATION (FIG. 8-)

The next cross section is introduced to show the decussation of the superior cerebellar peduncle ( $\mathrm{XScp}$ ) preparatory to its entrance into the red nucleus. The section selected discloses this decussation at its maximum and affords another illustration of the relatively poor development of the cercbello-rubro-spinal connections, which implies a low degree of organiza- 
tion in coordinative control. This section also shows the caudal extremity of a large mass of gray matter situated ventral to the mesial fillet (Mf), namely, the substantia nigra $(\mathrm{Sbn})$. This mass, as in lemur, assumes

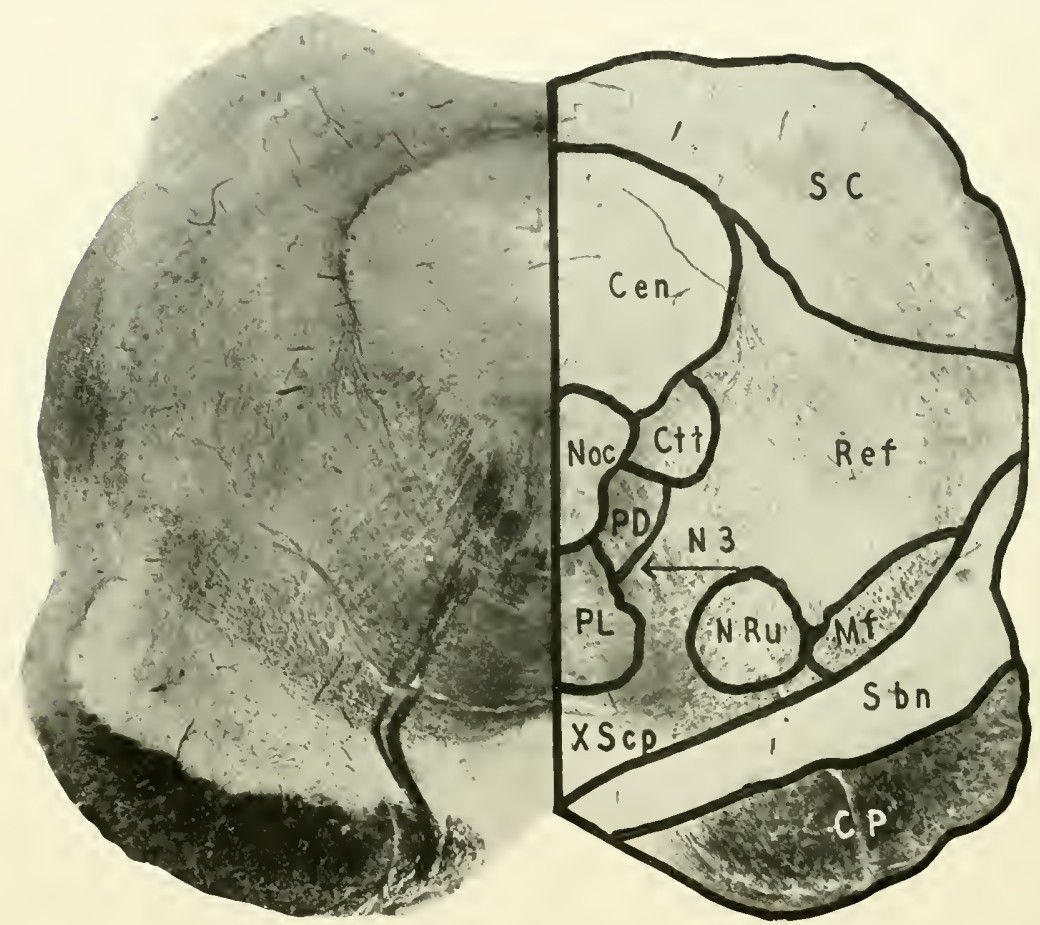

FIG. 8-. MARMOSET, LEVEL OF THE SUPERIOR CEREBELLAR PEDUNCULAR DECUSSATION.

CEx, Central Gray Matter; CP, Cerebral Peduncle; Crt, Central Tegmental Tract; MF, Mlesial Fillet; noc, Oculomotor Nucleus; NRU, Nucleus Ruber; N3, Oculomotor Nerve; PD, Predorsal Bundle; PL, Posterior Longitudinal Fasciculus; ReF, Reticular Formation; SC, Superior Colliculus; sBN, Substantia Nigra; XscP, Decussation of the Superior Cerebellar Peduncle. [Accession No. 146. Section 162. Actual Size o $\times 7 \mathrm{~mm}$.]

large proportions. If the substantia nigra is concerned with the control of certain automatic associated movements, as has been presumed by many 
authorities, then it is fair to believe that the marmoset is possessed of a high degree of automatic associative control. Such a presumption would accord well with its recognized inferiority in the development of volitional control of movement. The animal, being poorly provided with such control, would of necessity require a considerable degree of automatic and associative regulation to carry on the performances essential to its natural motor adjustments. The probability of this supposition is further attested by the large size of the basal ganglia in the forebrain. These structures, emprising the globus pallidus and catudate nuclei, are usually regarded as part of the mechanism essential to the control of automatic associated movements. The pyramidal nerve fibers are now collected in a compact bundle accompanied by other axons representing the pallio-ponto-cerebellar system. A large mass of decussating fibers dorsal to the central gray matter constitutes the commissure of the superior colliculi. The central gray matter ( Cen), much enlarged in size, surrounds the small aqueduct of Sylvius. Ventromesially it contains the nucleus oculomotorius (Noc). A superficial layer of gray matter forms the stratum griseum superficiale of the superior colliculus, while lateral to this the brachium conjunctivum posticum is extending forward to the mesial genieulate body.

\section{LEVEL OF T11E SUPERIOR COLLICULUS (FIG. 88)}

At the level of the superior colliculus the most distinguishing feature is the appearance of the tectal plate of the midbrain whose surface relief has previously been referred to as particularly prominent. The superior colliculus itself presents a definite degree of stratification reminiscent of the optic Iobes in the birds, reptiles, amphibia and fish. The most supcrficial layer consists of the stratum griseum superficiale. This part of the brain still retains some of its original dominance in visual function. While it is true that much or most of the higher visual syntheses have their representation 
in the cerebral eortex of the occipital lobe, it is probably true also that the superior collieulus maintains its primordial arrangement in the interest of certain primitive and immediate rellex reactions in response to visual stimuli.

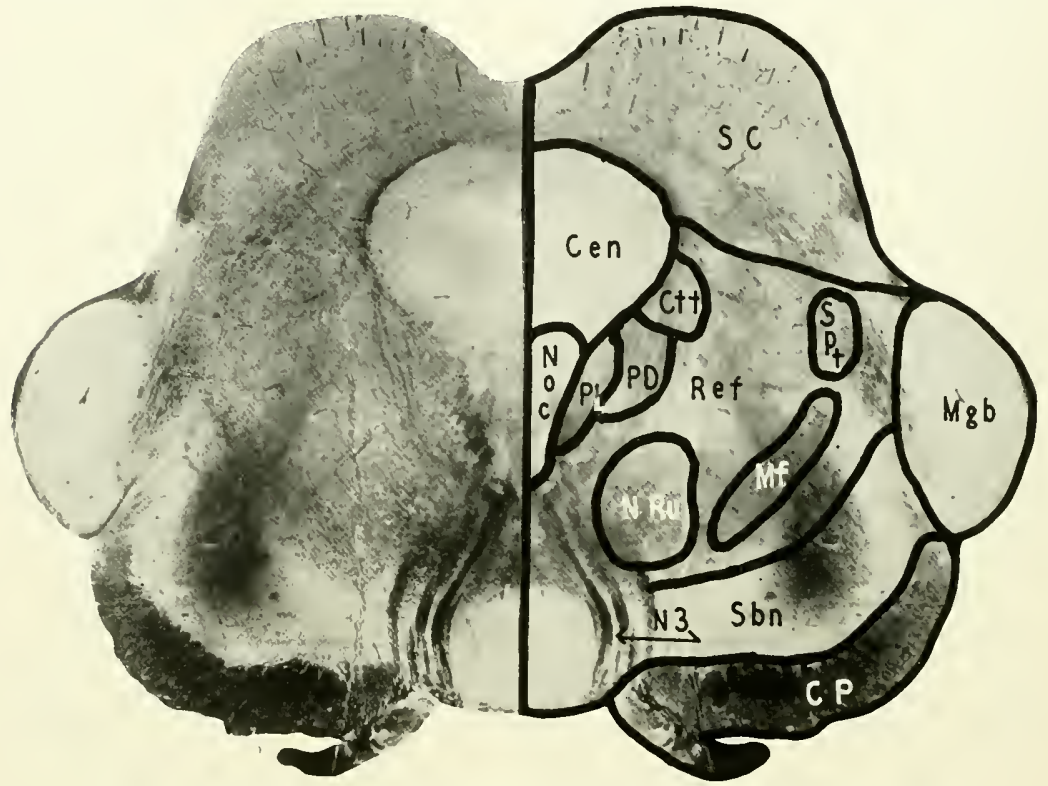

FIG. 88. MARMOSET. LEVEL OF THE SLPERIOR COLLICLLUS.

CEx, Central Gray Matter; CP, Cerebral Peduncle; CTT, Centrat Tegmental Tract; MF, Mesial Fillet; MGB, Ilesial Geniculate Budy; Noc, Oculomotor Nucleus; NRu, Nucleus Ruber; N3, Oculonotor Nerve; PD, Predorsal Bundle; PL, Posterior Longitudinal Fasciculus; REF, Reticular Formation; sBN, Substantia Nigra; sc, Superior Colliculus; SPT, Spinothalamic Tract. [Accession No. 146. Section 167. Actual Size ro $\times 6 \mathrm{~mm}$.]

In this particular, the case is similar to that of the inferior colliculus which has apparently retained much of its primordial auditory function. The necessity for quick, decisive motor responses as a reaction to visual stimuli on the part of the marmoset must be obvious. Most of the animal's reactions to visual stimulation result in very rapid movements of the head, eyes, neck and limbs. 
These movements have much of the quality of immediate rellex responses. They resemble but little the motor reactions characterized by any marked degree of deliberate reflection in selecting alternative courses of action which may arise in connection with any given situation. For this reason apparently the superior colliculus retains much of its primitive conspicuity as well as much of its original structural organization. The evidence afforded by marmoset indicates that vision has attained only partial telencephalization in this form.

Also of importance is the appearance of the large substantia nigra ( $\mathrm{Sbn}$ ) which again points to the possibility of the animal's large endowment in automatic associative control of movement. In the most ventral portion of the basis are the fibers constituting the cerebral peduncle (CP). A lateral protrusion in juxtaposition to the substantia nigra is the mesial geniculate body $(\mathrm{Mgb})$ along whose periphery is situated the brachium conjunctivum posticum containing auditory fibers on their way from the inferior colliculus. Dorsal to the mesial fillet ( $\mathrm{If}$ ) is a collection of large motor cells, the nucleus ruber ( $N R u$ ), which gives origin to the rubrospinal tract, the connecting link between the cerebellum and the final common pathway of the cerebrospinal axis. The central gray matter $(\mathrm{Cen})$ is now greatly increased in size and surrounds a snall aqueduct of Sylvius. At its ventromesial angle it contains the nucleus oculomotorius ( $\mathrm{Noc}$ ) which gives rise to the third cranial nerve supplying all the muscles of the eyeball with the exception of the external rectus and the superior oblique. An important feature of this nucleus is the lact that it is very poorly supplied with internuclear or commissural fibers. The two nuclei maintain a certain degree of independence. This perhaps is no more marked than in lemur, but it is distinctly more pronounced than in the higher apes and man. It is probable that marmoset possesses but a small degree of power to converge the visual axes and in consequence has a limited amount of stereoscopic vision. Passing forward from the nucleus of the third nerve are its emergent fibers. 


\section{LEVEL OF THE OPTIC CHIASM (FIG. 80)}

At the level of the optic chiasm the identifying feature is the decussation in the optic pathway $(\mathrm{Opt})$. The optic thatamus is also represented. This is

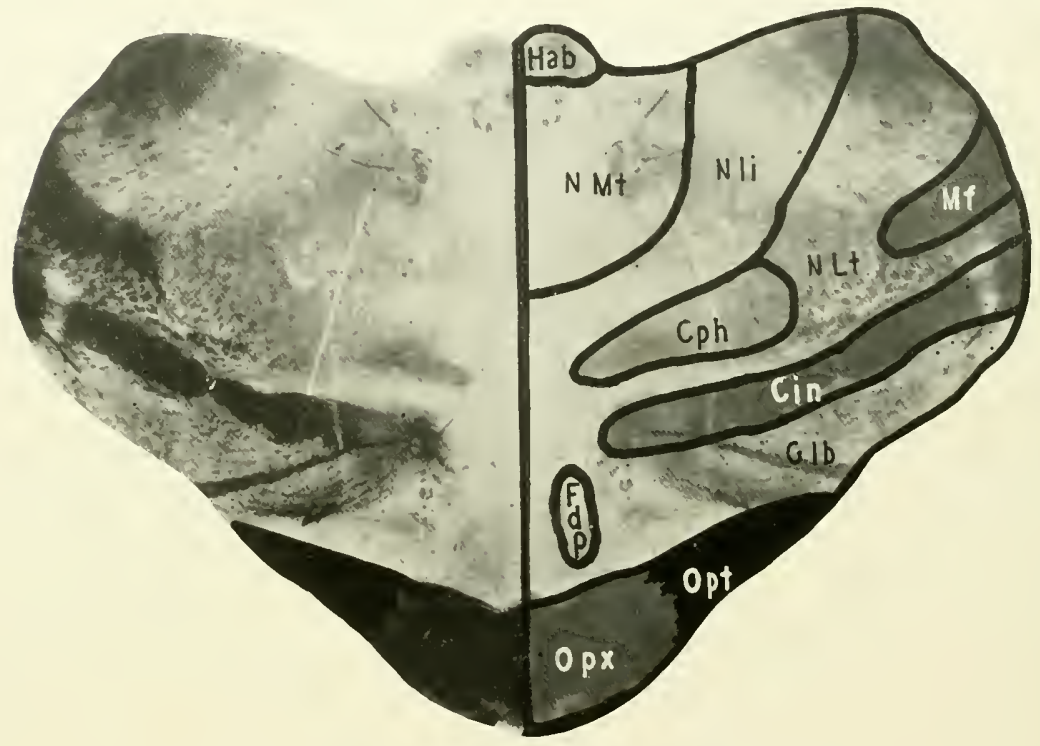

FIG. 89. MARMOSET. LEIEL OF THE OPTIC CHIASM.

CIN, Internal Capsule; CPH, Corpus Iypothalamicum; FDP, Descending Pillars of the Fornix; GLB, Globus Pallidus; нав, Nuclcus Ilabenulae; Mr, Mesial Fillet; NMT, Mledial Nucleus of the Thalanus; Ni, Internal Lateral Nucleus of the Thalamus; NLI, External Lateral Nucleus of the Thalamus; opt, Optic Tract; opx, Optic Chiasm. [Accession No. $14^{6}$. Section 219 . Actual Size $19 \times 12$ mm.]

an important structure inasmuch as it represents the last great relay station for the conduction of all types of sensibility with the exception of the olfactory sense. Situated ventral to the main nuclei of the optic thalamus is a diffuse collection of fibers and gray matter, the corpus hypothalamicum 
$(\mathrm{Cph})$. The nucleus which forms the central core of this body is surrounded by two fields of white matter constituting field $\mathrm{H}_{1}$ and field $\mathrm{H}_{2}$ of Forel. Lateral to the optic thalamus and the nucleus hypothatamicus is a dense

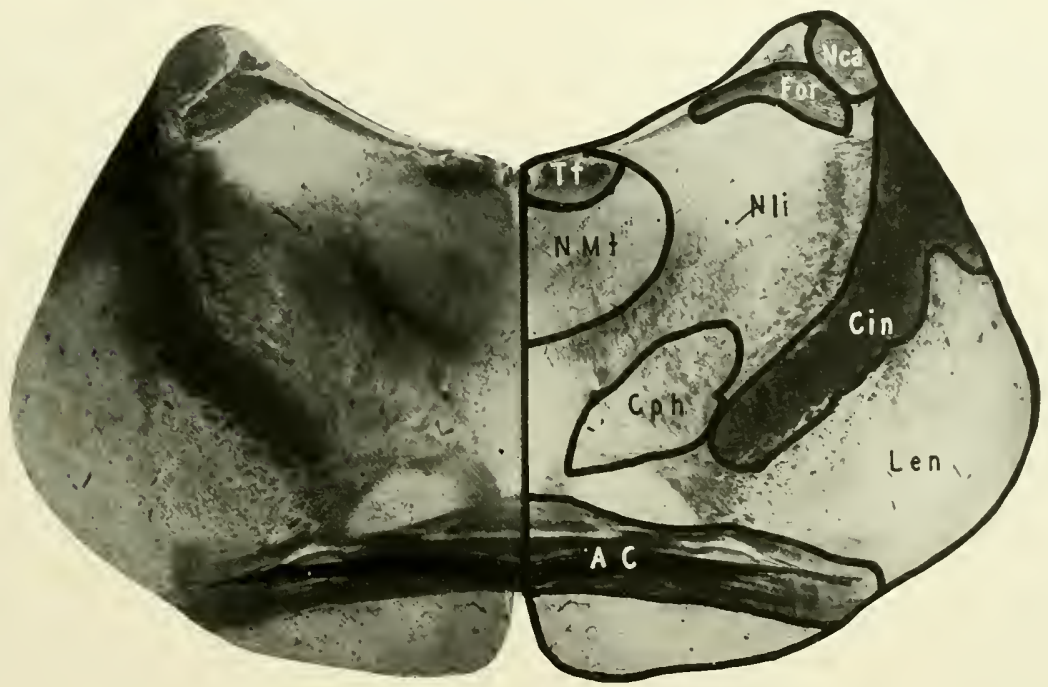

FIG. 9O. MARMOSET. LEVEL OF THE ANTERIOR COMMISSURE.

AC, Anterior Commissure; CIN, Internal Capsule; $\mathrm{CPH}$, Corpus Hypothalamicum; For, Fornix; LEN, LenticuIar Nucleus; NCA, Caudate Nucleus; ммт, Medial Nucleus of the Thalamus; NLI, Internal Lateral Nucleus of the Thalamus; $\pi$, Taenia Thalami. [Accession No. 146. Section 241. Actual Size $15 \times 6 \mathrm{~mm}$.]

band of medullary substance comprising a portion of the internal capsule (Cin); while ventral to this massive bundle of myelinized axons is a large nuclear mass, the globus pallidus ( Glb). This structure takes its importance from the fact that it is one of the main basal ganglia regulating automatic associated movements. Immediately mesial to the globus pallidus is a bundle of faintly staining fibers, the descending pillars of the fornix ( $\left.F d_{p}\right)$. 


\section{LEVEL OF THE ANTERIOR COMMISSLRE (FIG. gO)}

At the level of the anterior commissure ( $\mathrm{AC}$ ) is illustrated the anterior extremity of the optic thalamus and a portion of the tuberculum anticum thalami $(\mathrm{N} M \mathrm{t})$. Lateral to the thalamus is a dense mass of fibers representing the internal capsule ( $\mathrm{Cin}$ ), lateral to which is a portion of the corpus striatum, the lenticular nucleus (Len). 


\author{
Chapter VI
}

\title{
RECONSTRUCTION OF THE GRAY MATTER IN THE BRAIN STEM OF CALLITHRIX JACCHUS
}

\section{Dorsal Sensory Nuclei}

HE nucleus of Goll first becomes apparent in the reconstruction as a
small sessile projection connected with the dorsal surface of the cen-
tral gray column. It rapidly extends dorsally as a narrow prolongation of the central gray column and presents at its extremity a latcrally flaring termination. It is situated close to the midline and separated from the dorsal median septum by a thin lamina of fibers. At a somewhat higher level a small collection of gray matter appears in the dorsal portion of the reticular formation. This consists of the nucleus of Burdach which, like the sensory nucleus of Goll, rapidly expands as it extends cephalad. Attached laterally to the main nuclear mass and presenting the characteristic arboreal form seen in the lemur is the external nucleus of Burdach (Blumenau). The effect of the opening of the fourth ventricle is already apparent in the divergent arrangement of the overhanging dorsal masses of the nuclei of Goll and Burdach. As these nuclei take their origin from the central gray column and the reticular formation mesial to the point of confluence betwecn the dorsal gray column of the spinal cord and the central gray column, the former is slowly and gradually shifted laterally, and its cxpanded cap, the substantia gelatinosa Rolandi, under the influence of the opening ventricle, shows a smilar lateral inclination. The transition from the Rolandic to the trigeminal gelatinosa occurs without sharp line of demarcation except that the trigeminal gelatinosa is constantly increasing in size as it ascends. In outline this structure presents a serrated surface, the indentations being occupied by the 
descending trigeminal tract fibers. The nuclear mass itself is triangular in form, its apex being mesially directed, while the base is disposed laterally in contact with the descending trigeminal fibers. The nucleus continues upward in its frxed location so constant as to afford an orienting point for other internal structures of the bulb, to the midpontine level where, after expanding, it rapidly undergoes contraction and disappears.

\section{The Inferior Olivary Nucleus}

Reconstruction of this mass of gray matter resembles very closely the nucleus as deseribed in lemur. The inferior olive forms a flat, double ribbon with its single plication situated ventrolaterally and close to the surface of the brain stem. Its free extremities are directed dorsomesially and turn slightly dorsally at their terminations. There is little indication of the development of the secondary plications characteristic of this nuclear mass in the higher primates.

The approach of the nucleus to the surface occasions a slight elevation which corresponds to the relief of the olive so conspicuous in the human brain stem. The chief olivary nucleus, as in the lemur, is poorly cleveloped. The plicated turn of the nucleus appears somewhat thicker and heavier than the two limbs of the nuclear mass formed by the accessory olivary nuclei which stretch dorsomesially toward the median raphe of the oblongata.

The inferior olivary nucleus in the marmoset, as in the lemur, consists mainly of the two accessory nuclei. The main inferior olivary nucleus is only suggested. The ventral accessory nucleus appears at the upper decussational level of the pyramidal tract as a flattened band directed mesially and slightly dorsally. It ascends, diminishing in width and approaching the midline. The dorsal accessory nucleus is a mass of nuclear material which rapidly flattens out and is embedded in the ventral surface of the reticular formation. It also tends to approach the midline and attenuates as it ascends. 
The main olivary nucleus appears as a small mass of nuchear material between the actual extremities of the accessory olives with which it fuses. It presents no plications.
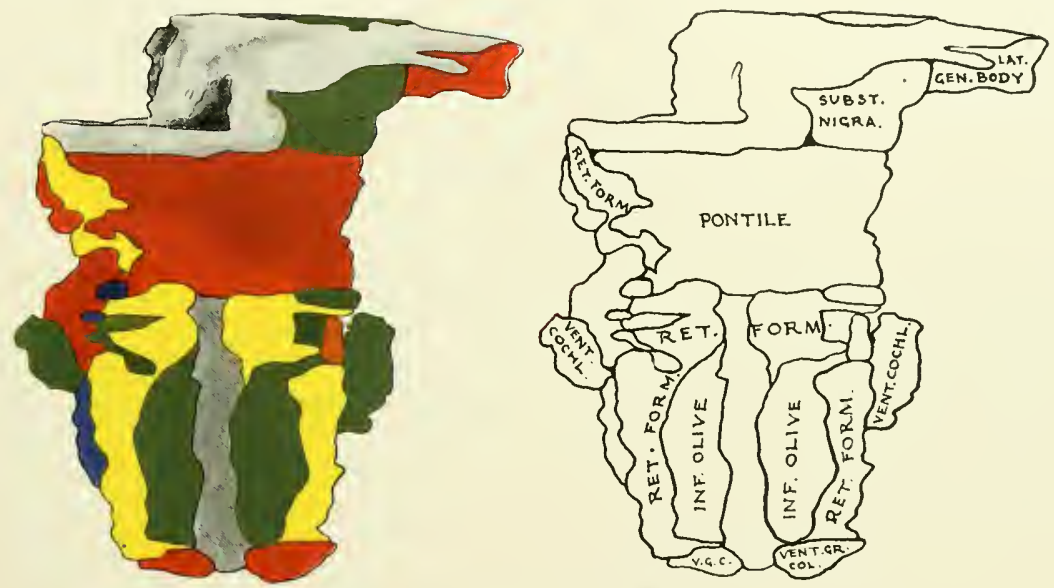

FIG. 91. IENTRAL SURFACE OF THE GRAY MATTER OF THE BRAIN STEM, CALLITHRIX JACCHUS.

Key to Diagram. 1NF. olive, Inferior Olive; lat. gen. body, Lateral Geniculate Body; pontile, Pontile Nuclei; REt. Form., Reticular Formation; subst. Nigra, Substantia Nigra; vevt. Cochl., Ventral Cochlear Nucleus; v. G. C. and veNr. GR. COL., Ventral Gray Column.

\section{The Reticular Formation}

Reconstruction of the reticular formation reveals in general the same characteristics found in the lemur. It provides a matrix through which numerous fasciculi make their way. It is surrounded, except dorsally, by fiber masses passing upward or downward through the stem. Dorsally it comes into contact and merges with the subependymal gray matter which appears as the continuation of the central gray column. The reticular formation originates as small masses of nuclear material appearing between the decussating bundles of the pyramidal tract, which, as these fibers are 
gradually drawn together into a compact bundle, eoalesee to form a recognizable structure.

In the pontile levels, the reticular formation is separated from the deep layer of the pontile nuclei by the trapezoid body. This structure at higher levels forms a part of the lateral boundary of the reticular formation.

The disposition of the reticular formation in marmoset is similar to that in lemur. It supplies the main gray mass of the brain stem, affording a matrix in which condensations of nuclear material appear as discrete nuclei. The lateral nuclei of the reticular formation and the superior olivary nuclei are but little better developed than in the lemur. In the region of the midbrain the nucleus ruber appears as a special condensation of the reticular formation, surrounded by its fiber capsule derived from the superior cerebellar peduncle and the striato-rubral tract. In its dorsomesial aspect there are embedded suceessively the nuclei of the mesial somatic motor cell column, the hypoglossal, the abdueens, the trochlear and the oculomotor nuclei. The vestibular nuclei appear, as in the lemur, in the dorsolateral angle of the tegmentum. The nucleus of Deiters first appears as a wedge between the reticular formation and the central gray matter. The cephalic extremity of the reticular formation comes into rather intimate relation with the substantia nigra but does not fuse with it. Actual fusion takes place between the reticular formation and the zona incerta of the diencephalon and with the caudoventral portion of the central thalamic gray matter.

\section{The Pontile Núclel}

In reconstruetion these nuclear masses appear rather abruptly at the level of the trapezoid body. There is no substantial indication of the areiform nuclei in the oblongata which appear in higher primates as caudal prolongations of the pontile nuclei along the ventral surface of the pyramidal traets. The pontile nuclei appear as bilateral nuclear masses mainly disposed 
mesially and laterally to the descending pallio-pontile and pallio-spinal systems of fibers. These mesial and lateral nuclear masses are connected by relatively light bridges of nuclear material. There is but little evidence
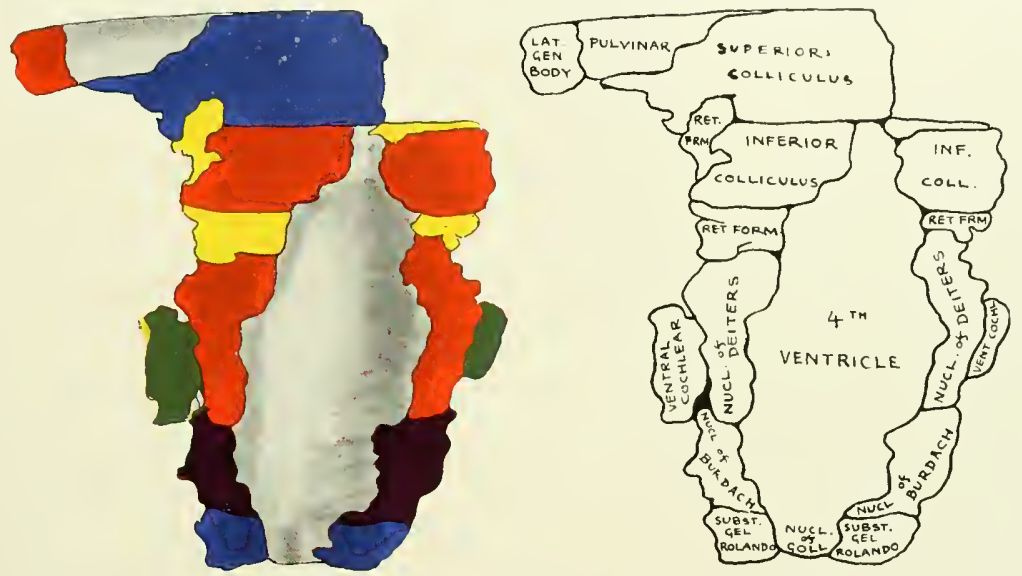

FIG. 92. DORSAL SURFACE OF THE GRAY MATTER OF THE BRAIN STEM, CALLITHRIX JACCHUS.

Key to Diagram. INF. Coll., Inferior Colliculus; Lat. GeN. Body, Lateral Geniculate Body; Nucl. OF BURDach, Nucleus of Burdach; Nucl. Of Deiters, Nucleus of Deiters; Nucl. of Gol.L, Nucleus of Goll; REt. FRM. and RET. ForM., Reticular Formation; SUbST. GEL. ROLANDO, Substantia Gelatinosa of Rolando: VENT. cochl., Ventral Cochlear Nucleus.

pointing toward the development of the invading strips of nuclear material so richly present in the higher forms. Cephalically the pontile nuclei become continuous with the relatively massive substantia nigra chiefly by means of the mesial and lateral condensations of nuclear material already mentioned in the study of the brain stem of Iemur as the lateral and mesial buttresses.

\section{The Vestibular Nuclei}

Reconstruction of this mass of nuclear substance discloses a small, wedge-shaped area lying between the nuclei of the columns of Goll and Bur- 
dach, the central gray matter and the reticular formation at the dorsolateral angle of the latter. The large nucleus of Deiters first appears at the Iower decussational level of the pyramidal tract and, rapidly expanding, reaches its greatest diameter at the midventricular level of the brain stem. From this region it diminishes as higher levels are approached. As it diminishes in bulk the vestibular nuclei increase to their maximum and then recede to give place to the cochlear nuclei. At the midventricular level the nucleus of Deiters and the nucleus of Schwalbe both begin to diminish. The vestibular muclei are carried upward about the lateral walls of the ventricle by the small nucleus of von Bechterew which is so poorly differentiated as to make adequate representation in this model impossible.

\section{The Cochlear Nuclei}

As shown in the reconstruction, the cochlear complex consists of both the ventral cochlear nucleus and the dorsal cochlear nucleus which are situated clirectly ventral to the subependymal gray matter of the floor of the lateral recess of the fourth ventricle. The dorsal cochlear nucleus is situated in the lateral ventricular recess and is more or less oval in outline. It extends for a short distance above and below the limits of the lateral ventricular recess. It also extends mesially for a short distance under the ventricular gray matter.

\section{The Substantia Nigra}

The reconstruction of this mass of gray matter resembles the nucleus found in lemur. It appears to be continuous with the deep layer of the pontile nuclei, supported throughout its entire transverse extent by this lamina, while at its extremities it rests upon the lateral and mesial buttresses of the pontile nuclei. Mesially it is connected with its fellow of the opposite side by the undifferentiated ventral interpechncular gray matter. In its lateral por- 
tion is found the discrete nucleus described in connection with lemur, from which nerve fibers pass dorsomesially into the mesencephatic tegmentum. Its cephalic termination is uncertain as it seems to attenuate and disappear
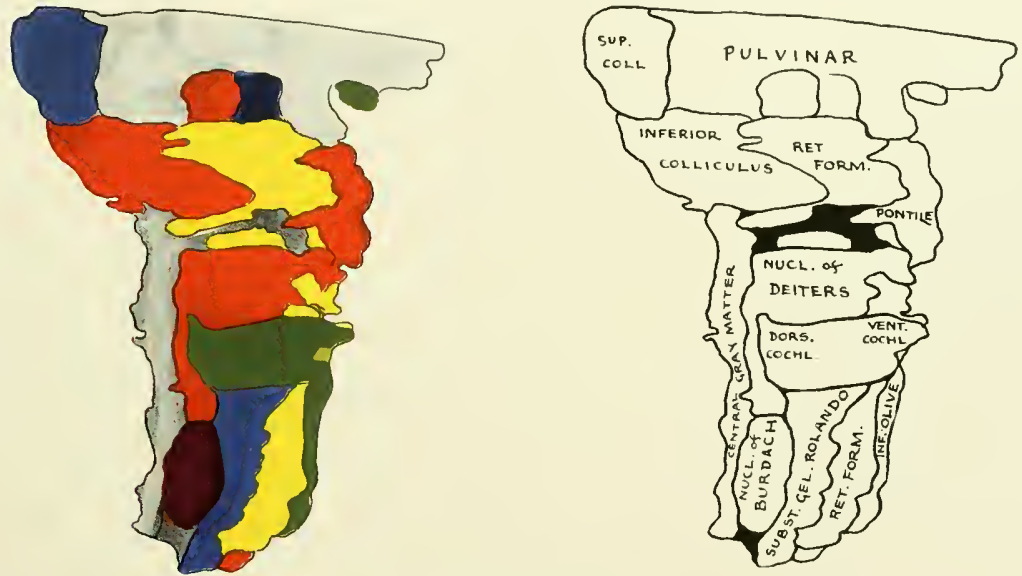

FIG. 93. LATERAL SURFACE OF THE GRAY MATTER OF THE BRAIN STEM, CALLITHRIX JACCHUS.

Key to Diagram. dors. Cochl., Dorsal Cochlear Nucleus; inf. Olive, Inferior Olive; Nucl. Of Burdach, Nucleus of Burdach; Nucl. OF Deiters, Nucleus of Deiters; PONTILE, Pontile Nuclei; REt. ForM., Reticular Formation; subst. gel. Rolando, Substantia Gelatinosa of Rolando; sup. Coli., Superior Colliculus; veNt. COCHL., Ventral Cochlear Nucleus.

with no continuity with the thalamic structures, although it appears to be replaced by the zona incerta of the diencephaton.

\section{The Colliculi}

In the reconstruction, the inferior colliculus arises at the lower mesencephalic level from the reticular formation laterally and the tectal gray matter surrounding the upper extremity of the fourth ventricle mesially. It is separated from the superior cerebellar peduncte by a narrow lateral prolongation from the reticular formation of the mesencephalic tegmentum. Mesially 
it is in contact with the dorsal undifferentiated gray matter surrounding the ventricle and the Sylvian aqueduct, while its lateral cxtremity appears to rest upon the lateral and dorsal extensions of the reticular formation. The inferior colliculus is quite massive and presents a marked expansion on the dorsal surface of the mesencephalon. It is separated from the superior colliculus by a narrow groove in which the reticular formation underlying the inferior colliculus comes to the surface. The superior colliculus is a massive structure and presents a well-marked surface elevation on the dorsal aspect of the mesencephalon. It also is continuous mesially with the undifferentiated dorsal gray matter. Laterally it comes into close relation with the inferior brachium and ventrally with the dorsal extensions of the reticular formation. Cephalically the superior colliculus does not make contact with the thalamic nuclei, being separated from them by a dorsal extension of the reticular formation.

\section{The Central Gral Matter}

In the reconstruction, the sheet of gray matter in the floor of the fourth ventricle seems featureless and is disposed as a flat, smooth stratum. The underlying structures produce no marked prominences or depressions in its surface. As the midbrain is approached, the floor of the ventricle decreases in size, the side walls and roof approach each other and thicken materially so that when the aqueduct of Sylvius is formed, the lateral walls and roof present subependymal gray matter fully as bulky as the gray matter of the floor of the aqueduct. Cephalically the contral gray matter is continuous with the gray matter underlying the ependymal lining of the third rentricle, forming the mesial group of the thalamic nuclei. The dorsal gray matter is continuous with the epithalamic structures, the ventral gray matter with the hypencephalic region. 


\section{Chapter VII}

\section{MYCETES SENICULUS, ITS BRAIN AND BEHAVIOR}

Its Position among the Primates; Measurements and Brain Indices; Surface Appearance of the Brain; Internal Structure of the Brain Stem in Cross Section

7 PHE Cebidae are in general much larger than the Hapalidae and more ape-like in appearance than the lemurs. The tail, although short in a few instances, is usually long and definitely prehensile. It is not covered with hair at its extremity and in this part especially manifests its prehensile characters. These animals vary considerably in size; some species are but little larger than the marmoset, while the larger members of the family are only a little smaller than some of the old-world monkeys. In general they are about as large as a fox-terrier. The development of the tail attains its highest degree of specialization in the spider monkey (Ateles) and the woolly monkey (Lagothrix). In these Cebidae the tail is so much differentiated in its prehensile function as to justify speaking of it as a fiftb band. By means of this fifth hand the monkey swings its way along among the branches, procuring many advantageous positions of the body which enable it to use both fore- and hindlimbs in the manner of hands. It can reach, grasp and even hurl small objects by means of its tail. When not in use, the tail is carried erect over the head. This highly specialized sensory organ adds an important element to the behavioral organization of the animal, some evidence of which must be reflected in the central nervous system.

The howling monkeys constitute an interesting group of the Cebidae. While they are not possessed of a tail so highly differentiated as the spider monkeys, this organ none the less has great efficiency in its prehensile 


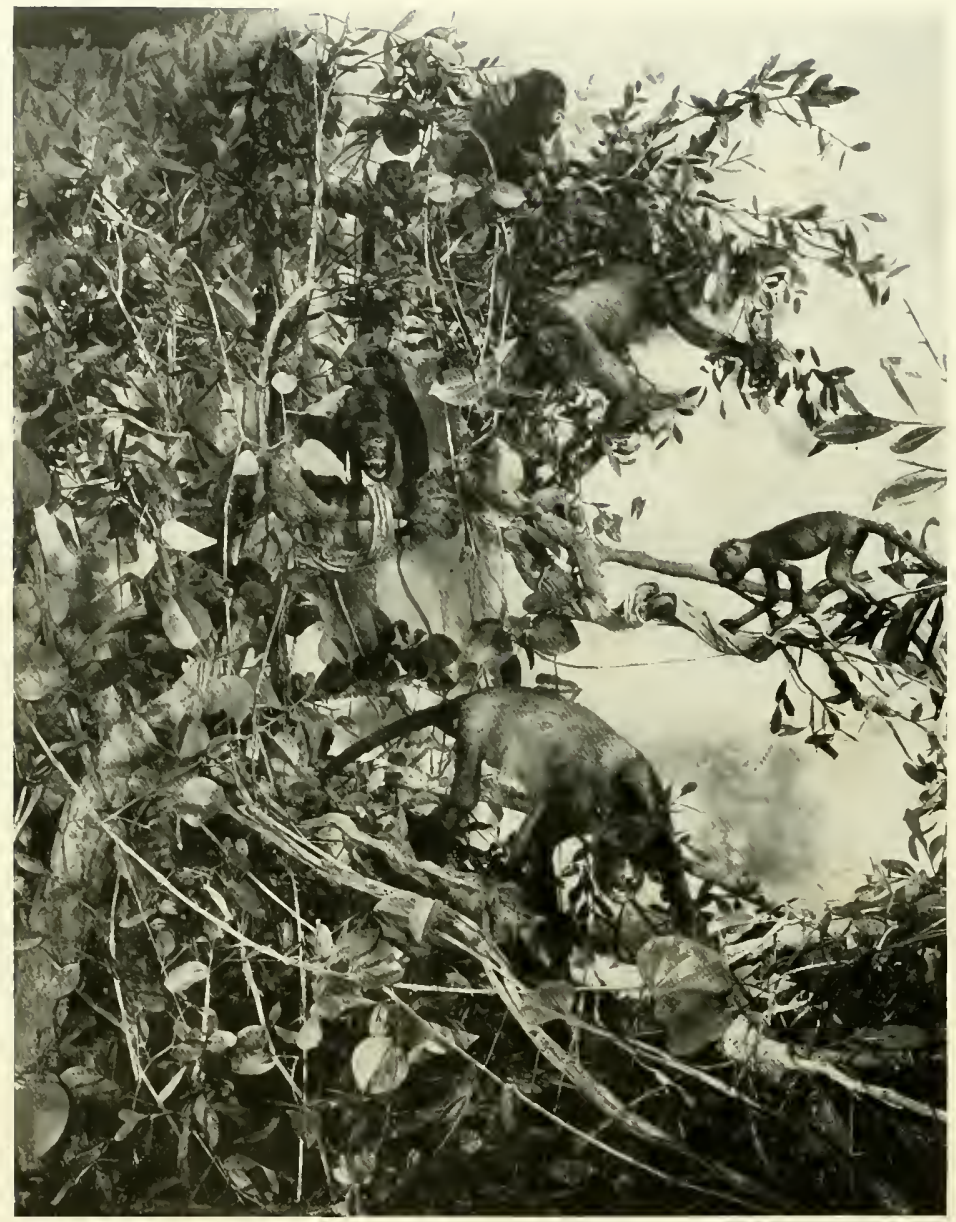

Courtesy, American Museum of Natural Histor

FIG. 94. HABITAT GROUP OF MYCETES SENICULUS, THE RED HOWLING MONKEY OF SOUTH AMERICA. 
capacities. In addition to this specialization, the howling monkeys have a sinus-like diverticulum in the larynx larger than that of any of the other Anrerican monkers. The hyoid bone is much enlarged and eontains a cavern-
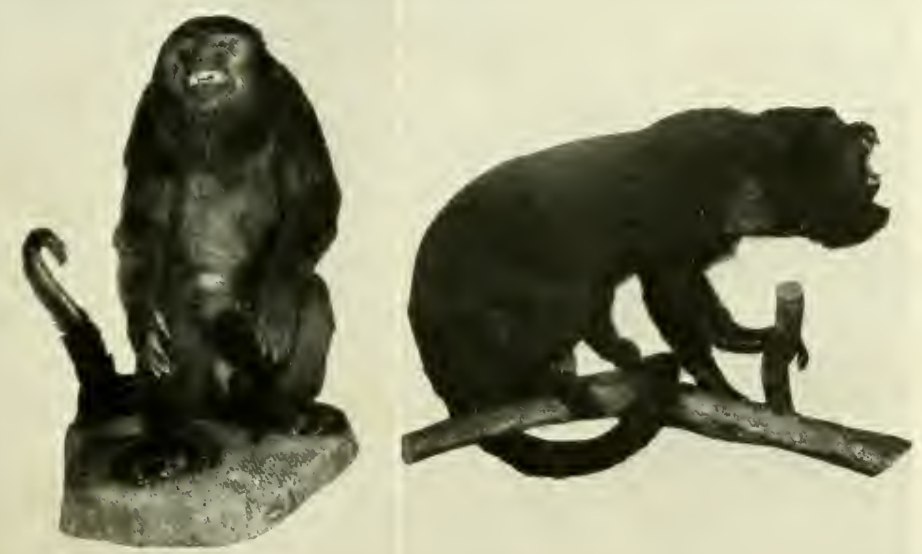

Courtesy. American Museum of Natural History.

FIGS. 95 AND 96 . TWO VIEWS OF MYCETES SENICULUS, THE RED HOWLING MONKEY OF SOUTH AMERICA, SHOWING ITS PREHENSILE TAIL.

ous dilatation, while the mandible, in order to protect the underlying structures, is likewise unusually large and heavy (Fig. 9j).

The howling monkeys also possess an opposable thumb and welldeveloped toes. They are described as being the most ferocious of South American monkeys, at the same time having the lowest degree of intelligence. Their mournful howlings are often audible for miles around. Their cries are supposed to be employed as a means of defense in order to intimidate their enemies. Their vocal powers are so awe-inspiring that the 
natives have associated certain almost supernatural attributes with these animals. For example, it is clained that these monkeys are capable of forming a bridge to span a river by means of their tails. But while the prehensile

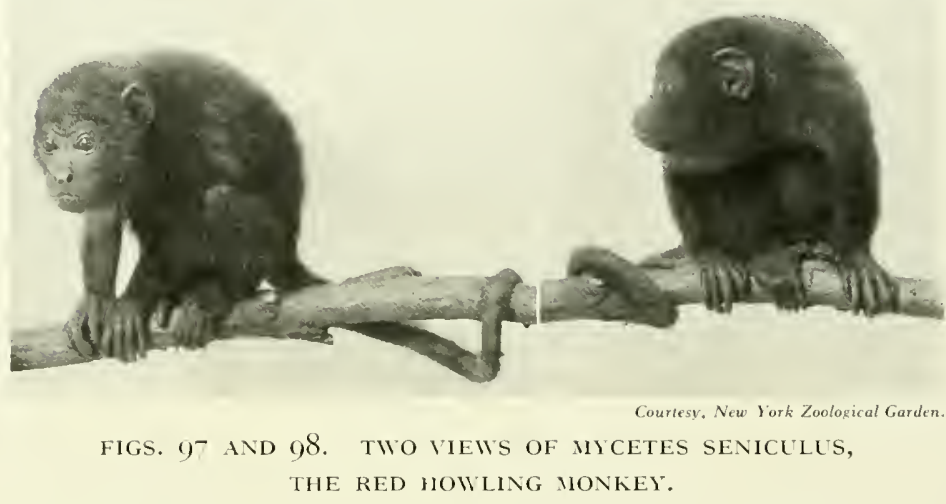

tail makes possible many feats of locomotion little short of miraculous, it seems clear that these bridge-building propensities are quite legendary. Not only is the thumb of mycetes well developed and opposable, but the hand as a whole is highly specialized. The face is naked with the exception of a heavy beard which hangs beneath the chin. The movements of the howling monkey are relatively slow when compared to those of the lemur or even of the spider monkey. The animals appear to be sullen in temper, and are practically untamable, soon dying in captivity. Whether or not their intelligence is of a low order and their adaptability to conditions outside of their natural cmiromment small, it is certain that they are among the least attractive of all the primates in disposition. Their fur is usually black, but in some cases it is brown or reddish-brown. In most species the sexes are alike in color. 


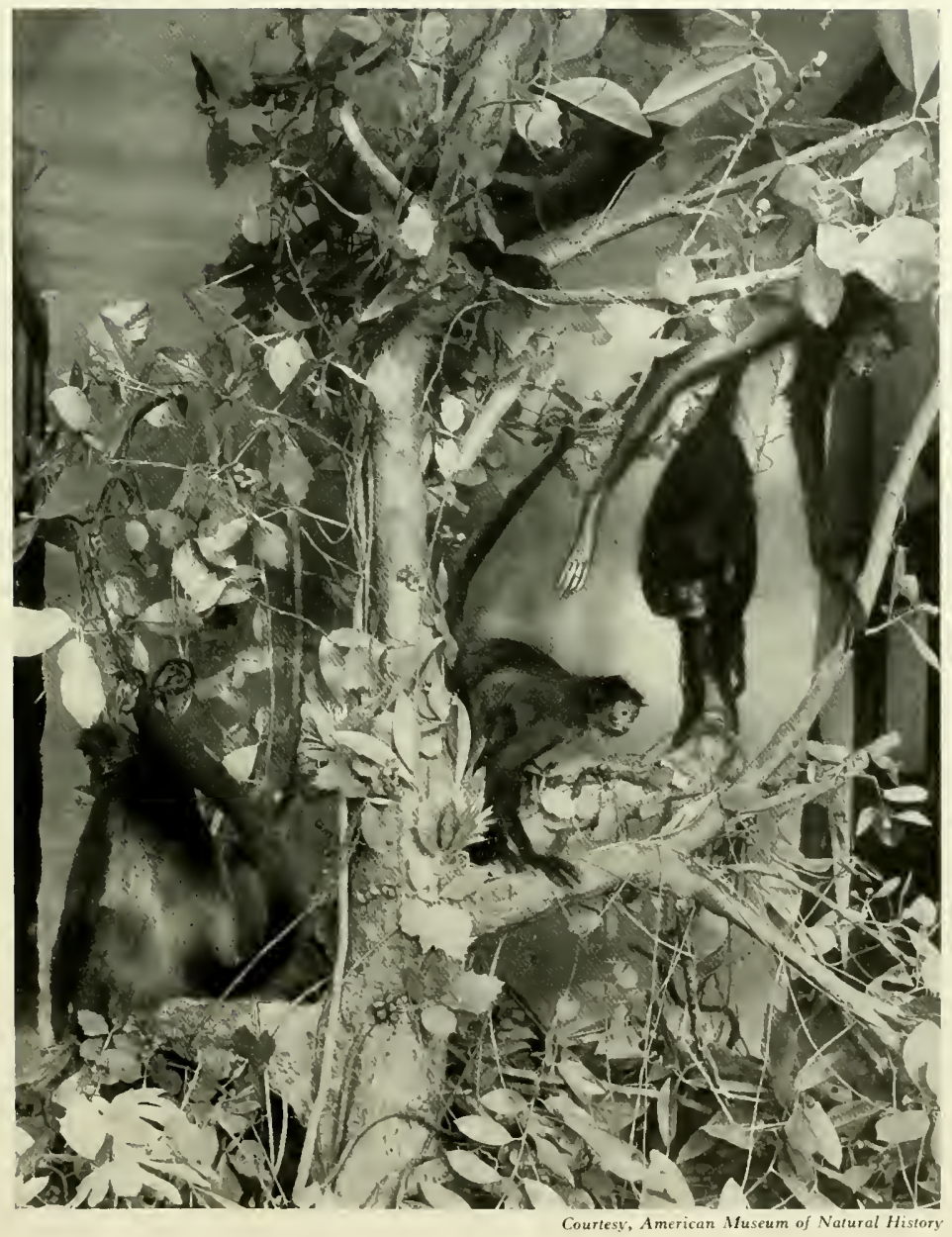

FIG. 99. HABITAT GROUP OF ATELES ATER, THE SPIDER MONKEY. 
The species here described is Mycetes senieulus, also known as Alouatta seniculus, the red howling monkey. The red howler has its habitat in Cartagena and Colombia. It lives in the forests near the Rio Negro but has also

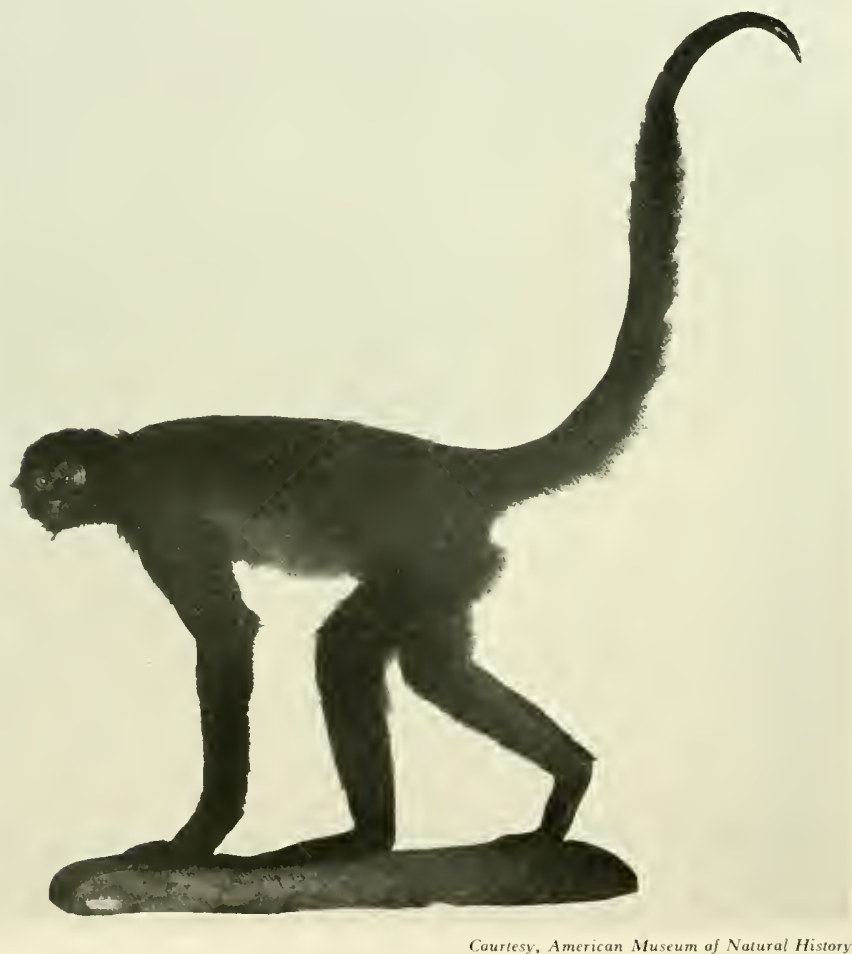

FIG. IOO. ATELES ATER, THE SPIDER MONKEY, SHOWING PREHENSILE TAIL.

been described in Brazil. The animal's fore- and hindlimbs are about of equal length, that is, between ten and twelve inches long. The length of the body, 
exclusive of the tail, is from eighteen to twenty inches, while the tail is about thirty inches in length. When these howlers are seen in the forests, three or four of them are usually together on the topmost branches of the trees. They live largely on fruit, although, like other South American monkeys, they are said to feed also on caterpillars and insects.

\section{General Behavioral Tendencies - Thorndyke's Observations}

In connection with the general behavioral tendencies of Mycetes seniculus, observations made by Professor Edwin L: Thorndyke, of Columbia University, upon several South American monkeys of the Cebus type (species not stated) are of particular importance. They represent the pioneer effort in the attempt to investigate by accurate psychological method certain aspects of simian behavior. Professor Thorndyke was chicfly interested in the manner in which monkeys may vary from other mammals in the general mental functions revealed by their methods of learning, as well as how they may vary from adult civilized human beings. He recognized three different modes by means of which knowledge may be acquired, namely, learning by trial and accidental success, learning by imitation, and learning by ideation. In the latter case the situation calls up some idea which then arouses the act or may in some way modify it. This, in fact, is the method of learning obviously employed in all advances of civilization.

Professor Thorndyke devised certain more or less complicated experiments by means of which he tested the monkeys. These tests consisted chictly of boxes with pegs, bolts, single bars, double bars, hooks, strings and wooden plugs. He arranged the plugs or loops in various combinations, the mastery of which was essential to release the animal from confmement or to admit it to a goal containing food. The tests made with such apparatus yielded negative results and made it clear that monkeys do not learn by reasoning. They do, however, form more associations and associations of 
greater variety than other mammals. Their combination of such associations is remarkably slow and incffective in providing any new bchavioral acquisition by this means. Nor were experiments involving the discrimination of

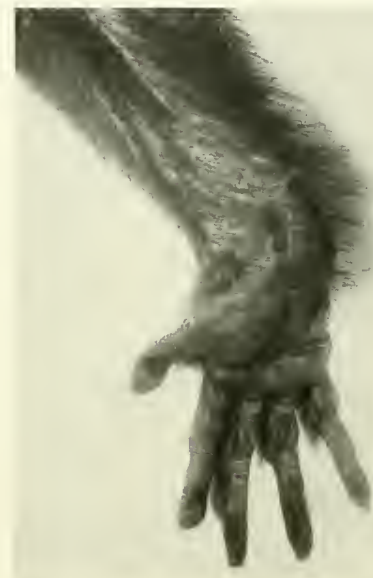

FIGS. IOI AND 102

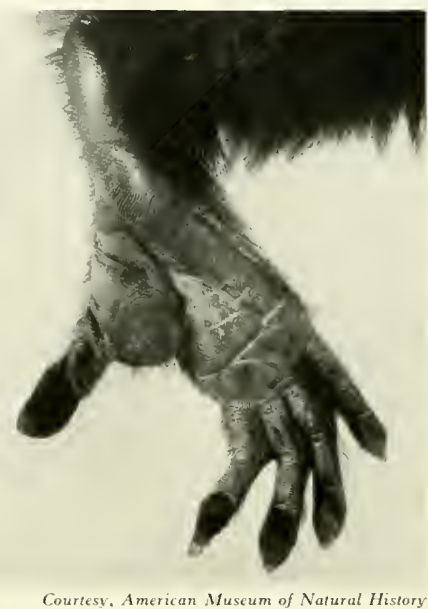

Courtesv, American Museum of Natural History

LEFr. Palmar surface of hand, showing digitation, well-developed balls of fingers and long, opposable thumb. Rıghr. Plantar surface of foot showing fairly well-developed heel and narrow sole, long finger-like toes, with long, prehensile hallux.

certain signals designed to set in motion definite lines of action any more productive of evidence. In experiments under the influence of human tuition the problem was can the monkey learn and does he commonly learn and do things, not by mere selection of the act from amongst the acts done by him, but by getting some idea and then himself providing the act because it is associated in his mind with that idea? As the result of these tests, the conclusion was a negative one. The records which were carefully made show no signs of any influence of tuition to which the animal was subjected. The systematic experiments designed to detect the presence of the ability to learn from 
human beings were almost unanimous against this possibility. Theoretically, it is likely that monkeys learn more from watching each other than from watching human beings. Professor Thorndyke's observations in this

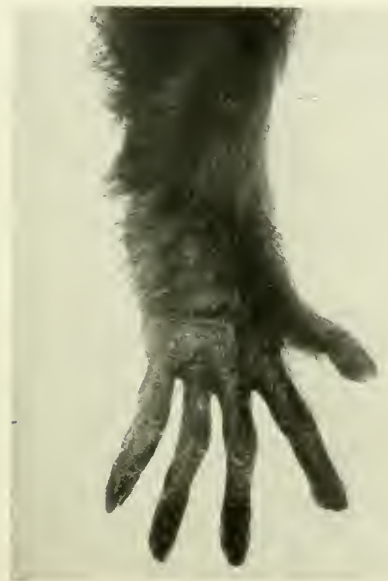

FIGS. IO3 AND IO4. HAND AND FOOT OF MYCETES SENICULUS.

LEFT. Dorsum of hand showing well-marked fingers and finger-nails.

Right. Dorsum of foot showing well-developed hallux, long finger-like toes and prominent toe-nails.

connection were somewhat limited. They do not seem to favor the hypothesis that these monkeys have any general ability to learn to do things by seeing them done by others, even of their own kind. This question is still to some extent an open one, requiring much more extensive study than it has yet received.

Concerning the general mental development of monkeys, Thorndyke believes that they represent a certain advance from the generalized mammalian type toward man. This is particularly true of their sensory equipment and their localized vision. Their motor equipment provides for the coor- 
dinated movements of the eye and the hand. In their method of learning, associative processes are quicker in formation of associations and there is a greater number of such associations as well as greater delicacy, complexity
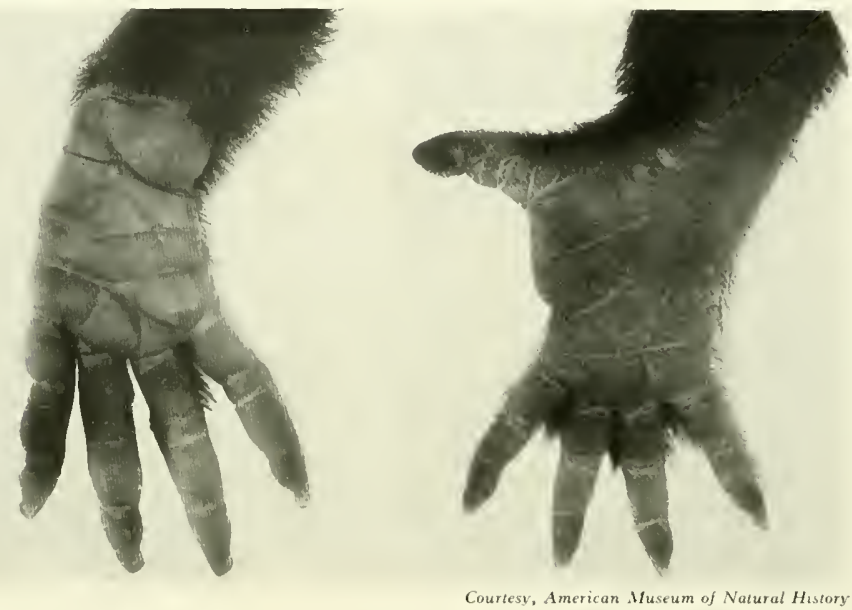

FIGS. IOj AND IO6. HAND AND FOOT OF SPIDER MONKEY.

LEFT. Palmar surface of hand showing well-developed palm, marked digitation with complete absence of the thumb.

Right. Plantar surface of the foot showing long sole, small heel, short opposable hallux and long toes.

and permanency in their representation. Yet, in spite of this increase as compared with lower mammals, these associations fail in their full significance as utilizable behavioral components, probably because they lack close interassociation. Thorndyke feels that there is nothing surprising in the comparative absence of free jdeas in these monkeys. The only demonstrable inteflectual advance of the monkeys over the mammals in general is the change from a few, narrowly confined, practical associations to a far greater assortment of them. This fact may turn out to be at the bottom of the 
only demonstrable advance in man. It is in reality an advance due to the brain acting with increased delicacy, bringing in its train the functions which distinguish human mental faculty from that of all other animals.
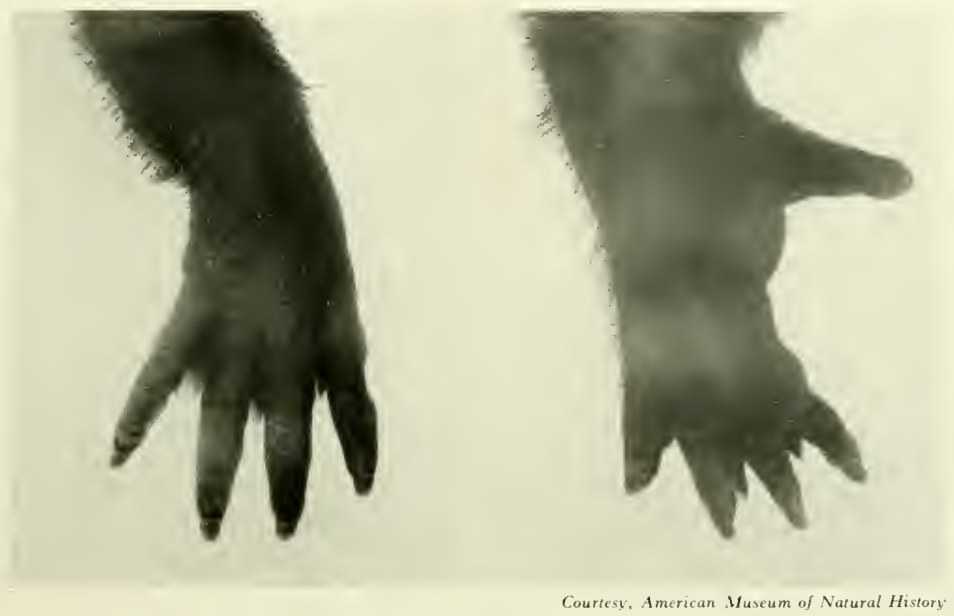

FIGS. IO7 AND IO8. HAND AND FOOT OF SPIDER MONKEY.

LEFT. Dorsum of hand, showing well-developed fingers and finger-nails, absenee of the thumb.

Right. Dorsum of the foot showing long metatarsus, long toes, opposable hallux, well marked toe-nails.

Brain Measurements and Indices in Mycetes Seniculus Diameters of the skull

Occipito-nasal. .................... $-8 \mathrm{~mm}$.

Bitemporal. . . . . . . . . . . . . . $t^{2} \mathrm{~mm}$.

Length of the brain case. ..............

Brain, including cerebellum and brain stem

Longitudinal. . . . . . . . . . . . . . . $; 2 \mathrm{~mm}$.

Transverse...................... to $\mathrm{mm}$. 
Total weight of the brain............... 24.; gms.

Water displacement of the brain.......... 24.5 c.c.

IV eight of the forebrain. ................ 20 gms.

Weight of the midbrain. ............... I gm.

Weight of the hindbrain.............. 3.j gms.

On the basis of these figures, the following indices were computed for the several divisions of the brain:

Forebrain index 8ı.6 per cent

Midbrain index 4.8 per cent

Hindbrain index I 3.6 per cent

These indices definitely place the animal in the manual group.

Surface Appearance of the Brain in Mrcetes Seniculus

THE FISSURAL PATTERN

The surface of the hemispheres of the brain is gyrencephalic with the most marked fissuring in the parietal, temporal and occipital regions. The frontal lobe has but few sulci. The fissure of Sylvius is a prominent landmark upon the lateral surface. Below and parallel to it is the superior temporal fissure, separating an inferior from a superior temporal convolution. Several interrupted sulci in the parietal region occupy the position of the interparietal fissure, while a small sulcus in the usual position of the fissura semilunaris separates the occipital from the parietal lobe. A short precentral sulcus extends horizontally from the sagittal line toward the fissure of Sylvius. The ventral extremity of this fissure falls considerably short of the latter sulcus. While this fissure may be easily discerned as the frssure of Rolando, its perpendicular relation to the great longitudinal fissure is reminiscent of the fissura cruciata of lower mammals. One deep fissure is present in the rostral 

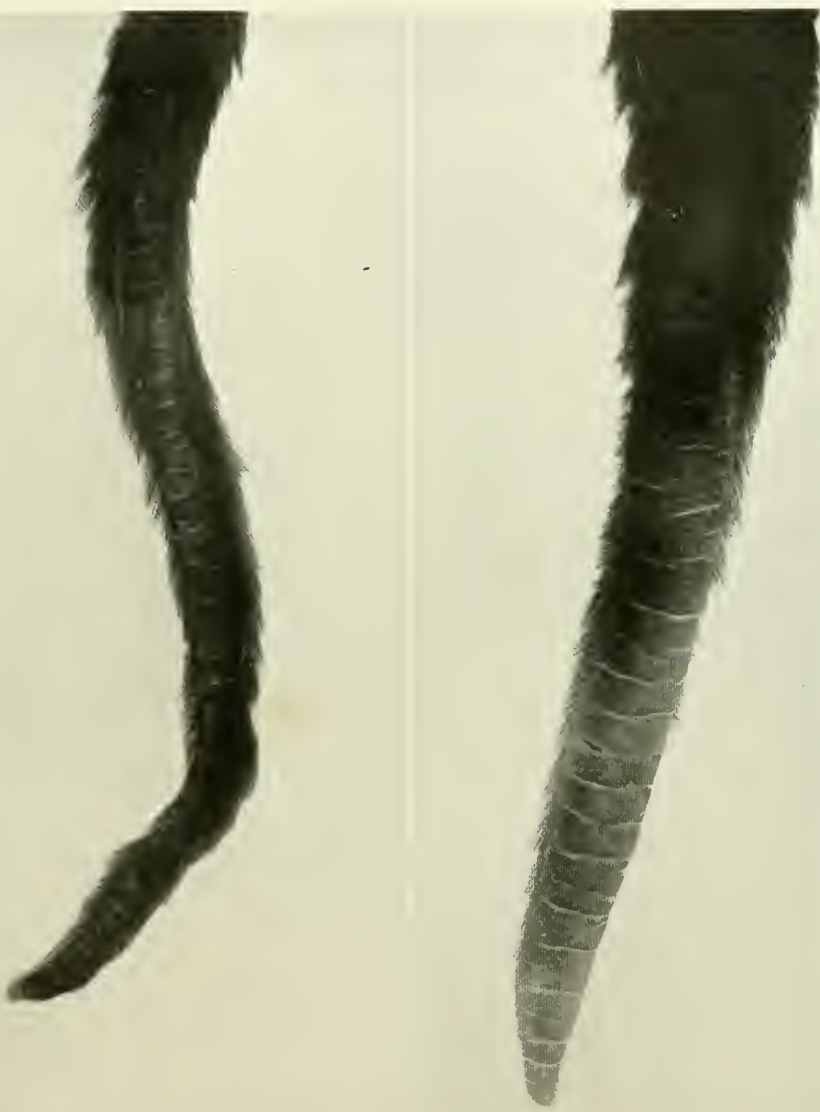

Courtesy, American Museum of Natural Hisiory

MYCETES SENICULUS, HOWLING MONKEY

ATELES ATER, SPIDER MONKEX

FIGS. IO9 AND I IO. DISTAL EXTREMITIES OF PREHENSILE TAILS.

The rugae in the tails indicate the high degree of sensory specialization in consequence of which these monkeys are able to employ the tail as a "fifth hand."

[203] 
extremity of the frontal lobe separating a small inferior frontal convolution from a large superior frontal convolution. Several indefinite, interrupted sulci are seen on the orbital surface of the frontal lobe. Upon the mesial surface
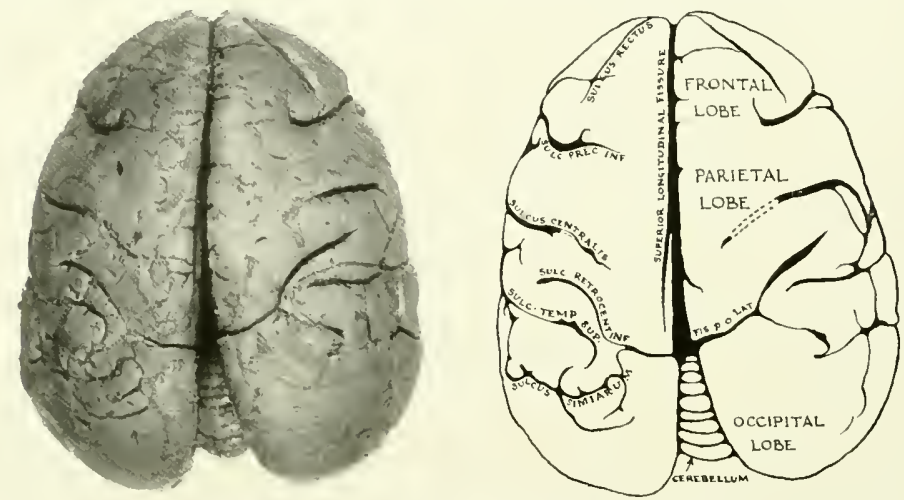

FIG. III. DORSAL SURFACE OF BRAIN, MYCETES SENICLLUS.

[Actual Length, +6 mm.]

Key to Dlagram. FIS. P. O. 1A]., Fissura Parietooccipitalis Lateralis; sulc. Prec. INF., Sulcus Precentralis Inferior; sulc. RetroceNt, INF., Sulcus Retro-centralis Inferior; Sulc. Jemp. SuP., Sulcus Temporalis Superior.

the indication of the general line and direction of the marginal sulcus is indicated by a series of interrupted fissures. The calcarine and collateral fissures are well defined, as is also the supracallosal fissure. The corpus callosum is larger than in marmoset and lemur. The splenium in particular is somewhat thicker than in the lower forms already considered.

The lateral appearance of the hemisphere in mycetes gives the impression of a marked advance as compared with lemur and marmoset. The brain is definitely gyreneephalic and its fissural patterns are rendered conspicuous by the appearance of the well-marked Sylvian sulcus which is no longer dominated by any suggestion of a circumsylvian arrangement of convolutions. Although there is no such marked development in the sulcus simiarum 
as appears in the more advanced primates, the inception of this fissure is seen in the occipital Iobe. The central fissure extends from the vertex of the hemisphere downward and forward toward the main Sylvian fissure in the
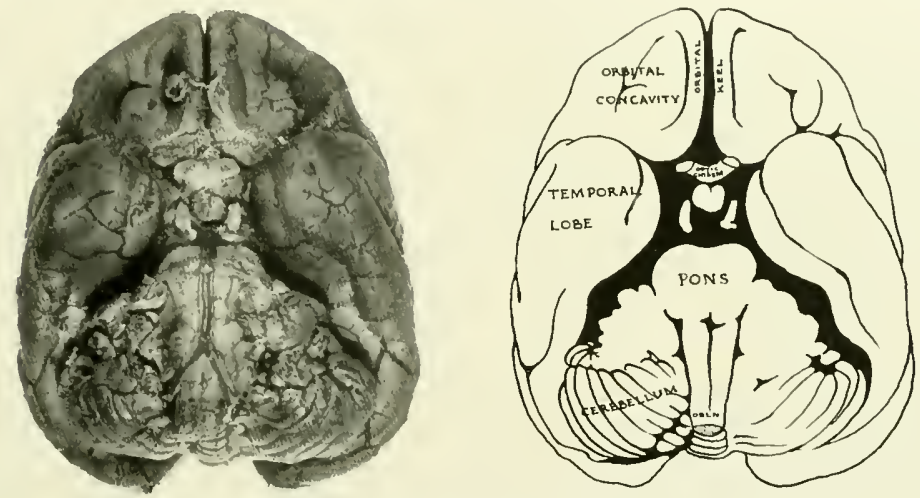

FIG. I12. BASE OF BRAIN, MICETES SENICULUS.

[Actual Length, $46 \mathrm{~mm}$.]

hey to Diagram. OBLn., Oblongata.

region between the parietal and frontal areas. Its angulation with the superior longitudinal sinus is something less than $90^{\circ}$. Likewise, the angle of the Sylvian suleus with the base line of the brain is a little less than $45^{\circ}$, showing a general disposition on the part of this fissure to depart from its primitive vertical position and incline itself more toward the horizontal.

\section{LOBATION OF MYCETES' BRAIN}

The lobation in the mycetes' brain is much more distinct than in the primates below it. This is due to the fact that the major sulci are all well defined. A short fissure indicates the position of the precentral sulcus in the frontal area, and deep fissures situated well forward toward the orbital surface inclicate the positions of the sulcus precentralis inferior and the sulcus 
frontomarginalis. The parictal lobe is well demarcated, its caudal boundary being established by the inception of the sulcus simiarum. The occipital lobe as a whole shows consickerably more expansion than in the case of lemur or
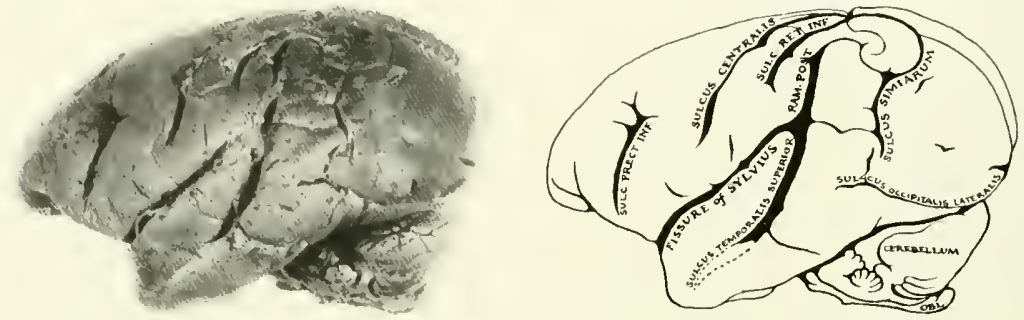

FIG. I I3. LEFT LATERAL SURFACE OF BRAIN, MYETES SENICULUS.

[Actual Length, $52 \mathrm{~mm}$.]

Key to Dlagram. obl., Oblongata; ram. post., Ramus Posterior of Superior Temporal Sulcus; sulc. PRECT. INF., Sulcus Precentralis Inferior; SUдc. RET. INF., Sulcus Retrocentralis Inferior.

marmoset. The temporal lobe, perhaps more than any other portion of the Iateral surface, shows a tendency toward that progressive advance which eventuates in the fully developed characters of this region in the primate brain.

THE BASAL SURFACE OF THE HEMISPHERE AND THE OCCIPITAL CONCAVITY

On the basal surface of the hemisphere, the two orbital concavities are well defmed, as are also the interorbital keels. The olfactory bulb and tract show a considerable decline in prominence and are detachable as far back as the trigonum olfactorium. The lateral root of the olfactory tract is much less prominent than in either the marmoset or the lemur, indicating in a general way the tendency toward deflorescence in the development of the olfactory central mechanism.

The occipital concavity is pronounced in mycetes due both to the expansion of the lateral lobes of the cercbellum and the further expansion of the 
occipital lobe of the hemisphere itself. This concavity is deepest in the midline where it appears as the postsplenial fossa for the accommodation of the protuberant superior vermis of the cerebellum.
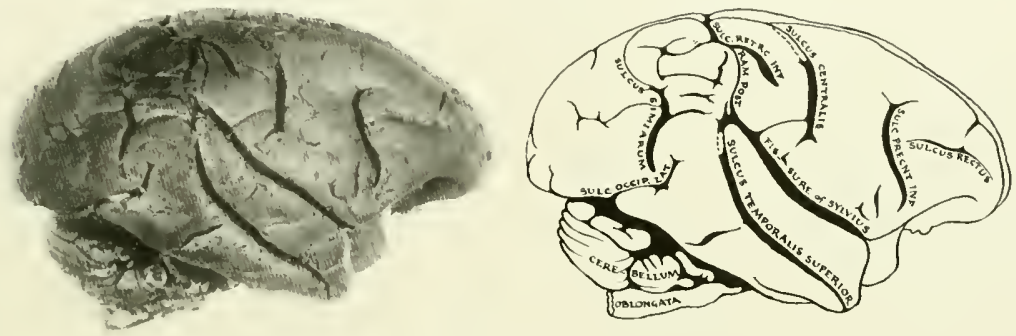

FIG. I I 4. RIGHT LATERAL SURFACE OF BRAIN, MYCETES SENICULUS.

[Actual Length, $52 \mathrm{~mm}$.]

Key to Diagram. rail. Post., Ramus Posterior of Superior Temporal Sulcus; sulc. Occip. Lat., Sulcus Occipitalis Lateralis; sulc. PRECNT. INF., Sulcus Precentralis Inferior; sulc. Retrc. inf., Sulcus Retrocentralis Inferior.

\section{THE CEREBELLUM}

In the cerebellum, certain advances are prominent, consequent primarily upon the pronounced expansion of the lateral lobes. The tentorial surface is entirely overhung by the occipital lobes. It is gabled from its lateral extremity toward the median ridge-pole formed by the vermis cerebelli. The interfolial sulci pass without interruption from the vermis to the Iateral lobes. On the occipital surface the expansion of the hemispheres of the cerebellum is an even more conspicuous feature. The inferior vermis still occupies a prominent position on this surface but its proportions are recluced to about a sixth of the entire expanse of this region. In the lower forms the vermis constitutes a third of this area. Two decp paramedian sulci interrupt the passage of the interfolial fissures from the vermis to the lateral lobes. A feature of much importance in connection with the petroso-ventricular surface of the cerebellum in mycetes is the extreme development of the flocculus. 
The flocculus in these monkeys, as well as in Ateles and Lagothrix, all species in which the prehensile tail has become highly specialized, reaches a higher point of development than in any other primates. Whether this prominence
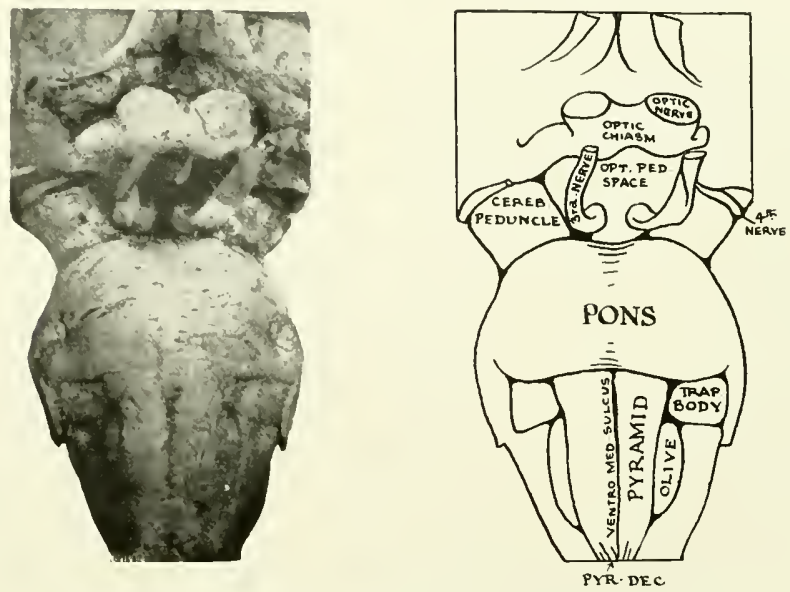

FIG. I I j. VENTRAL SURFACE OF BRAIN STEM, MYCETES SENICULUS. [Actual Length, $34 \mathrm{~mm}$.]

Key to Diagran. Cereb. Peduncte, Cerebral Peduncle; opt. PEd. SPace, Opticopeduncular Space; pyr. DEC., Pyramidal Decussation; trap. Bodr, Trapezoid Body; ventro. Med. sulc, Ventromedian Sulcus.

of the flocculus is due, as has been thought to be the case by several authorities, to the additional responsibilities imposed upon the coordinative mechanism in response to the prehensile tail is a question needing further investigation.

\section{THE BRAIN STEM}

The surface markings of the brain stem in mycetes are more pronounced than in lemur or marmoset, atthough in only a few instances do they attain the prominence observed in the higher apes. The oblongata upon its ventral 
surface presents a ventromesial sulcus which is interrupted at its caudal extremity by the decussating pyramidal fibers. Upon either side of this sulcus are two well-defined pyramids indicating an animal having a wider
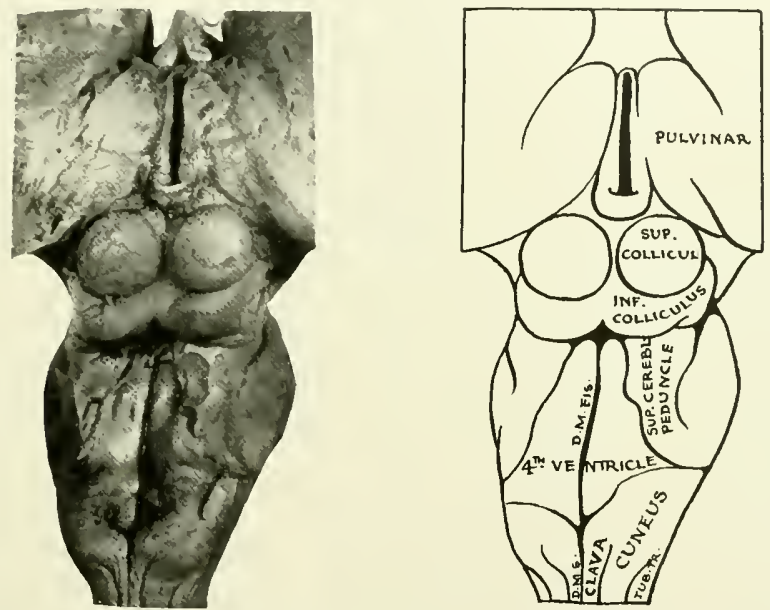

FIG. I 16. DORSAL SURFACE OF BRAIN STEM, MYCETES SENICULUS. [Actual Length, $34 \mathrm{~mm}$.]

Key to Diagran. D. M. FIS., Dorsomedian Fissure; D.M.s., Dorsomedian Sulcus; INF. Colinculus, Inferior Colliculus; sup. Cerebl. peduncle, Superior Cerebellar Peduncle; sup. Collitcul.., Superior Collicultus; тUв. TR., Tuberculum Trigemini.

range of volitional control than is true of either lemur or marmoset. Lateral to each pyramid is a small olivary eminence.

The clorsal surface is characterized by the presence of the two enlargements representing the columns of Goll and Burdach. While both of these structures are prominent, the column of Goll is slightly larger than the column of Burdach. The increment in the column of Goll reflects the appearance of a highly developed prehensile tail which adds what some authorities 
speak of as a fifth hand to the animal's motor and sensory equipment. Although the prehensile adaptability of the tail in mycetes is hardly to be compared with that of the woolly monkey (Lagothrix) or the spidermonkey (Ateles), the caudal appendage of the howling monkey is an important organ which the animal uses with great deftness and skill. The increment in the column of Burdach is also significant. It reflects a further differentiation of the hand with the consequent development of new motor capacities as well as aequisitions in manual dexterity and precision. In proportion as the forelimb has emancipated itself from its limitations as a locomotor organ, it has expanded its potentialities in mastery of the environment and has added immeasurably to the upbuilding of new behavioral reactions.

One feature of the brain stem which differentiates myectes from lemur and marmoset is the size of the cerebellum.

The pons Varolii is also considerably larger than in the lower forms. The inference from the size of the cercbellum in conjunction with that of the pons seems to be that mycetes possesses a fairly wide range of acquired skilled movements and employs the forelimbs for other purposes than those of locomotion.

\section{DORSAL ASPECT OF THE MIDBRAIN}

The dorsal aspect of the midbrain presents two well-defined sets of collicular eminences whose prominence suggests the persistence of certain visual and anditory functions primordially vested in the mesencephalon.

\section{Internal Structure of the Brain Stem in Mycetes Seniculus}

The internal structure of the brain stem in mycetes gives the impression that all of the structures thus far recognized as criteria in estimating behavioral reactions stand out with a clearness of definition not observed in either the marmoset or the lemur. 
LEVEL OF THE PYRAMIDAL DECUSSATION (IIG. IIT)

At the level of the pyramidal decussation the chief feature is the crossing of the pyramidal bundles and the effect which these crossing fibers have upon

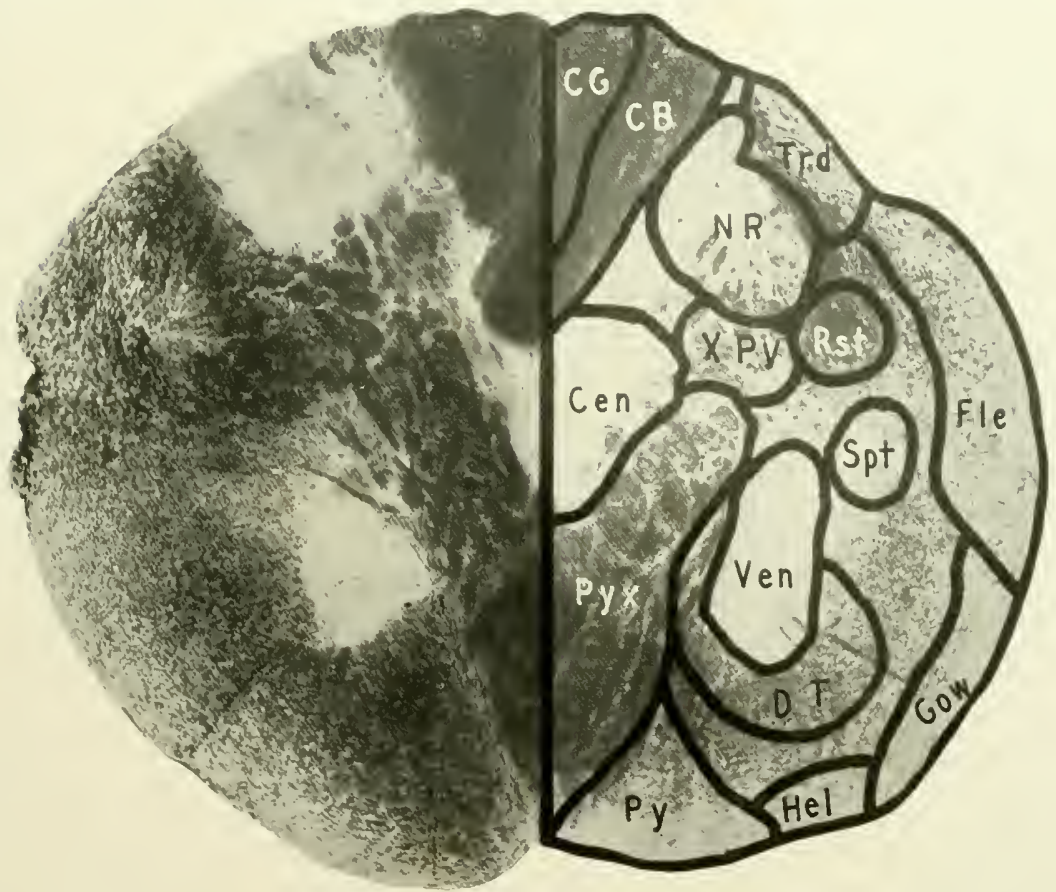

FIG. 117. MCETES SENICULUS. LEVEL OF THE PYRAMIDAL DECUSSATION. Св, Column of Burdach; CEN, Central Gray Matter; CG, Column of Goll; DT, Dciterso-spinal Tract; FLE, Dorsal Spinocerebellar Tract; gow, Ventral Spinoccrebellar Tract; Her, Spino-olivary Tract of Helweg; NR, Nucleus of Rolando; Py, Pyramid; prx, Pyramidal Decussation; RST, Rubrospinal Tract; spt, Spinothalamic Tract; TRD, Descending Trigeminal Tract; ven, Ventral Gray Column; xpy, Crossed Pyramidal Tract. [Accession No. 1 48 . Section 15. Actual size $8 \times 7 \mathrm{~mm}$.]

the arrangement of the gray matter. The decussation is shown at Pyx. Its final accomplishment and the formation of a crossed pyramidal tract about 
to pass into the spinal cord is indicated at XPy, while the as yet uncrossed portion of the pyramid is shown at $\mathrm{Py}$. Compared with the lower forms, both the pyramid and the bundles constituting its decussation are larger in relation to the rest of the cross section. This increase in size of the pyramidal system is significant of accessions to volitional control over the muscles, more particularly the muscles of the upper extremities.

The importance of this accession is noteworthy since this animal, when compared with those already discussed, has developed a highly specialized hand. Even in this particular, however, it has some of the defects characteristic of the higher anthropoid apes, especially in the relative shortness of the thumb when compared with the other digits. Nevertheless, because the thumb is opposable, it gives a defmitely new capacity to the upper extremity as a grasping organ and thus induces a train of consequences with far-reaching influences. The hand now becomes an instrument for analyzing elements in the environment. It assumes new activities in grasping and manipulating objects, and finally in submitting them to closer visual scrutiny. The manual development, because it adds to the equipment of these animals by providing a more efficient means for exploring their surroundings, inaugurates the processes necessary to the eventual psychological differentiation between what is intrinsically part of the animal and what is definitely external to it. Thus an important step in constructing the psychic clements which distinguish between self and extra-self is established. The large size of the pyramidal decussation indicates an increment in volitional control especially demanded to meet the new motor possibilities of a well-dereloped hand.

Quite as much does the expansion of a dorsal sensory ficld denote functional increments in the sphere of sensibility in consequence of manual and caudal development. The additions in the column of Goll (CG) represent sensory expansions mainly due to the prehensile tail which, if it has not attained in mycetes the degree of functional differentiation warranting the 
designation of a fifth hand, as in Ateles, none the less has become an organ possessed of delicate sensory discrimination. It is probable that the acquisition of such a prehensile tail is chiefly accountable for expansion in the column of Goll since no further provocative specialization has occurred in the hind leg or foot.

One particular development in connection with the nucleus of Goll is the median unpaired nucleus of Bischoff which is situated at the caudal extremity of the dorsal sensory nuclei in juxtaposition to the dorsal median septum. This nucleus was first described by Bischoff in I 899 . It develops according to Zechandelaar in animals possessed of tails used prehensilely, as in the spider monkeys, or as supporting organs in the kangaroos, or as in the Cetacea acting as propelling organs. Its presence in mycetes indicates the high degree of development of the prehensile tail in this animal.

Even more striking, however, is the expansion of the column of Burdach (CB) at this level, for which but one interpretation scems reasonable, namely, the appearance of a highly differentiated hand. The degree of discriminative sensibility now vested in the cutaneous and subcutaneous structures of this hand provides the ability to estimate the consistency of objects, to detect differences in their size and shape, texture and temperature, moisture and dryness, as well as other physical qualities which may be appreciated by manual contact. To these sensory discriminations are added others equally important because they take their significance from the sensory impressions created by new ranges of motion. These new possibilities suggest that there has evolved from the simple and but little differentiated forelimb originally specialized for locomotion, an organ so highly modified that it may almost be considered new. New also are the avenues of contact with life which the hand has created, adding immeasurably to the stream of behavioral reactions of which the animal is capable. 
It is difficult to estimate all of the far-reaching consequences of this progressive differentiation of the upper extremity. The advent of the hand not only brings into existence an acquisitive explorer in the environment, but, by releasing the forelimbs from the responsibility of locomotion, it influences profoundly both the posture and the method of locomotion itself. In such a capacity it dictates new tendencies in the selection of habitat. It provides new means of defense and offense and thus may affect the matter of food supply and metabolism. It becomes an instrument of investigation and contrivanee, the creator of a wide range of gestures and hence of symbols, and finally through its agency as a means of communication, leads on to the vocal accompaniments which ultimately eventuate in verbal speech. Having such importance in the synthesis of reactions which characterize the complex output of behavioral performances in the highest form, the significance of this dorsal sensory field in the oblongata cannot be orerestimated. There may be certain difficulties, perhaps, in maintaining that the progressive differentiation of the hand has been one of the chief factors in the later expansions of consciousness. Yet it must be clear that the animal possessed of such a discriminating organ requires more extensive sensory areas in the brain than the animal not similarly equipped. The increase of these sensory areas which represent the acquisitions of manual discrimination cannot fail to have a widespread influence by amplifying the sensory syntheses which enter into consciousness. The growing importance of the hand as a sensory organ is witnessed by another interesting fact. The portion of the dorsal sensory field in mycetes allotted to the transmission of sensory impulses from the head and face is not proportionally large when compared with the areas for receiving impressions from the extremities. Both the substantia gelatinosa and the descending trigeminal tract ( $\mathrm{Trd}$ ) are relatively smaller than in lemur or marmoset. The apparent reason for this relative decrease in prominence may be sought in the fact that the face and head have lost some of their 
primitive responsibility in the direction of loconotion. This function, in part at least, is now delegated to the more effectively organized receptive organ, the hand.

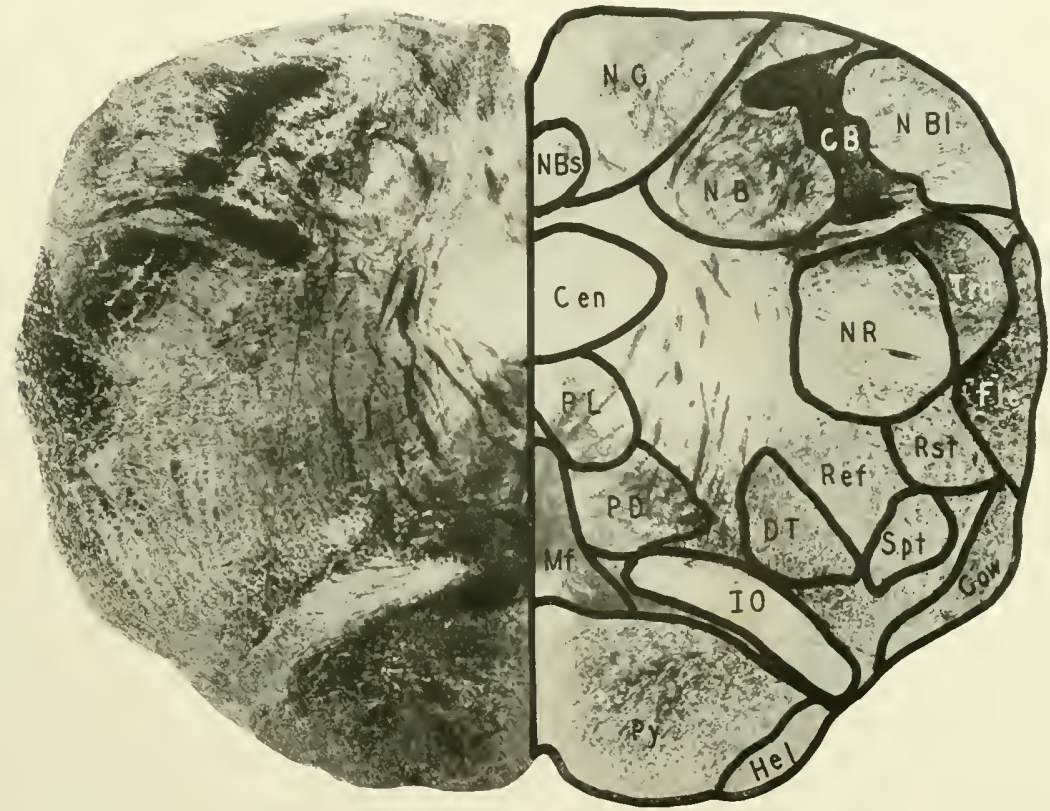

FIG. II 8. MYCETES SENiCulus. LEVEl. OF THE CAUDAl EXTREMITY OF THE INFERIOR OLIVE.

CB, Column of Burdach; CFN, Central Gray Matter; DT, Deiterso-spinal Tract; F-LE, Dursal Spinocerebellar Tract; Gow, Ventral Spinocerebellar Tract; HeL, Spino-olivary Tract of Hlelweg; Io, Inferior Olive; MF, Mesial Fillet; NB, Nucleus of Burdach; NBs, Nucleus of Bischoff; NBL, Nucleus of Bímenau; NG, Nucleus of Goll; NR, Nucleus of Rolando; PL, Posterior Longitudinal Fasciculus; PD, Predorsal Bundle; Pr, Pyramid; REF, Reticular Formation; RST, Rubrospinal Tract; SPT, Spinothalamic Tract; TRD, Descending Trigeminal Tract. [Accession No. 148. Section 85. Actual Size $1 \mathrm{I} \times 7 \mathrm{~mm}$.]

LEVEL OF THE CAUDAL EXTREMITI OF THE INFERIOR OLIVE (FIG. I I8)

At the level of the caudal extremity of the inferior olive, a clearer impression is obtained of the relative size of the pyramidal system (Py). There 
is evidently a defnite accession in motor control, particularly in the regulation of the movements in the upper extremity. The structures in the dorsal sensory field have occasioned a general broadening in the diameters in this region and also show an increase both in fiber richness and extensiveness of the nuclear collections. The nucleus of Goll (NG) appears to be of about the same size as the nucleus of Burdach (NB). The increase in the latter element indicates the addition of a highly dereloped hand. A corresponding increase in the mesial fillet (Mf) indicates inerements in the function of discriminative sensibility already made apparent by expansions in the dorsal columns of Goll and Burdach. A feature of much importance is the inferior olivary body (IO) whose caudal extremity is seen in this section. Here, as elsewhere in the brain stem of mycetes, the olivary body fails to show that clearcut delimitation characteristic of those forms in which the structure attains its highest differentiation. Since the inferior olive is functionally active in the coordination of simultaneous movements in head, eyes and forearm, and sinee it facilitates the coordination of all skilled learned performances, it should be more highly specialized in this species than in the lemur or marmoset. Differentiation of the olive depends upon the extent to which cooperative movements of the eyes, head and hand are coordinated. Such morements manifest an increase of effectiveness in proportion as the animal is able, by means of the hand, to bring objects into closer scrutiny by the eyes. This ocular, cephatic and brachial adjustment is also proportional to the degree of accuracy with which head and ye movements are capable of following manipulations of the hand.

The substantia gelatinosa of Rolando (NR) and its accompanying descending tract of the fifth nerve ( $\mathrm{Trd}$ ) are prominent features at this level although comparatively smaller than in lenur or marmoset. The significance of this apparent decrease in the sensory representation of the head 
and face has already been identified as a concomitant development dependent upon the increase in functional capacity of the hand for directing locomotion.

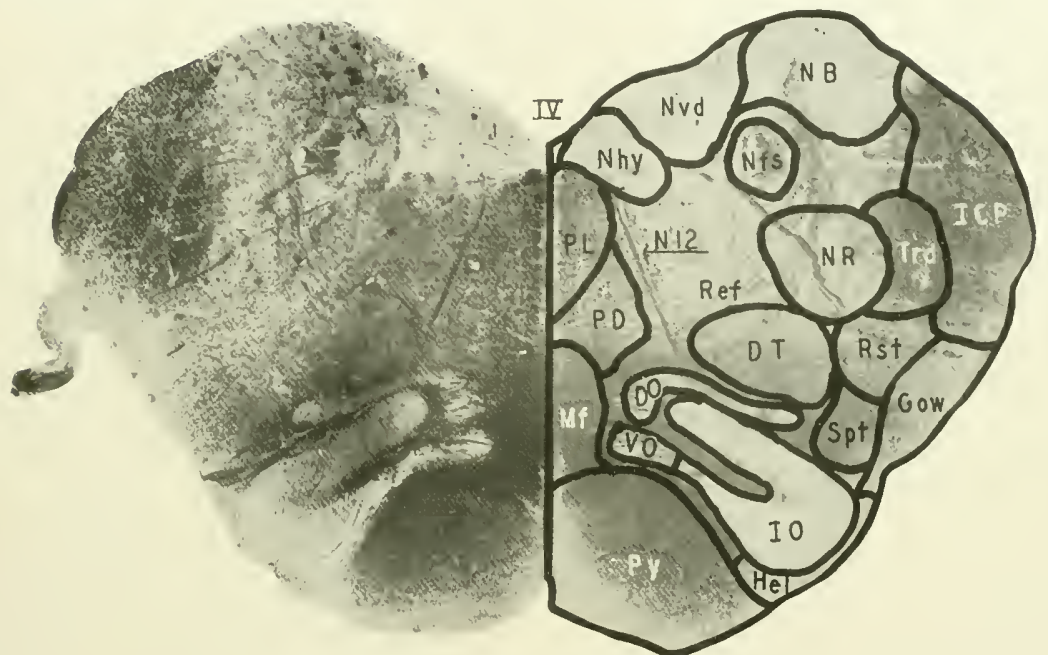

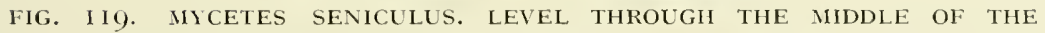
INFERIOR OLIVE.

Do, Dorsal Olive; DT, Dciterso-spinal Tract; Gow, Ventral Spinocerebellar Tract; 11EL, Spino-olivary Tract of Helweg; ICP, Inferior Cerebellar Peduncle; IO, Inferior Olive; MF, Nesial Fillet; NB, Nucleus of Burdach; NFS, Facial Nucleus; NHr, Nucleus Hypoglossus; nr, Nucleus of Rolando; Nvd, Dorsal Vagal Nucleus; NI 2, Hypoglossal Nerve; PD, Predorsal Bundle; PL, Posterior Longitudinal Fasciculus; Py, Pyramid; Ref, Reticular Formation; RST, Rubrospinal Tract; SPT, Spinothalamic Tract; TRD, Descending Trigeminal Tract; vo, Ventral Accessory Olive; 1v, Fourth Ventricle. [Accession No. 148. Section 145. Actual Size $13 \times 6 \mathrm{~mm}$.]

LEVEL THROUGH THE MIDDLE OF THE INFERIOR OLIVE (FIG. I I9)

At the level through the middle of the inferior olive ( $\mathrm{IO}$ ) it is evident that the unfolding which has occurred in the olivary body is considerable. Not only is there an increase in size, but there is also a tendency for this structure to assume the characteristic outline which identifies it in the higher species. It has, however, little of the convoluted appearance so prominent in the larger simians. It is now possible to recognize the main olive and several 
accessory portions, i.e., the rentral accesson olive (VO) and the dorsal accessom olive (DO). The significance of this increase both in definition and size of the olivary body may be understood in relation to the animal's

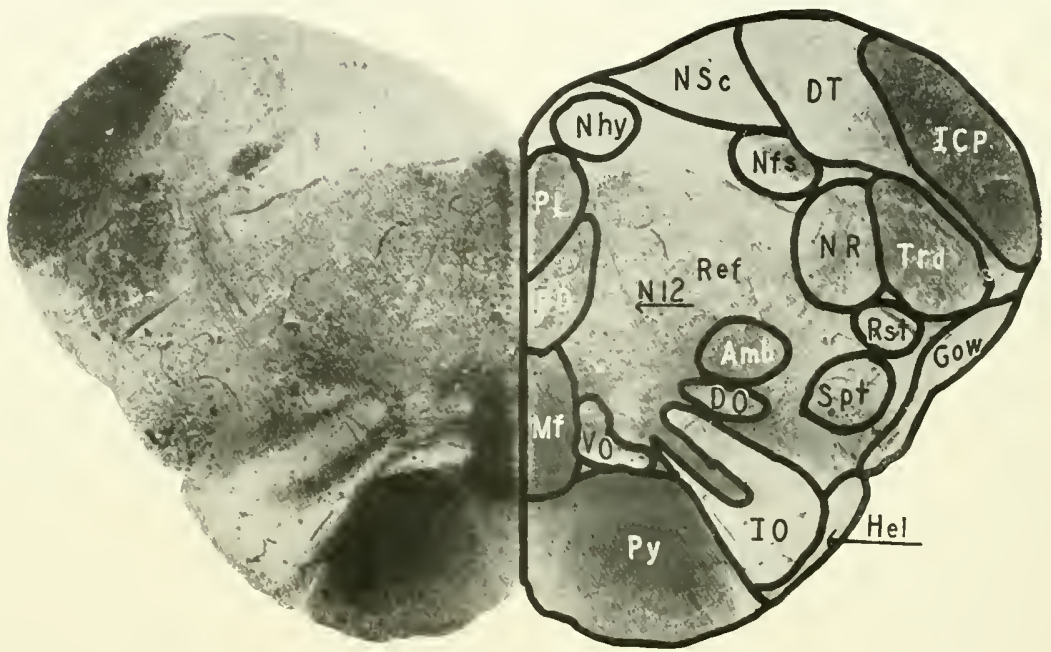

FIG. 120. MYCETES SENICULUS. LEVEL OF THE NUCLEUS AMBIGULS.

Aмв, Nuclcus Ambiguus; DT, Deiters' Nucleus; Do, Dorsal Accessory Olive; Gow, Dorsal Spinoccrebellar Tract; HeL, Spino-olivary Tract of Helweg; ICP, Inferior Cerebellar Peduncle; 10, Inferior Olive; MF, MesiaI Fillet; NHY, Ilypoglossal Nucleus; NFs, Fascicultu Solitarius and Nucleus; Nsc, Nucleus of Schwalbe; Nr, Nucleus of Rolando; N12, Hypoglossal Nerve; PL, Posterior Longitudinal Fasciculus; PD, Predorsal Fasciculus; PY, Pyramid; REF, Reticular Formation; RST, Rubrospinal Tract; SPT, Spinothalamic Tract; TRD, Descending Trigeminal Tract; vo, Ventral Accessory Olive. [Accession No. 148. Section 155. Actual Size $13 \times 6 \mathrm{~mm}$.

need of more accurate coordination in the simultaneous movements of eyes, head and hands for the new motor attainments acquired in consequence of manual development.

LEVEL OF THE NUCLEUS SUPPLYING MOTOR FIBERS TO THE IARYNX (FIG. I 2O)

At this level, a noteworthy specialization appears in the more fixed portion of the brain stem which throughout the primate series, generally speak- 
ing, shows little or no adaptive modification. In the case of the howling monkey, however, there is a striking degree of prominence in the development of those nuclei which have control of the larynx and hence regulate voice production. It is perhaps not surprising to find the nucleus ambiguus ( $\mathrm{Amb}$ ) as well as the dorsal vagal nucleus, which are related to motor and sensory control of the larynx, highly developed in these animals. One of the characteristic physiological features of mycetes is the terrifying sound which they produce by their vocal organs and from which they have earned their distinguishing cognomen, howling monkeys.

LEVEL OF THE VESTIBLLAR AND CEREBELLAR NiLLEI (FIGS. 121 AND 122 )

Here at the level of the vestibular and cerebellar nuclei there are indications of the extent to which the balancing and coordinating mechanisms have developed. The vestibular nuclei, comprising the triangular nucleus of Schwalbe (NSc) and the nucleus of Deiters (ND), occupy a position in relation with the floor of the fourth ventricle; while the cerebellar nuclei, which consist of the nucleus dentatus $(\mathrm{Ndt})$ and the nucleus fastigii ( $\mathrm{Nfg}$ ), are situated in the medullary substance of the vermal portion of the cerebellum. The vestibular nuclei are important receiving centers of the balancing mechanism and this, to a certain extent, is also true of the nucleus fastigii, which is connected with the vestibular mechanism by means of the juxtarestiform body. The dentate nucleus, on the other hand, has quite a different significance, being an index of the degree of efferent conduction provided for the cerebellar impulses. The major outflow from the cerebellum is conveyed by axons which take their origin in the dentate nucleus and constitute the superior cerebellar peduncle. Thus, the nucleus dentatus offers a means of estimating the relative amount of coordinative influence which the cerebellum is capable of contributing to the muscles. 
In regard to this nucleus in mycetes, it is important to note that the structure is not only more extensive in its general dimensions, but also begins to manifest, even if in a somewhat ill-defmed manner, that tendency toward

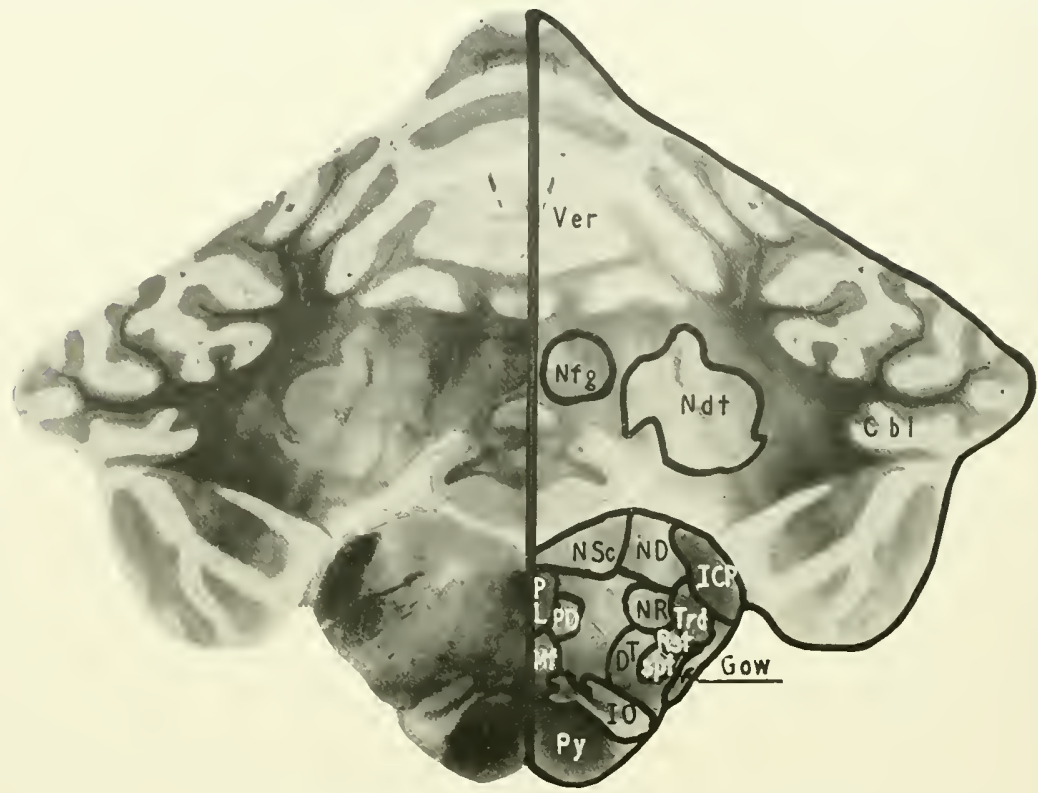

Fig. I 21. MYCETES SENICULUS. LEVEL OF THE CEREBELLAR NUClei.

CBL, Lateral Lobe of Cercbellum; DT, Deitersal Tract; Gow; Ventral Spinocerebellar Tract; ICP, Inferior Cercbellar Peduncle; 10, Inferior Olive; MF, Mcsial Fillet; ND, Deiters' Nucleus; NDT, Dentate Nucleus; NFG, Nucleus Fastigii; NR, Nucleus of Rolando; nsc, Nucleus of Schwalbe; PD, Predorsal Bundle; PL, Posterior LongitudinaI Fasciculus; pY, Pyramid; RST, Rubrospinal Tract; SPT, Spinothalamic Tract; TRD, Descending Trigeminal Tract; VER, Vermis of Cerebellum. [Accession No. I 48 . Section I6o. Actual Size $13 \times 9 \mathrm{~mm}$.]

convolution of its surfaces typical of the higher primates. It may be inferred, therefore, that the degree of coordination which the cerebellum furnishes to the somatic muscles is greater in mycetes than in lemur or marmoset. The necessity of such coordination becomes clear in the light of the increased 
complexity in motion resulting from a greater volitional control of the upper extremities. Not alone is this increment in coordination essential to the newer movements of the hand, but now there is the further need of coordina-

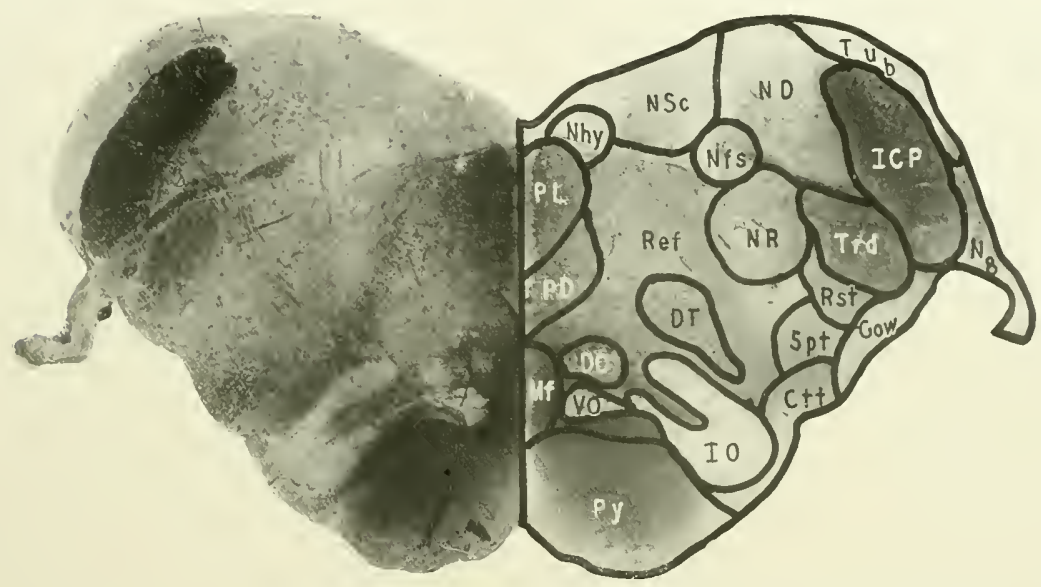

FIG. I 22. MYCETES SENICULUS. LEVEL OF THE VESTIBLLAR NLCLEI.

CтT, Central Tegmental Tract; DT, Deiterso-spinal Tract; Do, Dorsal Accessory Olive; cow, Ventral Spinocerebellar Tract; ICP, Inferior Cerebellar Peduncle; Io, Inferior Olive; MF, Mesial Fillet; ND, Deiters' Nucleus; NFs, Facial Nucleus; Nsc, Nucleus of Schwalbe; N8, Auditory Nerve; NR, Nucleus of Rolando; NHY, Nucleus Hypoglossus; PD, Predorsal Bundle; PL, Posterior Longitudinal Fasciculus; Pr; Pyramid; Ref, Reticular Formation; RST, Rubrospinal Tract; SPT, Spinothalamic Tract; TRD, Descending Trigeminal Tract; TUB, Tuberculum Acusticum; vo, Ventral Accessory Olive. [Accession No. 148. Section 165. Actual Size, $13 \times 5 \mathrm{~mm}$.]

tion arising from the fact that the animal depends more upon the hindlimbs in locomotion than do the lower forms. This requirement applies likewise to the partial attainment of the upright posture as well as the animal's tendency to sit upon its haunches and to balance itself in this position.

All of these factors demand a more extensive development of coordinative as well as equilibratory control, and this control in its more remote secondary effects has aided in the development of the hand. 
LEVEL OF THE EMERGENCE OF THE SIXTH NERIE (FIG. 123)

A remarkable feature at this level of the brain stem in mycetes is the appearance in its entire course of the emergent root of the abducens nerve.

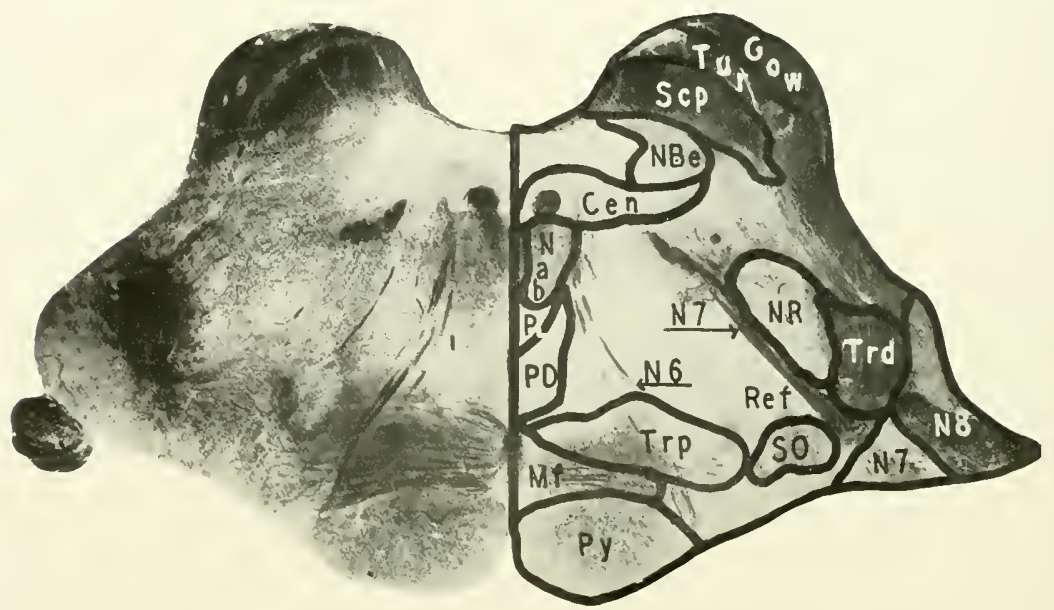

FIG. I 23. MYCETES SENICULUS. LEVEL, OF EMERGENCE OF SIXTH NERVE.

C.EN, Central Gray Matter; Gow, Ventral Spinocerebellar Tract; we, Mesial Fillet; NAB, Abducens Nucleus; NBE, Nucleus of Bechterew; NR, Nucleus of Rolando; N6, Abducens Nerve; N7, Facial Nerve; N8, Auditory Nerve; PD, Predorsal Bundle; PL, Posterior Longitudinal Fasciculus; Py, Pyramid; REF, Reticular Formation; scP, Superior Cerebellar Peduncle; so, Superior Olive; TRD, Descending Trigeminal Tract; TRP, Trapezoid Body; TUR, Tractus Uncinatus of Russel (Hlook Bundlc). [Accession No. I 8 . Section 230. Actual Size $18 \times 7 \mathrm{~mm}$.]

The origin of this nerve is seen in its nucleus ( $\mathrm{Nab}$ ), and its course dircetly ventrad through the trapezoid body $(\operatorname{Tr} \mathrm{p})$, the lateral portion of the mesial lillet ( $\mathrm{If}$ ), and its emergence lateral to the pyramid ( Py), are exceptional in cross sections of the primate stem. No fibers of the pons are as yet apparent at this stage. The central gray matter ( Cen) is a narrow zone, which in its mesial angle contains the sccond portion of the facial nerve $\left(N_{7}\right)$. Its lateral angle is continuous with the nucleus of Bechterew ( $\mathrm{NBe}$ ). 
Situated above this latter nucleus are three important bundles of fibers superimposed one above the other: the superior cerebellar peduncle $\left(S_{c p}\right)$, the fasciculus uncinatus of Russel ( Tur), and the ventral spinocerebellar tract (Gow). The trapezoid body (Trp) and mesial fillet (Mf) lie dorsal to the pyramid (Py). At the lateral apex of the corpus trapezoideum is the superior olive ( $\mathrm{SO}$ ). The emergence from the axis of the seventh nerve in its relation to the fibers of the eighth nerve is clearly seen ( $\mathrm{N}-$ and $N 8$ ).

LEVEL THROUGH THE MDDLE OF THE PONS VAROLII (FIG. I24)

At the level through the middle of the pons Varolii a clearer conception may be gained regarding the size and complexity of the pons. The stratum superficiale is a dense and relatively broad bundle of fibers making its way transversely into the middle cerebellar peduncle ( M c p). Dorsal to this is the stratum complexum containing the scattered bundles of the pyramidal system ( Py), some transverse fibers and the pontile nuclei ( PN). In the most dorsal position is a fairly wide zone constituting the stratum profundum, the transverse pontile fibers of which also enter the middle cerebellar peduncle.

Perhaps at no other level is it possible to obtain so comprehensive a view of the animal's motor capacity from structural indices. The size of the pons, including its transverse fibers and nuclear masses, indicates a degree of cortical expansion in the cerebral hemisphere much above that attained by lemur or marmoset. It is significant of an animal capable of considerable coordinative control, one which has developed the lateral lobes of the cerebellum more than is to be observed in Iower mammals. Because of this development it has acquired control over the upper extremity such as is commensurate only with animals possessing a more or less highly developed hand. Nor is all of the prominence attained by the pons Varolii directly ascribable to the increased demands in coordinative regulation of the upper extremity. These demands may be a primary incident or even an incentive to the 
increase in coordinative control of the body as a whole. Such additional control in coordination would arise from the profound readjustments occasioned by the assumption of the semi-erect posture and partial biped locomotion

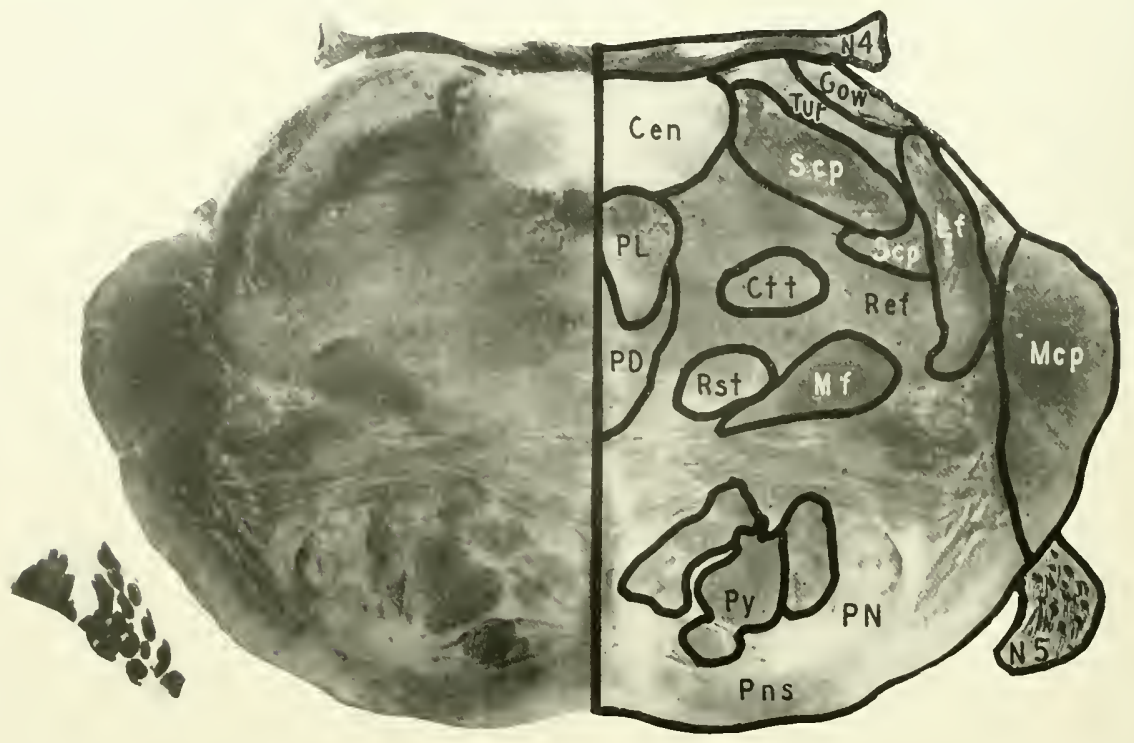

FIG. 124. MYCETES SENICULUS. LEVEL THROUGH MIDDLE OF PONS VAROLII. CEN, Central Gray Matter; CTT, Central Tegmental Tract; cow, Ventral Spinocercbellar Tract; 1.F, Lateral Fillet; MF, Mesial Fillet; MCP, Niddle Cerebellar Peduncle; N4, Trochlear Nerve; N5, Trigeminal Nerve; PD, Predorsal Bundle; PL, Posterior Longitudinal Fasciculus; PN, Pontile Nuclei; PNs, Pons; Pv; Pyramid; REF, Reticular Formation; RST, Rubrospinal Tract; sCP, Superior Cerebellar Peduncle; tur, Tractus Uncinatus of Russel (Hook Bundle). [Accession No. 148. Section 305. Actual Size $15 \times 10 \mathrm{~mm}$.

as well as from the fact that the animal is now able to sit upon its haunches and in this position use its hands for various new purposes. Similarly the scattered bundles of the pyramidal system, although disseminated amidst the stratum complexum, present a greater degree of prominence in mycetes than they do in lemur or marmoset. They afford the basis for a structural 
index to contrast the probable difference in volitional control inherent in these several species. That the quantitative difference favors mycetes, there can be no doubt. Its behavioral reactions both in its learned and automatic movements are consonant with this estimation.

On the boundary between the stratum profundum and the remainder of the brain stem is the mesial fillet (MI) whose size is considerably above that observed in lemur and marmoset. Here it is possible to form an idea of the relative volume of this ascending sensory pathway and so estimate the degree to which the animal has developed its diseriminative sensibility. The increments to this ascending fasciculus of fibers appear to be due to additional sensory contributions arising in the upper extremity and particularly in the hand.

In the tegmentum lateral to the mesial fillet is the lateral fillet (Lf), now approaching the inferior colliculus for another relay in the pathway of hearing. A dense bundle situated mesial to the lateral fillet constitutes the superior eerebellar peduncle (Scp) which affords an opportunity of estimating to what extent the animal is equipped with a conduction system for coordinative control of the muscles. The superior cerebellar peduncle is larger than in lemur or marmoset, from which it may be inferred that mycetes is possessed of a more highly claborated coordinating control.

The central gray matter ( Cen) surrounds the much reduced ventricular canal, the aqueduct of Sylvius. The roof of the aqueduct is formed by the medullary velum in which are the decussating fibers of the trochlear nerve $\left(\mathrm{N}_{+}\right)$on their way to the superior oblique muscle of the eyeball. Ventral to these fibers is the aseending or mesencephalic root of the fifth nerve.

LENEL OF THE INFERIOR COLLICULUS (F1G. 125 )

At the level of the inferior colliculus several features of importanee make their appearanee. Among these is the tectal specialization of the inferior 


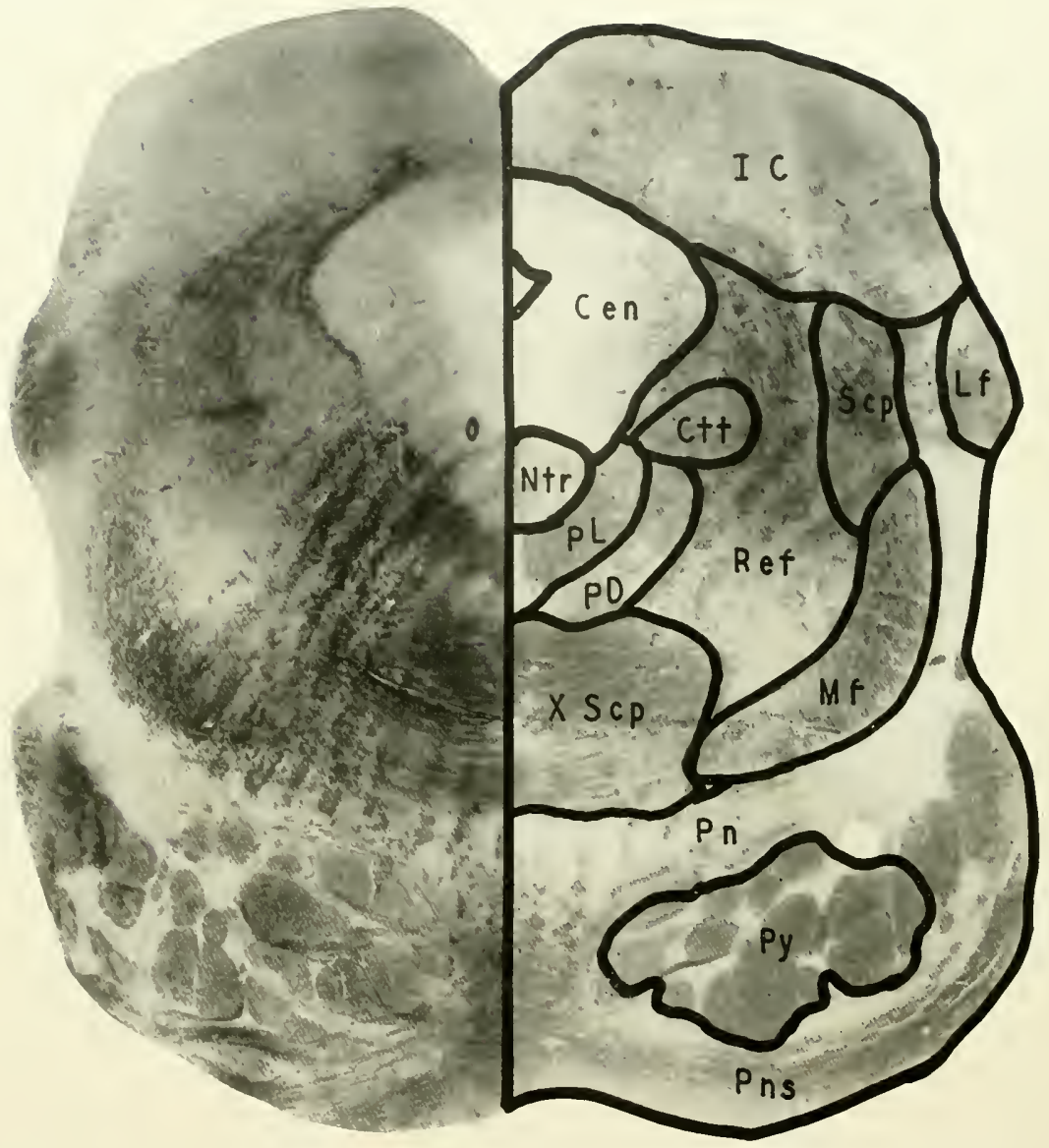

FIG. I25. MYCETES SENICULUS. LEVEL OF THE INFERIOR COLLICULUS. CEN, Central Gray Matter; crt, Central Tegmental Tract; IC, Inferior Colliculus; LF, Lateral Fillet; MF, Mesial Fillet; NTR, Nucleus Trochlearis; PD, Predorsal Bundle; P., Posterior Longitudinal Fasciculus; PN, Pontile Nuclei; PNS, Pons; Py, Pyramid; Ref, Reticular Formation; scP, Superior Cerebellar Peduncle; $\mathrm{xscP}$, Crossing of the Superior Cerebellar Peduncle. [Accession No. 48 . Section $3^{-0}$. Actual Size $13 \times 13 \mathrm{~mm}$.] 
colliculus (IC). The histological organization of the tectal region discloses a stratification almost as complex as in lemur and marmoset and thus suggests a functional capacity in the primitive correlating center of hearing of similar importance. The relation of such a correlating center to direct antomatic acts of defense and offense in response to auditory stimuli appears clear. The necessity for such a relation is as important in this arboreal animal as in those already considered.

A prominent feature at this level is the extensive substantia nigra, an index suggesting the probable persistence of many highly complex automatic associated movements. Ventral to the mesial fillet is the pons Varolii (Pns). It contains the pallio-ponto-cerebellar system of fibers as well as the fibers of the pyramidal system which latter, as in the oblongata, are not aggregated in a single bundle. From the size of these two fiber systems it is apparent to what extent the aninal is endowed with volitional control from the cerebral cortex, as well as what concurrent cerebellar impulses must accompany the volitional stream which designs, initiates, directs and finally inhibits all voluntary movements.

\section{LEVEL OF THE SUPERIOR COLLICULUS (FIG. I 26)}

At this level, the superior colliculus (SC) serves as an important relay in the pathway of vision. Since there is still a considerable histological organization in this tectal region, it seems probable that the superior colliculus (SC) may retain some of its primitive visual function. That much of this function, however, has now been delegated to the oceipital lobe is evident by the pronounced development in the calcarine or visual area of the cerebral cortex. The ventral portion of the central gray matter contains the nucleus oculomotorius ( Noc) whose fibers pass forward and inward to the oculomotor sulcus from which they emerge to supply all of the extrinsic muscles of the eycball with the exception of the superior oblique and external 
rectus muscles. This nerve also supplies all of the intrinsic ocular muscles and the levator muscle of the upper eyelid. It is especially significant that the commissural connections of the nucleus oculomotorius are much more prom-

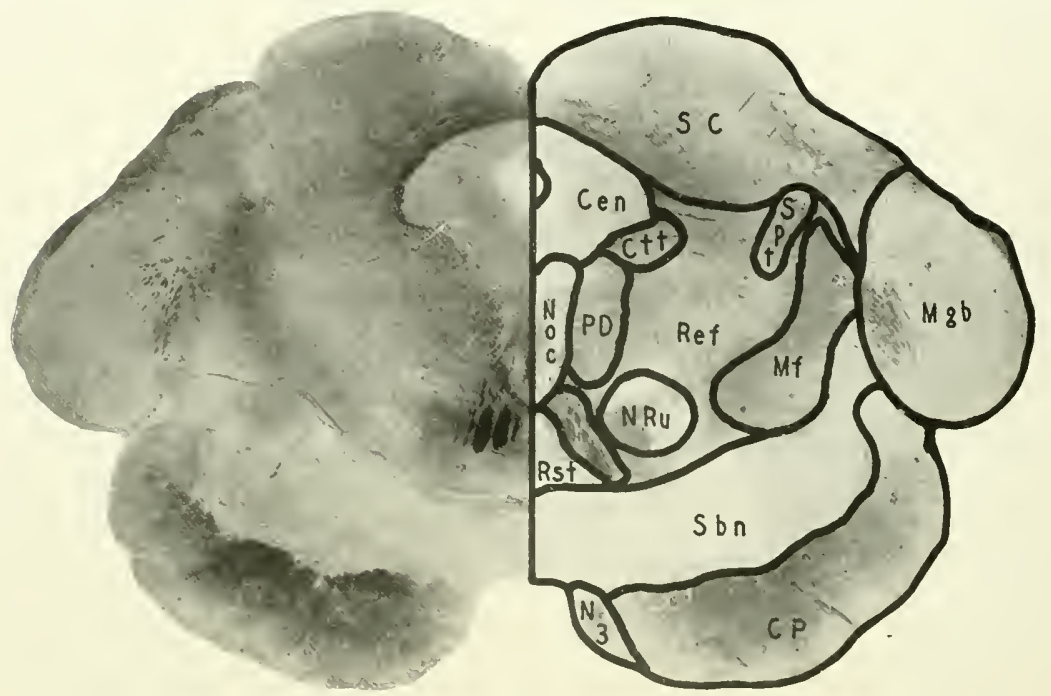

FIG. I26. MYCETES SENiCULUS. LEVEL OF THE SLPERIOR COLliClLUS. CEN, Central Gray Matter; CTt, Central Tegmental Tract; CP. Cerebral Peduncle; MgB, Mesial Geniculate Body; Mf, Mesial Fillet; noc, Oculomotor Nucleus; NRu, Nucleus Ruber; N3, Oculomotor Nerve; PD, Predorsal Bundle; ReF, Reticular Formation; RSt, Rubrospinal Tract; sc, Superion Colliculus; sBn, Substantia Nigra; spt, Spinothalamic Tract. [Accession No. 1 48 . Section 401 . Actual Size $18 \times 9 \mathrm{~mm}$. ]

inent than in lemur or marmoset, thus implying a closer association in interocular movements of the eyes and thereby securing a nearer approach to binocular vision and stereoscopic fusion.

Ventrolateral to the nucleus oculomotorius is the red nucleus (NRu) which is fairly well defmed. It constitutes a relay station in the course of the cerebello-spinal pathway. From it arise the frbers of the rubrospinal tract (Rst) over which pass impulses necessary to muscular coordination. In 
this sense the size of the nucleus ruber furnishes some clue of the coordinating capacity of the animal. Although more prominent than in marmoset or lemur, the nucleus ruber is not so large as in some of the higher apes, which

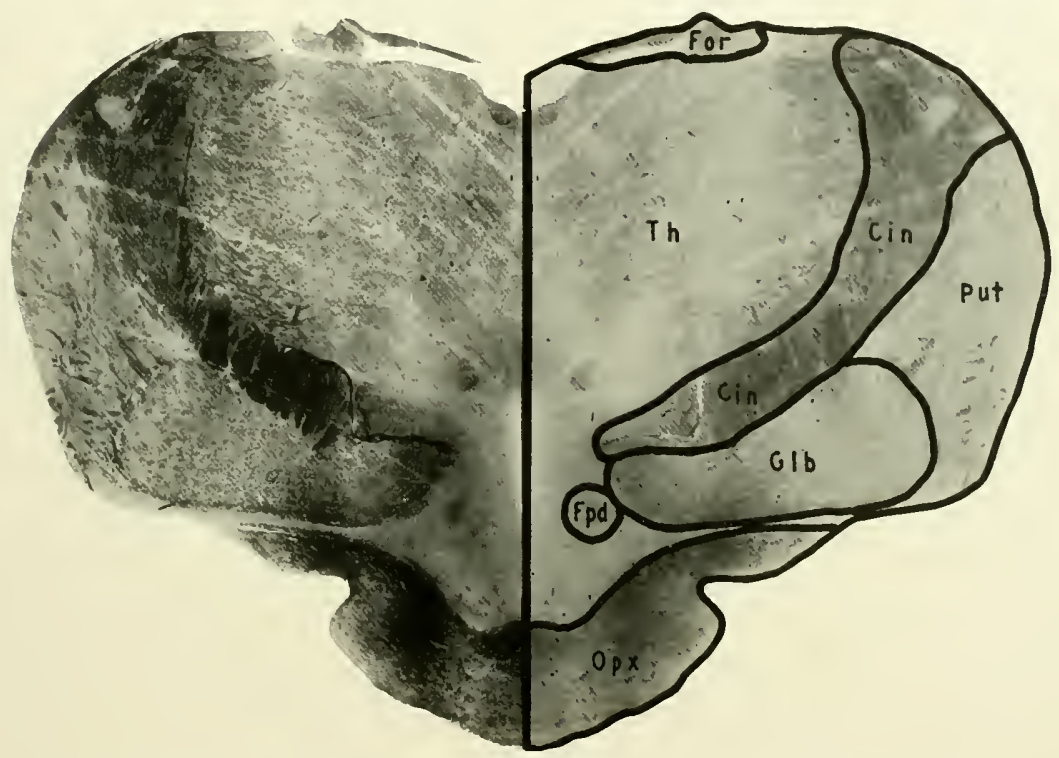

FIG. 12:- MYCETES SENICULUS. LEVEL OF THE OPTIC CHIASM.

CrN, Internal Capsule; FoR, Fornix; FPD, Descending Pillar of Fornix; glB, Globus Pallidus; opx, Optic Chiasm; put, Putamen; тн, Thalamus. [Accession No. 148. Section 501 . Actual Size $28 \times 17 \mathrm{~mm}$.]

seems to justify the supposition that the animal's coordinative powers lie somewhere intermediate between the fowest and highest differentiation in the primate series.

LEIEL OF THE OPTIC CHIASM (FIG. I 27)

At this level the section shows the changes incident to the appearance of the optic thalamus ( $\mathrm{Th}$ ) and the optic decussation ( $\mathrm{Opx}$ ). The massive 
structure of the internal capsule $(\mathrm{Cin})$ is indicative, as are the cerebral peduncle and the pons Varolii, of the high degree of neokinetic expansion exhibited by these monkeys. The behavioral patterns have become greatly

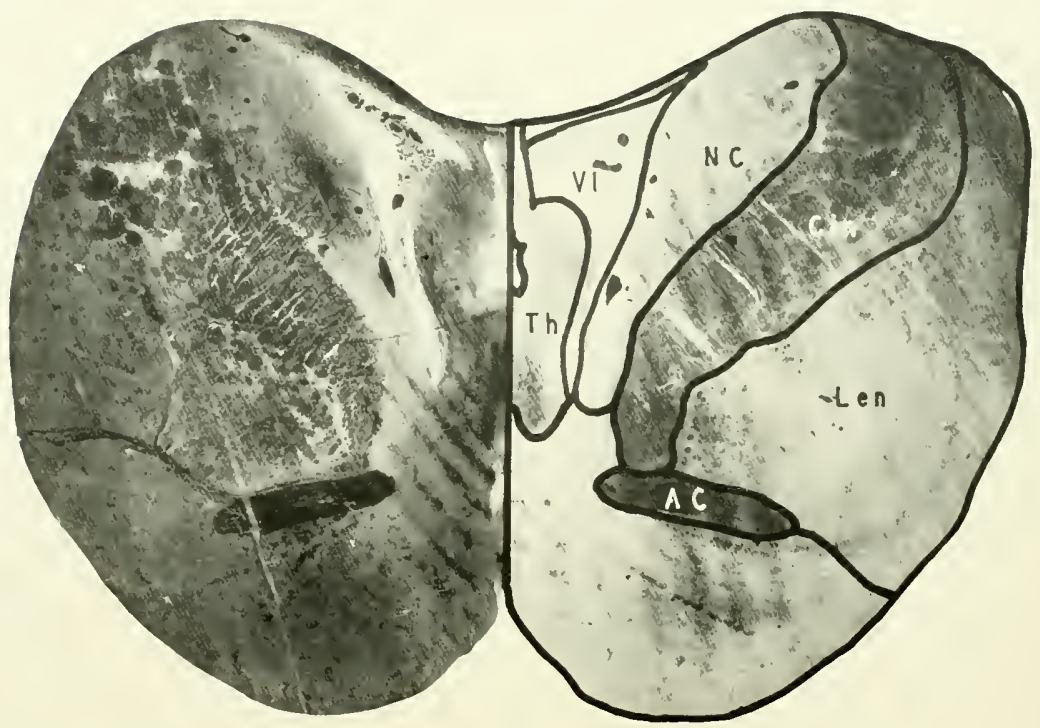

FIG. 128. MCETES SENICLLUS. LEVEL OF THE ANTERIOR COMMISSLRE. AC, Anterior Commissure; CIN, Internal Capsule; LEN, Lenticular Nucleus; NC, Caudate Nucleus; TH, Thalamus; vL, Lateral Ventricle. [Accession No. 148 . Section 575. Actual Size $28 \times 13 \mathrm{~mm}$.]

amplified in consequence of those new capacities made available by quadrumanous differentiation. To this new range of capacity should also be added those facilities of motion made possible through the prehensile tail, also spoken of as the fifth hand. Bordering on the outer surface of the internal capsule are the two major divisions of the lenticular nucleus, namely, the globus pallidus (GIb) and the putamen (Put). 
LEVEL OF THE ANTERIOR COMMISSURE (FIG. I 28)

At the level of the anterior commissure the brain stem has terminated in its cephalic extremity. The upper limit of the optic thalamus $\left(\mathrm{Th}_{\mathrm{h}}\right)$ is seen as it forms the anterior thalamic tuberele. The anterior commissure (AC) is approaching the midline from either side, about to establish communication between the two halves of the axis. The other important structures in this level are indicated by corresponding letters in the captions. 



\section{Chapter VIII}

\section{RECONSTRUCTION OF THE GRAY MATTER IN THE BRAIN STEMI OF MICETES SENICULUS}

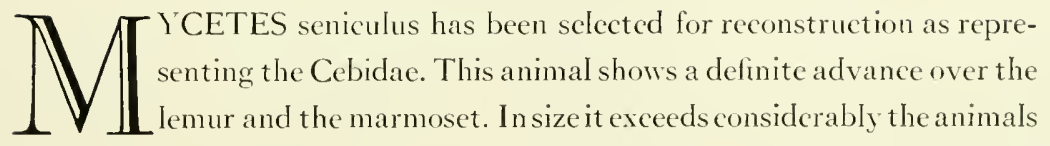
already mentioned and the brain reconstruction is correspondingly larger than that found in either of the two preceding forms.

\section{The Dorsal Sensory Nuclei}

The nucleus of Goll first appears in the reconstruction as a dorsal extension of the central gray matter between the point of attachment of the dorsal gray column to the central gray column and the dorsal median septum. The nuclear mass is a narrow prolongation from the contral gray column. It rapidly extends dorsally until it forms a core in the column of Goll. The nucleus is narrow, laterally compressed and presents a somewhat bulbous enlargement at its dorsal tip. At a somewhat higher level than the origin of the nucleus of Goll there appears on the dorsal margin of the central gray column a flat, sessile condensation in the central gray columm which is the beginning of the nucleus of Burdach. At about the same level in the dorsal portion of the mass of white fibers forming the column of Burdach appear isolated masses of gray matter which coalesce and become attached to the dorsal extension of the central gray column to form the external nucleus of Burdach. The arboreal character of this nucleus, already mentioned in lemur, is further developed in mycetes.

The appearance of the nuclei of Goll and Burdach between the dorsomedian septum and the point of confluence of the dorsal gray column with the 
central gray column tends further to separate this latter structure from the midline. As the mass of the dorso-medullary nuclei reaches its maximum, the substantia gelatinosa Rolandi which at this point is passing over into the substantia gelatinosa trigemini is shifted into its fixed lateral position. The heavy dorsal mass of the nucleus of Burdach overhangs laterally the substantia gelatinosa trigemini. The central gray matter at this point has merged to a considerable extent with the reticular formation. The transition between the substantia gelatinosa Rolandi and the substantia gelatinosa trigemini is essentially without a definite line of demareation. As the latter structure is traced upward, however, it constantly increases in size except for the constriction found at the midolivary level, termed the waist of the trigeminal nucleus.

\section{The Inferior Olivary Nucleus}

In the reconstruction, the inferior olivary nucleus shows a moderate advance over that found in the Lemur mongoz and the Callithrix jacehus. A slight indication of secondary plication now appears in the dorsal and ventral branches of the U-shaped nucleus. The ventral aceessory olivary nucleus appears at about the point at which the dorsal sensory nuclei begin to assume proportions of any size. It first manifests itself as a round collection of gray material which flattens out obliquely from before backward and inward. At a somewhat higher level, isolated gray islands appear which coalesce and form a similar parallel flattened band, the dorsal accessory olivary nucleus. The chief nucleus appears between the lateral extremities of this aceessory nucleus as a rounded mass which rapidly assumes the form of a loop, the ventral branch of which fuses with the lateral extremity of the ventral accessory nucleus, while the dorsal branch is continued mesially parallel to the dorsal accessory nucleus. The mesial extremities of the accessory nuclei then fuse together. The acessory nuclei attenuate and dis- 
appear leaving the loop of the chief nucleus to continue upward for a short distance, after which it also comes to an end. The location of the inferior olivary nucleus corresponds to that already described in lemur and marmoset.
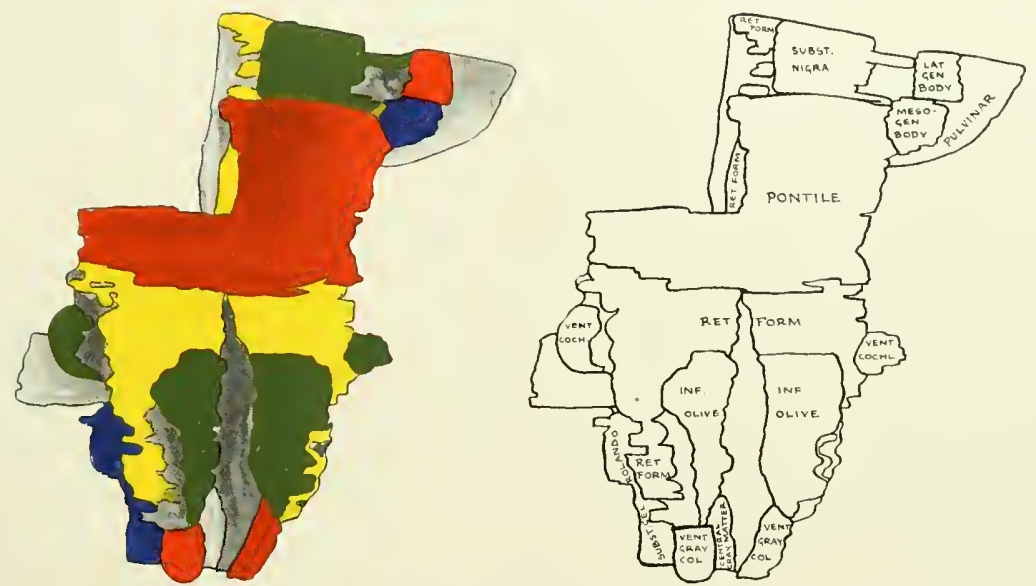

FIG. 129. VENTRAL SURFACE OF THE GRAY MATTER OF TIIE BRAIN STEM, MICETES SENICULUS.

Key to Diagram. iNf. OltVe, Inferior Olive; lat. GeN. Bodr, Lateral Geniculate Body; Meso-gex. Bodr, Mesial Geniculate Body; Pontile, Pontile Nuclei; Ret. Foru., Reticular Furmation; SU bSt. Gel. ROLANdo, Substantia Gelatinosa of Rolando; sUbst. nigra., Substantia Nigra; venr. Coch. and vevt. Cucil., Ventral Cochlear Nucleus; veNt, GRAY col., Ventral Gray Column.

The base of the nucleus approaches the ventrolateral angle of the brain stem, producing a slight elevation on the surface which corresponds to the olivary body found on the surface of the brain stem.

\section{The Reticular Formation}

The reconstruction of this mass of gray and white matter appears as a discrete body at about the middle of the pyramidal decussation. It is separated from and lateral to the ventral gray column. It rapidly increases in size 
as the ventral gray columns diminish and finally the latter disappear by merging into the former at the level of the inferior olivary nucleus.

The reticular formation furnishes the same matrix for the ascending and descending fiber tracts found in the two preceding forms. It is surrounded on all sides, except the dorsal, by the ascending and descending fiber bundles of the stem. It is connected laterally with the mesial surface of the substantia gclatinosa trigemini and clorsally with the bases of the nuckei of Goll and Burdach. Embedded in it ventromesially is the dorsal lamina of the inferior olivary nucleus together with the dorsal accessory olivary nucleus. As the reticular formation procecds upward in the stem it gradually increases in size until it assumes considerable proportions. The nucleus lateralis of the reticular formation and the superior olive are developed to a somewhat greater extent than in lemur and mamoset. In the region of the midbrain the pronounced nuclear condensation of the reticular formation appears in the form of the nucleus ruber which is developed to a somewhat greater extent in this form than in either of the preceding types. In the dorsal aspect of the reticular formation are successively embedded the nuclei of the mesial somatic motor cell column, namely, the hypoglossus nucleus in the region of the medulla, the nucleus abducentis in the midpontile region, the nucleus trochlearis and the nucleus oculomotorius in the mesenccphalic segment.

In the dorsolateral angle, between the subependymal gray matter and the cephalic extremity of the nucleus of Burdach, appears a condensation in the reticular formation which gives rise to the vestibular complex. The nucleus of Deiters separates the subependymal gray matter and the nucleus of Burdach, reaching its maximum diameter at a level somewhat above the midventricular level of the stem. At this point the triangular nucleus of Schwalbe appears, and continues upward in its characteristic position. The nucleus of von Bechterew lies lateral and dorsal to the rest of the vestibular complex in the lateral wall of the fourth ventricle. The reticular 
formation seems to be continuous with the matrix of gray matter from which develop the zona incerta of the diencephalon and also that of the less differentiated hypencephalic gray matter.
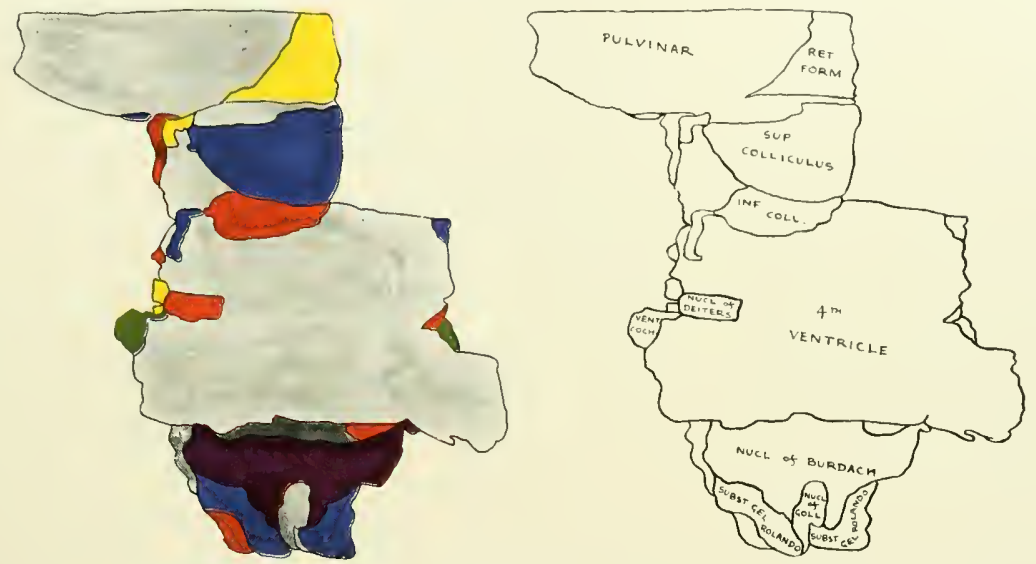

FIG. 130. DORSAL SURFACE OF THE GRAY MATTER OF THE BRAIN STEM, MYCETES SENICULU'S.

Key to Diagram. INF. coll., Inferior Colliculus; Nucl. OF Burdach, Nucleus of Burdach; Necl. Of deiters, Nucleus of Deiters; nuCl. OF GOLl, Nucleus of Goll; RET. FORM., Reticular Formation; SUbST. GEL. ROLANDO, Substantia Gelatinosa of Rolando; sup. Colliculus, Superior Colliculus; veNt. CoCH., Ventral Cochlear Nucleus.

\section{The Pontile Nuclei}

In reconstruction the pontile nuclei appear rather abruptly at the level of the trapezoid body. The nucleus is considerably more massive than in the Lemur mongoz and the Callithrix jacchus, and is tunnelled on both sides by the descending pyramidal and pallio-pontile tracts. This again produces the typical arrangement of a lateral and mesial buttress, connected ventrally and dorsally by the superficial and the deep layer of the pontile nucleus. The simple arrangement of the nucleus as found in the Lemur mongoz and the marmoset now becomes somewhat more complicated with masses of nuclear 
material beginning to infiltrate into the bundles of the prramidal and the pallio-pontile system of fibers, producing a lacework of nerve cells and fibers between the mesial aspects of the two Iateral and mesial buttresses. As is the case in the lemur and marmoset, the deep layer of the pontile nuclei, together with the dorsal portions of the lateral and mesial buttresses, becomes continuous with and serves, so to speak, as a support for the mesencephalic substantia nigra.

\section{The Vestibular Complex}

The reconstruction of this nucleus has been described as a condensation in the reticular formation arising at about the middle of the ventricular portion of the medulla oblongata. It appears first as a small, wedge-shaped mass of gray matter between the subependymal gray matter of the ventricular floor and the clorsal mass of the mucleus of Burdach. The nucleus of Deiters expands rapidly, becoming roughly triangular in shape, with its base upon the subependymal gray matter, its lateral boundary facing toward the nucleus of Burdach, and its mesial boundary toward the subependymal ventricular gray matter and the reticular formation. The triangular nucleus of Schwalbe continues somewhat cephalad to the lateral ventricular recess and then merges with the reticular formation. The nucleus of von Bechterew is situated in the lateral wall of the fourth ventricle cephalad to the lateral recess. This last nucleus is so fecbly developed as to be scarcely demonstrable in the model.

\section{The Cochlear Complex}

As reconstructed, this nuclear collection conforms with the type found in the lemur and the marmoset. It represents a trough which covers the caudal, lateral and cephalic aspects of the entering cochlear nerve root. The 
cochlear nerve, therefore, is uncovered by this nuclear material mesially where it comes into contact with the brain stem. The nuclear material extends along the course of the nerve, interspersed between its fibers. As
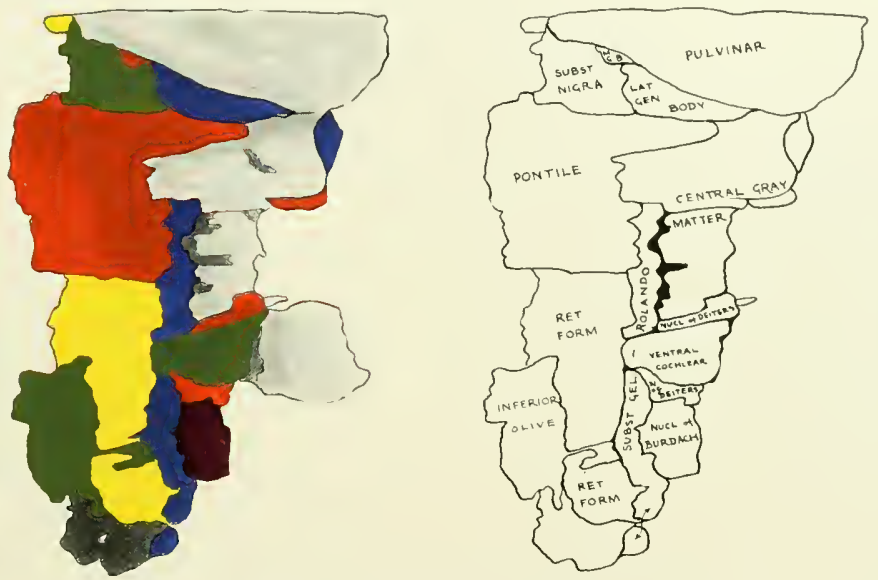

FIG. I3I. LATERAL SURFACE OF THE GRAY MATTER OF THE BRAIN STEN, MYCETES SENICULUS.

Key to Diagra.r. Lat. gev. Bodr, Lateral Geniculate Body; M.g.B., Mesial Geniculate Body; Nucl. of Burdach, Nucleus of Burdach; N. OF deiters and vecl. of deiters, Nucleus of Deiters; povtile, Puntile Nuclei; Ret. Forı., Reticular Formation; subst. gel. Rolando, Substantia Gelatinosa of Rolando; scbst. nigra, Substantia Nigra; ventral cochlear, Ventral Cochlear Nucleus.

the cochlear nerve is traced into the dorsolateral angle of the brain stem another nuclear complex connected with the cochlear apparatus makes its appearance-the dorsal cochlear nucleus which lies in the lateral recess of the fourth ventricle, dorsal to the vestibular complex. This nucleus is triangular in shape, its base resting upon the subependymal gray matter, its lateral border directed toward the inferior and middle cerebellar peduncles and its mesial border lying against the vestibular complex. 


\section{The Slbstantia Nigra}

In the reconstruction, the substantia nigra appears in the reticular formation of the mesencephalon, apparently as a cephalic specialization in the deep layer of the pontile nueleus. Mesially it is eontinuous with the undifferentiated interpeduncular gray matter. Ventrally its surface is somewhat corrugated by the passage of the fibers of the pes pedunculi. Dorsally it is in contact with the reticular formation of the mesencephalon which is demarcated from it by the circumferential fiber bundles of this nuclear mass. $\checkmark$ entrolaterally in its upper portion it is hollowed out to a marked extent by a special nuclear accumulation which takes place in this part of the substantia nigra. This nuelear mass presents a tangle of myelinated nerve fibers which seem to rise in the nucleus and pass into the reticular formation of the mesencephalon. Cephalically it is continuous with the subthalamic gray structures of the dieneephalon and laterally it merges with the reticular formation in which are embedded the mesial genieulate bodies.

\section{The Colmiculi}

The reconstruction of the inferior colliculus appears as a rapidly inereasing mass of gray matter supported laterally by the dorsal extension of the retieular formation. Dorsally it is continuous with the dorsal gray matter of the tectum which separates it from its fellow of the opposite side and also provides a pathway for the inferior collicular commissure. At the midmeseneephalic level the inferior colliculus diminishes in thickness and gradually disappears. In this region the dorsal extension of the tegmental reticular formation comes to the surface of the tectum of the midbrain. Immediately suceeding this, the superior colliculus appears, situated in an essentially similar position to that occupied by the inferior colliculi. Its dorsal and rentral extremities receive the same type of support as that of the 
inferior colliculus and it is connected across the midline with its fellow of the opposite side by the superior collicular commissure.

\section{The Nucleus Ruber}

In the reconstruction, the nucleus ruber in Mycetes seniculus appears as a fairly well-differentiated and eneapsulated mass of gray matter in the mesial portion of the reticular formation. It receives at its caudal extremity the decussating fibers of the superior cerebellar peduncle. These fibers are continued about the nueleus, forming a capsule for it and many of them are continued upward beyond the nucleus ruber into the subthalamie region. The upper extremity of the nucleus ruber projects into the diencephalic reticular formation and the nueleus in general seems to be located at a more cephalic level than is the case in the human brain sten.

\section{The Central Gray Matter}

The reconstruction of the central gray matter as first observed in the higher cervical level is somewhat cordiform in outline and is separated from the ventral gray column. To its dorsolateral corners are attached the bases of the dorsal gray columns. As the ventricular level is approached a narrow prolongation of gray matter is seen to arise from its dorsal aspect and passing along the dorsal median septum it divides into two tongue-shaped processes which flare out on either side. This is the frrst indication of the opening of the fourth ventricle. The central gray matter passes outward and backward, gradually flattening into a narrow ribbon of gray matter lying under the ependyma of the fourth ventriele. There the narrow strip of gray matter is continued around the lateral boundaries of the ventricle, both in the inferior and superior medullary velum so that the entire ventricular cavity is surrounded by material derived from or continuous with the original central gray matter. The gray matter of the floor of the fourth ventricle is relatively 
smooth and presents little modelling of its surface in the lower half of the fourth ventricle. The walls are formed successively by the nuclei of Goll and Burdach and the nucleus of Deiters. In the upper half of the ventricle the floor shows a single well-marked and rounded medial eminence produced by the mass of gray matter forming the nucleus abducentis, close to the midline and just above a line joining the two lateral recesses of the fourth ventricle. As the upper portions of the ventrick are approached the walls rapidy contract to form the narrow aqueduct of Sylvius which traverses the mesencephalon. The central gray matter of the mesencephalon is considerably thicker than that found in the lemur or the marmoset. It contains the dorsal extension of the trochlear and oculomotor nuclei which lie embedded in the dorsal region of the mesencephalic tegmentum. At the upper extremity of the mesencephalon the central gray matter is directly continuous with the subependymal gray matter of the third ventricle and the mesial thalamic muclei. The most dorsal portion of the central gray matter in the mesencephalon is continuous with the epithalamic group of structures, while the most ventral portion is continuous with the hypencephatic structures. 


\section{Chapter IX}

\section{COMPARATIVE SUMIIARY OF STRUCTURES HAVING EVOLUTIONAL SIGNIFICANCE IN THE BRAIN STEMS OF THE LOWER PRINATES}

A Critical Comparison of the Pramidal Tract, Oliran Bodr, Dorsal Sensory Nuclei, Vestibular, Cerebellar and Pontile Nuclei, the Midbrain Colliculi and Oculomotor Decussation. Their Erolutional Significance in Relation to Beharior

Comparative Review of Structural and Behavioral Adaptations

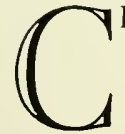
ERTAIN homologous constituents in the brain stem of the lemur, tarsier, marmoset and howling monkey manifest a measurable variability in structural definition and relative size. Such variations seem to harmonize with equally well-defined modifications in the sensory and motor equipment utilized by these animals in their highly dillerentiated behavioral adjustments. That these variables should particularly involve the neokinetic elements of behavior might be presupposed from the fact that the progressive addition of new motor complexes paved the way to the highest erolutional development.

1. The Pyramidal Tract in Relation to the Voluntary Control of the Extremities with Especial Reference to the Hand

Especially striking are the variations indicative of modification in volitional influence over the somatic muscles. The pyramidal system has quite as much significance with regard to the degree of neokinetic activity as the cortex of the cerebral hemispheres. In direct proportion as the phyletic expansion of the neopallium has made possible new accessions of highly 
skilled performances, the pyramidal tract has progressively enlarged. Although this neopallial expansion adds new cortical fields for more extensive sensory correlation, its ultimate object is the behavioral expression made manifest in the total motor output from the cerebral cortex. The possibilities of increasing the richness of sensory associations within a single sphere of sensibility such, for example, as vision, are obviously dependent upon increase in its structural substratum. But when combinations of sensibility such as those within the realm of vision are incorporated in compound association of sight and hearing, taste and smell and all qualities of somatic sensibility, then even vaster possibilities for sensing the world are open to the animal. In its turn, this gradually advancing conquest of the environment through avenues of the senses must find expression in new currents of behavior. It might be expected that the afferent convergence of this sensory influx would require a correspondingly expanded channel in the efferent pyramidal system. Indeed, it seems surprising that the pyramidal tract is no larger than it actually is. The pyramid, however, is a newcomer in the central axis. It is essentially a mammalian character in the brain, particularly implicated in the differentiation of the appendicular musculature. Its influence over the axial muscles, although potential, seldom reaches a high degree of specialization. The impulses which it conducts are preeminently concerned in the execution of such skilled performances as belong to the group of complex learned reactions. Undoubtedly the most highly organized of these skilled acts are dependent upon the operation of the distal portions of the upper and lower extremities, namely, the feet and toes, the hands and fingers. It is in relation to the progressive adaptation apparent in the upper extremity that the pyramidal system is most intimately connected. Substantially little change occurs in the specialization of the fower extremity in the lower primates. The addition of the prehensile tail in certain of the South American monkeys undoubtedly requires an accession in volitional control 
of the caudal musculature. It is by no means so insistent in its demands as the rapidly differentiating struetures of the upper extremity and particularly of the hand.

In lemur and tarsius, the pyramidal tract appears to be less prominent than in Mycetes seneculus. Such is the case also in marmoset. The hand of this animal has made a hesitating advance toward manual differentiation, and its pyramidal tract is hence less conspicuous than in the howling monkey.

\section{PLANIMETRIC COEFFICIENT}

These facts are clearly illustrated by certain coefficients which show that the pyramidal area in proportion to the remainder of the cross section of the oblongata is greater in the howling monkey than in either marmoset, tarsius or lemur.

The numerical expression of this proportion may be termed a planimetric coefficient. It is obtained by means of projection drawings of the cross section in which the structure to be measured appears, the projection being produced at a fixed magnification. The area occupied by the structure under consideration is then determined by means of the planimeter, and in a similar manner the area of the hemisection in which the structure lies. The ratio of the structure whose coefficient is sought to the total hemisection of the axis is then computed and the figure taken to represent the planimetric coefficient. The planimetric coefficients of the pyramidal tract in lemur, tarsius, marmoset and mycetes are:

Planimetric Coefficients of Pyramidal Tract in Lower Primates

\begin{tabular}{|c|c|c|}
\hline & Species & Coefficient \\
\hline Lemur & & .110 \\
\hline Tarsier & & .032 \\
\hline Marmoset & & .064 \\
\hline Mycetes & & $.13^{-}$ \\
\hline
\end{tabular}


While these frgures may not in any sense be accepted as definitive, they afford a close approximation to an actual estimate of the fact. From them it seems permissible to conclude that the increment in pyramidal volume is proportional to that increasing demand for control of motor performances made possible through the progressive development of the hand.

MORPHOLOGICAL CONSIDERATIONS IN THE COMPARISON OF

UPPER EXTREMITIES IN MAN AND LOWER PRIMATES

If the human upper extremity be accepted as the structural standard in the process of manual differentiation, certain morphological conditions must be taken into account when comparing these parts of the human body with similar structures in the lower primates. Among these conditions are the proportional length of the arm to the body, of the forearm to the arm, of the hand to the forearm, as well as the proportions of the metacarpals and phalanges, particularly the proportion of the metacarpal bones and phalanges of the thumb. There should likewise be included the degree of opposability of the thumb and the differentiation of the fingers, especially the finger nails and the cutaneous pads connected with the distal phalanges. Estimated in the light of such criteria, the upper extremity in lemur and tarsius falls considerably short of complete manual differentiation. In the case of marmoset, the proportions in the major segments in the limb are at variance with the accepted human standard. The differentiation of the fingers is particularly primitive. The thumb is short, the cutaneous pads of the distal phalanges correspond more with the conditions presented by animals possessed of claws, and finally, the nails upon the fingers are much more claw-like than in any other form of primate.

In mycetes, however, the behavioral reactions made possible through differentiation of the hands place the animal much closer to man than lemur, tarsier or marmoset. The performances of the howling monkey are 
much more humanoid than those of the still lower primates. Although it is distinctly subhuman in its manual achievements, none the less mycetes must be assigned a place well up in the class of definite manual differentiation.

11. Tile Inferior Olivary Nucleus in Relation to the Regulation of Movements in the Eres, Head and Hands

FUNCTION OF THE INFERIOR OLIVARY NUCLEUS

There is considerable doubt concerning the function of the inferior olivary nucleus. Certain facts regarding it, however, are fairly obvious. The olive must be closely related in function to the cerebellum since its major connections are with that organ. The axons arising in the olivary substance pass as olivo-cerebellar fibers to the inferior cerebellar peduncle and thus reach the vermis and lateral cerebellar lobes. Whatever the precise function of this structure may be, its intimate association in the cerebellar reflex are implies an activity related to the coordinative control of somatic musculature. The inferior olive is intercalated as a relay nucleus in some important pathway whose impulses are destined to the cerebellum. It apparently serves to diffuse these impulses more extensively and thus bring into operation larger fields of cerebellar tissue in the interest of a highly specialized coordinating activity.

Von Bechterew and other neurophysiologists are largely agreed that the inferior olive is functionally concerned with static coordination. Experiments upon dogs in which the olive of one side has been injured cause a peculiar paralysis of the eye muscles with irregular nystagmic movements and simultaneous torsion of the body about its long axis. This torsion inmediately follows the injury and attains its highest degree directly after the operation. In the course of time the torsion becomes less pronounced and the paralysis of the eye muscles together with the nystagmus diminishes. 
Recent experimental work upon cats (Pike), in which the inferior olive on one side was destroyed, produced almost identical results with those reported by Bechterew. The paratysis of the eve muscles, the nystagmus and the torsion of the body were all prominent symptoms as a result of discrete olivary lesions. The most striking feature of the disorder, however, was the torsion which took place in the trunk and neck. This torsion determined such a position of the body that the head and face of the animal together with its forelimbs were pointed one way, while the hind extremities pointed in the opposite direction. Repetitions of this experiment produced similar effects in all cases. These experiments were controlled in such a way as to exclude the results of injury to neighboring structures. The conchsion that the inferior ofive is involved in the coordinative control of the eye, neck and arm musculature seemed unavoidable.

Clinico-pathological observation sheds little light upon this problem. Cases in which lesions of the olive have been observed are usually masked by encroachment of the pathological process upon some important afferent or efferent tracts in the oblongata. They have in no instance been discrete enough to permit of valid deductions regarding the function of this structure on the basis of pathological alteration in man.

\section{CONNECTIONS OF THE INFERIOR OLIIE}

These connections are of much significance in this question. The outstanding fibers related to the olivary body are those already mentioned as constituting the olivo-cerebellar pathway. These fibers undoubtedly establish the ultimate connection between the olive and various portions of the cerebellar cortex including both the remis and the lateral lobes. Another important connection is the central tegmental tract which lies along the ventrolateral aspect of the olive and may be traced upward in the tegmentum of the pons into the midbrain in the region of the nucleus oculomotorius and 
mesencephalic nucleus of the trigeminal nerve. $\mathrm{B}_{\mathrm{y}}$ some authorities this latter nucleus is accredited with proprioceptive functions, receiving sensory stimuli from the ere muscles. Some fibers of the central tegmental tract may pass further cephalad in the direction of the basal ganglia of the cndbrain and also into the posterior commissure. The main bulk of this bundle seems to terminate in the region of the nucleus oculomotorius. By means of it connection is established between the chief nuclei concerned in regulation of ocular movements and the inferior olive. A small and poorly myclinized tract of fibers may be traced to the olive from origins in the cervical and upper thoracic segments of the spinal cord. This is the spino-olivary tract of Helweg.

In the absence of other ascending or descending connections with this important nuclear structure it scems probable that the olivary nucleus is an intermediary station for impulses received from the muscles of the eye, neck, upper extremity and perhaps the upper portion of the trunk. It ultimately delivers these impulses to the cerebellum. Such connections might well serve the purposes of simultaneous coordination in the ocular, neck, arm and upper trunk muscles. The fact that experimental lesions of the inferior olive disturb the coordination of the eye muscles and cause pathological torsion in the neck lends support to the theory that one of the functions of the olive, if not its chief function, is simultaneous coordination of cye, head and hand movements and also the performance of all highly skilled acts. This presumption is borne out by the progressive increase in the conspicuity of the olive while passing from the lowest of the primates to the highest members of this order.

\section{OLIVARY SUBDIVISIONS}

Morphologically several olivary subdivisions have been recognized. These include a portion which is old, often referred to as the paleo-olive, and 
a portion which is more recent, the nen-olire. Each of these subdivisions has been aceredited with special connections in the cercbellum. This question, however, does not necessarily coneern the phyletic problem at present under consideration. Discrete differentiation of olivary segments and the significance of their connections are matters needing further investigation before their real value as to the function of this nuclear structure is determined. Although there is undoubted significance underlying the distinction between the phyletically old and new portions of the inferior olivary body, attention is here directed to the evolutional unfolding which has involved the structure as a whole rather than any changes affecting its individual parts.

EYE, HEAD AND HAND MOVEMENTS, AND THEIR CONTROL

As the hand gains in its capacity to utilize motor patterns, the wider becomes its range of purposive reactions. The process of acquiring manual performances, as well as the actual execution of them once they have been acquired, necessitates certain directive influences. Vision especially becomes an important supplementary and even dominating factor. Without the aid of sight many complex movements of the hands could not be learned, and quite as certainly many acts would be deprived of their full effectiveness if the eyes did not supply the proper idea of distance and perspective.

Accepting the cooperation of vision as cssential to the organization and performance of many highly skilled acts acquired by the animal, especially those resulting in the movements of the hands and fingers, it becomes clear that a close functional inter-relation between the movements of the eyeball and of the hand must exist. It is necessary that the visual axes hold in focus the movements of the hand during the performance of acts which have been acquired with the supplementary cooperation of the visual function. In this sense the cye and the hand become essentially one organ, since the movements in the one must follow and harmonize with the movements 
in the other. The muscular structures producing these movements must therefore be integrated in a manner to produce this harmony of motor effect. Indeed, certain other effectors are also implicated in this intricate activity which manifests itself in these simultaneous movements of eye and hand. To this group belong the muscles of the trunk which sustain the body in definite positions to support the movements of the head upon the neck. The muscles of the hindlimbs should also be included in this category. The animal, especially in the erect posture, requires the cooperation of the hindlimbs in support of the body thus to provide a stable basis for the eye and head movements which accompany the movements of the hand.

Sivergic Units. One of the requisites for the precise control of such an extensive grouping of muscles in the body is the muscular coordination in each of the groups cooperating to produce the result. Such coordination is dependent upon the proper intermuscular relation of all the specialized groups forming the agonist and antagonist muscles. Within each group there exists, under normal conditions, a definite relationship in regard to muscular tension. When one muscle, it may be a flexor, contracts to produce a flexor movement, its antagonistic extensor likewise contracts to such an extent as to impose a check or guiding effect upon the muscle producing the movement. Each group of muscles because it acts in this manner to maintain this specialized intermuscular relation, has been called a stnergic unit. The entire body musculature is composed of synergic units which maintain this defnite intermuscular relation when in action.

To secure such coordinate movement, one entire division of the central nervous system is set apart, namely, the cerebellum. Under the control of this organ the synergic units of the body are maintained in their proper relations to each other. By the same means proper intermuscular relation between the synergic units is established. The function which regulates coordinated action in the muscles of the body is known as stnergia. In the 
operation of this function, the cerebellum must be in touch from instant to instant with the varying degrees of muscle tension existing in each muscle group of the body. Cerebellopetal fibers furnish a physical means by which such communication is established. A continuous stream of afferent impulses is thus passing to the cerebellum during all phases of muscular activity. It is interesting in this connection to note that most of the afferent pathways to the cerebellum from the musculature of the body pass upward in the spinocerebellar tracts whose destination is the vermis cerebelli. This portion of cerebellar organization is chiefly concerned with the axial and paraxial musculature, while the lateral lobes are much more engaged with the coordinative control of the appendicular muscles in the limbs. All of the ascending cerebellar frbers are believed to receive intermediate relay in precerebellar nuclei.

The column of Clark in the spinal cord is an example of such a precerebellar nucleus. It receives peripheral afferent fibers and in turn gives rise to fibers which constitute the dorsal spinocerebellar tract. A similar longitudinal nucleus, perhaps not so discretely limited as the column of Clark, provides a relay for the fibers constituting the ventral spinocerebellar tract. Both of these precerebellar nuclei have the form of long cell columns extending through many segments of the spinal cord. In this respect they differ from the inferior olive which is also a conspicuous precerebellar nucleus. Th is nucleus is saccular in form and limited to the segments of the oblongata.

\section{EYOLUTIONAL EXPANSION IN THE INFERIOR OLIVE}

Such expansion as occurs in the spinal precerebellar nuclei must in the main be longitudinal. It is for this reason less conspicuous than the expansion in the inferior olive which is largely in the transverse diameters. The progressive cnlargement of the inferior olivary nucleus in passing from the lowest of the primate order to man gives the impression of a much more striking evolutional process than is the case with other precerebellar nuclei. The olive is also more impressive from the fact that in its expansion there is 
a tendency for it to become more definitely convoluted. This feature goes hand in hand with the progressive development of the lateral cerebellar lobes. Those forms having the largest cerebellar hemispheres have also the most highly convoluted inferior olivary nuclei.

The parallelism in derelopment of olive and lateral lobes of the cerebellum depends upon the fact that the inferior olivary nucleus, in its capacity of a precerebellar relay station, sends its fibers not only to the vermis, but in very large measure to the lateral lobes as well. Such is not the case with the precerebellar nuclei situated in the spinal cord. Most of the fibers arising in Clark's column, as well as those which give origin to the ventral spinocerebellar tract, have their destination in the vermis of the cerebellum. The contrasts drawn by these morphological facts are not without evolutional significance. The spinocerebellar tracts represent a portion of the musculature with striking phyletic constancy, namely, the axial muscles of the body. The representation of these muscles when projected upon the cerebellar cortex requires for its elaboration the limited areas of the vermis only. This musculature is restricted to the trunk, the neck and such axial structures as those innervated by certain of the cranial nerves. No such functional limitation prescribes the representation of the inferior olivary body as a precerebellar nucleus. It expands as the cerebellar lobes expand, and having a widespread connection with them, exists in response to portions of the muscular system whose representation when projected upon the cerebellar cortex reaches all areas both in the vermis and in the lateral cerebellar lobes.

Factors Underling Progressive Expansion of Inferior Olivary Nucleus. Apparently the same dynamic influences which have produced expansion in the cerebellar hemispheres are operative in the progressive expansion of the inferior olivary nucleus. Were it possible to select the one most compelling factor underlying such influence, it would doubtless be the progressive differentiation of the forclimb. This differentiation does not alone 
participate in the intimate specializations occurring in the upper extremity. It invokes those widespread adaptations concurrent with and consequent upon the liberation of the forelimb from the responsibilities of locomotion. It enters into the complete assumption of upright posture, and modification of the lower extremities essential to this purpose, together with acquisitions of muscular coordination incident both to bipedal locomotion and the perfection of manual dexterity.

Planimetric and Longitudinal Coefficients of Olivary Body. Evidence in support of this view is lound even in the lower primates where the comparison of lemur, tarsier, marmoset and mycetes reveals a progressive expansion in the inferior olive. The accompanying tabulation of the planimetric coeflicients of the nucleus indicates a volumetric increment of soo per cent or more in the olive of mycetes as compared with the other three species.

Longitudinal coefficients of the olivary body, however, show even more extensive change. Together these two mensurations confirm the supposition that the inferior olive almost entirely expresses its expansion transversely within the limits of the oblongatal segments. It differs from the spinal precerebellar nuclei whose expansion is chiefly longitudinal. From a functional comparison of these four species it is clear that manual performances in mycetes are more complex and have a greater range of adaptability. As a structural indicator of this phyletic progress the inferior olive is of especial significance. It denotes the proficiency attained in the simultaneous movements of the eyes, head and hands.

Coefficients of the Inferior Oline in the Lower Primates

\begin{tabular}{|lccc|}
\hline & Species & Planimetric & Longitudinal \\
\hline Lemur & .060 & .290 \\
\hline Tarsier & .042 & .180 \\
\hline Marmoset & .038 & .230 \\
\hline Mycetes & .120 & .260 \\
\hline
\end{tabular}


i11. The Dorsal Sensory Nuclei in Their Relation to Discrimunative Sensibility in the Extremities

THE NUCLEI OF GOLL, BURDACH AND BLUMENAL

The nuclear structures in the dorsal columns, intercalated in the pathway of discriminate sensibility, assume importance in relation to motor performances which depend upon kinesthetic organization. The more perfectly an animal senses the movements and postures in the several parts of its body, the more completely is it able to adjust these parts to complex motor patterns. A low degree of kinesthetic organization is indicative of a limited range of reaction patterns. No specialized area of the nervous system affords a more illuminating index concerning the discriminative sensory influx than the dorsal sensory freld. Experiments and clinical pathology havedemonstrated a discrete division in the oblongatal nuclei of these dorsal columns. Discriminative impulses from the muscles, tendons, joints and bones in the leg and tail find relay stations in their advance toward the ccrcbral cortex, in the nucleus of Goll. Similarly, impulses from the upper extremity are relayed in the nucleus of Burdach. The ancillary nucleus of Blumenau, known also as the lateral nucleus of Monakow, is connected with the nucleus cuneatus. It presents certain features in which it differs from the dorsal nuclei. Its staining reaction is more intense and its cells are somewhat larger. Various opinions have been held concerning its relay function, both for and against the belief that it is intermediary in advancing impulses from the upper extremity to the cerebellum. It seems clear, particularly in lemur and mycetes, that many fibers do make their way to the inferior cercbellar peduncle from this nucleus of Blumenau. It is equally certain that the pathway itself gains in prominence with the increasing degree of differentiation in the hand. While, therefore, no final opinion may be expressed with reference to the function of the nucleus of Blumenau, there is evidence to show that it serves as a relay for certain 
impulses which arise in the proprioceptive organs of the upper extrenity and are destined for the cerebellum.

\section{THE NLCLEL'S OF ROLANDO}

The remaining nuclear structure in the dorsal sensory field, namely, the nucleus of Rolando (substantia gelatinosa trigemini) accounts for the sensory innervation of the face and of the head rostral to the interparietal line. The exact course followed by the fibers conveying discriminative sensibility from the trunk, from the neek and from the back of the head is not so well understood as the tracts which constitute the main conduction pathway from the extremities. It is possible that many of the fibers from the trunk, back of the head and neck make their way to the oblongata after relay in the reticular formation. They may ineorporate themselves in the great sensory pathways of the dorsal columns to receive their relay in the nuclei of Goll, Burdach and Rolando. In any event, these axial fibers, representing the distribution of the dermatomes in the trunk, in the neck and in the back of the head, constitute more or less constant factors which in all probability vary but little from species to species. It is unlikely that any great variation, either in the capacity of sensibility or in the demands for conduction, arises in connection with the trunk. Such modifications as do oceur appear to be induced by conspicuous variables such as the tail, the forelimb and the hindlimb. Thus it might be expected that in an animal possessed of a highly efficient prehensile tail, the nucleus of Goll would appear in larger dimensions than in an animal either possessed of no tail or having one of much more generalized functional significance. The chicf nuclear expression connected with the development of the tail appears in the nucleus of Bischoff. The development of the hand affects the column of Burdach which is much larger in animals having a high degree of manual differentiation than in those manifesting but slight differentiation of this kind. 
In the case of the Rolandic nucleus, although the area of innervation which it represents (the face and ventral portion of the head) is exquisitely axial in its distribution, this sensory territory of the body manifests considerable functional variability according as the animal depends more or less upon the head and face for the direction of its locomotion.

A COMPARISON OF THE NLCLEI OF GOLL AND OI BLRDACH

IN THE LOWER PRIMATES

A comparison of the nucleus of Goll in lemur, tarsier, marmoset and mycetes shows an actual increase in size in the howling monkey, aceounted for largely by the presence of a well-defined nucleus of Bischoff. In lemur the planimetric coefficient of the nucleus gracilis (GoII) is 4.1 per cent, in tarsius 2.6 per cent, in marmoset, 6.8 per cent, while in mycetes it reaches 13.1 per cent. All of these forms are essentially quadrumanal. Such inerease as oceurs in the size of the nucleus of Goll in these species cannot be attributed to essential modifications in the hindimbs which, specialized as they are for arboreal life, become more or less standardized by this fact. They are subject to but little variation. The motive of this nuclear expansion must in consequence be sought elsewhere than in the specialization of the feet. The most obvious possibility to suggest itself is the specialization of the tail. In lemur, tarsier and marmoset the planimetric coefficient of the nucleus gracilis shows no striking differences. In these animals the tail serves as a balancing and steering organ. It has developed no prehensile qualities and thus has beeme but little specialized in its sensory capacity. As might be presumed, such an organ would not exert much influence tending to effect expansion in the sensory receiving nuclei.

In the case of mycetes, however, the nucleus of Goll shows a marked expansion. Its coefficient cxceeds that of marmoset by nearly - per cent and that of lemur by 9 per cent. Such a striking advance as this must have more 
than passing significance. The skillfull manner in which mycetes employs its tail supplies the reason for calling this organ a fifth hand, and hence the need of sensory specialization in this caudal appendage is evident. The tail at its tip is free of hair and presents upon its ventral surface parallel rugae not unlike the rugous markings upon the balls of the fingers and thumb. The animal utilizes its tail not merely for purposes of suspension during locomotion, but in many selective acts in which sensory discrimination is necessary. A tail manifesting such a degree of deftness at once implies the acquisition of a large series of motor patterns. It must therefore be the case that the kinesthetic development in connection with the movements of the tail is largely expanded in mycetes as compared with lemur, tarsier and marmoset.

This expansion, however, is not confmed to the nucleus of Goll. The planimetric coefficients of the nucleus of Burdach show a similar increase in the size. In lemur this nucleus has a planimetric coefficient of 4.9 per cent, in tarsius, of 2.9 per cent, in marmoset, of 4.3 per cent. In mycetes, the coefficient is 11.3 per cent. Since the nucleus of Burdach, in the main, represents the discriminative sensory influx from the upper extremity, the hand would prove a dy namic factor most likely to exert an influence favoring expansion. In lemur, manual specialization is not far advanced. The thumb is short and lacks much of the opposability characteristic of the higher species. Both the forearm and arm are short and do not approximate the ideal proportions of human conditions. In fact, the forelimb is still so largely implicated in locomotion that it possesses many characters inherent in a locomotor organ. Even more submanual is the forelimb in tarsier and marmoset. In both of these species, differentiation of the hand has made ineffectual attempts in the direction of human conditions. In the case of mycetes, however, the forelimb has advanced decisively toward ultimate manual differentiation. Its locomotor offices have been supplemented by the use of the prehensile tail which, permitting suspension of the body, affords opportunities for the freer use of the 
hand in acts of discrimination and selection. The planimetric coeflicient of the nueleus of Burdach in mycetes is slightly in excess of that of any of the other primates including the anthropoids and man. This observation will be more fully discussed in connection with humanoid and human manual differentiation. In general, it seems to indicate a strong adaptive influence operating in direct response to the specialization of the prehensile tail which has largely freed the forelimbs from funetions of locomotion. It has thus made them available for more complex motor patterns utilized in exploring and further dominating the environment. The tabulation of coefficients follows:

Planinetric Coefficients of Dorsal Sensory Nuclei in Lower Primates

\begin{tabular}{|c|c|c|c|}
\hline & Species & Goll & Burdach \\
\hline Lemur & & $.04 I$ & .049 \\
\hline Tarsier & & .026 & .029 \\
\hline Marmoset & - & .068 & .043 \\
\hline Mycetes & & .131 & .113 \\
\hline
\end{tabular}

Longitudinal Coefficients of Dorsal Sensory Nuclei in Lower Primates

\begin{tabular}{|c|c|c|}
\hline Species & Goll & Burdach \\
\hline Lemur & 200 & .290 \\
\hline Tarsier & .190 & .240 \\
\hline Marmoset & $.19^{\circ}$ & .210 \\
\hline Mycetes & .210 & .280 \\
\hline
\end{tabular}

Advances in the nucleus of GoII and more especially in the median nueleus of Bischoff are measurably demonstrable in mycetes as an apparent response to the adaptive modifications incident to the development of the prehensile tail. This species, compared with lemur, tarsier and marmoset, shows a similar advance in the nueleus of Burdach as a consequenee of further manual differentiation. 


\section{The V'estibular Neclei in Their Relation to the BalanCING MECHANISI}

\section{EXTREME SENSITIYENESS OF THE BALANCING MECHANISM}

This group of nuclei is fundamentally associated with the function of balancing, that is, the maintaining of the body in the optimum physiological posture, or of righting the body in the event that it may for any reason be forced out of this posture. This posture itself, although subject to numerous modifications, presents a lairly well-generalized pattern in all vertebrates. In accordance with this pattern the animal's best posture appears that in which the ventral surface of the body is nearest to the surface of the earth. This is true of fish, amphibia, reptiles and birds. From such a posture these animals may most readily initiate locomotion or remain in a resting phase preparatory to locomotion. This posture in mammals, with a few notable exceptions, such as the sloth and the bat, is similar to that in the lower vertebrates. Any slight deflection in this posture tends to cause extensive disorganization in the animal's behavior. Such disturbances may be artificially induced by pathological lesions affecting the nervous system, more particularly that part of the nervous system connected with the proprioceptive organization upon which balancing function depends. Thus the destruction of one semicircular canal will so thoroughly disorganize the animal's capacity for assuming and maintaining the optimum physiological posture as to make locomotion impossible. It also makes the animal incapable of maintaining itself in any position.

The mechanism upon which this important activity depends must needs be highly organized. This applies to its receptor organs as well as its central representation for receiving, converting and transmitting postural impulses to the musculature. Furthermore, this mechanism is extremely sensitive to many conditions of habitat and behavioral adjustments. Aquatic, aerial and 
arboreal adaptations create the most marked variations in this neural apparatus. The responsibility of the balancing mechanism in animals whose life is spent largely in flight, soaring to great heights, or dropping swiftly from the air to alight upon the branches of trees is most exacting. It necessitates a mechanism capable of adjusting the body in postures most advantageous for the use of wings. Adaptation to arboreal life imposes similar requirements upon the balancing apparatus. Such of the apes, for example, as are able to live almost exclusively in the trees and move from place to place by leaping, climbing and swinging, require a most delicately adjusted mechanism in the interest of equilibration.

\section{FUNCTION OF THE VESTIBULAR NUCLEI}

The primary centers associated with balancing are the vestibular nuclei. These nuclei serve as the chief receiving stations for the impulses flowing inward from the semicircular canals, the utricle and saccule. It is probable that even from the earliest stages of life these nuclear groups in the oblongata exert a pronounced influence upon the behavior of the young animal. With rare exceptions among the mammals, the power to assume and maintain the optimum physiological posture derelops shortly after birth. The structural substratum for the early maturing of this function is found in the fact that the nerve fibers permitting communication between the proprioceptive organs have consummated their connections with the vestibular nuclei. These nuclei are in turn provided with efferent fibers necessary to the conduction of impulses destined to the somatic muscles. In this manner the reflex ares essential to maintain the physiological optimum posture are completed. The vestibular nuclei in the oblongata are, of themselves, sufficient to mediate the reflex impulses necessary to the proper balance of the body. On the other hand, there is evidence that many myclinized fibers, even at an early period of development, pass to the region of the inferior colliculus. These axons 
have connections with the lower portions of the neuraxis. They provide a series of rellex ares which participate in the balaneing function. That this function is primarily the coneern of the lower segments of the brain below the dieneephalon and endbrain seems clear on the basis of ontogenesis. It may be held with a fair degree of ecrtainty that the reflex ares made possible through the restibular nuclei of the oblongata, perhaps supplemented by influenees from the midbrain, are sufficient for a considerable period of time in early life to carry on the reflex activities involved in the balancing reaction.

\section{CONNECTIONS OF THE VESTIBLLAR NLCLE1}

The several connections of the vestibular nuclei become of eonsiderable importance in this relation. These nuclei, as atready indicated, are primarily connected by afferent fibers with the proprioceptors of the internal ear, including the saceule, the utricle and the semicireular eanals. The nuelei have connection with the vermis of the cerebellum through the corpus juxtarestiforme, also with the midbrain, particularly the oculomotor nuclei.

\section{COMPARATIVE DIMENSIONS OF DEITERS' AND SCHWALBE'S AREA IN THE LOWER PRIMATES}

Conclusions based on comparative dimensions of Deiters' and Schwalbe's nuclei respectively indicate that the funetion of balancing and maintaining the optimum physiological posture in all of the primates is essentially the same in effeetiveness. Deiters' nueleus throughout the primate series manifests strikingly constant dimensions. It presents a maximum variation in its planimetric coefficients of less than 7 per cent, while its variation in its longitudinal coeflieients does not exceed + per cent. In the case of Schwalbe's nucleus apparently the same constancy obtains in so far as the planimetric coeflicient is coneerned, although in the comparative longitudinal measurements of the nueleus a variational range of 6 per cent 
has been noted. The following tables give the planimetric and longitudinal coeffeients of Deiters' and Schwabe's nuclei in lemur, tarsier, marmoset and mycetes:

Planimetric Coefficients of the Vestibllar Nuclei in Lemur, Tarsier, Marmoset and Mycetes

\begin{tabular}{|c|c|c|}
\hline & Deiters' Nucleus & Schwalbe's Nucleus \\
\hline Lemur & .082 & 045 \\
\hline Tarsier & 180 & 062 \\
\hline Narmoset & .077 & .060 \\
\hline Mycetes & .114 & 090 \\
\hline
\end{tabular}

Longitudinal Coefficients of the Vestibllar Nuclei in Limur, Tarsier, Marmoset and Mycetes

\begin{tabular}{|c|c|c|}
\hline Species & Deiters' Nucleus & Schwalbe's Nucleus \\
\hline Lemur & .21 & .21 \\
\hline Tarsier & 22 & .17 \\
\hline Marmoset & .21 & .20 \\
\hline Mycetes & .18 & 15 \\
\hline
\end{tabular}

Such differences as do exist in the vestibular nuclei of these species appear to favor tarsius, a fact which denotes how much more this animal depends upon its balancing function than lemur, mycetes or marmoset. This added functional responsibility may be inherent in tarsius due to its locomotion. In mycetes the problem in balancing includes not only the ordinary locomotion of climbing, leaping and swinging by hands and feet, but also embraces the many postures of the body incident to suspension by means of the tail. Mycetes is capable of assuming positions in which the body is suspended while the hands and feet are freed for other activities than those of locomotion. In such positions as these the requirements of balance must be considerably greater than in other primates. The entire process of motor 
adjustment, operating upon a less stable base in mycetes calls into play the functional activity of additional balancing reflexes. While the problems of balancing change from species to species according as the locomotor adaptation is made to arboreal, terrestrial or intermediate modes of life, the demands for equilibration remain essentially the same throughout the primate order. This undoubtedly is due to the fact that although the equilibratory requirements of arboreal life may recede as the animal approaches nearer to a strictly terrestrial habit of living, these are replaced by new requisites for balancing induced by the gradual assumption of the erect posture, by bipedal and ultimate plantigrade locomotion.

That tarsius and mycetes should exeed other primates in the size of their vestibular areas is due to the fact that they have added to their balancing equipment certain ancillary mechanisms essential to peculiarities in their locomotor specializations. Such specializations are conditioned by the prehensile tail or saltatory locomotion.

In their general evolutional significance, the vestibular nuclei do not contribute so striking an example of progressive unfolding as is the case with many other structures. Nevertheless, their high specialization in mycetes is worthy of note. They reveal to what extent the development of a plastic character like the prehensile tail may influence so fundamental a function as that regulating the equilibrium of the body. The absence of such a tail in lemur, tarsier and marmoset seems to make this differential element all the more signifreant in the evolutional sense.

\section{v. The Cerebellar Nuclei and the Nucleus Ruber in Relation to Coordination}

Several relatively prominent cell groups are recognized and distinguished as cerebellar nuclei. They include the nucleus dentatus, nucleus emboliformis, nucleus globosus and nucleus fastigii. The globosal and fastigial nuclei belong 
to what may be called the medial cerebellar division. The nucleus dentatus and nucleus emboliformis, occupying a more lateral position, are regarded as constituents of the lateral cerebellar lobes. In the preceding descriptions attention has been directed particularly to the mucleus globosus and the nucleus dentatus. Reconstructions of these structures have indicated how difficult it is to make intrinsic distinctions between the medial and the lateral nuclei. For this reason the dentate nucleus has been taken to include both the nucleus dentatus and the nucleus cmboliformis, while the nucleus globosus includes the globosal and fastigial nuclei.

The division of these nuclei into a medial and lateral group has much functional significance. The lateral group is in large measure representative of the appendicular muscles of the body, that is, the upper and lower extremities. That this group represents the appendicular muscles exclusively is not the case; but the main expansions in the nucleus dentatus occur in response to the progressive differentiation of motor capacity in the arms and legs.

THE DENTATE NUCLEUS AND ITS CONCURRENT EXPANSION WITH THE CEREBELLUM AND CEREBRAL HEMISPHERES

Inasmuch as the dentate nucleus is the principal relay station for efferent impulses from the cerebellum, its significance in connection with this organ as a whole becomes correspondingly important. From the physiological point of view, it is fairly well established that conditions demanding greater coordinative control of the musculature have their structural response in definite expansions of the cerebellum. A comparison of different species of mammals discloses the fact that the central or vermal portions of the cerebelIum have participated less in such development than the lateral lobes. This is especially notable in the primates, in which the proportional size of the cerebellar hemispheres in relation to the vermis progressively increases in 
passing from the lowest apes to man. Concomitant with this increase of the lateral cerebellar lobes, there is an expansion in the dentate nucleus. The reason for this growth in the cercbellar hemispheres and the corresponding development of the dentate nueleus is found in the connections of the coordinating organ. The vermis of the cerebellum has a much more restricted connection than the lateral lobes. It is in communication by means of afferent spinocerebellar tracts with the spinal cord and by olivo-cerebellar fibers as well as vestibulo-cerebellar fibers, with the oblongata. The lateral lobes, on the other hand, although connected with the axial portions of the brain by olivo-cerebellar fibers, receive their main tributaries from the pallio-pontocercbellar fibers. These axons connect the lateral lobes of the cerebellum with the hemispheres of the cerebrum. In other words, the verm is of the cerebellum responds to such inllux of impulses as may arise from cerebellar representation in the spinal cord and oblongata; whereas the cerebellar hemispheres are responsive to more complex neural syntheses created within the cerebral cortex as well as in the oblongata. Impulses from these latter sources appear to require more expansive cerebellar receiving areas for their claboration than is afforded by the vermal cortex. It is apparent that the phyletic growth of the cerebral hemispheres has determined cerebellar expansion. In proportion as the cerebral cortex becomes more highly convoluted, the connections between the cerebral and cerebellar hemispheres entarge and the lateral lobes of the cerebellum expand correspondingly.

The case of the vermis is different. Its connections are exchisively segmental, that is to say, with the definitely segmented portions of the central axis. The lateral cerebellar lobes, although possessing certain segmental connections, establish their preeminent communications by means of suprasegmental fibers. These latter fibers arise in portions of the central nervous system which have developed as superstructures over and above the primordial segmented neuraxis. 
The dynamic significance of the suprasegmental divisions of the nervous system has already been mentioned in relation to the actual and potential expansions of the brain. Such expansions provide further opportunity for increasing the range and number of neural syntheses in the progressive adaptations of animal behavior. To find the dentate nucleus expanding concurrently with the lateral hemispheres of the cerebellum and the cerebral hemispheres reflects the tendency of this nucleus to participate in the progressive development of behavioral adaptation. For this reason, the nucleus may be accepted as a reliable index of the coordinative adaptability of the animal. Its size, proportions and definition may, to an extent at least, be taken to indicate the range and intricacy of behavioral adjustments of which an animal is capable.

Connections of the Dentate Nucleus. As the gateway of impulses passing out of the cerebellum, the nucleus dentatus has its major connection, by way of the supcrior cerebellar peduncle, with the red nucleus in the midbrain. This latter structure acts as an intermediate relay for the conduction of impulses through the axis to their various levels of distribution in the brain stem and spinal cord. The course of the fibers constituting the superior cerebellar peduncle need not be considered in detail further than to observe that by means of two major decussations, both of which occur in the midbrain, the final connection between the dentate nucleus and the muscles is ipsilateral. In this way, the dentate nucleus on one side distributes the impulses from the cerebellum to the musculature of the corresponding side of the body. Acting thus in the capacity of the chief efferent distributing station in the cercbcllum, the dentate nucleus is connected by means of cortico-dentate fibers with areas in the lateral lobes of the cerebellum as well as in the vermis. Syntheses of coordinating impulses arising in the cortex of the vermis and the lateral lobes thus make their way to the dentate nucleus and here find an outlet for their stabilizing influence over the muscles of the body. 


\section{THE NUCLEUS GLOBOSUS}

The nucleus globosus chiefly represents the central or vermal portions of the cerebellum. Functionally the rermis is more rigidly fixed and responds but little to the progressive expansions of behavior. It supplies coordinative control to the axial muscles which show considerably less adaptive variation than the appendicular muscles. These axial muscles determine primordial postural patterns of the body. From the phyletic standpoint their coordinative control is paleostatic. It has been present from the beginning of vertebrate organization when trunkal movements were the preeminent requisites of locomotion. The expansion of such trunkal movements, as behavior becomes progressively more complicated, is relatively small in comparison with that of limb movements. Hence the nucleus globosus shows no marked degree of development concomitant with the cerebellar lobes or the cerebral hemisphcres. In this respect it differs from the dentate nucleus.

\section{COMPARISON OF NUCLEUS DENTATUS AND NUCLEUS GLOBOSUS IN LOWER PRIMATES}

In general, the dentate nucleus appears most prominent in such animals as are possessed of the most highly differentiated forelimbs. These nuclear relations when applied to the lemur, tarsier, marmoset and mycetes are made clear in the planimetric and longitudinal coefficients as shown in the tables:

Planimetric Coefficients of the Nuclels Dentatus and Nucleus Globosus in Lemur, Tarsier, Marmoset and Mycetes

\begin{tabular}{|c|c|c|}
\hline Spccies & Nucleus Dentatus & Nucleus Globosus \\
\hline Lemur & .110 & .030 \\
\hline Tarsier & .059 & .037 \\
\hline Marmoset & .077 & .050 \\
\hline Mycetes & .130 & .032 \\
\hline
\end{tabular}


Longitudinal Coefficients of the Nucleus Dentatus and Nucleus Globosus in Lemur, Tarsier, Marmoset and Mícetes

\begin{tabular}{|c|c|c|}
\hline Species & Nucleus Dentatus & Nucleus GIobosus \\
\hline Lemur & .230 & .10 \\
\hline Tarsier & .180 & .10 \\
\hline Narmoset & .150 & .10 \\
\hline Mycetes & .150 & . 10 \\
\hline
\end{tabular}

Although the differences in these species are not so striking as in the higher primates, they are sufficient to indicate that an evolutional process has impressed its influence upon the chief cerebellar nuclei.

\section{THE NUCLEUS RUBER}

The nucleus ruber of the midbrain, which is the principal relay station between the cerebellum and the spinal cord, is particularly sensitive to eerebellar expansion. It thus offers a reliable index of augmentation in coordinative control over the muscles. Since the red nucleus receives impulses directly from the nucleus dentatus, the increments in the former should be proportional to those in the latter. In other words, if there does occur an actual increase in the coordinative control arising from the cerebellum both the dentate and the red nuclei should show increments of expansion in their coefficients pari passu. That such is not the fact, either in the lower primates or in the primate series as a whole, calls for explanation. In comparing the red nucleus in the lower primates, there is a progressive rise from the lemur to mycetes. This increase is so pronounced as to leave little question of a progressive expansion in this nuclear structure of the midbrain. The longitudinal coefficients likewise show a corresponding increment in the nucleus. 
These coefficients are shown in the accompanying table:

Coefficients of the Red Neclels in tue Loner Primates

\begin{tabular}{|l|cc|}
\hline & Species & \multicolumn{2}{c|}{ Planimetric Longitudinal } \\
\hline Lemur & .012 & .090 \\
\hline Tarsier & .025 & 160 \\
\hline Marmoset & .044 & 150 \\
\hline Mycetes & .081 & .140 \\
\hline
\end{tabular}

LACK OF STRICT FUNCTIONAL PARALLELISM BETWEEN

THE DENTATE NUCLEUS AND THE NUCLEUS RUBER

One fact, however. is not sat isfactorily explained by these data; namely, that the increment of the dentate nucleus in these species does not vary pari passu with the increment of the red nueleus. This disparity probably indicates a lack of strict functional parallelism between the dentate nucleus on the one hand and the nucleus ruber on the other. Such disparity may be explained by certain fibers arising in the red nucleus and passing forward through higher levels to the suprasegmental portions of the axis. There is also some evidence to indicate that eertain desending fibers from the region of the corpus striatum connect the latter body to subadjacent aggregations of gray matter in the nucleus ruber. This nucleus may, therefore, represent a composite relay station into which enter impulses not only from the cerebelhum, but certain others from the corpus striatum as well. Thus, insofar as the functional significance of the red nucleus is concerned, variations in the increment of its expansion are perhaps less important as bearing upon the function of coordination than those of the dentate nucleus. It may be regarded, however, as collateral evidence in retation to this function, and is not without value in estimating the progressive changes going forward in the cerebellum. 
i. The Postile Nuclei in Their Relation to the Regulation of Skilled Movemexts, Particularly in the Upper Extremity

\section{THE PONS VAROLII AS AN INDEX OF INTELLIGENCE}

A statement to the effect that the pons Varolii may be held as an index of the intelligence possessed by an animal has found considerable acceptance. Allowing for a degree of exaggeration inherent in all such axiomatic formulations, it may be safe to say that the pons Varolii does to a large extent indicate the proficiency attained in the power, range and complexity of skilled performances. A structure, therefore, providing direct indications of such specializations would de facto represent the extent to which the intelligence has been developed.

The size of the pons Varolii varies conspicuously in the different orders of mammals. Being primarily a mammalian strueture, it has its fowest representation in those animals which have least developed the use of the fore or hind legs. Its highest development occurs in those animals in which the forelimbs have become freed from the function of earrying the body, and are employed for purposes other than those of locomotion. Thus, while in monotremes and edentates the pons Varolii is small to the point of being almost negligible, in primates and most particularly in man, it reaches its greatest dimensions. The physiological substratum underlying this variation in size is defmite and significant. In the execution of any learned skilled performance, it has been shown that there is need for the encurrence of two simultaneous streams of innervation. In the first place, the design, pattern, extent and duration of the act must be devised and directed; its incentive must be constructed and its limitations prescribed. While the stream of nerve impulses constituting this synthesis is being built up and distributed to the effector structures, a sccond stream must run parallel with it in order to maintain the proper coordination of the muscles. That the incentive syn- 
thesis to any such skilled or learned performance has its origin in many areas of the cerebral cortex is the generally accepted opinion of the present day. According to this view each specialized area of the neopallium may, and probably does, participate in the formulation of this incentive synthesis. Kinesthetic sense, general body sense, vision, hearing, the sense of smell and even of taste, together with certain higher discriminative faculties imparting the elements of judgment, all contribute to this composite body of impulses which finally combine to form an incentivesynthesis. The genesis of thesecond of the two concurrent streams is of equal importance. Each major functional region of the cerebral cortex-such, for example, as the frontal, the parietal, the temporal and the occipital lobe gives rise to a group of fibers which, becoming collected, enter the corona radiata, thence pass through the internal capsule, into the cerebral peduncle, and ultimately end in the pons Varolii. These are known as the pallio-pontile fibers. Their several subdivisions are specifically indicated as the fronto-pontile, parieto-pontile, temporo-pontile and occipito-pontile contingents. Each contingent by means of synapses in a large nuclear mass of the pons Varolii, the pontile nuclei, gains ultimate connection with the lateral lobes of the cerebellum.

Those animals with small-sized lobes of cerebral cortex contribute correspondingly small contingents to this pallio-ponto-cerebellar system. In consequence, not only the number of fibers entering into the pons from this source, but the size of the pontile nucleus necessary to rclay them therein, is correspondingly small. Hence, the animal equipped with a small cerebral cortex must of necessity have a small pons Varolii. From the functional standpoint, the smallness of the pons is indicative of an animal poorly equipped in the more complex varieties of skilled learned performances. For these reasons the size of the pontile nuclei may also be employed as a reliable index in estimating the degree of expansion in the cerebral cortex, and, from such estimation, in arriving at an opinion as to the degree to which that animal 
has developed its skilled performances. No more cogent indicator of these specialized functional capacities may be obtained in the brain stem. It is, perhaps, even safe to say that of aII the structures in this part of the central nervous system, none is more reliable or important than these nuclei lodged in the pons Varolii.

COMPARISON OF PONTILE NUCLEI IN LOWER PRIMATES

In looking to this structure, therefore, for evidence of evolutional unfolding, a comparison of the lower primates gives the impression of a progressive development in this nuclear group of the pons. Such a structural expansion is also well borne out physiologically by the increment manifested by these animals in their acquired learned reactions, and more particularly those performances executed by means of the upper extremities.

A tabulation showing the planimetric coefficients of the pontile nuclei in lemur, tarsier, marmoset and mycetes is appended. From it, a progressive expansion is readily apparent, mycetes having nuclei nearly twice the size of lemur. There may be some question concerning the relatively high pontile differentiation manifested by the marmoset, an animal known to possess a manual differentiation quite inferior to that of lemur. In spite of this apparent discrepancy, there is a strong probability that the entire family of Hapalidae employ the upper extremities in a much more hand-like manner than is true of the lemurs. Hence, while structurally the hands in the marmo-

Coefficients of the Pontile Nuclei in the Lower Prinates

\begin{tabular}{|lcc|c|}
\hline & Species & Planimetric & Longitudinal \\
\hline Lemur & .055 & .270 \\
\hline Tarsier & .057 & .330 \\
\hline Marmoset & .095 & .230 \\
\hline Mycetes & .03 & .350 \\
\hline
\end{tabular}


set have not attained the degree of specialization that characterizes lemur or mycetes, they still have developed sufficient capacity for complex skilled performances to necessitate relatively extensive pontile nuclei.

\section{THE PALLIO-PONTO-CEREBELLAR STSTEM}

By means of the relay already referred to in the pons, the pallio-pontile system is continued into the cerebellum. This entire collection of fibers constitutes what is termed the pallio-ponto-cerebellar system. As these fibers take their origin in the cerebral cortex, they are spread out much in the manner of a fan, becoming convergent as they approach the internal capsule and cerebral peduncle. In their termination, also, they agin spread out in relation with the lateral lobes of the cerebellum. Thus, the pallio-pontocerebellar system has in relation with it two conspicuous fan-like radiations: one at its origin in the cerebral cortex, another in its termination in the lateral lobe of the cerebellum. This is a morphological fact of much significance, clearly indicating as it does the many regions of the cerebral cortex which are comnected with all areas of the lateral lobe of the cerebellum. Furthermore, as the cerebral hemisphere increases in size and prominence during the course of evolutionary expansion, this pallio-ponto-cerebellar system likewise gains in volume. It contains a greater number of fibers in those animals possessed of large hemispheres with well-defined lobation than in those species in which lobation is more inconspicuous. It follows that in proportion as the number of fibers arising in the cerebral cortex is large, so also are the pontile nuclei.

Functional Significance of Pallio-ponto-cerebellar Fibers. Clinical and pathological conditions have not as yet yelded satisfactory evidence concerning the functional significance of these pallio-ponto-cerebellar fibers. The reason for this deficiency is not far to seek. Lesions in the cercbral hemisphere near the surface of the cortex where these fibers take origin, in 
the corona radiata, or in the internal capsule where they become convergent toward the cerebral peduncle, are not discrete enough to produce exclusive involvement of the pallio-pontile axons. Thus it is that no pure disturbance involving this system has yet been observed. Either the motor system or some part of the sensory system is simultaneously implicated by the lesion, and so adds elements which raise doubts as to the specific function of the pallio-ponto-cerebellar fibers. Experimental lesions are complicated by similar difficulties. Neither of these sources of information has, therefore, contributed as much as might be desired in revealing the functional activities of this system of axons. On the other hand, the increase of this system in animals capable of highly complex performances bespeaks a function which has a capacity for expansion directly proportional to the evolutional development of the cerebral hemispheres.

The origin of these fibers in the cerebral cortex, their relay in the pons Varolii, their termination in the lateral cerebellar lobes, declare them to be of utmost importance in some phase of neural activity whose mechanism ultimately depends upon the cerebellum. This mechanism at the same time has as its essential coadjutor the controlling influence of the cerebral cortex. Whether it be finally decided that the functional capacity of the pallioponto-cerebellar system is primarily in the interest of coordination for the performance of complex skilled acts, or whether it is shown that there exists some other reciprocal relation between the cerebellum on the one hand and the cerebral cortex on the other, is a question for further investigation to decide. In either case the pontile nuclei serve as a relay for a great system of fibers arising in the neopallium. They show progressive expansion in proportion to the evolutional development of the endbrain. Such expansion goes hand in hand with the acquisition of more complex, more numerous, more varied voluntary performances, capable of producing a greater continuity in action. That motor activities characterized by such qualities as these are 
most conmonly seen in those animals which have gamed comparative freedom of the upper extremities from the offices of locomotion, is obviously true without further proof. As a corollary to the supposition that the pontile nuclei increase in proportion as the animal becomes more capable in its skilled performances, it is true that these nuclei also expand as greater manual differentiation is attained. It may therefore be held that the progressive increment in this important group of nuclei bears definite relation to the increasing capacities acquired as the forelimbs become more precisely capable of manual function.

\section{vii. The Colliclli of the Midbrain in Their Relation to Sight AND HEARING}

THEIR PRIMORDIAL EMINENCE IN ALDITORY AND VISUAL FUNCTIONS

The history of the mesencephalon indicates that this portion of the brain has presided over many important functions during the development of the phylum. It quite as clearly demonstrates the waning predominance of this part of the brain as evolution proceeded. Not the least important among the earlier functions of the midbrain are those connected with the special senses of sight and hearing. One portion of the midbrain has become so highly specialized for visual function that it has earned for itself in many of the lower forms of vertebrates the title of optic lobe. Thus in the fish, in the amphibia, in the reptiles and in the birds the optic lobe is one of the most conspicuous parts of the entire brain. Similarly, a more caudal portion of the roofplate of the midbrain beame highly differentiated in connection with the function of hearing. But one of the most striking marks of progress as the brain found opportunity to provide for more ample syntheses in the different sensory spheres is the manner in which the midbrain has lost its ancient prestige. Those extensive areas developed in relation to sight and hearing 
have gradually been deprived of their morphological prominenee. In the primates they appear as inconspicuous elements covered by the greatly expanded endbrain.

In viewing the mesencephalon and its two sets of colliculi, the fact of their primordial eminence in auditory and visual functions must not be overlooked. Nor must it be considered a sudden departure from an old arehitectural design that these two areas of the brain have passed into relative insignificance. It was by the gradual delegation of visual and auditory functions to other regions that the midbrain became progressively reduced. Apparently the object of this delegation of former power to other areas was to enlist the activities of a more promising region. There was need of sufficient expansion to accommodate the rapidly growing demand for more complex associations in the realms of sight and hearing. The portion of the nervous system which has shown the chief propensity in this direction is the telencephalon. It is the last of all the brain constituents to make its appearance in the process of individual as well as phyletic development. In the endbrain there are special regions known respectively as the auditory or temporal area and the visual or occipital area, which have largely taken over the functions of hearing and of vision.

\section{COMPARISON OF THE INFERIOR COLLICULI OF LOWER PRIMATES}

Yet in spite of this assumption of functional activity on the part of the cerebral hemispheres, the midbrain has not altogether given over its original autonomy. A comparison of the lower primates, such as lemur, with man clearly reveals this fact. Even a comparison of elosely allied lower primates indicates that to some extent at least the progressive delegation of function from the colliculi to the endbrain is in process. The inferior colliculus, connected with hearing, shows in its planimetric coefficients a definite decline almost as striking in its longitudinal coefficients. 
Coefficients of the Inferior Colliculi in the Lower Primates

\begin{tabular}{|c|c|c|c|}
\hline & Species & Planimetric & Longitudinal \\
\hline Lemur & & 223 & 210 \\
\hline Tarsier & & 230 & 1 to \\
\hline Marmoset & & 210 & 200 \\
\hline Mycetes & & .182 & 190 \\
\hline
\end{tabular}

Coefficients of the Superior Colliclli in the Lower Primates

\begin{tabular}{|c|c|c|c|}
\hline & Species & Planimetric & Longitudinal \\
\hline Lemur & & .140 & 200 \\
\hline Tarsier & & $.33^{7}$ & 290 \\
\hline Narmoset & & .154 & 130 \\
\hline Wricetes & & 161 & 130 \\
\hline
\end{tabular}

This would seem to signify that the auditory function in passing from the lower to the upper end of the primate order has tended to lose much of its immediate reactive force; in other words, auditory impulses transmitted to the central nervous system become progressively more in need of the supervision by higher synthetizing areas. In these newer cortical areas they are more complexly associated, more extensively evaluated. Apparently the need of immediate reflex reactions in response to auditory stimulation is less important to the higher members of the primate group than to the lower species. Thus a sudden sound coming into the lemur's field of consciousness immediately provokes a motor response in the interest of defense or escape. The same sound arising in the auditory sphere of man is first subjected to more extensive associational review before action is determined by it. The difference in behavior manifested by these two types of reaction indicates that a greater number of alternatives are possible to man than to such a low form of primate as the Iemur. In the latter, the quick response to auditory stimuli would undoubtedly prove more to its advantage than a course of 
action in which the stimuli are first submitted to a process of deliberation and selection.

The decline of the superior or visual colliculus is more comspicuous in the planimetric coefficients than that of the inferior colliculus; and furthermore the longitudinal coefficients of the superior colliculus show a definite decrease in myeetes if compared either with the lemur or tarsius. The inference seems fair that the superior colliculus in tarsius is vested with more visual function than is the ease with the other lower primates here discussed. The decrease, therefore, in prominence of both the inferior and superior colliculus, al though it is not strikingly shown in the coefficients, must be acecpted as marking the inception of that gradual process whose final consummation is seen in the conditions observed in man. These two midbrain structures, even within the circumscribed limits of the primate order, disclose a most illuminating record regarding the evolutional process which has taken place in the brain stem. Unlike other structures heretolore considered, their evidence speaks in favor of waning function, of a steady delegation of this function to other expanding portions of the brain. For those who may wish to realize more clearly how this part of the midbrain reveals the course of evolution, a review of the conditions in the mesencephalon of the lower forms of vertebrates is especially recommended. Here may be seen the huge optic lobes of the fish, the bird or the reptile. Their size compared with the relatively insignificant colliculi of primates speaks strongly in favor of an evolutionary deflorescence which reaches its fmal stages in the human brain.

\section{vili. The Oculomotor Decussation in Relation to Binocular Vision}

Another notable feature in the mesencephalic portion of the brain stem is the internuclear connection between the two main cell groups in the oculomotor nucleus. This nucleus, involved as it is in the regulation of oculomotor 
movements, affords the final common pathway for all ocular innervation with the exception of the superior oblique and the external rectus muscles. The important feature concerning the internuclear connection of this cell group arises from the fact that in the higher grades of the primate series, the nuclei of the two sides are most extensively connected by means of commissural and decussating fibers. From the functional standpoint this interrelationship between the nuclei denotes an increasing capacity for coordinating the nerve impulses arising in the two nuclear groups. Thus, the oculomotor nucleus which is provided with the least abundant intercommunication between the groups on the two sides would, in comparison with a similar nucleus having an extensive intercommunication, be capable of far less conjugate control of the muscles moving the two eves.

RELATION OF CLOSE INTERNUCLEAR CONNECTIONS TO BINOCULAR VISION

The serial increase in this internuclear connection appears to be attended by an increasing degree of binocular vision possessed by the animal. The establishment of such vision primarily requires that the visual axes of the two eyes shall be capable of maintaining the primary position of Listing, that is to say, in parallel. Also for the purposes of near vision with close focusing they should have the capacity of convergence to a certain degree at least. These two positions of the visual axes, in parallel and in convergence, depend upon the cooperative action of the oculomotor muscles of the two eyes. There must be a simultaneous adjustment in the contractural tension, not only in holding the gaze fixed upon distant and nearby objects, but in moving the eyes through horizontal as well as vertical ares.

The more closely, therefore, the oculomotor nuclei of the two sides are interrelated by means of connections between them, the more likely is such harmony of action to be obtained in the oculomotor movements. Conse- 
quently, the presence of a rich internuctear communication vouchsafes the more accurate conjugation of the visual axes in the interest of binocular vision.

The Nucleus of Perlia. The late Dr. John Hunter has demonstrated that a special medial group of cells, the nucleus of Perlia, develops especially in the interest of ocular convergence. The nucleus is small or absent in animals possessing little or no attainment of binocular stereoscopic vision. It is present in tarsius but in that species is much smaller than in the higher primates whose vision is effectively stereoscopic. The phyletic significance of this nucleus of Perlia corresponds closely with that of the interoculomotor decussation.

\section{EFFECT OF DENELOPMENT OF STEREOSCOPIC YISION}

The development of stercoscopic vision has profound cffects which far exceed the limits of visual sensibility alone. It actuafly produces a decisive influence upon the development of motion. It particularly affects those movements which depend for their exccution upon the fine, minute and precise skilled acts employed in performances acquired and directed by vision. There are certain acts which may be acquired only if vision is capable of directing and furnishing the proper spatial relations for their guidance. Such a highly skilled performance as taking aim at a distant mark could be acquired and executed only with visual assistance. Acts in which a definite aim or direction is a prerequisite, although the distance from the eyes may be but slight, also require a similar degree of visual supervision.

COMPARISON OF THE VISUAL FUNCTION OF THE LOWER PRIMATES

A great number of skilled performances depend, therefore, upon the function of vision. They are often rendered impossible or defective if for any reason they are deprived of visual guidance. The largest number of these 
skilled acts pertain to those performanees demanding the greatest manual dexterity, such, for example, as handwriting, carving, painting and the use of instruments, implements and tools. Many skilled performances of a much simpler nature are also dependent upon vision for their acquisition and retention. This fact may be discerned in the infant learning to grasp, hold and manipulate objects. It is, however, only as these manual activities become more complex, more exacting in their precision, that the full degree of visual regulation is demanded by them. The advantage of stereoseopic vision in producing perspective and proper distance relations then becomes indispensable. In other words, stereoscopic vision should be regarded as a fundamental contribution to the upbuilding of the most highly complex performances. Even in the fower primates this tendency is clearly observed, as the coefficients of the oculomotor decussation show. Thus, in the lemur, whose eyes are widely separated and in which the need of extensive stereoscopic vision is not pronounced, the oculomotor decussation occupies but 16 per cent of the entire oculomotor nucleus. Only a relatively small portion of this aggregation of cells is brought into close internuclear relation. This fact accords with the relatively limited degree of skilled performanees of which these animals are capable. In tarsius it was impossible to estimate the extent of the decussation by mensuration. The animal possesses a certain degree of binocular vision which, however, is probably not stereoscopic.

Marmoset, on the other hand, shows a more extensive intermuclear connection. Its longitudinal coefficient of the oculomotor decussation is 38 per cent, a fact which is quite in keeping with the closer relation of the eyes and a greater range of finer movements. It would seem, therefore, in this comparison, that binocular vision is more essential to the marmoset than to the lemur.

A still more striking difference is apparent in the mycetes, the oculomotor decussation of which occupies 69 per cent of the entire length of the 
oculomotor nucleus. This at once indicates a group of nuclei which have much closer internuncial relation by means of the decussating and commissural fibers. Mycetes exceeds lemur, tarsius and marmoset in its range of acquired skilled movements. It has a much more hand-like disposition in the motor adaptations of its forclimbs. The tabulation of the longitudinal eocfficients of the oculomotor decussation is:

Longitudinal Coefficients of Oculomotor Decissation in Lower Primates

\begin{tabular}{|c|c|}
\hline & Coefficient \\
\hline Lemur & 160 \\
\hline \multicolumn{2}{|l|}{ Tarsier } \\
\hline Marmoset & 380 \\
\hline Mycetes & 690 \\
\hline
\end{tabular}

\section{The Important Factor in Progressive Development}

\section{of Behavioral Activity}

If the progressive development in behavioral activity traced through these lower primates could be attributed to a single factor it might be ascribed to an increase in discriminative sensibility. The evidence of such an increase is apparent in comparing lemur, tarsius, marmoset and mycetes. The influx of new volumes of sensory stimuli from such an organ as the developing hand or ineident to the progressive differentiation of the prehensile tail could not fail to introduce new powers for the direction of motion. It would inevitably expand kinesthetic memories and associations. By enriching the entire field of somesthetic sensation it supplies the basis for new motor activities. This expansion in discriminative sensibility is not limited to those impulses arising from the somesthetic receptors alone. Vision and hearing have likewise undergone a similar extension. The combinations and intercombinations among these several types of sensory perception thus 
provide dynamic material for a progressively widening sphere of behavioral performance.

The physiological substratum of such sensory augmentation may be seen in increments to the rolitional control of manual movements. It is also witnessed in the expansion of coordinative regulation whose structural representation is seen in the close interrelation between the cerebellum and the great fields of sensory perception in the cerebral cortex. It is apparent in the progressive development of coordination for simultancous movements of head, eye and hand, structurally indicated by increase in the inferior olivary nucleus. The increment in the oculomotor decussation introduced for binocular and stereoscopic vision renders still more exact those skilled movements made possible through the influx of new sensory impressions. It leads directly to the upbuilding of more extensive kinesthetic associations.

The dynamic incentives determining all of this expansion may not yet be discerned with clearness. If, however, some motive were presumed to direct the structural design of this process, its goal might well be that instrument with which to dominate the enviromment most effectively, such for example as the hand. 
PART II

THE INTERMEDIATE PRIMATES 



\section{INTRODUCTION TO PART II}

$\mathbb{V}$ ALID objections may be urged against the establishment of such a group as the intermediate primates upon phylogenctic or paleontological grounds. But there are substantial reasons for this distinction because of cert ain likenesses in these brains. It should be understood, however, that such similarities as do exist in the encephalon of this intermediate group do not constitute an entirely satisfactory basis for the far-reaching generic associations which this grouping would seem to imply. They offer convenient criteria for distinguishing between those forms possessed of brains having relatively the most simple design, and those which have become more complex in their constitution.

The intermediate primates comprise all of the old-world monkeys with the exception of the three great man-like apes. According to this classification, the Hylobatidae or gibbons fall into the intermediate class. This group also comprises all of the members of the great family of Lasiopygidae, which includes the baboons, mandrils, the macaques and cynocephalous monkeys, the mangabeys, gucnons and langeurs. The most forcible objection which might be urged against the inclusion of the gibbons in this group is their many striking anthropoid characters. A survey of the central nervous system of these primates, however, would seem to justify the view that they are in many respects more primitive than the man-like apes. In size of brain and in configuration of fissural pattern, the gibbons come much nearer to the lasiopygidal species than they do to the higher anthropoids.

The species of intermediate primates selected for description include Papio cynocephalus (Cynocephalus babuin), one of the large dog-headed baboons, Pithecus rhesus (Macacus rhesus), the common macaque of India, and Hylobates hoolock, the gibbon. 
Many features in the behavior of the gibbon are noteworthy and raise the animal to a plane somewhat above that of the other intermediate primates. In hylobates the brachiating type of locomotion has replaced the pronograde specialization of lower primates. Swinging from the branches by the fong arms has obviously produced a greater erectness of the body in the gibbon than is true of any other intermediate primates. The gibbon also is able to stand, walk and even rum in the erect posture. This hylobate erectness therefore appears to be the first step toward the manlike specializations of bipedal locomotion and bimanal differentiation. These facts warrant the recognition of the Proanthropoid Stage as represented by the gibbons. 


\section{Chapter X}

\section{PAPIO CYNOCEPHALUS, THE COMMION DOG-HEADED BABOON, ITS BRAIN AND BEHAVIOR}

Its Position among the Primates; Measurements and Brain Indices; Surface Appearance of the Brain; Internal Structure of the Brain Stem in Cross Section

THE genus Papio comprises all of the cynocephalous or dog-headed baboons. In general, these animals possess a massive body, and the

1 adult is remarkable for its great strength. As a group they are considered the lowest of the old-world or Catarrhine monkeys. Because of their large size and great strength they are looked upon as dangerous animals particularly as they have aggressive, ugly natures.

\section{Appearance of the Baboon}

The face and head of the baboon are dog-like and elongated; the nostrils have a canine disposition. The tail is variable in Iength but never prehensile and sometimes only rudimentary. It is usually carried with a curve near the basal extremity arching away from the body, the remainder of the tail hanging straight downward. All species of papio possess callosities or fleshy pads about the buttocks which in some instances are of large size and brilliantly colored, the coloring being intensified especially in the femates during the mating season. At such times these callosities in the females may increase in size so that they cover nearly the entire ghuteal region. Along the rostrum of certain species there develop bony ridges elevated nearly to the level of the eyes. These ridges are most common in the males and the skin over them is brightly colored. In the mandril the skin over the ridge is of a bluish color, while the skin on the bridge of the nose is red. This coloring adds considerably 
to the almost repulsive and unattractive countenance of the animal. The fore- and hindlimbs are of nearly equal length. The species run on their hands and feet with the eyes direeted downward so that they are obliged to elevate

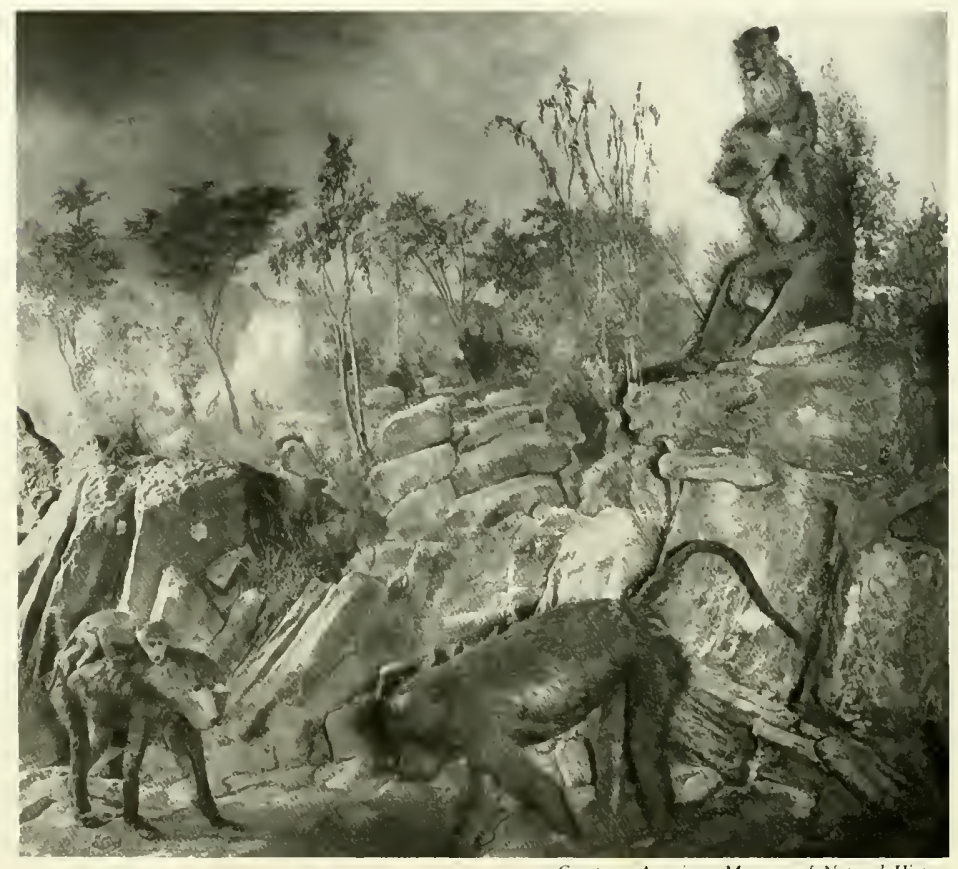

Courlesy. American Museum of Nalural Historv

FIG. I 32. HABITAT GROUP. BABOON AT THE WATER HOLE.

the large, overhanging eyebrows in the attempt to look upward and forward. The palms of the hands together with the soles of the feet are laid flat upon the ground in walking and rumning. The thumb is long and prominent, reaching to the middle of the first joint of the forefinger. The neck is also relatively long, and cheek pouches are present. 
The Baboon's Habits in the Wild State and in Captivity

These apes are gregarious and usually go in large herds. Often as many as a hundred individuals gather together in one herd. Beeause of their large

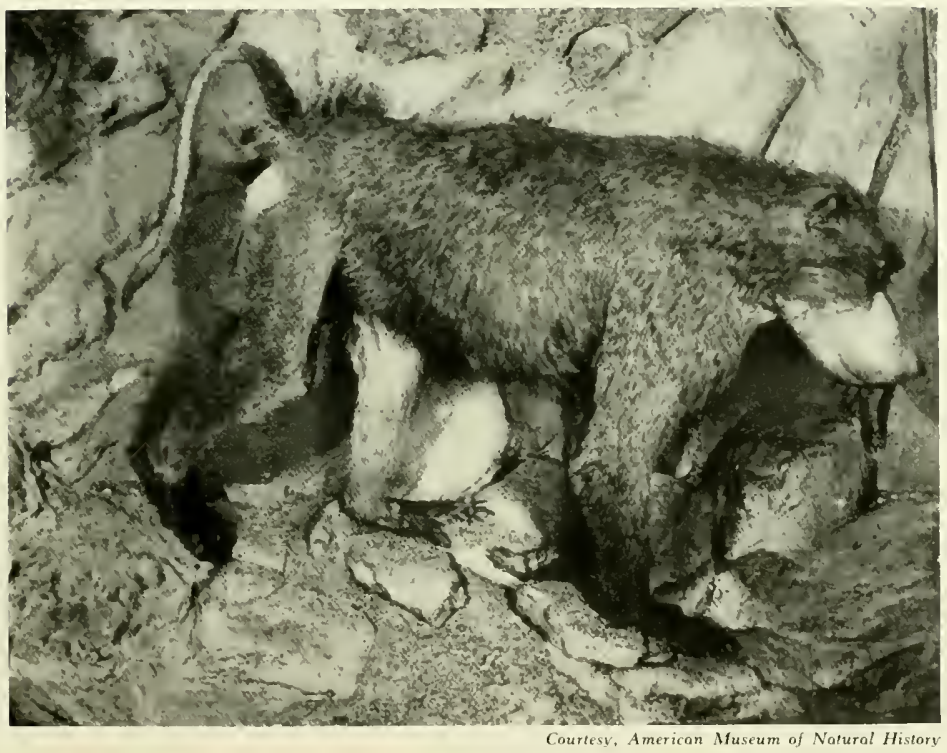

FIG. 133. PAPIO CYNOCEPHALUS (BABOON).

number and aggressive dispositions, they become formidable antagonists especially when irritated or disturbed. They have eanines which, being long and sharply pointed, are capable of inflicting dangerous and severe wounds. They appear to possess a series of articulate sounds, various in kind, resembling barks, grunts or even sereams. Sometimes they make low and subducd murmurs, imparting to them various inflections which are comprehended at onee by members of the herd. Often the slightest murmur of warning made by one member of the herd will determine a eourse of aetion on the part of all 
the rest. This is especially true in determining flight when the animals are engaged either in pillage or mischief. Occupied in such operations as this, an outpost is usually set in some favorable point from which to give notice of the
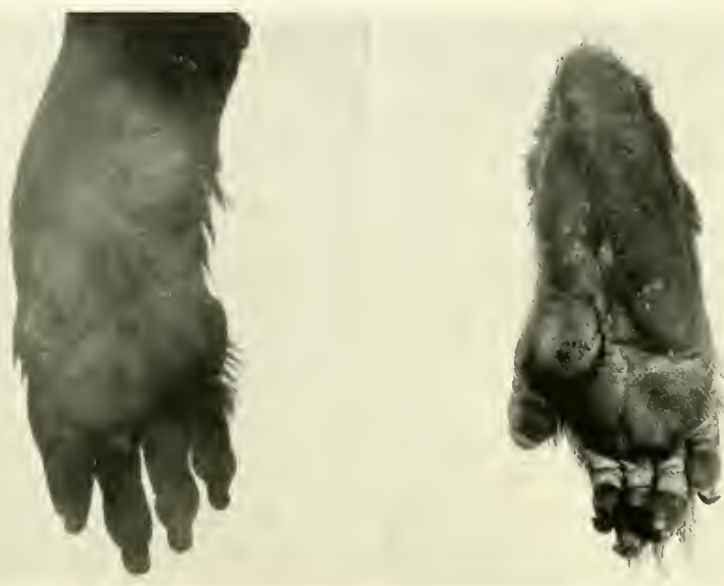

Courtesy, American Museum of Natural History

FIGS. 134 AND 135. HAND AND FOOT OF PAPIO CYNOCEPHALUS.

Left. Palmar surface of the hand showing broadening of the palm with a hect-like development in the hypothenar eminence, shortening of the digits including the thumb. These changes are in adaptation to terrestrial life.

Right. Plantar surface of the foot showing shortening of the twes, broad ball of the foot and narrow heel, modifications for terrestrial lucomotion.

approach of a foe and thus enable the marauders to escape.

These baborns inhabit rocky places such as ravines, erags, or hilly promontories where grass and trees are scanty. Their favorite abodes are usually in such places as are surrounded by wide plains which enable them to lie in wait for an opportune moment to commit some pillaging exeursion on a garden or field and at the same time to have all opportunity to escape on the approach of danger. They are much given to malicious mischief of this 
kind, for which reason they are both feared and despised by the inhabitants of the country which they infest. If attacked, they often turn upon their pursuers and in some instances inflict serious wounds upon their assailants.
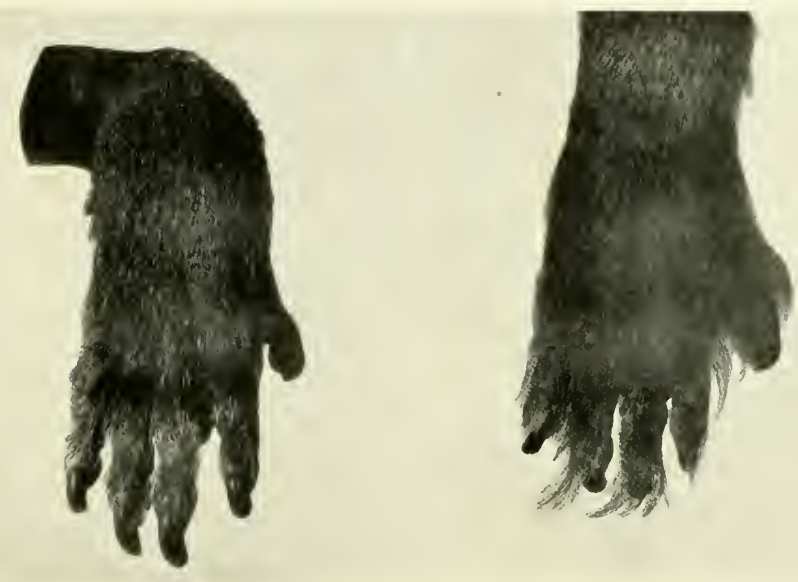

Courtesv, American Museum of Natural History

FIGS. 136 AND 137. HAND AND FOOT OF PAPIO CYNOCEPHALUS.

LEFr. Dorsum of hand showing shortening ofthe digits including the thumb, an evident adaptation tolocomotion upon the ground.

Right. Dorsum of foot, showing short toes, tong toe nails, evidences of terrestrial adiptation.

Some species of papio, however, prefer to live in the dense forests and $\operatorname{cimb}$ readily even in the loftiest trees. Those which live in the more open country show a marked agility in clambering among the rocks and gaining inacessible heights or positions with safety. The baboon is practically omnivorous although its chief food consists of roots, fruits, reptiles and insects. To procure these they are continually searching and turning over stones underneath which the desired objects of their quest may lie concealed.

In captivity the animals are both surly and unfriendly; even those born 
in captivity and reared thus are more difficult to approach and much less teachable than most of the other apes. They are as vindictive, treacherous and unlikeable because of their disagreeable dispositions as they are repellent to look at because of their more or less grotesque appearance. In teachability they show none of that quick adjustment which is observed in most of the other monkeys. This fact, combined with their general tendency toward savage reactions, has carned for them the reputation of being the lowest of the old-world monkeys.

Their manual dexterity is limited to a marked degree, due probably to the fact that the forelimbs are used in locomotion more than in any other function. It is probable that their vision is less endowed with stereoseopic, binocular adjustments than is true of the apes who carry the body in such a manner that the head is for the most part in an upright position. The baboons seldom assume the erect position even when supporting the body with the forclimbs upon some adjacent object. They do, however, sit upon their haunches in a somewhat crouched position but not nearly so freely as many of the other genera of Lasiopygidae.

\section{Geographical Distribltion}

The genus Papio is confuned almost exchsively to northern Africa and Arabia. The species investigated in this study is Papio cynocephahus, also spoken of as Cynocephatus babuin. The yellow baboon's habitat is Mediterranean Africa, Nubia, central and castern Africa.

\section{Measurements and Indices of Papio Cynocephalus}

Individuals of this species vary considerably in size and some of the older males are as large as members belonging to the dark-colored baboons. In general, the body length is about 1500 or $1600 \mathrm{~mm}$. The tail is from 700 to $730 \mathrm{~mm}$. long. 
Occipito-nasal length of the skull ........ I-3 $\mathrm{mm}$.

Interparietal width............... $62 \mathrm{~mm}$.

Width of the brain case........... $87.3 \mathrm{~mm}$.

The dimensions of the brain including the cerebellum and brain stem are:

Longitudinal . . . . . . . . . . . . . . $90 \mathrm{~mm}$.

Transverse (temporo-parietal or intertemporal).. $70 \mathrm{~mm}$.

Total weight of the brain.............. 12-. $\mathrm{gm}$.

Total water displacement.............. 12j.o c.c.

Neight of the forebrain (including cnd-brain..... $109.0 \mathrm{gm}$.

and interbrain)

Weight of the midbrain............. $2.2 \mathrm{gm}$.

Weight of the hindbrain.............

Upon the basis of these figures, the following encephalic indices have been computed for the several divisions of the animal's brain:

Forebrain index................ 83.0

Midbrain index................. 3.0

Hindbrain index................. I 4.0

The forebrain index of 83 assigns the baboon to that class of animals possessed of a brain which indicates a fairly well-advanced manual development.

\section{Surface Appearance of the Brain in Papio Crnocephalus} LOBES AND FISSURES

The brain is definitely gyrencephalic in type. The lobation is distinct, the demarcation between the frontal and parietal lobes being clearly drawn by a prominent fissure of Rolando (Fig. I38). This fissure leaves the superior longitudinal fissure almost at right angles, much in the manner of the 
cruciate fissure in lower mammals. It has a slight obliquity forward, however, approximating the general angulation of this fissure with the superior longitudinal fissure as seen in the higher anthropoids and man. The frontal lobe
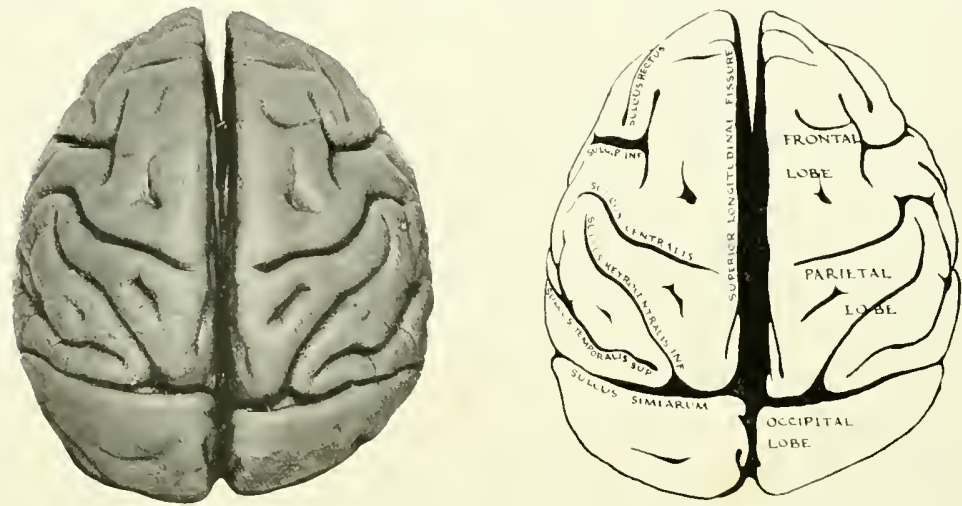

FIG. 138. DORSAL SLRFACE OF BRAIN, PAPIO CINOCEPIIALUS. [Actual Length $77 \mathrm{~mm}$.]

hey to Diagram. sulc. P. INF., Sulcus Precentralis Inferior; Sulcus retrocentralis inf., Suleus Retrocentralis Inferior; sulcus tempokalis sup., Sulcus Temporalis Superior.

stands out sharply because of its marked orbital concavity into which the orbital plate of the frontal bone projects. A well-defined fissure of Sylvius extends backward and upward, more oblique in its general direction than that seen in the lower primates. It demarcates a well-defined temporal lobe the tip of which projects forward for a considerable distance, giving it particular prominence in front of the horizontal limb of the fissure of Sylvius. A well-marked and long sulcus simiarum separates the parietal from the occipital lobe, thus completing the topographical outline of the major hemispheral lobes on the convexity of the brain. The convolutions and fissures are richest and most complex in the parietal and temporal lobes, and least pronouneed in the frontal lobe. The entire surface configuration and the arrangement of the fissures produce the impression of a gyrencephalic pat- 
tern of moderate simplicity, in which, however, may be discerned all the characters which later are more perfectly outlined in the higher anthropoid hemispheres.
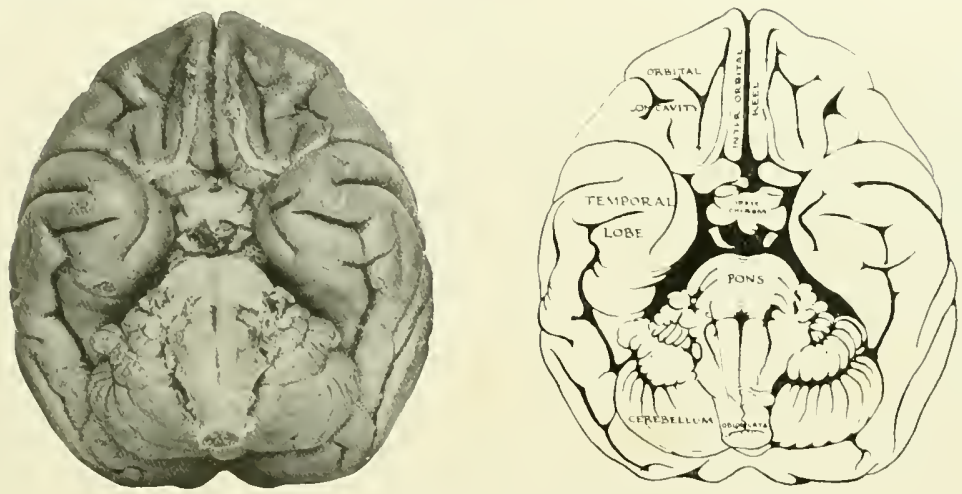

FIG. 139. BASE OF BRAIN, PAPIO CYNOCEPHALUS. [Actual Length $77 \mathrm{~mm}$.]

ORBITAL AND CEREBELLAR CONCAVITIES

As already indicated, the orbital concavity is a prominent feature, as is the case in all lower primates. Its inner margin is formed by a marked interorbital keel, along which the olfactory tract and bulb extend, the tract being readily detachable up to the olfactory trigone. The incipience of a gyrus rectus may be discerned in the beginning of an olfactory sulcus. The angulation of the chiasm with the tracts is typical of the primates. The cerebellar concavity is especially deep and most pronounced in the median line caudal to the splenium of the corpus callosum.

THE ENDBRAIN AND OTHER STRUCTURES

The basal surface of the endbrain, in its convolutional pattern, shows a distinct but somewhat feeble design typical of this region in the Anthro- 
poidea in general. The orbital surface of the frontal, the sphenoidal surface of the temporal and the occipital surface of the occipital lobe all have indications of the typical fissures found in these regions. Several deep
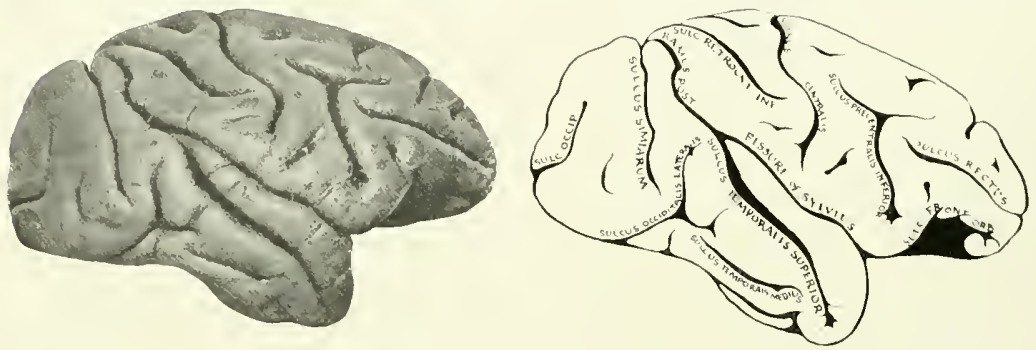

FIG. I fo. RIGHT LATERAL SURFACE OF BRAIN, PAPIO CYNOCEPHALLS. [Actual Length $89 \mathrm{~mm}$.]

Key to Diagram. Ramus post., Ramus Porterior of Sulci Temporali Superior; sulc. Front. ORB., Sulcus Frontalis Orbitalis; sulc. OCcip., Sulcus Occipitalis (Inferior); sulc. Retrocnt. INF., Sulcus Retrocentralis Inferior.

annectent gyres may be brought to light by the separation of the Sylvian fissure as well as the presence of a faintly outlined series of radiating gyres constituting the island of Reil. One deep anncetent gyre is found midway between the two extremities of the sulcus semilunaris.

\section{THE CEREBELLUM}

The cerebellum in Papio is characterized by the relatively large size of the vermis which as yet manifests little tendency to lose its surface prominence. The tentorial surface is sharply gabled, the vermis rising as a marked median ridge-pole. The interfolial sulci are directly continuous without fissural interruption from vermis to lateral lobe. On the occipital surface two paramedian sulci separate the vermis from the lateral lobes, although the former have a marked prominence on this surface. The petroso-ventricular surface presents three elements: (I) a high elevated portion of the supcrior vermis, (2) the ventricular portion, and (3) the petrosal area. 
THE BRAIN STEM

Numerous features upon the sereral surfaces of the brain stem in baboon make this portion of the central nerrous system stand out in
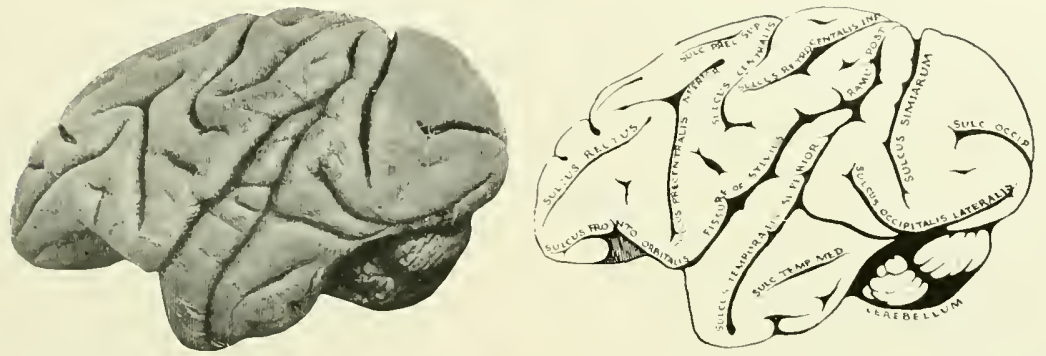

FIG. $1+1$. LEFT LATERAL SURFACE OF BRAIN, PAPIO CYNOCEPHALIS. [Actual Length $89 \mathrm{~mm}$.]

Key to Diagram. Ramus post., Ramus Posterior of Sulcus Temporalis Superior; sulc. occip., Sulcus Occipitalis (Inferior); sulc. Prec. Sup., Sulcus Precentralis Superior; sulcus Retrocentralis iNF., Sulcus Retrocentralis Inferior; sulc. TEmp. med., Sulcus Temporalis Medius.

greater prommence than is the case with any of the lower primates.

The Овlongata. The oblongata presents a well-marked intermediate sulcus and two ventrolateral sulci upon its ventral surface. Two well-defined pyramidal elevations lie upon either side of the ventromedial sulcus at its cephalic extremity; while the pyramids themselyes are separated from two well-defmed inferior olivary eminences by the ventrolateral sulci. The pyramids, as in other forms, taper caudally as they approach the lower extremity of their decussation, and in this area a faint indication of interlacing bundles interrupting the ventral sulcus may be seen, indicating the position of these crossing fibers. The relative prominence of the pyramidal elevations as well as the olivary eminences, as compared with the lower primates, signifies a progressive increase in the dimensions of these structures, and thus probably bespeaks some extension in the functions with which these two 
structures are concerned. In other words, the cortico-spinal connection for the transmission of voluntary impulses shows expansion in the baboon as compared to the lower forms. The relative increase in the size of the
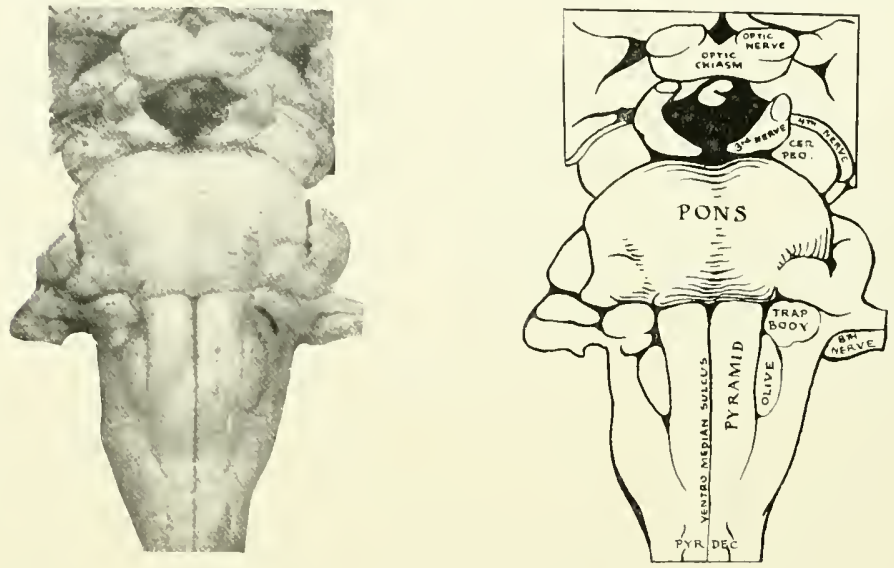

FIG. I f2. IENTRAL SLRFACE OF BRAIN STEM, PAPIO CINOCEPHALUS.

[Actual Length $46 \mathrm{~mm}$.]

Key to Diagram. Cer. Ped., Cerebral Peduncle; Pyr. dec., Pyramidal Decussation; trap. Body, Tianezoid Body.

inferior olive points to an expansion in the reflex control of simult aneous movements of eyes, head and hands as well as in all skilled, learned performances.

The Povs Varolil. On the ventral surface of the oblongata, at its cephalic extremity, there appears the usual expansion of the neuraxis, the pons Varolii. A sharp line of cemarcation separates the oblongata from the pons, the bulbopontile sulcus. A small blind recess at the point where the ventromedian sulcus meets the bulbopontile sulcus, forms the foramen caecum posticum. From the sulcus between the inferior olive and the pyramid, fubers of the twelfth nerve emerge from the oblongata. At the cephalic extremity of the pons is the optico-peduncular space bounded in front by 
the optic chiasm and optic tracts and caudally by the convergent fibers of the cerebral peduncles. In this space is lodged the tuber cinereum, bearing the attachment of the infundibular stalk and the mammillary bodies.
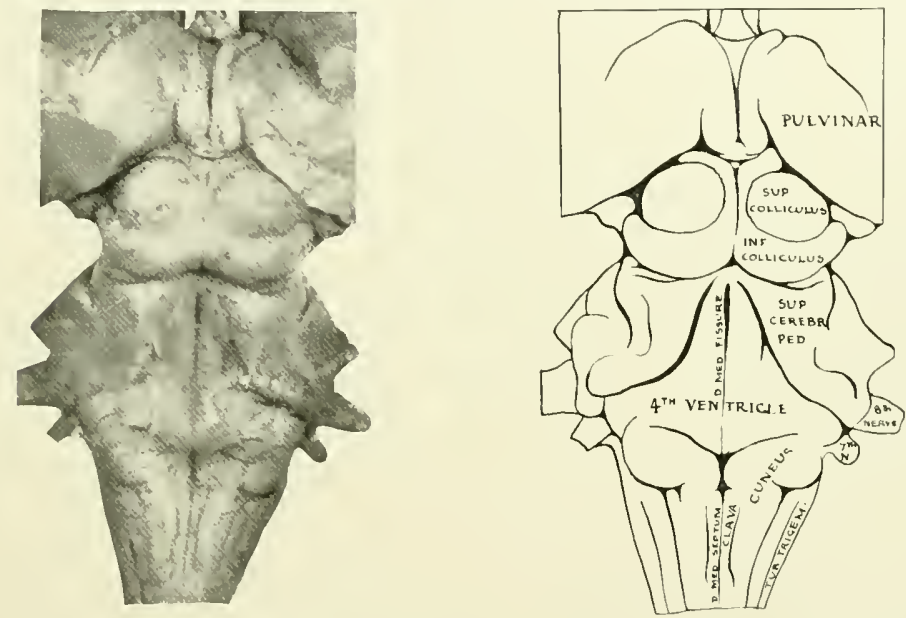

FIG. I +3. DORSAL SLRFACE OF BRAIX STEM, PAPIO CYNOCEPHALLS. [Actual Length $46 \mathrm{~mm}$.]

hey to Diagram. D. Med. Fisstre, Dorsomedian Fissure: D. Med. Septen, Dorsomedian Septum; iNf. Colliculus, Inferior Coilliculus; Sup. CEREbr. PED., Superior Cerebellar Peduncle; sup. COlliculus, Superior Colliculus; тив. тRigen., Tuberculum Trigemini; 7 ти N., Seventh Nerve.

A well-defined median groove along the ventral surface of the pons marks the position of the basilar artery. The pons itself has attained more prominence in baboon than in the lower primates, and this may be taken as indicative of extensions in the pallio-pontile connection, in turn signifying an increase in the cerebral cortex. Since it is by means of the pons that ultimate connection between the cerebral hemisphere and the cerebellum is established, functionally, this connection as it is at present understood concerns itself with the coordination of the extremities. It is believed that 
the increased size of the pons thus indicates a greater capacity on the part of the animat to execute a wider range and a larger number of more highly skilled acts.

The Dorsal Aspect of the Brain Stex. Upom removal of the cerebellum, the dorsal aspect of the oblongata shows its two typical areas, the ventricular and the infraventricular regions, the ventricular area forming the inferior triangle of the fouth ventricle. In the infraventricular portion of the dorsal surface, the dorsomedian septum separates the two halves of the axis in the midline.

Upon either side of the dorsomedian septum, two eminences of considerable size appear. These eminences are the clava and the cuneus, which represent the projections caused by the presence of the dorsal sensory nuclei. Both of these eminences extend cephalad in the direction of the ventricular portion of the oblongata and gradually divaricate as the alar plates separate to expose the inferior angle of the floor of the fourth ventricle. This inferior angle of the ventricle is bounded by a fairly prominent clevation of the clava; while the cuneus is slightly more conspicuors. Both of these elevations decrease in prominence as they approach their cephalic extremities, and at the level of the lateral recess have disappeared as relief markings on the clorsal aspect of the medulla oblongata. The relative increase in development of the cuncus undoubtedly indicates accessions to sensory representation due to the further development of the forelimb and particularly the differentiation of the hand. The fact that the clava has not kept pace with the expansion of the cuneus bears out the lack of further sensory differentiation taking place in the caudal areas of the animal. Thus, although the hindlimb is highly specialized for purposes of locomotion and particularly of climbing either in trees or over rocky emincnces, the failure to develop a prehensile tail - and in fact, as in some species, to have no more than a mere caudal rudiment - reduces the influx of nerve impulses arising in the hinder 
portions of the animal. This influx is certainly much inferior in volume to that of such forms as are possessed of definitely prehensile tails, like mycetes, spider monkeys and woolly monkeys. The tail in baboon, when developed to any extent, has its importance as a steering or balancing organ, a function much less exacting than that requiring the discriminative elements of sensibility possessed by a prehensile organ.

Further cephalad, the dorsal surface of the axis presents the metencephalon and the cephalic continuation of the fourth ventricle, which is bounded upon either side by high elevations caused by the middle and superior cerebellar peduncles. In the floor of the fourth ventricle the markings are fairly conspicuous. The median sulcus extends from the obex to the beginning of the iter and is well defined. It is crossed at about its middle by marked striae acusticac entering the floor of the ventricle at the level of the lateral recess. The inferior triangle of the fourth ventricle contains a depression upon either side of the median sulcus indicating the position of the nucleus hypoglossus, Iateral to which the fovea vagi appears in plain outline. It is difficult to discern any specialization corresponding to the area postrema or the area plumiformis. In the cephalic triangle of the fourth ventricle the markings are less distinct, although immediately above the striae acusticae may be seen a slight elevation, the eminentia abducentis. There is no region in this portion of the ventricle which may be identified as the locus coeruleus. The elevations of the vestibular area are fairly well marked.

The midbrain along its dorsal aspect presents a characteristic quadrigeminal plate with the median sulcus intersected at right angles by the intercollicular sulcus. At the cephalic extremity of the median sulcus is the triangular depression, the fovea pinealis, in which is lodged the epiphysis. The quadrigeminal plates are well developed, the superior colliculi being 
larger in all diameters than the inferior colliculi, athough both of these sets of elevations as compared with the fower primates are relatively less prominent.

The Ventral Surface of the Midbrain. On the ventral surface of the midbrain are the two prominent bundles of fibers which constitute the cerebral peduncles which appear in marked retief. This increase in prominence as compared with the lower primates is due to the fact that the cerebral cortex is more highly developed in the baboon and supplies a greater number of fibers entering both the pyramidal and the pallio-ponto-cerebellar systems. This in itself has an important physiological bearing in that it signifies an increased wealth in skilled movements as well as an advance in the coordination of the animal's movements.

The Lateral Aspect of the Brain Sten. The markings upon the lateral aspect of the brain stem are fairly pronounced. In the oblongata two prominent eminenees may be seen on the lateral surface, the tuberculum acusticum and the tuberculum trigeminum, the latter being in less conspicuous relief than the former. The tuberculum acusticum merges with the corpus restiforme without a sharp line of demarcation. In the mesencephaton the mesial genieulate body forms a prominent elevation upon the lateral surface.

Internal Structure of the Brain Stem in Papio Cinocephalls

Many features already described in the surface relief of the brain stem acquire even more significant proportions in cross section. These sections in the brain stem of the baboon furnish a survey of the internal strueture at all of its most critical levels. In the discussions, the physiological significance of the several structures described will be considered bricfly, since their general significance will be summarized at the conclusion of this part of the work. As in the case of the lower primates, it would be quite impossible to give in full detail all of the features contained in each one of the cross 
sections. It has been the purpose rather to select those structures which are more plastic in the evolutional sense and therefore show a greater range of morphological variability than the archaic, rigidly fixed portions of the brain stem. This makes necessary the omission of much description which might be desired by students requiring a general histological review of the various levels of the axis, and white such omission is to be regretted, the inclusion of more histological detail would so encumber the text as to make it needlessly burdensome.

LEVEL OF THE PIRAMIIA DECLSSATION (FIG. I 44 )

At this level the most conspicuous features of the oblongata are the crossing fibers of the pyramidal system ( $\mathrm{P}_{\mathrm{y} x}$ ) which have brought about the separation of the ventral gray column (Ven) from the central gray matter (Cen). The width of the pyramidal fascicles forming the decussation is relatively broad, giving the impression of a pyramidal tract with considerable capacity for voluntary control over the animal's musculature.

In the dorsal fields another feature of importance appears in the caudal extremity of the nucleus of Goll (NG). This nucleus lies immediately lateral to the dorsomedian septum and is surrounded by a dense bundle of fibers constituting the column of Goll (CG). This extensive freld of myelinized fibers arises from levels in the spinal cord particularly representing the dermatomes in the skin and the proprioceptors in the muscles, joints and bones of the lower extremities and tail. The column of Goll (CG) is separated from the next adjacent bundle of fibers, the column of Burdach (CB), by a fairly well-defined dorsal paramedian sulcus. The column of Goll is considerably less in its dimensions than the column of Burdach, warranting the inference that the influx of stimuli conducted by way of Burdach's fasciculus is of greater volume than that passing in by way of the 
fasciculus of Goll. The functional significance of this disparity in the size of the two columns becomes apparent when the relation of Burdach's tract with the upper extremity, and especially its manual portion, is recalled.

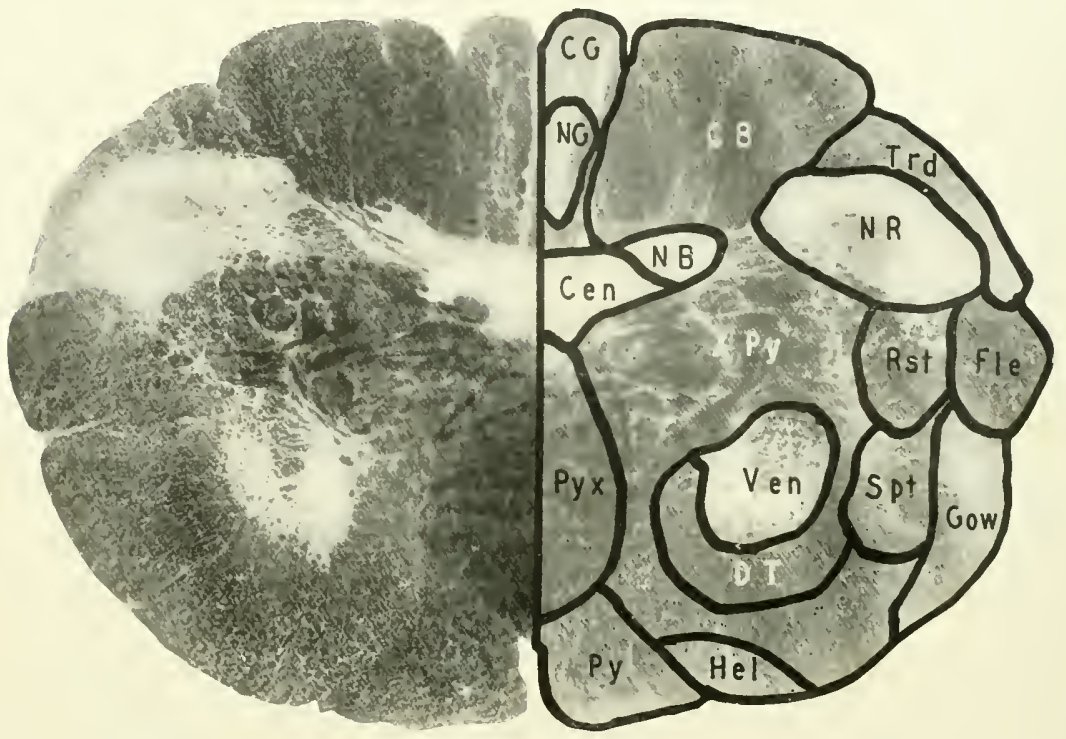

FIG. I 4 ४. BABOON. LEVEL OF THE PYRAMIDAL DECUSSATION.

CB, Column of Burdach; CEN, Central Gray Matter; CG, Column of Goll; DT, Deiterso-spinal Tract; FLE, Dorsal Spinocerebellar Tract; Gow, Ventral Spinocerebellar Tract; HIEL, Spino-olivary Tract of Helweg; NB, Nucleus of Burdach; NG, Nucleus of Goll; NR, Nuclens of Rolando; Py, Pyramid; Pyx, Pyramidal Decussation; RST, Rubrospinal Tract; SPT, Spinothalamic Tract; TRD, Descending Trigeminal Tract; VEN, Ventral Gray Colunn; xpy, Crosscd Pyramidal Tract. [Accession No. 150. Scction 5. Actual Size $10 \times 6 \mathrm{~mm}$.]

A collection of gray matter forming one of the most conspicuous elements in the dorsal fields at this level is the extensive substantia gelatinosa trigemini (NR) which acts as a relay station for all fibers bearing impressions from the trigeminal areas of the face and head. This area of innervation corresponds to the region anterior to the interparietal line. 
The influx of sensory impressions from this region depends upon the degree to which the head is used in directing the animal's locomotion and as an instrument for exploration of its environment. As a rule, animals provided with hair-covered facial areas and long vibrissae, such as the rodents and the cat family, are equipped with a large receiving center for sensory stimuli arising in this region of the body. In animals whose facial cutancous areas are more or less completely denude of hair, in which also the hand and fingers have more or less taken the place of the face for the purposes of directing locomotion, there is a tendency for the substantia gelatinosa trigemini to decrease in size. The substantia gelatinosa in the baboon is somewhat less conspicuous than it is in the several lower primates; and certainly much less prominent than in the carnivores or rodents. It is, however, more massive than in such forms as have come to depend almost exclusively upon the hand for the direction of their movements.

The entire dorsal field in this section, as in the case of the other species already considered, is significant as representing the complete discriminative sensory territory of the body. The portion of this field adjacent to the dorsomedian septum conveys impressions from the tail, lower extremity and lower portions of the trunk; the next succeding portion serves the upper trunk, arm and hand, while the most lateral portion represents the trigeminal areas of the face and head. It scems apparent in contrasting these three areas which serve as indices to the sensory influx from the main discriminative areas of the body, that the intermediate zone, or column of Burdach, is probably the largest of the three in baboon, thus strongly suggesting that the hand, forearm and arm have taken precedence from the standpoint of sensory importance over the other areas of the body.

Immediately lateral to the substantia gelatinosa, and lying upon the circumference of the axis, is a dense bundle of fibers representing the descend- 
ing tract of the fifth or trigeminal nerve ( $\mathrm{Trd}$ ). Other structures of interest at this level should be noted; among them, the ventral gray column ( Ven) cut off from its former attachment to the central gray matter (Cen) by the crossing fibers of the pyramidal system ( $\mathrm{P}_{\mathrm{yx}}$ ).

Dorsal to the ventral gray column are the scattered bundles of the pyramidal tract $\left(\mathrm{Py}_{\mathrm{y}}\right.$ ) as they are beginning to make their descent from the oblongata into the spinal cord. Passing through these scattered pyramidal bundles in a transverse direction are a few of the emergent fibers of the eleventh pair of cranial nerves, the spinal accessory nerve. Along the margin of the section is a narrow band of medullary substance, constituting the cireumferential zone. This consists of the major ascending spinocerebellar fibers ( Fle, Gow). Lying internal to the circumferential zone, bordering upon the ventral gray column, is the intermediate zone containing in the central portion among other tracts, the two descending Deiterso-spinal tracts (DT) and in its more lateral portion, the spinothalamic and rubrospinal tracts (Spt, Rst).

LEYEL OF THE CALDAL EXTREMITY OF THE NLCLEUS OF BURDACH (FIG, $14 j$ )

At this level an illustration is introduced to show more clearly certain nuclear details in the dorsal sensory freld. In this section, perhaps better than elsewhere, morphological specializations in the dorsal columns are evident. Thus in close relation to the dorsomedian septum is the nuclear mass constituting the nucleus of Goll ( $\mathrm{NG}$ ), lateral to which is the nucleus of Burdach (NB), and finally in the most lateral position, the substantia gelatinosa (NR). These three structures represent the relay stations for all the sensory afferent frbers coming from the head and neck, upper trumk and arm, lower trunk, leg and tail. Their preemption of the dorsal field gives the region its predominantly sensory character. On either side of 
the ventromedian suleus may be seen some of the crossing bundles of the pyramidal system and also some bundles prior to decussation (Pyx). A large mass of gray matter adjacent to the ventral gray and apparently

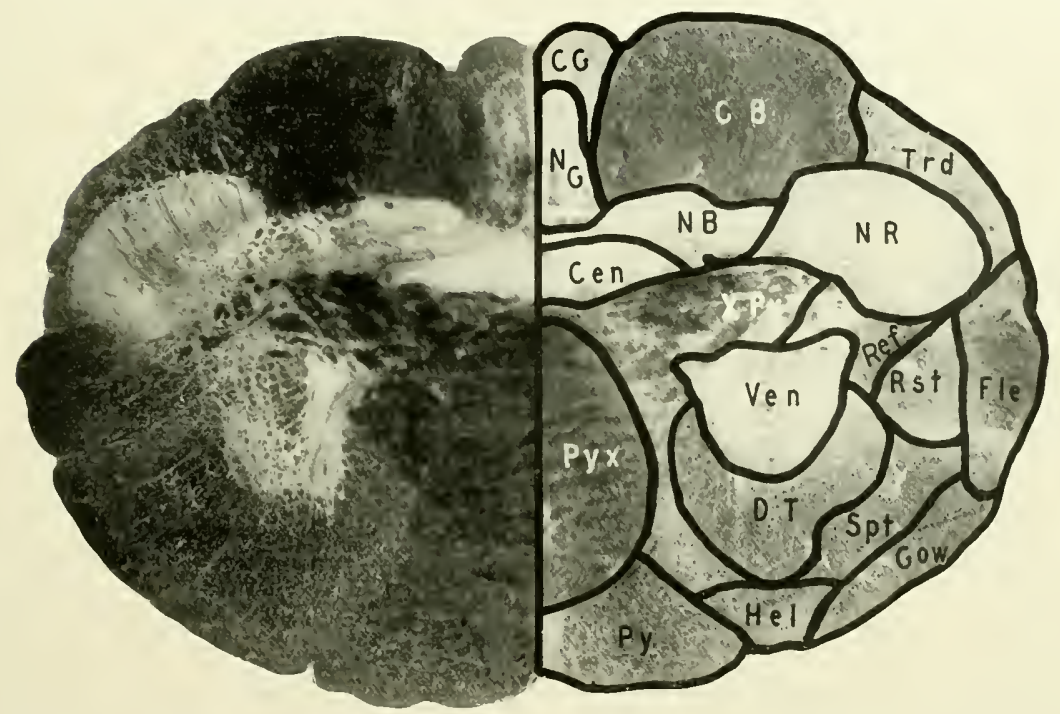

FIG. $14 \vec{j}$ BABOON. LEVEL OF CAUDAL EXTREMITY OF NLCLEUS OF BURDACH. CB, Column of Burdach; CEN, Central Gray Matter; CG, Column of Goll; DT, Deiterso-spinal Tract; FLE, Dorsal Spinocerebellar Tract; Gow, Ventral Spinocerebellar Tract; HeL, Spino-olivary Tract of I felweg; NB, Nucleus of Burdach; Ng, Nucleus of Goll; NR, Nucleus of Rolando: Py, Pyramid; pyx, Pyramidal Decussation; REF, Reticular Formation; RSt, Rubrospinal Tract; SPT, Spinothalamic Tract; TRD, Descending Trigeminal Tract; ven, Ventral Gray Column; xpy, Crossed Pyramida] Tract. [Accession No. 150. Section 25. Actual Size $12 \times 8 \mathrm{~mm}$.]

continuous with the substantia gelatinosa as well as the nucleus of Burdach, is the griseal portion of the reticular formation (Ref). Surrounding the reticular formation are the fibers constituting the intermediate medullary substance, and, upon the periphery of this area, the circumferential zone. 
LEVEL OF CAUDAL EXTREMITY OI INFERIOR OLIVARI NUCLELS (FIG. I 46 )

At this level the appearance of the cross section has undergone considerable change. The principal modification arises from the fact that a new mass of gray matter, hitherto not present, has made its appearance. This is the inferior olivary nucleus ( $\mathrm{IO}$ ) which lies dorsolateral to the fiber bundles comstituting the pyramid ( $\mathrm{Py}_{\mathrm{y}}$ ). Dorsal to the chief olive is a smaller mass of gray matter, the dorsal accessory olive, while dorsal to the pyramid and ventromesial to the inferior olivary nucleus are some transversely disposed fibers extending mesially toward the raphe. These are fibers of the mesial fillet (Mf) which have undergone decussation. The decussating fibers forming the mesial fillet constitute the internal areuate fibers, most of which take origin in the nucleus of Goll and form that portion of the fillet which here represents the lower extremity and tail. The pyramid ( $\mathrm{Py}$ ) now has the appearance of a dense bundle occupying the most ventromesial portion of the section. Its diameters, both transverse and antero-posterior, give it the proportion of about i to 8 of the entire section. This ratio furnishes some idea as to the relative importance of the pyramidal system in the regulation of motion, particularly if taken in reference to the corresponding system of fibers in the lemur where the proportion appeared to be about 1 to $I 0$. This increment in the pyramidal system signifies a functional increase in the regulation of roluntary motion.

The dorsal nuclei of Goll and Burdach ( NG, NB) are much increased in size as compared with the lower sections. The mucleus of Burdach (NB) appears to be considerably larger than the nucleus of Goll ( NG), especially if estimated in connection with a number of scattered accessory nuclei surrounding it and usually known as the lateral nucleus of Blumenau. This increase in the nucleus of Burdach still further bears out the impres- 
sion that the volume of sensory impulses reaching the central nervous system from the upper extremity is larger than that from either the head and face or the lower extremity and tail. The substantia gelatinosa (NR) is

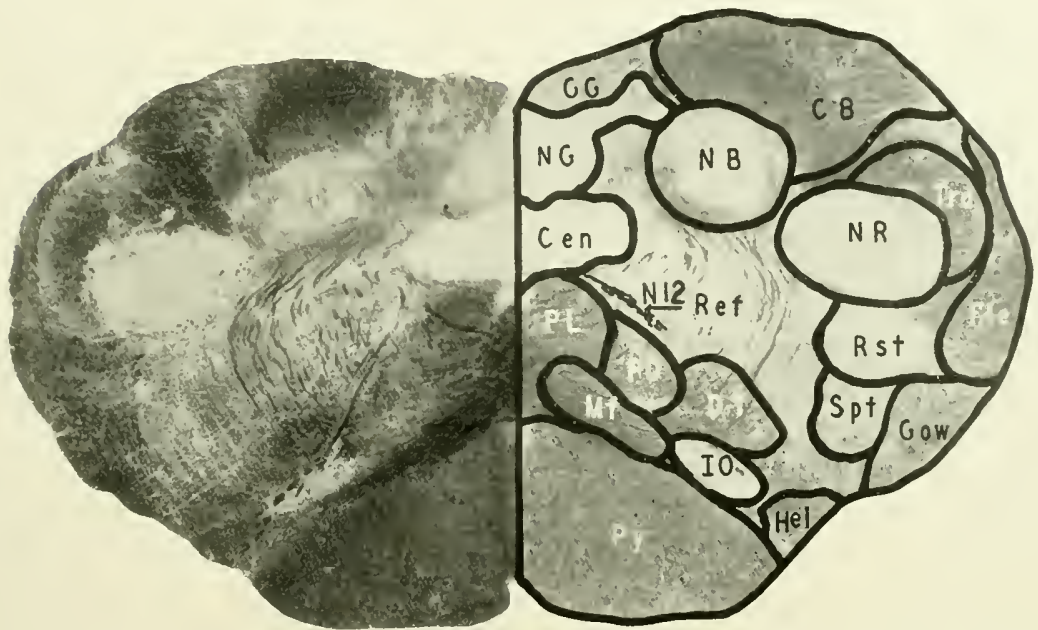

FIG. If6. BABOON. LEVEL OF THE CAUDAL EXTREMITY OF THE INFERIOR OLIVARY NUCLEUS.

CB, Column of Burdach; CEN, Central Gray Matter; CG, Column of Goll; DT, Deiterso-spinal Tract; Fle, Dorsal Spinocercbellar Tract; Gow, Ventral Spinocerebellar Tract; Hes, Spino-olivary Tract of Helweg; 10, Inferior Olive; MF, Mesial Fillet; NB, Nucleus of Burdach; NG, Nucleus of Goll; NR, Nucleus of Rolando; VI 2, Hypoglossal Nerve; PD, Predorsal Bundle; PL, Posterior Longitudinal Fasciculus; Pr; Pyramid; REF, Reticular Formation; RST, Rubrospinal Tract; SPT, Spinothalamic Tract; TRD, Descending Trigeminal Tract. [Accession No. 150 . Section 65. Actual Size $1.4 \times 8 \mathrm{~mm}$.]

surrounded by a large descending trigeminal tract ( Trd). Dorsal to the decussating fibers of the mesial fillet (Mf) and ventral to the central gray matter $(\operatorname{Cen})$ is an important bundle of fibers, the posterior longitudinal fasciculus (PL).

Ventrolateral to the central gray matter may be seen a large collection of gray substance constituting the griseal portion of the reticular formation 
( $R$ ef). This is penetrated by numerous internal arcuate fibers extending in Iong sweeping curves from the region of the nucleus of Burdach, forward, inward and backward, to enter the decussation of the mesial fillet. Passing diagonally through the reticular formation, and intersecting the arcuate fibers, are axons of the twelfth nerve ( $\mathrm{N}_{12}$ ) as they are making their way from the hypoglossal nucleus at the ventrolateral border of the central gray matter.

\section{LEVEL THROLGH THE MIDDLE OF THE INFERIOR OLIVE (F1G, I 47 )}

At this level the most conspicuous feature is the appearance of the somewhat irregular mass of gray matter lying in the ventral field of the section, but separated from the midline by the interposition of the pyramid ( $\mathrm{Py}_{\mathrm{y}}$ ) and the mesial fillet (Mf). This mass of gray matter is the inferior olivary nucleus (IO). It appears as a somewhat irregular, convoluted, saccular structure whose fundus is directed toward the periphery and causes a slight elevation upon the surface already referred to as the olivary eminence of the oblongata. Its hilus is directed dorsomesially; its cavity is filled with heavily myelinized axons. Into it and through it pass arcuate fibers, many of them in the direction of the restiform body on their way to the cerebellum. Ventromesial to the olive is a detached portion, the mesial accessory olive (VO), and dorsal to it a small detached portion, the dorsal accessory olive (DO). The significance of this large nuclear mass in the oblongata has already been discussed. Its probable relation to the function of controlling the simultancous movements of head, eyes and hand and of facilitating the coordination of all skilled learned performances has been commented upon and the reasons for ascribing such regulation to it have previously been given (see page 253). Its increase in prominence, both as to size and configuration, when compared with the lower primates, speaks for an expansion in the function over which it presides. 
The central gray matter $(\mathrm{Cen})$ is less extensive than it is in the preceding section. It now forms the floor of the widely opened fourth ventricle and contains in its rentromesial portion the nucleus hypoglossi (Nhy).

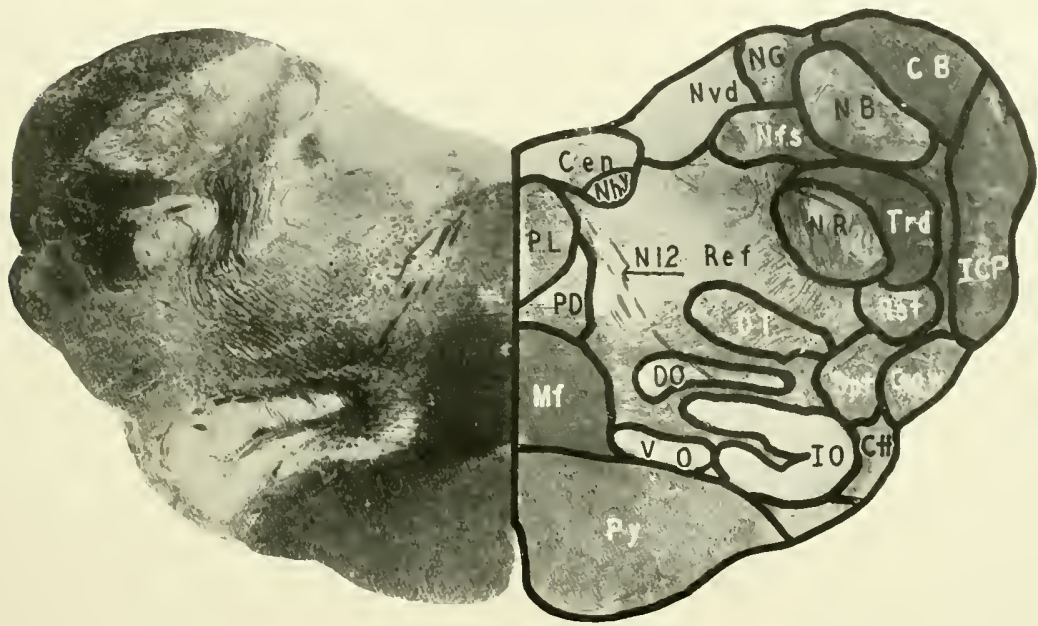

FIG. I 47 . BABOON. LEVEL THROUGH THE MIDDLE OF THE INFERIOR OLJVE.

CB, Column of Burdach; CEN, Central Gray Matter; Ctт, Central Tegmental Tract; Do, Dorsal Accessory Olive; DT, Deiterso-spinal Tract; Gow, Ventral Spinocerebellar Tract; ICP, Inferior Cerebellar Pcdunclc; IO, Inferior Olive; MF, Mlesial Fillet; NB, Nucleus of Burdach; NFs, Nucleus Solitarius; NHy, Hypoglossal Nucleus; NR, Nucleus of Rolando; NG, Nucleus of Goll; NVD, Dorsal Vagal Nucleus; NI2, Ilypoglossal Nerve; PD, Predorsal Bundle; PL, Posterior Longitudinal Fasciculus; Pr, Pyramid; REF, Reticular Formation; RST, Rubrospinal Tract; sPT, Spinothalamic Tract; TRD, Descending Trigeminal Tract; vo, Ventral Accessory Olive. [Accession No, 150 . Section 133. Actual Size $15 \times 6 \mathrm{~mm}$.]

Emergent fibers of the twelfth nerve ( $\mathrm{N}_{12}$ ) leave this nucleus from its mesial side passing ventrolaterally outward, many of them to penetrate the inferior olivary nucleus, finally emerging from the ventrolateral suleus of the oblongata. In the central gray matter ( Cen) lateral to the nucleus hypoglossi is the extensive dorsal nucleus of the vagus or pneumogastric nerve into which a number of entering fibers make their way ( $\mathrm{Nvd}$ ). 
Lateral to the dorsal nucleus of the pnemogastric nerve is a compact oval bundle constituting the fasciculus solitarius ( $\mathrm{Nfs}$ ). Some entering fibers of the pnemogastric nerve are seen approaching the fasciculus solitarius. This nucleus belongs to the communis system. It conveys deseending fibers, ultimately entering into relation with the nucleus of the fasciculus solitarius which is connected with the sense of taste. It also receives fibers from the glossopharyngeal nerve, supplying the tongue, and a large contingent from the pars intermedia of Wrisberg connected with the facial nerve. Ist phyletie constancy in the primates seems to argue that the sense of taste undergoes but little material alteration throughout this order of rertebrates.

Lateral to the central gray matter, but separated by a narrow strand of fibers, is the cephalic remmant of the nucleus of Goll (NG), which at this level is much attemuated. In a more lateral position is the mucleus of Burdach (NB). Ventral to the nucleus of Burdach is the substantia gelatinosa (N R) surrounded on its external surface by the dense bundle of the descending trigeminal tract ( $\mathrm{Tr}$ ). Oceupying a peripheral position in the dorsolateral aspeet of the oblongata is a bundle of fibers which has been assembled from several sururces and consists of axons, all of which are destined to enter the cerebcllum. This structure is the restiform body or inferior cerebellar peduncle (ICP). The griseal portion of the reticular formation (Ref) lies ventral to the central gray matter $(\operatorname{Cen})$ and is penetrated by many areuate fibers, most of which have their origin in the nucleus of Burdach (NB). These arcuate fibers pass forward and inward from the median line to decussate in the raphe and ascend as part of the mesial fillet ( $\mathrm{If}$ ). Some of these arcuate frbers pass through the inferior olivary mucleus. Immediately adjacent to the median raphe and ventral to the central gray matter is the posterior longitudinal fasciculus (PL) in front of which lies the collected mass of the ascending axons constituting the mesial fillet (Mf), while the dense bundles of axons comprising the pyramid ( $P_{y}$ ) lie 
ventral to the fillet. Ventrolateral to the griseal portion of the reticular formation is the intermediate medullary substance which contains the Deiterso-spinal tracts (DT) as well as the rubrospinal and spinothatamic tracts (Rst, $\left.S_{p} t\right)$. On the periphery of this region is a narrow band of fibers, the circumferential zone, containing the ventral spinocerebellar tract (Gow). Immediately contiguous to the ventrolateral surface of the inferior olivary nucleus is the well-defined bundle of the central tegmental tract $(\mathrm{Ctt})$ which connects this nuclear mass with structures in the midbrain.

\section{LEVEL OF THE VESTIBULAR NUCLEl (FIG, $1+8)$}

Here a prominent alteration takes place. This change involves the final disappearance of certain sensory elements which at lower levels are predominant features in the oblongata, with the substitution therefore of certain sensory elements having a totally different function. The latter come to occupy positions topographically identical with the sensory elements which they now replace. If the dorsal portion of the oblongata of the lower levels is notable for a single feature, it is the presence of the sensory relay stations which take up and pass on those stimuli for discriminative sensibility arising in the tail, in the extremities, in the trunk and neck. Having fulfilled their mission by thus providing for the complete relay of these sensory impulses, the nuclei of Goll and Burdach disappear in order to make way for other sensory receiving stations which transmit impulses from proprioceptive receptors of a highly specialized variety. These latter receptors are lodged in the semicircular canals, in the utricle and saccule of the internal ear. They are related to a function intimately concerned with the maintenance of body balance. Two large nuclei, collectively forming the vestibular area of the oblongata, thus replace the nuclei of Goll and Burdach.

The second nuclear mass, by the addition of which this level becomes notable, is situated in a stifl more lateral position and receives fibers also from 
the internal ear, but in this case, from the cochlear portion of it. It is intimately associated with the function of hearing. These collections of eells in the oblongata form the cochlear nuclei, one of which appears as the

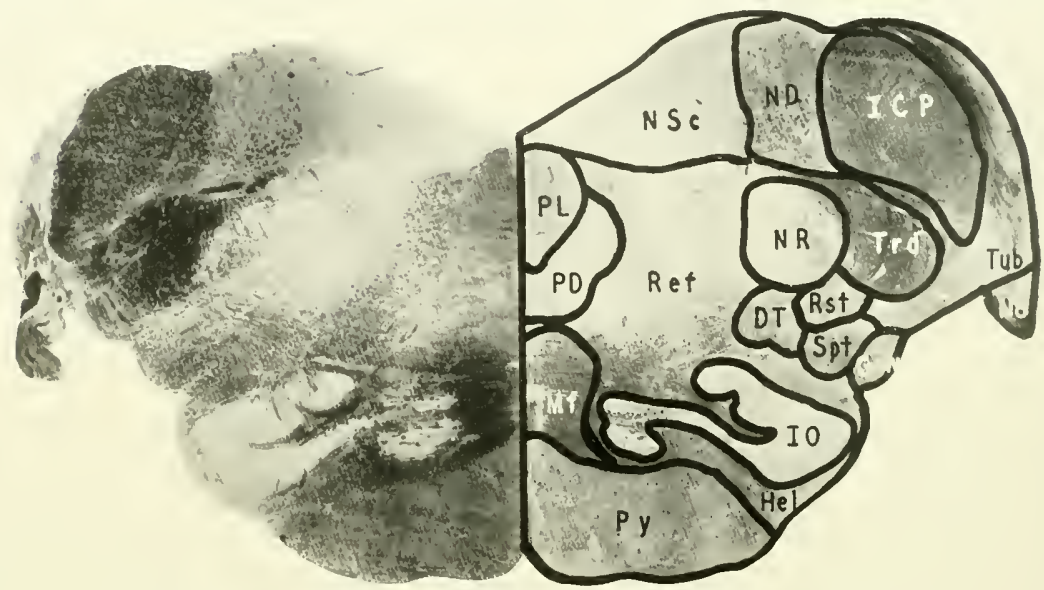

FIG. I 48 . BABOON. LEVEL OF THE VESTIBULAR NUCLEI.

DT, Deiterso-spinal Tract; Gow, Ventral Spinocerebellar Tract; HEL, Spino-olivary Tract of Helweg; ICP, Inferior Cerebellar Peduncle; וo, Inferior Olive; MF, Mesial Fillet; ND, Deiters' Nucleus; NR, Nucleus of Rolando; Nsc, Nucleus of Schwalbe; N8, Auditory Nerve; PD, Predorsal Bundle; PL, Posterior Longit udinal Fasciculus; py, Pyramid; Rer, Reticular Formation; RSt, Rubrospinal Tract; SPT, Spinothalamic Tract; TRD, Descending Trigeminal Tract; тUв, Tuberculum Acusticum. [Accession No. 150. Section 175. Actual Size I6 $\times 7 \mathrm{~mm}$.]

large protuberance on the dorsolateral aspect of the axis forming the tuberculum acusticum $(\mathrm{Tub})$. The vestibular nuclei comprise the triangular nucleus of Schwalbe (NSc) which oceupies a position in the central gray matter in the dorsal field immediately below the floor of the fourth ventricle; and a nucleus consisting of large cells dispersed among which are many bundles of axons, Deiters' nucleus (ND). Both of these vestibular nuclei receive fibers from the vestibular portion of the ear, and although the exact function of each nucleus is not adequately understood, pathological 
Iesions in and cxperimental disturbances of the two nuclear structures produce definite disturbances in equilibration. These two nuckei, therefore, have becn accepted as the principal relay stations for transmission of impulses neeessary to the maintenance of the proper balance of the body. They thus become of primary importance in relation to the balancing mechanism. Their size and general relations with reference to the rest of the section furnish some conception of the degree of balaneing function necessary to the animal in order to maintain its cquilibratory adjustments.

The dimensions of the restibular nuclei in the baboon indicate that its balaneing requirements have not increased materially orer those of the lower primates; in fact, the numerical estimation of the area occupied by these structures would show the vestibular nuclei to be somewhat smaller than in any of the species already considered. This may be due to the fact that the baboon carries on its life for the most part upon the ground, and with the exception of a few species is not a tree-living animal. It has not acquired the erect posture, and hence has placed no further obligations upon the balancing mechanism due to maintaining cquilibrium on two feet.

In the extreme lateral region of the medulla oblongata is a portion of the enlargement forming the tuberculum acusticum ( $\mathrm{T} u \mathrm{~b}$ ) into which enter fibers from the cochlear division of the eighth nerve. Immediately mesial to these fibers are some vestibular fibers which take origin in the proprioceptors of the vestibule of the internal car. These fibers pass inward and backward; some of them lateral to the descending tract of the fifth nerve, some of them actually penetrating this bundle to reach the nucleus of Deiters (ND). Situated between the tuberculum acusticum (Tub) and Deiters' nucleus (ND) is the large oval bundle of densely compacted neurons comprising the restiform body (ICP). In this bundle are assembled the ascending fibers from the spinal cord and oblongata on their way to the cerebellum. The fibers arise from several different sources and 
convey to the cerebellum afferent impulses necessary for the proper maintenance of cercbellar function. In this sense, the size of the restiform body (ICP) becomes significant as an index to the degree of aflerent influx received by the cerebellum. The size of this strueture in the baboon indicates an animal fairly well equipped in coordinative control, although by no means as abundantly supplied in this respect as many of the higher apes.

Ventral to Deiters' nucleus is a dense mass of descending fibers, the descending trigeminal tract ( Trd) and mesial to it, the substantia gelatinosa (NR). Ventral to the latter structure is a large diffuse mass of gray matter constituting the nucleus facialis for the innervation of the facial musculature. From this nucleus are seen streaming inward and backward a number of myelinated fibers in spray-like fashion toward the floor of the fourth ventricle. This is the first portion of the facial nerve.

The most ventral portion of the section is oecupied by the compact bundles of the pyramidal tract (Py) immediately dorsal to which is a collection of crossing fibers constituting the secondary axons of the auditory pathway.

\section{LEVEL OF THE CEREBELLAR NLCLEI (FIG. I 49 )}

At the level of the cerebellar nuclei several structures which afford a basis for estimating the functional capacity of the cerebellum make their appearance. These are the cerebellar nuclei. A portion only of the cerebellum is shown in this section. The organ itself consists of a central division, the vermis (Ver), and two lateral lobes or hemispheres ( $\mathrm{Cbl}$ ). The mesial portion of the lateral lobes, the medullary vestibule, is shown. Situated in this vestibule is an irregular, difluse collection of gray matter, the nucleus dentatus $(\mathrm{Ndt})$. In this nucleus arise most of the fibers leaving the cerebellum, thus providing the major efferent path for cerebellar impulses concerned in coordinative control of the muscles. The size and 
configuration of the dentate nucleus are therefore of significance as showing to what degree the cerebellum contributes to the function of coordination. This nucleus in lower primates is a diffuse mass of cells

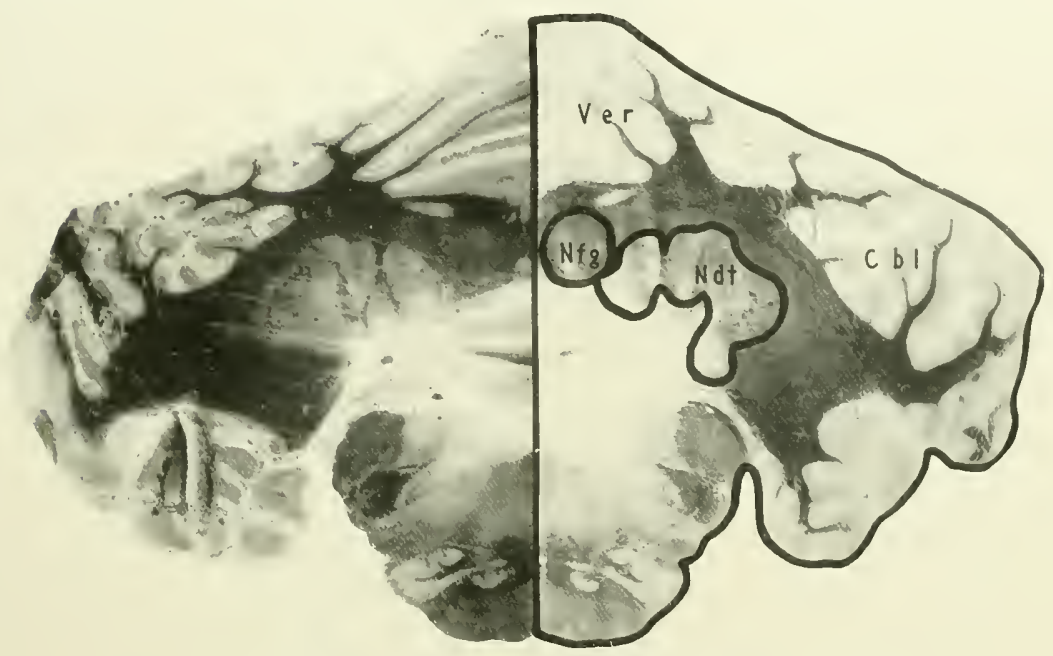

FIG. I 49. BABOON. LEVEL OF THE CEREBELLAR NUCLEI.

CB1, Cerebellum; ndt, Cerebellar Nuclei, Lateral Group; NFG, Cerebellar Nucici, Mlesial Group; ver, Vermis Cerebelli. [Accession No. 150. Section 163. Actual Size $37 \times 23 \mathrm{~mm}$.]

without convolutions, but in higher primates it is much convoluted and clearly circumscribed. The relatively small, irregular and non-convoluted nuclei of this group may be considered as primitive, since a comparative study of mammalia, or even of the primates, shows a distinct tendency for the dentate nucleus to become more indefinite in its outline and pattern, the Iower the animal's position in the scale. Thus, a dentate nucleus with little or no convolution belongs to an animal whose cerebellar function is limited insofar as it does not regulate a complex series of highly skilled acts, but is organized for the more simple muscular reactions, such as seen in the 


\section{THE INTERMIEDIATE PRIMATES}

quadruped type. The dentate nucleus of the baboon belongs to this latter group. It is typical of an animal with strong quadrupedal tendencies and possessed of no very wide range of highly skilled, learned motor performances.

Mesiaf to the dentate nucleus is another collection of nerve cells. This is the nucleus fastigii. The fibers arising and terminating in this nucleus in the main make their way by the juxtarestiform body to connections with nuclei in the floor of the fourth ventricle, especially those which receive impulses from the semicircular canals, utricke and saccule. The nucleus fastigii is in this sense a structure most intimately concerned with the function of balancing and may be regarded as one of the higher stations for equilibratory control. Its comparatively large size in baboon has a significance not unlike that of Deiters' nucleus, indicating a considerable need on the part of the animal for a highly organized balaneing mechanism.

The vermis of the cerebellum ( Ver) is primarily concerned with the coordinative control of those muscles of the body which surround the axis and are immediately adjacent to it. This part of the ccrebellum varies least phyletically, in this respect being quite unlike the lateral lobes which show in marked clegree a variability in size, in richness of foliation, in expansive tendency, all of which appear to bear a direct relation to the degree of functional capacity manifested by the upper and lower extremites.

\section{LEVEL OF THE EMERGENT FIBERS OF THE SIXTH NERVE AND THE CAUDAL FIBERS OF THE PONS VAROLII (FIG. IjO)}

At this level certain striking changes have occurred, more particularly those marked by the presence of many transversely disposed fibers crossing the ventral surface of the axis and forming the pons Varolii. Chief among these fibers, and the most ventral in position, are those forming the stratum superficiale pontis, dorsal to which are the scattered fasciculi of the pyramidal system $\left(\mathrm{Py}_{\mathrm{y}}\right)$. Dispersed among the bundles of the pyramidal tract is the 
caudal extremity of the pontile nuclei (PN). Another feature of this level is the gradual diminution in size of the fourth ventricle now approaching its cephalic extremity and about to join the aqueduct of Sylvius. Its roof is

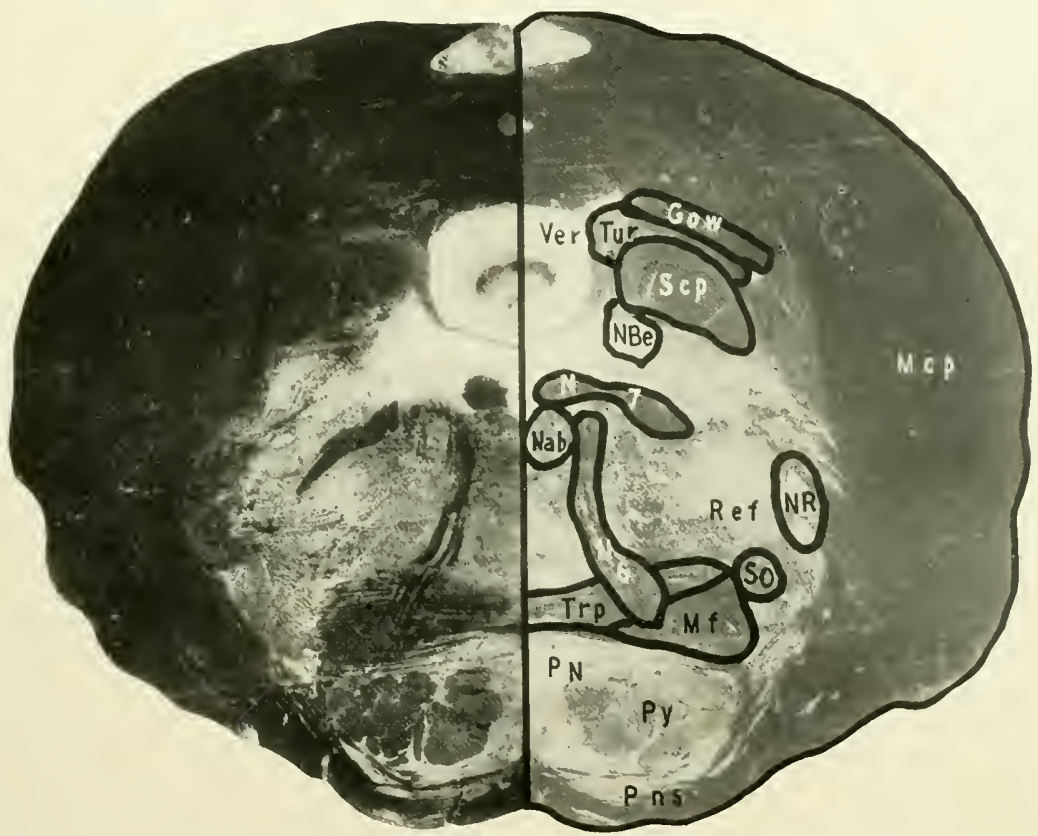

FIG. I 50 . BABOON. LEVEL OF THE EMERGENT FIBERS OF THE SIXTH NERIE AND THE CALDAL FIBERS OF THE PONS VAROLII.

Gow, Ventral Spinocerebellar Tract; мCP, Middle Cerebellar Peduncle; MF, Mesial Fillet; NaB, Abducens Nucleus; NBE, Nucleus of Bechterew; NR, Nucleus of Rolando; 6 , Abducens Nerve; N-, Facial Nerve; Px, Pontile Nuclei; pNs, Pons Varolii; PY, Py ramid; ReF, Reticular Formation; scP, Superior Cerebellar Peduncle: so, Superior Olive; TRP, Trapezoid Body; TUR, Tractus Uncinatus of Russel (Ilook Bundle); VrR, Vermis Cerebelli. [Accession No. i jo. Section 25-. Actual Size $22 \times 16 \mathrm{~mm}$.]

formed by a thin medullary layer, the superior medullary velum, resting upon which is the central portion of the vermis of the cerebellum ( $\mathrm{Ver}$ ). 
This section is notable also as showing the beginning of the middle cerebellar peduncle ( $\mathrm{Mcp}$ ) which consists of the collected axons arising from the pontile nuclei to complete the connection between the hemispheres of the cerebrum and the cerebellum.

Two of the cranial nerves also appear in the section. One is the sixth cranial nerve (N6), whose nucleus abducens ( $\mathrm{Nab}$ ) lies immediately beneath the floor of the ventricle in the central gray matter. A large number of myclinated fibers pass forward from the nucleus to emerge at the bulbopontile sulcus. The nervus facialis (seventh cranial nerve) ( $\left.\mathrm{N}_{7}\right)$, in its second portion, appears as a circular bundle of fibers inmediately beneath the floor of the fourth ventricle and mesial to the nucleus abducentis. The fourth portion of this nerve is seen extending obliquely forward and outward from the nucleus abducentis, and rumning a course divergent with the emergent fibers of the abducens nerve. The seventh nerve supplies the muscles of facial expression, while the sixth nerve innervates the external rectus musele of the eyeball.

In the lateral angle of the ventricular space, and oceupying a position immediately lateral to the subependymal gray matter, is a collection of eells constituting one of the vestibular nuclei, the nucleus of Bechterew (NBe). Dorsal to the nucleus of Bechterew, and forming the lateral boundary of the ventricular space, is a large oval mass of myelinated fibers, the superior cerebellar peduncle $(\mathrm{Scp})$. This bundle represents the aggregation of nerve frbers for impulses arising in the cercbellum and destined for the several lower levels of the neuraxis in the interest of coordinative control of the muscles. Along its dersal aspect is a narrow bundle of deeply myelinized libers, the ventral spinocerebellar tract ( $\mathrm{Gow}$ ), which follows this circuitous course to enter the cercbellum.

In the central portion of the section are the crossing fibers constituting the upper portion of the trapezoid body ( Trp) at whose lateral extremity is a nuclear collection, the superior olivary body ( $\mathrm{SO}$ ). In a position lateral 
to the emerging fibers forming the fourth portion of the seventh nerve is the substantia gelatinosa (NR), lateral to which is the now dense and compact mass of the descenching trigeminal tract.

IEVEL AT THE MIDDLE OF THE PONS VAROLII SHOWING THE ENTERING FIBERS OF TIH FIFTH OR TRIGEIINAL NERIE (FIG. I jI)

At this level several noteworthy features make their appearance. The most important of these is the broad band of entering libers of the fifth nerve which make their way through the middle peduncle (Mcp), passing backward as a dense mass of myclinated fibers $\left(\mathrm{N}_{j}\right)$, of which the more lateral portion constitutes the sensory root of the trigeminal nerve, and the more mesial, the motor root. Sensory fibers are seen terminating in the cephalic portion of the substantia gelatinosa which here is somewhat convoluted in its appearance and is for this reason spoken of as the convolutiones quinti (NR). Mesial to this, and somewhat ventrally placed, are the more densely myclinized bundles of fibers taking origin in a nuclear mass, the nucleus masticatorius of the fifth nerve (NM). Two of the chief connecting links of the cerebellum appear at this level. The first is the middle cerebellar peduncle ( Mep) situated in the most xentral position of the section and sweeping outward and backward to enter the cerebellum and distribute its fibers to the lateral lobes of that organ. It represents the connection of the cerebral cortex with the hemispheres of the cerebellum. The second great connecting link in the cerebellar system shown in this section is the superior cercbellar peduncle $(S c p)$ situated in the lateral extremity of the fourth ventricle and representing fibers arising in the dentate nucleus, which form the great efferent pathway for impulses out of the cerebellum, destined for lower levels of the neuraxis in the interest of coordinative control of the muscles. 
At the level of the middle of the pons Varolii ( Pns) this structure attains its full dimensions and shows its three major layers, the stratum superficiale, the stratum profundum and the stratum complexum. Scattered among these

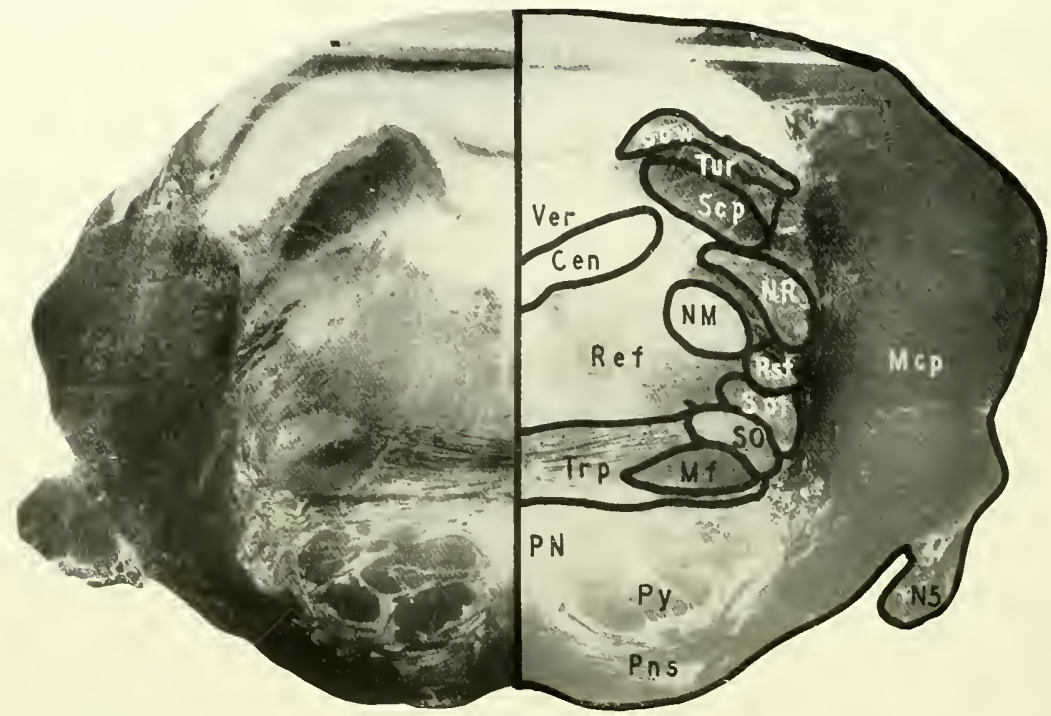

FIG. 1 II. BABOON. LEVEL AT THE MIDDLE OF THE PONS TAROLII, SHOWING THE ENTERING FIBERS OF THE TRIGEMINAL NERIE.

CEN, Central Gray Matter; Gow, Ventral Spinocerebellar Tract; MCP, Middle Cerebellar Peduncle; MF. Mesial Fillet; N5, Trigeminal Nerve; NM, Nucleus Masticatorius Trigemini; NR, Nucleus of Rolando; pN, Pontile Nuclei; pNs, Pons Varolii; Pyramid; py, Ref, Reticular Formation; Rst, Rubrospinal Tract; scp, Superior Ccrebellar Peduncle; so, Superior Olive; SPT, Spinothalamic Tract; TRP, Trapezoid Body; TLR, Tractus Uncinatus of Russel (I look Bundle); ver, Vermis. [Accession No. 150. Section 282. Actual Size $22 \times 16 \mathrm{~mm}$.]

transverse fibers of the stratum complexum are groups of separated bundles of the pyramidal system ( Py ) and the large nuclear aggregations of the pontile nuclei ( PN). All of the fibers emerging from the pons proceed laterally and, after relay in the pontile nucleus, assemble to form the middle cerebellar 
peduncle (Mcp) which passes into the cerebellum to terminate in the lateral lobes of this organ. The three layers of the pons, including the pontile nuclei and the scattered bundles of the pyramidal system, constitute the basis pomtis. The dorsal boundary of the base of the pons is established by the transversely disposed fasciculus forming the mesial fillet (Mf), dorsal to which is the tegmentum pontis. In the dorsal region of the section, immediately lateral to the much constricted fourth ventricle, is the superior cercbellar peduncle, and dorsal to this the uncinate tract of Russel ( T ur). A more heavily myelinized bundle lateral to this fasciculus is the ventral spinocerebellar tract on its way toward the verm is of the cerebellum (Gow). Some fibers of the motor root of the trigeminal nerve emcrge from the nucleus masticatorius (NM).

LEVEL OF THE INFERIOR COLLICULUS (FIGS. $1 ; 2$ AND I 33 )

At this level certain features make their appearance which alter the external configuration of the brain stem. Most prominent among these is the extreme narrowing of the ventricular cavity to form the beginning of the Sylvian aqueduct, while just above the roof of the aqueduct rise two elevations, one upon either side of a deep median sulcus. These structures constitute the inferior colliculi (IC), distinguishing characteristics of the quadrigeminal plate of the midbrain. Ventrally the convex contour of the section is undergoing some alteration due to the appearance of a deep median groove which tends to separate the two halves of the pons Varolii in the median line. This groove eventually becomes decper in the next succeding section to form a depression, the beginning of the interpeduncular space which separates one cerebral peduncle from the other. The separation is foreshadowed in this section on the ventral surface of the pons, where it is possible to discern the beginning of the right and left cercbral peduncles. The stratum superficiale pontis and the stratum profundum pontis are well 
marked at this level, as is also the stratum complexum containing many transverse pontile fibers scattered among which are the bundles of the pyramidal system (Py) and the large masses of the pontile nuclei (PN).

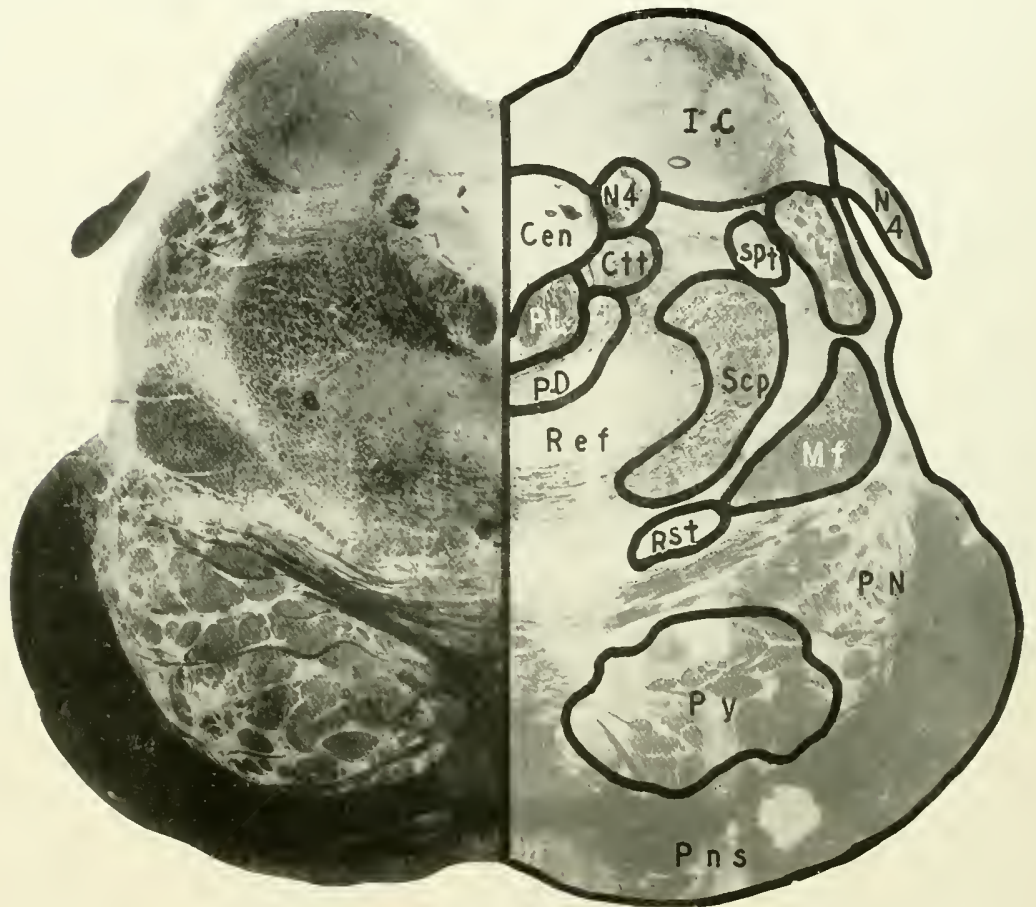

FIG. I 52. BABOON. LEVEL OF THE INFERIOR COLLICULUS SHOWING ALSO THE EMERGENCE OF THE FOURTH NERVE.

CEN, Central Gray Matter; CTT, Central Tegmental Tract; IC, Inferior Colliculus; LF, Lateral Fillet; MF, Nesial Fillet; N4, Trochlear Nerve; PD, Predorsal Bundle; PL, Posterior Longitudinal Fasciculus; PN, Pontile Nuclei; pNs, Pons Varolii; PY, Pyramid; REF, Reticular Formation; RST, Rubrospinal Tract; sCP, Superior Cerebellar Peduncle; spr, Spinothalamic Tract. [Accession No. 150 . Section 345. Actual Size $19 \times 15 \mathrm{~mm}$.]

The mesial fillet (Mf) forms the boundary line between the basal and tegmental portions of the axis. 
The major changes appear in the tectal portions of this section as the inferior colliculi ( IC). These structures are significant because of the relation they bear to the sense of hearing. They are primitive receiving

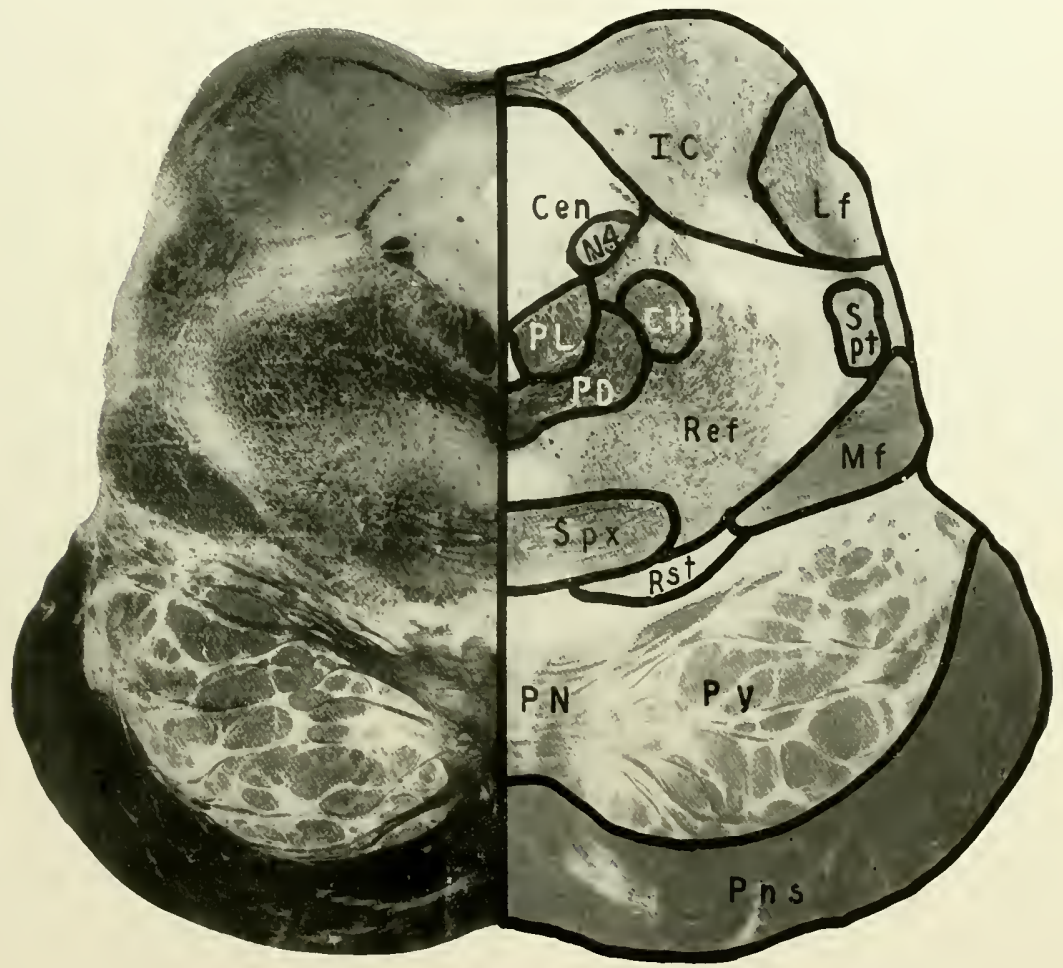

FIG. I j3. BABOON. LEVEL OF THE INFERIOR COLLICLLUS.

CEN, Central Gray Matter; CrT, Central Tegmental Tract; IC, Inferior Colliculus; LF, Lateral Fillet; MF, Mesial Fillet; N4, Trochlear Nerve; PD, Predorsal Bundle; PL, Posterior Longitudinal Fasciculus; PN, Pontile Nucleus; PNS, Pons Varolii; PY, Pyramid; REF, Reticular Formation; RST, Rubrospinal Tract; SPT, Spinothalamic Tract; spx, Crossing of Superio- Cerebellar Peduncle. [Accession No. 150. Section 351. Actual Size $19 \times 15 \mathrm{~mm}$. 
stations for this special sense. In the lower vertebrates they constitute the chief central nervous mechanism for auditory impressions, and in them the main elaboration of hearing is carried on. In most of the higher forms, particularly the mammals, these auditory colliculi have surrendered most of their original auditory function to cortical areas of the cerebral hemispheres. Their relatively large size in baboon is indicative of a retention of much of their primordial significance; while they are less conspicuous than in the lower primates, they are still larger than the corresponding structures in the large anthropoids and man. Undoubtedly they provide a rellex center for the immediate reflex translation of auditory stimuli into motor responses active in escape or defense. These structures also show a certain degree of their original stratification both in cells and fibers, being in this respect more conspicuous than in the higher anthropoids. From this it may be inferred that, while the auditory sense has been telencephalized to a certain extent in the baboon, it has not undergone an advance to the cortex as marked as that in the primates at the upper extremity of the series.

Lateral and ventral to the inferior colliculus is the collected mass of fibers constituting the lateral fillet ( Lf) bearing impulses over the secondary pathway of hearing to this primary receiving station in the midbrain. A large mass of transverse fibers, sweeping inward and forward toward the midline, comprises the two major divisions of the superior cerebellar peduncle ( $\mathrm{Spx}$ ) now about to undergo its complete decussation preparatory to entering the red nucleus. The pyramidal tract (Py) together with the descending fibers of the pallio-ponto-cerebellar tract is situated along the ventral aspect of the axis. Dorsal to the pyramidal tract and stretching partially across the section at its laterat extremity is a mass of gray matter containing cells of several different sizes, the dorsal extremity of the substantia nigra. The specific functions of this nuclear mass are not clearly understood; it is presumed to be essential 
to the regulation of certain automatic associated movements. The centrai gray matter (Cen), considerably enlarged as compared with the lower levels, surrounds the caudal extremity of the Sylvian aqueduct. At its lateral extremity are several scattered bundles of nerve fibers, the mesencephalic root of the trigeminal nerve, while mesial to this root along the ventral border of the central gray matter are two or more bundles of fibers, the descending fasciculi of the trochlear nerve $\left(N_{4}\right)$. Upon its extreme ventral aspect the central gray matter is bounded by fibers forming the fasciculus longitudinalis posterior (PL) and the fasciculus predorsalis (PD).

\section{LEVEL OF THE SUPERIOR COLLICULUS (FIG. $1 j+$ )}

At this level conspicuous mesencephalic structures make their appearance. Chief among these are the much reduced remnants of the opt ic lobes of the lower vertebrates. These lobes, formerly serving as the end stations of visual sensibility, still retain a degree of stratification reminiscent of their condition in the lower forms. At least three distinct strata of alternating cells and fibers may be discerned microscopically. During the process in which the optic lobes have progressively delegated their visual function to the occipital areas in the cerebral cortex, a similar supersedence by the cerebral hemispheres has involved the sense of hearing as well as those modalities of general body sensibility essential to the production of the most highly organized volitional movements. It is still evident even in the primate order that a slow transference from the visual region of the midbrain to the higher visual centers of the occipital tobe is in process.

Another element in this level is the oculomotor nucleus (Noc) which gives rise to the third cranial nerve whose frbers supply all but two of the muscles moving the eyeball. The fact that this oculomotor nucleus shows a relatively simple degree of development, particularly in its internuclear fibers, implies a relatively low degree of oculomotor organization. These 
conditions indicate that vision in the baboon is as yet only partially binocular and the convolutions in the occipital lobe bear out the presumption advanced on the strength of the interocular connections between the oculomotor

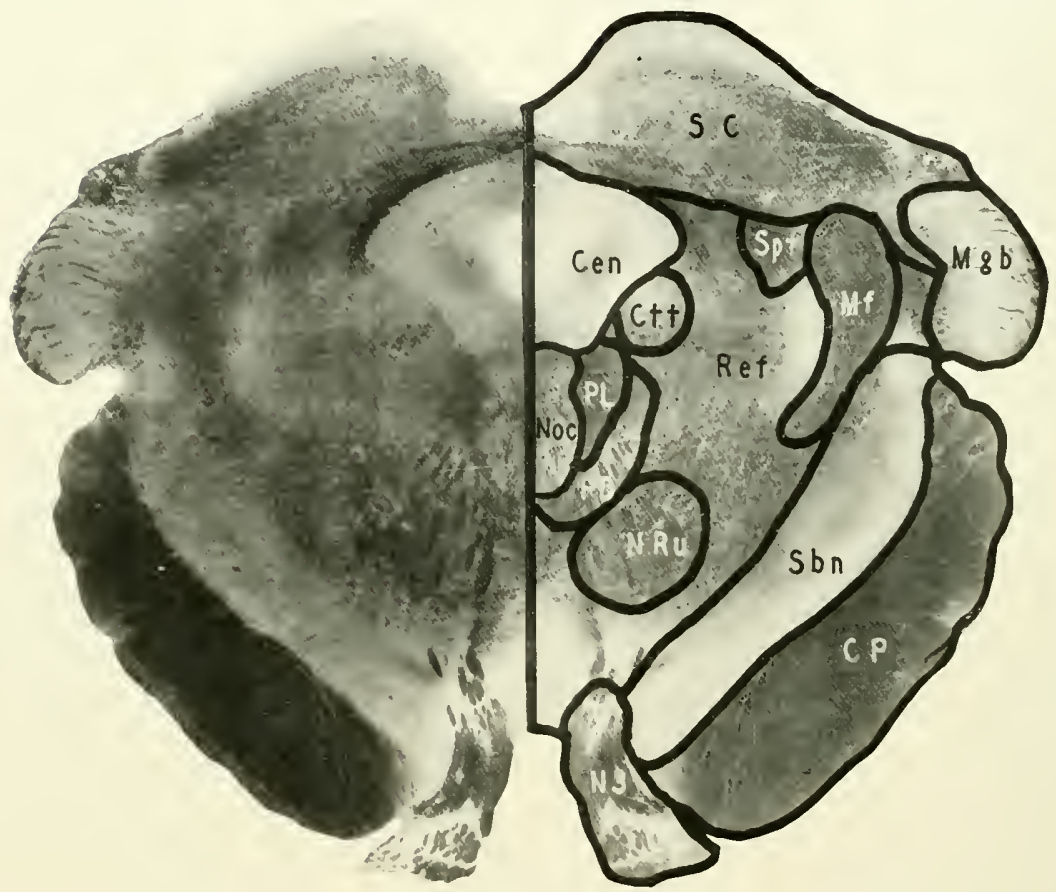

FIG. I j4. BABOON. LEVEL OF THE SUPERIOR COLLICULUS.

CEv, Central Gray Matter; ctT, Central Tegmental Tract; $\mathrm{CP}$. Cerebral Peduncle; MF, Mlesial Fillet; MGB, Mesial Geniculate Body; Noc, Oculomotor Nucleus; NRU, Nucleus Ruber; N3, Oculomotor Nerve; PD, Predorsal Bundle; PL, Posterior Longitudinal Fasciculus; REF, Reticular Formation; sBn, Substantia Nigral Sr, Superior Colliculus; Spt; Spinothalamic Tract. [Accession No. 1 so. Section 341. Actual Size $19 \times 12 \mathrm{~mm}$.;

nuclei. The fibers of the oculomotor nerve $\left(\mathrm{N}_{3}\right)$ sweep forward and outward from the nucleus in their course to emerge from the brain stem in the sulcus oculomotorius. Ventrolateral to the central gray matter (Cen) 
is the reticular formation ( $R \mathrm{ef}$ ), to which attention is directed because of its diffuse, indefinite character. In the ventromesial portion of the reticular formation, in the region through which the radiating fibers of the oculomotor nerve are passing, an ill-defned aggregation of gray matter represents the nucleus ruber (NRu). The indefinite character of this nucleus in baboon, as well as in the lower primates, is a fact of considerable significance. The nucleus merges with the adjacent reticular formation, producing the impression that it is but a part of this formation. To this point reference will be made subsequently in considering the reticular formation as a diffuse matrix in the tegmental portion of the stem out of which certain discrete structures take their origin or seem to emerge by more exact delimitation of their boundaries. In this light, the reticular formation throughout the entire series of primates assumes a greater evolutional importance than is usually attributed to it. This region of the brain stem is usually regarded as a terra incognita and hence passed over without especial comment because of its diffuse and nondescript character. To us it appcars more as the territory on the outshirts of advancing specialization, out of which, as the evolutional process goes forward, there develop more highly organized structures. It is not unlike a suburban area in the outlying regions of a community whose structural organization is progressively encroaching upon the as yet undeveloped fields. That so much of reticular formation still remains even in the highest primates might perhaps argue further potentialities of differentiation in this diffuse neural material. However this may be, emphasis is laid upon the gradual recession of a poorly differentiated portion of the axis, and its equally gradual replacement by more highly organized structures as the physical and physiological factors of evolution make their appcarance in the progress of the primates.

Ventrolateral to the formatio reticularis is a large and broad freld of gray matter containing cells of several sizes, the substantia nigra ( $\mathrm{Sbn}$ ), to 
which has been attributed the regulation, in part at least, of certain automatic associated movements. Immediately dorsal to the substantia nigra is the mesial fillet (Mf). In a position in front of the oculomotor nucleus

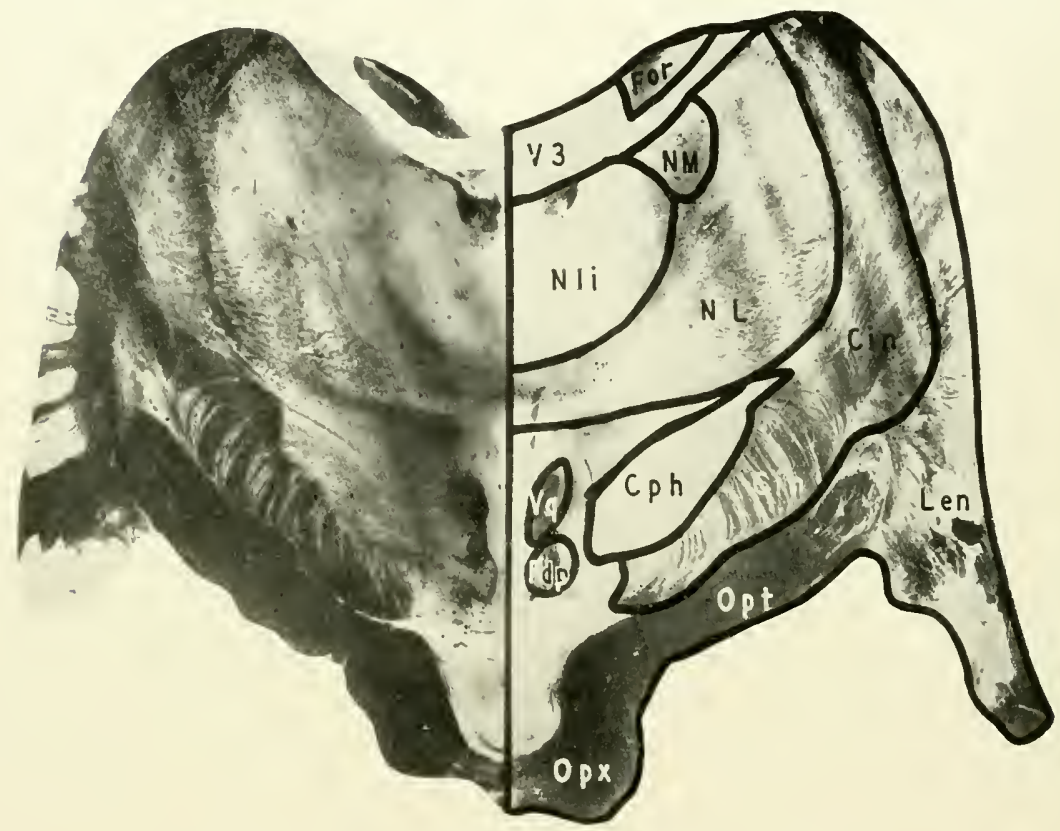

FIG. Ijj. BABOON. LEVEL OF THE OPTIC CHIASM.

CIN, Internal Capsule; CPH, Corpus Hypothalamicum; FDP, Descending Pillar of Fornix; For, Fornix; LEN, Lenticular Nucleus; NL, Lateral Nucleus of the Thalamus; NLI, Nucleus Thalami Lateralis Internus; NM, Nucleus Medialis Thalami; opx, Optic Chiasm; opt, Optic Tract; vo, Fasciculus of Vicq D'Azyr; v3, Third Ventricle. [Accession No. 1 so. Section 525. Actual Size $33 \times 21 \mathrm{~mm}$.]

are two systems of decussating fibers, one, the dorsal decussation of Meynert through which the emergent fibers of the third nerve make their way toward the surface, and ventral to this is the ventral decussation of Forel. These decussations represent respectively the crossing fibers arising in the 
reticular formation of the midbrain to enter the predorsal bundle on the one hand, and other clecussating descending fibers arising in the red nucleus to form the rubrospinal tract.

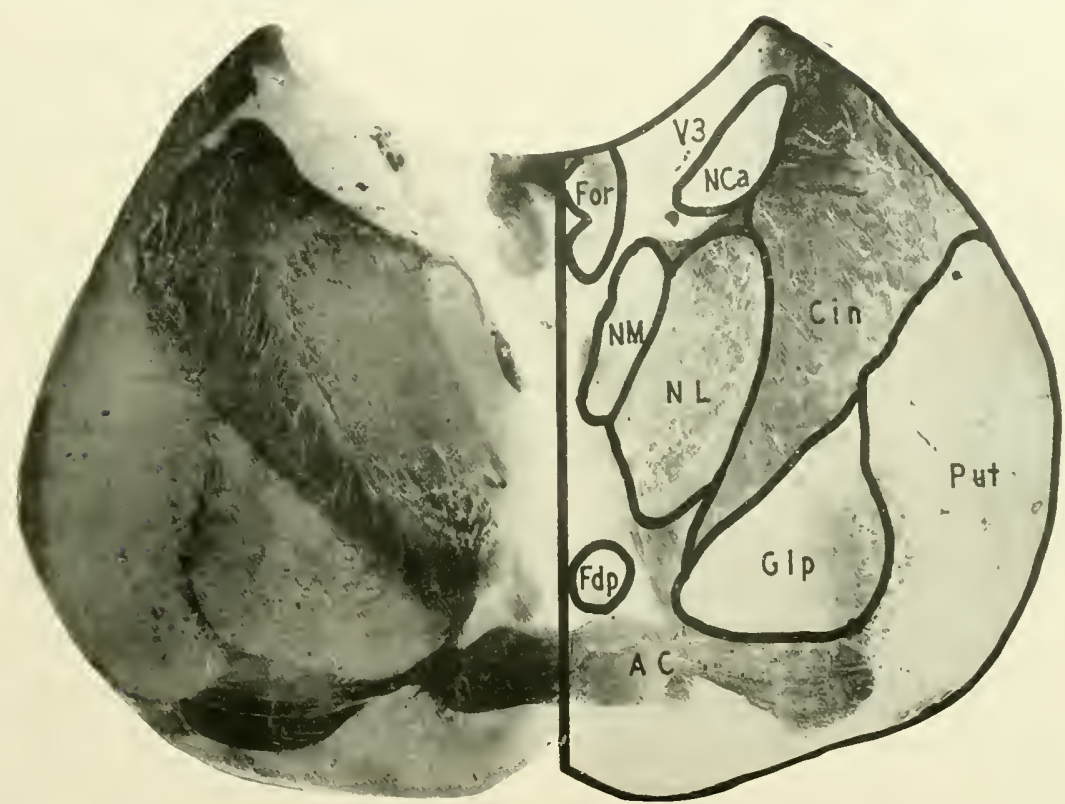

FIG. 1 ; 6. BABOON. LEVEL OF THE ANTERIOR COMMISSURE.

AC, Anterior Commissure; Civ, Internal Capsule; FDP, Descending Pillar of the Fornix; For, Fornix; GLP, Globus Pallidus; NCA, Caudate Nucleus; NL, Lateral Nucleus of the Thalamus; Nm, Nucleus Medialis Thalami; PUT, Putamen; 13, Third Ventricle. [Accession No. 150. Section 627. Actual Size $38 \times 21 \mathrm{~mm}$.]

A lateral projection in the cross section indicates the position of the mesial geniculate body ( $M g \mathrm{gb})$, one of the relay stations for the auditory pathway in its further course to the cortical region for hearing in the cerebral hemisphere. 
LEVEL OF THE OPTIC CHIASM (FIG. I jj)

Here the erossing fibers of the optic pathway are the identifying feature $\left(\mathrm{Opx}_{\mathrm{px}}\right)$. The optic thalamus appears as another element, which represents the last relay station for all the pathways of sensibility with the exception of the olfactory sense. In it ends the mesial fillet, and from it start those axons serving to carry the last relay of the somesthetic pathway from the thalamus to the parietal lobes of the cercbral cortex. Dorsal to the optic chiasm are crossing fibers which constitute the supraoptic commissure of Meynert. In a position dorsal to the optic tract $(\mathrm{Opt})$ is a dense mass forming the internal capsule ( $\mathrm{Cin}$ ). Bordering upon these fibers of the capsule is a collection of striated gray and white matter, the lenticular nucleus ( Len). This, being one of the most important portions of the endbrain, does not properly belong to the deseription of the brain stem. The remaining structures of interest in this section are indicated in the legend beneath the frgure.

LEVEL OF THE ANTERIOR COMMISSURE (FIG. I ;6)

At this level the section illustrates the cephalic limit of the brain stem, the last remaining structure of the anterior portion of the optic thalamus. The structures of topographical interest in this section are indicated by corresponding letters in the legend beneath the figure. 


\section{Chapter Xi}

\section{RECONSTRUCTION OF THE GRAY MATTER IN THE BRAIN STEM OF PAPIO CINOCEPHALUS}

\footnotetext{
7 HE reconstruction of the brain stem of Papio cynocephalus begins in the lower medullary region. The higher levels of the spinal cord 1 are not represented in the model.
}

In the lowest levels of the reconstruction the contour of the central gray matter is already considerably flattened from before backward and drawn into an almost directly lateral position. The central gray matter gives rise at its dorsolateral extremities to the flattened cervix of the dorsal horn which expands at its termination into a more or less oval-shaped substantia gelatinosa trigemini. This structure has already assumed its lateral position which it maintains throughout the rest of its extent.

\section{The Dorsal Medullary Nuclei}

The nucleus of the column of Goll consists of a narrow, Iaterally flattened ribbon of gray matter projecting dorsally from the body of the central gray matter. It is separated from its fellow of the opposite side by a thin investment of white matter. The nucleus continues cephalad for some distance without showing any material change until the caudal extremity of the inferior olivary nucleus is reached, when it rapidly expands, presenting a heavy mass of overhanging gray matter which is directed laterally under the influence of the opening of the fourth ventricle.

The nucleus of the column of Burdach makes its first appearance just below the level at which the nucleus of the column of Goll begins its rapid increase in size, as a thickened condensation in the dorsal surface of the central gray matter. This small mass of gray matter rapidly increases in size, expanding dorsally and Iaterally into a relatively rich arborescent 
mass which is constantly shifted laterad by the opening of the fourth ventriele until it overhangs, to a considerable extent, and almost obscures from view the substantia gelatinosa trigemini. The nucleus of the column of Goll reaches its maximum development at the level where the fourth ventricle opens and from that point it diminishes rather rapidly in size. It is gradually obscured from view by the floor of the fourth ventricle which approaches closely to the dorsal mass of the nucleus of the column of Burdach, thus submerging the nucleus of the column of Goll.

The nucleus of the column of Burdach continues upward to a lerel considerably above the opening of the fourth ventricle, foming a part of the lateral boundary of the ventricle. As a result of its lateral displacement it overhangs the substantia gelatinosa trigemini. It rather abruptly diminishes in size and disappears at about the mid-level of the inferior olivary nucleus.

The substantia gelatinosa trigemini extends upward in the lateral region of the neuraxis, increasing in all of its diameters to the inferior olivary level where it is covered over by the lateral overhanging extensions of the nucleus of the column of Burdach. It reappears embedded in the reticular matrix between the ventrolateral nuclei of the reticular formation and the nucleus of Deiters. At this level it undergoes a reduction which has been constantly found in this nucleus and which may be termed the "waist of the trigeminal nucleus." From this point upward it increases in size until its maximum is reached as the caput of the nucleus in the mid-pontile level. It then suddenly undergoes reduction and disappears in the upper pontile region where it presents on its mesial surface the rounded, obliquely directed masticatory motor nucleus of the trigeminal complex.

\section{The Inferior Olivary Nucleus}

In the reconstruction of the inferior olivary nueleus there is evident a considerable advance over that seen in the preceding forms. The ventral 
accessory olive makes its appearance at about the upper level of the pyramidal condensation as a flat band of gray matter disposed transversely with a slight inclination dorsally at its mesial extremity. The mesial extrem-
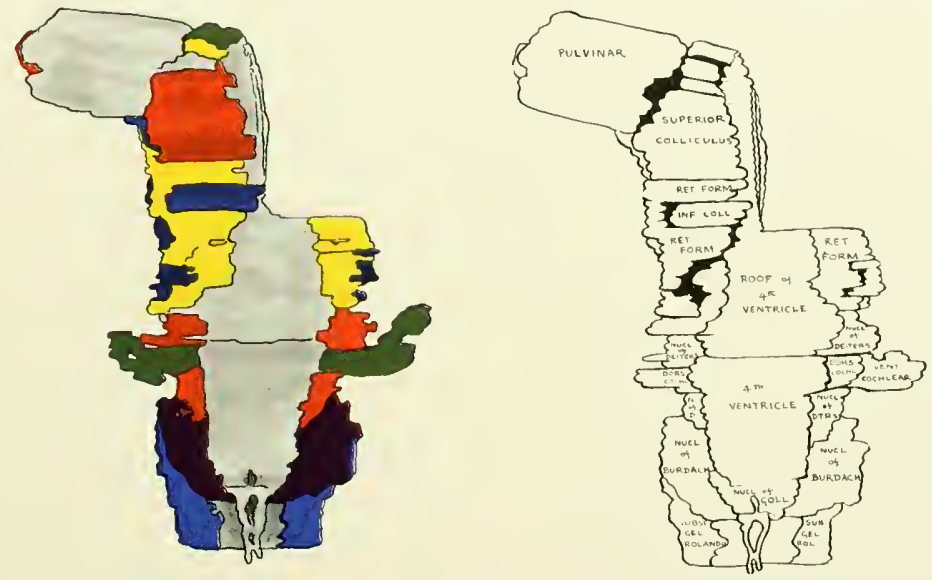

FIG. I F7. DORSAL SURFACE OF THE GRAY MATTER OF THE BRAIN STEM, PAPIO CINOCEPHALUS.

Key to Dlagram. Dors. Cochl., Dorsal Cochlear; inf. Coll., Inferior Colliculus; Nucl. of Burdach, Nucleus of Burdach; N. OF D., NUCL. OF DEITERS and NUCLEUS OF DTRS., Nucleus of Deiters; NUCL. OF GOLL, Nucleus of Goll; RET. Form., Reticular Formation; SUb. GEl. kOL. and SUbST. GEl. ROLANdo, Substantia Gelatinosa of Rolando; Vent. Coch., Ventral Cochlear Nucleus.

ity of the nucleus turns backward upon itself, forming a hook-shaped mass of gray matter.

The dorsal accessory olive appears only slightly above the level of the appearance of the ventral accessory nucleus as a small lamina of gray matter which continues upward as a rather narrow strand. At the level of appearance of the main mass of the inferior olivary nucleus it expands into a narrow flattened sheet which lies dorsal to the hilus of the inferior olivary nucleus. The main nucleus appears as the fourth ventricle begins to open in the form of a loop-shaped layer of gray matter, presenting a few simple reduplica- 
tions. These reduplications appear mainly in the dorsal limb of the loop. The fundus is relatively thick and the ventral branch of the nucleus is somewhat curved, with its concavity directed forward. The mesial extremity points almost directly toward its fellow of the opposite side. The dorsal branch of the loop is less extensive than the ventral branch, presenting the reduplications already mentioned. The hilus of the nucleus is bridged over by the dorsal accessory olivary nucleus. The whole olivary complex extends upward to a point just below the lateral recess of the fourth ventricle, where it rapidly reaches its termination.

The dorsal accessory olivary nucleus and the dorsal branch of the inferior olivary mucleus throughout their entire extent lic in relatively close contact with the ventral surface of the reticular formation, out of which they seem to differentiate.

\section{The Reticular Formation}

As reconstructed, the reticular formation appears as a flattened lamina of gray matter applied to the lateral surface of the ventral gray column. It extends dorsally and laterally to come into contact with the substantia gelatinosa trigemini. It increases rapidly in bulk, proportionally with the decrease in the ventral gray column which gradually becomes absorbed by the expanding reticular formation. In the midoblongatal region, the reticular formation forms a relatively massive structure, somewhat triangular in shape, its base being applied to the central gray matter mesially, and to the base of the nucleus of the column of Burdach laterally, while its apex extends between the inferior olivary complex and the substantia gelatinosa trigemini.

Continuing from this point upward, the reticular formation undergoes a considerable reduction, due chicfly to the increasing development of the inferior olivary nucleus, until the upper limit of this structure is 
reached, where it increases rapidly in size, thus becoming the main mass of the pontile tegmentum. It is irregular in its surface outline, being indented by the dorsal surface of the inferior olivary nucleus and the dorsal accessory
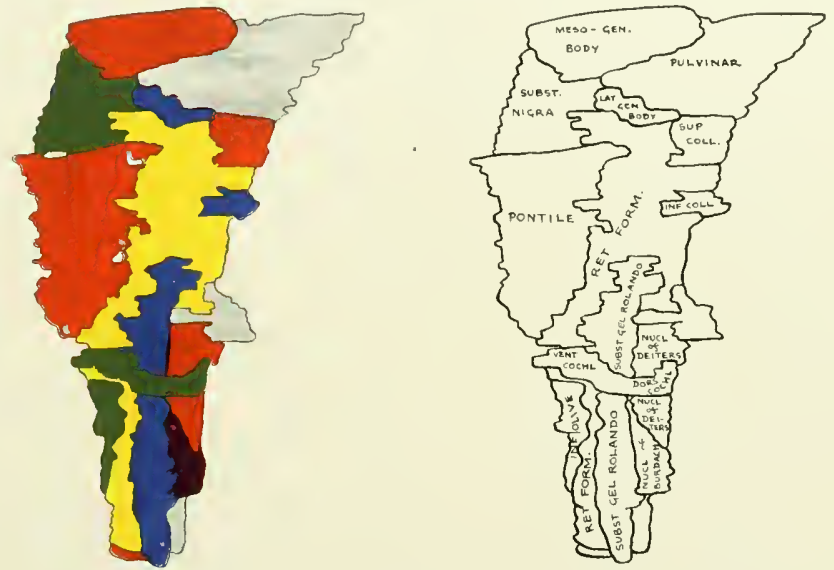

FIG. I j8. LATERAL SURFACE OF THE GRAY MATTER OF THE BRAIN STEM, PAPIO CYNOCEPHALUS.

Key to Diagram. dors. Cochl., Dorsal Cochlear Nucleus; inf. COll., Inferior Colliculus; inf. Olive, lnferior Olive; LAT. GeN. BoDY, Lateral Geniculate Body; Meso-gen. BodY, Mesial Geniculate Body; NuCl. of Burdach, Nucleus of Burdach; Necl. of Delters, Nucleus of Deiters; pontile, Pontile Nuclei; Ret. FOrn., Reticular Formation; SUbSt. Gel. Rolando, Substantia Gelatinosa of Rolando; subst. Nigra, Substantia Nigra; sup. coll., Superior Colliculus; vent. cochl., Ventral Cochlear Nucleus.

nucleus, laterally by the substantia gelatinosa trigemini and dorsolaterally by the base of the nucleus of Burdach and the beginning of the vestibular area.

Above the inferior olivary nucleus the reticular formation shows the effect of the developing trapezoid body by presenting a relatively deep excavation on its ventral surface close to the midline, in which the main mass of the trapezoid body lies.

Laterally the reticular formation is in contact with the substantia gelatinosa trigemini and above the level of this nuclear mass it presents a 
very irregular surface marked by the entrances and exits of the various fiber bundles connected with the colliculi and by the development of the lateral fillet from the trapezoid body. The course of this fiber tract can be definitely followed along the lateral surface of the reticular formation.

Immediately prior to the formation of the lateral fillet the trapezoid body occupies a considerable excavation in the central portion of the ventral surface of the reticular formation. This bay is limited by mesial and lateral extensions of the reticular formation which pass ventrally to afford contact with the dorsal layer of the mesial and lateral buttresses of the pontile nucleus.

Cephatad to the inferior colliculus the inferior brachium makes its appearance as the continuation of the acoustic pathway and produces a groove on the surface of the mesencephalic reticular formation at which level this fiber bundle acts as the next relay to the mesial geniculate body. As the tract approaches this nuclear mass it again sinks into the reticular matrix and is covered oxer by a lateral extension of the retieular formation.

Dorsally the reticular formation is in close contact with the body of the eentral gray matter, separated from it, however, by the circumferential condensation of fibers mainly represented by the posterior longitudinal faseiculus, the predorsal bundle and the mesial fillet. In the cephalic portion of the pontile tegmentum the reticular formation is disposed in a rather irregular fashion, presenting a lateral thin lamina overlying the superior cercbellar peduncle as it enters the upper part of the tegmentum from the superior medullary velum.

As the pontile reticular formation is sueceeded by that of the mesencephalon the circumferential layer of the reticular formation beeomes relatively thicker, whereas the central mass of the reticular formation diminishes in bulk due to the fact that the superior cercbellar peduncle is approaching the midline. 
In the lower mesencephalic areas this process has continued so far that the central mass of the reticular formation gradually disappears and its mass assumes a much greater expansion. In this region of the mesencephaton the
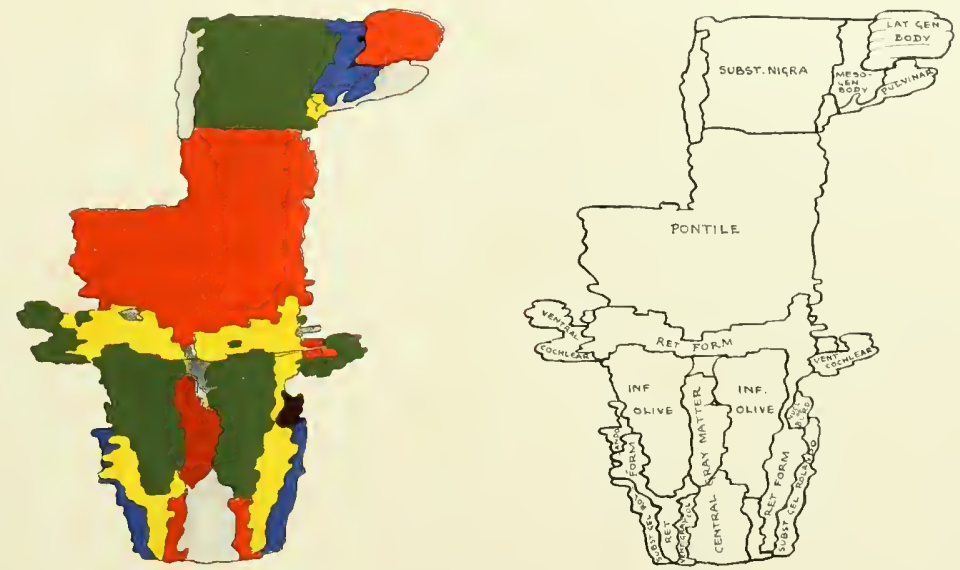

FIG. 159. VENTRAL SURFACE OF THE GRAY MATTER OF THE BRAIN STEM, PAPIO CYNOCEPHALUS.

Key to Diagrai, INF. Olive, Inferior Olive; Lat. GeN. Body, Lateral Geniculate Body; meso-gen. Boty, Mesial Geniculate Budy; nucl. of Burd., Nucleus of Burdach; pontulf, Puntile Nuclei; Ret. Foru., ReticuIar Formation; subst. Gel. rol. and subst. gel. rolando, Substantia Gelatinosa of Rolando; subst. Nigra, Substantia Nigra; vent. Cochlear, Ventral Cochlear Nucleus; IENT. grar col., Ventral Gray Column.

reticular formation now gives place to a specialization forming the large nucleus ruber which is surrounded by a capsule derived from the superior cerebellar peduncle and descending striato-rubral fibers. The lateral mass of the reticular formation follows closely the course of the superior cerebellar peduncle as this structure approaches the nucleus ruber. Above the level of the red nucleus the reticular formation again reaches the midline and its most central portion assumes its original position and importance. It is separated from the central gray matter by the longitudinally coursing median bundles 
of the posterior longitudinal fasciculus and the circumferential reticular fiber collections.

\section{The Pontile Nuclei}

In reconstruction, the pontile nuclei present an arrangement essentially similar to that found in the lower forms. The formation of a superficial and a deep layer comnected at their mesial and lateral extremities by masses of gray matter which represent the lateral and mesial buttresses is present also in the baboon. The ventral and lateral surfaces of the pontile muclei correspond accurately with the surface anatomy of the pons itself.

Mesially the mesial buttresses come into contact with one another at the median raphe. Caudally the pontile nuclei arise in poorly developed areiform nuclei which extend downward for a short distance upon the ventral surface of the collected pyramidal tract. At the median line the mesial buttresses are carried backward to eome into contact with the ventromesial angle of the reticular formation. A similar arrangement exists on the lateral aspect where the lateral buttresses extend backward to become continuous with the ventrolateral angle of the reticular formation. Between these two points of conIluence, mesial and lateral, the deep layer of the pontile nucleus is separated from the ventral surface of the reticular formation by the bay in which lies the trapezoid body.

The simple arrangement found in the lower forms is becoming more and more complex and the interior of the pontile nucleus is found to be traversed by numerous strands of nuclear material which pass from one side to the other, breaking up the pallio-spinal and pallio-pontile systems of fibers into smaller constituent masses.

As the stem is followed upward, the deep layer of the pontile nuclei is found to become directly continuous with the central portion of the substantia nigra, while the mesial and lateral portions of the substantia nigra rest upon the mesial and lateral buttresses of the pontile nuclei. 


\section{Tine Vestibular Conplex}

The vestibular complex appears on the surface of the reconstruction at about the mid-olivary level of the brain stem. It is at first a small triangular mass of gray matter Iocated between the lateral surface of the central gray matter and the cephalic extremity of the nucleus of Burdach. As it increases in extent and bulk it acts as a wedge separating the upper extremity of the nucleus of the column of Burdach from the central gray matter. It rapidly increases in its ventrodorsal extent until it becomes a prominent feature in the dorsolateral portion of the tegmentum. Its ventral continuation forms a rather sharp, wedge-shaped mass of gray matter which presents on its lateral surface a distinct concavity in which is lodged the inferior cerebellar peduncle. This portion of the complex is formed by the nucleus of Deiters which reaches its maximum extent at the midventricular level. From this point upward it rapidly diminishes in size, being replaced by the more dorsally situated nucleus of Schwalbe, the triangular nucleus of the vestibular complex. This structure lies dorsal and somewhat mesial to the nucleus of Deiters, mesial to the dorsal cochlear nucleus and crossed by the fibers of the striae acusticae. The triangular nucleus is continued upward for a short distance, gradually attenuating in size and merging with the indilferent reticular matrix of the lateral walls of the fourth ventricle above the level of the lateral ventricular recess. The third element in the vestibular nuclei, the nucleus of Bechterew, is found in the lateral wall of the ventricular formation and is not represented in the reconstruction.

\section{The Cochlear Complex}

The cochlar complex seems to be definitely less developed than that in the three preceding forms. The nuclear masses are relatively small and poorly developed. The same arrangement is found to be present, that is, the almost complete investment of the entering nerve fibers.by the nuclear 
masses, the mesial surface only of the entering nerve remaining uneovered by nuclear material so that the trough arrangenent of the nucleus about the entering nerve stem is maintained. The dorsal cochlear nucleus, which is situated dorsal to the triangular nucleus of Schwalbe of the vestibular complex, is also relatively small and lies in contact with the lateral extremity of the eentral gray matter of the floor of the fourth ventricle. The strands of gray matter connecting the ventral and the dorsal cochlear nuclei are also poorly developed.

\section{The Colliculi}

The colliculi, which are situated in the tectum of the midbrain, appear relatively well developed in the baboon. The superior colliculus is clearly much more extensive than the inferior colliculus, the former being at least three times the vertical height of the latter. The inferior colliculus arises from the dorsal extension of the reticular formation at the point of junction between the mesencephalon and the metencephalon. It is relatively inconspicuous and is separated from the larger superior colliculus by the intercollicular furrow, where the lateral extension of the reticular formation comes to the surface of the stem. At its lateral and upper extremities the inferior colliculus presents the beginning of the inferior brachium which passes from the inferior colliculus directly to the mesial geniculate body. The inferior colliculus is continuous laterally with the reticular formation, while dorsally it is continuous with the indifferent dorsal gray matter of the mesencephalon.

The superior colliculus arises, as does also the inferior, by a specialization in the tectum of the mesencephalon. It is in contact dorsally with the indifferent dorsal gray matter lying in the median line. Laterally it is separated from the reticular formation by the development at this point of the superior brachium which serves to connect the superior colliculus with 


\section{RECONSTRUCTION OF PAPIO CINOCEPIHALUS $3+5$}

the Iateral geniculate body. Mesially both of these nuclear structures are continued across the midline by the respective superior and inferior collicular commissures.

\section{Tue Substantia Nigra}

In the reconstruction, the substantia nigra appears as a relatively massive nuclear collection in the pes pedunculi of the mesencephalon. Situated ventral to it and leaving impressions upon its ventral surface are located the pallio-spinal and pallio-pontile fiber systems. It is supported ventrally by the heavy mesial and lateral buttresses of the pontile nuclei and throughout its transverse extent by the deep layer of the pontile nucleus, from a specialization of which it seems to take origin. It is in contact mesially with the indifferent interpeduncular gray matter and is piereed at this junction by the emerging fibers of the oculomotor nerve. Its dorsal surface is concave dorsally and is separated from the reticular formation of the mesencephalic tegmentum by the circumferential fiber accumulations which appear in this region, coursing longitudinally and transversely through the stem. At its Iateral extremity it presents a nuclear condensation, from which fibers pass into the mesencephalic tegmentum. Laterally it sends a prolongation toward the mesial and lateral geniculate bodies. As the diencephalon is approached, the substantia nigra diminishes in size and seens to merge with the zona incerta of the diencephalon.

\section{The Nucleus Ruber}

The nucleus ruber appears in reconstruction in the more cephalic portion of the mesencephalic tegmentum and is encapsulated by the fibers received from the decussation of the superior cerebellar peduncle. It is fairly well developed and of moderate size. Its cephalic extremity approaches, if it does not fuse with, the reticular formation of the diencephalon. 


\section{The Central Gray Matter}

The central gray matter in the baboon is first found as a transversely arranged mass of gray matter which laterally receives the base of the cervix of the dorsal horn. Dorsally the central gray matter presents the formation of the nucleus of the column of Goll which has already been described as narrow laminae of gray matter in the mesial dorsal white column, while the lateral extension of the gray matter forms the dorsal horns. From the point of confluence of the dorsal horn with the central gray matter of the reticular formation, the uucleus of the column of Burdach arises. As the stem is followed upward, the lateral trend of these gray masses becomes evident, the substantia gelatinosa trigemini passing outward into the lateral meridian of the cord, the nucleus of the column of Goll passing laterally and conforming to the opening of the lower extremity of the fourth ventricle, white between the nucleus of the column of Goll and the substantia gelatinosa trigemini appears the nucleus of the column of Burdach.

The body of the central gray matter rapidly flattens out into a narrow band of gray matter which forms the floor of the fourth ventricle. This floor is practically featureless, being, as in the other forms, almost smooth in the Inver half of the ventricke. It gradually increases in its lateral extent until the level of the lateral recess is reached, from which point it then begins to contract.

The floor of the upper half of the fourth ventricle presents the medial elevation corresponding to the development of the nuclear mass forming the nucleus of the sixth cranial nerve, the nucleus abducentis. The walls of the upper half of the ventriele rapidly approach each other and at the junction of the metencephalon and the mesencephalon, the central gray matter again constitutes the relatively heavy wall surrounding the aqueduct of Sylvius. Ventrally this central gray matter is continued forward into a relatively long tongue of gray matter which passes forward in 
the midline of the meseneephalon. Laterally the central gray matter forms a heavy structure which gradually becomes more circularly arranged about the aqueduct of Sylvius. In the lower portion of the mesencephalon the nucleus trochlearis appears as a specialization in the ventral prolongation of gray matter which we have found passing forward from the ventral aspect of the central gray matter. A similar arrangement is found also in the upper part of the mesencephalon where the nucleus oculomotorius likewise develops in a ventral prolongation on either side of the midline. As the interbrain is approached the eentral gray matter elongates ventrally, forming the long, thin sheet of gray matter surrounding the aqueduct of Sylvius in its dorsal portion. This extension ventrally is produced by its approach toward its confluence with the caudal portion of the third ventricle. Where the mesencephalon becomes continuous with the diencephalon the gray matter surrounding the aqueduct of Sylvius becomes continuous with the subependymal gray matter lining the third ventricle and the gray matter forming the mesial group of nuclei of the diencephalon. The central gray matter forming the roof of the Sylvian aqueduct becomes continuous with the structures in the roof of the diencephaton, while the floor of the aqueduct and the undifferentiated medial interpeduncular gray matter merges with the hypencephalic region. 



\section{Chapter Xil \\ PITHECUS RHESUS, MACACUS RHESUS, ITS BRAIN AND BEHAVIOR}

Its Position among the Primates; Measurements and Brain Indices; Surface Appearance of the Brain; Internal Structure of the Brain Stem in Cross Section

7 HE genus Pithecus, which includes the macaques, is distributed throughout India, as far north as Cashmere and Tibet, extending

L southward to the island of Ceylon and eastward to the Bay of Bengal. These monkeys are also found in upper and lower Burma, Siam, Cochin China and the Malay Peninsula, as well as certain adjacent islands.

\section{Appearance and Behavior of the Macaques}

In general, the macaques are much smaller in size than the baboons and for the most part are tree-living animals, inhabiting the jungle and forests usually on the borders of human habitation. In many species the face is free of hair, the ears prominent and protruding above the vertex of the skull. Some species are heavily bearded about the face and have a mane extending down the back of the head. The species here described, Macacus rhesus (Pithecus rhesus), is the common macaque of northern India which is held in great veneration by the Hindus, although not considered sacred by them. In certain of their temples large numbers of these macaques are kept and given the freedom of the building where they become bold and often troublesome.

The head of the macaque is much less dog-shaped than that of the baboon. The eyes are closely set and the animal is prone to take a sitting posture on its haunches, holding the head upright so that the eyes are 
directed forward. Its attitude in sitting is distinctly humanoid, while its attention and gaze convey the impression of an attentive scrutiny of all that seems to hold its interest in its environment. Its nose is short with a fairly well-defined nasal bridge and nostrils directed downward characteristic of the Catarrhine type. The lips are thin and the upper one particularly long, giving a tendency toward a muzzle-shaped eonfiguration of the snout. The fore- and hindlimbs are of about equal length. The forelimbs are equipped with a well-differentiated hand, although the thumb is somewhat shorter than in the baboon. The toes, especially the great toe, have nuch more the arrangement of fingers, and the animal thus presents definite quadrumanal differentiation.

Its movements are quick and cleft. It changes from one position to another with surprising swiftness and in an instant makes its way from the ground to some high altitude either in the trees or some other safe retreat. These monkeys are gregarious and tend to go together in herds, often of eonsiderable size. If captured young, the animal is easily trained and quickly learns many and often amusing tricks. It is full of mischief and curiosity. Macaques frequently beeome an actual nuisance in the neighborhood of towns where they may live in large numbers. When adult, they sometimes become quite ill-tempered, often savage, even to the extent of attacking the inhabitants without much provocation. For the most part they live in eultivated tracts, along the banks of streams, affecting rather than avoiding the habitations of man. These monkeys seem to have little fear of their human neighbors; indeed, they appear to enjoy the opportunity of molesting the inhabitants by many annoying pranks. Sometimes their attentions are vigorously resented and they are made the object of vindictive attacks. Their reaction on such occasions is most like that of tantalizing small boys who take an almost idiotic delight in the vain efforts of their pursuers to overtake them, and continue their aggravating anties in order to prolong the 


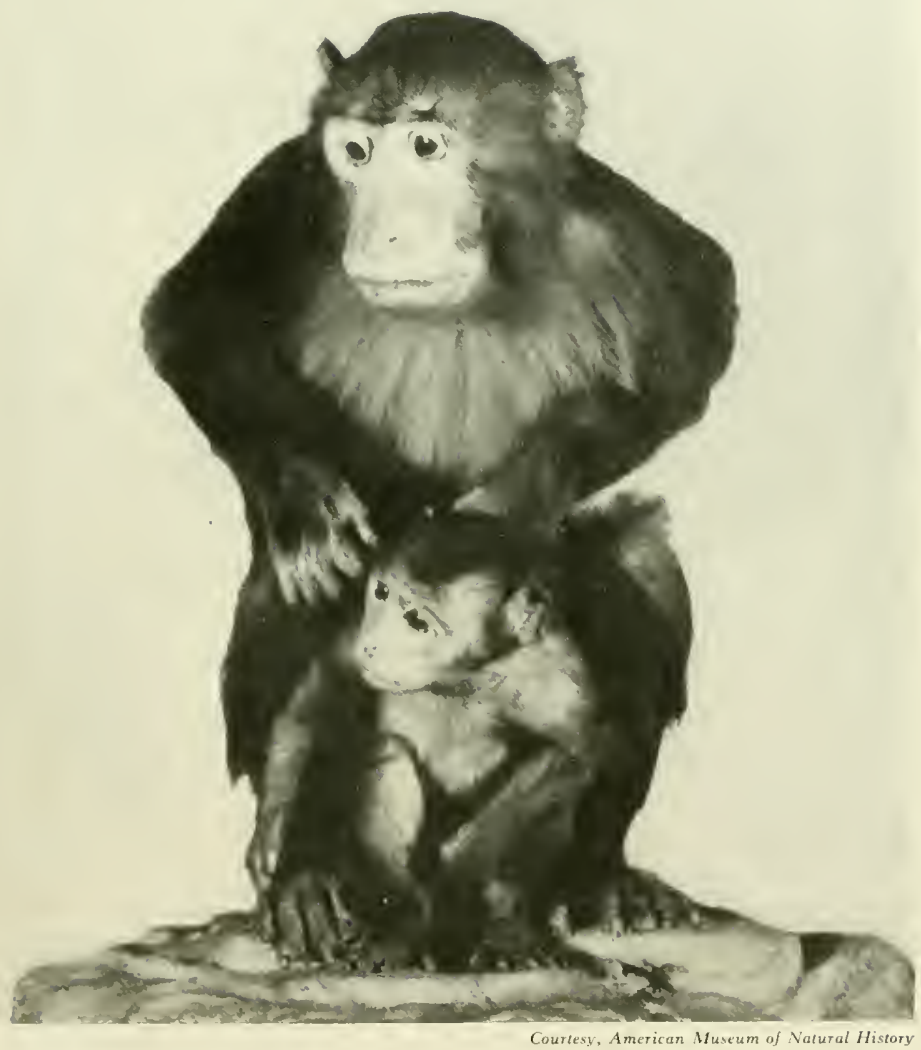

FIG. 160. MACACUS RHESLS. FLLL-GROWN MONKEY AND YOUNG. 
pleasures of the futile pursuit. If, perchance, threatened with capture, they often resort to concerted action in which a number of members of the group will take part in defending or rescuing one of the herd, which may be in diff-
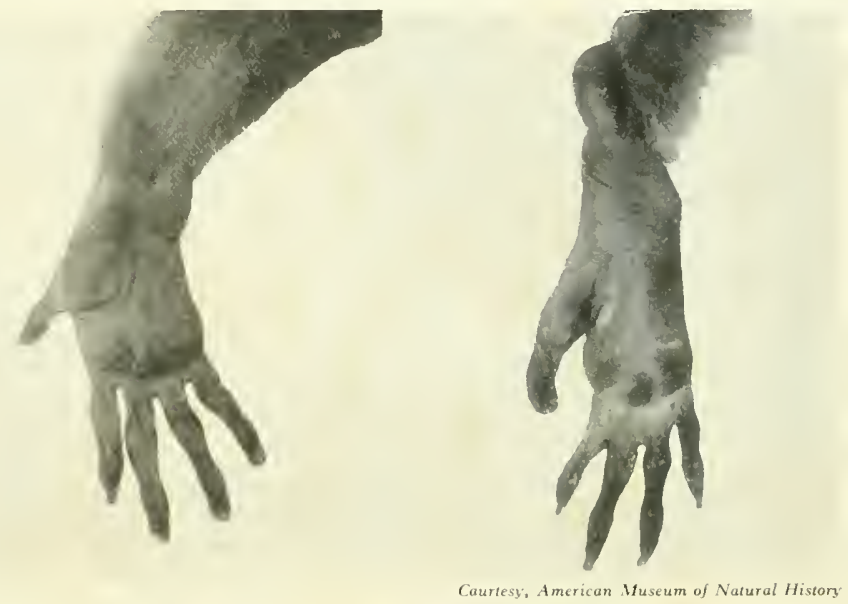

FIGS. I6I AND I62. HAND AND FOOT OF MACACUS RHESUS.

LEFT. Palmar surface of hand showing well-developed manual characteristics, pronounced digitation, short but opposable thumb.

Right. Plantar surface of foot showing well-developed heel, narrow sole, long, narrow toes, and long opposable hallux.

culties. Among themselves they are constantly on the move. Repose seems totally foreign to their behavioral program. Scampering, swinging, chattering, screaming, they go all day long among the trees, either without design in their actions, or changing some lleeting purpose so frequently that their entire formula of behavior has the appearance of ceaseless, kaleidoscopic motion. They are very quarrelsome, constantly fighting or teasing each other. And here, as in all of their activities, the object of their anger, the victim of their mirth is as quickly shifted as their fleeting attention. They feed upon spiders 
and many other kinds of insects, besides fruits and berries. Their motor mechanism not only adapts them to rapid movements among the branches of the trees, but enables them to travel with great speed over the surface of the
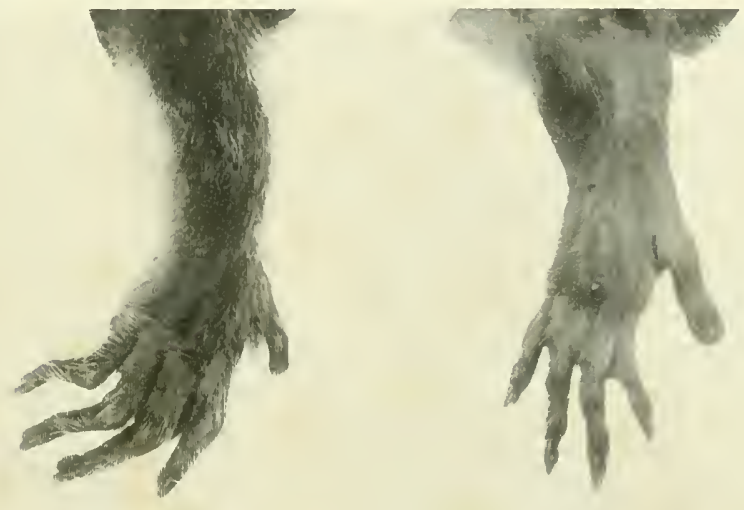

Courtesy, American Museum of Nulural History.

FIGS. I63 AND 164. HAND AND FOOT OF MACACUS RHESUS.

LEFT. Dorsum of hand showing long slender fingers and well-developed finger-nails.

Rıснт. Dorsum of foot, showing liarge, opposible great toe, well-marked digitation, pronounced toe-nails and general hand-like characters of the foot.

ground. Having no fear of the water, they are able to swim for long clistances. As compared with the baboons, they show a greater mental alertness.

\section{Psychological Studies upon the Pithecus Monkeys}

Not a little exact psychological study has been devoted to the several species of pithecus monkeys, particularly concerning their ability to learn, their mentality and their capacity for development of ideation. Kinnaman (1902), in his "Pithecus Rhesus," presents valuable data concerning the 
learning process, sensory discrimination, number reaction and tests of imitation. The results indicate a considerably higher level of intelligence than that ascribed by Thorndyke to the new-world monkeys which he subjected to such tests. Kinnaman found evidence also of general ideas and reasoning, both of which, however, are of a low order. Hobhouse $(1915)$ in a further contribution to our knowledge of the mental life of monkeys, in tests that were admirably adapted to gauge the ideational capacity of these subjects, agrees in general with Kinnaman that the macacus monkey is possessed of definite ideas.

In $1008 \mathrm{~J}$. B. Watson, testing the imitative ability of Macacus rhesus, found relatively little evidence of other than extremely simple forms of ideation. On the other hand, Haggerty (Igog), after much more extended investigation of several species of this family, obtained convineing evidence of ideation and imitative behavior, and concluded that these simian forms depended upon a certain degree of ideational experience. Witmer (1910), in confirmation of Haggerty's results, reports definitely imitative behavior in Pithecus irus; while the work of Shepherd (1910) agrees elosely, so far as the evidence of ideation is concerned, with Thorndyke. The species employed in his tests was Macacus rhesus; all of which, he believed, exhibited ideas of a low order or something closely corresponding to ideas. Franz (1907-1911), in consequence of his study upon these monkeys, was able to throw no special light upon the problem of ideation, although his mode of approach took into account rather the function of various portions of the brain than the accurate description of various features of behavior. In 1911, Hamilton, investigating both Macacus rhesus and Macacus irus, employed an ingenious quadruple choice method which showed that the two monkeys exhibited a fairly adequate type of ideational response.

Yerkes, in his extensive monograph, "The Mental Life of Monkeys and Apes" (1916), was able to disclose results which contrast rather sharply 
with those of previous observers. This no doubt was in consequence of more systematic, longer study, and experimental methods better suited to reveal the problem-solving ability on the part of the monkey. Yerkes' interest eentered on the question of the ability of the animal to learn the solution of relational problems of varying difficulties. On the basis of these observations, he constructed curves of leaming. The Pithecus monkeys which he employed yielded evidenee of ideation, and Yerkes agrees with Thorndyke that free ideas are seanty in these animals; though ideas may develop, they are rather conerete and definitely attached than free. He believes that the general conclusions of previous experimental observers have done no real injustice to the ideational ability of monkeys. It is clear, however, that there are extreme differenees in the mental development of different species of monkeys. The slow process of monkeys in the solution of their problems is quite surprising, however, but their success in such solutions is really less rapid than that of many of the lower mammals, such for example as the pig.

\section{Measurements and Indices of Macacus Ruesus}

Total length of the animal............. I $260 \mathrm{~mm}$.

Length of tail. .................... $330 \mathrm{~mm}$.

Total length of the skull. ............. $1+5 .+\mathrm{mm}$.

Occipito-nasal length............... I1; $; \mathrm{mm}$.

Breadth.................... $96.3 \mathrm{~mm}$.

Intertemporal width............... jo $\mathrm{mm}$.

Brain case..................... $67.6 \mathrm{~mm}$.

The brain is distinctly gyrencephalic in type, with the fissure of Sylvius, the fissure of Rolando and the sulcus simiarum constituting the chicf fissural landmarks upon the convexity. As compared with baboon, the convolutions and fissures are about equal in prominence and size. 
The dimensions of the brain including the cerebellum and brain stem are:

Longitudinal........

Transverse or interparictal

Total weight of the brain.........

Weight of the forebrain

Weight of the midbrain...

Weight of the hindbrain
$-8 \mathrm{~mm}$.

6- $\mathrm{mm}$.

$126 \mathrm{gms}$.

109 gms.

2 gms.

$1 ;$ gms.

Total volume of the brain, determined by water dis-

placement.

120 c.c.

Volume of the forebrain......

100 c.c.

2 c.c.

18 c.c.

Upon the basis of these figures, computed on the weight of the several portions of the brain, the following encephalic indices were determined for the several divisions of the brain:

Forebrain index................. $8+8_{+}$per cent

Midbrain index.................. 2 per cent

Hindbrain inckex. ................. I . . per eent

Macacus rhesus, possessing as it does a forebrain index of $8+$ per cent, comes by this reason naturally into the group of those animals in which manual development has progressed to a marked degree. This index places the animal distinctly higher than any of the lower primates.

Surface Appearance of the Brain in Macacus Rhesus LOBES AND FISSURES

The convexity of the hemisphere shows much the same characteristic arrangement as in baboon. The region of cortex comprised between the 
fissure of Rolando and the sulcus simiarum, namely the parietal lobe, is much more richly convoluted than any other portion of the surface of the macacus brain. The temporal lobe, which is a continuation of the parietal Iobe ventrally, also has a marked degree of fissural richness. The area of cortex lying in front of the fissure of Rolando, namely the frontal lobe, is the least richly fissured and convoluted portion of the brain. The orbital surface presents a well-defined interorbital keel upon either side of which is a deep orbital indenture characteristic particularly of the simian brain. The fissures upon this surface correspond closely to those observed in the baboon. The occipital lobe, especially caudal to the sulcus simiarum, shows less convolution even than the frontal area, but this lack of fissural pattern, so conspicuous on the lateral convexity of this region of the brain, is offset by the relative richness in fissures on the mesial and ventral surfaces of the occipital lobe. The ventral surface presents a deep cerebellar concavity. In general, it may be said that the cortex of the macacus brain corresponds closely to that of the baboon, its lobation being essentially the same and its fissures occupying similar positions. If there is any essential difference between these two types of cerebral hemisphere, it is to be found in the greater richness in convolutional pattern of the macacus. The hemispheres of both of these species give a convincing representation of the simian type of brain, even in such minor details as the temporo-sphenoidal incisure and the ventral projection of the tip of the temporal lobe. In both instances, the occipital lobe overhangs and completely covers the tentorial surface of the cerebellum, a feature which is so typically primate that it may be regarded as a primarily identifying character of this order.

THE CEREBELLUM

The cerebellum presents a tentorial surface which, however, is more convex from side to side than is the case in the still higher primates. Its vermal 
ridge-pole also has a higher elevation. The foliation upon this surface is typical of all simians, i.e., continuous across the midline over the vermis into the lateral lobe without suleal interruption. The occipital surface presents a
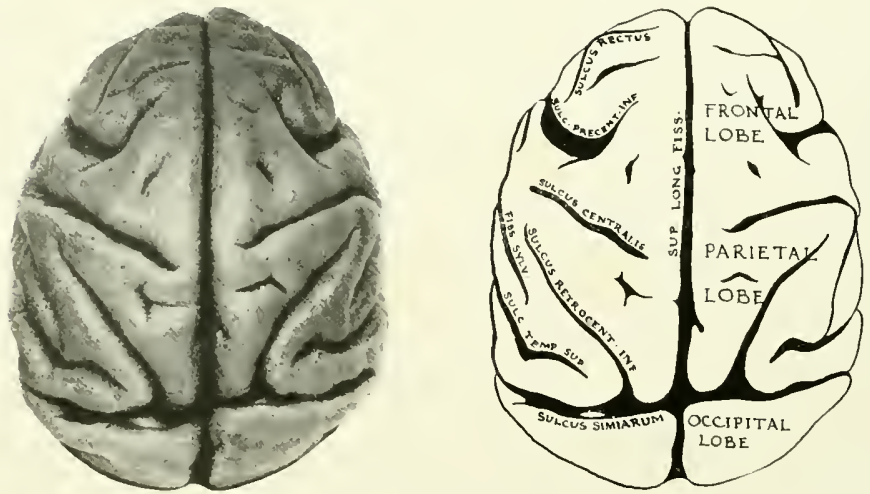

FIG. I6;. DORSAL SURFACE OF BRAIN OF MACACUS RHESUS. [Actual Length $56 \mathrm{~mm}$.]

hey to Diagran. Fiss. Sylv., Fissure of Sylvius; sulc. precent. inf., Sulcus Precentralis Inferior; sulc. RetroceNt. INF., Sulcus Retrocentralis Inferior; sulc. TEMP, SuP., Sulcus Temporalis Superior; sup. Long. FISS., Superior Longitudinal Fissure.

definite median clevation, the vermis, separated from the lateral lobes by the two paramedian sulei; so that the vermal folia here are separated by definitely longitudinal sulei, while there is a deflection of the suleal lines in the lateral lobes, downward and forward. The petroso-ventricular surface of the cerebcllum presents its characteristic features. At its lateral extremity is the cerebello-pontile angle in which is contained a flocculus of moderate size. The remaining portion of this surface is in rclation with the roof of the fourth ventricle, and thus, until the oblongata is removed by disscetion, concealed from view. 
TIIE BRAIN STEM

In general, the outline of all of the structures appearing upon the surface of the brain stem in macacus has a clearness of definition which is not the case
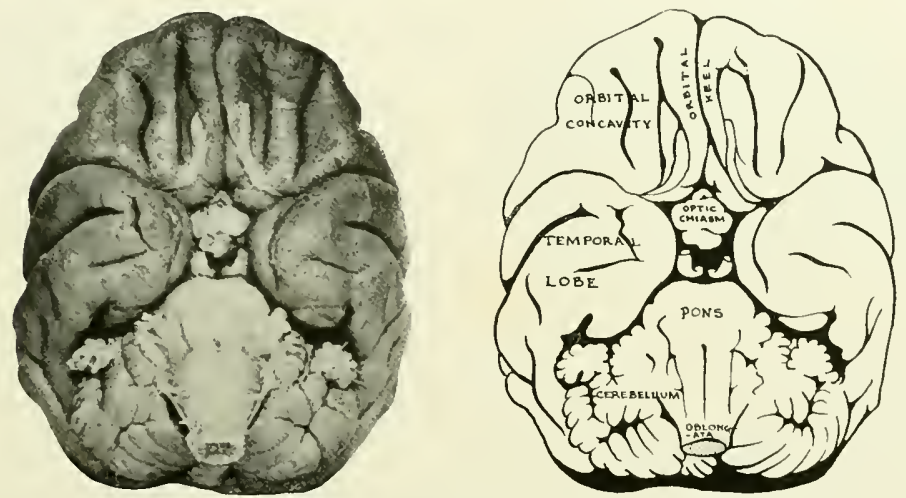

FIG. 166. BASE OF BRAIN OF MACACUS RHESUS. [Actual Length $; 6 \mathrm{~mm}$.]

in the lower primates, nor even in more closely allied species, such as Papio cynocephalus.

The Obloxgata. The oblongata, upon its ventral surface, presents a well-defined ventromedian sulcus, flanked upon either side by two pronounced elevations, the pyramids. These pyramids extend from their bases which are situated above, in relation with the lower border of the pons Varolii, downward, and gradually attenuate to an apex, where many of the fibers pass inward and backward to form the pyramidal decussation. Lateral to the pyramid, and separated from it by a well-defmed sulcus, is the eminence produced by the inferior olivary nucleus. The sulcus separating this structure from the pyramid is the pre-olivary sulcus. Dorsally the olivary eminence is bounded by the post-olivary sulcus separating it from a rather faintly outlined elevation on the lateral surface, 
which becomes more prominent as it extends toward the pons, the restiform body. In the cephalic region of this lateral area is another protuberance, the tuberculum acusticum.
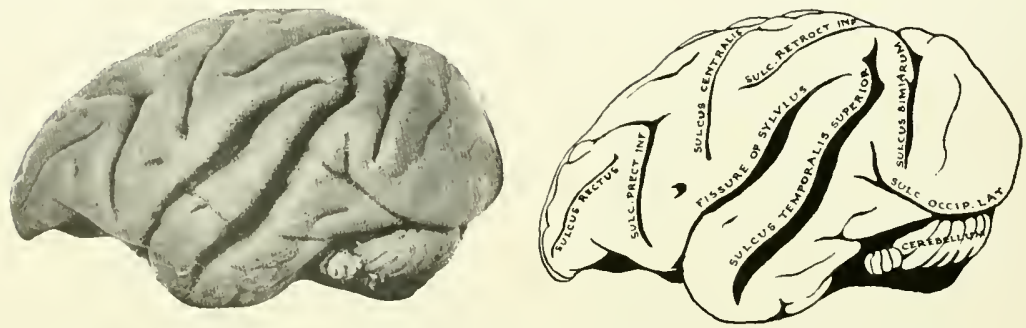

FIG. 167. LEFT LATERAL SLRFACE OF BRAIN, MACACUS RHESLS. [Actual Length $62 \mathrm{~mm}$.]

hex to Diagram. sulc. occip. lat., Sulcus Occipitalis Lateralis; sulc. Prect. INF., Sulcus Precentralis Inferior; sUlC. Retroct. INF., Sulcus Retrocentralis.

The dorsal surface of the oblongata presents its two characteristic divisions, the ventricular and the infraventricular portions. The infraventricular portion is dircetly continuous with the cervical portion of the spinal cord. As the inferior angle of the fourth ventricle is approached, two slight elevations appear on either side of the dorsal septum. The most mesial of these is the clava, an eminence produced by the presence in the dorsal ficld of the nucleus of Goll. At a slightly higher level, separated from the clava by the dorsal paramedian sulcus, a second elevation, the cuneus, makes its appearance. Both of these elevations extend cephalad following the general divergence occasioned by the opening of the ventricular space. Of these two elevations, which represent respectively the nucleus of Goll and the nucleus of Burdach, the latter is definitely larger than the former. The significance of this inequality arises from the fact that in macacus, the forelimb and hand, having gained predominance as sensory organs over 
the tail and leg, require more extensive relay stations for the conduction of sensory impulses to the brain.

The ventricular portion of the oblongata presents a eharacteristic
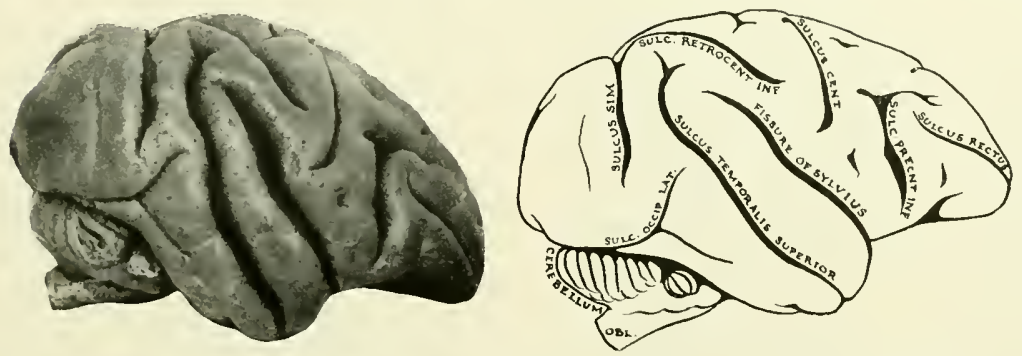

FIG. I68. RIGHT LATERAL SURFACE OF BRAIN, MACACUS RHESLS. [Actual Length $62 \mathrm{~mm}$.]

Key to Diagran. obl., Oblongata; sulcus Cint, Sulcus Centralis; Suzc. occip. zat., Sulcus Occipitalis Lateralis; sclic. PRecnt. INF., Sulcus Precentralis Inferior; sulc. Retrocent. INF., Sulcus Retrocentralis Inferior; sclcus sim., Sulcus Simiarum.

arrangement, in the divarication of the alar plates and the opening of the ventricular space. The caudal angle is directed downward and covered for a short distance by a remunt of the central gray matter, the obex. The floor of the ventricle is bounded caudally by two high walls produced by the elevations of the cuneus and clava. These, however, gradually become reduced in height until they reach the level of the lateral recess, where they are on the same plane as the ventricular floor. This floor is divided longitudinally by a deep median sulcus. Upon cither side of this suleus, in the lower angle of the ventricle, is the trigonum hypoglossi in the position of the hypoglossal nucleus, and lateral to it, the suleus limitans separating the trigonum hypoglossi from the forea vagi, the latter marking the position of the dorsal ragal nucleus. No area postrema or area plumiformis could be discerned in the speeimens examined. 
Cephalad to the eminences of the cuncus and clava, apparently in direct continuity with them as they become attenuated and reach the level of the ventricular floor, is a marked elevation. This is the restibular area
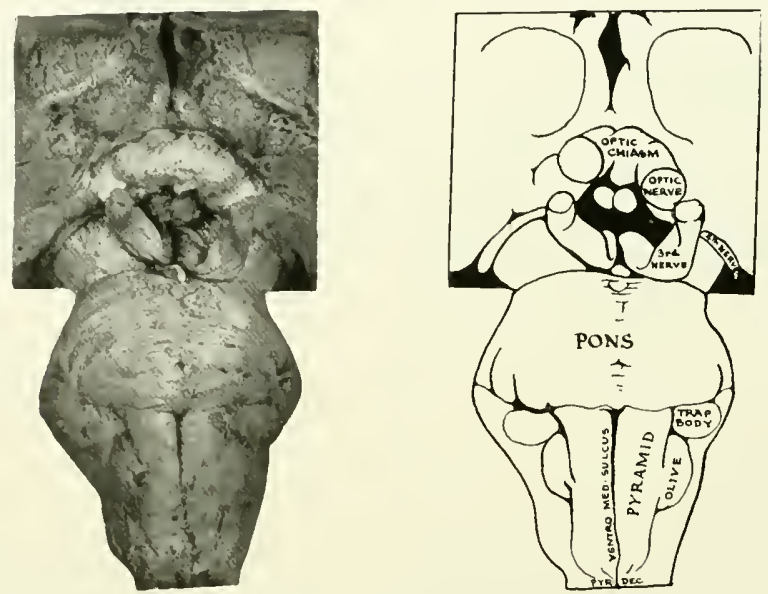

FIG. I69. VENTRAL SURFACE OF BRAIN STEM, MACACLS RHESUS. [Actual l.ength, 4+ mm.]

Key to Diagram. trap. bodr, Trapezoid Body; pyr. dec., Pyramidal Decussation; ventro med. sulcus. Ventromedian Sulcus.

which contains the nuclear elements for the receipt of impulses from the internal ear and particularly related to the balancing mechanism. The marked prominence of this elevation in the relief of the floor of the ventricle is indicative of the degree to which macacus depends upon its balancing function. A boundary between the cephalic and caudal triangles of the fourth ventricle is produced by the crossing striae acusticae, many of which pass obliquely downward, while a few ascend, passing over the outer surface of the restibular eminences. The apex of the cephalic angle of the ventricle marks the point of transition into the caudal orifice of the Sylvian aqueduct. 
Immediately above the aqueduct appears the quadrigeminal plate of the midbrain, characteristically arranged in four symmetrieal clevations, two of which are caudal in position, the inferior colliculi, while the two cephatic
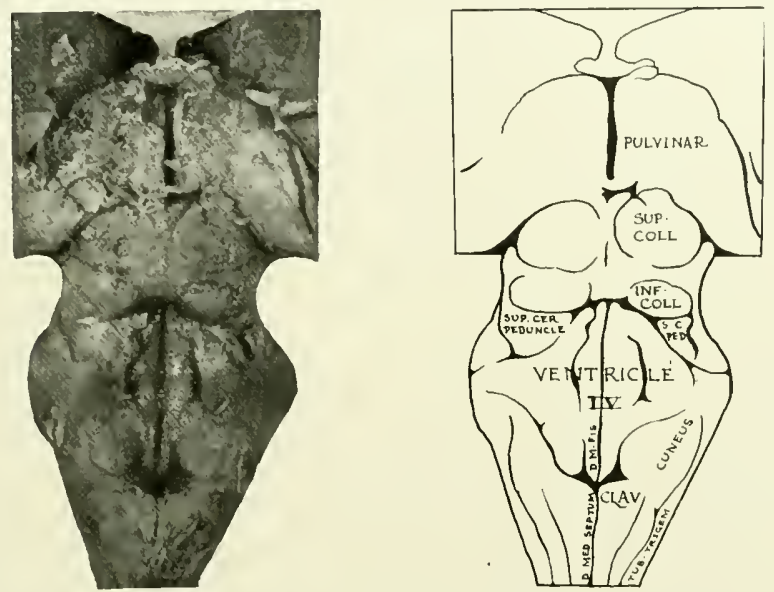

FIG. J7O. DORSAL SURFACE OF BRAIN STEM, MACACUS RHESLS. [Actual Length, $44 \mathrm{~mm}$.

Key to Diagram. Clav., Clava; D. M. Fis., Dorsomedian Fissure; D. Med. Septum, Dorsomedian Septum; INF. Coll., Inferior Colliculus; sup. CER. PEDUnCle and S. C. PEd., Superior Cerebellar Peduncle; stP. Corl.., Superior Colliculus; tUB. TRIgEM., Tuberculum Trigemini.

elevations form the superior colliculi. These latter are about twice the size of the inferior colliculi, and slightly more elevated as tectal structures. The four elevations are separated Iongitudinally by the median intercollieular suleus, and transversely by the transverse intereollicular suleus. On the lateral surface of the midbrain, extending from the inferior colliculus, is the brachium conjunetivum posticum which terminates in the mesial geniculate body. A similar brachium extends forward from the superior colliculus to terminate in the lateral geniculate body. 
The Pons Varolir. Upon the ventral surface of the brain stem the oblongata is separated from a broad and llat band of transverse nerve libers, the pons Varolii, by a well-defined bulbopontile suleus, whieh latter marks the point of emergence from the brain stem of the sixth, seventh and eighth nerves. The prominence of the pons denotes the degree of development in the cerebral cortex, especially the neopallium, both as to its general proportions as well as the richness of its convolutional and fissural patterns. From the functional standpoint such a pontile development justifies the conclusion that macacus is an animal endowed with a rather extensive range of skilled performances; and furthermore, eapable of acquiring many learned reactions quite unknown in many of the lower primates. Indeed, such prominenee in the pons bespeaks an animal capable of more complex behavior than its next Jower eongener, the baboon. This seems to be borne out by the reactions of these animals observed in captivity. The antagonism and stubbormness of the baboon in resisting attempts to teach it are in marked contrast to the rapid, almost uncanny acquisitiveness of the macacus tribes in learning new, if often most aggravating tricks.

The ventral surface of the pons presents a fairly decp groove which contains the basilar artery. This surface terminates abruptly at its cephalic limit in the pontopeduncular suleus where the cerebral peduncles begin. By their divergenee, these peduneles ereate the interpeduncular space, which is limited above by the optie chiasm, and bounded laterally by the two cerebral peduncles and optic tracts. It contains the mammillary bodies, the region of attachment of the infundibular stalk and the tuber einereum.

The Cerebral Peduncles. The cerebral peduncles, as is the case of the pons Varolii, are more clearly defined than in the lower species, and this distinetness gives the impression of an animal possessed of more discretely organized behavioral reactions. This feature is of importance as it is one of the 
most considerable factors in weighing the evidence concerning the relation of the primates, one to another.

\section{Internal Structure of the Bran Stem of Macacus Rhesus}

Although the levels at which the sections have been selected in the macacus brain do not exactly correspond with those of the baboon, they are so chosen as to cover all of the major features atready discussed in the brain stem of the other primates. The impression is gained at once that all of the chief nuclear structures stand out with a clearer definition than is the case in any of the previous specimens. This increasing clarity is a distinguishing feature in the higher primates and man.

\section{LEVEL OF THE PYRAMIDAL DECUSSATION (FIG. I-1)}

At the level of the pyramidal decussation the outstanding feature is the crossing fibers of the pyramidal bundles and the influence which this decussation produces upon the arrangement of the gray matter. The crossing pramidal fasciculi ( $\mathrm{Pyx}_{\mathrm{y}}$ ) sweep backward and outward from their initial position on one side of the axis, across the midline to a lateral position on the opposite side, where they finally take up their descending course as the crossed pyramidal tract. In the process of crossing, these pyramidal fibers have followed such a course as to separate the ventral gray column (Ven) from the central gray matter $(\mathrm{Cen})$. The distance between these two elements of the gray matter is considerably greater than in any of the other primates thus far considered, conveying the impression that the pyramidal system is itself more voluminous than in the lower forms. This impression needs corroboration before it can be maintained that the pyramidal system in macacus is really more extensive and thus provides more ample conduction for voluntary control over the somatic musculature. The ventral gray matter ( $\mathrm{e}$ en) occupies a position about midway 


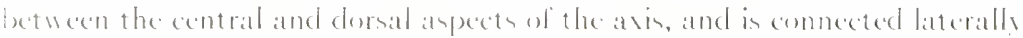
he an elongated, isthmus-lihe area 1 ith a somen hat expanded dorsal horn

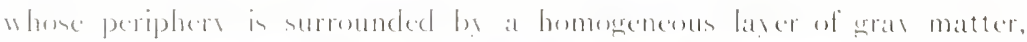

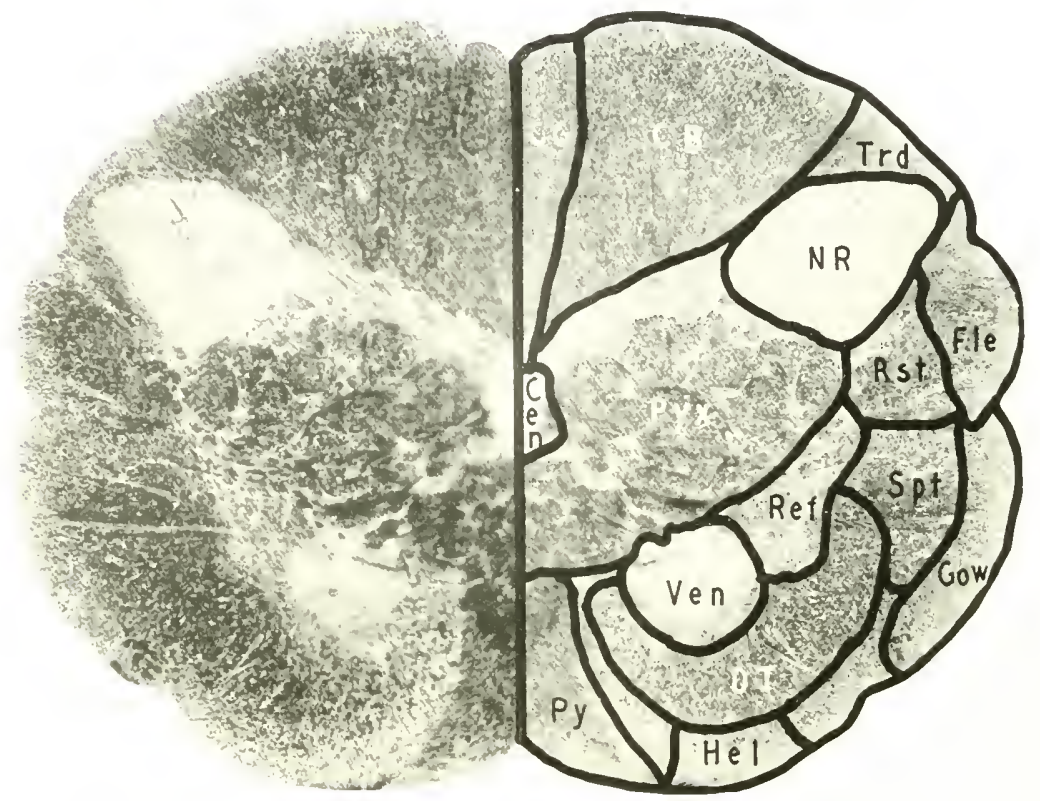

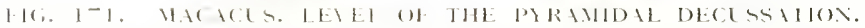

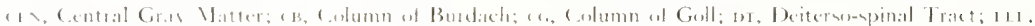

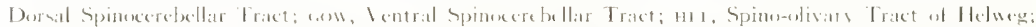

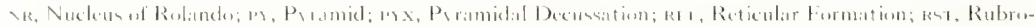

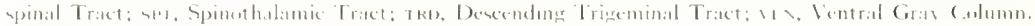
Acression No. 140. Section 10. Actual Size 11). - mm.

the substantia gelatinosa trigemini (NR). The narrow, isthmus-like band of gral matter connecting this latter with the contral writy matter forms the cervis of the dorsal horn. Dorsal to the central wray matter (Cen), the corvix of the dorsal horn and the substantia gelationest trigemint is the 
expansive dorsal freld in which, however, there is as yet no sign of those great sensory nuclei which serve as relays for the sensory pathways from the extremities. The broad expanse of this dorsal sensory field indicates the

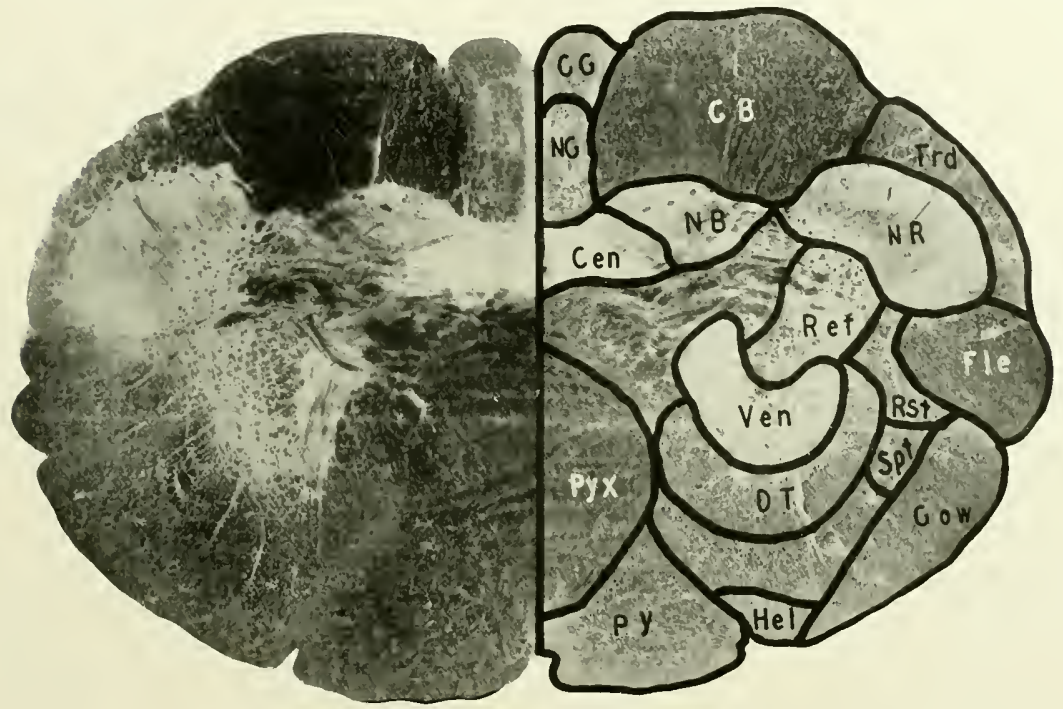

FIG. I-2. MACACLS. LEVEL OF CALDAL LIMIT OF DORSAL SENSORY NLCLEI. CEN, Central Gray Matter; cB, Column of Burdach; CG, Column of Goll; Dr, Deiterso-spinal Tract; FLE. Dorsal Spinocerebellar Tract; Gow; Ventral Spinocerebellar Tract; hel, Spino-olivary Tract of llelweg: мB, Nucleus of Burdach; NG, Nucleus of Goll; NR, Nucleus of Rolando; Py, Pyramid; Pix, Pyramidal Decussation; REF, Reticular Formation; RST, Rubrospinal Tract; sPT, Spinothalamic Tract; TRD, Descending Trigeminal Tract; veN, Ventral Gray Column. [Accession No. 149. Scction 35. Actual Size $12 \times 8 \mathrm{~mm}$.]

capacity of the central axis for the conduction of sensory impulses concerned in discriminative sensibility. The faint indenture on the dorsal aspect of the section indicates the position of the dorsal paramedian sulcus which separates the column of Goll (CG) from the column of Burdach (C.B), thus furnishing some idea as to the relative dimensions of these two tracts. 
Judgment based upon this comparison would favor the pathway from the upper extremity as being more capacious for the purpose of sensory conduction. Should this impression be borne out by other facts, the inference that there has been an advance in the upper extremity, and especially the hand as a sensory organ, seems justified. This fact, taken in conjunction with the relatively small size of the substantia gelatinosa, as compared with the similar structure in other species, implies that the facial region of the animal has lost some of its original importance as a sensory directing organ. In all probability much of this function has been delegated to more capable sensory structures of the forclimb, particularly the hand.

Occupying the most ventral position in the cross section is the pyramidal system (Py $\mathrm{x}$ ), most of whose fibers are actively engaged in the process of decussation. On the ventrolateral aspect of the section, lying external to the ventral gray matter, is the medullary substance constituting the circumferential zone, and mesial to this is the intermediate zone.

LEVEL OF THE CAUDAL LIMT OF THE INFERIOR OLIVARY NLCLEUS (FIG. IT3)

At this level certain striking changes have appeared. Most notable among these is a dense mass of gray matter situated in the ventral portion of the section, the caudal extremity of the inferior olivary nucleus (IO). In the dorsal sensory field there appear the huge masses of nuclear substance constituting respectively the nucleus of $\mathrm{Goll}(\mathrm{NG})$ and the nucleus of Burdach (NB). A feature of the inferior olivary nucleus is the sharper definition of its borders, giving it the appearance of a more discrete structure than in any of the lower species. This nucleus, as is the case with other structures, conveys the impression of an emergence from a more primitive indefiniteness. The central gray matter (Cen) surrounding the central canal occupies a position somewhat more dorsal than in the previous level, indicating a general tendency of this portion of the gray substance to move 
into the position ultimately oceupied by the floor of the fourth ventricle. Dorsal to it, and adjacent to the dorsomedian septum, is a large nuclear mass surrounded by a small capsule of medullary substance, the nucleus

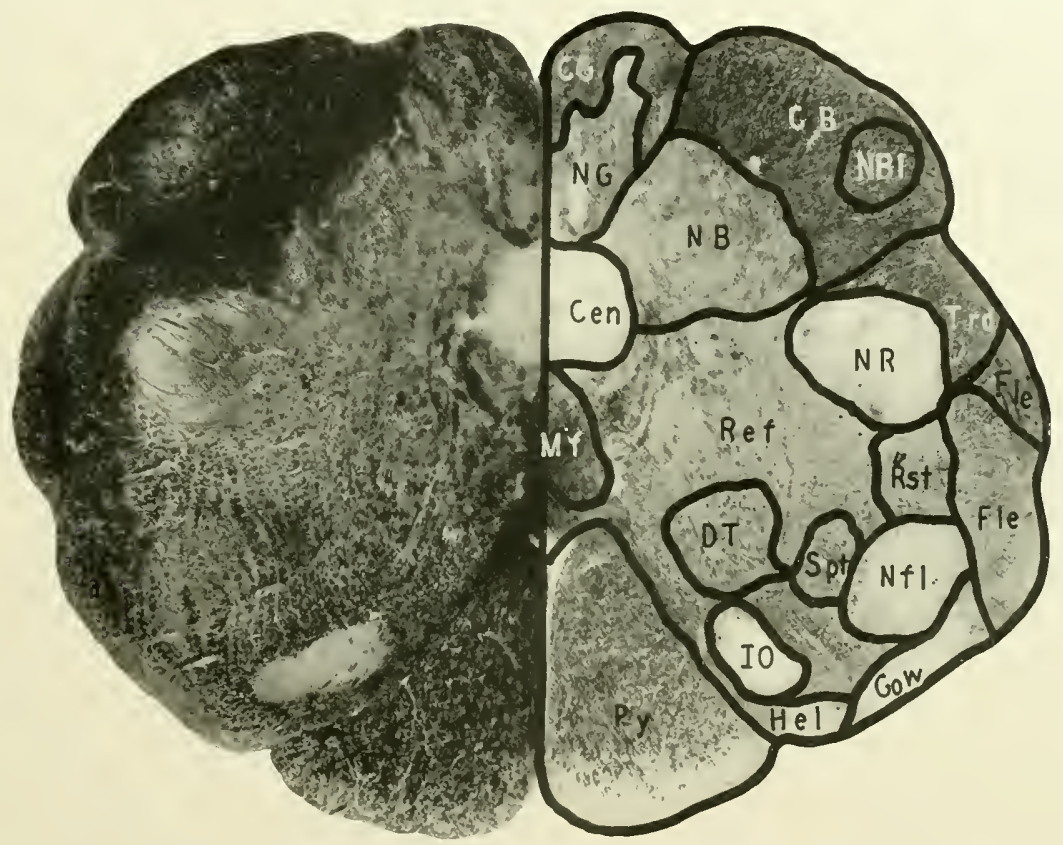

FIG. I73. MACACUS. LEVEL OF CAUDAL LIMIT OF INFERIOR OLIVARY NLCLEUS. CEN, Central Gray Mattcr; CB, Column of Burdach; CG, Column of Goll; DT, Deiterso-spinal Tract; FLE, Dorsal Spinocerebellar Tract; gow, Ventral Spinocerebellar Tract; rEL, Spino-olivary Tract of Helweg; 10 , Inferior Olive; MF, Mesial Fillet; NB, Nuclets of Burdach; NBL, Nucleus of Blumenau; NG, Nucleus of Goll; NFt, Facial Nucleus; NR, Nucleus of Rolando; Py, Pyramid; REF, Reticular Formation; RsT, Rubrospinal Tract; spr, Spinothalamic Tract; TRD, Descending Trigeminal Tract. [Accession No. I 49. Section go. Actua] Size I I $\times 8 \mathrm{~mm}$.]

of Goll (NG), lateral to which, and separated from it by the dorsal paramedian sulcus, is the much larger nucleus of Burdach (NB). De- 
tached from this nucleus and lying near the periphery is an equally large sensory element, the nuckeus of Blumenau (NBl). In a position ventral to the nuckeus of Blumenau is the substantia gelatinosa trigemini (NR), in contact externally with the fibers of the descending trigeminal tract (Trd). It is now possible to compare with more eritical sense the relative dimensions of the three major elements entering into the dorsal sensory field, and representing the dermatomic areas of the body from the soles of the feet to the crown of the head. These elements serve all modalities of discriminative sensibility within the same limits. It is apparent that the column and nucleus of Burdach far exceed in size either of their neighboring structures, the nucleus of Goll (NG) situated mesial to them, or the substantia gelatinosa trigemini (NR) situated ventral to them. On the strength of this comparison, the conclusion seems tenable that the hand and upper extremity have gained much in prominence as sensory organs over the head and face, which latter are only meagerly represented by the relatively small receiving station of the trigeminal nerve. Quite as much do the lower extremity and tail suffer by the comparison as sensory organs. In Macacus rhesus the tail is short and has developed none of that prehensile specialization which characterizes the new-world monkeys.

Passing through the substantia gelatinosa trigemini (NR) are a few emergent fibers of the eleventh nerve, making their way toward the periphery of the axis. The fibers of the twelfth nerve are also seen taking origin in the ventral portion of the eentral gray matter (Cen) and passing forward in the direction of the inferior olivary nucleus $(10)$. The reticular formation (Ref) occupies a central position, and through it pass a number of internal arcuate fibers from the nucleus of Burdach, while large masses of decussating fibers extend inward from erigins in the nucleus of Goll and pass mesially to enter the decussation of the mesial fillet (Mlf). The collected bundle of the pyramid ( $\mathrm{Py}_{\mathrm{y}}$ ) occupics its usual ventromesial position and affords a clear 
idea as to the relative size of the pyramidal system in macacus. Lying upon the ventrolateral periphery is the circumferential zone, mesial to which is the intermediate zone. Some external arenate fibers, apparently arising in the nucleus of Burdach, pass forward and inward to their decussation in the median raphe.

LEVEL THROUGH THE MIDDLE OF THE INFERIOR OLIVARY NUCLEUS (FIG. I7- 4 )

At this level, the addition to the inferior olive (IO) of the mesial and dorsal accessory olives (DO, VO) is clearly shown. This level, however, is more signifieant as again inclicating the relative sizes of the three major nuclear relay stations for discriminative sensibility, oeeupying the dorsal sensory freld. Thus the nucleus of Goll (NG) appears much smaller as compared with the nueleus of Burdach ( NB), and the aecessory nucleus of Blumenau ( $\mathrm{NBI}$ ) (now showing some of its points of confluenee with the more mesial cell mass of Burdach). It offers an almost convincing argument that the leg, foot and tail have been superseded in their sensory importance by the development of the forclimb and particularly the hand. The relative functional significance of the forelimb and hand, as represented by the nuclei of Burdach and Blumenau (NB, NBI), when compared with the sensory signifieance of the face and head, is elearly illustrated in the relatively small size of the substantia gelatinosa trigemini (NR).

The section also indicates the manner in which the internal arcuate frbers, arising in the nucleus of Goll, sweep forward and inward as dense collected bundles, skirting the ventral gray matter, while those arcuate fibers arising from the nucleus of Burdach make their way by longer, more graceful ares, in less dense compact bundles, penetrating in their passage the main mass of the reticular formation. Fibers of the twelfth nerve ( $\mathrm{N}_{12}$ ) (nervus hypoglossus) make their way from their nucleus (Nhy) in the ventral portion of the eentral gray matter to the inferior olivary nucleus 
(IO). Dorsal to the pyramid ( $P_{y}$ ), and containing at this level many of the compact bundles of clecussating fibers, is the mesial fillet ( $\mathrm{If}$ ) as its fibers are beginning to turn cephatad after crossing the median raphe.

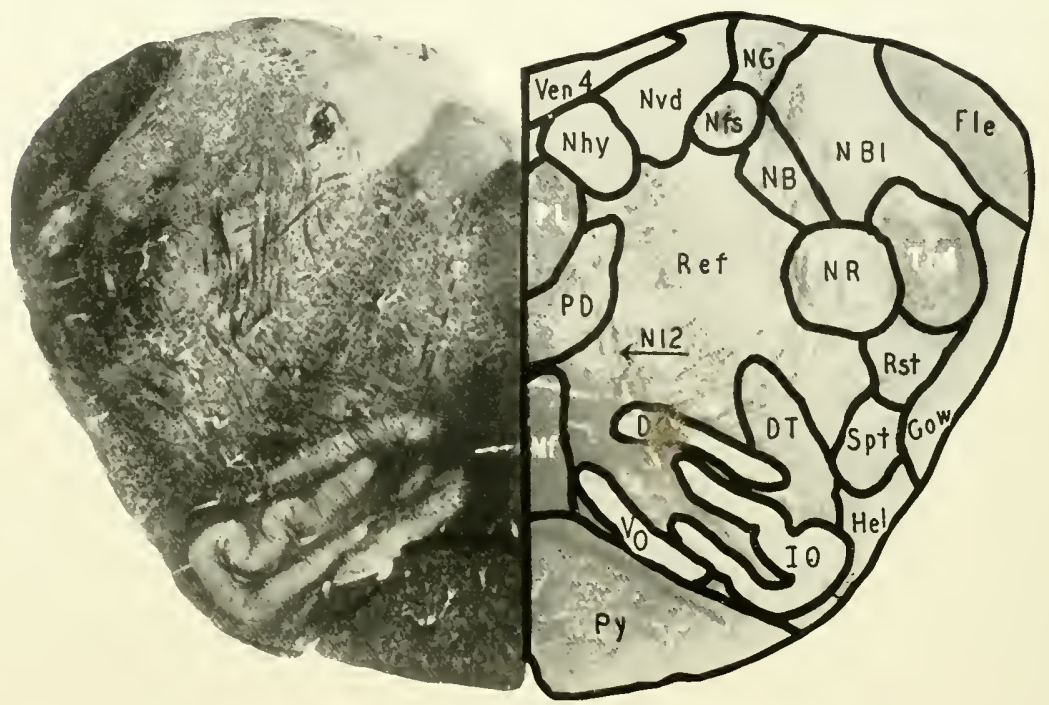

FIG. 174. MACACUS. LEYEL THROUGH MIDDLE OF INFERIOR OLIYARY NUCLEUS.

Do, Dorsal Accessory Olive; pr, Deiterso-spinal Tract; fle, Dorsal Spinecerebellar Tract; gow, Ventral Spinocerebellar Tract; HEL, Spino-olivary Tract of Ifelweg; 10. Inferior Olive; MF, Mesial Fille1; NB, Nucleus of Burdach; NBL, Nucleus of Blumenau; NFS, Facial Nucleus; NG, Nucleus of Goll; NHr, Hypoglossal Nucleus; NvD, Dorsal Vagal Nucleus; NR, Nucleus of Rolando; N12, Hypoglossal Nerve; PD, Predorsal Bundle; PL, Posterior Longitudinal Fasciculus; PY, Pyramid; REF, Reticular Formation; Rst, Rubrospinal Tract; SPT, Spinothalamic Tract; TRD, Descending Trigeminal Tract; vEX 4. Fourth Ventricle; vo, Ventral Accessory Olive. [Accession No. 149. Section 141. Actual Size $13 \times 8 \mathrm{~mm}$.]

LEYEL OF THE VESTIBULAR NUCLEI (FIG. 17j)

Here the cross section shows considerable alteration in its general configuration. Its diameters in the dorsal portion of the field are more extensive than in the lower seetions. This is primarily due to the widening of the 
fourth ventricle and the appearance of the lateral recesses. Among the intrinsic modifications of the axis at this level is a group of nuclei connected with the internal car, serving as relays for impulses concerned with the special

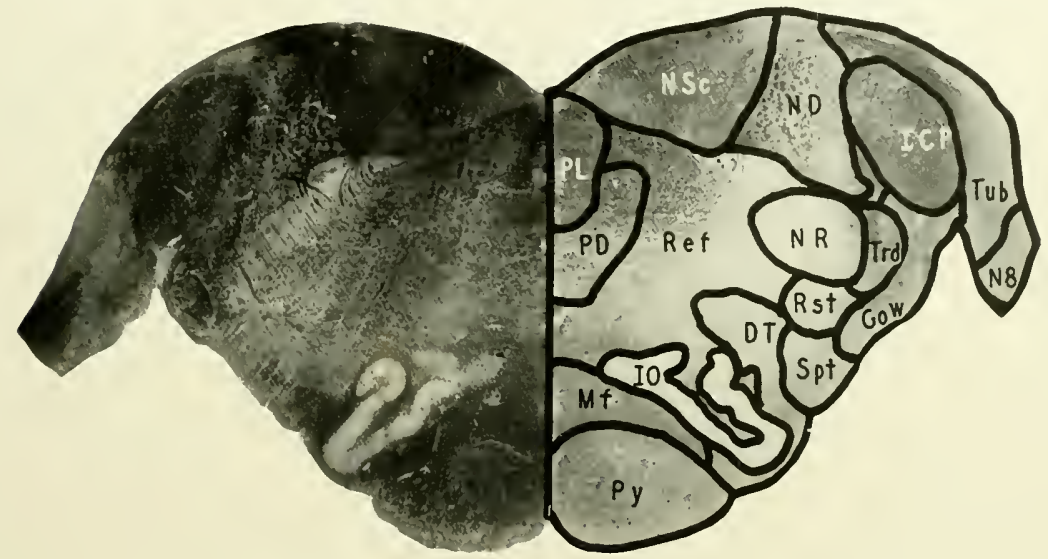

FIG. ITj. MACACUS. LEVEL OF THE VESTIBLLAR NLCLEI.

DT, Deiterso-spinal Tract; Gow, Ventral Spinocerebellar Tract; ICP, Inferior Cerebellar Peduncle; 10, Inferior Olive; MF, Mesial Fillet; ND, Deiters' Nucleus; NR, Nucleus of Rolando; NSc, Nucleus of Schwalbe; N8, Auditory Nerve; PD, Predorsal Bundle; PL, Posterior Longitudinal Fasciculus; Pr, Pyranrid; REF, Reticular Formation; RST, Rubrospinal Tract; SPT, Spinothalamic Tract; TRD, Descending Trigeminal Tract; $T(B$, Tuberculum Acusticum. [Accession No. 149. Section 185. Actual Size $17 \times 7 \mathrm{~mm}$.]

sense of hearing as well as with the balancing mechanism. Situated immediately beneath the floor of the fourth ventricle, underlying the ependymal gray matter, is a large triangular nucleus of small cells, the nucleus of Schwalbe (NSc), and lateral to this a nucleus containing many large cells, the nucleus magnocellularis of Deiters (ND). Dispersed among the cells of the latter nucleus are many small bundles of heavily myelinated axons. These two nuclei constitute the vestibular complex. They have assumed, in a general way, the positions occupied in the lower levels by the nuclei of Goll and Burdach. In the topographical relation of these 
nuclear masses there is complete correspondence in all species thus far considered, from which it must be apparent that a defmite principle of structural design regulates the manner in which this series of nuclei is laid down in the brain stem.

Beginning at the caudal extremity of the oblongata are the nuelei for discriminative sensibility in the tail, leg and arm; immediately succeeding them in the next higher levels are the nuclear relay stations receiving impulses from the proprioceptors of the internal ear in the interest of the balancing function. Thus a superposed series of nuclei in the clorsal field of the oblongata in the form of a long column makes provision for the primary relay of all sensory impulses arising in the proprioceptors of the body. This is not merely of generic significance, for its long antecedent history in the vertcbrates makes it a matter of phyletic moment.

Lateral to Deiters' nucleus, forming the extreme lateral structure of the cross section, is an elevation on the surface of the axis, the tuberculum acusticum ( $\mathrm{Tub}$ ), into which enter some of the fibers of the cochlear division of the eighth nerve (N8). This elevation forms a relay station in the pathway of hearing, so that in the tuberculum acusticum and the two vestibular nuclei at this level, the chief receiving stations for the eighth nerve appear. Interposed between the tuberculum acusticum and Deiters' nucleus is a dense oval bundle of fibers constituting the restiform body (ICP).

Mention shoukl be made of the large size of the vestibular area, inasmuch as this clement of the brain stem causes a pronounced elevation in the floor of the fourth ventricle. Furthermore, the large dimensions of the vestibular complex in macacus indicate to what extent the balaneing function is necessary in this animal.

Another feature at this level is the inferior olivary nucleus (IO) connected with which are the two accessory olivary nucler. Attention is especially called to the clear-cut boundaries of the inferior olivary nucleus 
in macacus, together with the tendency toward definite convolution of its structure, which appears in this species in such conspicuous development for the first time. This tendency to convolution is one which will be seen to beeome more marked as the higher apes are approached. It reaches its greatest degree of complexity in the brain stem of man.

The pyramid ( $\mathrm{P}_{y^{*}}$ ) occupies its typical ventromesial position and affords an opportunity for estimating its size in relation to the rest of the cross section. Dorsal to it are the bundles constituting the mesial fillet ( $M \mathrm{f}$ ). Ventral to Deiters' nucleus, faintly outlined at this level, is the substantia gelatinosa trigemini (NR), upon the lateral border of which are the collected fibers of the descending trigeminal tract ( $\mathrm{Trd}$ ). The reticular formation ( $R \mathrm{ef}$ ) is quite extensive and is penctrated by many arcuate fibers apparently arising in the nucleus of Deiters, $t o$ form the Deiterso-spinal tracts. If one feature may be signalized at this level, it is the great prominence of the vestibular complex with the implication which it bears eoncerning the balancing function of the macacus.

\section{LEIEL OF THE CEREBELLAR NUCLEI (FIG. I-6)}

At the level of the cerebellar nuclei the organization of the cerebellar connection is indicated. The two groups of cerebellar nuclei, namely, the mesial and the lateral groups, have all of that indefmite development which characterizes the lower primates. The lateral group $(\mathrm{Ndt})$, whose largest constituent part is the nucleus dentatus, is a more or less diffuse mass of nuclear substance surrounded by medullary tissue. It has none of the convoluted appearance or discrete outline prominent in the higher anthropoids. In this respect it allies itself closely with all the lower forms previously described.

Situated in the roof of the fourth ventricle is the mesial nuclear group ( $\mathrm{Nfg}$ ), consisting of the nucleus fastigius and the nucleus globosus. Between 
the mesiat and lateral groups of the cerebellar nuclei are the massive bundles of the juxtarestiform body. The roof of the fourth ventricle is formed by the nuclear substance of the cerebellar nuclei. Its floor still contains a large

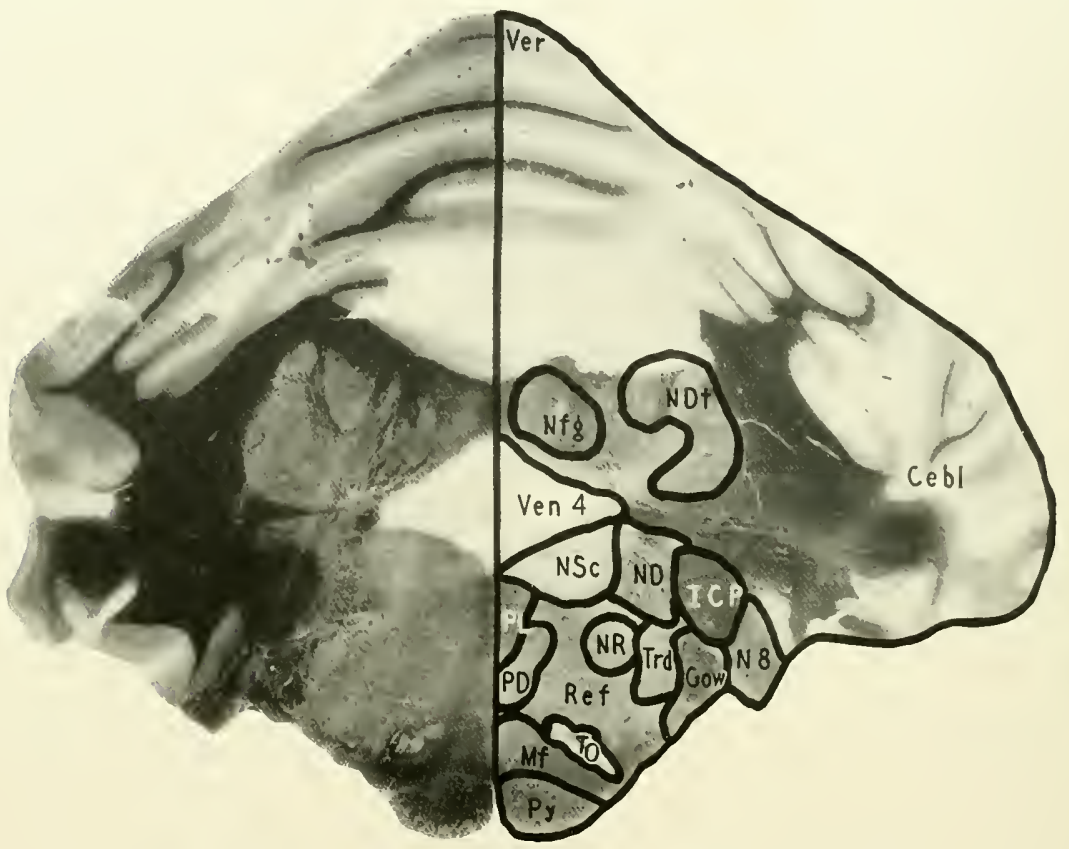

FIG. I 6 . MACACUS. IEIEL OF THE CEREBELLAR NLCLEI.

CEBr, Curebellum; gow, Ventral Spinocerebellar Tract; ICP, Inferior Cerebellar Peduncle; IO, Inferior Olive; MF, Mesial Fillet; ND, Nucleus of Deiters; NDT, Cercbellar Nuclei, Lateral Group; NFG, Cerebellar Nuclei, Mesial Group; NR, Nucleus of Rolando; Nsc, Schwalbe's Nucleus; N8, Auditory Nerve; PD, Predorsal Bundle; PL, Posterior Longitudinal Fasciculus; Pr, Pyramid; REF, Reticular Formation; trd, Descending Trigeminal Tract: VEk, Cerebellar Vermis; VEN 4, Fourth Ventricle. [Accession No. 1 fo. Section rgo. Actual Size $18 \times 15 \mathrm{~mm}$.]

representation of Schwalbe's and Deiters' nuclei (NSc, ND). The other elements at this level are indicated by letters in the caption. 
The principal feature illustrated by this region is the dilfuse character of the cerebellar nuclei. This condition at once indicates an animal poorly provided with coordinative control over its skilled acts. This view of the behavior of macacus is further supported by the relatively small size of the lateral cerebellar lobes. The animal is noted, and even notorious, for its prankishness and its great agility as a climber. It is possible to teach these monkeys many amusing tricks, so that the conception of them as limited in their neokinetic performances perhaps does them injustice. On the other hand, the fact should not be overlooked that however much they may improve by training, these animals use their new acquisitions but little in the ordinary routine of their lives, and escaping from captivity, rclapse to the simpler motor patterns essential to their primitive arboreal adaptations. It must, therefore, be accepted from such evidence as is elicited from the cerebellar muclei and their connections, that the macacus species are, in fact, capable of a very limited range of skilled learned performances which they habitually employ in the pursuits of their daily lives.

LEVEL OF EMERGENCE OF SIXTH CRANIAL NERVE, NERIUS ABDLCENS (FIG. I77)

At this level the section has undergone considerable change due to the appearance of the caudal portion of the pons Varolii (PN). This structure now adds a basal element to the neuraxis and makes distinguishable in it the basis and the tegmentum pontis. The pons here consists of but a few transverse fibers constituting the stratum superficiale pontis, and the caudal extremity of the pontile nuclei contained within which latter are the somewhat scattered fibers of the pyramidal system $\left(P_{y}\right)$. The lateral continuation of the superficial stratum of the pons enters into and forms part of the massive middle cerebellar peduncle (Mcp). The tegmentum at this level is separated from the basis by a number of transverse decussating fibers constituting the trapezoid body ( T r p ) in whose lateral extremity is the 
superior olivary body ( $\mathrm{SO})$. Lateral to this is a small portion of the nucleus facialis ( $\mathrm{Nf}$ ) which gives rise to the first portion of the seventh nerve, a series of line, myelinated fibers arranged more or less as a spray. It extends inward

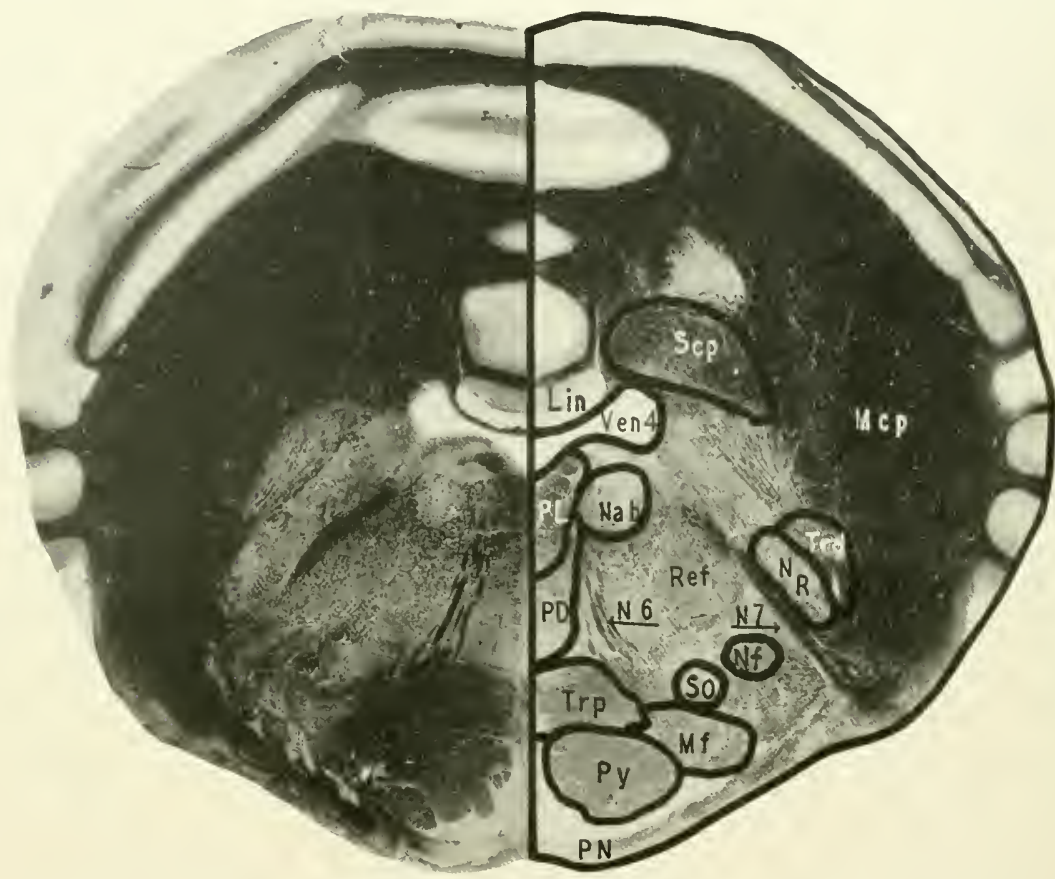

FIG. IT7. MACACUS. LEVEL OF THE ENERGENCE OF THE SIXTH NERVE. LiN, Lingula; мср, Middle Cerebellar Peduncle; Mr, Mesial Fillet; NAB, Nucleus Abducentis; $N F$, Faeial Nucleus; NR, Nucleus of Rolando; N6. Abducens Nerve; N7, Facial Nerve; PD, Predorsal Bundle: PL, Posterior Longitudinal Fasciculus; PN, Pontile Nuclei; Pr, Pyramid; REF, Reticular Formation; TRD, Descending Trigeminal Tract; scP, Superior Cerebdlar Peduncle; so, Superior Olive; TRD, Descending Trigeminal Traet; TRI?, Trapezoid Body; ven 4, Fourth Ventricle. [Accession No. 140. Section 230. Actual Sizc $20 \times 16 \mathrm{~mm}$.

and backward toward the floor of the fourth ventricle. From the superior olivary nucleus a similar, but somewhat larger spray of myelinized fibers 
extends backward and inward, apparently terminating in the large nuclear mass situated on the floor of the fourth ventricle. This latter is the nucleus abducentis ( $\mathrm{Nab}$ ) from which arise the fibers of the sixth nerve to supply the external rectus muscle of the eyeball. They pass successively through the reticular formation ( $R$ ef), the trapezoid body ( $\operatorname{Trp}$ ) and the stratum superficiale pontis. The fiber connections between the superior olive and nucleus of the sixth nerve constitute the superior olivary peduncle. Axons from the superior olive to the sixth nerve nucleus serve the purpose of turning the eyes reflexly toward the side from which the animal may hear a sudden or unexpected sound. The immediate deflection of the gaze toward the source of possible danger would thus be of service in the quick detection of an approaching enemy or other unfavorable factor.

Dorsomesial to the nucleus of the sixth nerve, lying between it and the subependymal gray matter on the floor of the ventricle, is a dense bundle of fibers constituting the second portion of the facial nerve $\left(\mathrm{N}_{-}\right)$) in its intramedullary course. The beginning of the third portion of this nerve, the genu facialis, lies immediately dorsal to the abducens nucleus, while the fourth division of the facial nerve forms a long bundle of heavily myelinized fibers extending obliquely forward and outward between the nucleus of the seventh neve and the substantia gelatinosa trigemini (NR).

Extending dorsolaterally from the substantia gelatinosa of Rolando is a radiating fasciculus which makes its way backward and inward toward the angle of the fourth ventricle where it apparently terminates in a heavily myclinized bundle of fibers, the fasciculus mesencephalici trigemini. The fourth ventricle is much reduced preparatory to transition into the Sylvian aqueduct. Above it lies the superior medullary velum upon which rests the lingular lobule of the cerchellum ( $\mathrm{Lin}$ ). Dorsal to the mesencephalic root of the fifth nerve is a dense bundle of fibers constituting the superior cerebellar peduncle $\left(\mathrm{S}_{\mathrm{c} p}\right)$. 
LETEL OF THE MIDDLE OF THE PONS VAROLII (FIG. I78)

Here this structure attains its full dimensions. It presents the three characteristic pontile layers, the stratum superficiale pontis, the stratum complexum pontis and the stratum profundum pontis. In the complex layer of the pons Varolii opportunity is aflorded to estimate the size and extent of the pontile nuclei (PN) which appear to be considerably larger than in baboon. The pyramidal fibers ( Py) occupy their usual position in the center of this layer, their bundles being much dispersed by the interposition of nuclear substance and many decussating fibers of this middle layer of the pons. The impression conveyed by the pons and its large nuclear mass is that it belongs to an animal possessed of relatively high coordinative control in its skilled movements.

Situated between the basis and the tegmentum, as the boundary line between these two, is the mesial fillet ( $\mathrm{If}$ ); lateral to the mesial fillet is the lateral fillet (Lf), in the dorsal extremity of which is the nucleus of the lateral fillet. A clense and large bundle of myelinized fibers in the extreme lateral position of the cross section is the middle cerebellar peduncle $(\mathrm{Mcp})$, while situated in the dorsolateral angle of the section is a smaller dense bundle of fibers which represents the superior cerebellar peduncle $(S c p)$. On the periphery over this bundle of fibers is a thin band of myelinized axons, the ventral spinocerebellar tract (Gow) on its way to the vermis of the cerebellum. Interposed between this latter tract and the superior cerebellar peduncle is the tractus uncinatus of Russel ( Tur). Ventral to the dense fasciculus forming the middle peduncle, many fibers enter the axis. These axons are the dorsal root fibers of the trigeminal nerve $(\mathrm{N} ;)$. Their course may be followed through the peduncle into the lateral aspect of the tegmentum where some of them end in relation with the cephalic extremity of the substantia gelatinosa trigemini (NR). 
The central gray matter (Cen) surrounds the much reduced ventricular space which is here approaching the caudal orifice of the Sylvian aqueduct. The major portion of the tegmentum is oceupied by the reticular

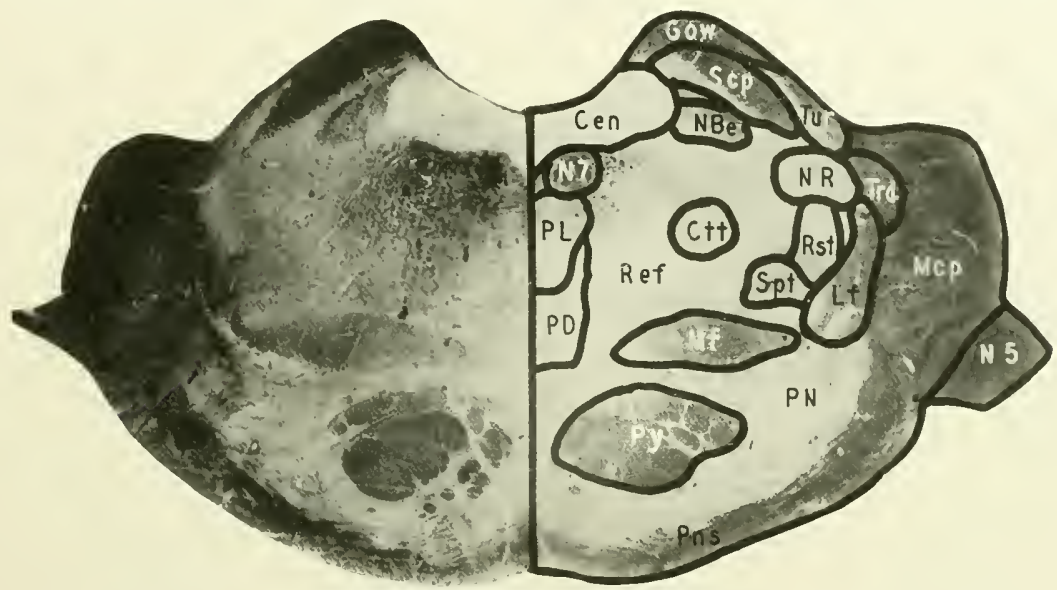

FIG. I-8. MACACUS. LEVEL THROLGH THE MIDDLE OF THE PONS VAROLII. CEN, Central Gray Natter; CrT, Central Tegmental Tract; gow, Ventral Spinocerebellar Tract; LF, Lateral Fillet; мCP, Middle Cerebellar Peduncle; мr, Mesial Fillet; NBE, Nucleus of Bechterew; NR, Nucleus of Rolando; No, Trigeminal Nerve; N-, Facial Nerve; PD, Predorsal Bundle; pr., Posterior Longitudinal Fasciculus; PN, Pontile Nuclei; PNs, Pons; Py, Pyramid; REF, Reticular Formation; Rst, Rubrospinal Tract; sCP, Superior Cerebellar Peduncle; SPT, Spinocerebellar Tract; TRD, Descending Trigeminal Tract; TuR, Tractus Uncinatus of Russel (Hook Bundle). [Accession No. 140. Section 280. Actual Size $21 \times 10 \mathrm{~mm}$.]

formation (Ref) in which are many transverse dccussating fibers, some of which at least appear to take origin in the substantia gclatinosa trigemini (NR), passing inward, it may be, as a sccondary pathway for the conduction of impressions from the face and head. These fibers appear to decussate, as is the case of all sensory axons, in the median raphc.

LEVEL OF THE EMERGENCE OF THE FOURTH OR TROCHLEAR NERVE (FIG. I79)

At this level, the neuraxis passes through its isthmial portion at or near the junction between the hindbrain and the midbrain. In its roofplate, which 
forms the superior medullary velum above the beginning of the Sylvian aqueduct (IT), are the decussating fibers of the fourth or trochlear nerve $\left(N_{4}\right)$ as they emerge from the brain stem. This nerve supplies the

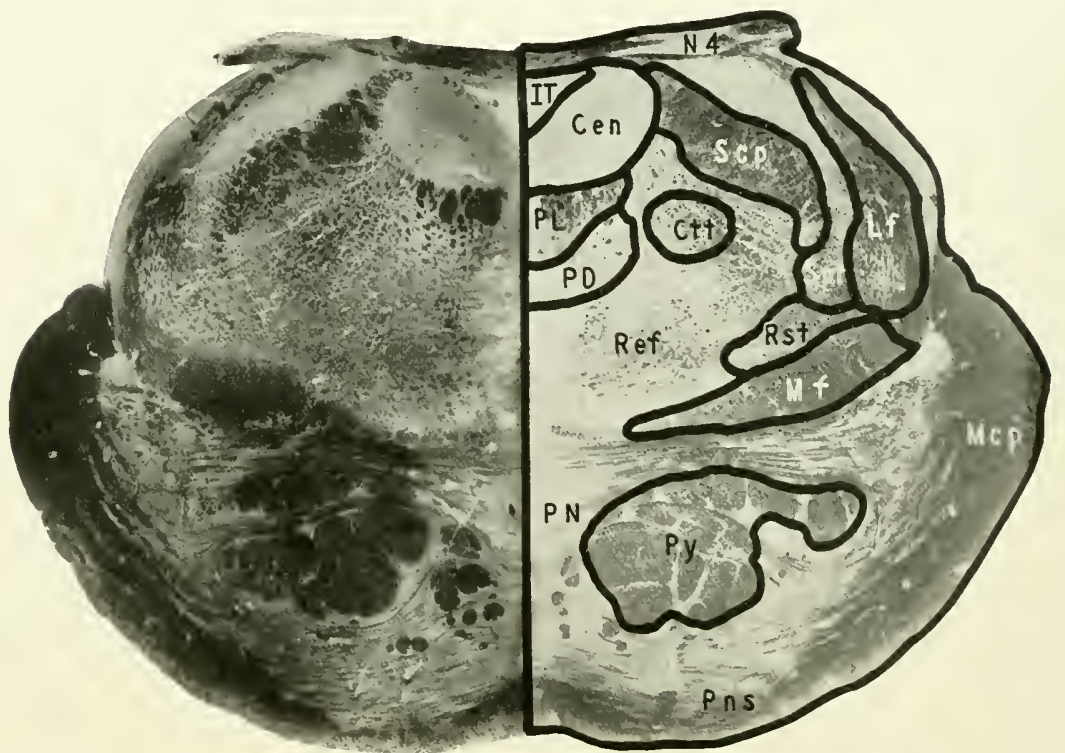

FIG. ITO. MACACUS. LEIEL OF THE EMERGENCE OF THE TROCHLEAR NERVE. CLN, Central Gray Matter; CTT, Central Tegmental Tract; $1 T$, Iter; Lr, Lateral Fillet; McP, Middle Cerebellar Peduncle; MF, Mesial Fillet; N4. Trochlear Nerve; PD, Predorsal Bundle; PL, Pusterior Longitudinal Fasciculus; PN, Pontile Nuclei; P\s, Pons; 1'Y, Pyramid; ReF, Reticular Formation; Rst, Rubrospinal Tract; scP, Superior Cercbellar Peduncle; SPT, Spinothalamic Tract. [Accession No. 14\%. Section 305. Actual Size $18 \times 12 \mathrm{~mm}$.

superior oblique muscte of the eyeball and is the only one of the cranial nerve series which undergoes eomplete decussation before its emergenee.

The three layers of the pons Varolii are still clearly defined and further opportunity is afforded to estimate the extensiveness of the pontile nuclei $(\mathrm{PN})$ whose bearing upon the derelopment of skilled movements has 
already been discussed. The fibers constituting the pyramidal system are still further separated by the interposition of transverse fibers and portions of the pontile nuclei. The tegmentum is separated from the basis here by the mesial fillet (Mf) at whose lateral extremity, and extending dorsally, is the fateral fillet (Lf). Mesial to the lateral fillet is a dense bundle, now somewhat scattered, the superior cerebellar peduncle ( $S$ ep), about to make its characteristic swing forward and inward preparatory to decussation in the midbrain. The reticular formation (Ref) occupies the major portion of the tegmentum. It contains at this level no specialized aggregations of nuclear differentiation.

\section{LEVEL OF THE INFERIOR COLLICULLS (FIG. 180)}

Here the contour of the axis is considerably altered by the appearance of two elevations on its dorsal surface. These elevations, the inferior colliculi (IC), still show a marked degree of stratification. Their prominence, together with this specialization, may be taken to indicate that some of the primitive function over which they preside is still retained. Fibers of the lateral fillet (Lf) have already entered into the inferior colliculus; still others are approaching it. The prominence of these tectal structures, both in surface relief and histological differentiation, doubtless indicates that some portion, at least, of the function of hearing still has representation in these primordial centers of that special sense. It is noteworthy, however, that the coefficients of this structure, as compared with the fower primates, show an actual decrease in size. This fact unquestionably implies that the telencephalizing process which is gradually transferring the major functional activities of the auditory sense to the temporal lobe of the cerebral hemisphere, has made considerable advance in such animals as the macaque, as a consequence of which the tectum of the midbrain has lost much of its primitive auditory dominance. The central gray matter ( $\mathrm{Cen}$ ) surrounds the Sylvian aque- 
duct. In its lateral portion are several dense bundles of myclinized axons representing the descending fibers of the trochlear nerve $\left(N_{t}\right)$ as they pass downward to the level of their final decussation before emergence from

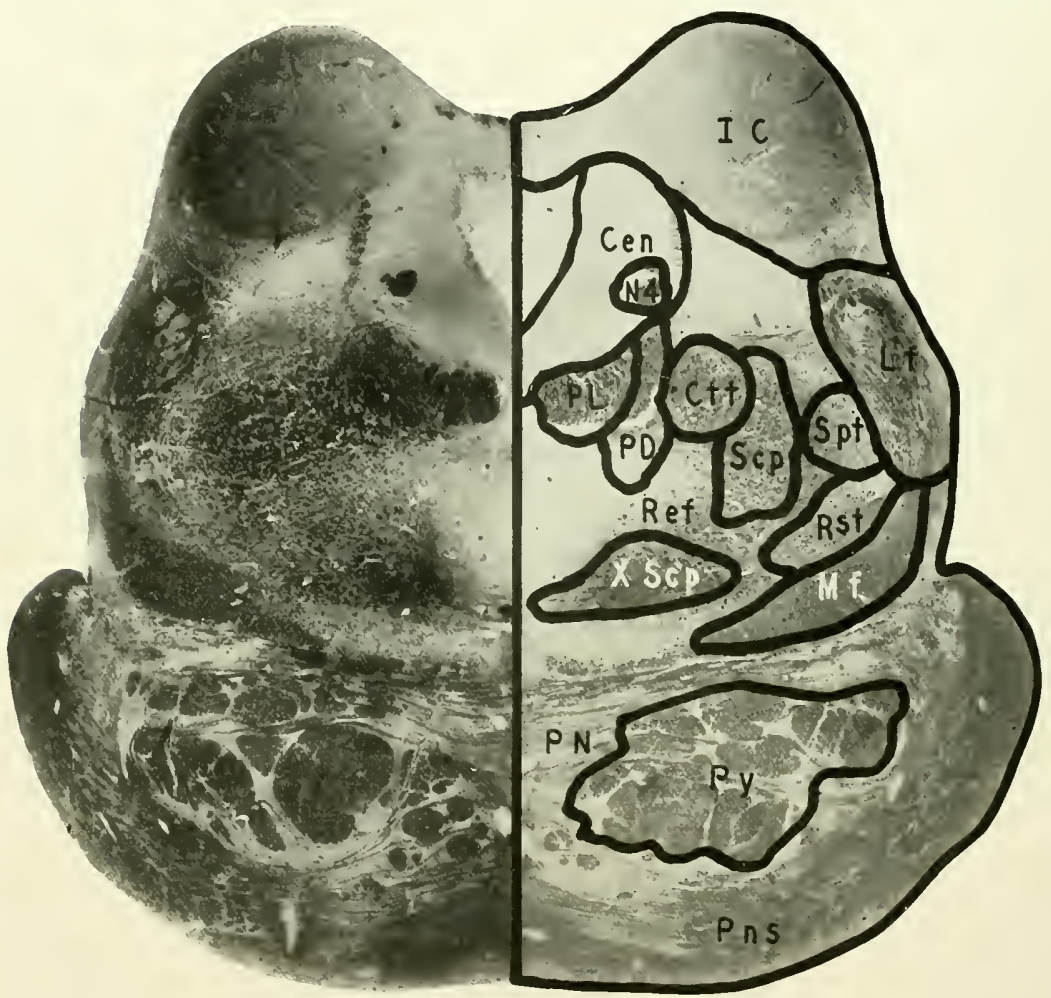

FIG. I 80, MACACUS. LEVEL OF THE INFERIOR COLLICULUS.

CEx, Central Gray Natter; ctr, Central Tegmental Tract; IC, Inferior Colliculus; LF, Lateral Fillet; MF, Mesial Fillet; N4. Trochlear Nerve; PD, Predorsal Bundle; PI, Postcrior Longitudinal Fasciculus; PN, Pontile Nuclei; Pvs, Pons; PY, Pyramid; REF, Reticular Formation; RST, Rubrospinal Tract; SCP, Superior Cerebellar Peduncle; SPT, Spinothalamic Tract; xscr, Crossing of the Superior Ccrebellar Peduncle. [Accession No. 149 . Section 320. Actual Size $16 \times 14 \mathrm{~mm}$.] 
the superior medullary velum. Lateral to the fibers of the trochlear nerve, on the outer border of the central gray matter, are the seattered fibers from the tractus mesencephaticus trigemini. The territory occupied by the reticular formation (Ref) has at this level become somewhat obscured by the fibers of the superior cerebellar peduncle ( $S e p$ ) which are sweeping inward and forward, about to undergo their decussation before coming into relation with the red nucleus. This decussation is shown as fully developed in the next section. The boundary between the basis and tegmentum, as at other levels, is indicated by the presence of the mesial fillet (Mf) from whose lateral angle extend dorsally many of the fibers of the lateral fillet ( $L f$ ), now making their way to the inferior colliculus.

The basis of the section still contains some of the typical characteristics of the pons Varolii in its three layers and also the disseminated condition of the pyramidal fibers ( $\mathrm{Py}_{\mathrm{y}}$ ). There is, however, a tendeney for the ventral groove of the pons to deepen and thus to foreshadow the beginning divergence of the basal portion of the axis, which ultimately occurs in the cercbral peduncles where an assemblage of the cortico-pontile fibers takes place and the pyramidal axons are reassembled into a single compact bundle.

\section{LEVEL OF THE SUPERIOR COLLiCULUS (FiG. I $8 \mathrm{I}$ )}

Here the contour of the brain stem has again undergone modification due to the appearance of two fairly prominent elevations on its dorsal aspect and the development of a wide cleft in the middle portion of its base. These elevations are the superior colliculi (SC) which represent remnants of the optic lobes of the lower vertebrates. That they are reduced in size in all mammals, particularly the primates, is significant of the fact that the process of telencephalization of the visual function has been steadily progressing since the advent of mammalian forms. Visual function has for the most part been taken over by the occipital lobe in the cerebral hemisphere, a 
386 THE INTERMEDIATE PRIMATES

portion of the endbrain which in the primates has gained particular prominence. In all probability, little of the primordial responsibility for the function of vision is still vested in the superior colliculi. This portion of the brain,

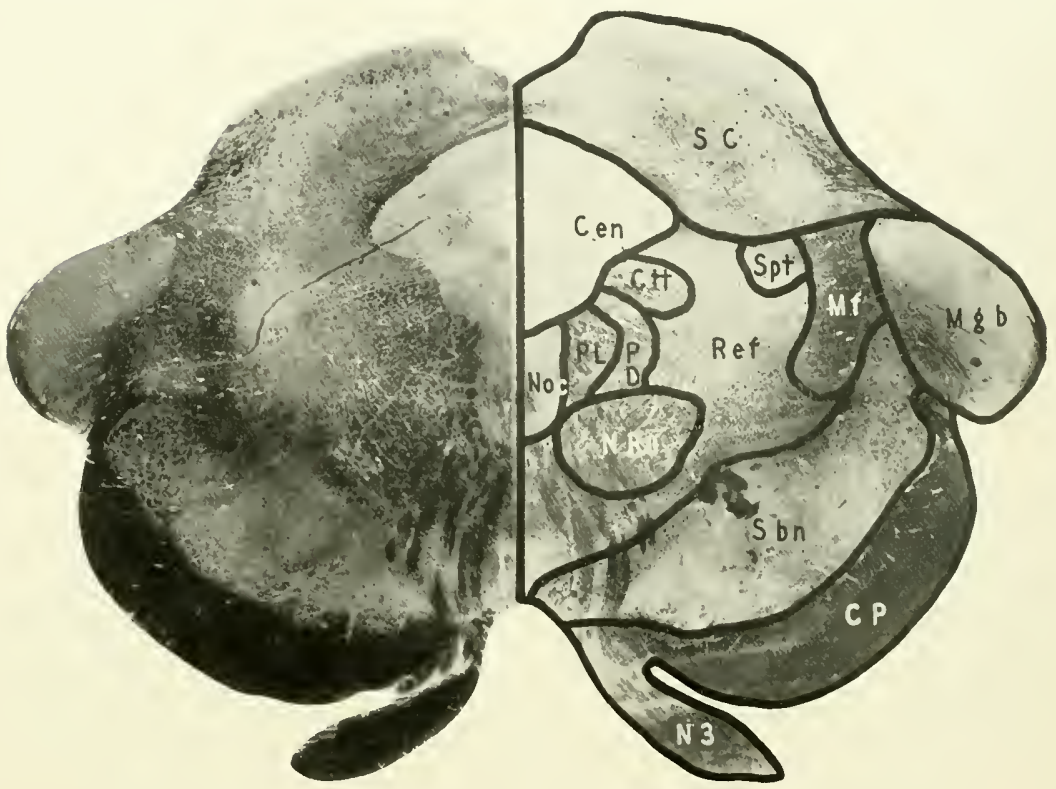

FIG. ISI. MACACUS. LEVEL OF THE SLPERIOR COI LICULUS.

Ci $>$, Central Gray Matter; CP, Cerebral Peduncle; CrT, Central Tegemental Tract; MF, Mesial Fillet; мGв, Mesial Geniculate Body; Noc, Oculomotor Nucleus; NRC, Nucleus Ruber; N3, Oculomotor Nerve; PD, Predorsal Bundle; PL, Posterior Longitudinal Fasciculus; Ref, Reticular Formation; sc, Superior Colliculus; sBv, Sulstantia Nigra; spt, Spinothalamic Tract. [Accession No. 140 . Section 388. Actual Size $19 \times 10 \mathrm{~mm}$.]

which for such a long period in vertebrate evolution held dominance in the animal's visual functions, has now become much reduced in this respect and appears as a relatively insignificant structural feature on the dorsal surface of the midbrain. As a matter of fact, the superior colliculus is somewhat larger than the inferior colliculus, and it still retains some degree of stratifica- 
tion reminiscent of its former high specialization when, as in the reptiles and birds, it presented fourteen distinct layers of alternating nerve cells and fibers.

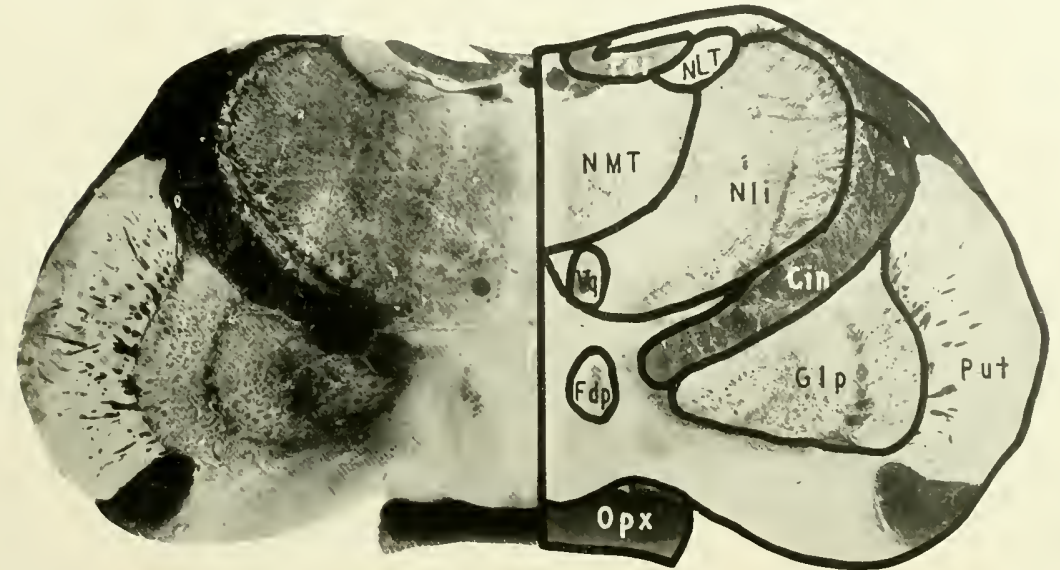

FIG. I82. MACACES. LEIEL OF THE OPTIC CHIASII.

as, Internal Capsule; Fdr. Descending Pillar of the Fornix; for, Fornix; glp, Glubus Pallidus; xl1, Nucleuts Lateralis Internis Thalami; NLT, Nuclcus Litteralis Thalami; NмT, Nucleus Mesialis Thalami; opx, Opt ic Decussation; ple, Putamen; vo, Fasciculus of Vieq d'Azyr. [Accession No. 149. Section 505. Actual Size $30 \times 16 \mathrm{~mm}$.

The central gray matter (Cen) surrounds the Sylvian aqueduct as it is approaching its cephalic orifice to communicate with the third ventricle. Its ventral portion is much prolonged by the appearance in it of an extremely important nuclear body, the nucleus aculomotorious (Noc) of the third cranial nerve from which emerge fibers destined for the oculomotor groove. Here they leave the stem en route to supply all of the muscles of the eyeball, both extrinsic and intrinsic, with the exception of the external rectus and the superior oblique. In the midline and connected with this nucleus are a number of fibers crossing from one side to the other. These are the commissural and decussating fibers associating the oculonotor nuclei. Their impor- 
tance has already been mentioned in connection with the derelopment of binocular vision. Special reference is made to them in this place in orcler that their significance in the animal's behavior may be subsecuently discussed.

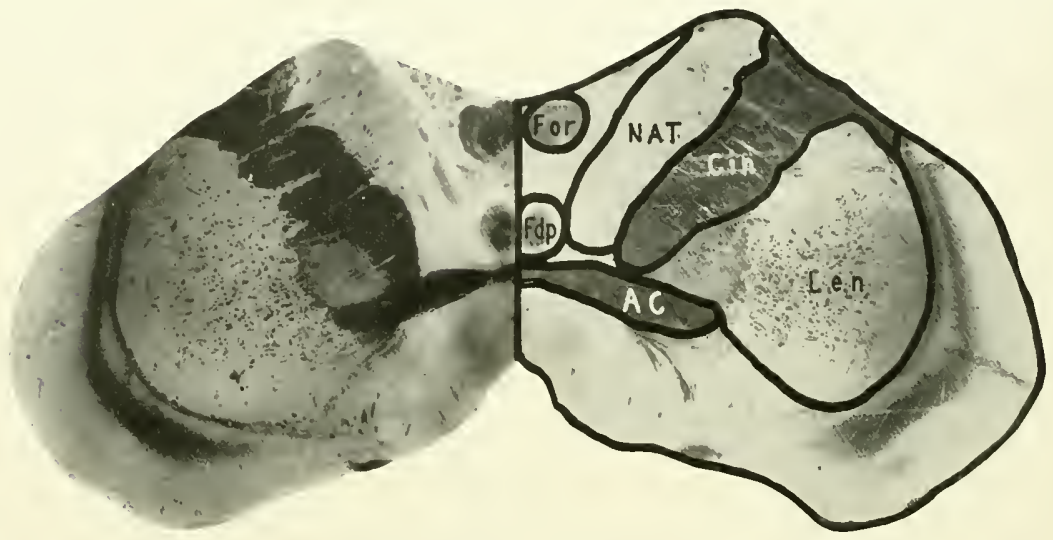

FIG. 183. MACACUS. LEVEL OF THE ANTERIOR COMMSSURE.

AC, Anterior Commissure; CIN, Internal Capsule; fDP, Descending Pillat of the Fornix; For, Fornix; LEN, Lenticular Nucleus: Nat, Nucleus Anterior Thalami. [Accession No. I 49 . Section 565 . Actual Size $30 \times 8 \mathrm{~mm}$.]

Ventral to the nucleus oculomotorius, although as yet poorly differentiated, is the nucleus ruber (NRu) through which some of the emergent fibers of the oculomotor nerve pass. Lateral to this nucleus is the reticular formation (Ref), whose general appearance suggests the lack of highly specialized differentiation in this species. The lateral portion of this formation is in continuation with a small protuberance on the lateral surface of the midbrain, the mesial geniculate body ( $M \mathrm{gb}$ ) which serves as one of the relay stations in the secondary pathway of hearing.

The ventral aspect of the section shows a cleft in and about the midline. This is the beginning of the optico-peduncular space which separates the basis into the right and left cerebral peduncles (CP). These 
peduncles now contain the compact bundles of the pallis-pontilesystem which connect the cerebral cortex with the lateral lobes of the cerebellum and the compact bundles of the cortico-spinal tract for the transmission of volitional impulses to the somatic musculature. Immediately dorsal to the cerebral peduncle is a large mass of gray matter, the substantia nigra ( $\mathrm{Sbn}$ ) whose relation to the control of certain automatic associated movements has been mentioned.

LEVEL OF THE OPTIC CHIASM (FIG. 182)

At this level, one of the most cephalic sections of the brain stem in this series is shown. A prominent feature is the optic thalamus which is separated by the internal capsule $(\mathrm{Cin}$ ) from the lenticular nuckeus, whose major portions are well illustrated, the globus pallidus (Glp) and the putamen $(\mathrm{P} u \mathrm{t})$. As this specialization in the diencephalic portion of the brainstem is highly complex and deserves separate treatment, the structures at this level are merely referred to as indicative of the marked changes in transition in the level at which the brain stem forms its final continuity with the endbrain. It is here that contact is ultimately established with those great expansions of the brain, the cerebral hemispheres. They, in later stages of evolution, have dominated all of the far-reaching modifications in behavior characteristic of mammals, reaching their consummate expression in the achievements of man. 



\title{
Chapter Xili
}

\section{RECONSTRUCTION OF THE GRAY MATTER IN THE BRAIN STEM OF PITHECUS RHESUS}

\begin{abstract}
7 HE reconstruction of this species follows the general lines already laid down in the preceding reconstructions. It is particularly valu-

L able as showing the structures in their interrelation and continuity, and thus furnishes a more realistic idea of the actual disposition of the more important masses of gray matter in the brain stem. So far as possible, the descriptions have been made almost exclusively objective, reserving the space for comment on the significance of the structures for the chapters on
\end{abstract} summaries and conclusions.

\section{The High Cervical Level of the Spinal Cord}

In the high cervical level of the spinal cord at which the reconstruction of the gray matter in macaeus begins, the ventral gray column is already detached from the central gray column by the decussating fibers of the pyramidal tract. In outline the ventral gray column is somewhat oval, directed ventrodorsally and laterally. It occupies the normal position of the ventral gray column in the spinal cord but is reduced in size. The central gray matter is somewhat cordiform in shape, presenting a ventral apex and a dorsal base from the lateral angles of which proceed the laterally compressed cervices of the dorsal gray column. The substantia gelatinosa trigemini is seated like a cap upon the dorsal cxtremity of the dorsal gray column and is already beginning to show the lateral swing occasioned by the appearance of the dorsal medullary nuclei and the opening of the fourth ventriele.

As the higher cervical segments pass into the lowermost levels of the oblongata, the ventral gray column becomes inereasingly interspersed with 
longitudinal fiber bundles and it gradually assumes the characteristic appearance of the reticular formation into which it merges.

\section{The Dorsal Sensori Nuclei}

The nucleus of the column of Goll makes its first appearance in the reconstruction as a prolongation from the central gray column, in close proximity to the dorsal median septum. It rapidly assumes its maximum ventrodorsal extent and proceeds upward as a gradually thickening mass of gray matter maintaining its median position and separated from its fellow of the opposite side by only a narrow encapsulation of white matter.

Somewhat above the level of the appearance of the inferior olivary nucleus, there appear scattered masses of gray matter in the column of Goll, lateral to the main mass of the nucleus of the column of Goll. They establish connection with the stem of the nucleus, which becomes somewhat rounded in shape and then rapidly expands laterally. This expansion continues laterally until the dorsal extremity of the nucleus assumes a massive appearance, oceupying the major portion of the dorsal aspect of the oblongata. It is somewhat arborescent in character, presenting masses of gray matter broken up and surrounded by penetrating, longitudinally coursing bundles of white fibers.

Ccphalically, the nucleus of the column of Goll comes to rather an abrupt termination and its most cephalic portion mesially forms part of the caudolatcral boundary of the fourth ventricle. Laterally it becomes submerged from view by the appearance in this region of the nucleus of Deiters.

The nucleus of the column of Burdach appears first as a rounded mass of nuclear material in the dorsal portion of the reticular formation. It is at this point encapsulated by fibers arising from the nucleus of Goll and, passing ventrally in an arcade as the internal arcuate fibers, gives rise to the mesial fillet. Increasing in size and extending dorsally at about 
the mid-level of the nucleus of the column of Burdach, there appears a secondary portion of the nucleus situated laterally in the collection of the ascending fibers of the column of Burdach, first as isolated groups of gray
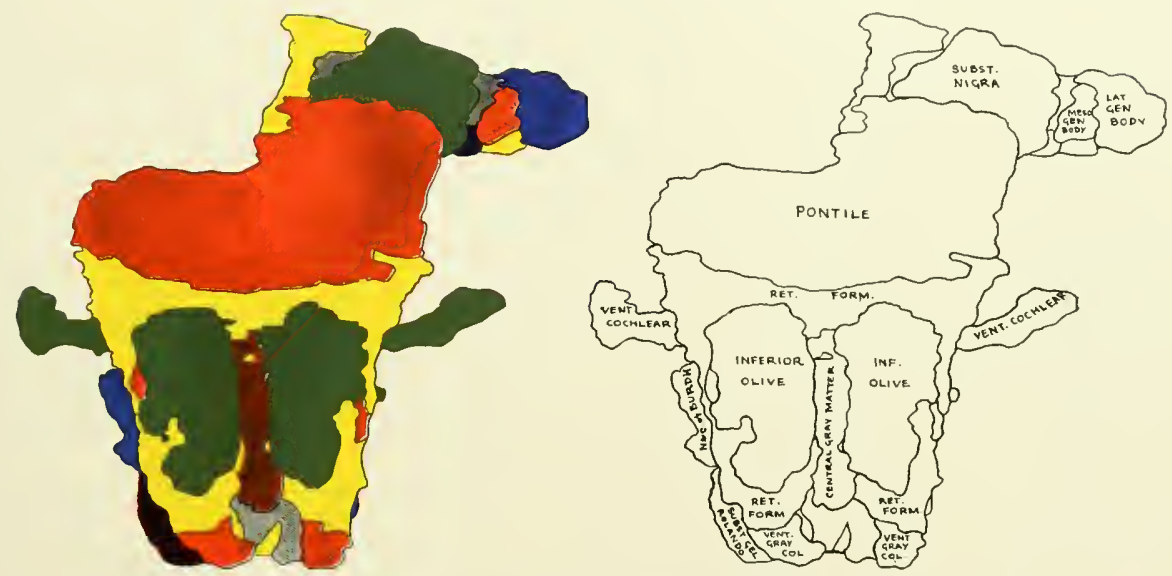

FIG. I84. VENTRAL SURFACE OF GRAY MATTER OF BRAIN STEM, PITHECUS RHESUS.

hey to Diagrair. INF. Olive, Inferior Olive; lat. GeN. body, Lateral Geniculate Body; meso-geN. Body, Mesial Geniculate Body; nuc. of Burdach, Nucleus of Burdach; pontil.F, Pontile Nuclei; Ret. Form., Reticular Formation; subst. gel. Rolando, Substantia Gelatinosa of Rolando; subst. nigra, Substantia Nigra; vent. Cochle ar, Ventral Cochlear Nucleus; vent. gray COL., Ventral Gray Column.

matter which coalesce to form a large nuclear mass lying lateral to the stem of the nucleus of the column of Burdach; this is the nucleus of Blumenau. It rapidly establishes connection with the mesial portion of the nucleus, its nuclear masses presenting the typical leafy arrangement characteristic of it. It extends laterally for a considerable distance and overhangs the prolongation of the substantia gelatinosa trigemini. Gradually encroached upon by the vestibular complex, it disappears from vicw, being entirely replaced by the vestibular nuclei. 
The substantia gelatinosa Rolandi has become relatively slender, and placed as a cap over the dorsal extremity of the dorsal gray column. As the highest cervical levels are approached, it is transformed, without any change whatsoever in its morphological characteristics, into the substantia gelatinosa trigemini which thereafter serves as a reception nucleus for the descending fibers from the Gasserian ganglion. It passes gradually in to the lateral position which is characteristic of this mass of gray matter in the primate brain, and at the level of the inferior olivary nucleus it assumes its permanent unchanging position. The nucleus continues upward, embedded more or less irregularly in the Iateral surface of the reticular formation, appearing at times on the surface and at other times being submerged from view by the reticular formation. The usual constriction which has previously been noticed is present at about the middle of the inferior olivary nucleus. Here the substantia gelatinosa trigemini reaches its minimum diameters. From this point it continues gradually to increase in size until it reaches its maximum at about the mid-ventricular region of the stem, above which it continues and develops its expanded cephalic extremity called the caput. Just prior to its disappearance it presents at its mesial and dorsal aspects the motor nucleus of the trigeminal nerve.

\section{The Inferior Olivary Nucleus}

The inferior olivary nucleus first appears in the reconstruction at the level of the cephalic extremity of the pyramidal decussation. Here it is a flattened lamina of gray matter directed mesially and slightly dorsally. This is the ventral accessory olivary nucleus (paleo-olive). It extends mesially and somewhat dorsally forming a flat ribbon with its mesial extremity twisted so as to lie in a more direct ventrodorsal diameter of the stem. It ends cephalically by merging with the dorsal extremity of the dorsal lamina of the inferior olivary aucleus. The main mass of the inferior olivary 
nucleus appears somewhat above this level, dorsolateral to the ventral accessory olive where it is a plicated fold of gray matter, its fundus directed toward the ventrolateral angle of the brain stem. It presents a
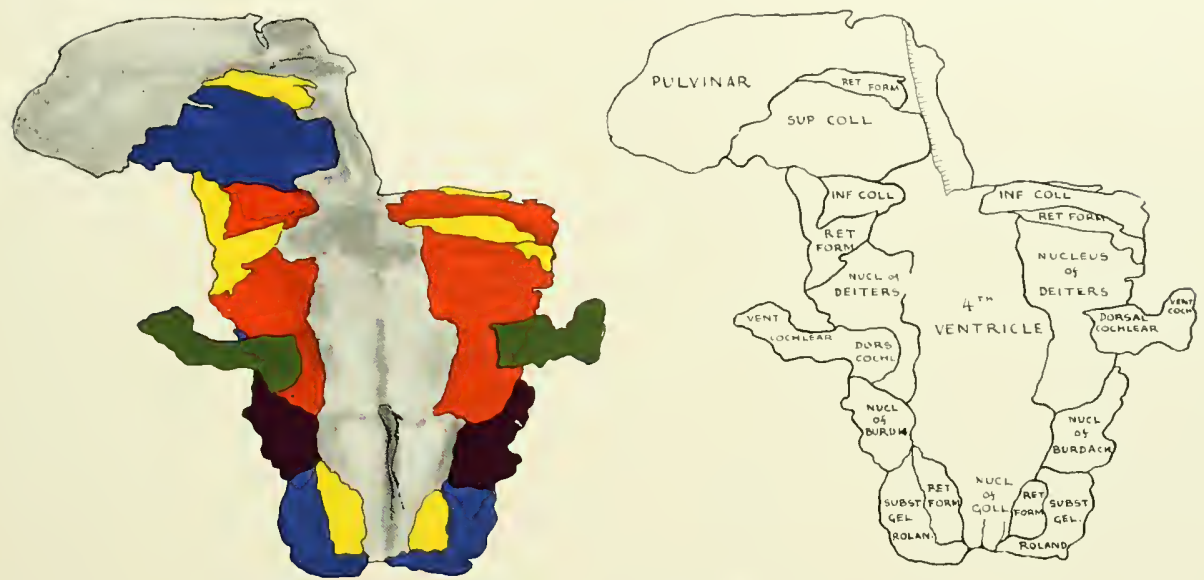

FIG. I85. DORSAL SURFACE OF GRAY MATTER OF BRAIN STEM, PITHECUS RHESUS.

Key to Diagram. dors. Cochl., Dorsal Cochlear Nucleus; inf. Coll., Inferior Colliculus; Nect. of Burd. and NuCl. OF BURDach, Nucleus of Burdach; NUCL. OF DEITERS, Nucleus of Deiters; NuCL. OF GOLL, Nucleus of Goll; RET. Form., Reticular Formation; Subst. Gel. Rol Avdo, Substantia Gclatinosa of Rolando; sup. COLL., Superior Colliculus; Vent. COCH, and vent. COCHLEar, Ventral Cochlear Nucleus.

tendency toward plication, an undulating strand of gray matter showing a number of bends and folds. The ventral lamina is almost devoid of reduplications, whereas the dorsal lamina shows an increased irregularity produced by the appearance of numerous reduplications. At a level somewhat above the appearance of the main mass of the inferior olivary nucleus, the dorsal accessory olivary nucleus appears as a flat, narrow strand of gray matter placed almost in the lateral meridian of the brain stem and overlying the hilus of the inferior olivary nucleus. It extends upward as a flat band in this 
same location and, gradually diminishing, it appears cephalically as a plug in the hilus of the nucleus. Before it completely disappears it scems to fuse with the dorsal extremity of the inferior olivary nucleus. The entire inferior olivary complex extends upward to a level somewhat below that of the middle of the ventricular eavity, where it ends rather abruptly, merging with the reticular formation in whose ventral surface it is embedded.

\section{The Reticular Formation}

The reticular formation, as shown in the reconstruction, begins in the upper cervical levels by the appearance of isolated groups of nerve cells lying dispersed between the fibers of the lateral portion of the decussating pyramidal tract. It here receives the termination of the ventral gray column and then rapidly becomes the outstanding feature in the tegmentum of the rhombencephalon. It is quite irregular in outline and presents embedded in its substance and along its surfaces the various condensations of gray matter forming the nuclei of the brain stem and the collections of nerve fibers which form the ascending and descending tracts. At its ventrolateral angle it presents the constant condensations of nuclear material which form the lateral nucleus of the reticular formation and the superior olive, the two latter structures being somewhat more prominent and extensive than those found in the preceding forms. On its ventral surface, extending from the eephalic limit of the pyramidal decussation to the mid-ventricular level of the stem, it forms a bed for the dorsal accessory olivary nucleus of the olivary complex which at its beginning emerges from, and at its termination disappears in the matrix of the reticular formation.

Laterally the reticular formation receives the substantia gelatinosa trigemini, while dorsomesially it affords attachment to the stem of the mucleus of the column of Goll and, somewhat more ecphalically, to the base of the nucleus of the column of Burdach. It continues upward, 
increasing in size, into the tegmentum of the pons, where it is massive in form and supplies the main bulk of the gray matter of the metencephalic tegmentum.
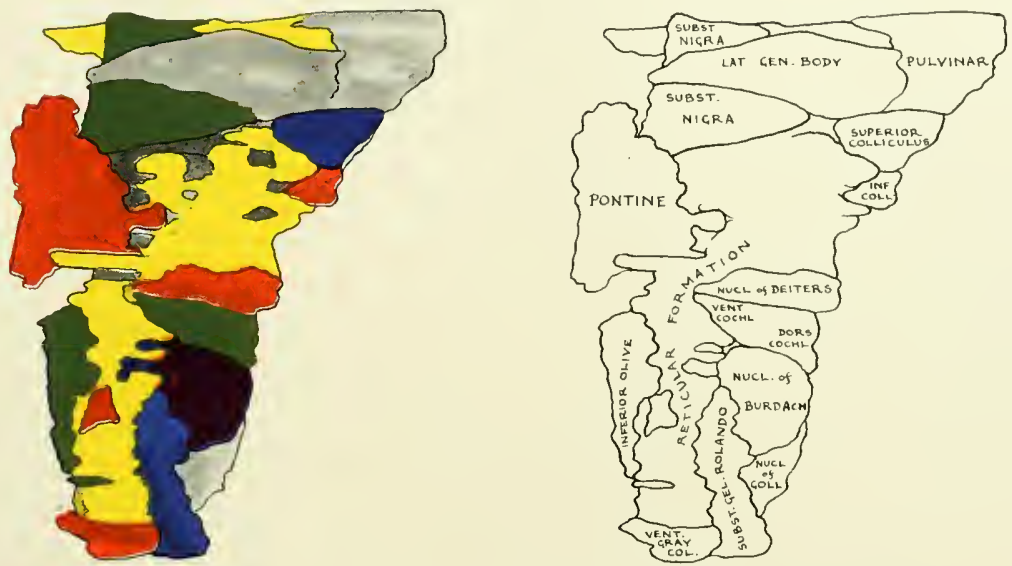

FIG. 186. LATERAL SURFACE OF GRAY MATTER OF BRAIN STEM, PITHECUS RHESUS.

Key to Diagram. dors, Cochl., Dorsal Cochlear Nucleus; inf. Coll., Inferior Colliculus; inf. Olive, Inferior Olive; LAT. GEN. BODY, Lateral Geniculate Body; NUC. OF BURDACH, Nueleus of Burdach; NUCL. OF deiters, Nucleus of Deiters; Nucl. of goll, Nucleus of Goll; PONtine, Pontile Nucleus; Subst. Gel. ROLANdo, Substantia Gelatinosa of Rolando; subst. Nigra, Substantia Nigra; vent. Cocul., Ventral Cochlear Nucleus; vent. Gray col., Ventral Gray Column.

Above the level of the olivary complex the ventral surface of the reticular formation recedes rather rapidly from the surface, providing along its ventral surface a deeply excavated bay for the lodgment of the trapezoid body, formed by the secondary fibers from the cochlear system. Continuing upward in the metencephalon the reticular formation gradually diminishes in bulk, separated at first from the deep layer of the pontile muclei by the trapezoid body. Above the level of the trapezoid body, the reticular formation comes into fairly close contact with the deep layer of the pontile nuclei and presents 
in this region the beginning mesencephalic appearance of the reticular formation with a large mesial mass and a lateral prolongation which turns outward and backward to encircle the superior cerebellar peduncle as it enters into the mesencephalic tegmentum from the superior medullary velum. Below the appearance of the inferior colliculi a prolongation of the reticular formation passes around dorsally toward the midline.

Into the space between the superior colliculus and inferior colliculus a similar prolongation of the mesencephalic reticular formation passes laterally from the lateral portion of the reticular formation almost to the midline, thus separating the colliculi from each other. Similarly, above the superior colliculus a rather narrow extension from the most cephalic region of the reticular formation again comes to the surface close to the junction between the mesencephalon and the diencephalon. In the mesencephalon itself, the major portion of the mesial mass of the reticular formation gives place to that most characteristic nucleus of the mesencephalon, the nucleus ruber; while the lateral portion extends upward and outward and at the junction of the mesencephalon with the diencephalon it forms a bed for the mesial surface of the mesial geniculate body and then fuses cephalically with the indifferent reticular formation of the diencephalon. In the upper medullary levels of the brain stem the dorsal surface of the reticular formation is in fairly close contact with the subependymal gray matter of the fourth ventricle and presents at its dorsolateral angle the specialization of the reticular formation, producing the vestibular complex.

\section{The Pontile Nuclei}

In the pontile nuclei of Macacus rhesus is found for the first time an extension indicating the arciform nuclei which appear as a flattened layer of gray matter applied to the ventral surface of the pyramid, conforming to its convex surface. This arciform nuclear formation is relatively poorly developed 
and almost immediately after its frrst identification the typical appearance of the pontile nuclei develops. In its most caudal portions the pontile nucleus appears as an incomplete ring of nuclear material deficient on the lateral aspect and surrounding the relatively undivided pyramidal tract frbers. As this structure is traced upward, the ring soon becomes complete, surrounding the pyramidal tract and the lowest fibers of the pallio-pontile system of fibers and, at the same time, there are distinctly developed the two layers of the pontile nucleus (the superficial and deep layers), which meet mesially and laterally the two buttresses of the pontile nuclei.

There is some indication of migration of masses of nuclear material in between the frbers of the two longitudinally coursing systems, producing a poorly developed, lace-like appearance. The pontile nuelei are materially heavier in formation and more extensive in distribution than in the preceding forms. As the mesencephalon is approached the mass of the pontile fibers gradually diminishes and the superficial layer contracts and extends further upward for only a short distance ventral to the developing cerebral peduncle. The deep layer becomes increasingly closer in proximity to the mesencephalic reticular formation and gradually is transformed into the substantia nigra.

\section{The Vestibular Complex}

The vestibular complex makes its appearance first as a wedge-shaped mass of gray matter inserted between the subependymal gray matter forming the floor of the fourth ventricle above the cephalic extremity of the nucleus of the column of Goll, and the mesial surface of the nucleus of the column of Burdach. Rapidly increasing in size, it replaces the nucleus of the column of Goll and displaces Iaterally the nucleus of the column of Burdach, thus bringing about through the agency of the opening of the fourth ventricle that lateral displacement which is characteristic of all of the dorsal medullary nuclei of the brain stem. 
The nucleus of Deiters' area rapidly increases in size and maintains its position of contact with the subependymal gray matter of the floor of the fouth ventricle. As the upper limit of the nucleus of the column of Burdach is approached, the nucleus of Deiters extends ventrally and laterally, assuming the position previously oceupied by the nucleus of the column of Burdach and presenting two prolongations - one mesial, closely applied to the lateral surface of the reticular formation, the other lateral and in close proximity to the entering root of the cochlear nerve. In this recess formed by the mesial and lateral prolongations is located the ascending nuclear mass of the substantia gelatinosa trigemini (Rolando). Somewhat above the mid-ventricular level of the stem it rapidly diminishes in size and is superseded by the triangular nucleus, the nucleus of Schwalbe, belonging to the vestibular complex which has first appeared just below the lateral recess of the fourth ventricle. The triangular nucleus rapidly becomes a comparatively bulky nucleus extending mesially to the base of the lateral wall of the ventricle, and laterally to the dorsolateral angle of the reticular formation. It extends upward almost to the upper limit of the meteneephalon and then disappears gradually. The ascending nucleus of the vestibular complex, the nucleus of Bechterew, is contained in the lateral wall of the fourth ventricle and is not of sufficient bulk to be represented satisfactorily in the reconstruction.

\section{The Cochlear Complex}

The cochlear complex of nuclei makes its appearance at the upper level of the inferior olivary nucleus as two masses of gray matter forming the ventral and dorsal cochlear nuclei. The large ventral cochlear nucleus is located at the extreme ventrolateral angle of the brain stem at the point of entrance of the cochlear nerve into the brain stem. It forms a fairly well-developed trough in which the cochlear nerve lies, eovering this nerve on its cephalic, lateral and caudal surfaces. 
The dorsal cochlear nucleus, on the other hand, appears below the lateral recess of the fourth ventricle as a more or less triangular mass of gray matter in the extreme dorsolateral angle of the brain stem. At this point it is located dorsal to the nucleus. A certain amount of nuclear material lies interspersed between the fibers of the cochlear nerve extending between and connecting the dorsal and ventral cochlear nuclei. The dorsal and ventral cochlear nuclei appear below the level of the cochlear nerve and extend to a level higher than that of the nerve itself.

\section{The Substantia Nigra}

The substantia nigra appears in the reconstruction as a well developed and extensive mass of gray matter situated dorsal to the cerebral peduncle and supported by the deep layer of the pontile nucleus and the mesial and lateral buttresses. Between these two structures it rests upon the deep layer of the pontile nucleus from which it seems to develop. It extends upward throughout the extent of the mesencephalon, diverging laterally and somewhat dorsally to terminate in a rounded, blunt extremity which is separated from the mesial geniculate body by a relatively small intervening mass of the reticular formation and the fiber capsule of the mesial geniculate body itself. At the junction of the mesencephalon and the diencephalon it comes to an end, separated from the corpus of Luys by a thin Iamina of white matter.

\section{The Colliculi}

The inferior colliculus constitutes a poorly developed structure lying as a crescentic mass of gray matter in the tectum of the mesencephalon. It is supported laterally by the lateral extension of the mesencephalic reticular formation, while mesially it lies against the undifferentiated dorsal gray matter. It is separated from the superior colliculus by a deep narrow 
extension of the reticular formation which passes between it and the superior collieulus. The inferior collieuli are closely connected across the midline by the commissure of the inferior colliculi. The superior collieular elevation is much more massive than the inferior colliculus and is similarly situated, being separated from the central gray matter surrounding the aqueduct of Sylvius by a thin lamina derived from the dorsal extension of the reticular formation. It is supported laterally by the same lateral extension of the mesencephalie reticular formation. Cephalieally it is limited by a thin extension of the lateral mesencephalic reticular formation. Mesially it rests against the undifferentiated dorsal gray matter and is connected with its fellow of the opposite side by the commissure of the superior collieulus.

\section{The Red Nucleus}

The nucleus ruber is contained within the mesial mass of the mesencephalic reticular formation and receives at its caudal extremity the decussating superior cerebellar peduncle which forms an eneapsulating mantle of white fibers about it, thus definitely outlining it from the surrounding matrix of the reticular formation. Although it is the outstanding feature of the meseneephalon, it is relatively poorly developed in this form, extending upward even beyond the junction of the meseneephalon and diencephalon into the substance of the latter division of the brain stem.

\section{The Central Gray Matter}

The central gray matter, as has been previously described, appears in the higher levels of the cervical cord as a roughly cordiform structure already separated from the ventral gray columns and providing a connection with the laterally compressed dorsal horns. Continuing upward in this same position, it gives origin to the dorsal extensions which form the nucleus of the column of Goll, and at a somewhat higher level it presents the 
condensations situated more laterally than the preceding, which extension forms the nucleus of the column of Burdach. At the point where the nucleus of the column of Goll begins to increase in size, there appears a narrow, tongue-shaped prolongation of the central gray matter, which gradually approaches the dorsal surface and then, extending laterally, the entire mass of the central gray matter is drawn dorsally and laterally flattening out to form the floor of the fourth ventricle. In its ventral surface lic embedded the dorsal nuclei of the ninth and tenth cranial nerves, and close to the midline appears the nucleus of the twelfth cranial nerve. Separating the gray matter of the ventricular floor and the reticular formation, lie the longitudinally coursing fiber bundles of the posterior fasciculus mesially, the fasciculus solitarius laterally, together with the peripheral condensation of fibers which circumferentially limits the reticular formation. In its lateral portion are cmbedded first the nucleus of the column of Goll, and then the nucleus of the column of Burdach, which become separated from contact with the central gray matter by the appearance of the nucleus of Deiters.

In the cephalic portions of the myeleneephalon and the metencephaton appcar small masses of gray matter connected with the ventral aspect of the central gray matter, extending along the tegmental raphe. These form a connected series of nuclei lying between the posterior longitudinal fasciculus, the predorsal bundle and the mesial fillet.

The central gray matter is in close contact with the vestibular and cochlear complexes throughout their extent in the metencephalon. At the upper limit of the triangular nucleus (Schwalbe) of the vestibular complex, the lateral walls of the ventricle begin to approach each other and as the upper extremity of the ventricle is reached, the floor, roof and lateral walls rapidly draw together and the aqueduct of Sylvius appears. The gray matter surrounding the aqueduct of Sylvius is relatively massive on all sides. Ventrally the central gray matter is continued forward as a long, tongue- 
shaped proeess which contains embedded within it the nuclei of the fourth and third cranial nerves. As the diencephalon is approached the ventral extension of the central gray matter rapidly inereases until the third ventricle appears where the central gray matter becomes continuous with the diencephalic subependymal gray matter and the mesial mass of the thalamic nuclei.

The floor of the fourth ventricle shows practically no modeling in its lower half. Above the level of the lateral recess, however, there appear a relatively well-marked eminences on either side of the midline, separated from each other by a rather deep suleus, representing the underlying condensation of the gray matter to form the nueleus of the sixth cranial nerve. 


\section{Chapter Nil}

\section{HYLOBATES HOOLOCK, THE GIBBON, ITS BRAIN AND BEHAVIOR}

Its Position among the Primales; The Proantbropoid Stage; Measurements and Brain Indices; Surface Appearance of the Brain; Intemal Structure of the Brain Stem in Cross Section

7 PE gibbons are usually assigned to the group of the higher anthropoids, with the qualification, however, that in this group they are

L the most remote from man. In the general appearance of their brain they ally themselves much more closely with the intermediate primates. This, in combination with the fact that by their adaptive radiation they have become equipped preeminently if not exclusively as tree dwellers, seems to justify their inclusion with those intermediate forms already described. The designation of them as "Hylobates" is intended to indicate how exquisitely these "tree walkers" are specialized for arboreal habitat. Their lives are passed in the trees, through whose branches they move with a swiftness and ease similar to those of a bird. When on terra firma they pass over its surface a little awkwardly, yet with much facility although compelled to balance themselves by holding their long arms over their heads and hastening their footsteps lest they fall. Once, however, their slender hands come in contact with the branches of a tree, a really marvelous change appears in their progression. Their uncertain gait is immediately replaced by a locomotion which has all the grace, the accuracy, the speed of a bird in flight.

\section{Appearance and Habits of the Gibbon}

The body and head of the gibbon are relatively small, little larger incleed, than some of the smaller macaques. The animal's legs are relatively short and it has no tail. The characteristic feature of the giblon is the 


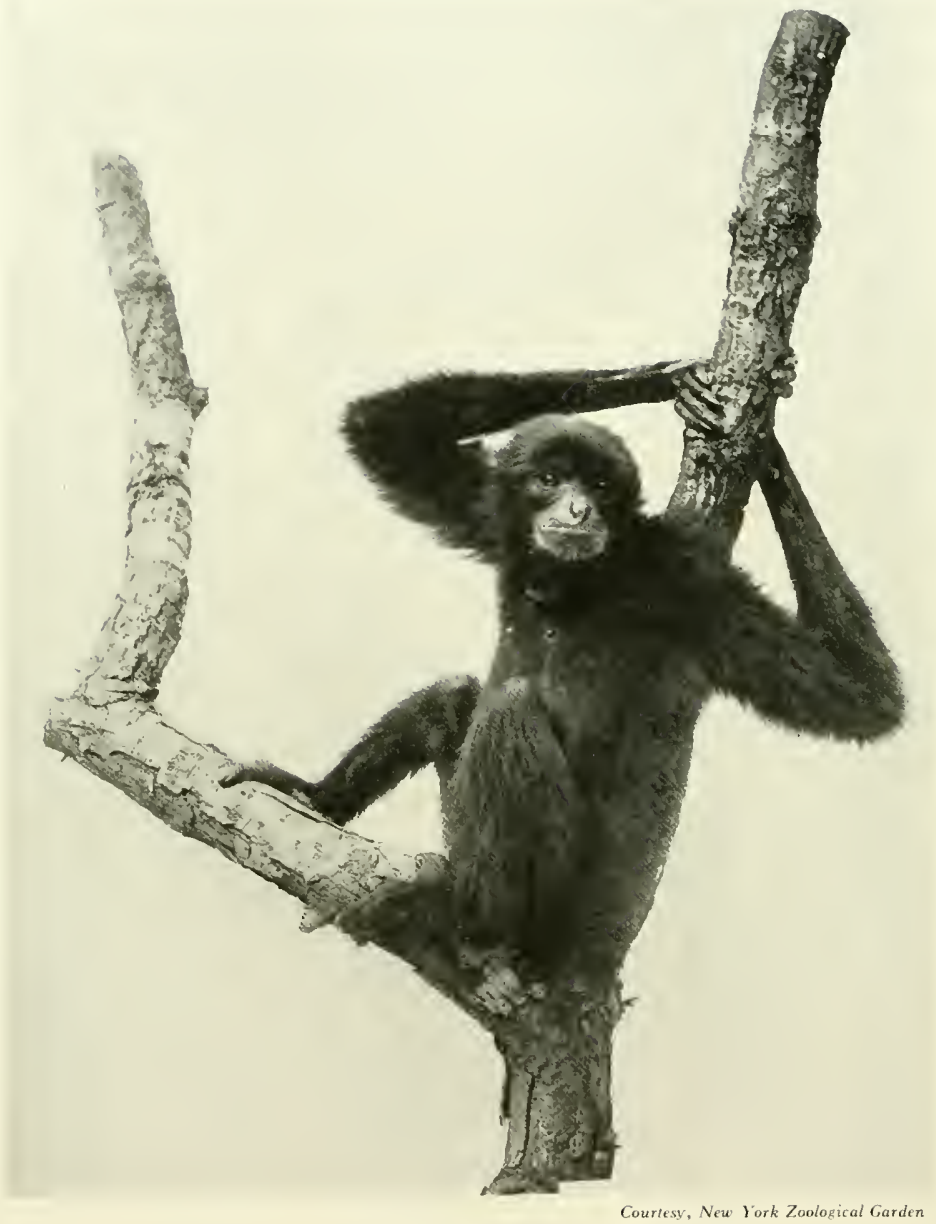

FIG. I8-. HYLOBATES SYNDACTYLUS (GIBBON). $[406]$ 
exeeptional length of the forearm which so increases the entire length of the arm that the tips of the fingers touch the ground when the animal stands erect. The slender hand is longer than the foot and the thumb is also long in

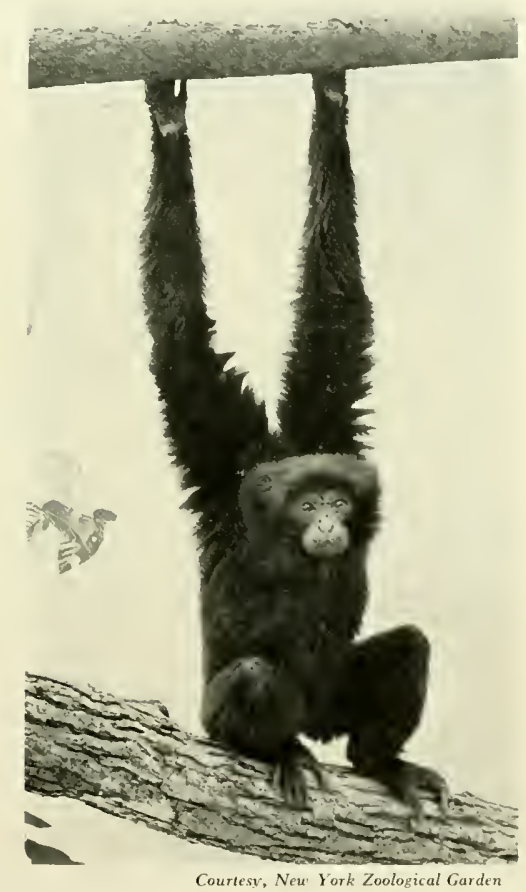

FIG. I 88. HYLOBATES SYNDACTYLUS (GIBBON).

Gibbon's brachiating locomotion has favored erect posture and thus initiated the Proanthropoid Stage.

proportion. These animals are possessed of remarkable vocal power and can be heard for great distances. They go in troupes and usually early in the morning give voice to their peculiar chorus of calls which seems to cease, as if regulated by some prearranged schedule, in the early forenoon. 
One young is produced at a time and the mother carries it under her body, the young one clinging to her fur with hands and fect. This appendage to the mother does not seem in the slightest to embarrass her progress as she
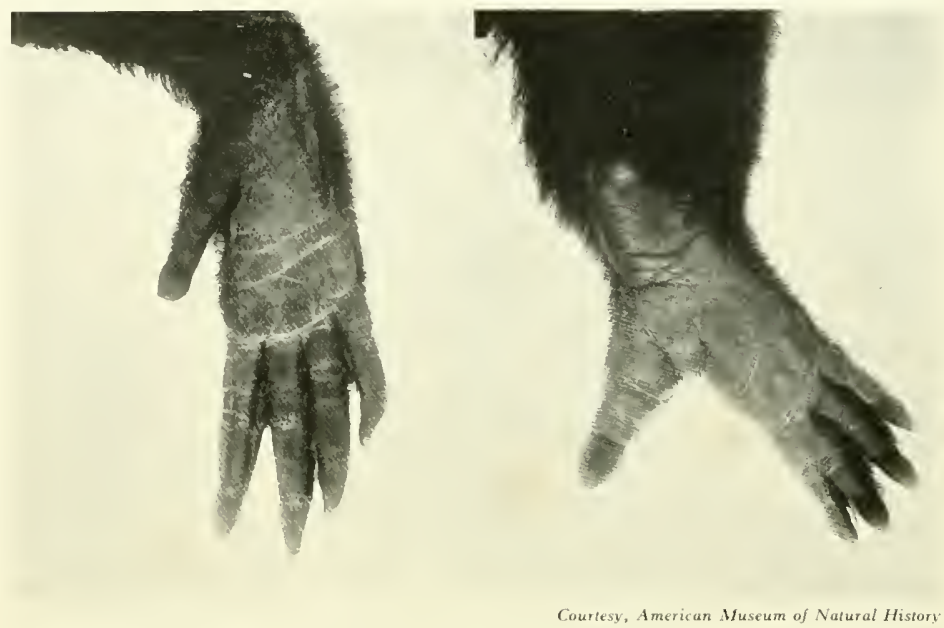

FIGS. I 89 AND IQO. HAND AND FOOT OF HYLOBATES SYNDACTYLUS.

LEFT. Palmar surface of hand, showing the extremely long fingers developed in consequence of brachiation.

The swinging type of locomotion of the gibbon has produced this specialization in the hand. Fingers show some syndactylism.

Rıcis. Plantar surface of the foot showing short heel, long, powerful great twe, marked syndactylism of second and third toes. The fout is adapted to prehensile purposes involved in the brachiating locomotion.

makes her way through the forest, executing her great swings from tree to tree which are just as prodigious in their length as those made by the unencumbered male.

The gibbon is a delicate animal and rarely survives long in captivity. By disposition it is gentle, often affectionate. It is not averse to handling by strangers and will come close to the sides of its cage with arms extended through the bars so that its hands may be grasped and stroked. The gibbons 
never leave the forest and are distributed for the most part throughout southern Asia and the adjacent islands. A few species venture from the inland jungles to the vicinity of the coast.
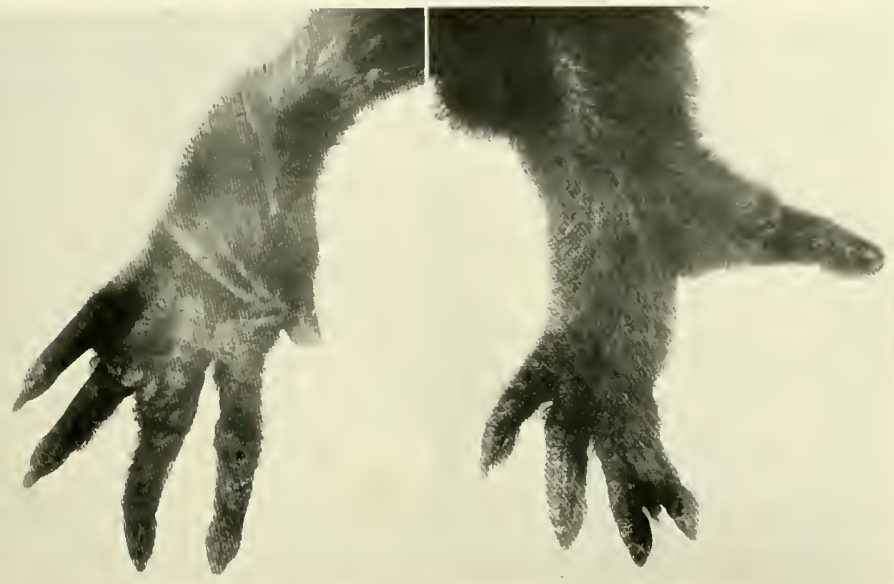

Courtesy, American Museum of Natural History

FIGS. I91 AND 192, HAND AND FOOT OF HYLOBATES SYNDACTYLLS.

LEFT. Dorsum of hand showing long, slender fingers, well-developed finger-nails, a hand adapted to grasping the branches in the gibbon's brachiating type of Jocomotion.

Right. Dorsum of foot showing marked hand-like characters in adaptation to brachiating locomotion. The long. powerful, opposable great toe is specialized for grasping purposes, coopcrating in this function especially with the syndactylic second and third toes.

THE HOOLOCK GIBBON

In general, the typical hue of the hoolock is black, although many varieties are found, as far as coloring is concerned. The hoolocks, however, are most consistent in this regard. Such variations in color as do occur affect the female more than the male. The hoolock gibbon is confined in its range to a limited district bounded by the Brahmaputra and Irawadi Rivers. It has a distinct aversion to water and cannot swin, a fact which 
probably cireumscribes its habitat to the region in which it is found. Like all members of this family, the hoolock is exquisitely arboreal. While it makes some successful efforts to progress over the surface of the ground, balaneing its body in a rather awkward manuer by holding its arms over its head, its life is passed essentially in the trees. The effects of brachiation are seen in the greater erectness of body which marks the beginning of the Proanthropoid Stage. Mr. Candler, who has studied the habits of this proanthropoid, gives such an interesting account of its actions, that it may be bere quoted in part:

"He [Hoolock] swings aloug to the thinnest part of the bough or to the slender end of a bamboo until it bends to his weight, then with a swing and a sort of a kick-olf, he flies through the air seizing another bough and swinging along it with the unerring aceuracy of a finished trapeze performer. I fancy he does very little walking in the wild state, for I bave never seen a wild Hoolock on the ground. Noreover, they are only found in the dense jungle where the ground is everywhere covered with tangled vegetation. It is puzzling to me why these anthropoids, being so entirely arboreal in habit, should be lacking in such a useful appendage as the tail. The Hoolocks are extremely shy and it is most difficult to watch them as they are concealed by leaves high up on the tops of the bamboo clumps or forest trees. You may hear their cries all around as you ride quickly along the jungle track; but the moment you leave the path or look up at them there is a dead silence and scarcely a leaf stirs until, tired of waiting, you move on again. The cry of the Hoolock is a characteristic sound in the Cachar jungle. It is a very pleasing note, rising and falling in intensity and reminding one somewhat in its rhytlum of a pack of beagles giving tongue on a scent, which is waxing and waung in strength as a larger or smaller number of the band join in the chorus. It is heard chiefly in the early morning, then all through the heat of the day there is silence but towards evening, as the sun sets, you may hear it again. 'Hoo'loo! Hoo'loo! Hoo'loo!' 
is supposed to describe the sound, but it is really quite indescribable in writing."

As in other species of apes, there is a special modification of the Iarymx which acts as a sort of resounding box and helps to make the sound carry, as it does, long distances. There is also a peculiar arrangement of the upper aperture of the larynx with its small and inadequate-looking epiglottis, which resembles more the arrangement in birds than the leaf-like epiglottis in man. Mr. Candler believes that the hoolocks work their ground systematically in their search for food just as the planter plucks one section of his tea to-day and another section in a different part of the garden to-morrow. For he has found them filling the air with their cries along a particular stretch of jungle road one day, while the next day not one was to be heard; and then, perhaps a week later, they were back again in the same place as at first.

Living as they do in fairly large communities, they are constantly on the move, and from what is known of their relatively good intelligence, it seems highly probable that their movements are guided by definite plans, and that they have some sort of governmental system. In Cachar, the tea planters often keep hoolocks for years at a time, permitting them to run about the compound quite frecly. Several such tame hoolocks were seen by Mr. Candler and were under his observation for several months at a time. They often would disappear and be away in the tree tops for days together, and at such times nothing tempts them to come down. But at length one of them seems to prefer to be sociable and he will then come and sit on the arm of a chair at breakfast, although he will never reach and snatch things off the table. His manners, in fact, are quite irreproachable, and he keeps himself remarkably clean. At sunset it is his custom to settle down to sleep, jammed tightly into the fork of a tree in a squatting position, usually with his arms over his head. In this semi-domesticated state, the hoolock seldom uses his voice, for he apparently finds no necessity for chattering or calling to his 
fellows. A long list of foods eaten by the hookek has been given by the numerous observers. This inchudes fruits, leaves, young shoots, spiders, insects, birds' eggs and young birds. But much of this may be considered as conjecture, athough there is good reason to believe that the diet is extremely varied. If captured young, the hoolock is readily tamed. It is gentle, well disposed and good tempered. It shows a marked intelligence, particularly in the acquisition of tricks and in adjusting itself to household regulation.

\section{Measurements and Indices of Hylobates Hoolock}

The measurements of the hoolock show on the average:

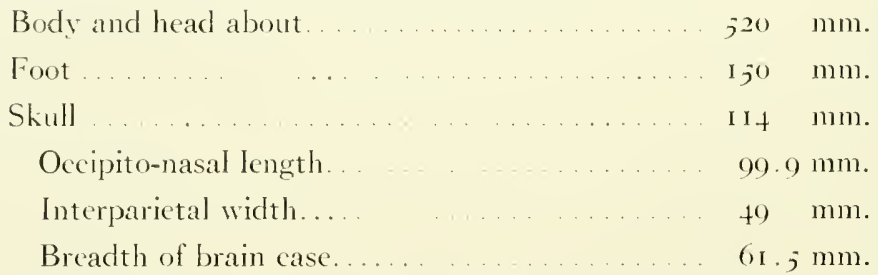

The dimensions of the brain including the cerebellum and brain stem are:

Longitudinal diameter . . . . . . . . . $6 . \ldots . \mathrm{mm}$.

Transverse diameter.............. ;j $\mathrm{mm}$.

The brain is distinctly gyrencephalic in type, presenting, as do the brains of all the other intermediate primates, a Sylvian, a Rolandic and a simian fissure, which distinctly set the boundaries of the frontal, parietal, temporal and occipital lobes upon the lateral convexity of the cerebral hemisphere.

The weights of the several divisions of the brain are:

Forebrain

Midbrain

Hindbrain

Total weight of brain. $j+$ gms.

2 gms.

13 gms.

69 gms. 
The water displacement of the brain is:

Forebrain

Midbrain

Hindbrain

Total displacement j3 c.e.

2 c.c.

Io c.e.

6j c.c.

The forcbrain indices, computed on the basis of weight and volume, give the gibbon a forebrain index of 81 per cent by weight and 82 per cent by volume, thus placing it in the group characterized by manual differentiation, although it occupies a rather low position in this group.

Surface Appearance of the Brain in Hrlobates Hoolock

From some of the more important superficial features on the external surface of the cerebral hemisphere, it is evident that the brain of the gibbon in many respects is little more advanced in its development than that of the baboon or macacus. The two great concavities on the basilar surface, i.e., the orbital concavity and the cerebellar concavity, are equally as pronounced as in the other intermediate primates. This fact in itself calls attention to the degree of limitation imposed upon the frontal and occipital lobes in their expansion. For it is almost in direct proportion as these basal concavities become less pronounced, that the two important territories of the cortex, the frontal and the occipital lobes, show progressive expansion. The olfactory bulb and tract are detachable as far back as the trigonum olfactorium. A rudimentary olfactory sulcus is present and a correspondingly rudimentary gyrus rectus. The interorbital kcel is prominent. As in the case of the baboon and the macacus, the boundaries of the superior longitudinal fissure tend to diverge as they approach the occipital pole. This divergence permits of a marked widening of the fissure which is in the interest of accommodating the superior vermis of the cercbellum whose appearance on the tentorial 
surface of this organ is sharply convex from side to side and thus forms a decisive ridge in the midline of the cerebellum. This ridge becomes progressively less distinet in the higher anthropoids and man. The divergence in
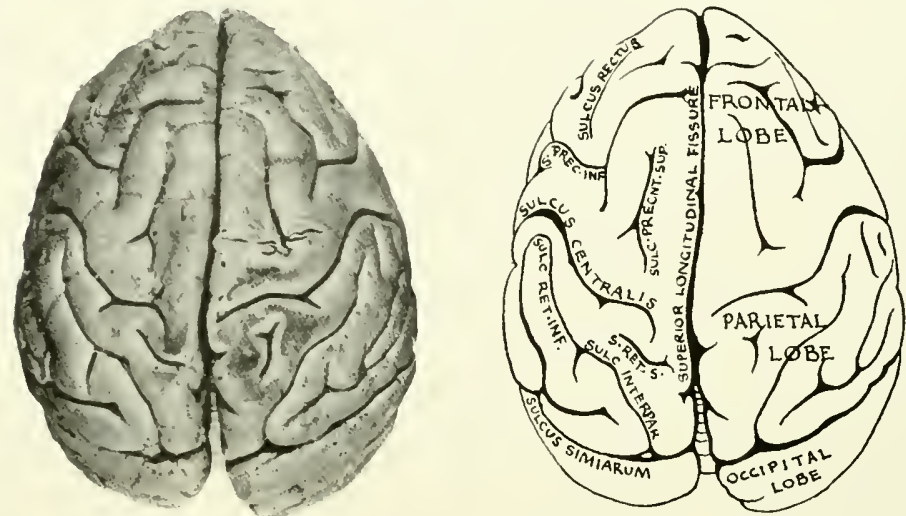

FIG. IO3 DORSAL SURFACE OF BRAIN, HYLOBATES HOOLOCK.

[Actual Length, $70 \mathrm{~mm}$.]

Key to Diagram. sulc. INTER-PaR., Sulcus Interparietalis, S. PRec. INF., Sulcus Precentralis Inferior; sulc. PRECNT, sup., Sulcus Precentralis Superior; sulc. RET. INF., Sulcus Retrocentralis Inferior; s. Ret. S., Sulcus Retrocentralis Superior.

the occipital portion of the superior Iongitudinal fissure of the higher forms gradually lessens, with the general eflect that the oceipital region eventually conceals from view the entire tentorial surface of the cerebellum.

\section{FISSURES AND LOBES}

In its fissures and Iobations, also, the eerebral hemisphere corresponds closely with the brain of the macaque and baboon. The fissure of Rolando separates the frontal and parietal lobes, the Sylvian fissure intervenes between the parietal and temporal lobes, the simian fissure separates the parietal and temporal lobes from the occipital lobe. The convolutional pattern in all 
of these cortical areas is relatively simple. It is most complex in the parictal region and least conspicuous in the frontal lobes. In respect to fissural development, the gibbon shows less advance than either the baboon or macacus.
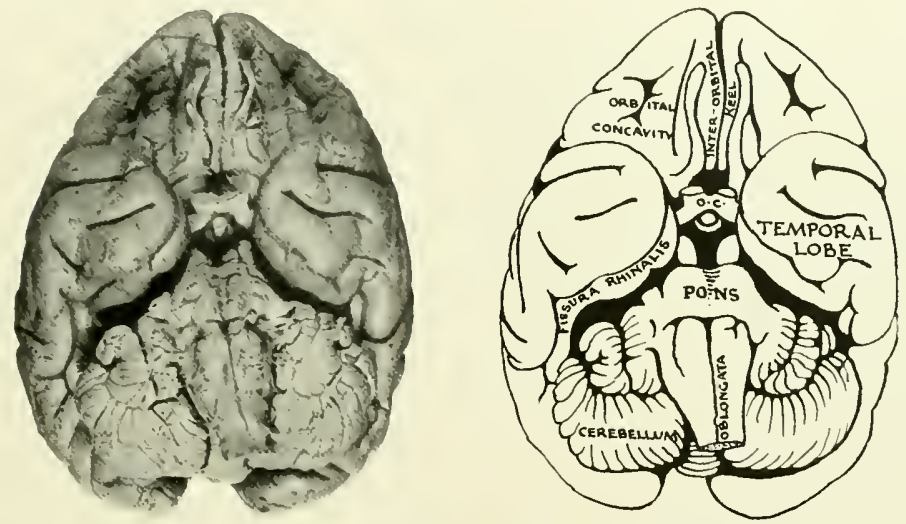

FIG. I94. BASE OF BRAIN, HYLOBATES HOOLOCK.

[Actual Length, $70 \mathrm{~mm}$.]

hey to Diagram. o.c., Optic Chiasm.

On the other hand, there is a richness of convolutional pattern in the occipital lobe, which does not exist in either of the other two forms. This fact, taken in conjunction with the much reduced appearance of the superior colliculus of the midbrain, indicates that in the gibbon most of the actual supervision of vision has been transferred to the occipital lobe. The fissures in the temporal Iobe, especially the superior temporal fissure, are well developed and this latter has connected with its extremity a well-defined angular gyrus.

The fissures of the basal surface of the frontal and temporal lobes give these regions an extremely simple appearance. In fact, the impression obtained from a survey of the cerebral hemispheres in gibbon allies this form nuch 
more closely with the group here identified as intermediate primates. Certainiy, the superficial appearance of the gibbon's cerebral hemisphere places between it and the more highly complex endbrain of the great anthropoid
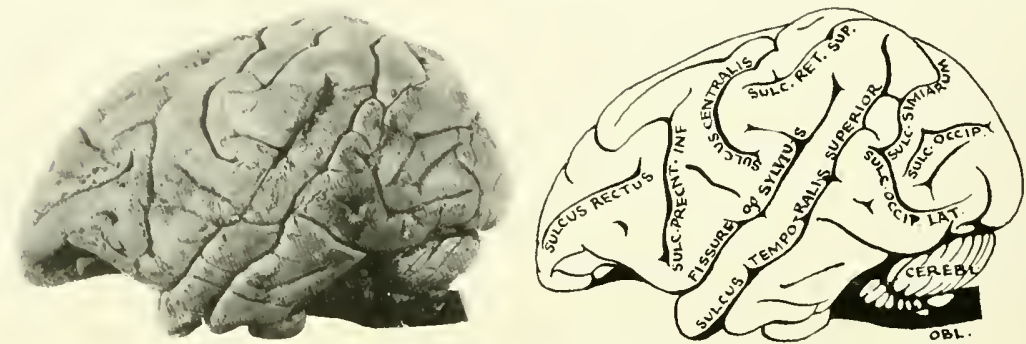

FIG. IOj. LEFT LATERAL SURFACE OF BRAIN, HYLOBATES HOOLOCK.

[Actual Length, $73 \mathrm{~mm}$.]

Key to Diagram. clrebl., Cerebellum; obl., Oblongata; sulc. occir., Sulcus Oceipitalis; sulc. occip. Lat., Suleus Occipitalis Lateralis; sulc. Precnt. INF., Sulcus Precentralis Inferior; StLC. RLT. SuP., Sulcus Retrocentralis Superior; sulc. simarui, Sulcus Simiarum.

apes a wide interval, so wide as to justify the opinion that in descent, the lineal relation between the gibbon and the anthropoid, however direct, must be quite remote.

\section{THE CEREBELLUM}

The cerebellum bears out this impression in conclusive manner. Its tentorial surface shows that marked convevity or gabling which culminates in a well-defined vermal ridge-pole. This feature is characteristic of intermediate and lower primates, but gradually disappears in the higher anthropoids. On the tentorial surface of the cerebellum the interfolial fissures pass from the vermis to the lateral lobe without sulcal interruption, while on the occipital surface, a paramedian sulcus upon either side of the vermis interrupts the folial sulci in their passage from the median to the lateral expansions of 
the cerebellum. The petroso-ventricular surface of the cerebeflum presents at fairly large floceulus oecupying the eerebello-pontile angle. The mesial portion of this surface is in relation with the roof of the fourth ventricle.
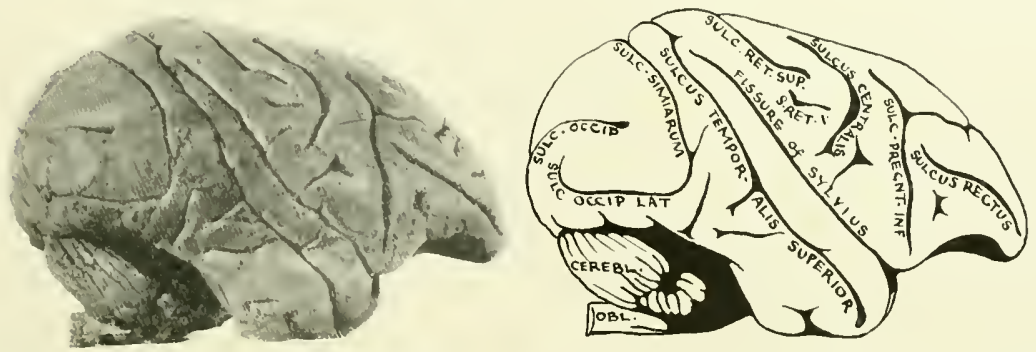

FIG. I06. RIGHT LATERAL SURFACE OF BRAIN, HYLOBATES HOOLOCK.

[Actual Length $73 \mathrm{~mm}$.]

Key to Diagram. Obl., Oblongata; sulc. occip., Sulcus Occipitalis; sulc. occip. Lat., Sulcus Occipitalis Lateralis; Cerebl., Cerebellum; sulc. Precnt. INF., Sulcus Precentralis Inferior; sulc, Ret. Sup., Sulcus Retrocentralis Superior; sulc. simiarum, Sulcus Simiarum; s. REt. I., Sulcus Retrocentralis Inferior.

\section{THE BRAIN STEM}

In the brain stem, the markings on the several surfaces in gibbon are more preeise than in the lower primates, and about equally as distinet as they are in the baboon and macacus.

The OBlongata. The oblongata upon its ventral surface presents a well-defined ventromedian sulcus, which is flanked upon either side by two fairly prominent pyramidal elevations. Lateral to the pyramid and separated from it by a pre-olivary suleus is a well-defined olivary eminence whose contour and proportions are somewhat more marked than in the other intermediate primates. The lateral surface shows the gradual gathering of the ascending spinocerebellar fibers until this collection forms the restiform body at the lower border of the pons Varolii. In the lower portion of the lateral surface there is a slight protuberance which marks the surface relief 
of the substantia gelatinosa trigemini. This trigeminal protuberance at the more cephalic levels of the oblongata is concealed from view by the superposition of the fibers entering the restiform body. In the most cephalic
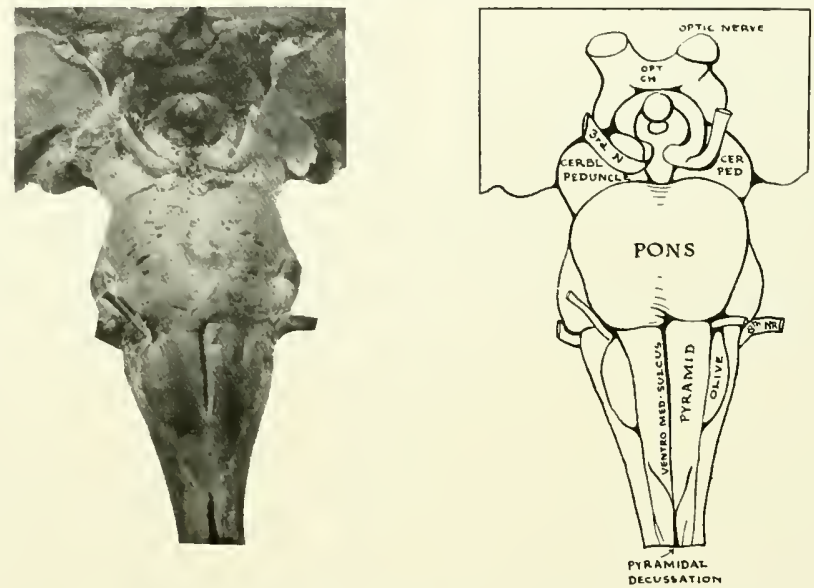

FIG. 197. VENTRAL SURFACE OF BRAIN STEM, HYLOBATES HOOLOCK. [Actual Length $45 \mathrm{~mm}$.]

Key to Diagram. Cer. Ped, and cerbl. Peduncle, Cerebral Peduncle; opt. Ch., Optic Chiasm; ventro MID. Sulcus, Ventromedian Sulcus; 3 RD N., Third Nerve; 8TH NR., Eighth Nerve.

portion of the lateral surface appears a well-defined protuberance, the tuberculum acusticum.

The dorsal surface of the oblongata presents its characteristic divisions, the ventricular and infraventricular portions. The infraventricular portion is characterized by the typical dorsomedian septum, upon cither side of which appears a well-defined clava, separated by a dorsal paramedian sulcus from an equally well-defined cuneus. These elevations on the dorsal surface indicate respectively the presence of the nucleus of Goll and the nucleus of Burdach. 
In the ventricular portion of the dorsal surface appears the caudal angle of the fourth ventricle bounded laterally by the elevations of the clava and cuneus. At the angle of this ventricle is the trigonum hypoglossi which
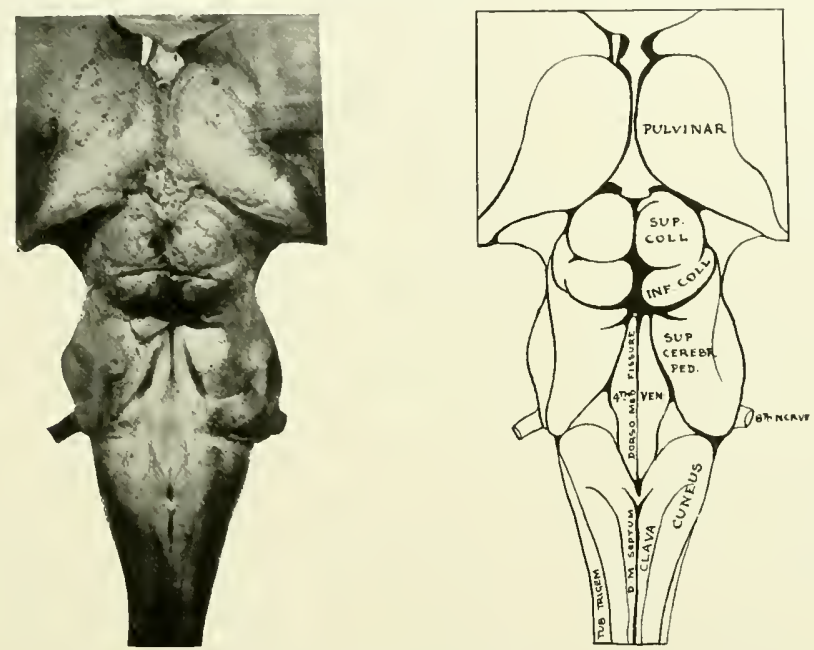

FIG. 198. DORSAL SURFACE OF BRAIN STEM, HYLOBATES HOOLOCK.

[Actual Length $45 \mathrm{~mm}$.]

Key to Diagran. D. M. Septum, Dorsomedian Septum; Dorso. Med. Fisslire, Dorsomedian Fissure; 1 NF. COLL., Inferior Colliculus; sup. CEREBr. PED., Superior Cerebellar Peduncle; sup. Col.., Superior Colliculus;

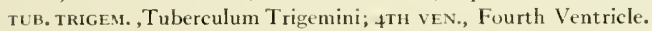

marks the position of the nucleus of the twelfth nerve; lateral to it, and separated from it by the sulcus limitans, is situated the fovea vagi above the dorsal nucleus of the pneumogastric nerve. The lateral walls constituted by the cuneus and clava become progressively reduced in altitude as they proceed cephalad, and funally at the level of the lateral recess have reached the plane of the ventricular floor. At this level, fibers of the eighth nerve make their entrance, as the striae acusticae; most of these fibers pass directly transversely inward to the dorsomedian sulcus. Below the level of the striac 
acusticac, a large elevation appears in the floor marking the vestibular area. This fact accords well with the exquisitely arboreal locomotion of the gibbon and reveals one of the most important regulating mechanisms which makes possible its almost bird-like passage through the forest. The median fissure becomes somewhat decper as the caudal orifice of the Sylvian aqueduct is approached, while the superior cerebellar peduncles, forming the lateral boundaries of the ventricle, converge toward the aqueduct. Immediately above this orifice of the aqueduct the trochlear nerve emerges from the brain stem after decussation in the superior medullary velum. At this level appear the characteristic developments in the rool of the midbrain, the collicular eminences. Of these, as in other primates, the inferior colliculi are the smaller. However, the disparity in size between these two sets of colliculi in gibbon is not so marked as in the macacus or baboon. This modification appears to be due to the fact that the superior colliculus has lost somewhat in prominence as compared with the other two intermediate primates. The correctness of this observation gains support from the more complex development of the occipital lobe in gibbon when compared with the baboon or macacus, thus implying a still further delegation of visual function to the oceipital lobe than in either of the other primates mentioned.

The Poxs Varoliz. Upon the ventral surface of the axis the pons Varolii appears as a fairly prominent transverse structure, separated from the oblongata by a well-defined bulbopontile sulcus. It is also separated at its cephalic extremity from the cerebral peduncles by the pedunculo-pontile sulcus. The pons in the gibbon gives the impression of a greater degree of prominence than in either the baboon or macacus. The inference based upon the pontile appearance accredits the gibbon with the possession of skilled movements more complex than either the baboon or the macacus.

The Ventral Surface of the Brain Stem. The brain stem upon its ventral surface terminates cephalically by the typical divergence of the two 
cerebral peduncles bounding the optico-peduncular space. From this space emerge the two oculomotor nerves and in it are contained the mammillary bodies, the attachment of the infundibular stalk and the tuber cincreum.

\section{Internal Structure of the Brain Stem in Hylobates Hoolock}

In some particulars the cross sections through the brain stem of gibbon show a slight advance, particularly in the definition of the structures when compared with the baboon and macacus. This applies more especially to the inferior olive and pontile nuclei. In other structural details the definition in gibbon does not show any marked degree of progress wer the other intermediate primates.

\section{LEVEL OF THE PIRAMIDAL DECUSSATION (FIG. 199)}

Here the crossing fibers of the pyramid (Pyx) determine a conspicuous rearrangement in the axis. The effects of this decussation are seen in several notable features. In the first place, the usual separation of the ventral gray column (Ven) from the central gray matter $(\mathrm{Cen})$ is clearly shown.

This behavior on the part of the pyramidal fibers is of particular interest. It is primarily a mammalian characteristic. Furthermore, the pallio-spinal comnection represented by the pyramid is peculiar to mammals. Decussation in conduction pathways of all varieties, whether intersegmental or projectional, is the almost invariable rule in the vertebrate nemraxis. It hether, as Cajal believes, such crossing was determined by the universal decussation of the optic fibers throughout this phytum, or whether some other factors have been operative, the irresistible tendency for long fiber tracts to pass from one side of the brain to the other has exerted its influence on the pyramidal system. The nerve fibers in this system are all newcomers among the conducting axons of the neuraxis. They were introduced by the mammals and represent the last accessional elements in the control of notor reactions. They are 
what may be called the modern agents of volitional behavior. According as their rolume is large or small, they indicate the directing capacity of the cerebral cortex and as such transmit the orders of the supreme motor author-

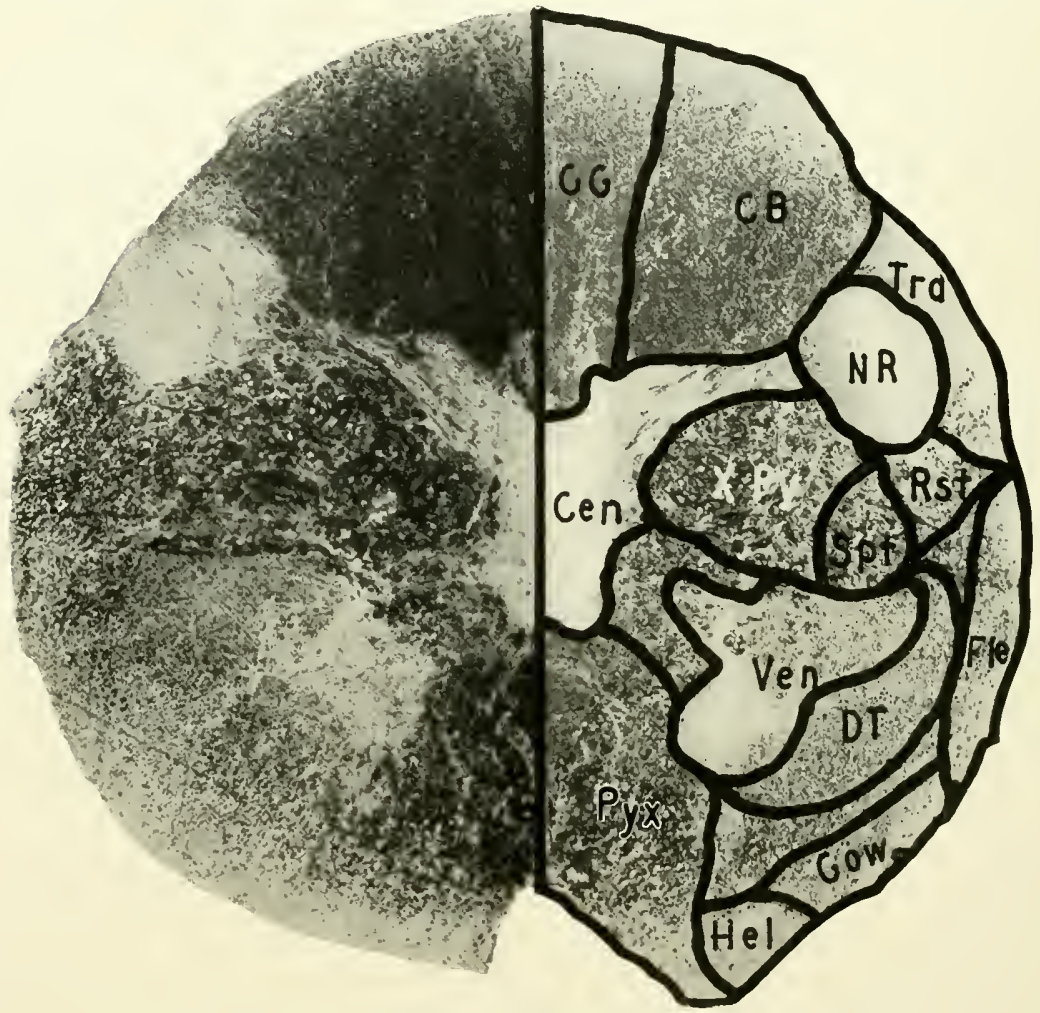

FIG. I99. GIBBON. LEVEL OF THE PYRAMIDAL DECUSSATION.

CEN, Central Gray Matter; CB, Column of Burdach; CG, Column of Goll; DT, Deiterso-spinal Tract; FLE, Dorsal Spinocerebellar Tract; Gow, Ventral Spinocerebcllar Tract; He,, Spino-olivary Tract of Helweg; NR, Nucleus of Rolando; prx, Pyramidal Decussation; RST, Rubrospinal Tract; spt, Spinothalamic Tract; TRD, Descending Trigeminal Tract; ven, Ventral Gray Matter; XPY, Crossed Pyramidal Tract. [Accession No. 1.1. Section 5. Actual size $8 \times 7 \mathrm{~mm}$.] 
ity. Regardless of their number, these pyramidal fibers have submitted themselves to the influences which determine decussation. They do not follow exactly the same manner of crossing in all mammals, nor is their decussation equally decisive in all species. In some instances the pyramid is small and the crossing fibers produce relatively inconspicuous changes in the oblongata. Where, as in the primates, the decussation is large, it occasions a most outspoken feature in cross section.

In certain mammals, the decussating fibers of the pyramid make their way almost furtively into the dorsal columns for their further descent into the spinal cord. In others, they cross with more conspicuity directly into the lateral columns. The primates, from one end of their order to the other, have established a fashion of pyramidal decussation which is distinctively their own.

With the possible exception of the transitional forms represented by lemur or of such anthropoid incipiency as is seen in the tarsicrs, the pyramidal system of primates is larger than in all other mammals. The secondary effects of pyramidal decussation are witnessed in the wide detachment of the ventral gray column (Ven) from the central gray matter (Cen) and also in the large field eventually occupied by the decussated fibers. This structural disposition and rearrangement is so decisive that any primate may be identified as such by the appearance of its pyramidal decussation.

Were these facts of structural importance alone, they might carry but little weight in the presence of so many other satisfactory identifying characters. It is their dynamic significance, however, that gives them their true value. In this sense, they indicate the degree of neopallial development in the brain and the extensions in the cortical control of behavior. Through the gradual expansion of this mammalian contribution to the nervous system, the primate has progressively advanced to the highest stages of differentiation. 
The dorsal sensory field is represented by the columns of Goll and of Burdach ( CG, CB), together with the substantia gelatinosa trigemini (NR) and its accompanying descending trigeminal tract (Trd). A comparison of these elements shows that the column of Burdach has increased in size relatively to the column of Goll. It is evident from this relation that the sensory teritories of the upper extremity of gibbon have gained in prominence. This advance is no doubt consequent upon the high degree of brachial and manual specialization essential to the animal's arboreal life. The comparatively small size of the muckeus of Rolando (NR) indicates that much of the tactile direction of locomotion must have been delegated to a more effective and highly specialized hand.

In the circumferential zone are the ascending fibers of the spinocerebetlar tracts (Fle, Gow). The medullary area surrounding the ventral gray column contains the Deiterso-spinal tracts (DT), while in the intermediate zone are the rubrospinal and spinothalamic tracts ( $R s t, S p t$ ).

LEVEL OF THE CALDAL EXTREMITY OF THE INFERIOR OLIVE (FIG. 2OO)

Here the transverse diameters of the axis have increased considerably. This change especially affects the dorsal sensory field, due to the appearance in it of the nuclei of $\mathrm{Goll}$ and Burdach ( NG, NB). Both nuclei are large but by actual measurement the nucleus of Burdach has a volume about twice that of Goll. The latter nucleus shows no median specialization characteristic of the nuckeus of Bischoff. Since the gibbon does not develop a tail, the absence of this unpaired median nucleus might be expected. Connected with Burdach's mucleus there is a large isolated mass which constitutes the nucleus of Blumenau ( $\mathrm{NBI}$ ). The substantia gelatinosa presents marked promincence and is in relation with a desecnding trigeminal tract ( $\operatorname{Trc}$ ) of considerable size. Estimated as a whole, the dorsal sensory field denotes the apportionment of the afferent influx. The territory of the upper extrem- 
ity has occasioned a notable increment in consequence of the animal's high specialization for arboreal life. The ultimate formula of the elements in the dorsal field of the great apes and man is here more nearly approached

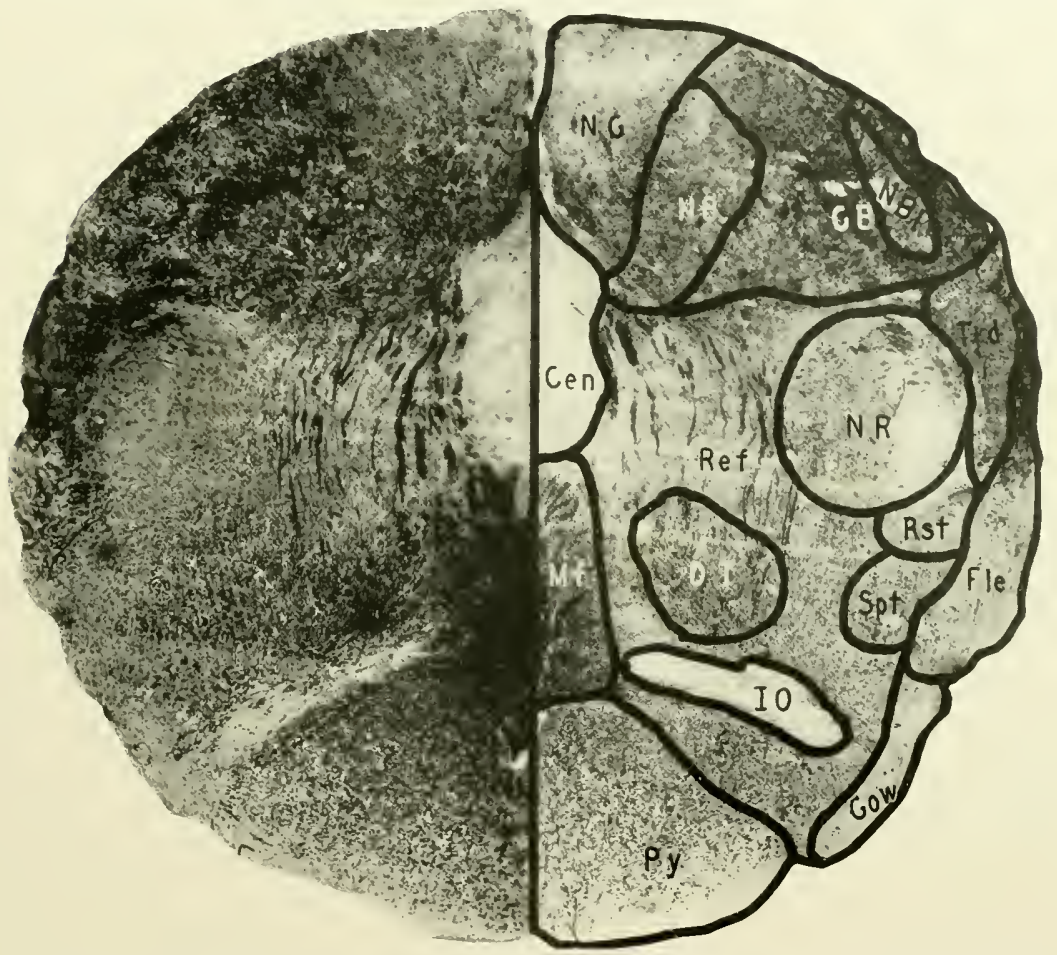

FIG. 200. GIBBON. LEIEL OF THE CAUDAL EXTREMITY OF THE INFERIOR OLIVE. $\mathrm{CB}$, Column of Burdach; CEN, Central Gray Matter; Dr, Deiterso-spinal Tract; Fle, Dorsal Spinocerebellar Tract; cow, Ventral Spinocerebellar Tract; 10, Inferior Olive; MF, Mesial Fillet; NB, Nucleus of Burdach; $\checkmark B L$, Nucleus of Blumenau; NG, Nucleus of Goll; NR, Nucleus of Rolando; pr, Pyramid; REF, Reticular Formation; RST, Rubrospinal Tract; spt, Spinothalamic Tract; TRD, Descending Trigeminal Tract. [Accession No. 14t. Section 16. Actual Size o $\times 8 \mathrm{~mm}$.]

than in any of the primates below this stage. Numerous coarse bundles of arcuate fibers arising in the nucleus of Goll pass forward toward 
the median raphe. In their passage they almost completely separate the central gray matter ( Cen) from connection with the substantia gelatinosa of Rolando (NR). The central gray matter has manifested decisive alteration. It is now almost quadrilateral in outline, with a narrow dorsal extension reaching backward into the dorsomedian septum. This tonguelike process indicates the preliminary rearrangement incident to the opening of the fourth ventricle. In the lateral field the circumferential zone is characterized by the usual dorsal migration of the spinocerebellar tracts. The dorsal spinocerebellar fasciculus (FIe) has changed its course to an oblique passage along the outer side of the descending trigeminal tract where its heavily myelinized fibers may be readily seen. The intermediate zone contains the rubrospinal ( $R s t)$, the spinothalamic $\left(S_{p} t\right)$ and the Deitersospinal (DT) tracts. The ventral field contains the fibers which constitute the pyramid $\left(\mathrm{P}_{y^{r}}\right)$, dorsal to which are the bundles of the posterior longitudinal and predorsal fasciculi. The internal arcuate libers, as they cross the raphe to form the mesial fillet (Mf), somewhat obscure the outlines of the predorsal and posterior longitudinal bundles. Dorsolateral to the pyramid is a slender body of gray matter, the caudal extremity of the inferior olive (IO). An extensive, diffuse reticular formation (Ref), lies between the olivary body and the nucleus of Rolando. It is traversed by numerous internal arcuate fibers and contains several indefinite nuclear aggregations, one constituting the lateral reticular nucleus and a second, the dorsal reticular nucleus.

A comparative survey of the six species of primates thus far considered must make impressive the striking similarities which characterize the corresponding cross sections of the brain stem. Level for level in lemuroid and anthropoid, the component structures are remarkably identical. So alike are they that the repeated descriptions of the several levels in the different species creates a degree of tedium felt by the reader no doubt as much as by 
the author. The repetition would be wholly objectionable and out of place were it not for one compelling argument which secms to make it justifrable. To recognize the morphological consistency, the unity of structural design, in the relation of so many intricate parts should suffice to dispel all doubts concerning the close gencric association of these animals. This unity is likewise convincing as to that influence which, working through the primate stock, has employed the same fundamental pattern to establish such conditions as result in progressively more efficient neural mechanisms and in better behavioral adjustments.

\section{LEVEL THROUGH THE MIDDLE OF THE INFERIOR OLIVE (FIGS. 201, 202)}

Here the most conspicuous feature is the appearance of the inferior olivary nucleus ( $\mathrm{IO}$ ). This structure here presents its full degree of development in the gibbon. Certain features of it are to be contrasted with the homologous structure in the lower primates. It has gained considerably in its general dimensions, both transversely and longitudinally. But its real increase in prominence is due to its more convoluted configuration characteristic of the human and humanoid structure. In connection with it are its two accessory olives ( $\mathrm{VO}, \mathrm{DO})$. The central gray matter occupies a position immediately beneath the floor of the fourth ventricle. In its most mesial portion it contains the nucleus of the twelfth nerve ( $\mathrm{Nhy}$ ), whose emergent fibers (NI2) pass from the nucleus and make their way ventrolatcrally toward the inferior olive. Lateral to the nucleus hypoglossus, and separated from it by the sulcus limitans in the central gray matter, is the dorsal nucleus of the pneumogastric nerve $(\mathrm{Nvd})$, some entering fibers of which may be discerned approaching this nuclear collection. Immcdiately adjacent and lateral to the dorsal nucleus of the tenth nerve is a dense collection of medullated fibers constituting the fasciculus solitarius surrounded by the nucleus of this fasciculus ( $\mathrm{Nfs}$ ). Ventral to the fasciculus solitarius is the 
substantia gelatinosa trigemini (NR), while lateral to it and contiguous with its external surface is the descending trigeminal tract ( $\operatorname{Trd}$ ). Occups ing the most dorsal position in the lateral field is the cephatic extremity of

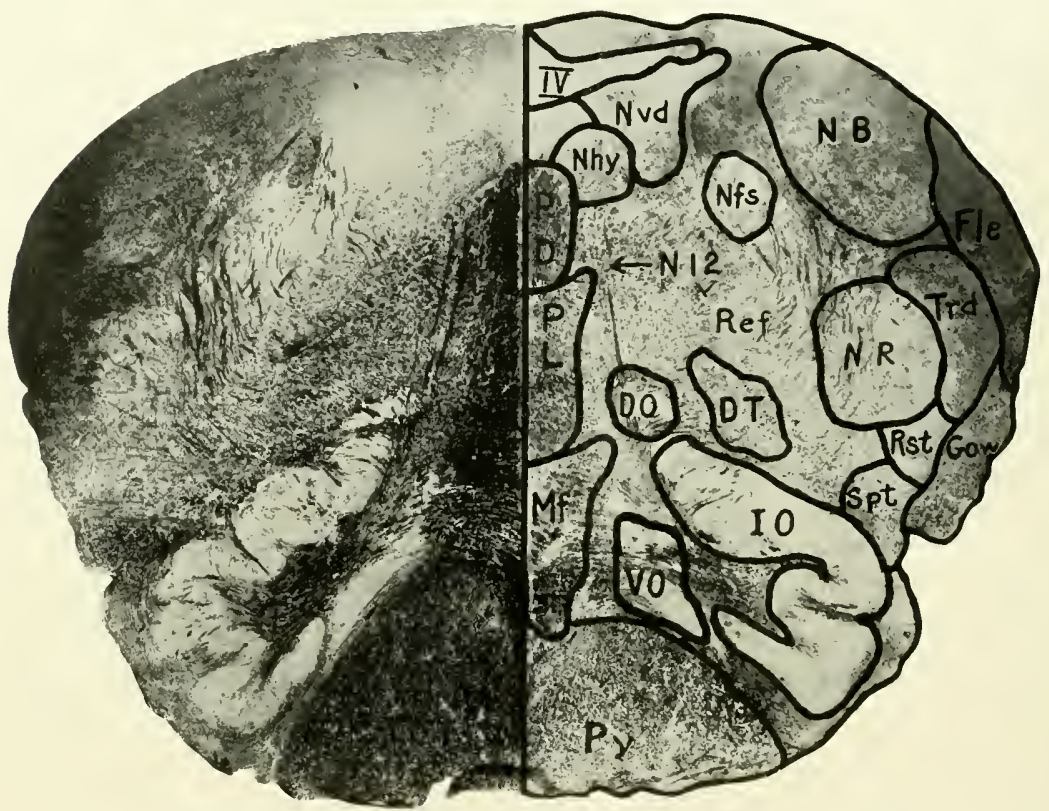

FIG. 2OI. GIBBON. LEVEL THROLGH THE MIDDLE OF THE INFERIOR OLITE. no, Dorsal Accessory Olive; DT, Deiterso-spinal Tract; fle, Dorsal Spinocerebellar Tract; guw, Ventral Spinocerebellar Tract; 10, Inferior Olive; N, Fourth Ventricke; MF, Mesial Fillet; NB, Nucleus of Burdach; NFs, Nucleus Fasciculus Solitarius; NHr, 1 ypoglossal Nucleus; NR, Nucleus of Rolando; Nrd, Dorsal Vagal Nucleus; N12, Twelfth or Hypoglossal Nerve; PD, Predorsal Bundle; PL, Posterior Longitudinal Fasciculus; PY, Pyramid; REE, Reticular Formation; RST, Rubrospinal Tract; SPT, Spinothalimic Tratet; TRD, Descending Trigeminal Tract; 10, Ventral Accessory Olive. [Accession No. 1+1. Section 101. Actual Size $1+X_{1}$ ro mm.]

the nucleus of Blumenau (NBI), from which numerous internal arcuate fibers make their way forward and inward through the reticular formation toward the median raphe, where they undergo decussation and enter the mesial fillet ( MI ) on their way to higher levels in the brain. 
The general size of the nucleus of Blumenau (NBI) is suggestive, as in other levels, of the degree to which the forelimb and hand have developed. Emphasis has been laid on the fact that the relatively poor development of

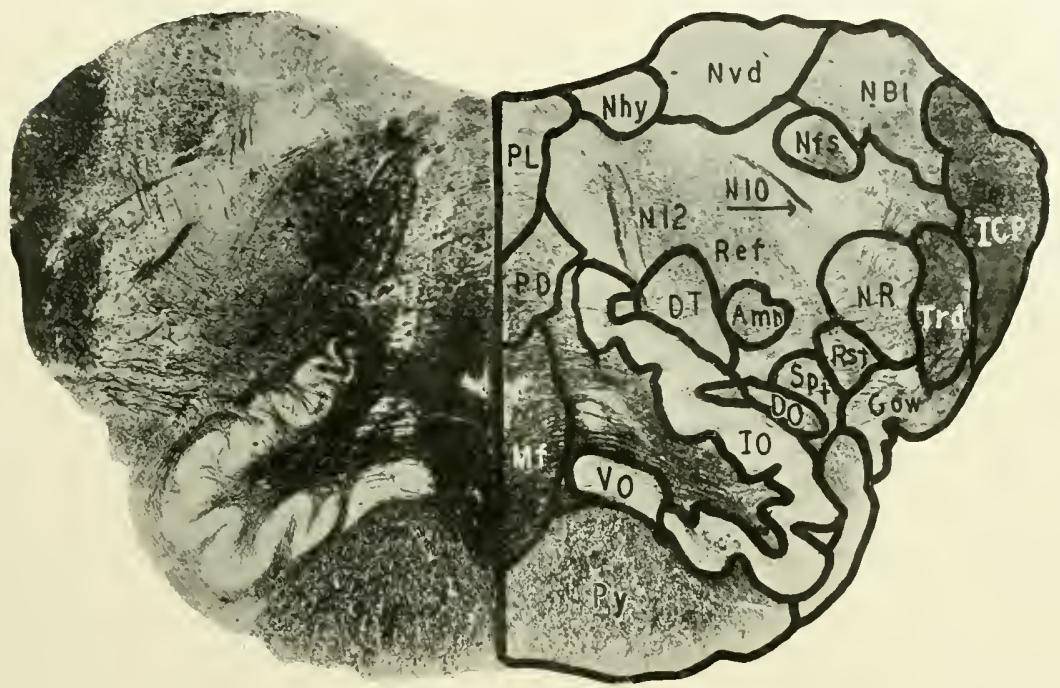

FIG. 202. GIBBON. LEIEL THROUGH THE MIDDLE OF THE INFERIOR OLINE.

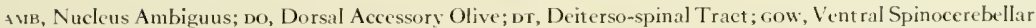
Tract; ıCP, Inferior Cerebellar Pedunele; 10, Inferior Olive; Mf, Mesial Fillet; Nвı, Nucleus of Blumenau; xrs, Nucleus Fasciculus Solitarius; xHr, llypoglossal Nucleus; NR, Nuclcus of Rolando; xvo, Dorsal Vagal Nucleus; Nıo, Tenth Cranial Nerve; vı2, Twelfth or Hypoglossal Nerve; PD, Predorsal Bundle; Pr, Pusterior Longitudinal Fasciculus; Py, Pyramid; REF, Reticular Formation; RST, Rubrospinal Tract; SPT, Spinothalitmic Tract; TRD, Descending Trigeminal Tract: vo, Ventral Accessory Olive. [Accession No. 1+1. Section 131. Actual Size $1+\times 8 \mathrm{~mm}$.]

the hindlimb in gibbon and the high degree of proficiency attained by the upper extremity and hand, especially for the purposes of its flect locomotion, give marked structural preponderance to the nucleus of Blumenau. The prominence of the inferior olivary nucleus compared with the other forms may also be in direct relation with the flight-like locomotion of the animal 
which has been likened to the most skillful attainment of expert trapeze performers. The execution of such acts would require most active and precise visual supervision, both in the recognition of distance as well as in the selection of adequate supports to sustain the weight of the body as the animal makes its prodigious swinging flights through space from one limb to another. Inaccuracy in visual judgment and particularly in the simultancous adjustment of head, eye and hand in the execution of such acts could not fail to be attended with catastrophe. Hence it is that the simultaneous movements of head, eye and hand become of greater importance to the gibbon than to the ground-living baboon or in the more conservative aerial feats of the macacus and allied monkeys.

The pyramid ( Py) occupies its characteristic position in the ventral area of the cross section, but from its general dimensions, it appears to show no marked increase over this structure in the macaque and baboon. The cross section bears out the general impression conveyed by the appearance on the external surface of the oblongata. It seems probable that, despite the high development of the hand and especially of the forearm in the adjustment to its arboreal locomotion, the gibbon has shown no great specialization in its skilled movements, generally speaking. The proficiency which the animal exhibits in the hindlimb would indicate less need for the volitional control in these parts of the body than that possessed by many of the other primates.

Dorsolateral to the olive is the reticular formation ( $R e f$ ) through which pass many of the internal arcuate fibers. Its definition is less well marked than in the other intermediate primates. At the lateral extremity of the section is a bundle of fibers constituting the restiform body (ICP) which is carrying spinoccrebellar and other ascending cerebellar fibers upward toward the cerebellum. 
LEVEL OF THE IESTIBULAR NUCLEI (FIG. 203)

At this level the configuration of the cross section is altered by the further marked enlargement of the fourth ventricle. Intricate changes depend

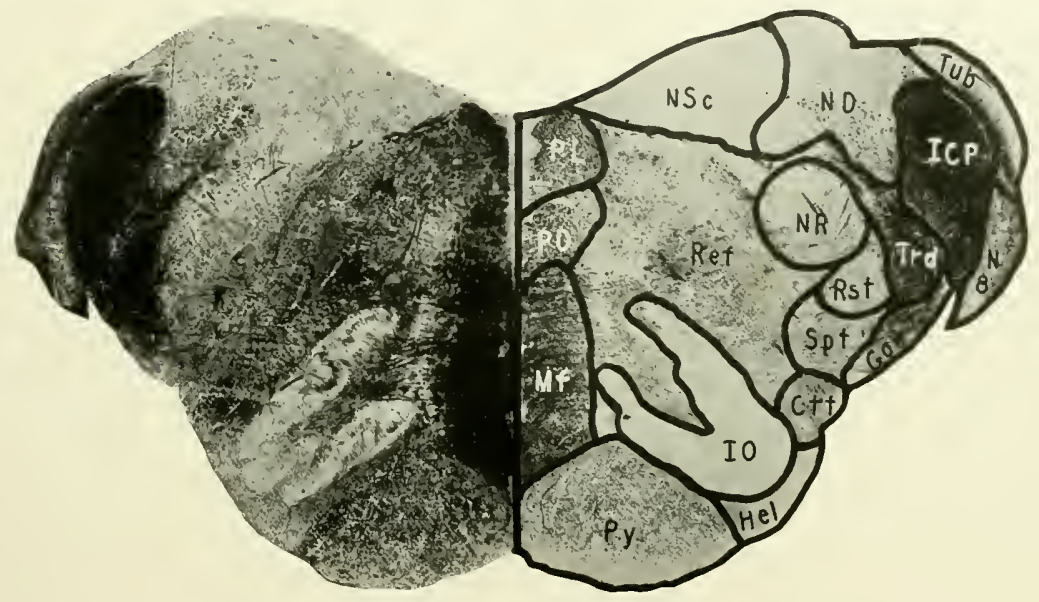

FIG. 203. GIBBON. LEVEL OF THE VESTIBULAR NUCLEI.

CTr, Central Tegmental Tract; gow, Ventral Spinocerebellar Tract; Het, Spino-olivary Tract of Helweg; ICP, Inferior Cerebellar Peduncle; IO, Inferior Olive; MF, Mesial Fillet; ND, Deiters' Nucleus; NR, Nucleus of Rolando; Nsc, Nucleus of Schwalbe; N8, Auditory Nerve; PD, Predorsal Bundle; PL, Posterior Longitudinal Fasciculus; PY, Pyramid; REF, Reticular Formation; RST, Rubrospinal Tract; SPT, Spinothalamic Tract; TRD, Descending Trigeminal Tract; тUв, Tuberculum Acusticum. [Accession No. 141. Section 141. Actua] Size $1+\times 7 \mathrm{~mm}$.]

upon the replacement of the dorsal columns of Goll and Burdach by the large elements of the vestibular area. The existence of a long proprioceptive column in the alar plate of the oblongata has already been noted. In its more caudal portion this column makes provision for the transmission of proprioceptive stimuli arising in the muscles and joints of the extremities and trunk. The cephalic division of this column serves in a similar capacity for the proprioceptors in the vestibular portion of the internal ear. This 
system is here represented by the nucleus triangularis of Sehwalbe (NSc) and the nucleus magnocellularis of Deiters (ND). Both of these nuclear structures are of relatively larger size than in most other primates and indicate a balaneing mechanism commensurate with the locomotor specialization of the gibbon's arboreal life.

The fibers of the restibular division of the acoustic nerve penetrate the substantia gelatinosa trigemini (NR) to enter Deiters' nucleus. Lateral to Deiters' nucleus is the corpus restiforme ( ICP) superposed upon which is the tuberculum acusticum $(\mathrm{Tub})$. The cireumferential area is now occupied largely by the rentral spinocercbellar tract (Gow), mesial to which is the heary bundle of the descending trigeminal tract (Trd). The inferior olivary nucleus with its aecessory bodies (IO) oecupies its characteristic position and has its usual form. There is evident in it a slightly greater tendency toward convolution than observed in lower primates. Its definition is, however, somewhat more hazy than is true of higher members of this order. Dorsolateral to the olive is the central tegmental tract $(\mathrm{Ctt})$, important because of its probable connections with the oculomotor nuclei and the mesencephalic root of the trigeminal nerve. This mesencephatic root, according to good authority, represents the proprioceptive conduction from the eye museles. In this light the eentral tegmental tract may be regarded as the intersegmental link between the primary midbrain nuclei which reecive afferent stimuli from ocular museles, and the inferior olivary nucleus. Immediately dorsolateral to the olive and mesial to the central tegmental tract is the spinothalamic tract $\left(S_{p} t\right)$, while ventral to the substantia gelatimosa trigemini ( $\mathrm{NR}$ ) is the rubrospinal tract (Rst). The reticular formation (Ref) is relatively large. Ventromesial to the olive is the pyramid $\left(\mathrm{Py}^{*}\right)$ and dorsal to it in succession toward the floor of the ventricle are the mesial fillet (Mf), the predorsal fasciculus (PD) and the posterior longitudinal fasciculus (PL). 
LEVEL OF THE CEREBELLAR NICLEI (I IG. 20

At this level the cerebellum contains evidenee of a decisive advance. This advance affects the cerebellar nuclei and particularly the nucleus den-

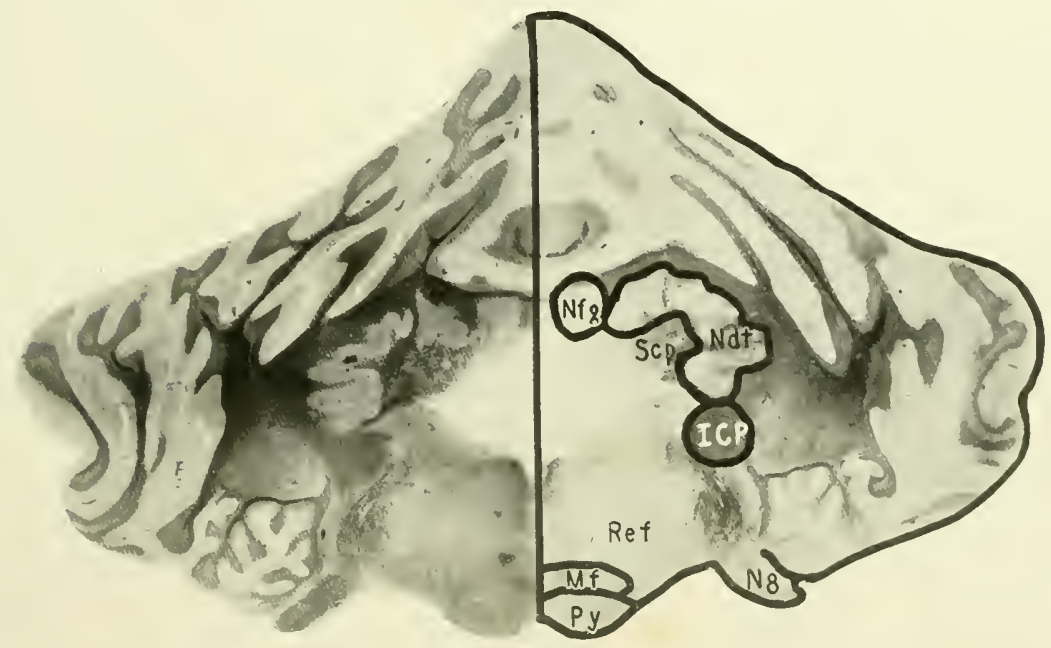

FIG. 204. GIBBON LEIEL OF THE CEREBELLAR NUCLEI.

ICP, Inferior Cerebellar Peduncle; MF, Mesial Fillet; XDt, Cerebellar Nuclei, Lateral Group; XFg, Cerebellar Nuclei, Mesial Group; N8, Auditory Nerve; Py, Pyramid; REF, Reticular Formation; scr, Superior Cerebellar Peduncle. [Accession No 1+1. Section 151. Actual Size $10 \times 12 \mathrm{~mm}$.]

tatus $(\mathrm{Ndt})$. For the first time this nucleus shows some of the tendencies in its development which foreshadow its ultimate configuration in the higher primates. In the species lower than gibbon, the dentate nucleus has had a more diffuse and somewhat amorphous appearance-amorphous at least in that it does not present any of those distinctive features which mark it in the great apes and man. A suspicion of the tendeney to become convoluted may be detected in the baboon and macacus. In general, however, this tendency in both of the latter species is confined to circumseribed and quite 
limited portions of the nuclear substance. The nucleus in the gibbon shows a number of well-defmed plications throughout its entire extent. Its hilus is dorsomesial in position and so disposed as to place its fundus in a ventromesial position. The general saccular arrangement of the nuclear substance is most pronouneed along the lateral wall. Here also the folia or convolutions are best defined. The mesial wall is much less developed in this respect. It gives the impression of a structure about to emerge from a diffuse matrix, but as yet not possessed of the definition characteristic of ultimate development. In this sense the dentate nucleus in gibbon represents a transitional stage in which only a portion of it has attained its full evolutional differentiation. It affords an illustration of one of the most important movements in the process of unfolding which appears both in ontogenetic and phylogenetic development. The gradual emergence of recognizable features out of a diffuse, more or less indefinite anlage is a rule in the genesis of all organs. The spinal cord, for example, in early fetal stages, shows but little differentiation of its principal histological features. In the early period of human development the dorsal and ventral gray columns are distinguishable but have many points of similarity. Only in infancy do these two columns of gray matter assume distinctive characters. During the first year of life it is often diffreult to distinguish histologically between the configuration of the several levels of the spinal cord, even when the comparison involves such widely removed portions as the cervical and sacral segments. Throughout adolescence and into adult life these differential features progressively assume their ultimate character. A similar process passing through gradient stages appears in the phyletic evolution of many structures in the nervous system. Such structures have their inception in a diffuse matrix and gradually acquire sharpness of defmition together with specific characteristics. The dentate nueleus in gibbon, therefore, is of great interest morphologically if for no other reason than representing a decisive stage of evolutional transition. Its physiological signif- 
icance is equally important. It indicates the pronounced accessions in coordinative control which this more advanced primate has acquired in response to its specialized arboreal locomotion. This detail of its neural organization approaches much nearer to the higher anthropoids than any of the intermediate primates.

Dorsomesial to the dentate nucleus is the nucleus fastigii ( $\mathrm{Nfg}$ ), while the inferior vermis projects into and fills most of the fourth ventricle.

\section{LEVEL OF THE EMERGENCE OF THE SIXTH CRANIAL NERIE (FIG. 20j)}

At this level the most important features in the section are the abducens nucleus $(\mathrm{Nab})$ situated in the floor of the fourth ventricle, the superior olive ( $\mathrm{SO}$ ) connected with the secondary cochlear pathway, and the appearance of the transverse fibers constituting the trapezoid body. The pyramid ( $\mathrm{Py}$ ) occupies its usual position in the ventromedian portion of the section, while dorsal to it, stretching across the section in a transverse manner, are many fibers connected with the secondary cochlear pathway of the trapezoid body. At the lateral extremity of this is a nuclear collection which forms the superior olive ( $\mathrm{SO}$ ), from which many fibers make their way to the nucleus abducentis. These latter constitute the peduncle of the superior olive, the fibers of which participate in reflex acts necessary to adjusting lateral gaze to the direction from which sudden sounds may arise. The importance of such a direct reflex mechanism is apparent when the needs of instantaneous visual detection are considered in relation to acts of self-protection. Mesial to the nucleus abducentis ( $\mathrm{Nab}$ ) are the fibers which form the second part in the emergent course of the facial nerve. Other fibers constituting the fourth part of this nerve sweep forward and outward, mesial to the substantia gelatinosa ( NR). The central gray matter occupies the floor of the fourth ventricle whose roof is formed by the vermis of the cerebellum. 
LEXEL THROLGH THE MIDDLE OF THE PONS VAROLII (FIG. 206)

At this level all three lavers of this structure may be discerned, namely, the stratum superficiale pontis, the stratum complexum pontis, containing

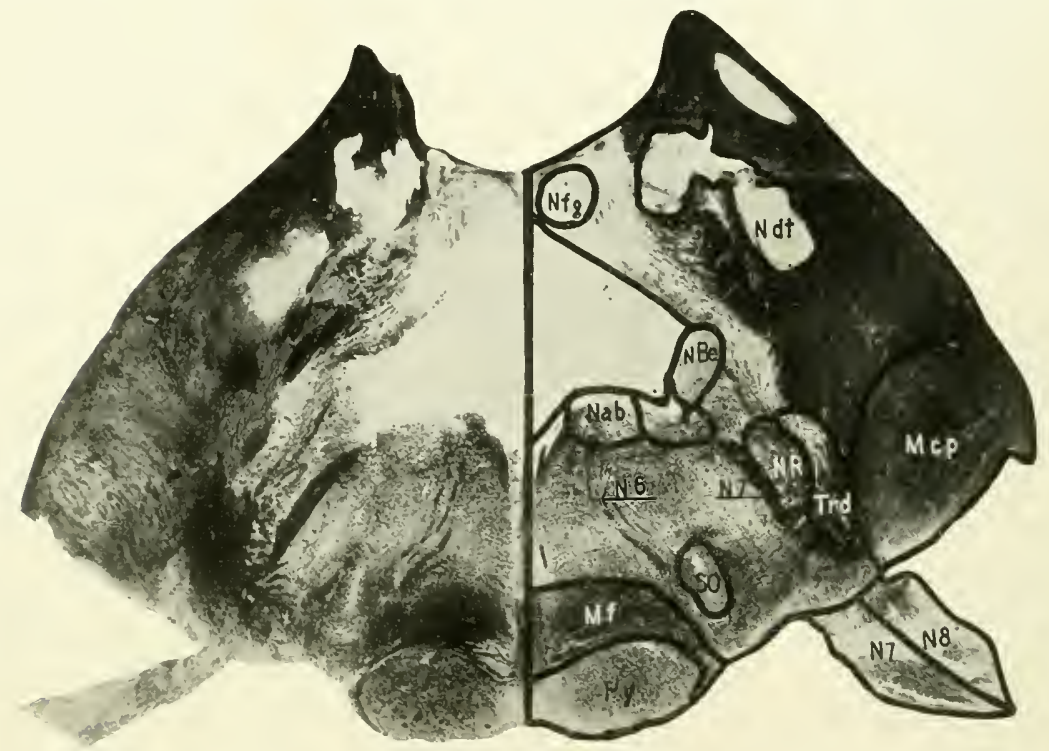

FIG. 20;. GIBBON. LEIEL OF THE EMERGENCE OF THE SIXTH CRANIAL NERVE. мсP, Middle Cerebellar Peduncle; uF, Mesial Fillet; NAB, Abducens Nucleus; NBE, Nucleus of Bechterew; Not, Gerebellar Nuclei, Lateral Group; NFg, Cercbellar Nuclei, Mesial Group; xk, Nucleus of Rolando; so. Abducens Nerve; N-, Facial Nerve; N8, Auditory Nerve; Py, Pyramid; so, Superior Olive; trd, Descending Trigeminal Tract. [Accession No. 1 1. Section 172. Actual Size $20 \times 16 \mathrm{~mm}$.]

some scattered fasciculi of the pyramid ( $P_{y}$ ), the pontile nuelear mass $(\mathrm{PN}$ ) and some decussating pontile fibers, and the stratum profundum pont is largely made up of transverse decussating fibers. At the lateral extremity of the section are the collected bundles constituting the middle cerebellar peduncle $(M \mathrm{cp})$. Entering the brain stem at this level near the mesial surface are some dorsal root axons of the fifth nerve $\left(N_{j}\right)$ on their way to the 
substantia gelatinosa. From this nucleus many fibers pass dorsally and mesially toward the angle of the fourth ventricle to form the tractus mesencephalicus trigemini.

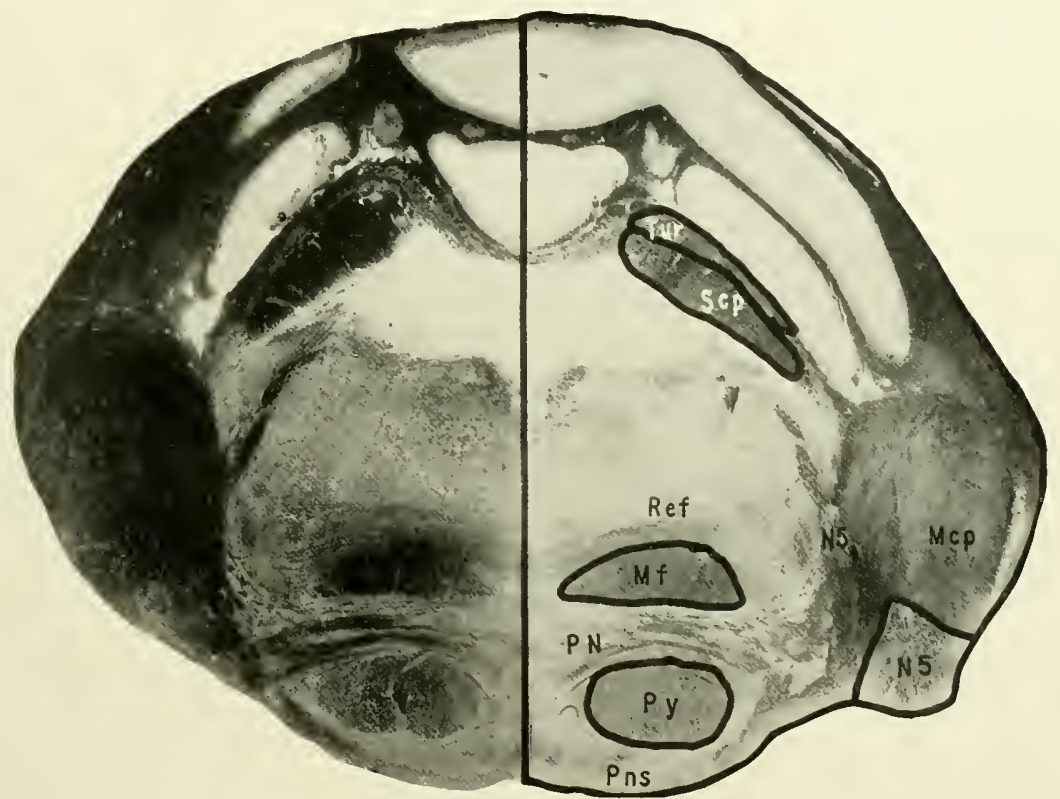

FIG. 206. GIBBON. LEVEL THROUGH THE MIDDLE OF THE PONS.

MCP, Middle Cerebellar Peduncle; MF, Mesial Fillet; N5, Trigeminal Nerve; PN, Pontile Nuclei; PNs, Pons; PY, Pyramid; ReF, Reticular Formation; SCP, Superior Cerebellar Peduncle; TUR, Tractus Uncinatus of Russel (Hook Bundle). [Accession No. 141. Section 186. Actual Size $21 \times 16 \mathrm{~mm}$.]

The central gray matter occupies a position immediately beneath the floor of the fourth ventricle and serves to form the lateral ventricular boundary. At the lateral extremity of the section is a dense mass of fibers, the superior cerebellar peduncle ( $\mathrm{Scp}$ ), an index concerning the degree of coordinative control which the animal possesses. Compared with the other intermediate primates, the gibbon shows no marked increase in this par- 
ticular. The boundary between the tegmentum and basis pont is is provided by the mesial fillet (Mf), while the reticular formation (Ref) show's no specialization of nuclear collections at this level.

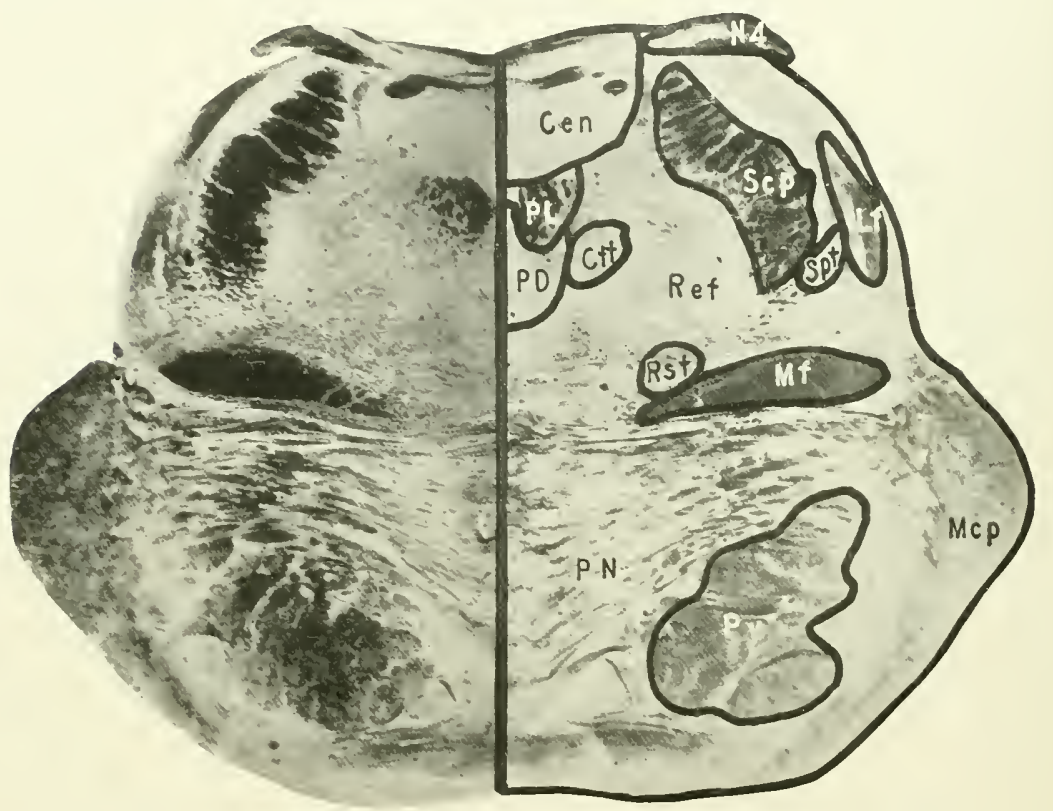

FIG. 20\% GIBBON. LEVEL OF THE EMERGENCE OF THE TROCILEAR NERVE.

CEN, Central Gray Matter; CTT, Central Tegmental Tract; LF, Lateral Fillet; MF, Mesial Fillet; MCP, Middle Cerebellar Peduncle; N屯, Trochlear Nerve; PD, Predorsal Bundlc; PL, Posterior Longitudinal Fasciculus; IP. Pontile Nuclei; Py; Pyramid; REF, Reticular Formation; RST, Rubrospinal Tract; sCP, Superior Cerebellar Peduncle; SPT, Spinothalamic Tract. [Accession No. 141. Section 250. Actual Size $16 \times 12 \mathrm{~mm}$.]

LEVEL OF THE EMERGENCE OF THE TROCHLEAR OR FOURTH CRANIAL NERVE (FIG. 207)

Here the appearance of the section has undergone considerable modification due to the fact that the axis is approaching its mesencephalic portion 
where the large space of the fourth ventricle is reduced as it approaches the aqueduct of Sylvius. The central gray matter ( Cen) surrounds the ventricle whose roof is formed by the superior medullary velum in which the fibers of the trochlear nerve $\left(\mathrm{N}_{t}\right)$ undergo complete decussation. These fibers subsequently emerge in relation with the dorsal aspect of the isthmus. In the central portion of the central gray matter ( $\mathrm{Cen}$ ) are the dense bundles constituting the fasciculus longitudinalis posterior (PL) and the fasciculus predorsalis (PD). Lateral to the central gray matter are the fibers constituting the superior cerebellar peduncle $(S c P$ ) preparatory to their inward deflection toward their decussation in the midbrain. Ventral to these fibers on the circumference of the axis is a mass of medullated axons forming the lateral fillet (Lf). At the boundary line between the tegmentum and basis are the transversely disposed bundles of the mesial fillet (Mf), while the general arrangement of the pons Varolii in its three characteristic layers appears ventral to this boundary. The pons here contains the stratum superficiale, the stratum complexum and the stratum profundum lying immediately ventral to the tegmental portion of the axis.

LEVEL OF THE INFERIOR COLLICULUS (FIG. 208)

Here the cross section shows further modification by the appearance of the two dorsal elevations constituting the primordial stations for the auditory pathway. These colliculi (IC) form a prominent elevation in the quadrigeminal plate of the midbrain and show a degree of stratification which harks back to their highest specialization in the lower vertebrates. Entering into the inferior colliculus are the collected bundles of the lateral fillet (Lf) which serves to convey impulses from the lower relay stations in the pathway of hearing. The central gray matter ( $\mathrm{Cen}$ ) completely surrounds the aqueduct of Sylvius. In its rentromesial portion is the caudal extremity of the 
nucleus trochlearis $(\mathrm{Ntr})$ from which the fourth cranial nerve arises $\left(\mathrm{N}_{t}\right)$. In the lateral border of the central gray matter are the scattered bundles forming the tractus mesencephalicus trigemini ( $T m t$ ), while in a ventral

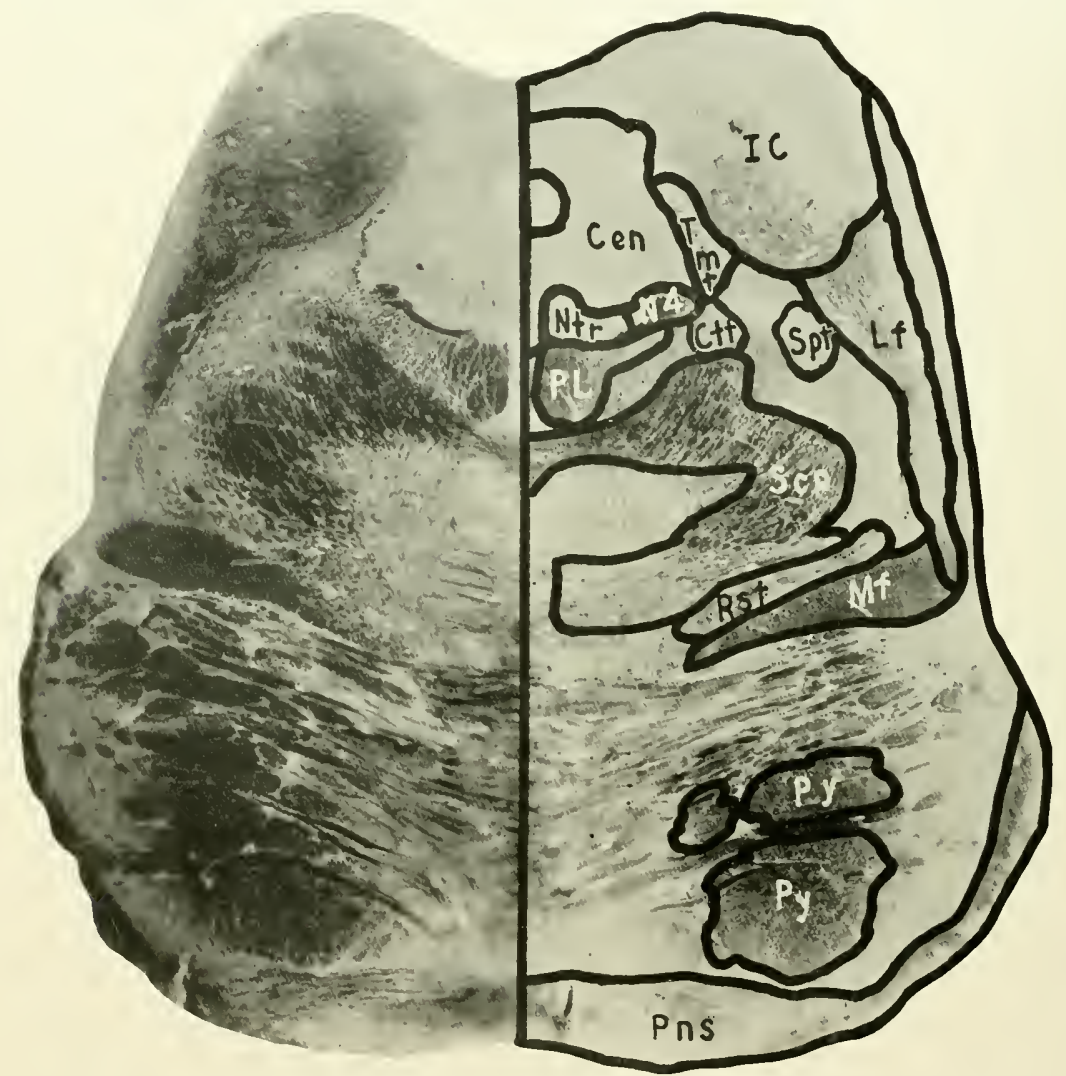

FIG. 208. GIBBON. LEVEL OF THE INFERIOR COLliCUluS.

CEN, Ccntral Gray Natter; CTT, Central Tegmental Tract; IC, Inferior Colliculus; LF, Lateral Fillet; MF, Mesial Fillet; NTr, Trapezoid Nucleus; N4, Trochlear Nerve; PL, Posterior Longitudinal Fasciculus; pxs, Pons; PY, Pyramid; RST, Rubrospinal Tract; SCP, Superior Cerebellar Peduncle; SPt, Spinothalamic Tract; TMT, Tractus Mesencephalici Trigemini. [Accession No. 141 . Section 278. Actual Size $16 \times 15 \mathrm{~mm}$.] 
position are fibers forming the posterior longitudinal fasciculus (PL) and the fasciculus preclorsalis.

The tegmentum at this level is separated from the basis by the fibers of the mesial fillet (Mf). The superior cerebellar pedunck ( $\mathrm{Scp}$ ) is about to make its decussation prior to entering the red nucleus.

LEVEL OF THE SLPERIOR COLliCULUS (FIGS, 209, 210)

At this level the configuration of the brain stem again shows some conspicuous alteration. This is chiefly due to the appearance of the two clevations in the dorsal region of the midbrain which form the superior coll liculi (SC). In a ventral position appears a divergence of the two cerebrapeduncles which forms the caudal boundary of the optico-peduncular space.

The superior colliculus in the gibbon, while somewhat less prominent than in the lower primates, even than in baboon or macacus, still retains a degree of its previous specialization. Traces of stratification may yet be detected in it. The central gray matter (Cen) surrounds the small ventricular space of the aqueduct of Sylvius and contains the nuclear specialization forming the nucleus oculomotorius ( $\mathrm{Noc}$ ). Near the median raphe many fibers from this nucleus cross to the opposite side, forming the oculomotor decussation. The nucleus ruber ( $\mathrm{NRu}$ ), whose large size and clear definition are in contrast with all other forms heretofore discussed, occupics its characteristic position in the tegmentum. Because of its much greater definition and its more apparent emergence from the surrounding reticular formation, it seems to show some further advance in the development of that system which has control over the coordinative regulation of movement. On the other hand, the functional duality of this nucleus as a relay station should be borne in mind. It is possible that its increments, both in definition and in size, are due to augmentation from the striatal structures of the endbrain rather than to an increase of fibers arising in the ccrebellum. In all probability, 
both factors have contributed some additional prominence to the red nucleus in gibbon. That its striorubral portion has undergone expansion might be expected in an animal presenting such complex arboreal locomotion. That

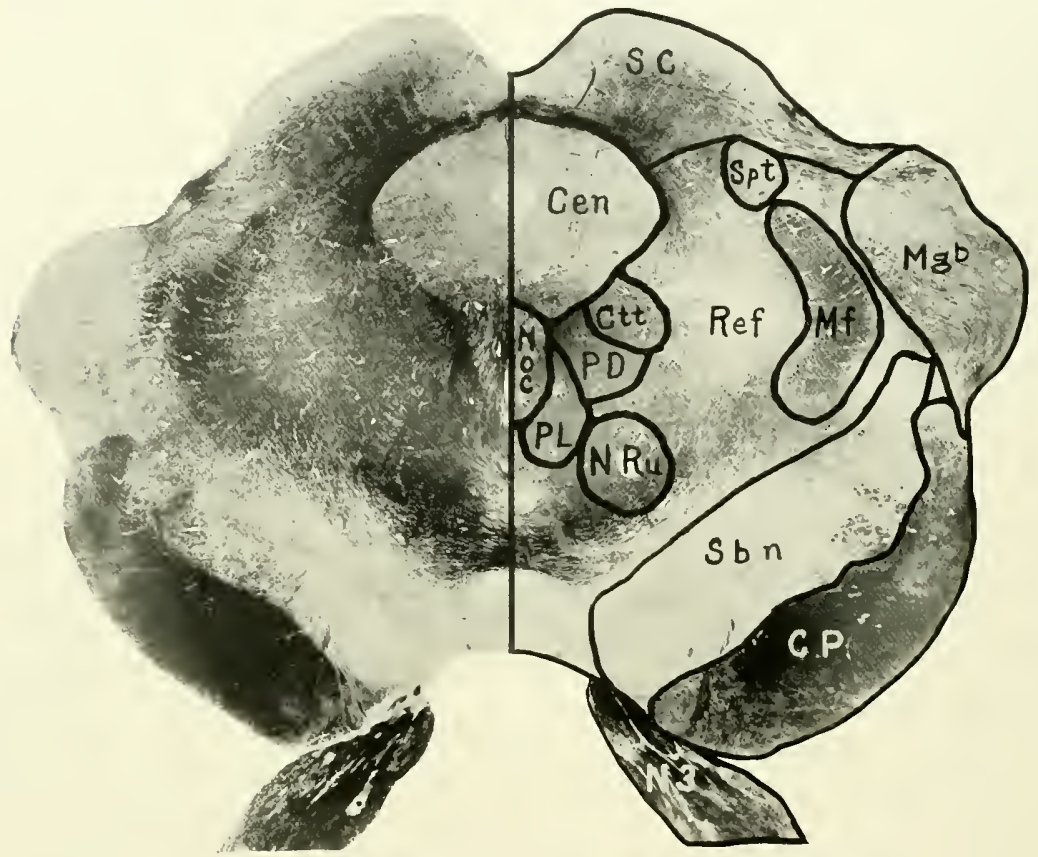

FIG. 209. GIBBON. LEVEL OF THE SLPERIOR COLLICULU'S.

CEN, Central Gray Matter; CP, Cerebral Pedurcle; ctr, Central Tegmental Tract; MF, Mesial Fillet; MGB, Mesial Geniculate Body; Noc, Nucleus Oculomotorius; NRC, Nucleus Ruber; N3, Oculomotor Nerve; PD, Predorsal Bundle; PL, Posterior Longitudinal Fasciculus; REF, Reticular Formation; sBn, Substantia Nigra; sc, Superior Colliculus; SPT, Spinothalamic Tract. [Accession No. 141 . Section 310 . Actual Size $30 \times 16 \mathrm{~mm}$.]

the cerebello-rubral portion of this nucleus has also undergone some expansion may be inferred from the increased size of the dentate nucleus. Surrounding the nucleus on all sides, and fying lateral to the central gray matter 
(Cen), is the reticular formation (Ref), in this species somewhat reduced in size and prominenee as compared with the Iower forms. This reduction is clue largely to the emergence from it of the large red nuclear mass. In the

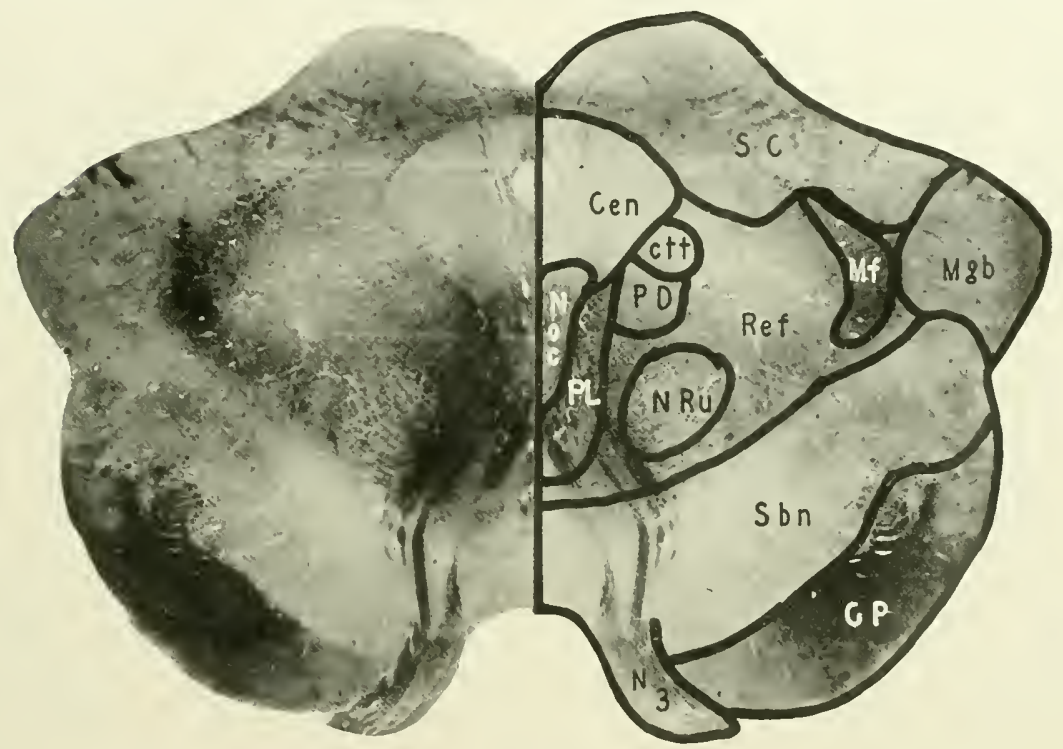

FIG. 210 . GIBBON. LEVEL OI THE SUPERIOR COLLICULUS.

CEN, Central Gray Matter; CP, Cerebral Peduncle; CTt, Central Tegmental Tract; MF, Mesial Fillet; MGB, Mesial Geniculate Body; Noc, Nucleus Oculomotorius; NRC, Nucleus Ruber; N3. Oculomotor Nerve; PD, Predorsal Bundle; PL, Posterior Longitudinal Fasciculus; REF, Reticular Formation; sBn, Substantia Nigra; sc, Superior Colliculus. [Accession No. 141. Section 350. Actual Size $30 \times 16 \mathrm{~mm}$.]

Iateral extremity of the section is seen a small protuberance, the mesial geniculate body $(\mathrm{Mgb})$. The gray matter of this nucleus seems to be confluent with another large nuclear aggregation situated ventromesial to it, the substantia nigra ( $\mathrm{Sbn}$ ). This mass of gray matter extends obliquely inward and forward from the region of the mesial geniculate body toward the optico-peduncular space and separates the basal portion of the mesen- 
cephaton from the tegmentum. The specific functions of the substantia nigra are vet surrounded by doubt, athough many writers attribute to it some regulating control of the automatic associated movements of the body.

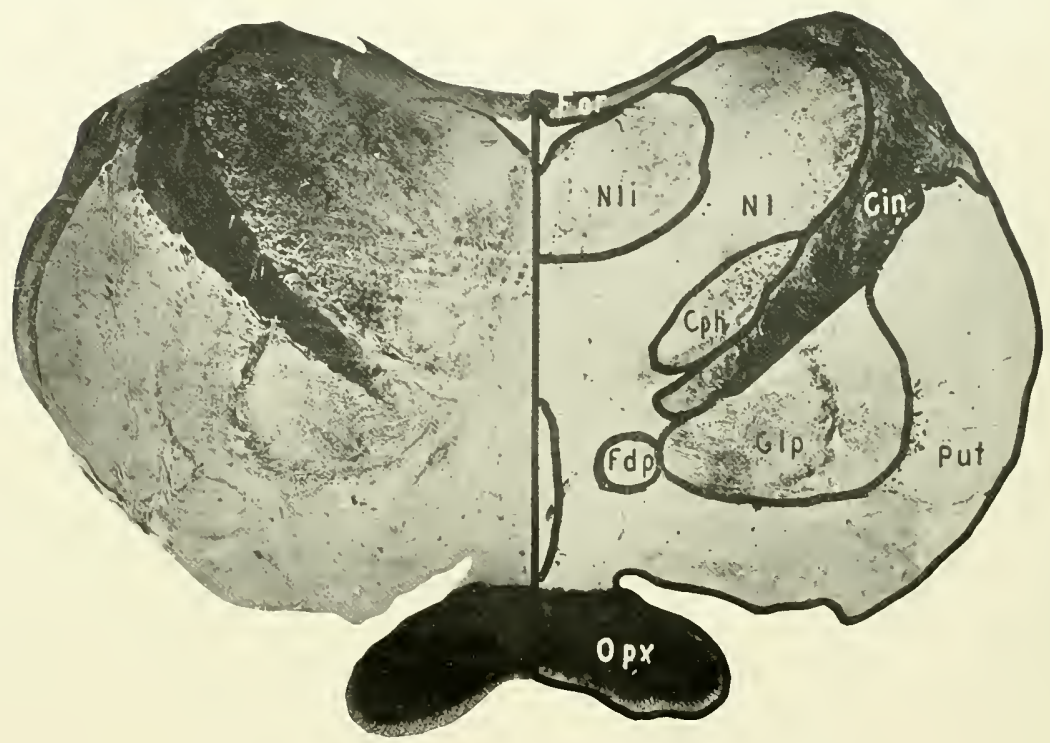

FIG. 2II. GIBBON. LEVEL OF THE OPTIC CHIASM.

CIN, Internal Capsule; CPH, Corpus Hypothalamicum; FDP, Descending Pillars of the Fornix; For, Fornix; GLP, Globus Pallidus; NL, Laterat Nucleus of the Thalamus; NLI, Internal Lateral Nucleus of the Thalamus; opx, Optic Chiasm; put, Putamen. [Accession No. 141. Section 440 . Actual Size $34 \times 19 \mathrm{~mm}$.]

This opinion is in part conjectural, although the structure as a whole has the appearance of a very important element in the brain stem.

\section{LEVEL OF THE OPTIC CHIASM (FIG. 2 I I)}

At this level the configuration of the section again shows those marked alterations due to the fact that the brain stem is about to reach its cephalic termination. Some of the massive lateral additions of the endbrain are already 
apparent. The most ventral structure in the cross section is the optic chiasm $\mathrm{O} p \mathrm{x}$ ) which takes the form observed in all primates, of a lateral clongation, together with the obtuse angulation in relation to the optic nerves and

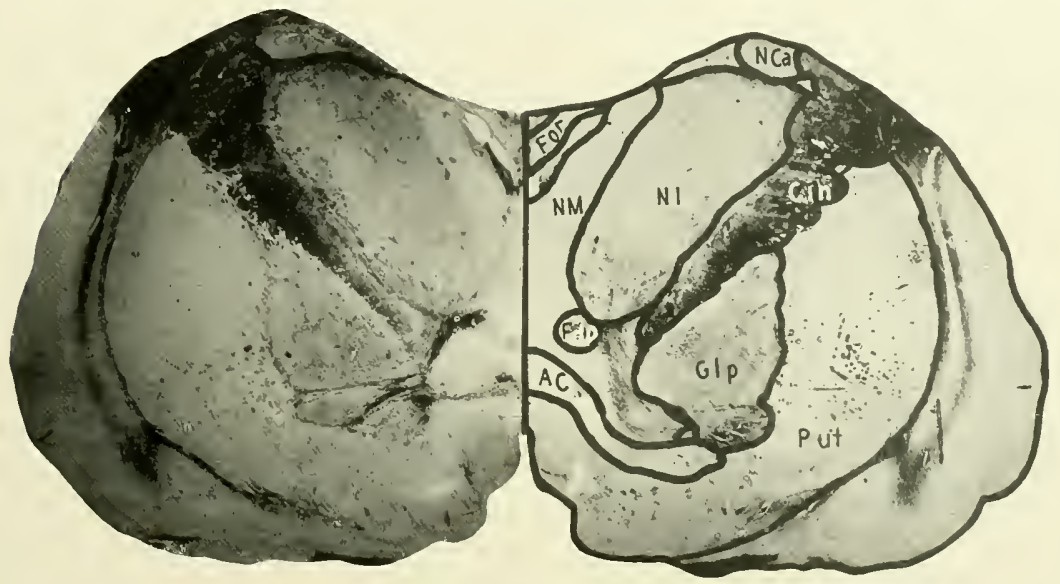

FIG. 2I2. GIBBON. LEVEL OF THE ANTERIOR COMMISSURE.

AC, Anterior Commissure; CIN, Internal Capsule; fDP, Descending Pillars of the Fornix; For, Fornix; GLP, Globus Pallidus; XCa, Nucleus Caudatus; N., Lateral Nucleus of the Thalamus; Nir, Nucleus Medialis Thalami; ptr, Putamen. [Accession No. 1 41 . Section 486 . Actual Size $34 \times 10 \mathrm{~mm}$.]

optic tracts. The supra-optic portion of the third ventricle appears immediately dorsal to the optic chiasm. Flanking either side of the ventricle are the dense masses of the optic thalami, which in turn are separated by the internal capsule ( $\mathrm{C}$ in) from the lenticular portion of the stratum. This nucleus presents its two characteristic portions, the mesial and darker area constituting the globus pallidus $\left(G I_{p}\right)$ and the outer and lighter portion, the putamen ( $\mathrm{Put}$ ). The evolutional significance of such complex elements as the thalamus and the lenticular nucleus could scarcely be approached within the limits of this work. 


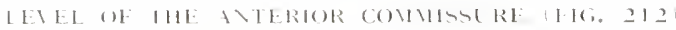

Here the brain stem, as consichered in this worh, comene to its aphalie termination. In the mid-sentral line is seen the marrew cledt of the third wotricke and bounding this an chevated portion of the thatamus, the tuberculum anticum, external to which is the cephalic portion of the hateral thatamic nucleos (N). This nucleus is separated by a dense mass of hearily myelinized fibers reposenting the anterion limb of the internal capsule Cin) from an expansine nuclear pertion of the corpus striatum Glp. Put). Passing ventrally inward tumard the midline, through this portion of the arpus striatum, are the estlected bundles forming the anterior commissure (AC). 


\section{Chapter NV}

\section{RECONSTRUCTION OF THE GRAY MATTER IN THE BRAIN STEMI OF HYLOBATES HOOLOCK}

HE reconstruction of the gray matter of the brain stem in Hylo-
bates hoolock begins at the caudal extremity of the inferior olivary
nucleus. The higher levels of the spinal cord are not represented in the model and the ventral gray columns have already merged with the reticular formation.

\section{The Dorsal Medullari Nuclei}

The nucleus of Goll, as first represented in the reconstruction, is already of considerable size. It is roughly quadrilateral in shape with a broad base which is confluent with the central gray matter. A large dorsal portion shows some tendency towards that lateral swing which characterizes these nuclei in the various brain stems atready studied.

The nucleus of Burdach arises slightly more cephalad than the nucleus of Goll, in fact, at about the same level as that in which the inferior olivary nucleus appears. Its origin is represented by a thickening at the point of junction between the nucleus of Goll and the central gray matter. The nucleus rapidly increases in size, expanding Iaterally and, at its periphery, somewhat ventrally, thus showing the same tendency towards the lateral swing evidenced by all of these dorsal nuclear structures. It soon reaches its maximum size and continues upward with little change. At the level of the lower third of the fourth ventricle it begins to diminish and rapidly comes to an end.

Directly ventral to the nucleus of Burdach is the substantia gelatinosa trigemini. This nucleus, as in other primates, has already reached a position widely removed from the midline. It is oval in outline with its long axis 
directed somewhat obliquely from before backward and inward. It rests in a space hollowed out of the reticular formation which surrounds it upon its dorsal, rentral and mesial surfaces. Dorsomesially the substantia gelatinosa trigemini is in relation first with the nucleus of Goll and, more cephalically, with the nucleus of Burdach.

In the gibbon, the constriction in the substantia gelatinosa trigemini described in connection with the other primate brain stems is well defined, occurring at about the level of the junction of the upper and middle thirds of the inferior olivary nucleus. Above this point the substantia gelatinosa again enlarges and passes upward in the lateral portion of the tegmentum to the upper metencephalic levels. At this point it expands to form its caput and is associated with the motor nucleus of the trigeminal nerve on its mesial aspect.

\section{The Inferior Olivari Nucleus}

In Hylobates hoolock the inferior olivary nucleus has become a relativety massive and prominent structure. It presents a great number of secondary plications in its surface. The fundus of the inferior olive is deep and wide, with a number of secondary loops. The accessory olivary nuclei are well developed. The ventral accessory nucleus begins below as a flat, elongated band applied to the inner half of the ventral branch of the main olivary nucleus. It extends upward in this position as a compressed lamina of gray matter lying between the inferior olivary nucleus and the pyramidal tract. As the ventral accessory nucleus is traced upward it gradually diminishes in size until it comes to an end apparently by fusing with the mesial extremity of the ventral branch of the principal nucteus.

The dorsal accessory olive begins at about the middle of the main olivary nucleus and appears as a narrow layer of gray matter applied to the inner portion of the dorsal branch of the olivary nucleus. It rapidly reaches its maximum transverse diameter and then, gradually diminishing, ends below 
the uppermost level of the main nucleus by fusing with the dorsal branch at its mesial extremity. The arrangement of the nucleus is similar to that found in other primates. Its deep surface lies embedded in the reticular for-
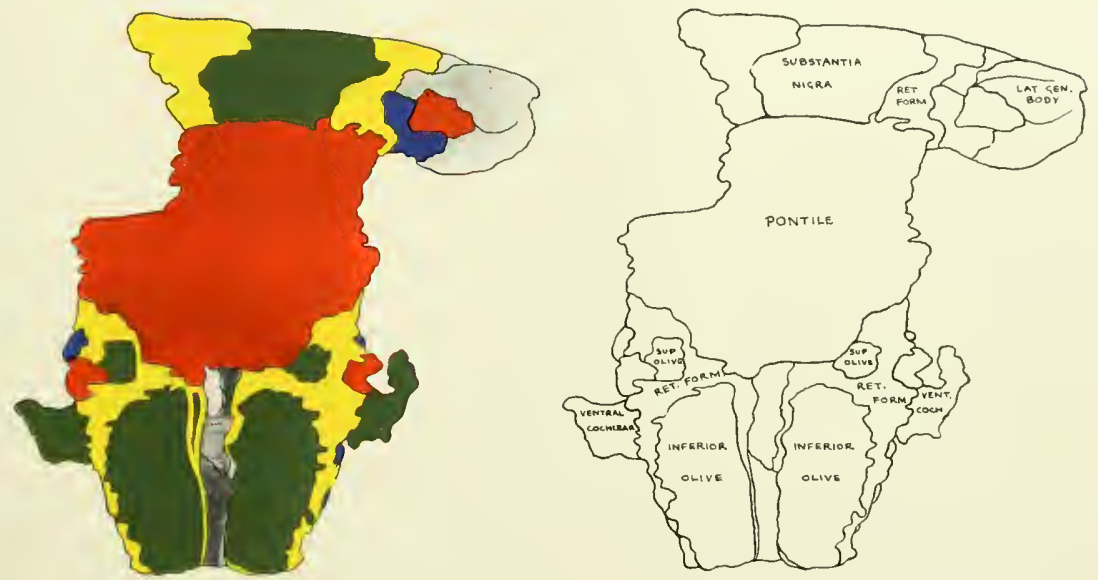

FIG. 213. VENTRAL SURFACE OF GRAY MATTER OF BRAIN STEM, HYLOBATES HOOLOCK.

Key to Diagram, iat. gen. body, Lateral Geniculate Body; pontule, Pontile Nuclei; ret. Form., Reticular Formation; sup. olive, Superior Olive; vent. Coch. and ventral cochlear, Ventral Cuchlear Nucleus.

mation and the dorsal extremities of its two laminae are turned dorsally at their mesial extremities. The hilus opens toward the contralateral inferior cerebellar peduncle.

\section{The Reticular Formation}

The ventral surface of the reticular formation in its oblongatal and lower pontile portions is, to a great extent, in contact with the inferior olivary nucleus. Lateral to the fundus of the olive the reticular formation approaches the surface of the stem and is uncovered except by peripheral fiber bundles. Dorsal to this uncovered area is the substantia gelatinosa 
trigemini which is situated in the dorsolateral angle of the reticular formation. Dorsal to the substantia gelatinosa this formation is covered by the fibers of the columns of Goll and Burdach and gives origin to the basal portions of the dorsal sensory nuclei. Mesially the reticular formation is continuous with the central gray matter. Its mesial surface is separated from its fellow of the opposite side by the longitudinal bundles situated adjacent to the raphe.

The pontile portion of the reticular formation on its ventral surface presents a deep excavation produced by the trapezoid body. Lateral to this excavation are located two nuclear masses of moderate size, forming the superior olive and the lateral reticular nucleus. As the superior cerebellar peduncle sinks deeper into the reticular formation, a lateral reticular prolongation envelops the peduncular bundle over its lateral surface.

In the mesencephalic portion of the neuraxis the reticular formation comes to the surface laterally, dorsal to the pontile nucleus and ventral to the colliculi. Somewhat ventral to the point at which the superior peduncle extends into the tegmentum is the pathway of the lateral fillet.

The reticular formation, as it is followed further upward in the stem, becomes more disseminated by the superior cerebellar peduncle. It is finally separated into a mesial and a lateral portion by the appearance within it of the red nucleus. At the level of this nucleus and above this level the reticular formation becomes irregular and forms a matrix in which the mesial geniculate body develops. In the diencephalon the reticular formation seems to merge with the zona incerta and other less well-defined nuclear masses in the caudal portions of the diencephaton.

\section{The Pontile Nuclei}

The pontile nuclei begin in the more cephalic levels of the medulla oblongata by the appearance of the arciform nuclei which partially surround the pyramidal tract on its ventral, mesial and dorsal aspects. This arciform 
development becomes more extensive until it eompletely invests the pyramidal tract, thus forming the superficial and deep layers of the pontile nucleus. The superficial layer is convex in contour, corresponding to the
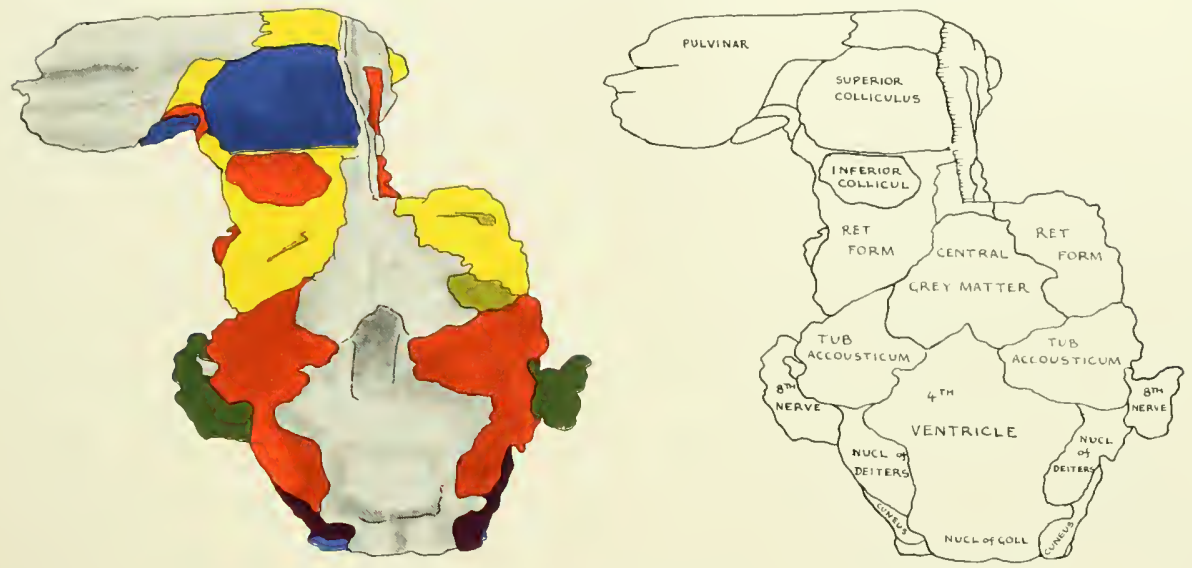

FIG. 2I . DORSAL SURFACE OF GRAY MATTER OF BRAIN STEM, HYLOBATES HOOLOCK.

Key to Diagram. iNferior coliticul, Inferior Colliculus; NeCl. OF Deiters, Nuclcus of Deiters; Nucl. OF GoLL, Nucleus of Goll; RET. ForM., Reticular Formation; тL B. Accousticum, Tuberculum Acusticum.

surface configuration of the pons itself, whereas the deep layer is more or less straight or somewhat concave. Dorsally the mesial buttress comes into approximate contact with the ventral surface of the reticular formation. The same arrangement is found in the lateral buttress which, although irregular in outline, comes into intimate relation with the ventrolateral angle of the reticular formation. Between these two points of fusion, however, the ventral surface of the reticular formation and the deep layer of the pontile nuclei are hollowed out by the trapezoid body.

The scattered collections of gray matter which pass across the tunnel formed within the pontile nuclei are considerably increased. Continuing 
upward, the superficial layer of the pontile nucleus remains in contact laterally with the ventral surface of the reticular formation of the mesencephalon. As the pontile nucleus approaches the isthmus mesencephali it rapidly decreases in size, leaving onty the deep nuclear layer which continues upward to merge directly with the substantia nigra.

\section{The Vestibular Complex}

The vestibular complex first comes to view on the surface of the reconstruction at the fower level of the fourth ventricle where it appears as a small, triangular, wedge-shaped mass, the nucleus of Deiters. It is intercalated between the lateral surface of the gray matter of the floor of the fourth ventricle and the eephalic portion of the nucleus of Burdach. The nucleus is continued forward, presenting a prolongation which overhangs the substantia gelatinosa trigemini. It reaches its maximum at about the midventricular level. At this point its dorsal surface is covered by the beginning development of the triangular nuckeus complex which gradually increases in size as the Deitersal nucleus diminishes. It passes upward in this position between the gray matter of the floor of the fourth ventricle and the reticular formation but diminishes in size as the upper pontile levels are approached. The nucleus of von Bechterew is continued from the upper portion of the triangular nucleus into the lateral wall of the fourth ventricle.

\section{The Cochlear Conplex}

The cochlear complex is relatively large in contrast to that of Cynocephalus babuin, which is relatively small. The ventral nucleus of hylobates is well developed, having the same trough-like formation present in the other members of the primate group. The roots of the nerves passing in to the nucleus are partially surrounded by the gray matter, leaving the mesial surface free. 
The dorsal cochlear nucleus is situated over the triangular nueleus of the vestibular complex and is connected with the ventral cochlear nucleus by numerous strands of gray matter. The size of the cochlear nucleus is
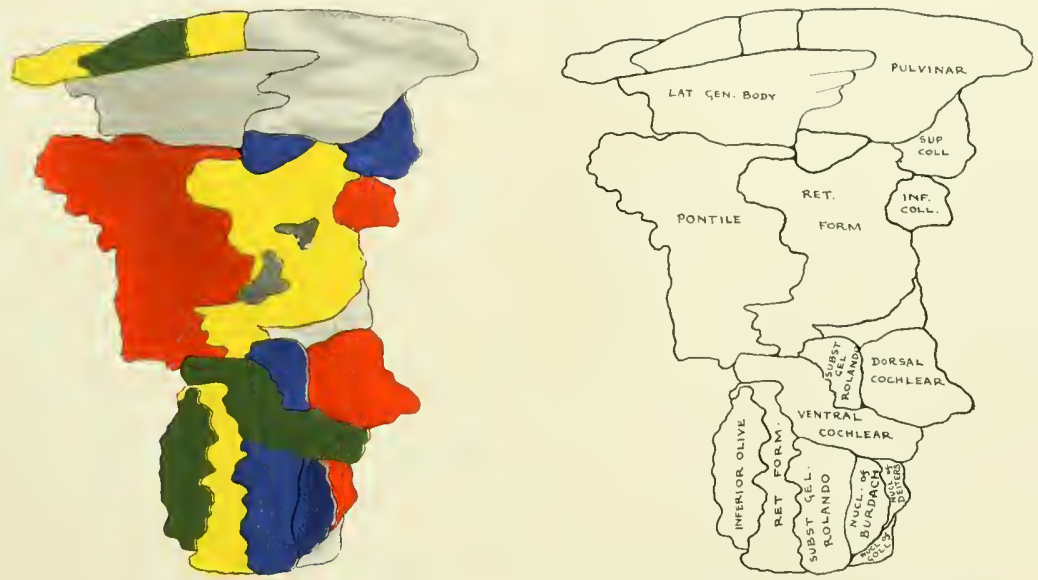

FIG. 2I \%. LATERAL SURFACE OF GRAY MATTER OF BRAIN STEM, HYLOBATES HOOLOCK.

Key to Diagram. dorsal cochlear, Dorsal Cochlear Nucleus; iNf. coll., Inferior Colliculus; Lat. gen. Bodr, Lateral Geniculate Body; NUCL. OF BURDACH, Nucleus of Burdach; NUCL. OF DEITERS, Nucleus of Deiters; nucl. of Golt, Nucleus of Goll; pontile, Pontile Nuclei; Ret. Form., Rcticular Formation; subst. Gel. Rolando, Substantia Gelatinosa of Rolando; sup. Coll., Superior Colliculus; ventral cochlear, Ventral Cochlear Nucleus.

commensurate with the relatively large size of the trapezoid body which produces a marked excavation in the ventral surface of the metencephalic reticular formation, separating this structure from the deep layer of the pontile nucleus.

\section{The Substantia Nigra}

The substantia nigra is developed as a massive nuclear collection by a specialization from the deep layer of the pontile nucleus. This deep Iayer 


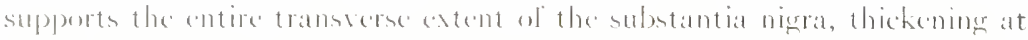
losth the mosial and lateral estremities, where the deep pontile laser luses

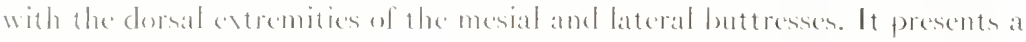

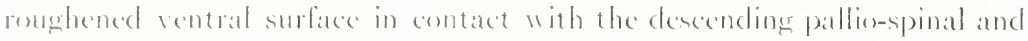
pallion-pontile tratets. Mlesially it is continumes with the indillerent inderpeluncular grat matter, while haterally it presconts a houted extremity which

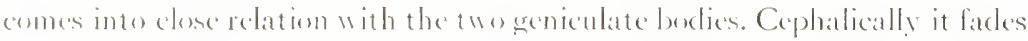

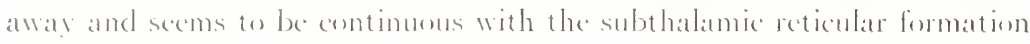
and the zonat incerta. The specialization in its lateral pertion, firm uhich merve dibers pass inter the terementum, is well developed.

\section{This: Conlacila}

These structures appear as specializations in the dorsal extension of the reticular formation in the mencencephatem. The inferion colliculus, of moderate size, rests upon the lateral extemsion of the retientar formation

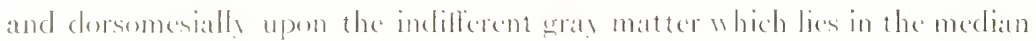
line. It is separated frem the superior enlliculus by the interenllienlar suleus

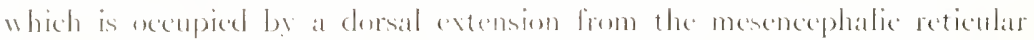
formation. The superior colficulus is much more extensive in size than the inferom colliculus and is smilarls situated. Its lateral extremity rests upon the lateral estemsion of the mesenecphalic reticular formation, while itsdorsal

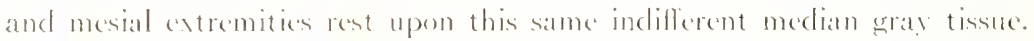

The ventral surlaces of both colliculi rest upon extensions of the mesencephalic reticular formation which prevents them from eoming into actual comtact with the central gray matter.

\section{THI: NlCLElS RIBLK}

The mudeus mber is only moderately developed in giblon, appearing in the cephalie portion of the mesenecphahon as a condensation in the mesial 
part of the reticular formation. It is fairly well differentiated from the surrounding reticular matrix by the fiber capsule derived from the superior cerebellar peduncle. It is spherical in shape, its cephalic extremity extending up to, if not into, the diencephalon.

\section{The Central Gray Matter}

The central gray matter in gibbon in the lowest levels of the reconstruction appears as a more or less rectangular mass of gray matter receiving at its ventrolateral angles the reticular formation, dorsally the nucleus of Goll and at its dorsolateral angles, the substantia gelatinosa Rolandi. Passing upward the central gray matter presents a flat condensation which represents the beginning of the nucleus of Burdach.

As the central gray matter is followed upward in the stem it passes dorsad and at the same time expands laterally, until it is disposed as a narrow, flat band Iying upon the dorsal surface of the reticular formation.

The fourth ventricle presents very little modelling in any part. Above the mid-point there is the suggestion of the nucleus abducentis, but this is not so well defined as it is in some of the other forms.

As the gray matter is flattening out from side to side, a long ventral prolongation stretches forward in the vicinity of the raphe. This prolongation of the central gray matter extends almost to the base of the pyramid. Above, it passes into the reticular formation which gradually approaches its fellow of the opposite side. In the upper portion of the pontile region the floor of the fourth ventricle begins to narrow towards the aqueduct of Sylvius. The lateral walls contract and finally the cavity of the ventricle is reduced to the small dimensions of the iter. At the same time, the gray matter surrounding the aqueduct increases in thickness and gradually becomes changed in diameter. In the ventral prolongation of the mesencephalic central gray matter arise the condensations of nuclear material which give rise to the 
fourth and third cranial nerve nuclei. The griseal investment of the aqueduct of Sylvius is relatively heavy. As the diencephalon is approached the ventrodorsal position of the central gray matter becomes more marked. Ventrally it is continuous with the indifferent interpeduncular gray matter which merges with that of the hypencephalic region. The lateral walls of the aqueduct of Sylvius are continuous with the subependymal gray matter lining the third ventricle. Dorsally the central gray matter fuses with the habenular region. 
CiAPTER IVI

\section{COMPARATIVE SUMMARY OF STRUCTURES HAVING EVOLUTIONAL SIGNIFICANCE IN THE BRAIN STENIS OF THE INTERMIEDIATE PRINIATES}

A Critical Comparison of the Pyramidal Tract, the Olinar Nucleus, the Dorsal, Vestibular, Cerebellar and Pontile Nuclei, the Midbrain Colliculi and Oculomotor Decussation. Their Erolutional Significance in Relation to the Bebavior of the Intermediate Primates. Comparison with Lower Primates

I N comparing the special structures of the brain stem selected because of their evolutional significance, the effort will first be made to contrast and then to note their comparative relation to the members of the lower primate group.

I. The Prramidal Sistem in Its Relation to Volitional Control, Especially of the Extremities

The pyramidal system, one of the most reliable indices concerning the degree of volitional control possessed by the animal, has attained such prominence that the motor cortex, and hence the motor capacity of these animals, may be assumed to represent a relatively high stage of development. Of these intermediate primates, the gibbon rather surprisingly reveals a planimetric coefficient considerably less than either the macacus or the baboon. This unexpected disclosure by means of measurement is partially suggested by casual inspection of the sections. It seems most likely that this disparity in pyramidal development arises from the fact that in gibbon, although most authorities place this animal in the group of the great anthropoids, the specialization of both fore- and hindlimbs is inferior to that of either macacus 
or baboon. As already noted, the hind legs are short and afford but imperfeet means of locomotion upon the ground. Its fore extremities, on the other hand, are most highly specialized for arboreal locomotion, the forearm being long, out of all proportion to the requirements essential for other skilled performances. The hand likewise has developed few of the more generalized capacities seen in other forms, but is particularly specialized as a swinging hook for reaching and grasping the branches as the animal makes its flight-like passage. In this light the behavioral development of gibbon accords well with the relatively low planimetric coefficient of its pyramidal development.

Both the macacus and the baboon are about equal in the planimetric coefficients of the pyramidal system, a fact which points to a behavioral development nearly on a par in these two species. Although macacus is essentially a tree-dwelling form, and the baboon mainly ground-living in habit, in both the development of hand and foot is essentially equal. Whatever slight advantage exists in the pyramidal comparison of the baboon and macacus favors the latter, a relation which is in harmony with the behavioral differences in these two forms. The macaque is recognized to be more adaptable and is measurably more teachable than the baboon. The motor specialization of the former would thus be expected to be somewhat greater than that of the latter.

The planimetric coefficients of the pyramidal system of the intermediate primates are given in the appended tabulation:

Planimetric Coefficients of the Primamidal Sistem

\begin{tabular}{|l|c|c|}
\hline & Species & Coefficient \\
\hline Gibbon & & .138 \\
\hline Macacus & .146 \\
\hline Baboon & .143 \\
\hline
\end{tabular}


Compared group for group, the intermediate primates show a greater planimetric coeflicient in the pyramidal system than the lower primates. In one instance alone does this lower group approach the intermediate one in this respect. Mycetes is about on a footing with gibbon. In general, the intermediate primates show a striking advance in the organization of the pyramidal system, although this increase in pyramidal volume is not surprising when the behavioral expansions of the group are brought into contrast with those of such forms as the lemur the tarsius and the marmoset. The closer approximation of mycetes to the figures of the intermediate species emphasizes again the high degree of specialization in these remarkable new-world monkeys. Because of their manual differentiation, as well as the functional capacity of their prehensile tails, they have developed a range of behavioral performances comparing favorably with their presumably more capable congeners of the old world.

\section{The Olivary Nucleus in Its Relation to the Regulation of Skilled Learned Performances}

The olivary nucleus, by comparison among the intermediate primates, clearly indicates that the gibbon has gained preeminence in this specialization of the brain. In definition of outline, in size, in degree of convolution, the olivary nucleus of the gibbon has every advantage over that structure in the macacus or baboon. From the functional standpoint, the conclusion may be drawn that in the simultaneous movements of the eye, head and hand, the gibbon has made a notable advance over its intermediate associates. This conclusion is in some respects difficult to reconcile with the known facts of the animal's behavior. It does not accord with the conception that the forelimb, particularly the hand, of the gibbon is in many particulars inferior to that of the other intermediate primates described. In no sense can it be said that the skilled manual performances of the gibbon are comparable with 
those of the macacus or the baboon, either in complexity of organization or degree of dexterity. This marked specialization in the regulation of simultancous movements in eye, hand and head seems to be supertluous in so far as the gibbon's behavioral attainments are concerned. There is that, however, in the skilled motor performances of this animal which may call for such spontancous coordination of oculo-cephalo-gyric movements as the gibbon manifests. The prodigious swings which it makes from branch to braneh in its flight-like locomotion demand, above everything else, the most accurate timing in the eoordination between the eye and hand.

The planmetric coefficients of macacus and baboon are practically ecpual; whatever little difference there may be favors the macaeus. The coefficients of the inferior olivary nueleus in the intermediate primates are given in the appended table:

Coefficients of the Inferior Olive in the Intermediate Prinates

\begin{tabular}{|c|c|c|c|}
\hline & Species & Planimetric & Longitudinal \\
\hline Gibbon & & I5j & .240 \\
\hline Macacus & & . 28 & .260 \\
\hline Baboon & & .125 & .220 \\
\hline
\end{tabular}

Contrasted with the lower primates the inferior olive shows a gain in prominence in the intermediate forms. Myeetes, however, as might be expeeted, approaches fairly close to the intermediate primates in this regard. It is strikingly inferior to the gibbon whose high degree of olivary expansion furnishes an element upon the strength of which it may lay claim to some degree of lineal propinquity to the three great anthropoids.

iil. The Dorsal Nuclei in Their Relation to Discriminative Sensibility in the Extremities and Tail

The nucleus of Goll, representative of the discriminative sensory influx from the tail and lower limbs, furnishes convincing contrast in the inter- 
mediate primates. In the first place, the median unpaired nucleus of Bischoff has entircly disappeared and no structure resembling this aggregation of cells in the dorsal column is found in any of the three species here compared. Inasmuch as this specialization of the dorsal sensory nucleus appears only in relation with those animals possessed of a prehensile tail or a tail used for propelling purposes, it has its importanee in this fact. The nucleus of Goll, according to its measurements, is larger in both the baboon and macacus than it is in the gibbon. This disparity is considerable and striking, for the nucleus in gibbon is about half as large as in the other two forms. The importance of this inequality is notable because the gibbon belongs to those apes which possess no tails; hence all of the sensory specialization pertaining to that organ, in the capacity either of a prehensile, a steering or a balancing structure, has disappeared in the Hylobate species. The volume of afferent impulses arising from the tail in the other monkeys has also decreased in the absence of prehensile function and by just so much the nucleus of Goll has become reduced in size. In addition, it must be borne in mind that the fower extremities in the gibbon are poorly developed; that is to say, the legs are not differentiated for locomotion on the ground. The main burden of locomotion falls upon the upper extremities inasmuch as the animal's extreme development of brachiation is the one outstanding physical feature of its motor organization, from which fact the family has received the name of "treewakkers." But their walking is conducted by means of the arms and hands rather than the legs and feet. Thus the gibbon's inferiority in the development of the column of Goll may be attributed to the dual effect of absence of a tail and certain functional depreciations in the lower extremities. The baboon and macacus, on the other hand, have a fair representation of nuclear substance in the nucleus of Goll, indicative of hind extremities equipped with adcquate proprioceptive organs for the receipt of discriminative impulses. The afferent influx from the tail makes a not inconsiderable addition, 
although in neither of these species does the tail differentiate as a prehensile organ. In this light, the relative dimensions of the nucleus of Goll in the three intermediate primates is significant in two of them, macacus and baboon, because the influx of discriminative impulses from the legs and tail is considerable. In the gibbon, which possesses no tail, sensory st imuli are correspondingly reduced. The coefficients of the nucleus of Goll in macacus, baboon and gibbon are shown in the appended tabulation:

Coefficients of The Nucleus of Goll in the Intermediate Prinates

\begin{tabular}{|lccc|}
\hline & Species & Planimetric & Longitudinal \\
\hline Gibbon & & 034 & .150 \\
Macacus & $0-6$ & .210 \\
Baboon & .086 & .210 \\
\hline
\end{tabular}

When the nucleus of Goll in the intermediate primates is compared with the corresponding structure in the lower primates, equally striking contrasts become apparent. In mycetes, for example, this nucleus is the most conspicuous. The preeminence of the nucleus of Goll in the howhing monkey is unquestionably related to the possession of a prehensile tail. Here again the specialization in structure brings about new spheres of activity in the realm of behavior. The nucleus of Goll in the gibbon has the smallest dimensions of all the primates thus far considered. Its size is even less than that of the lemur or marmoset. The specialization of the hindlimb, therefore, and the development of the tail, as these factors bear upon the influx of stimuli concerned in discriminative sensibility and thus become active in the organization of the animal's behavior, are clearly reflected in the primary receiving station for these impulses in the brain stem, the nucleus of Goll.

In the case of the nucleus of Burdach, however, the conditions are somewhat different. This nucleus in macacus is considerably larger than in either baboon or gibbon. In all probability the difference is to be attributed to the 
greater degree of differentiation in the upper extremity and hand. This interpretation gains in probability because in the baboon the fore extremity, particularly the hand, is influenced in its differentiation by adaptation to locomotion upon the ground, while in the gibbon the hand and upper extremity are peculiarly adapted to arboreal focomotion. In macacus, however, there is some degree of freedom of the hand which approaches more nearty the humanoid standard of a forelimb. Not in size alone, but quite as much in the spccialization of the accessory nucleus of Blumenau, the nucleus of Burdach shows a greater degree of differentiation in macacus than in gibbon or baboon. The planimetric coefficients of the nucleus of Burdach (including the accessory nucleus of Blumenau) in baboon, macacus and gibbon are shown in the appended tabulations:

Coefficients of the Nucleus of Burdach in the Intermediate Primates

\begin{tabular}{|c|c|c|}
\hline Species & Flanimetric & Longitudinal \\
\hline Gibbon & .068 & 150 \\
\hline Macacus & .086 & 290 \\
\hline Baboon & .065 & 290 \\
\hline
\end{tabular}

The longitudinal coefficients of the two dorsal groups of sensory nuclei are also given. From the latter tabulation, it is of interest to note the general consistency in length of both the nucleus of Goll and of Burdach. The only notable departure from this equality is the length of the nucleus of Goll in gibbon which is practically half that of the other forms. This fact accords with the observations already made on the basis of the planimetric coefficients of this nucleus in gibbon.

As compared with the nucleus of Burdach in the lower primates, the planimetric figures show that in mycetes this nucleus is larger than in all other forms either of the lower or the intermediate groups. This predominance of mycetes in both of its dorsal nuclear specializations must be 
considered as definitely influenced by the possession of a prehensile tail. The substantiation of this view in regard to the nucleus of Goll presents far fewer difficulties than is the case with the nueleus of Burdach, yet the wider range of motor performance made possible by the prehensile tail may be urged as the basis of greater manual freedom and hence an inerease in the volume of afferent influx from the upper extremity.

\section{iv. The Vestibular Nuclei and Their Relation to the Balancing Mechanism}

The functional eapacity of the balancing mechanism in the intermediate primates, particularly as indicated by the nucleus of Deiters, appears most prominent in the gibbon. This observation is borne out by the facts recorded in connection with the surface markings of the oblongata in which the large protuberances on the floor of the fourth ventricle occasioned by the vestibular area were noted (p. +20). This preeminence of the gibbon in the index of its balaneing mechanism is undoubtedly due to the adjustments necessary in its arboreal locomotion. The differences in these three species with reference to the triangular nucleus of Schwalbe are not great. It is probable that all three of these animals have nearly the same balancing problems to meet in their locomotion and station, which thus places their balancing mechanism about on a par. The coeflicients of the vestibular areas in the macacus, baboon and gibbon are given in the appended tabulation:

Coefficients of Deiters' Area in the Intermediate Primates

\begin{tabular}{|c|c|c|}
\hline Species & Planimetric & Longitudinal \\
\hline Gibbon & $.08_{5}$ & .140 \\
\hline Macacus & .075 & .110 \\
\hline Baboon & .060 & .110 \\
\hline
\end{tabular}


Coefficients of the Entire Vestibular Area in the Intermediate Prinates

\begin{tabular}{|c|c|c|c|}
\hline & Species & Planimetric & LongitudinaI \\
\hline Gibbon & & 1.77 & 140 \\
\hline Macacus & & .162 & .110 \\
\hline Baboon & & .155 & 110 \\
\hline
\end{tabular}

Even more interesting is the comparison of the intermediate primates with those of the lower group. This contrast shows how nearly equal the balancing problems in all of these primates are. In this respect, however, mycetes and tarsius exceed all of the other species. Here again, as in the case of the sensory nuclei of the dorsal column, it is undoubtedly the additional motor eapacity and locomotor specialization that bring into play new ranges of motion connected with balancing. These additions demand a more effective mechanism for this function. But among the intermediate primates the gibbon, because of its peculiar arboreal locomotion, has a slightly more differentiated mechanism than its congeners in this group.

\section{v. The Cerebellar Nuclei in Their Relation to the Coordination of Movements, Especially the More Complex Movements of The UpPer Extrenities}

Employing the dentate nucleus of the cerebellum as an important representative of coordinative function in the brain, it appears from a comparison of this structure in the intermediate primates that the baboon shows a somewhat greater specialization than either of the other forms. This comparison, which slightly favors the baboon, is somewhat perplexing at first glance. Taking into consideration the fact that this animal is specialized for rapid and almost perfect quadrupedal locomotion upon the ground, and at the same time has almost equal capacity for climbing in rocky places and even in the trees, it becomes evident that its musculature is in need of a high degree 
of coordinative control. Although it must be admitted that the manual diflerentiation in baboon, while in some respects superior to that of gibbon, is in no way better than that of macacus, nevertheless, the dual responsibility forced upon the forelimbs for locomotion upon the ground and in climbing entails a higher degree of specialization of corrdinative control than in either of the other two forms considered. Such an interpretation is none too strongly urged at this time. Other factors may be operative which as yet have not come to light. This explanation of the larger size of the dentate nucleus in the baboon than in the other intermediate primates, however, seems for the moment worthy of consideration. The coefficients of the dentate nucleus in the baboon, macaque and gibbon are given in the appended tabulation:

Coefficients of the Dentate Nucleus in the Intermediate Prinates;

\begin{tabular}{|c|c|c|}
\hline Species & Planimetric & Longitudinal \\
\hline Gibbon & .134 & .210 \\
\hline Macacus & .155 & .180 \\
\hline Baboon & .16 & .240 \\
\hline
\end{tabular}

It is perhaps of even greater significance that the nucleus dentatus in the intermediate primates is in all cases greater than the similar structure in the lower primates. This difference would indicate a definite expansion in cerebellar function in passing from these lower to the higher forms. It points to a probable increment in the coordinative control of the musculature since these animals are becoming more highly specialized in their motor capacity, which gives them a wider range of behavioral adaptation.

The nuclear specialization in the midbrain which receives, as a relay station, the superior cerebellar peduncle, namely, the red nucleus, bears out the fact already observed in relation with the nucleus dentatus. The nucleus ruber is larger in the baboon than in either the gibbon or macacus, thus 
intimating that the relaying structure in the efferent pathway of impulses out of the cerebellum varies with the dentate nucleus from which these impulses arise. The planimetric coefficients of the red nucleus are shown in the appended tabulations:

Planimetric Coefficients of the Red Nucleus in the Intermedite Primates

\begin{tabular}{|lcc|}
\hline & Species & Coeflicient \\
\hline Gibbon & & 051 \\
\hline Nacacus & 05 \\
\hline Baboon & 060 \\
\hline
\end{tabular}

As compared with the similar structure in the lower primates, the red nucleus is in general larger in the intermediate primates, with one exception, namely, Mycetes seniculus, which has a planimetric coefficient greater than in all of the other forms thus far described.

vi. Tile Pontile Nuclel and Their Relation to the Control of Skilled Movements, Especially Complex Manual Performances

A comparison of the pontile nuclei, those structures of the brain to which such importance has been attached as indicative of the animal's capacity for acquiring skilled motor performances, shows that the gibbon is slightly more advanced in the line of this development. This fact does not accord with the observations with reference to the nucleus of Burdach which furnishes the basis for estimating the volume of discriminative influx from the upper extremity and hand. It will be recalled that in this review, the macacus was accredited with a higher degree of manual differentiation than the other intermediate primates. On the other hand, the pontile nuclei seem to indicate that the gibbon is endowed with a high degree of specialization in its skilled movements, and is thus capable of a wider range of behavioral adaptation. It is difficult to reconcile this apparent discrepancy, since the macacus, by 
reason of the structural differentiation in its hand, its mode of life in the trees, its general reaction within its habitat seems to possess a wider range of motor differentiation. It may be that the gibbon is equally endowed in these respects. Being a more or less reelusive animal concerning whose behavior in the free state less is known than of the macacus, it is impossible to say at present that its manual performances are actually inferior to those of the macaque. The figures do not indieate such a condition. Yet it is possible that because of the rather low degree of differentiation in the lower limbs and feet, much more responsibility is imposed upon the forelimbs and hand. This, at least, would explain why the pontile nuclei are large in the gibbon. The division of labor between the hands and the feet in macacus and baboon is nearly equal; in the gibbon there is a marked inequality which gives the forclimb and the hand over-emphasis in their motor responsibility. The coeflicients of the pontile nuclei of the baboon, macaque and gibbon are given in the appended tabulation. While the planimeteric dimensions in gibbon are somewhat larger than in the other species, the estimated mass of the nuclei is nearly equal in them all.

Coefficients of the Pontile Nuclei in the Intermediate Primates

\begin{tabular}{|c|c|c|}
\hline Species & Planimetric & Longitudinal \\
\hline Gibbon & 200 & 260 \\
\hline Macacus & .150 & 340 \\
\hline Baboon & .164 & 310 \\
\hline
\end{tabular}

More significant, however, than the comparison between the several members of the intermediate primate group, is the fact that all of these three species show a definite superiority in their pontile nuclei over the lower primates. This superiority is striking and decisive, leaving no doubt that in 
passing from the lower to the higher group there has been a definite increment in these nuclear structures. The gibbon, for example, as compared with the lemur, shows a pontile nuclear aggregation nearly four times as large. These nuclei in the gibbon are nearly twice as large as they appear in either the marmoset or mycetes.

Whatever diffeulties, therefore, may seem to exist in estimating the relative importance of these nuclei so far as this is revealed by their dimensions in the intermediate group, there can be no doubt of the expansion in the pontile nuclei in passing from the lower to the intermediate primates. This may be accepted as indicative of a progressive functional inerement in manual specialization.

\section{vir. The Midbrain Colliculi in Relation to the functions of Sight AND HEARING}

The comparison of the inferior colliculi of the intermediate primates indicates a further decrease in functional prominence in these auditory relay centers. The inferior colliculus in gibbon is smaller and less well developed than in either the macacus or the baboon. That some of the primordial auditory function is still rested in the inferior colliculus and acts in the interest of immediate reflex adjustment to sudden noises or sounds is probably true. But the sense of hearing in gibbon appears to have undergone such amplifrcation in its associational values that the auditory area of the cerebral cortex has taken supersedence over the more primitive midbrain region originally active in auditory function. Both the macacus and baboon seem to have retained more of the immediate reflex organization based upon the sense of hearing than is the case in gibbon; they seem to depend less than the gibbon upon the transmission of auditory stimuli to the cerebral cortex before determining upon specific courses of action. This structure thus indicates the 
progressive advance which has taken place in telencephalization, a process which is one of the outstanding features in the evolution of the brain. Its consequences are seen in the advancement to the cerebral hemispheres of functions formerly vested in lower primordial portions of the neuraxis. The coefficients of the inferior colliculi are appended in the following tabulation:

Coefficients of the Inferior Colliculi in the Intermediate Primates

\begin{tabular}{|c|c|c|}
\hline Species & Planimetric & Longitudinal \\
\hline Gibbon & 130 & $0^{-0}$ \\
\hline Macacus & $1^{-5}$ & 190 \\
\hline Baboon & 150 & 080 \\
\hline
\end{tabular}

A comparison of the inferior colliculus of the intermediate group with that of the lower primates shows in even more striking manner the recession of this formerly prominent midbrain structure. It bears out the idea of a progressive evolution in the sense of hearing. This is indicated by the steady advance which the neural organization of this special sense has made in transferring its activities from a lower more limited area of the nervous system to a higher and more expansible territory.

The superior colliculus, somewhat less emphatically, gives similar evidence of gradual, progressive delegation of a highly specialized sensory function from lower to higher centers in the brain. The superior colliculus in the gibbon is smaller than either in macacus or baboon. This fact finds substantial confirmation in the external configuration of the occipital lobe where the fissural pattern in gibbon is richer and the convolutions more ample than in the other intermediate primates. The occipital area of the cerebral cortex in gibbon thus appears to be more actively concerned with the visual function than in macacus or baboon. The coefficients of the superior colliculus of macacus, baboon and gibbon are appended in the following tabulation: 
Coefficients of the Stperior Colliculi in the latermediate Primates

\begin{tabular}{|c|c|c|}
\hline Species & Planimetric & Longitudinal \\
\hline Gibbon & .132 & .090 \\
\hline Macacus & .158 & 130 \\
\hline Baboon & .173 & .130 \\
\hline
\end{tabular}

The comparison of the lower primates with those in the intermediate group develops a significant fact, namely, that the superior colliculus in baboon is more prominent, by virtue of its larger size, than in any of the other species. Although not aiming to over-estimate the value of a purely mensurational observation, it seems likely that the baboon, whose life is adapted to the open plains where danger may approach it from all directions and where the safeguarding by the visual as weIl as the auditory lookout becomes correspondingly important, requires a visual mechanism with greater possibilitics for immediate reflex responses. In this way visual impressions from all sides may at once produce such protective attitudes and defense reactions as to guarantee the animal's safety in its more exposed habitat. In the comparison of the lower primates with the intermediate group, the planimetric estimations do not give quite so convineing an account of telencephalization as is the case with the inferior colliculus; nevertheless, the evidence of such a proeess may be clearly diseerned. This portion of the midbrain is to be regarded as one of the important indices disclosing a defnite line of evolutional modification.

\section{vili. The Oculonotor Decussation in Relation to Binocular and Stereoscopic Vision}

This decussation between the oculomotor nuclei on the two sides of the brain forms one of the significant criteria indicative of the progressive adaptation of skilled acts. The connection between these two nuclei becomes 
essential to adapt the ocular muscles to the needs of binocular vision. Among the intermediate primates, the interocular connection is considerably more extensive in baboon than in macacus or gibbon. The explanation of this preeminence in the baboon may be sought in the nature of its habitat and mode of life. Living as it does in open places, its range of vision is consequently much wider and it requires adjustment to varying distances much more than is the case of the tree-dwelling animals whose visual ficlds are naturally circumscribed by the foliage among which they pass their lives. Thus in all probability, responding to the need for rapid adjustment of far and near vision, the baboon has developed a quality of visual perception which in certain respects surpasses that of the other intermediate primates. The longitudinal coefficients of the oculomotor decussation in baboon, macacus and gibbon are given in the appended tabulation:

Longitudinal Coefficients of Oculomotor Decussation in Intermediate Primates

\begin{tabular}{|c|c|}
\hline Species & Coefficient \\
\hline Gibbon & 660 \\
\hline Macacus & -10 \\
\hline Baboon & -90 \\
\hline
\end{tabular}

From these figures it is apparent that, when contrasted with the fower primates, the degree of internuclear connection in the nuclei controlling the eye muscles of the intermediate group is much greater. This denotes a specialization in visual function in passing upward from the lower extremity of this order, in the interest of a higher type of ocular control. It especially introduces those elements of vision which give more exact perception concerning distance, perspective and contour.

Were it possible to summarize all of the progressive specializations which have been noted, it might be said that whatever the variations and differences among the intermediate primates, these species as a group show defunite, even decisive advances in all of the structural details regulating the 
fundamental behavioral reactions. These details include the elements essential to voluntary control of the extremities with especial reference to the hand, the regulation of simultaneous movements of eyes, head and hand, the increments of discriminative sensibility dependent upon manual differentiation, the increase of coordinative control of the musculature, the expansion of the range of skilled movements, particularly manual performances, the telencephalization in control of the functions of sight and hearing, and the progressive development of binocular vision. 




